Dimitri Ara

Georges Maltsiniotis

JOINT ET TRANCHES POUR LES $\infty$-CATÉGORIES STRICTES 


\section{Dimitri Ara}

Aix Marseille Univ, CNRS, Centrale Marseille, I2M, Marseille, France.

E-mail : dimitri.ara@univ-amu.fr

Url : http://www.i2m.univ-amu.fr/perso/dimitri.ara/

Georges Maltsiniotis

Institut de Mathématiques de Jussieu, Université Paris 7 Denis Diderot, Case Postale 7012, Bâtiment Sophie Germain, 75205 Paris Cedex 13, France.

E-mail : georges.maltsiniotis@imj-prg.fr

Url : http://webusers.imj-prg.fr/ georges.maltsiniotis/

Classification mathématique par sujets (2000). — 18A25, 18D05, 18D20, 18G30, 18G35, 18G55, 55U10, 55U15.

Mots clefs. - $\infty$-catégories de Gray, $\infty$-catégories strictes, catégories monoïdales, catégories monoïdales localement bifermées, complexes dirigés augmentés, joint, nerf de Street, orientaux, produit tensoriel de Gray, tranches, transformations lax.

Key words and phrases. - Gray $\infty$-categories, strict $\infty$-categories, monoidal categories, locally biclosed monoidal categories, augmented directed complexes, join, Street's nerve, orientals, Gray tensor product, slices, lax transformations. 


\title{
JOINT ET TRANCHES POUR LES $\infty$-CATÉGORIES STRICTES
}

\author{
Dimitri Ara, Georges Maltsiniotis
}

\begin{abstract}
Résumé. - Le but de cet article est de développer une théorie du joint et des tranches pour les $\infty$-catégories strictes. À deux $\infty$-catégories strictes, on en associe une troisième qu'on appelle leur joint. Cette opération est compatible au joint usuel des catégories à troncation près. On montre que le joint définit une structure de catégorie monoïdale sur la catégorie des $\infty$-catégories strictes et qu'il commute aux limites inductives connexes en chaque variable. En particulier, on obtient l'existence de certains adjoints à droite; ces adjoints définissent des tranches $\infty$-catégoriques, en un sens généralisé. On énonce des conjectures de fonctorialité du joint et des tranches par rapport aux transformations lax et oplax supérieures et on démontre des premiers résultats dans ce sens. Ces résultats sont utilisés dans un autre travail pour établir un théorème A de Quillen $\infty$-catégorique. Enfin, dans un appendice, on revisite le produit tensoriel de Gray $\infty$-catégorique. Un des principaux outils utilisés dans ce travail est la théorie des complexes dirigés augmentés de Steiner.
\end{abstract}

Abstract (Join and slices for strict $\infty$-categories). — The goal of this paper is to develop a theory of join and slices for strict $\infty$-categories. To any pair of strict $\infty$-categories, we associate a third one that we call their join. This operation is compatible with the usual join of categories up to truncation. We show that the join defines a monoidal category structure on the category of strict $\infty$-categories and that it respects connected inductive limits in each variable. In particular, we obtain the existence of some right adjoints; these adjoints define $\infty$-categorical slices, in a generalized sense. We state some conjectures about the functoriality of the join and the slices with respect to higher lax and oplax transformations and we prove some first results in this direction. These results are used in another paper to establish a Quillen Theorem A for strict $\infty$-categories. Finally, in an appendix, we revisit the Gray tensor product of strict $\infty$-categories. One of the main tools used in this paper is Steiner's theory of augmented directed complexes. 



\section{TABLE DES MATIÈRES}

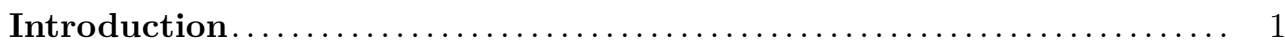

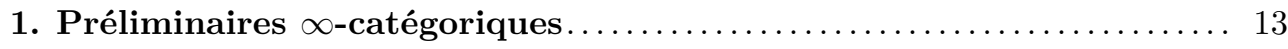

2. Rappels et compléments sur la théorie de Steiner............ 21

3. Limites inductives de complexes de Steiner................. 35

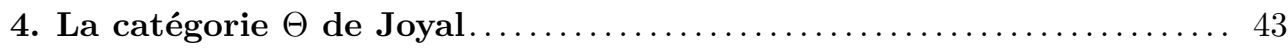

5. Extension de structures de catégorie monoïdale à la Day ......... 49

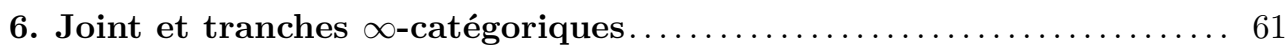

7. Une application : construction du nerf de Street............. 85

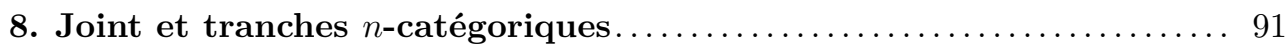

9. Description explicite des tranches au-dessous d'un objet........ 99

10. Fonctorialités des tranches : résultats pour les complexes........111

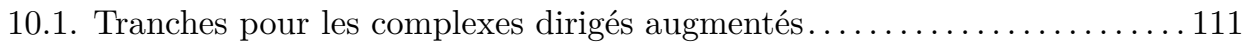

10.2. Morphisme associé à un triangle ............................. 117

10.3. Fonctorialité des morphismes associés aux triangles. . . . . . . . . . . . 121

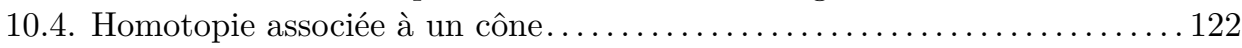

10.5. Fonctorialités des morphismes associés aux cônes............... 124

11. Fonctorialités des tranches : résultats pour les $\infty$-catégories.......131

11.1. Un lemme pour se ramener aux complexes dirigés augmentés . . . . . . 131

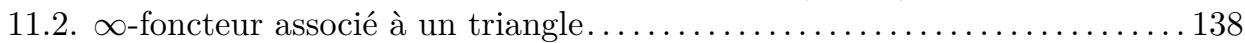

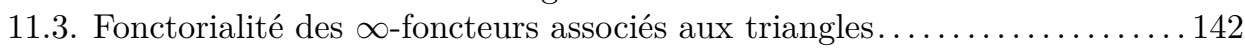

11.4. Transformation oplax associée à un cône..................... 144

11.5. Fonctorialités des transformations oplax associées aux cônes . . . . . . 147 
A. Produit tensoriel $\infty$-catégorique ............................ 153

B. Compléments sur les transformations oplax .................. 167

B.1. Description de la $\infty$-catégorie des cylindres................... 167

B.2. Transformations oplax et produit tensoriel...................... 177

B.3. Composition verticale des transformations oplax................. 182

B.4. Homotopies et transformations oplax .......................... 184

B.5. Joint et transformations oplax................................. 189

B.6. Suspension et transformations oplax......................... 191

C. Fonctorialités des tranches : conjectures..................... 195

Références..................................................213

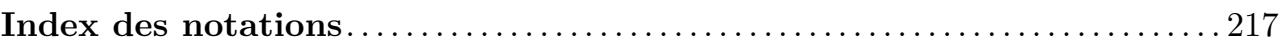

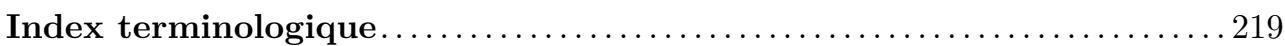




\section{INTRODUCTION}

Ce travail, même s'il en est essentiellement indépendant, est issu d'un projet consacré à la théorie homotopique des $\infty$-catégories strictes, projet constitué actuellement des textes $[\mathbf{5}],[\mathbf{2}],[\mathbf{6}],[\mathbf{7}],[\mathbf{8}],[\mathbf{3}]$ et $[\mathbf{9}]$, ainsi que de l'article [21] de Gagna. Ces travaux sont motivés par le fait que les $\infty$-catégories strictes fournissent des modèles des types d'homotopie plus proches de l'intuition géométrique que ceux fournis par les catégories. Une description détaillée de ce projet et de ses motivations se trouve dans l'introduction de [7]. C'est en travaillant sur un théorème A de Quillen pour les $\infty$-catégories strictes, résultat principal de [7] et [8], que le besoin de définir une théorie du joint et des tranches $\infty$-catégoriques généralisées s'est fait sentir. En effet, non seulement l'énoncé même du théorème A fait intervenir des tranches du type $c \backslash C$, où $c$ est un objet de $C$, mais surtout, sa démonstration, déjà pour les 2-catégories strictes, fait intervenir des tranches de la forme $c \backslash C$, où $c$ est un $n$-simplexe du nerf de $C$ (voir la preuve de [16, théorème 2.16], preuve inspirée des références originales $[\mathbf{1 3}]$ et $[\mathbf{1 4}])$. La motivation initiale du présent travail était de fournir les outils pour définir et étudier ces tranches généralisées pour les $\infty$-catégories en vue d'une démonstration d'un théorème $\mathrm{A} \infty$-catégorique. Néanmoins, les notions de joint et de tranches $\infty$-catégoriques sont, nous semble-t-il, des notions fondamentales de la théorie des $\infty$-catégories strictes dont l'intérêt dépasse largement les applications qui les ont motivées.

Commençons par rappeler la situation pour le joint et les tranches en dimension 1 , c'est-à-dire pour les catégories. Si $A$ et $B$ sont deux catégories, on définit une nouvelle catégorie $A \star B$, appelée le joint de $A$ et $B$, de la manière suivante. Le graphe sousjacent à $A \star B$ est le graphe sous-jacent à la somme disjointe $A \amalg B$ auquel on adjoint une flèche $j_{b, a}$ de $a$ vers $b$ pour tout couple $(a, b)$ formé d'un objet $a$ de $A$ et d'un objet $b$ de $B$. Les identités et la composition sont définies de la manière évidente. On obtient ainsi un foncteur

$$
\begin{aligned}
\mathcal{C} a t \times \mathcal{C} a t & \rightarrow \mathcal{C} a t \\
(A, B) & \mapsto A \star B,
\end{aligned}
$$

où $\mathcal{C} a t$ désigne la catégorie des petites catégories. On vérifie facilement que le joint définit une structure de catégorie monoïdale sur $\mathcal{C} a t$ d'unité la catégorie vide. Cette 
structure n'est pas bifermée mais est localement bifermée au sens suivant : pour toutes petites catégories $A$ et $B$, les foncteurs

$$
\begin{array}{rlrl}
\mathcal{C} a t & \rightarrow A \backslash \mathcal{C} a t & \mathcal{C} a t & \rightarrow B \backslash \mathcal{C} a t \\
B & \mapsto\left(A \star B, \iota_{1}: A \rightarrow A \star B\right)
\end{array} \quad \begin{array}{ll}
A & \mapsto\left(A \star B, \iota_{2}: B \rightarrow A \star B\right),
\end{array}
$$

où

$$
A \stackrel{\iota_{1}}{\longrightarrow} A \star B \stackrel{\iota_{2}}{\longleftarrow} B
$$

désignent les foncteurs canoniques, admettent des adjoints à droite. On obtient ainsi des foncteurs

$$
\begin{aligned}
A \backslash \mathcal{C} a t & \rightarrow \mathcal{C} a t \\
(C, u: A \rightarrow C) & \mapsto u \backslash C
\end{aligned} \text { et } \quad \begin{aligned}
B \backslash \mathcal{C} a t & \rightarrow \mathcal{C} a t \\
(C, v: B \rightarrow C) & \mapsto C / v
\end{aligned}
$$

qu'on appelle respectivement les foncteurs tranches généralisées au-dessous et audessus. Explicitement, si $u: A \rightarrow C$ est un foncteur, les objets de la catégorie $u \backslash C$ sont les cônes inductifs sur le diagramme $u$ et les morphismes sont les morphismes de cônes en un sens évident. De même pour la catégorie $C / v$ et les cônes projectifs. Si $c$ est un objet de $C$, on peut considérer $c$ comme un foncteur $c: e \rightarrow C$, où $e$ désigne la catégorie finale, et la catégorie $c \backslash C$ au sens précédent n'est autre que la tranche usuelle. Ainsi, les tranches $u \backslash C$ sont des tranches généralisées au sens où on considère des tranches au-dessous d'un diagramme quelconque à valeur dans $C$ et non pas seulement d'un objet de $C$.

Ce point de vue sur les tranches ne joue traditionnellement pas un rôle important en théorie des catégories, sans doute parce que les objets en jeu sont simples à décrire explicitement. Néanmoins, le joint et ses deux adjoints sont au cœur de la théorie des quasi-catégories (voir par exemple [24]) et sont un outil précieux pour obtenir la structure de catégorie de modèles de Joyal [25] sur les ensembles simpliciaux.

Le résultat principal de cet article est la généralisation du formalisme du joint et des tranches à la catégorie $\infty$-C at des $\infty$-catégories strictes. On définit un foncteur

$$
\begin{aligned}
\infty-\mathcal{C} a t \times \infty-\mathcal{C} a t & \rightarrow \infty-\mathcal{C} a t \\
(A, B) & \mapsto A \star B
\end{aligned}
$$

qu'on appelle le joint $\infty$-catégorique. Ce foncteur est compatible au joint 1-catégorique au sens suivant : si $A$ et $B$ sont deux catégories vues comme des $\infty$-catégories, alors leur joint $\infty$-catégorique $A \star B$ est une 3-catégorie dont le tronqué 1-catégorique, obtenu en appliquant l'adjoint à gauche du foncteur d'inclusion de $\mathcal{C} a t$ dans $\infty$ - $\mathcal{C}$ at, est le joint 1-catégorique usuel. En effet, le joint $\infty$-catégorique $A \star B$ s'obtient à partir du joint 1-catégorique en ajoutant, pour tout objet $a$ de $A$ et toute flèche $g: b \rightarrow b^{\prime}$ de $B$, une 2-flèche dans le triangle formé de $g, j_{b, a}$ et $j_{b^{\prime}, a}$, pour toute flèche $f: a \rightarrow a^{\prime}$ de $A$ et tout objet $b$ de $B$, une 2 -flèche dans le triangle formé de $f, j_{b, a}$ et $j_{b, a^{\prime}}$ et, pour toute flèche de $A$ et toute flèche de $B$, une 3 -flèche dans le tétraèdre formé des 
triangles du type précédent, et en quotientant par les relations évidentes. Notre joint $\infty$-catégorique est par ailleurs compatible au joint des ensembles simpliciaux (voir par exemple [24, section 3]) au sens où, si $X$ et $Y$ sont deux ensembles simpliciaux et si $c_{\infty}$ désigne l'adjoint à gauche du nerf de Street [36], on a un isomorphisme canonique de $\infty$-catégories $c_{\infty}(X \star Y) \simeq c_{\infty}(X) \star c_{\infty}(Y)$.

On montre que le joint $\infty$-catégorique définit une structure de catégorie monoïdale sur $\infty$-C at d'unité la $\infty$-catégorie vide et que cette structure est localement bifermée. Ainsi, pour toute $\infty$-catégorie $A$ et toute $\infty$-catégorie $B$, les foncteurs

$$
\begin{aligned}
& \infty-\mathcal{C} a t \rightarrow A \backslash \infty-\mathcal{C} a t \\
& B \mapsto\left(A \star B, \iota_{1}: A \rightarrow A \star B\right) \\
& \infty-\mathcal{C} a t \rightarrow B \backslash \infty-\mathcal{C} a t \\
& A \mapsto\left(A \star B, \iota_{2}: B \rightarrow A \star B\right)
\end{aligned}
$$

où

$$
A \stackrel{\iota_{1}}{\longrightarrow} A \star B \stackrel{\iota_{2}}{\longleftarrow} B
$$

désignent des $\infty$-foncteurs canoniques, admettent des adjoints à droite. On obtient ainsi des foncteurs

$$
\begin{aligned}
& A \backslash \infty-\mathcal{C} a t \rightarrow \infty-\mathcal{C} a t \\
& (C, u: A \rightarrow C) \mapsto u \backslash C \\
& \text { et } \\
& B \backslash \infty-\mathcal{C} a t \rightarrow \infty-\mathcal{C} a t \\
& (C, v: B \rightarrow C) \mapsto C / v \text {. }
\end{aligned}
$$

Le premier foncteur définit les tranches $\infty$-catégoriques généralisées au-dessous. Si c est un objet de $C$, on peut considérer $c$ comme un $\infty$-foncteur $c: e \rightarrow C$, où $e$ désigne la $\infty$-catégorie finale, et on obtient une tranche $c \backslash C$ de $C$ au-dessous de l'objet $c$. Dans ce cas particulier, on décrit explicitement la $\infty$-catégorie $c \backslash C$ et on retrouve les formules connues décrivant les cônes $n$-catégoriques (voir [30] pour le cas, plus général, des cylindres).

Nous avons décidé de réserver la notation $C / v$, ainsi que la terminologie « tranches au-dessus », à une variante de la $\infty$-catégorie $C / v$. La catégorie $\infty$ - $\mathcal{C} a t$ possède un automorphisme remarquable qui envoie une $\infty$-catégorie $C$ sur la $\infty$-catégorie $C^{\circ}$ obtenue en renversant le sens des $i$-flèches de $C$ pour tout $i>0$. Le joint n'est pas compatible à cet automorphisme et on obtient un foncteur

$$
\begin{aligned}
\infty \text { - Cat } \times \infty \text { - } \text { a } a t & \rightarrow \infty-\mathcal{C} a t \\
(A, B) & \mapsto\left(B^{\circ} \star A^{\circ}\right)^{\circ}
\end{aligned}
$$

qui définit une seconde structure de catégorie monoïdale sur la catégorie des $\infty$-catégories. Ce foncteur permet d'obtenir comme ci-dessus des foncteurs

$$
\begin{aligned}
& A \backslash \infty-\mathcal{C} a t \rightarrow \infty-\mathcal{C} a t \\
& (C, u: A \rightarrow C) \mapsto u \backslash C \quad \text { co } \quad \text { et } \quad(C, v: B \rightarrow C) \mapsto C / v \quad \text {. }
\end{aligned}
$$

C'est ce second foncteur qui définit les tranches $\infty$-catégoriques généralisées au-dessus. 
Le choix de privilégier les $\infty$-catégories $u \backslash C$ et $C / v$ par rapport aux $\infty$-catégories $u \backslash C$ et $C / v$ est motivé par le fait que les premières admettent une description beaucoup plus simples que les dernières. Par ailleurs, on a des isomorphismes canoniques

$$
C / v \simeq\left(v^{\circ} \backslash C^{\circ}\right)^{\circ} \quad \text { et } \quad \stackrel{\text { co }}{u \backslash C} \simeq\left(C^{\circ} \stackrel{\text { co }}{/} u^{\circ}\right)^{\circ} .
$$

Si $A$ et $B$ sont deux $n$-catégories strictes, pour un $n \geqslant 0$, leur joint est une $(2 n+1)$-catégorie stricte. En tronquant cette $(2 n+1)$-catégorie en dimension $n$ (c'est-à-dire en appliquant l'adjoint à gauche du foncteur d'inclusion), on obtient une $n$-catégorie $A \star_{n} B$ qu'on appelle leur joint $n$-catégorique. On montre qu'on définit ainsi une structure de catégorie monoïdale sur $n$-C at, la catégorie des $n$-catégories strictes, et que cette structure est localement bifermée comme ci-dessus. Lorsque $n=1$, on retrouve la structure de catégorie monoïdale définie par le joint 1-catégorique usuel.

Pour construire ce joint $\infty$-catégorique, on adopte une stratégie inspirée, d'une part, d'une esquisse de construction du produit tensoriel de Gray $\infty$-catégorique donnée par Street dans [40, section 9] et, d'autre part, d'idées de Steiner pour construire ce même produit tensoriel basées sur sa théorie des complexes dirigés augmentés [32].

La théorie de Steiner associe à tout complexe dirigé augmenté, c'est-à-dire à tout complexe de chaînes de groupes abéliens en degrés positifs augmenté et muni en chaque degré d'un sous-monoïde des chaînes, une $\infty$-catégorie stricte. Un des résultats importants de [32] donne des conditions suffisantes pour que la $\infty$-catégorie ainsi associée soit libre au sens des polygraphes. La théorie de Steiner permet ainsi de décrire en termes de complexes de chaînes une sous-catégorie pleine de $\infty$ - $\mathcal{C}$ at, que nous appellerons la catégorie des $\infty$-catégories de Steiner fortes, qui contient notamment la catégorie $\Theta$ de Joyal [23], les orientaux de Street [36] et les cubes $n$-catégoriques. (Une théorie alternative permettant de décrire ces $\infty$-catégories de manière combinatoire est la théorie des complexes de parité de Street $[\mathbf{3 7}, \mathbf{3 8}]$.)

Afin de construire le joint $\infty$-catégorique, on commence par décrire, en termes de produit tensoriel de complexes de chaînes, le joint de deux complexes dirigés augmentés, s'inspirant d'une construction analogue due à Street [37] dans le cadre des complexes de parité. On obtient alors une structure de catégorie monoïdale sur la catégorie des complexes dirigés augmentés. On montre que cette structure induit une structure de catégorie monoïdale sur la catégorie des $\infty$-catégories de Steiner fortes. Il s'agit ensuite d'étendre cette structure à la catégorie de toutes les $\infty$-catégories strictes. Pour cela, suivant la stratégie de Street pour définir le produit tensoriel de Gray [40, section 9], on utilise un théorème d'extension de structures de catégorie monoïdale à la Day $[\mathbf{1 9}, \mathbf{2 0}]$. Notre structure de catégorie monoïdale n'étant pas bifermée mais seulement localement bifermée, les résultats de Day ne s'appliquent pas tels quels. On est donc conduit à généraliser un théorème de Day au cas localement bifermé. Enfin, la partie la plus délicate de la construction du joint $\infty$-catégorique 
est la vérification des hypothèses de ce théorème à la Day. Il s'agit essentiellement de construire à la main les tranches $u \backslash C$ et $C \stackrel{\text { co }}{v}$ dans le cas où les sources de $u$ et $v$ sont des $\infty$-catégories de Steiner fortes, et de vérifier que ces tranches ont les propriétés universelles attendues.

Dans un appendice, on construit le produit tensoriel de Gray $\infty$-catégorique en suivant une stratégie analogue. Rappelons que le produit tensoriel de Gray $\infty$-catégorique est une généralisation $\infty$-catégorique du produit tensoriel introduit par Gray dans [22] sur la catégorie des 2-catégories strictes. Ce produit définit une structure de catégorie monoïdale bifermée sur $\infty$-C at. Le produit tensoriel de Gray $\infty$-catégorique a été construit pour la première fois par Al-Agl et Steiner [1] , généralisant une construction analogue pour les $\infty$-groupoïdes due à Brown et Higgins [12]. Deux constructions alternatives sont données par Crans dans sa thèse [18]. Dans [32], Steiner propose une nouvelle construction qui a l'avantage d'être relativement explicite. Néanmoins, la preuve de [32] est incomplète. En effet, dans la preuve de son théorème 7.3, Steiner affirme qu'il est évident que son produit tensoriel commute aux limites inductives, sous-entendant que cette commutation est formelle, ce qui n'est pas le cas. Steiner nous a cependant affirmé savoir compléter cette preuve, esquissant un argument [35]. Dans l'appendice A, on propose une démonstration alternative en adoptant la stratégie qu'on a utilisé pour construire le joint. En particulier, on utilise de manière cruciale un théorème de Day et, comme dans le cas du joint, la partie la plus délicate du travail consiste à vérifier que les hypothèses du théorème de Day sont satisfaites.

On formule de très générales conjectures de fonctorialité du joint et des tranches. Pour cela, on introduit la notion de $\infty$-catégorie de Gray, catégorie enrichie dans la catégorie des $\infty$-catégories strictes munie du produit tensoriel de Gray. (On définit également au passage la notion de $\infty$-sesquicatégorie.) La $\infty$-catégorie de Gray para-

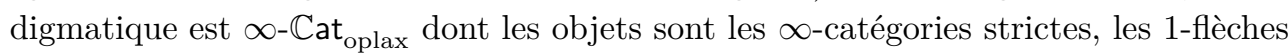
les $\infty$-foncteurs stricts, les 2-flèches les transformations oplax et les flèches supérieures les transformations oplax supérieures. De même, on introduit la notion de catégorie de Gray gauche, catégorie enrichie dans la catégorie des $\infty$-catégories strictes munie du produit tensoriel $(C, D) \mapsto D \otimes C$ obtenu en transposant celui de Gray. La $\infty$-catégorie de Gray gauche paradigmatique est $\infty$ - $\mathbb{C a t}_{\text {lax }}$ obtenue en utilisant cette fois les transformations lax. On conjecture l'existence de tranches pour les $\infty$-catégories de Gray et les $\infty$-catégories de Gray gauches au-dessus ou au-dessous d'un objet. En particulier, si $C$ désigne une $\infty$-catégorie stricte, on disposerait de $\infty$-catégories de Gray

$$
C \backslash \infty-\mathbb{C a t}_{\text {oplax }} \text { et } \infty-\mathbb{C a t}_{\text {oplax }} / C
$$

et de $\infty$-catégories de Gray gauches

$$
C \backslash \infty-\mathbb{C a t}_{\mathrm{lax}} \text { et } \infty-\mathbb{C a t}_{\mathrm{lax}} / \mathrm{co} \text {. }
$$


On conjecture que les foncteurs $\bullet \star C$ et $C \star \bullet$ proviennent de $\infty$-foncteurs de Gray et de Gray gauches (c'est-à-dire des foncteurs enrichis) respectivement

$$
\begin{aligned}
& \bullet \star C: \infty-\mathbb{C a t}_{\text {oplax }} \rightarrow C \backslash \infty-\mathbb{C a t}_{\text {oplax }} \\
& C \star \bullet: \infty-\mathbb{C a t}_{\text {lax }} \rightarrow C \backslash \infty-\mathbb{C a t}_{\text {lax }}
\end{aligned}
$$

et que l'association $(A, A \stackrel{u}{\rightarrow} C) \mapsto u \backslash C$, selon qu'on la considère comme un foncteur en $C$ ou en $A$, provient de $\infty$-foncteurs de Gray gauches et de Gray respectivement

$$
\begin{gathered}
A \backslash \infty-\mathbb{C a t}_{\text {lax }} \rightarrow \infty-\mathbb{C a t}_{\text {lax }} \\
\left(\infty-\mathbb{C a t}_{\text {lax }}{ }^{\text {co }} C\right)^{\circ} \rightarrow \infty-\mathbb{C a t}_{\text {oplax }},
\end{gathered}
$$

où ${ }^{\circ}$ est une dualité transformant une $\infty$-catégorie de Gray gauche en une $\infty$-catégorie de Gray (et réciproquement). Dans ce texte, on s'intéresse spécifiquement à la dernière conjecture, c'est-à-dire au $\infty$-foncteur de Gray $\left(\infty-\mathbb{C a t}_{\text {lax }} / C\right)^{\circ} \rightarrow \infty$-Cat ${ }_{\text {oplax }}$, car c'est celui-ci qui nous permet de démontrer un théorème $\mathrm{A} \infty$-catégorique dans [8]. Explicitons en petite dimension à quoi correspond l'action d'un tel $\infty$-foncteur sur les cellules :

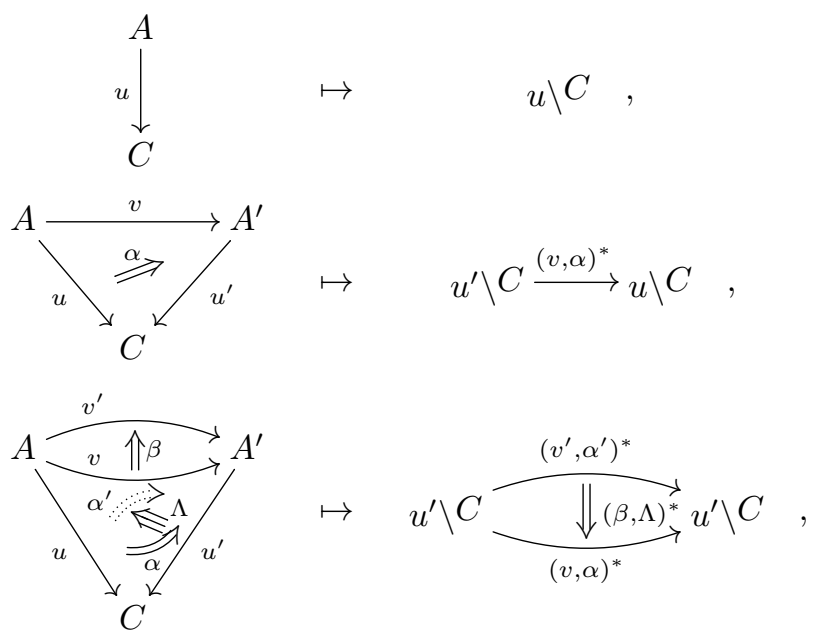

où les 2-flèches et 3-flèches à gauche du signe « $\mapsto »$ sont des transformations lax et des 2-transformations lax respectivement, et la 2-flèche à droite de ce signe est une transformation oplax.

Ces conjectures sont étayées par le fait que les conjectures analogues pour les complexes dirigés augmentés (vérifiant une hypothèse anodine) sont vraies. Dans ce texte, nous démontrons ces analogues en petites dimensions. Plus précisément, nous montrons qu'on peut définir une correspondance comme dans l'illustration ci-dessus en remplaçant les $\infty$-catégories par des complexes dirigés augmentés, les $\infty$-foncteurs par des morphismes de complexes dirigés augmentés, les transformations oplax par 
des homotopies et les transformations lax par des antihomotopies, notion duale à celle d'homotopie. On montre par ailleurs la sesquifonctorialité de cette correspondance.

Un des résultats principaux de cet article est l'extension de cette correspondance en basse dimension aux $\infty$-catégories strictes, malheureusement sous des hypothèses restrictives (mais suffisantes pour obtenir notre théorème $\mathrm{A} \infty$-catégorique dans [8]). On montre qu'on peut définir une correspondance comme dans l'illustration ci-dessus si on se restreint aux diagrammes obtenus en composant un diagramme comme dans l'illustration, où $A, A^{\prime}$ et $C$ sont des $\infty$-catégories de Steiner fortes et $u^{\prime}$ est un monomorphisme vérifiant une hypothèse technique, par un $\infty$-foncteur quelconque $C \rightarrow C^{\prime}$ (en particulier, on ne demande pas que $C^{\prime}$ soit une $\infty$-catégorie de Steiner forte). On montre que cette correspondance est sesquifonctorielle.

Ces résultats, dans le cas des complexes dirigés augmentés, sont obtenus en produisant des formules explicites et par le calcul. Cette approche s'adapte a priori mal au cas des $\infty$-catégories mais nous montrons qu'on peut déduire le cas des $\infty$-catégories de celui des complexes dirigés augmentés. Pour ce faire, on utilise, d'une part, la densité de la sous-catégorie pleine de $\infty$-Cat formée des $\infty$-catégories de Steiner fortes et, d'autre part, un résultat, démontré dans ce texte, de stabilité des $\infty$-catégories de Steiner fortes par certaines sommes amalgamées. C'est des hypothèses de ce dernier résultat que provient la condition restrictive sur $u^{\prime}$.

Après que nous avons rendu publique une première version de ce texte, Dominic Verity nous a signalé que ses travaux sur les ensembles compliciaux permettraient également de définir un joint $\infty$-catégorique. Dans [42, section 3], Verity définit un joint des ensembles stratifiés, ensembles simpliciaux munis de la donnée supplémentaire de simplexes distingués dits fins. Il nous a affirmé qu'on pourrait montrer, en utilisant un théorème de Day, que ce joint induit un joint sur la sous-catégorie réflexive formée des ensembles compliciaux. Or, en vertu de la conjecture de Street-Roberts qu'il a démontrée [41], les ensembles compliciaux sont équivalents aux $\infty$-catégories strictes. Il est probable que ce joint $\infty$-catégorique serait isomorphe au nôtre, même si la comparaison ne semble pas triviale.

Organisation de l'article. - Le premier chapitre est consacré aux préliminaires sur les $\infty$-catégories strictes. On fixe nos notations et conventions et on rappelle quelques constructions classiques. On définit la notion standard de $\infty$-catégorie stricte engendrée librement au sens des polygraphes et on dégage un résultat de commutation aux limites inductives de ces $\infty$-catégories qui jouera un rôle important dans la suite du texte. On définit la notion de $\infty$-transformation oplax en donnant des formules explicites et on étudie quelques moyens de composer ces $\infty$-transformations oplax.

Dans le deuxième chapitre, on rappelle la théorie des complexes dirigés augmentés de Steiner et on y apporte quelques compléments. On définit les foncteurs $\lambda$ et $\nu$ de Steiner qui forment un couple de foncteurs adjoints entre la catégorie des $\infty$-catégories 
strictes et la catégorie des complexes dirigés augmentés. On introduit les termes « complexe de Steiner » et « complexe de Steiner fort » pour désigner ce que Steiner appelle les complexes dirigés augmentés à base unitaire sans boucle ou fortement sans boucle. On rappelle les deux résultats fondamentaux de la théorie de Steiner : le foncteur $\nu$ restreint aux complexes de Steiner est, d'une part, pleinement fidèle et, d'autre part, à valeurs dans la catégorie des $\infty$-catégories libres au sens des polygraphes. On apporte ensuite les compléments suivants. On définit la notion de complexe dirigé augmenté décent, notion peut-être plus pertinente que celle de complexe dirigé augmenté. On définit des endofoncteurs de dualité de la catégorie des complexes dirigés augmentés et des notions de troncations pour ces complexes. On étudie les compatibilités entre ces constructions et les foncteurs $\lambda$ et $\nu$ de Steiner. Enfin, on introduit les notions d'homotopie et d'antihomotopie entre morphismes de complexes dirigés augmentés et, plus généralement, celles de $n$-homotopie et de $n$-antihomotopie, et on décrit quelques opérations sur ces objets.

Dans le troisième chapitre, on décrit les limites inductives de complexes de Steiner et on étudie la commutation du foncteur $\nu$ à celles-ci. On introduit la notion de système de Steiner et on montre que le foncteur $\nu$ commute aux limites inductives de ces systèmes. On définit la notion d'inclusion rigide ordonnée entre complexes de Steiner et on montre qu'une somme amalgamée de complexes de Steiner forts faisant intervenir des inclusions rigides ordonnées forme un système de Steiner. Le premier résultat joue un rôle important dans la construction même du joint et le second dans les propriétés de fonctorialité du joint.

Le quatrième chapitre est consacré à la catégorie $\Theta$ de Joyal. On la définit comme une sous-catégorie pleine de la catégorie des $\infty$-catégories strictes en utilisant la notion de sommes globulaires. On indique comment les préfaisceaux sur $\Theta$ compatibles à ces sommes globulaires induisent des $\infty$-catégories. On étudie la restriction du foncteur $\lambda$ de Steiner à $\Theta$. On montre que $\Theta$ est dans l'image du foncteur $\nu$ de Steiner en utilisant les résultats du chapitre précédent (ce résultat était déjà connu de Steiner [34]).

Dans le cinquième chapitre, on s'intéresse à la question suivante : pour définir une structure de catégorie monoïdale sur une catégorie, sous quelles conditions suffit-il de le faire sur une sous-catégorie dense? Un théorème de Day répond à cette question pour les catégories monoïdales bifermées. On généralise ce résultat à ce qu'on appelle les catégories monoïdales localement bifermées qui, si la catégorie sous-jacente est localement présentable, sont les structures pour lesquelles le produit tensoriel commute aux limites inductives connexes en chaque variable. C'est ce résultat qu'on utilisera dans le chapitre suivant pour construire le joint.

Le sixième chapitre est le chapitre central de l'article. C'est dans celui-ci qu'on construit le foncteur joint et les tranches généralisées. On commence par des rappels sur la structure de catégorie monoïdale bifermée sur la catégorie des complexes dirigés augmentés définie par le produit tensoriel. On en déduit, grâce à un jeu de 
suspensions déjà présent sous une autre forme dans [37], une nouvelle structure de catégorie monoïdale sur cette même catégorie; on appelle le produit de cette structure le joint. On montre que cette structure est localement bifermée et qu'elle induit une structure de catégorie monoïdale sur la catégorie des complexes de Steiner forts. On transporte cette structure sur la sous-catégorie pleine de $\infty$-Cat formée des $\infty$-catégories de Steiner fortes et on vérifie que les hypothèses de notre théorème à la Day dégagé dans le chapitre précédent sont satisfaites; c'est le contenu de la proposition 6.27, proposition clé de ce chapitre. On en déduit l'existence d'une structure de catégorie monoïdale localement bifermée sur la catégorie des $\infty$-catégories strictes. On obtient ainsi un foncteur joint et des foncteurs tranches. On finit le chapitre par une étude des compatibilités du joint et des tranches avec les dualités de la catégorie des $\infty$-catégories strictes.

Dans le septième chapitre, on montre comment on peut définir les orientaux de Street et le nerf de Street formellement à partir des propriétés de notre joint $\infty$-catégorique, idée apparaissant déjà dans [37]. Plus précisément, on montre que la $\infty$-catégorie finale est munie d'une unique structure de monoïde pour la structure de catégorie monoïdale définie par le joint et que l'objet cosimplicial associé à ce monoïde est l'objet cosimplicial de Street. On démontre par ailleurs que l'adjoint à gauche du nerf de Street est un foncteur monoïdal pour le joint simplicial et le joint $\infty$-catégorique.

Dans le huitième chapitre, on montre que la structure de catégorie monoïdale sur la catégorie des $\infty$-catégories strictes définie par le joint induit par troncation une structure de catégories monoïdales sur la catégorie des $n$-catégories strictes pour tout $n \geqslant 0$. On obtient de plus que cette structure est localement bifermée. On vérifie que pour $n=1$ on retrouve le joint et les tranches 1-catégoriques usuels.

Le but du neuvième chapitre est de décrire par des formules explicites les tranches au-dessous d'un objet qui ont été définies abstraitement dans le sixième chapitre. En particulier, pour $n=2$, on retrouve les formules définissant les tranches qui apparaissent déjà dans la littérature.

Le dixième chapitre est consacré aux tranches des complexes dirigés augmentés et à leurs propriétés de fonctorialité. On définit explicitement des tranches généralisées pour les complexes dirigés augmentés et on montre que celles-ci ont la propriété universelle attendue. On étudie des propriétés de fonctorialité de l'opération tranche. On montre qu'à tout triangle commutatif à une antihomotopie près, on peut associer un morphisme entre les tranches associées et qu'à tout cône commutatif à une 2-antihomotopie près, on peut associer une homotopie entre les morphismes entre les tranches associées. On étudie les compatibilités de ces constructions aux différentes compositions.

Le onzième chapitre contient d'importants résultats de fonctorialité des tranches $\infty$-catégoriques, analogues $\infty$-catégoriques des résultats du chapitre précédent. On 
montre qu'à tout triangle de $\infty$-foncteurs commutatif à une transformation lax près se factorisant par un triangle de complexes de Steiner forts (et satisfaisant une hypothèse technique), on peut associer un $\infty$-foncteur entre les tranches $\infty$-catégoriques associées. De même, on montre qu'à tout cône satisfaisant une condition analogue on peut associer une transformation oplax. Enfin, on étudie les compatibilités de ces constructions aux différentes compositions. Tous ces résultats sont obtenus à partir des résultats analogues pour les complexes dirigés augmentés. Cette réduction au cas des complexes dirigés augmentés utilise de manière cruciale les résultats sur les sommes amalgamées de complexes de Steiner forts établis dans le troisième chapitre.

L'appendice A est consacré au produit tensoriel de Gray $\infty$-catégorique. On applique les techniques utilisées précédemment pour le joint pour construire le produit tensoriel de Gray et montrer qu'il définit une structure de catégorie monoïdale bifermée sur la catégorie des $\infty$-catégories strictes. On définit les $\infty$-catégories strictes des foncteurs lax et oplax comme Hom interne à droite et à gauche. On discute les compatibilités du produit tensoriel et de ces deux Hom internes avec les dualités de la catégorie des $\infty$-catégories strictes. On montre que, comme dans le cas du joint, le produit tensoriel induit par troncation une structure de catégorie monoïdale bifermée sur la catégorie des $n$-catégories strictes pour tout $n \geqslant 0$.

Le but de l'appendice B est de montrer l'équivalence entre la définition concrète des $\infty$-foncteurs oplax donnée dans le premier chapitre et la notion abstraite définie en termes du produit tensoriel $\infty$-catégorique. Ceci nous permet de définir la composition verticale des transformations oplax sans donner explicitement de formules. On utilise par ailleurs cette équivalence pour associer à toute homotopie de complexes dirigés augmentés une transformation oplax de $\infty$-catégories et on étudie les propriétés de cette correspondance. Pour finir, on étudie le lien entre les $\infty$-catégories $\mathrm{D}_{1} \otimes C, \mathrm{D}_{0} \star C$ et $\Sigma C$, où $\mathrm{D}_{0}$ et $\mathrm{D}_{1}$ désignent les $\infty$-catégories associées aux ensembles ordonnés $\{0\}$ et $\{0<1\}$, et $\Sigma$ désigne la suspension, introduite dans cet appendice.

Enfin, dans l'appendice C, on dégage des conjectures de fonctorialités du joint et des tranches dont les résultats du douzième chapitre sont des cas particuliers. On introduit la notion de $\mathcal{V}$-sesquicatégorie, où $\mathcal{V}$ est une catégorie munie d'un foncteur vers la catégorie des ensembles. Dans le cas où $\mathcal{V}$ est la catégorie $\infty$ - $\mathcal{C}$ at des $\infty$-catégories strictes munie du foncteur ensemble des objets, on obtient la notion de $\infty$-sesquicatégorie. On introduit par ailleurs les notions de $\infty$-catégorie de Gray et de $\infty$-catégorie de Gray gauche, $\infty$-catégories enrichies dans $\infty$-C at munie respectivement du produit tensoriel de Gray et du transposé de ce produit tensoriel. On donne

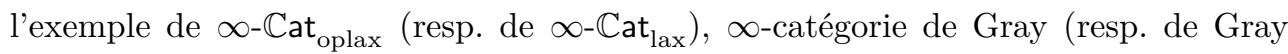
gauche) dont les objets sont les $\infty$-catégories strictes, les 1-flèches les $\infty$-foncteurs stricts et les $i$-flèches pour $i>1$ les $(i-1)$-transformations oplax (resp. lax). On conjecture l'existence d'une $\infty$-catégorie de Gray (resp. de Gray gauche) tranche audessus ou au-dessous d'un objet d'une $\infty$-catégorie de Gray (resp. de Gray gauche). On 
conjecture que les foncteurs joint et tranches $\infty$-catégoriques, vus comme foncteurs en une de leurs deux variables, proviennent de $\infty$-foncteurs de Gray ou de Gray gauches (c'est-à-dire de foncteurs enrichis) entre des tranches de $\infty$-Cat ${ }_{\text {oplax }}$ et $\infty$-Cat lax $_{\text {lax }}$.

Remerciements. - Les auteurs remercient Andrea Gagna pour les nombreuses coquilles qu'il a débusquées dans une version préliminaire de ce texte, ainsi que pour l'exemple 3.4 qui lui est dû et qui montre que la première rédaction du troisième chapitre était légèrement incorrecte.

Notations et terminologie. - Si $\mathcal{C}$ est une catégorie, on notera $\mathrm{Ob}(\mathcal{C})$ la classe de ses objets. La catégorie opposée à $\mathcal{C}$ sera notée $\mathcal{C}^{\circ}$. Si $\mathcal{D}$ est une seconde catégorie, la catégorie des foncteurs de $\mathcal{C}$ vers $\mathcal{D}$ sera notée $\operatorname{Hom}(\mathcal{C}, \mathcal{D})$. On notera $\mathcal{E}$ ns la catégorie des ensembles et $\mathcal{A} b$ la catégorie des groupes abéliens. Si $A$ est une petite catégorie, on notera $\widehat{A}$ la catégorie des préfaisceaux sur $A$, c'est-à-dire la catégorie $\underline{\operatorname{Hom}}\left(A^{\circ}, \mathcal{E} n s\right)$ des foncteurs de $A^{\circ}$ vers la catégorie des ensembles.

On dira qu'une catégorie est connexe si elle 0-connexe. Autrement dit, on ne considérera pas la catégorie vide comme une catégorie connexe. Ainsi, lorsqu'on parlera de limites inductives connexes, on supposera que la catégorie source du système inductif est non vide. 



\section{CHAPITRE 1}

\section{PRÉLIMINAIRES $\infty$-CATÉGORIQUES}

1.1. - On notera $\infty$-Cat la catégorie des petites $\infty$-catégories strictes et des $\infty$-foncteurs stricts entre celles-ci. Toutes les $\infty$-catégories et tous les $\infty$-foncteurs considérés dans ce texte seront stricts et on se permettra donc d'omettre l'adjectif « strict ». Sauf mention expresse du contraire, ces $\infty$-catégories seront de plus supposées petites. Si $C$ est une $\infty$-catégorie, pour $i \geqslant 0$, on notera $C_{i}$ l'ensemble de ses $i$-flèches ou $i$-cellules. Si $x$ est une $i$-flèche pour $i>0$, on notera respectivement $s(x)$ et $t(x)$ les $(i-1)$-flèches source et but de $x$. Plus généralement, pour $j$ tel que $0 \leqslant j \leqslant i$, on notera $s_{j}(x)$ et $t_{j}(x)$ la $j$-source et le $j$-but de $x$, c'est-à-dire les $j$-flèches source et but itérés de $x$ en dimension $j$. On dira que deux $i$-flèches $x$ et $y$ sont $j$-composables si on a $s_{j}(x)=t_{j}(y)$. Si $x$ et $y$ sont $j$-composables, on notera $x *_{j} y$ leur composé qu'on appellera parfois $j$-composé. Si $x$ est une $i$-flèche de $C$, on notera $1_{x}$ la $(i+1)$-flèche identité de $x$. On dira parfois qu'une $(i+1)$-flèche de la forme $1_{x}$, pour $x$ une $i$-flèche, est triviale.

Afin de simplifier certaines formules, on adoptera les conventions suivantes. Pour tous $i, j>k \geqslant 0$, si $x$ est une $i$-flèche de $C, y$ une $j$-flèche de $C$ et qu'on a l'égalité $s_{k}(x)=t_{k}(y)$, on notera $x *_{k} y$ la $l$-flèche, où $l$ est le maximum de $\{i, j\}$, définie par

$$
x *_{k} y= \begin{cases}1_{x} *_{k} y & \text { si } i \leqslant j, \\ x *_{k} 1_{y} & \text { si } i \geqslant j,\end{cases}
$$

où $1_{z}$, pour $z=x, y$, désigne l'identité itérée de $z$ en dimension $l$. Par ailleurs, si $i<j$, on considérera que l'opération $*_{i}$ est prioritaire sur l'opération $*_{j}$ de sorte qu'on a

$$
x *_{i} y *_{j} z=\left(x *_{i} y\right) *_{j} z \quad \text { et } \quad x *_{j} y *_{i} z=x *_{j}\left(y *_{i} z\right),
$$

lorsque ces formules ont un sens. 
1.2. - Soit $n \geqslant 0$. On considérera une $n$-catégorie $C$ comme une $\infty$-catégorie dont les ensembles de $j$-flèches $C_{j}$ pour $j>n$ ne contiennent que des cellules triviales. On notera $n$-C at la sous-catégorie pleine de $\infty$-C at formée des $n$-catégories.

Le foncteur d'inclusion $n$-C at $\hookrightarrow \infty$-C at des $n$-catégories dans les $\infty$-catégories admet un adjoint à gauche et un adjoint à droite qu'on notera respectivement

$$
\tau_{\leqslant n}^{\mathrm{i}}: \infty-\mathcal{C} a t \rightarrow n-\mathcal{C} a t \quad \text { et } \quad \tau_{\leqslant n}^{\mathrm{b}}: \infty-\mathcal{C} a t \rightarrow n-\mathcal{C} a t .
$$

On appellera $\tau_{\leqslant n}^{\mathrm{i}}$ le foncteur $n$-tronqué intelligent et $\tau_{\leqslant n}^{\mathrm{b}}$ le foncteur $n$-tronqué bête. Explicitement, si $C$ est une $\infty$-catégorie, on a

$$
\tau_{\leqslant n}^{\mathrm{i}}(C)_{i}= \begin{cases}C_{i} & \text { si } i<n, \\ C_{n} / \sim & \text { si } i \geqslant n,\end{cases}
$$

où $\sim$ est la relation d'équivalence sur $C_{n}$ définie par $« x \sim y$ s'il existe un zigzag de $(n+1)$-flèches de $C$ entre $x$ et $y »$, et

$$
\tau_{\leqslant n}^{\mathrm{b}}(C)_{i}= \begin{cases}C_{i} & \text { si } i<n, \\ C_{n} & \text { si } i \geqslant n,\end{cases}
$$

les compositions et identités étant héritées de celles de $C$ de la manière évidente.

On identifiera souvent $\tau_{\leqslant n}^{\mathrm{b}}(C)$ à une sous- $\infty$-catégorie de $C$ via le morphisme d'adjonction $\tau_{\leqslant n}^{\mathrm{b}}(C) \hookrightarrow C$.

Par ailleurs, le foncteur $\tau_{\leqslant n}^{\mathrm{b}}$ admet lui-même un adjoint à droite : le foncteur qui associe à une $n$-catégorie $C$ la $(n+1)$-catégorie dont le $n$-tronqué bête est $C$ et telle que pour toutes $n$-flèches $x$ et $y$, il existe exactement une $(n+1)$-flèche de $x$ vers $y$. En particulier, le foncteur $\tau_{\leqslant n}^{\mathrm{b}}$ commute aussi bien aux limites projectives qu'aux limites inductives.

1.3. - Soit $C$ une $\infty$-catégorie. Un ensemble multiplicatif de cellules de $C$ est un ensemble $M$ de cellules de $C$ satisfaisant aux deux conditions suivantes :

(a) pour tout $i \geqslant 0$ et toute $i$-flèche $x \operatorname{de} C$, si $x$ appartient à $M$, alors la $(i+1)$-flèche $1_{x}$ appartient à $M$;

(b) pour tous $i>j \geqslant 0$ et tout couple $x, y$ de $i$-flèches $j$-composables de $C$, si $x$ et $y$ appartiennent à $M$, alors la $i$-flèche composée $x *_{j} y$ appartient à $M$.

On dit qu'un ensemble $E$ de cellules d'une $\infty$-catégorie $C$ engendre $C$ par compositions si l'ensemble de toutes les cellules de $C$ est le plus petit ensemble multiplicatif de cellules de $C$ contenant $E$. Dans ce cas, $E$ contient nécessairement l'ensemble des objets de $C$ et, pour tout $i>0$, toute $i$-flèche de $C$ est un composé d'un nombre fini de $i$-flèches qui sont dans $E$ ou sont des identités itérées de cellules appartenant à $E$.

1.4. - Soient $C$ une $\infty$-catégorie et $E$ un ensemble de cellules de $C$. Pour $i \geqslant 0$, posons $E_{i}=E \cap C_{i}$. On dira que $E$ engendre librement $C$ au sens des polygraphes si

(a) $E_{0}=C_{0}$; 
(b) pour tout $i \geqslant 0$, toute $\infty$-catégorie $D$, tout $\infty$-foncteur $u: \tau_{\leqslant i}^{\mathrm{b}}(C) \rightarrow D$ et toute application $f: E_{i+1} \rightarrow D_{i+1}$ tels que, pour tout $x$ dans $E_{i+1}$, on ait

$$
s(f(x))=u(s(x)) \quad \text { et } \quad t(f(x))=u(t(x)),
$$

il existe un unique $\infty$-foncteur $u^{\prime}: \tau_{\leqslant i+1}^{\mathrm{b}}(C) \rightarrow D$ tel que

$$
u_{\mid \tau_{\leqslant i}^{\mathrm{b}}(C)}^{\prime}=u \quad \text { et } \quad u_{\mid E_{i+1}}^{\prime}=f .
$$

Proposition 1.5. - Soient $C$ une $\infty$-catégorie et $E$ un ensemble de cellules de $C$ qui l'engendre librement au sens des polygraphes. Alors $E$ engendre $C$ par compositions.

Démonstration. - Pour $i \geqslant 0$, on pose $E_{i}=E \cap C_{i}$ et $E_{\leqslant i}=\cup_{0 \leqslant j \leqslant i} E_{j}$. On va montrer par récurrence sur $i \geqslant 0$ que l'ensemble $E_{\leqslant i}$ engendre $\tau_{\leqslant i}^{\mathrm{b}}(C)$ par compositions, ce qui prouvera la proposition. Pour $i=0$, l'assertion est évidente grâce à la condition (a) du paragraphe 1.4. Supposons donc l'assertion démontrée pour $i$ et démontrons-la pour $i+1$. Soit $D$ la sous- $(i+1)$-catégorie de $\tau_{\leqslant i+1}^{\mathrm{b}}(C)$ dont les $j$-flèches, pour $0 \leqslant j \leqslant i$, sont les $j$-flèches de $C$ et dont les $(i+1)$-flèches sont les composés d'éléments de $E_{i+1}$ et d'identités itérées de $j$-flèches de $C$ pour $0 \leqslant j \leqslant i$. Par hypothèse de récurrence, l'ensemble $E_{\leqslant i+1}$ engendre $D$ par compositions. Il suffit donc de montrer que $\tau_{\leqslant i+1}^{\mathrm{b}}(C)=D$ ou, de manière équivalente, que l'inclusion $v: D \hookrightarrow \tau_{\leqslant i+1}^{\mathrm{b}}(C)$ admet une section. Par définition, on a une inclusion $u: \tau_{\leqslant i}^{\mathrm{b}}(C) \hookrightarrow D$ et une inclusion $f: E_{i+1} \hookrightarrow D_{i+1}$ satisfaisant aux hypothèses de la condition (b) du paragraphe 1.4. On en déduit l'existence d'un $\infty$-foncteur $u^{\prime}: \tau_{\leqslant i+1}^{\mathrm{b}}(C) \rightarrow D$ tel que

$$
u_{\mid \tau_{\leqslant i}^{\mathrm{b}}(C)}^{\prime}=u \quad \text { et } \quad u_{\mid E_{i+1}}^{\prime}=f .
$$

La restriction de $v u^{\prime}$ à $\tau_{\leqslant i}^{\mathrm{b}}(C)$ est donc l'inclusion $\tau_{\leqslant i}^{\mathrm{b}}(C) \hookrightarrow \tau_{\leqslant i+1}^{\mathrm{b}}(C)$ et la restriction à $E_{i+1}$ l'inclusion $E_{i+1} \hookrightarrow C_{i+1}$, ce qui, en vertu de la partie unicité de la condition (b) du paragraphe 1.4 , prouve que $u^{\prime}$ est une section de $v$, ce qu'il fallait démontrer.

Proposition 1.6. - Soient $P: A \rightarrow \infty$-Cat un foncteur de source une petite catégorie $A, C$ une $\infty$-catégorie et $\alpha$ une transformation naturelle de $P$ vers le foncteur constant de valeur $C$. On suppose fixé, pour tout objet a de A, un ensemble $E_{a}$ engendrant librement la $\infty$-catégorie $P(a)$ au sens des polygraphes, ainsi qu'un ensemble $E$ engendrant librement la $\infty$-catégorie $C$ au sens des polygraphes. On suppose que

(a) pour tout objet a de $A$, on a $\alpha_{a}\left(E_{a}\right) \subset E$;

(b) pour toute flèche $f: a \rightarrow b$ de $A$, on a $P(f)\left(E_{a}\right) \subset E_{b}$;

(c) l'application $\lim _{a \in A} E_{a} \rightarrow E$ induite par $\alpha$ est une bijection.

Alors le $\infty$-foncteur $\lim _{a \in A} P(a) \rightarrow C$ induit par $\alpha$ est un isomorphisme de $\infty$-catégories.

Démonstration. - Pour tout $i \geqslant 0$, on pose $E_{i}=E \cap C_{i}$ et, pour tout objet $a$ de $A$, $E_{a, i}=E_{a} \cap P(a)_{i}$. Par hypothèse, pour tout $i \geqslant 0$, l'application $\lim _{A} E_{a, i} \rightarrow E_{i}$ 
induite par $\alpha$ est bijective. On va montrer par récurrence sur $i$ que, pour tout $i \geqslant 0$, le tronqué bête (défini au paragraphe 1.2)

$$
\tau_{\leqslant i}^{\mathrm{b}}(\underset{A}{\lim } P) \simeq \underset{A}{\lim _{\leqslant}} \tau_{\leqslant i}^{\mathrm{b}} P \longrightarrow \tau_{\leqslant i}^{\mathrm{b}}(C)
$$

du $\infty$-foncteur $\lim _{A} P \rightarrow C$ induit par $\alpha$ est un isomorphisme, ce qui prouvera la proposition. Pour $\overrightarrow{i=}=0$, cela résulte de la bijectivité de l'application $\lim _{A} E_{a, 0} \rightarrow E_{0}$ et du fait que, en vertu de la condition $(a)$ du paragraphe 1.4 , on a $C_{0}=E_{0}$ et $P(a)_{0}=E_{a, 0}$ pour tout $a$ dans $A$. Supposons l'assertion établie pour un $i \geqslant 0$ et prouvons-la pour $i+1$. Soient $D$ une $\infty$-catégorie et $\beta: \tau_{\leqslant i+1}^{\mathrm{b}} P \rightarrow D$ une transformation naturelle de but le foncteur constant de valeur $D$. Il s'agit de montrer qu'il existe un unique $\infty$-foncteur $v: \tau_{\leqslant i+1}^{\mathrm{b}}(C) \rightarrow D$ tel que, pour tout objet $a$ de $A$, on ait $\beta_{a}=v \circ \tau_{\leqslant i+1}^{\mathrm{b}}\left(\alpha_{a}\right)$. En vertu de l'hypothèse de récurrence, il existe un unique $\infty$-foncteur $u: \tau_{\leqslant i}^{\mathrm{b}}(C) \rightarrow D$ tel que, pour tout objet $a$ de $A$, la restriction de $\beta_{a}$ à $\tau_{\leqslant i}^{\mathrm{b}}(P(a))$ soit égale à $u \circ \tau_{\leqslant i}^{\mathrm{b}}\left(\alpha_{a}\right)$. D'autre part, la bijectivité de l'application $\lim _{A} E_{a, i+1} \rightarrow E_{i+1}$ implique l'existence d'une unique application $f: E_{i+1} \rightarrow D_{i+1}$ telle que, pour tout objet $a$ de $A$ et tout $x_{a}$ dans $E_{a, i+1}$, on ait $\beta_{a}\left(x_{a}\right)=f\left(\alpha_{a}\left(x_{a}\right)\right)$. Or, pour tout $x$ dans $E_{i+1}$, il existe un objet $a$ de $A$ et $x_{a}$ dans $E_{a, i+1}$ tel que $x=\alpha_{a}\left(x_{a}\right)$. On a donc

$$
\begin{aligned}
s(f(x)) & =s\left(f\left(\alpha_{a}\left(x_{a}\right)\right)\right)=s\left(\beta_{a}\left(x_{a}\right)\right)=\beta_{a}\left(s\left(x_{a}\right)\right) \\
& =u \alpha_{a}\left(s\left(x_{a}\right)\right)=u\left(s\left(\alpha_{a}\left(x_{a}\right)\right)\right)=u(s(x))
\end{aligned}
$$

et, de même, $t(f(x))=u(t(x))$. Comme $E$ engendre librement $C$ au sens des polygraphes, en vertu de la condition $(b)$ du paragraphe 1.4, il existe donc un unique $\infty$-foncteur $v: \tau_{\leqslant i+1}^{\mathrm{b}}(C) \rightarrow D$ tel que

$$
v_{\mid \tau_{\leqslant i}^{\mathrm{b}}(C)}=u \quad \text { et } \quad v_{\mid E_{i+1}}=f .
$$

Il reste à prouver que, pour tout objet $a$ de $A$, on a $\beta_{a}=v \circ \tau_{\leqslant i+1}^{\mathrm{b}}\left(\alpha_{a}\right)$. Or, on a les égalités

$$
\beta_{a \mid \tau_{\leqslant i}^{\mathrm{b}}(P(a))}=u \circ \tau_{\leqslant i}^{\mathrm{b}}\left(\alpha_{a}\right)=v_{\mid \tau_{\leqslant i}^{\mathrm{b}}(C)} \circ \tau_{\leqslant i}^{\mathrm{b}}\left(\alpha_{a}\right)=\left(v \circ \tau_{\leqslant i+1}^{\mathrm{b}}\left(\alpha_{a}\right)\right)_{\mid \tau_{\leqslant i}^{\mathrm{b}}(P(a))}
$$

et, pour tout $x_{a}$ dans $E_{a, i+1}$, on a $\beta_{a}\left(x_{a}\right)=f\left(\alpha_{a}\left(x_{a}\right)\right)=v\left(\alpha_{a}\left(x_{a}\right)\right)$ et donc

$$
\beta_{a \mid E_{a, i+1}}=\left(v \circ \tau_{\leqslant i+1}^{\mathrm{b}}\left(\alpha_{a}\right)\right)_{\mid E_{a, i+1}} .
$$

Comme $E_{a}$ engendre librement $P(a)$ au sens des polygraphes, en vertu de la partie unicité de la condition $(b)$ du paragraphe 1.4 , on a donc $\beta_{a}=v \circ \tau_{\leqslant i+1}^{\mathrm{b}}\left(\alpha_{a}\right)$, ce qui achève la démonstration.

Remarque 1.7. - On vérifie facilement que la proposition précédente reste vraie si on remplace l'hypothèse « $E_{a}$ engendre librement $P(a)$ au sens des polygraphes » par l'hypothèse plus faible « $E_{a}$ engendre $P(a)$ par compositions ». En revanche, on doit toujours supposer que l'ensemble $E$ engendre librement $C$ au sens des polygraphes. On n'aura pas besoin de ce résultat dans la suite. 
1.8. - Soit $C$ une $\infty$-catégorie. On définit une $\infty$-catégorie $C^{\circ}$, appelée le dual de $C$, en inversant le sens des $i$-flèches de $C$ pour tout $i>0$. On vérifie immédiatement que l'application $C \mapsto C^{\circ}$ définit un endofoncteur involutif $D: \infty$-Cat $\rightarrow \infty$-Cat.

Plus généralement, pour toute partie $J$ de $\mathbb{N}^{*}=\mathbb{N} \backslash\{0\}$, on définit un endofoncteur involutif $D_{J}: \infty$-C at $\rightarrow \infty$-C at qui envoie une $\infty$-catégorie sur la $\infty$-catégorie obtenue en inversant le sens des $i$-flèches pour tout $i$ dans $J$. On appellera $D_{J}(C)$ le $J$-dual de $C$. Par définition, on a $D=D_{\mathbb{N}^{*}}$.

Deux autres dualités jouent un rôle particulièrement important. Si $J$ est l'ensemble des entiers strictement positifs impairs, on appellera le J-dual le dual impair et on désignera $D_{J}(C)$ par $C^{\text {op }}$. Si $J$ est l'ensemble des entiers strictement positifs pairs, on parlera du dual pair et on désignera $D_{J}(C)$ par $C^{\mathrm{co}}$. Notons que si $C$ est une $\infty$-catégorie, on a $C^{\circ}=\left(C^{\text {op }}\right)^{\text {co }}=\left(C^{\text {co }}\right)^{\text {op }}$. Pour cette raison, la $\infty$-catégorie $C^{\circ}$ est parfois notée $C^{\text {coop }}$ dans la littérature.

1.9. - Soient $u, v: C \rightarrow D$ deux $\infty$-foncteurs. Une prétransformation oplax $\alpha$ de $u$ vers $v$ consiste en la donnée, pour tout $i \geqslant 0$ et toute $i$-flèche $x$ de $C$, d'une $(i+1)$-flèche

$$
\alpha_{x}: \alpha_{t_{i-1}(x)} *_{i-1} \cdots *_{1} \alpha_{t_{0}(x)} *_{0} u(x) \longrightarrow v(x) *_{0} \alpha_{s_{0}(x)} *_{1} \cdots *_{i_{-1}} \alpha_{s_{i-1}(x)}
$$

de $D$. (Pour lire ces formules, il faut garder en tête les conventions énoncées au paragraphe 1.1 : en particulier, pour $k<l$, l'opération $*_{k}$ est prioritaire sur l'opération $*_{l}$.)

Ainsi, si $x$ est un objet de $C$, on dispose d'une 1-flèche

$$
\begin{gathered}
u(x) \\
\alpha_{x} \downarrow \\
v(x)
\end{gathered}
$$

de $D$; si $x$ est une 1-flèche de $C$, on dispose d'une 2-flèche

$$
u\left(s_{0}(x)\right) \stackrel{u(x)}{\alpha_{s_{0}(x)}} u\left(t_{0}(x)\right)
$$

de $D$; si $x$ est une 2-flèche de $C$, on dispose d'une 3-flèche

$$
u\left(s_{0}(x)\right) \frac{u\left(s_{1}(x)\right)}{u(x) \Downarrow} u\left(t_{0}(x)\right)
$$


de $D$ de source $\alpha_{t_{1}(x)} *_{1}\left(\alpha_{t_{0}(x)} *_{0} u(x)\right)$ et de but $\left(v(x) *_{0} \alpha_{s_{0}(x)}\right) *_{1} \alpha_{s_{1}(x)}$; etc.

Une telle prétransformation oplax est une transformation oplax si elle satisfait aux axiomes de fonctorialité suivants :

(a) pour tout $i \geqslant 0$ et toute $i$-flèche $x$ de $C$, on a

$$
\alpha_{1_{x}}=1_{\alpha_{x}}
$$

(b) pour tous $i>j \geqslant 0$ et tout couple $x, y$ de $i$-flèches $j$-composables de $C$, on a

$$
\begin{aligned}
& \alpha_{x *_{j} y}=\left(v\left(t_{j+1}(x)\right) *_{0} \alpha_{s_{0}(y)} *_{1} \cdots *_{j-1} \alpha_{s_{j-1}(y)} *_{j} \alpha_{y}\right) \\
& *_{j+1}\left(\alpha_{x} *_{j} \alpha_{t_{j-1}(x)} *_{j-1} \cdots *_{1} \alpha_{t_{0}(x)} *_{0} u\left(s_{j+1}(y)\right)\right) .
\end{aligned}
$$

Remarque 1.10. - On montrera dans l'appendice B (voir notamment le corollaire B.2.6) que les transformations oplax entre $\infty$-foncteurs de $C$ vers $D$ peuvent s'interpréter en termes du produit tensoriel de Gray $\infty$-catégorique. Plus précisément, en notant $\mathrm{D}_{1}$ la catégorie associée à l'ensemble ordonné $0<1$, de telles transformations oplax correspondent aux $\infty$-foncteurs $\mathrm{D}_{1} \otimes C \rightarrow D$, où $\otimes$ désigne le produit tensoriel de Gray (voir le paragraphe A.16), ou encore, par adjonction, aux $\infty$-foncteurs $C \rightarrow \underline{\text { Hom }}_{\text {lax }}\left(\mathrm{D}_{1}, D\right)$ (voir le paragraphe A.18).

Remarque 1.11. - La $\infty$-catégorie $\underline{\operatorname{Hom}}_{\mathrm{lax}}\left(\mathrm{D}_{1}, D\right)$ de la remarque précédente est isomorphe à la $\infty$-catégorie $H D$ des cylindres dans $D$ introduite par Métayer dans [30] (voir notre remarque B.1.16). Ainsi, les formules définissant les transformations oplax se trouvent déjà dans [30].

1.12. - Soient $u, v: C \rightarrow D$ deux $\infty$-foncteurs. Une transformation lax de $u$ vers $v$ est une transformation oplax de $u^{\text {co }}: C^{\text {co }} \rightarrow D^{\text {co }}$ vers $v^{\text {co }}: C^{\text {co }} \rightarrow D^{\text {co }}$.

Remarque 1.13. - On verra dans l'appendice B (voir le corollaire B.2.7) que les transformations lax entre $\infty$-foncteurs de $C$ vers $D$ correspondent aux $\infty$-foncteurs $C \otimes \mathrm{D}_{1} \rightarrow D$.

Remarque 1.14. - Si $u, v: C \rightarrow D$ sont deux $\infty$-foncteurs, on vérifie facilement qu'une transformation oplax de $u^{\circ}$ vers $v^{\circ}$ n'est autre qu'une transformation oplax de $v$ vers $u$; on en déduit qu'une transformation oplax de $u^{\text {op }}$ vers $v^{\text {op }}$ est une transformation lax de $v$ vers $u$.

Dans ce texte, on privilégiera les transformations oplax aux transformations lax. On laisse le soin au lecteur d'expliciter la notion de transformation lax et de dualiser les énoncés concernant les transformations oplax en des énoncés sur les transformations lax.

1.15. - Soit $u: C \rightarrow D$ un $\infty$-foncteur. On vérifie immédiatement qu'on définit une transformation oplax $1_{u}$ de $u$ vers $u$ en posant, pour toute cellule $x$ de $C$,

$$
\left(1_{u}\right)_{x}=1_{u(x)}
$$


On appellera cette transformation oplax la transformation oplax identité de $u$.

1.16. - Soient $v_{0}, v_{1}: C \rightarrow D$ deux $\infty$-foncteurs et $\alpha$ une transformation oplax de $v_{0}$ vers $v_{1}$. Soit $u: B \rightarrow C$ un $\infty$-foncteur. On vérifie immédiatement qu'on définit une transformation oplax $\alpha * u$ de $v_{0} u$ vers $v_{1} u$ (qui sont des $\infty$-foncteurs de $B$ vers $D$ ) en posant, pour $x$ une cellule de $B$,

$$
(\alpha * u)_{x}=\alpha_{u(x)} .
$$

De même, si $w: D \rightarrow E$ est un $\infty$-foncteur, on définit une transformation oplax $w * \alpha$ de $w v_{0}$ vers $w v_{1}$ (qui sont des $\infty$-foncteurs de $C$ vers $E$ ) en posant, pour $x$ une cellule de $C$,

$$
(w * \alpha)_{x}=w\left(\alpha_{x}\right) .
$$

1.17. - Fixons une $\infty$-catégorie $C$. Soient $(A, v: A \rightarrow C)$ et $(B, w: B \rightarrow C)$ deux $\infty$-catégories au-dessus de $C$ et $u_{0}, u_{1}: A \rightarrow B$ deux $\infty$-foncteurs au-dessus de $C$. Autrement dit, on a un diagramme

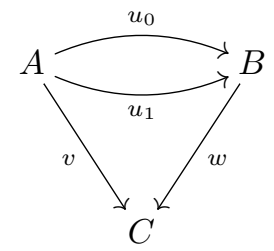

de $\infty$-foncteurs formé de deux triangles commutatifs. On dira qu'une transformation oplax $\alpha$ de $u_{0}$ vers $u_{1}$ est au-dessus de $C$ si $w * \alpha$ est la transformation oplax identité de $v$. 



\section{CHAPITRE 2}

\section{RAPPELS ET COMPLÉMENTS SUR LA THÉORIE DE STEINER}

Le but de ce chapitre est d'exposer la théorie développée par Steiner dans [32] et d'y apporter quelques compléments.

2.1. - Dans ce texte, par « complexe de chaînes » on entendra toujours « complexe de chaînes de groupes abéliens en degrés positifs ». On rappelle qu'un élément homogène d'un complexe de chaînes $K$ est un élément de la somme disjointe ensembliste des $K_{n}$ pour $n \geqslant 0$. Le degré d'un élément homogène $x$, c'est-à-dire l'unique $n$ tel que $x$ appartienne à $K_{n}$, sera noté $|x|$.

2.2. - Un complexe dirigé augmenté est un triplet $\left(K, K^{*}, e\right)$, où

$$
K=\cdots \stackrel{d_{n+1}}{\longrightarrow} K_{n} \stackrel{d_{n}}{\longrightarrow} K_{n-1} \stackrel{d_{n-1}}{\longrightarrow} \cdots \stackrel{d_{2}}{\longrightarrow} K_{1} \stackrel{d_{1}}{\longrightarrow} K_{0}
$$

est un complexe de chaînes (de groupes abéliens en degrés positifs), $e: K_{0} \rightarrow \mathbb{Z}$ est une augmentation (on a donc $e d_{1}=0$ ) et $K^{*}=\left(K_{n}^{*}\right)_{n \geqslant 0}$ est la donnée pour tout $n \geqslant 0$ d'un sous-monoïde $K_{n}^{*}$ du groupe abélien $K_{n}$. (On ne demande aucune compatibilité entre la différentielle et ces sous-monoïdes.) On appellera les sous-monoïdes $K_{n}^{*}$, pour $n \geqslant 0$, les sous-monoïdes de positivité de $K$.

Un morphisme de complexes dirigés augmentés est un morphisme de complexes de chaînes augmentés qui respecte les sous-monoïdes de positivité. Plus précisément, si $\left(K, K^{*}, e\right)$ et $\left(K^{\prime}, K^{\prime *}, e^{\prime}\right)$ sont deux complexes dirigés augmentés, un morphisme du premier vers le second est donné par un morphisme de complexes de chaînes $f: K \rightarrow K^{\prime}$ tel que $e^{\prime} f_{0}=e$ et $f_{n}\left(K_{n}^{*}\right) \subset K_{n}^{\prime *}$ pour tout $n \geqslant 0$. On notera $\mathcal{C}_{\text {da }}$ la catégorie des complexes dirigés augmentés.

On désignera souvent, par abus de notation, un complexe dirigé augmenté par son complexe de chaînes sous-jacent.

2.3. - On définit un foncteur

$$
\lambda: \infty-\mathcal{C} a t \rightarrow \mathcal{C}_{\mathrm{da}}
$$


de la manière suivante.

Soit $C$ une $\infty$-catégorie. Pour $i \geqslant 0$, le groupe abélien $\lambda(C)_{i}$ est défini par les générateurs

$$
[x], \quad \text { pour } x \text { une } i \text {-flèche de } C,
$$

soumis aux relations

$$
\left[x *_{j} y\right]=[x]+[y], \quad \text { pour } 0 \leqslant j<i \text { et } x \text { et } y \text { des } i \text {-flèches } j \text {-composables. }
$$

Le sous-monoïde de positivité $\lambda(C)_{i}^{*}$ est le sous-monoïde engendré par les générateurs $[x]$ pour $x$ une $i$-flèche de $C$. Pour $i>0$, la différentielle $d_{i}: \lambda(C)_{i} \rightarrow \lambda(C)_{i-1}$ est définie par

$$
d([x])=[t(x)]-[s(x)], \quad \text { pour } x \text { une } i \text {-flèche de } C .
$$

Enfin, l'augmentation $\lambda(C)_{0} \rightarrow \mathbb{Z}$ est l'unique morphisme qui envoie, pour tout objet $x$ de $C$, le générateur $[x]$ sur 1 . On vérifie immédiatement qu'on obtient bien ainsi un complexe dirigé augmenté.

Si $u: C \rightarrow D$ est un $\infty$-foncteur, on définit, pour tout $i \geqslant 0$, un morphisme de groupes abéliens $\lambda(u)_{i}: \lambda(C)_{i} \rightarrow \lambda(D)_{i}$ en envoyant un générateur $[x]$, pour $x$ une $i$-flèche de $C$, sur le générateur $[u(x)]$. On vérifie immédiatement qu'on obtient ainsi un morphisme de complexes dirigés augmentés $\lambda(u): \lambda(C) \rightarrow \lambda(D)$ et que $\lambda$ ainsi défini est bien un foncteur.

2.4. - On définit un foncteur

$$
\nu: \mathcal{C}_{\mathrm{da}} \rightarrow \infty-\mathcal{C} a t
$$

de la manière suivante.

Soit $K$ un complexe dirigé augmenté. Pour $i \geqslant 0$, les $i$-flèches de $\nu(K)$ sont les tableaux

$$
\left(\begin{array}{llll}
x_{0}^{0} & \ldots & x_{i-1}^{0} & x_{i}^{0} \\
x_{0}^{1} & \ldots & x_{i-1}^{1} & x_{i}^{1}
\end{array}\right)
$$

tels que

(a) $x_{k}^{\varepsilon}$ appartient à $K_{k}^{*}$ pour $\varepsilon=0,1$ et $0 \leqslant k \leqslant i$;

(b) $d\left(x_{k}^{\varepsilon}\right)=x_{k-1}^{1}-x_{k-1}^{0}$ pour $\varepsilon=0,1$ et $0<k \leqslant i$;

(c) $e\left(x_{0}^{\varepsilon}\right)=1$ pour $\varepsilon=0,1$;

(d) $x_{i}^{0}=x_{i}^{1}$.

La structure de $\infty$-catégorie sur $\nu(K)$ est définie de la manière suivante. Soit

$$
x=\left(\begin{array}{llll}
x_{0}^{0} & \ldots & x_{i-1}^{0} & x_{i}^{0} \\
x_{0}^{1} & \ldots & x_{i-1}^{1} & x_{i}^{1}
\end{array}\right),
$$


pour $i \geqslant 0$, une $i$-flèche de $\nu(K)$. Si $i>0$, les sources et buts de $x$ sont donnés par les tableaux

$$
s(x)=\left(\begin{array}{llll}
x_{0}^{0} & \ldots & x_{i-2}^{0} & x_{i-1}^{0} \\
x_{0}^{1} & \ldots & x_{i-2}^{1} & x_{i-1}^{0}
\end{array}\right) \quad \text { et } \quad t(x)=\left(\begin{array}{llll}
x_{0}^{0} & \ldots & x_{i-2}^{0} & x_{i-1}^{1} \\
x_{0}^{1} & \ldots & x_{i-2}^{1} & x_{i-1}^{1}
\end{array}\right) .
$$

Pour tout $i \geqslant 0$, l'identité de $x$ est le tableau

$$
1_{x}=\left(\begin{array}{cccc}
x_{0}^{0} & \ldots & x_{i}^{0} & 0 \\
x_{0}^{1} & \ldots & x_{i}^{1} & 0
\end{array}\right) .
$$

Enfin, si

$$
x=\left(\begin{array}{llll}
x_{0}^{0} & \ldots & x_{i-1}^{0} & x_{i}^{0} \\
x_{0}^{1} & \ldots & x_{i-1}^{1} & x_{i}^{1}
\end{array}\right) \quad \text { et } y=\left(\begin{array}{llll}
y_{0}^{0} & \ldots & y_{i-1}^{0} & y_{i}^{0} \\
y_{0}^{1} & \ldots & y_{i-1}^{1} & y_{i}^{1}
\end{array}\right)
$$

sont deux $i$-flèches $j$-composables pour $i>j \geqslant 0$, on pose

$$
x *_{j} y=\left(\begin{array}{cccccc}
y_{0}^{0} & \ldots & y_{j}^{0} & x_{j+1}^{0}+y_{j+1}^{0} & \ldots & x_{i}^{0}+y_{i}^{0} \\
x_{0}^{1} & \ldots & x_{j}^{1} & x_{j+1}^{1}+y_{j+1}^{1} & \ldots & x_{i}^{1}+y_{i}^{1}
\end{array}\right) .
$$

On vérifie qu'on définit bien ainsi une $\infty$-catégorie.

Si $x$ est une $i$-flèche de $\nu(K)$ pour un $i \geqslant 0$, on notera $x_{k}^{\varepsilon}$, pour $0 \leqslant k \leqslant i$ et $\varepsilon=0,1$, les composantes du tableau

$$
x=\left(\begin{array}{llll}
x_{0}^{0} & \ldots & x_{i-1}^{0} & x_{i}^{0} \\
x_{0}^{1} & \ldots & x_{i-1}^{1} & x_{i}^{1}
\end{array}\right) .
$$

On désignera par $x_{i}$ l'élément $x_{i}^{0}=x_{i}^{1}$ et, pour $k>i$ et $\varepsilon=0,1$, on posera $x_{k}^{\varepsilon}=0$.

Si maintenant $f: K \rightarrow K^{\prime}$ est un morphisme de complexes dirigés augmentés, on vérifie qu'on définit un $\infty$-foncteur $\nu(f): \nu(K) \rightarrow \nu\left(K^{\prime}\right)$ par la formule

$$
x=\left(\begin{array}{cccc}
x_{0}^{0} & \ldots & x_{i-1}^{0} & x_{i}^{0} \\
x_{0}^{1} & \ldots & x_{i-1}^{1} & x_{i}^{1}
\end{array}\right) \mapsto f(x)=\left(\begin{array}{llll}
f\left(x_{1}^{0}\right) & \ldots & f\left(x_{i-1}^{0}\right) & f\left(x_{i}^{0}\right) \\
f\left(x_{1}^{1}\right) & \ldots & f\left(x_{i-1}^{1}\right) & f\left(x_{i}^{1}\right)
\end{array}\right) .
$$

On vérifie immédiatement que $\nu$ ainsi défini est bien un foncteur.

Théorème 2.5 (Steiner). — Les foncteurs

$$
\lambda: \infty-\mathcal{C} a t \rightarrow \mathcal{C}_{\mathrm{da}}, \quad \nu: \mathcal{C}_{\mathrm{da}} \rightarrow \infty-\mathcal{C} a t
$$

forment un couple de foncteurs adjoints.

Démonstration. - Voir [32, théorème 2.11].

2.6. - Une base d'un complexe dirigé augmenté $K$ est un ensemble gradué $B=\left(B_{i}\right)_{i \geqslant 0}$ tel que, pour tout $i \geqslant 0$,

(a) $B_{i}$ est une base du $\mathbb{Z}$-module $K_{i}$;

(b) $B_{i}$ engendre le sous-monoïde $K_{i}^{*}$ de $K_{i}$. 
On identifiera souvent une base $B=\left(B_{i}\right)_{i \geqslant 0}$ à l'ensemble $\coprod_{i \geqslant 0} B_{i}$.

Soit $K$ un complexe dirigé augmenté. On définit une relation de préordre $\leqslant$ sur $K_{i}$ en posant

$$
x \leqslant y \stackrel{\text { déf }}{\Longleftrightarrow} y-x \in K_{i}^{*} .
$$

On vérifie immédiatement que si $K$ admet une base, cette relation de préordre est une relation d'ordre et que les éléments de $B_{i}$ sont les éléments minimaux de $\left(K_{i}^{*} \backslash\{0\}, \leqslant\right)$. Ainsi, si $K$ admet une base, cette base est unique.

On dira que le complexe dirigé augmenté $K$ est à base s'il admet une base (nécessairement unique).

2.7. - Fixons $A$ un groupe abélien libre muni d'une base $B$. Soit

$$
x=\sum_{b \in B} x_{b} b
$$

un élément de $A$. On appellera support de $x$ l'ensemble

$$
\operatorname{supp}(x)=\left\{b \in B \mid x_{b} \neq 0\right\} \text {. }
$$

Notons $A^{*}$ le sous-monoïde de $A$ engendré par $B$. On définit deux éléments $x_{+}$et $x_{-}$ de $A^{*}$ par

$$
x_{+}=\sum_{\substack{b \in B \\ x_{b}>0}} x_{b} b \quad \text { et } \quad x_{-}=-\sum_{\substack{b \in B \\ x_{b}<0}} x_{b} b .
$$

On a alors $x=x_{+}-x_{-}$.

En particulier, si $K$ est un complexe dirigé augmenté admettant une base $B=\left(B_{i}\right)_{i \geqslant 0}$, pour tout $x$ dans $K_{i}$ pour un $i \geqslant 0$, on dispose, en appliquant le paragraphe précédent au groupe abélien $K_{i}$ muni de sa base $B_{i}$, d'une notion de support de $x$ et d'éléments $x_{+}$et $x_{-}$de $K_{i}^{*}$.

2.8. - Soit $K$ un complexe dirigé augmenté admettant une base $B=\left(B_{i}\right)_{i \geqslant 0}$. Pour $i \geqslant 0$ et $x$ un élément de $K_{i}$, on définit un tableau

$$
\langle x\rangle=\left(\begin{array}{ccccc}
\langle x\rangle_{0}^{0} & \langle x\rangle_{1}^{0} & \ldots & \langle x\rangle_{i-1}^{0} & \langle x\rangle_{i}^{0} \\
\langle x\rangle_{0}^{1} & \langle x\rangle_{1}^{1} & \ldots & \langle x\rangle_{i-1}^{1} & \langle x\rangle_{i}^{1}
\end{array}\right),
$$

où les $\langle x\rangle_{k}^{\varepsilon}$ sont définis par récurrence descendante sur $k$ de $i$ à 0 :

$-\langle x\rangle_{i}^{0}=x=\langle x\rangle_{i}^{1}$

$-\langle x\rangle_{k-1}^{0}=d\left(\langle x\rangle_{k}^{0}\right)_{-}$et $\langle x\rangle_{k-1}^{1}=d\left(\langle x\rangle_{k}^{1}\right)_{+}$pour $0<k \leqslant i$.

On vérifie facilement que ce tableau est une $i$-flèche de $\nu(K)$ si et seulement si, d'une part, $x$ appartient à $K_{i}^{*}$ et, d'autre part, on a les égalités $e\left(\langle x\rangle_{0}^{0}\right)=1=e\left(\langle x\rangle_{0}^{1}\right)$. On posera par ailleurs $\langle x\rangle_{i}=x$ et $\langle x\rangle_{k}^{\varepsilon}=0$ pour tout $k>i$ et $\varepsilon=0,1$. Lorsque $\langle x\rangle$ est une $i$-flèche, ces conventions sont compatibles avec celles du paragraphe 2.4 . 
On dit que la base $B$ de $K$ est unitaire si, pour tout $i \geqslant 0$ et tout $x$ dans $B_{i}$, le tableau $\langle x\rangle$ est une $i$-flèche de $\nu(K)$, ce qui revient à dire qu'on a l'égalité $e\left(\langle x\rangle_{0}^{0}\right)=1=e\left(\langle x\rangle_{0}^{1}\right)$.

On dit qu'un complexe dirigé augmenté est à base unitaire s'il est à base et que son unique base est unitaire. Si un complexe dirigé augmenté $K$ est à base unitaire, pour tout élément $x$ de la base de $K$, on appelle la cellule $\langle x\rangle$ de $\nu(K)$ l'atome associé à $x$.

2.9. - Soit $K$ un complexe dirigé augmenté admettant une base $B$. Pour $i \geqslant 0$, on note $\leqslant_{i}$ la plus petite relation de préordre sur $B\left(=\coprod_{i} B_{i}\right)$ satisfaisant

$$
x \leqslant_{i} y \quad \text { si } \quad|x|>i,|y|>i \text { et } \operatorname{supp}\left(\langle x\rangle_{i}^{1}\right) \cap \operatorname{supp}\left(\langle y\rangle_{i}^{0}\right) \neq \varnothing .
$$

On dit que la base $B$ est sans boucle si, pour tout $i \geqslant 0$, la relation de préordre $\leqslant_{i}$ est une relation d'ordre.

On dit qu'un complexe dirigé augmenté est à base sans boucle s'il est à base et si sa base est sans boucle.

2.10. - On appellera complexe de Steiner un complexe dirigé augmenté à base dont la base est unitaire et sans boucle.

On appellera $\infty$-catégorie de Steiner une $\infty$-catégorie dans l'image essentielle de la restriction du foncteur $\nu: \mathcal{C}_{\mathrm{da}} \rightarrow \infty$ - $\mathcal{C}$ at à la catégorie des complexes de Steiner. Le théorème suivant affirme que le foncteur $\nu$ induit une équivalence de catégories entre la catégorie des complexes de Steiner et celle des $\infty$-catégories de Steiner.

Théorème 2.11 (Steiner). — Pour tout complexe de Steiner K, le morphisme d'adjonction

$$
\lambda(\nu(K)) \rightarrow K
$$

est un isomorphisme. En particulier, la restriction du foncteur $\nu: \mathcal{C}_{\mathrm{da}} \rightarrow \infty-\mathcal{C}$ at à la catégorie des complexes de Steiner est un foncteur pleinement fidèle.

Démonstration. - Voir [32, théorème 5.6].

Théorème 2.12 (Steiner). - Soit $K$ un complexe de Steiner. Alors la $\infty$-catégorie $\nu(K)$ est engendrée librement au sens des polygraphes par ses atomes, c'est-àdire par les $\langle x\rangle$, où $x$ varie dans la base de $K$.

Démonstration. - Voir [32, théorème 6.1].

2.13. - Soit $K$ un complexe dirigé augmenté admettant une base $B$. On notera $\leqslant_{\mathbb{N}}$ la plus petite relation de préordre sur $B$ satisfaisant

$$
x \leqslant_{\mathbb{N}} y \quad \text { si } \quad x \in \operatorname{supp}\left(d(y)_{-}\right) \text {ou } y \in \operatorname{supp}\left(d(x)_{+}\right),
$$

où, par convention, $d(b)=0$ si $b$ est dans $B_{0}$. On dit que la base $B$ est fortement sans boucle si la relation de préordre $\leqslant_{\mathbb{N}}$ est une relation d'ordre. 
On dit qu'un complexe dirigé augmenté est à base fortement sans boucle s'il est à base et si sa base est fortement sans boucle.

Proposition 2.14 (Steiner). - Soit $K$ un complexe dirigé augmenté admettant une base B. Si la base B est fortement sans boucle, alors elle est sans boucle.

Démonstration. - Voir [32, proposition 3.7].

2.15. - On appellera complexe de Steiner fort un complexe dirigé augmenté à base dont la base est unitaire et fortement sans boucle. En vertu de la proposition précédente, un complexe de Steiner fort est un complexe de Steiner. On notera $\mathcal{S} t_{\mathrm{f}}$ la sous-catégorie pleine de la catégorie des complexes dirigés augmentés formée des complexes de Steiner forts.

On appellera $\infty$-catégorie de Steiner forte une $\infty$-catégorie dans l'image essentielle de la restriction du foncteur $\nu: \mathcal{C}_{\mathrm{da}} \rightarrow \infty$-C at à la catégorie des complexes de Steiner forts. En vertu du théorème 2.11, le foncteur $\nu$ induit donc une équivalence de la catégorie des complexes de Steiner forts vers la catégorie des $\infty$-catégories de Steiner fortes.

Exemple 2.16. — L'exemple suivant montre que la classe des $\infty$-catégories de Steiner fortes est strictement incluse dans celle des $\infty$-catégories de Steiner. Considérons la 3-catégorie $C$ définie par le diagramme

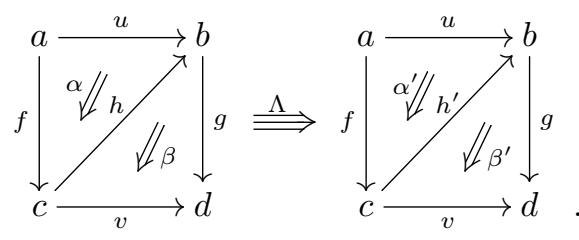

On vérifie facilement que $\lambda(C)$ est un complexe de Steiner, de base les éléments de $\lambda(C)$ correspondant aux cellules « génératrices » de $C$ (c'est-à-dire celles qui sont nommées dans le diagramme ci-dessus), et que le morphisme canonique $C \rightarrow \nu(\lambda(C))$ est un isomorphisme. Néanmoins, il n'est pas vrai que $\lambda(C)$ soit un complexe de Steiner fort : en effet, on a

$$
\beta \leqslant_{\mathbb{N}} \Lambda \leqslant_{\mathbb{N}} \alpha^{\prime} \leqslant_{\mathbb{N}} h^{\prime} \leqslant_{\mathbb{N}} b \leqslant_{\mathbb{N}} g \leqslant_{\mathbb{N}} \beta,
$$

où $\leqslant_{\mathbb{N}}$ désigne la relation du paragraphe 2.13 et où on a identifié les cellules génératrices de $C$ avec les éléments de la base de $\lambda(C)$ leur correspondant.

Dans la suite de ce chapitre, on introduit quelques compléments sur la notion de complexe dirigé augmenté.

2.17. - On dira qu'un complexe dirigé augmenté $K$ est décent si, pour tout $x$ dans $K_{0}^{*}$, on a $e(x) \geqslant 0$. Notons que tout complexe dirigé augmenté $K$ à base unitaire, 
et donc en particulier tout complexe de Steiner (et donc tout complexe de Steiner fort), est décent puisque, par définition, si $x$ est dans la base de $K_{0}^{*}$, on a $e(x)=1$.

2.18. - Soient $K$ un complexe dirigé augmenté et $J$ une partie de $\mathbb{N}^{*}=\mathbb{N} \backslash\{0\}$. On définit un complexe dirigé augmenté $D_{J}(K)$, appelé le $J$-dual de $K$, de la manière suivante :

- pour tout $n \geqslant 0$, on a

$$
D_{J}(K)_{n}=K_{n} \quad \text { et } \quad D_{J}(K)_{n}^{*}=K_{n}^{*} ;
$$

- $D_{J}(K)$ a même augmentation que $K$;

- la différentielle $d^{\prime}$ de $D_{J}(K)$ est donnée, pour $n \geqslant 1$, par

$$
d_{n}^{\prime}= \begin{cases}-d_{n} & \text { si } n \text { appartient à } J, \\ d_{n} & \text { sinon. }\end{cases}
$$

On vérifie immédiatement que $K \mapsto D_{J}(K)$ définit un endofoncteur involutif $D_{J}: \mathcal{C}_{\mathrm{da}} \rightarrow \mathcal{C}_{\mathrm{da}}$ de la catégorie des complexes dirigés augmentés.

Comme dans le cas des $\infty$-catégories, trois dualités jouent un rôle particulièrement important. Si $J$ est l'ensemble des entiers strictement positifs impairs, on appellera le $J$-dual le dual impair et on désignera $D_{J}(K)$ par $K^{\mathrm{op}}$. Si $J$ est l'ensemble des entiers strictement positifs pairs, on parlera du dual pair et on désignera $D_{J}(K)$ par $K^{\text {co }}$. Enfin, si $J$ est l'ensemble des entiers strictement positifs, on appellera le $J$-dual le dual et on désignera $D_{J}(K)$ par $K^{\circ}$.

Proposition 2.19. - Pour toute partie $J$ de $\mathbb{N}^{*}$, les carrés de foncteurs
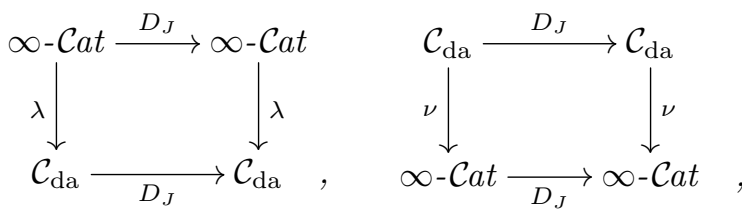

où les foncteurs horizontaux sont ceux définis au paragraphe précédent et au paragraphe 1.8, sont commutatifs à isomorphisme canonique près. En particulier, pour toute $\infty$-catégorie $C$, on a des isomorphismes canoniques

$$
\lambda\left(C^{\mathrm{op}}\right) \simeq(\lambda(C))^{\mathrm{op}}, \quad \lambda\left(C^{\mathrm{co}}\right) \simeq(\lambda(C))^{\mathrm{co}} \quad \text { et } \quad \lambda\left(C^{\circ}\right) \simeq(\lambda(C))^{\circ},
$$

et, pour tout complexe dirigé augmenté $K$, on a des isomorphismes canoniques

$$
\nu\left(K^{\mathrm{op}}\right) \simeq(\nu(K))^{\mathrm{op}}, \quad \nu\left(K^{\mathrm{co}}\right) \simeq(\nu(K))^{\mathrm{co}} \quad \text { et } \quad \nu\left(K^{\circ}\right) \simeq(\nu(K))^{\circ} .
$$

Démonstration. - Puisque $D_{J}$ est une involution, ce foncteur est son propre adjoint à gauche comme à droite. La commutativité à isomorphisme près des deux carrés de l'énoncé est donc équivalente par adjonction et il suffit de vérifier la commutativité du premier. 
Soit $C$ une $\infty$-catégorie. Par définition, pour $i \geqslant 0$, le groupe abélien $\lambda\left(D_{J}(C)\right)_{i}$ est engendré par les $[x]$, pour $x$ dans $D_{J}(C)_{i}=C_{i}$, soumis aux relations $\left[x *_{j}^{\prime} y\right]=[x]+[y]$, pour $x$ et $y$ des $i$-flèches composables pour la $j$-composition $*_{j}^{\prime}$ de $D_{J}(C)$. Si $j+1$ n'appartient pas à $J$, la composition $*_{j}^{\prime}$ est la composition $*_{j}$ de $C$. Si $j+1$ appartient à $J$, alors $x$ et $y$ sont composables pour $*_{j}^{\prime}$ si et seulement si $y$ et $x$ sont composables pour $*_{j}$ et on a alors $x *_{j}^{\prime} y=y *_{j} x$. Puisque $[x]+[y]=[y]+[x]$, les relations définissant $\lambda\left(D_{J}(C)\right)_{i}$ sont donc les mêmes que celles qui définissent $\lambda(C)_{i}$. Puisque par définition, on a $D_{J}(\lambda(C))_{i}=\lambda(C)_{i}$, on obtient donc l'égalité

$$
\lambda\left(D_{J}(C)\right)_{i}=D_{J}(\lambda(C))_{i} .
$$

On a également

$$
\lambda\left(D_{J}(C)\right)_{i}^{*}=D_{J}(\lambda(C))_{i}^{*}
$$

puisque ces sous-monoïdes sont engendrés par les mêmes générateurs. Enfin, si $i>0$ et $x$ appartient à $C_{i}$, la différentielle $d^{\prime}$ de $\lambda\left(D_{J}(C)\right)$ envoie $[x]$ sur $\left[t^{\prime}(x)\right]-\left[s^{\prime}(x)\right]$, où $s^{\prime}$ et $t^{\prime}$ désignent la source et le but dans $D_{J}(C)$. Si $i$ n'appartient pas à $J$, on a $s^{\prime}(x)=s(x)$ et $t^{\prime}(x)=t(x)$, et donc $d^{\prime}([x])=d([x])$, où $d$ désigne la différentielle de $\lambda(C)$. Si $i$ appartient à $J$, on a $s^{\prime}(x)=t(x)$ et $t^{\prime}(x)=s(x)$, et donc $d^{\prime}([x])=-d([x])$, ce qui montre que $\lambda\left(D_{J}(C)\right)$ et $D_{J}(\lambda(C))$ ont même différentielle. L'égalité des augmentations étant évidente, ceci achève la démonstration.

2.20. - Soit $K$ un complexe dirigé augmenté. On appellera dimension de $K$ le plus petit entier $n \geqslant 0$ tel que $K_{i}=0$ pour tout $i>n$. Si un tel $n$ n'existe pas, on dira que $K$ est de dimension infinie. Pour $n \geqslant 0$, on notera $n-\mathcal{C}_{\text {da }}$ la sous-catégorie pleine de $\mathcal{C}_{\text {da }}$ formée des complexes dirigés augmentés de dimension au plus $n$.

Le foncteur d'inclusion $n-\mathcal{C}_{\text {da }} \hookrightarrow \mathcal{C}_{\text {da }}$ admet un adjoint à gauche et un adjoint à droite qu'on notera respectivement

$$
\tau_{\leqslant n}^{\mathrm{i}}: \mathcal{C}_{\mathrm{da}} \rightarrow n-\mathcal{C}_{\mathrm{da}} \quad \text { et } \quad \tau_{\leqslant n}^{\mathrm{b}}: \mathcal{C}_{\mathrm{da}} \rightarrow n-\mathcal{C}_{\mathrm{da}} .
$$

On appellera $\tau_{\leqslant n}^{\mathrm{i}}$ le foncteur $n$-tronqué intelligent et $\tau_{\leqslant n}^{\mathrm{b}}$ le foncteur $n$-tronqué bête.

Explicitement, si $K$ est un complexe dirigé augmenté, on a

$$
\tau_{\leqslant n}^{\mathrm{i}}(K)_{i}=\left\{\begin{array}{ll}
K_{i} & \text { si } i<n, \\
K_{n} / d\left(K_{n+1}\right) & \text { si } i=n, \\
0 & \text { si } i>n,
\end{array} \quad \text { et } \quad \tau_{\leqslant n}^{\mathrm{i}}(K)_{i}^{*}= \begin{cases}K_{i}^{*} & \text { si } i<n, \\
\overline{K_{n}^{*}} & \text { si } i=n, \\
0 & \text { si } i>n,\end{cases}\right.
$$

où $\overline{K_{n}^{*}}$ désigne l'image de $K_{n}^{*}$ dans $K_{n} / d\left(K_{n+1}\right)$, et

$$
\tau_{\leqslant n}^{\mathrm{b}}(K)_{i}=\left\{\begin{array}{ll}
K_{i} & \text { si } i \leqslant n, \\
0 & \text { si } i>n,
\end{array} \quad \text { et } \quad \tau_{\leqslant n}^{\mathrm{b}}(K)_{i}^{*}= \begin{cases}K_{i}^{*} & \text { si } i \leqslant n, \\
0 & \text { si } i>n,\end{cases}\right.
$$

les différentielles et augmentations étant héritées de celles de $K$ de la manière évidente. 
2.21. - Fixons un entier $n \geqslant 0$. Si $C$ est une $n$-catégorie, il est immédiat que $\lambda(C)$ est un complexe dirigé augmenté de dimension au plus $n$. De même, si $K$ est un complexe dirigé augmenté de dimension $n$, il est immédiat que $\nu(K)$ est une $n$ catégorie. Les foncteurs $\lambda$ et $\nu$ induisent donc des foncteurs

$$
\lambda: n-\mathcal{C}_{\mathrm{da}} \rightarrow n-\mathcal{C} a t \quad \text { et } \quad \nu: n-\mathcal{C} a t \rightarrow n-\mathcal{C}_{\mathrm{da}}
$$

et ceux-ci forment un couple de foncteurs adjoints. Par définition, les carrés
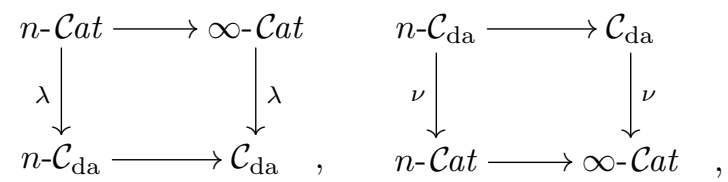

où les flèches horizontales désignent les foncteurs d'inclusion, sont commutatifs.

Proposition 2.22. - Pour tout entier $n \geqslant 0$, les carrés
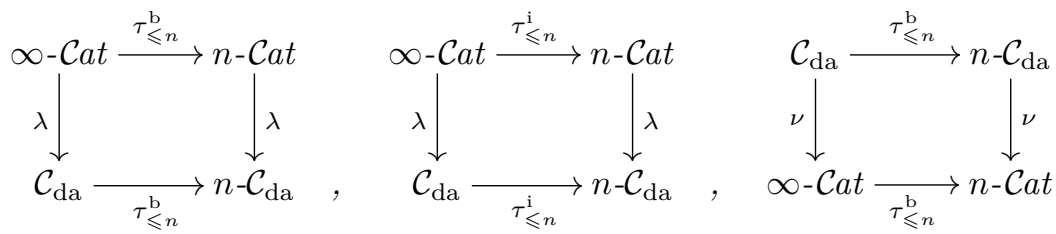

sont commutatifs à isomorphisme canonique près.

Démonstration. - L'existence des flèches de comparaison dans ces carrés résulte formellement de la commutativité des deux carrés du paragraphe précédent. Les commutativités à isomorphisme près du premier et du troisième carrés sont évidentes. Enfin, la commutativité à isomorphisme près du deuxième carré résulte par adjonction de la commutativité du second carré du paragraphe précédent.

Proposition 2.23. - Soit $K$ un complexe dirigé augmenté à base. Pour tout entier $n \geqslant 0$, le morphisme canonique

$$
\tau_{\leqslant n}^{\mathrm{i}} \nu(K) \rightarrow \nu \tau_{\leqslant n}^{\mathrm{i}}(K)
$$

est un isomorphisme.

Démonstration. - Explicitons la flèche de comparaison $\alpha: \tau_{\leqslant n}^{\mathrm{i}} \nu(K) \rightarrow \nu \tau_{\leqslant n}^{\mathrm{i}}(K)$. Par adjonction, puisque $\nu \tau_{\leqslant n}^{\mathrm{i}}(K)$ est une $n$-catégorie, il s'agit de définir un $\infty$-foncteur $\nu(K) \rightarrow \nu \tau_{\leqslant n}^{\mathrm{i}}(K)$. On obtient ce $\infty$-foncteur en appliquant le foncteur $\nu$ au morphisme canonique $K \rightarrow \tau_{\leqslant n}^{\mathrm{i}}(K)$. Montrons que $\alpha: \tau_{\leqslant n}^{\mathrm{i}} \nu(K) \rightarrow \nu \tau_{\leqslant n}^{\mathrm{i}}(K)$ est un isomorphisme. Pour $i<n$, il est évident que $\alpha_{i}$ est une bijection. Pour conclure, il suffit donc de montrer que $\alpha_{n}$ est une bijection. Décrivons explicitement 
$\alpha_{n}:\left(\tau_{\leqslant n}^{\mathrm{i}} \nu(K)\right)_{n} \rightarrow\left(\nu \tau_{\leqslant n}^{\mathrm{i}}(K)\right)_{n}$. Une $n$-flèche de $\tau_{\leqslant n}^{\mathrm{i}} \nu(K)$ est la classe d'équivalence d'un élément

$$
\left(\begin{array}{cccc}
x_{0}^{0} & \cdots & x_{n-1}^{0} & x_{n}^{0} \\
x_{0}^{1} & \cdots & x_{n-1}^{1} & x_{n}^{1}
\end{array}\right)
$$

de $\nu(K)_{n}$ pour la plus petite relation d'équivalence identifiant

$$
x=\left(\begin{array}{cccc}
x_{0}^{0} & \ldots & x_{n-1}^{0} & x_{n}^{0} \\
x_{0}^{1} & \ldots & x_{n-1}^{1} & x_{n}^{1}
\end{array}\right) \quad \text { et } y=\left(\begin{array}{cccc}
y_{0}^{0} & \ldots & y_{n-1}^{0} & y_{n}^{0} \\
y_{0}^{1} & \ldots & y_{n-1}^{1} & y_{n}^{1}
\end{array}\right)
$$

s'il existe

$$
z=\left(\begin{array}{cccc}
z_{0}^{0} & \cdots & z_{n}^{0} & z_{n+1}^{0} \\
z_{0}^{1} & \cdots & z_{n}^{1} & z_{n+1}^{1}
\end{array}\right)
$$

dans $\nu(K)_{n+1}$ tel que $x=s(z)$ et $y=t(z)$. Concrètement, cette dernière condition signifie qu'on a

$$
\left(\begin{array}{ccc}
x_{0}^{0} & \ldots & x_{n-1}^{0} \\
x_{0}^{1} & \ldots & x_{n-1}^{1}
\end{array}\right)=\left(\begin{array}{lll}
y_{0}^{0} & \ldots & y_{n-1}^{0} \\
y_{0}^{1} & \ldots & y_{n-1}^{1}
\end{array}\right)
$$

et qu'il existe $z_{n+1}$ dans $K_{n+1}^{*}$ tel que $d\left(z_{n+1}\right)=y_{n}^{1}-x_{n}^{0}$. On vérifie facilement que la condition définissant la relation d'équivalence engendrée s'obtient de la même manière mais en demandant que $z_{n+1}$ soit dans le sous-groupe de $K_{n+1}$ engendré par $K_{n+1}^{*}$. (Plus précisément, la clôture symétrique s'obtient en demandant que $z_{n+1}$ soit dans $K_{n+1}^{*}$ ou dans $-K_{n+1}^{*}$, et la clôture transitive de la clôture symétrique en demandant que $z$ soit dans $K_{n+1}^{*}+\left(-K_{n+1}^{*}\right)$ qui n'est autre que le sous-groupe engendré par $K_{n+1}^{*}$.) Or, puisqu'on a supposé le complexe $K$ à base, ce sous-groupe est $K_{n+1}$ tout entier. Par ailleurs, une $n$-flèche de $\nu \tau_{\leqslant n}^{\mathrm{i}}(K)$ est un tableau

$$
\left(\begin{array}{llll}
x_{0}^{0} & \ldots & x_{n-1}^{0} & t_{n}^{0} \\
x_{0}^{1} & \cdots & x_{n-1}^{1} & t_{n}^{1}
\end{array}\right),
$$

où les $x_{i}^{\varepsilon}$, pour $0 \leqslant i<n$ et $\varepsilon=0,1$, sont dans $K_{i}^{*}$, et $t_{n}^{0}=t_{n}^{1}$ est dans l'image de $K_{n}^{*}$ dans $K_{n} / d\left(K_{n+1}\right)$, satisfaisant aux conditions du paragraphe 2.4. L'application $\alpha_{n}$ envoie la $n$-flèche donnée par la classe de

$$
\left(\begin{array}{cccc}
x_{0}^{0} & \ldots & x_{n-1}^{0} & x_{n}^{0} \\
x_{0}^{1} & \ldots & x_{n-1}^{1} & x_{n}^{1}
\end{array}\right)
$$

sur la $n$-flèche

$$
\left(\begin{array}{cccc}
x_{0}^{0} & \ldots & x_{n-1}^{0} & \overline{x_{n}^{0}} \\
x_{0}^{1} & \ldots & x_{n-1}^{1} & \overline{x_{n}^{1}}
\end{array}\right),
$$

où $\overline{x_{n}^{0}}=\overline{x_{n}^{1}}$ désigne l'image de $x_{n}^{0}=x_{n}^{1}$ dans $K_{n} / d\left(K_{n+1}\right)$. Notre description de la relation d'équivalence définissant les $n$-flèches de $\tau_{\leqslant n}^{\mathrm{i}} \nu(K)$ montre que cette application est une bijection, ce qui achève la démonstration. 
Remarque 2.24. - Il n'est pas vrai en général que le morphisme de l'énoncé précédent soit un isomorphisme si on ne fait aucune hypothèse sur le complexe dirigé augmenté $K$; un contre-exemple pour $n=0$ est donné par le complexe de chaînes normalisé de l'ensemble simplicial $\Delta_{1}$ muni des sous-monoïdes de positivité $\mathbb{N}^{2} \subset \mathbb{Z}^{2}$ et $0 \subset \mathbb{Z}$ en degré 0 et 1 respectivement.

2.25. - Soient $f, g: K \rightarrow L$ deux morphismes de complexes de chaînes. Rappelons qu'une homotopie $h$ de $f$ vers $g$ consiste en la donnée de morphismes de groupes abéliens

$$
h_{i}: K_{i} \rightarrow L_{i+1}, \quad \text { pour } i \geqslant 0,
$$

satisfaisant à la condition

$$
d_{i+1} h_{i}+h_{i-1} d_{i}=g_{i}-f_{i}, \quad \text { pour } i \geqslant 0,
$$

en convenant que $h_{-1}=0$ et $d_{0}=0$. Pour $i=0$, on a donc

$$
d_{1} h_{0}=g_{0}-f_{0} .
$$

2.26. - Soient $f, g: K \rightarrow L$ deux morphismes de complexes dirigés augmentés. Une homotopie $h$ de $f$ vers $g$ est une homotopie entre les morphismes de complexes de chaînes sous-jacents satisfaisant à la condition supplémentaire suivante :

$$
h_{i}\left(K_{i}^{*}\right) \subset L_{i+1}^{*}, \quad \text { pour } i \geqslant 0 .
$$

Soient $f, g: K \rightarrow L$ deux morphismes de complexes dirigés augmentés. Une antihomotopie de $f$ vers $g$ est une homotopie de $f^{\text {co }}: K^{\text {co }} \rightarrow L^{\text {co }}$ vers $g^{\text {co }}: K^{\text {co }} \rightarrow L^{\text {co }}$. Explicitement, une antihomotopie $h$ de $f$ vers $g$ consiste en la donnée de morphismes de groupes abéliens

$$
h_{i}: K_{i} \rightarrow L_{i+1}, \quad \text { pour } i \geqslant 0,
$$

satisfaisant aux conditions suivantes :

- pour tout $i \geqslant 0$, on a

$$
d_{i+1} h_{i}-h_{i-1} d_{i}=(-1)^{i}\left(g_{i}-f_{i}\right),
$$

en convenant toujours que $h_{-1}=0$ et $d_{0}=0$ (en particulier, pour $i=0$, on a $\left.d_{1} h_{0}=g_{0}-f_{0}\right)$;

- pour tout $i \geqslant 0$, on a

$$
h_{i}\left(K_{i}^{*}\right) \subset L_{i+1}^{*} .
$$

Remarque 2.27. - On verra dans l'appendice B (voir le paragraphe B.4.1) qu'une homotopie entre morphismes de complexes dirigés augmentés de $K$ vers $L$ correspond à la donnée d'un morphisme de $\lambda\left(\mathrm{D}_{1}\right) \otimes K$ vers $L$, où $\mathrm{D}_{1}$ désigne la catégorie associée à l'ensemble ordonné $\{0<1\}$ et $\otimes$ le produit tensoriel des complexes dirigés augmentés (voir le paragraphe 6.2). On en déduira qu'on obtient la notion d'antihomotopie en inversant les deux facteurs de $\lambda\left(\mathrm{D}_{1}\right) \otimes K$, c'est-à-dire en considérant des morphismes de $K \otimes \lambda\left(\mathrm{D}_{1}\right)$ vers $L$. 
2.28. - Plus généralement, on va définir une notion de $n$-homotopie de complexes dirigés augmentés pour tout $n \geqslant 0$. Soient $K$ et $L$ deux complexes dirigés augmentés. On définit simultanément par récurrence sur $n \geqslant 0$ les notions de $n$-homotopie de $K$ vers $L$ et de $n$-homotopies de $K$ vers $L$ parallèles comme suit. Une 0 -homotopie de $K$ vers $L$ est un morphisme de complexes dirigés augmentés de $K$ vers $L$. Deux telles 0-homotopies sont par définition toujours parallèles. Si $n>0$, une $n$-homotopie $H$ de $K$ vers $L$ est la donnée de deux $(n-1)$-homotopies $h$ et $k$ parallèles de $K$ vers $L$, ainsi que de morphismes de groupes abéliens

$$
H_{i}: K_{i} \rightarrow L_{i+n}, \quad \text { pour } i \geqslant 0,
$$

satisfaisant aux conditions suivantes :

- pour tout $i \geqslant 0$, on a

$$
d_{i+n} H_{i}-(-1)^{n} H_{i-1} d_{i}=k_{i}-h_{i},
$$

en convenant que $H_{-1}=0$ et $d_{0}=0$ (en particulier, pour $i=0$, on a l'égalité $\left.d_{n} H_{0}=k_{0}-h_{0}\right)$;

- pour tout $i \geqslant 0$, on a

$$
H_{i}\left(K_{i}^{*}\right) \subset L_{i+n}^{*}
$$

On dit alors également que $H$ est une $n$-homotopie de $h$ vers $k$. Deux $n$-homotopies sont parallèles si elles vont toutes deux d'une même $(n-1)$-homotopie vers une même $(n-1)$-homotopie.

Pour $n=1$, on retrouve la notion d'homotopie de morphismes de complexes dirigés augmentés.

On définit de même une notion de $n$-antihomotopie pour tout $n \geqslant 0$ en remplaçant la relation liant $H$ à $h$ et $k$ dans la définition d'une $n$-homotopie par l'égalité

$$
d_{i+n} H_{i}-H_{i-1} d_{i}=(-1)^{i}\left(k_{i}-h_{i}\right), \quad \text { pour } i \geqslant 0,
$$

en convenant toujours que $H_{-1}=0$ et $d_{0}=0$ (en particulier, pour $i=0$, on a $\left.d_{n} H_{0}=k_{0}-h_{0}\right)$. On retrouve également, pour $n=1$, la notion d'antihomotopie.

Remarque 2.29. - Les notions de $n$-homotopie et $n$-antihomotopie peuvent également s'interpréter en termes du produit tensoriel des complexes dirigés augmentés (voir la remarque B.4.3).

On verra au paragraphe C.30 que les complexes dirigés augmentés munis des $n$-homotopies (resp. des $n$-antihomotopies), pour $n \geqslant 0$, forment une $\infty$-sesquicatégorie au sens du paragraphe C.3 et même une $\infty$-catégorie de Gray (resp. une $\infty$-catégorie de Gray gauche) au sens du paragraphe C.16. Dans la suite de ce chapitre, on décrit quelques unes des opérations de ces structures.

Dans la suite de ce chapitre, on fixe $K$ et $L$ deux complexes dirigés augmentés. 
2.30. - Soit $h$ une $n$-homotopie (resp. une $n$-antihomotopie) de $K$ vers $L$, pour un $n \geqslant 0$. On définit une $(n+1)$-homotopie (resp. une $(n+1)$-antihomotopie) $1_{h}$ de $h$ vers $h$ en posant, pour $i \geqslant 0$,

$$
\left(1_{h}\right)_{i}=0 .
$$

On appellera $1_{h}$ l'homotopie identité (resp. l'antihomotopie identité) de $h$.

En particulier, si $f: K \rightarrow L$ est un morphisme de complexes dirigés augmentés, on dispose d'une homotopie $1_{f}$ de $f$ vers $f$ et d'une antihomotopie, également notée $1_{f}$, de $f$ vers $f$.

2.31. - Soient $h$ et $h^{\prime}$ deux $(n-1)$-homotopies (resp. deux $(n-1)$-antihomotopies) de $K$ vers $L$, pour un $n \geqslant 1$, et soit $H$ une $n$-homotopie (resp. une $n$-antihomotopie) de $h$ vers $h^{\prime}$. Si $g: J \rightarrow K$ est un morphisme de complexes dirigés augmentés, on vérifie immédiatement, par récurrence, qu'on définit une $n$-homotopie (resp. une $n$-antihomotopie) $H g$ de $h^{\prime} g$ vers $h g$ (qui vont de $J$ vers $L$ ) en posant, pour $i \geqslant 0$,

$$
(H g)_{i}=H_{i} g_{i} .
$$

De même, si on a un morphisme de complexes dirigés augmentés $g: L \rightarrow M$, on définit une $n$-homotopie (resp. une $n$-antihomotopie) $g H$ de $g h$ vers $g h^{\prime}$ (qui vont de $K$ vers $M$ ) en posant, pour $i \geqslant 0$,

$$
(g H)_{i}=g_{i+n} H_{i} .
$$

2.32. - Soient $h_{0}, h_{1}$ et $h_{2}$ trois $(n-1)$-homotopies (resp. trois $(n-1)$-antihomotopies) de $K$ vers $L$, pour un $n \geqslant 1$, soient $H$ une $n$-homotopie (resp. une $n$-antihomotopie) de $h_{0}$ vers $h_{1}$ et $H^{\prime}$ une $n$-homotopie (resp. une $n$-antihomotopie) de $h_{1}$ vers $h_{2}$

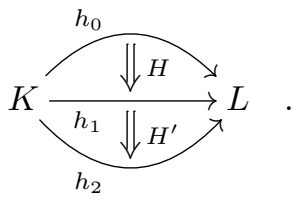

On vérifie immédiatement qu'on définit une $n$-homotopie (resp. une $n$-antihomotopie) $H^{\prime}+H$ de $h_{0}$ vers $h_{2}$ en posant, pour $i \geqslant 0$,

$$
\left(H^{\prime}+H\right)_{i}=H_{i}^{\prime}+H_{i} .
$$

2.33. - Soient $f_{0}, f_{1}$ et $f_{2}$ trois $(n-2)$-homotopies (resp. trois $(n-2)$-antihomotopies) de $K$ vers $L$, pour un $n \geqslant 2$, soient $h$ et $k$ deux $(n-1)$-homotopies (resp. deux $(n-1)$-antihomotopies) de $f_{0}$ vers $f_{1}$, et $h^{\prime}$ et $k^{\prime}$ deux $(n-1)$-homotopies (resp. deux $(n-1)$-antihomotopies) de $f_{1}$ vers $f_{2}$, et soient enfin $H$ une $n$-homotopie (resp. une $n$-antihomotopie) de $h$ vers $k$ et $H^{\prime}$ une $n$-homotopie (resp. une $n$-antihomotopie) 
de $h^{\prime}$ vers $k^{\prime}$

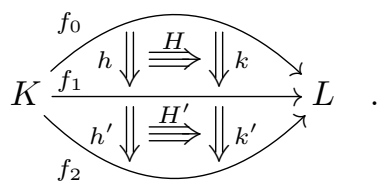

On vérifie immédiatement qu'on définit une $n$-homotopie (resp. une $n$-antihomotopie) $H^{\prime}+H$ de $h^{\prime}+h$ vers $k^{\prime}+k$ (voir le paragraphe précédent) en posant, pour $i \geqslant 0$,

$$
\left(H^{\prime}+H\right)_{i}=H_{i}^{\prime}+H_{i} \text {. }
$$

On prendra garde au fait qu'on représente par « + deux opérations différentes sur les $n$-homotopies et $n$-antihomotopies (voir le paragraphe précédent pour la première), ces opérations correspondant aux composition en codimension 1 et 2 respectivement. Néanmoins, le contexte rendra toujours clair l'opération désignée par « + » Par ailleurs, dans ce texte, nous n'utiliserons l'opération introduite dans ce paragraphe que dans le cas particulier où $H$ ou $H^{\prime}$ est une identité.

2.34. - Soient $f, g: K \rightarrow L$ et $f^{\prime}, g^{\prime}: L \rightarrow M$ des morphismes de complexes dirigés augmentés et soient $h$ une homotopie (resp. une antihomotopie) de $f$ vers $g$ et $h^{\prime}$ une homotopie (resp. une antihomotopie) de $f^{\prime}$ vers $g^{\prime}$

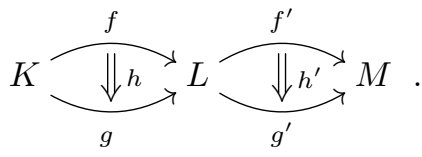

On définit une 2-homotopie (resp. une 2-antihomotopie) $h^{\prime} h$ de $h^{\prime} g+f^{\prime} h$ vers $g^{\prime} h+h^{\prime} f$ (resp. de $g^{\prime} h+h^{\prime} f$ vers $h^{\prime} g+f^{\prime} h$ ) en posant, pour $i \geqslant 0$,

$$
\left(h^{\prime} h\right)_{i}=h_{i+1}^{\prime} h_{i} .
$$

Vérifions-le dans le cas des antihomotopies (c'est celui que nous utiliserons dans ce texte). Pour $i \geqslant 0$, avec les conventions adéquates pour le cas $i=0$, on a

d'où l'assertion.

$$
\begin{aligned}
d_{i+2} h_{i+1}^{\prime} h_{i}-h_{i}^{\prime} h_{i-1} d_{i} & =d_{i+2} h_{i+1}^{\prime} h_{i}-h_{i}^{\prime} d_{i+1} h_{i}+h_{i}^{\prime} d_{i+1} h_{i}-h_{i}^{\prime} h_{i-1} d_{i} \\
& =\left(d_{i+2} h_{i+1}^{\prime}-h_{i}^{\prime} d_{i+1}\right) h_{i}+h_{i}^{\prime}\left(d_{i+1} h_{i}-h_{i-1} d_{i}\right) \\
& =(-1)^{i+1}\left(g_{i+1}^{\prime}-f_{i+1}^{\prime}\right) h_{i}+(-1)^{i} h_{i}^{\prime}\left(g_{i}-f_{i}\right) \\
& =(-1)^{i}\left[\left(h_{i}^{\prime} g_{i}+f_{i+1}^{\prime} h_{i}\right)-\left(g_{i+1}^{\prime} h_{i}+h_{i}^{\prime} f_{i}\right)\right],
\end{aligned}
$$




\section{CHAPITRE 3}

\section{LIMITES INDUCTIVES DE COMPLEXES DE STEINER}

Le but de ce chapitre est de dégager des résultats de commutation du foncteur $\nu: \mathcal{C}_{\mathrm{da}} \rightarrow \infty$-C $a t$ de Steiner à certaines classes de limites inductives.

3.1. - On peut montrer que la catégorie des complexes dirigés augmentés est localement présentable. En particulier, elle est cocomplète. Dans la suite du texte, nous aurons besoin d'une description explicite de ses limites inductives. Soit donc $F: I \rightarrow \mathcal{C}_{\text {da }}$ un foncteur de source une petite catégorie $I$. Notons $\left(K(i), K(i)^{*}, e(i)\right)$ le complexe dirigé augmenté $F(i)$. On définit un complexe dirigé augmenté $\left(K, K^{*}, e\right)$ de la manière suivante. Le complexe de chaînes $K$ est la limite inductive des complexes de chaînes $K(i)$. Rappelons que cette limite inductive est calculée degré par degré. Pour $n \geqslant 0$, le sous-monoïde $K_{n}^{*}$ de $K_{n}$ est le sous-monoïde engendré par les images des $K(i)_{n}^{*}$ par les morphismes $K(i)_{n} \rightarrow K_{n}$. Enfin, l'augmentation $e$ est donnée par la propriété universelle de $K_{0}=\lim _{i \in I} K(i)_{0}$. On vérifie facilement que $\left(K, K^{*}, e\right)$ est bien la limite inductive de $F$.

3.2. - Soient $K$ et $L$ deux complexes dirigés augmentés à base. Un morphisme $f: K \rightarrow L$ sera dit prérigide s'il envoie tout élément de la base de $K$ sur un élément de la base de $L$. (On rappelle que si un complexe dirigé augmenté admet une base, cette base est unique.) On dira que $f$ est rigide si, de plus, pour tout élément $b$ de la base de $K$, on a $f\left(\langle b\rangle_{k}^{\varepsilon}\right)=\langle f(b)\rangle_{k}^{\varepsilon}$, pour $\varepsilon=0,1$ et $0 \leqslant k \leqslant|b|$. Lorsque $K$ est à base unitaire, cette dernière condition signifie exactement que, pour tout élément $b$ de la base de $K$, on a $\nu(f)(\langle b\rangle)=\langle f(b)\rangle$. On verra plus loin (proposition 3.10) que les notions de morphismes prérigides et rigides coïncident lorsque $f$ est un monomorphisme.

Remarque 3.3. - Si $f: K \rightarrow L$ est un morphisme rigide entre complexes de Steiner, de sorte que $\nu(K)$ et $\nu(L)$ sont en vertu du théorème 2.12 engendrés librement au sens des polygraphes par leurs atomes, le $\infty$-foncteur $\nu(f): \nu(K) \rightarrow \nu(L)$ est rigide 
au sens où il provient d'un morphisme de polygraphes. Autrement dit, ce $\infty$-foncteur respecte les générateurs au sens des polygraphes de $\nu(L)$ et $\nu(K)$ donnés par les atomes.

Exemple 3.4. - L'exemple suivant montre qu'un morphisme prérigide n'est pas nécessairement rigide, même entre complexes de Steiner forts. Considérons les 2-catégories

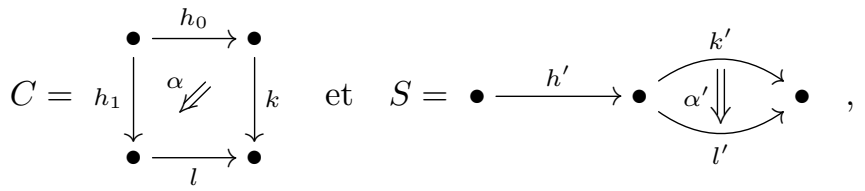

et le 2-foncteur $u: C \rightarrow S$ défini par

$$
u\left(h_{0}\right)=u\left(h_{1}\right)=h^{\prime}, \quad u(k)=k^{\prime}, \quad u(l)=l^{\prime} \quad \text { et } \quad u(\alpha)=\alpha^{\prime} *_{0} h^{\prime} .
$$

On vérifie facilement que le morphisme $f=\lambda(u): \lambda(C) \rightarrow \lambda(S)$ est prérigide. En particulier, on a $f([\alpha])=\left[\alpha^{\prime}\right]$. Néanmoins, on n'a pas $\nu(f)(\langle[\alpha]\rangle)=\left\langle\left[\alpha^{\prime}\right]\right\rangle$. Par exemple, on a $f\left(\langle[\alpha]\rangle_{1}^{0}\right)=\left[k^{\prime}\right]+\left[h^{\prime}\right]$ mais $\left\langle\left[\alpha^{\prime}\right]\right\rangle_{1}^{0}=\left[k^{\prime}\right]$. On verra plus loin que les 2-catégories $C$ et $S$ sont des $\infty$-catégories de Steiner fortes (cela résulte des propositions 4.13 et A.3, ainsi que de l'isomorphisme $C \simeq \nu\left(\lambda\left(\mathrm{D}_{1}\right) \otimes \lambda\left(\mathrm{D}_{1}\right)\right)$, où $\mathrm{D}_{1}$ désigne la catégorie associée à l'ensemble ordonné $\{0<1\})$. En particulier, les complexes $\lambda(C)$ et $\lambda(S)$ sont de Steiner forts et le $\infty$-foncteur $\nu(f)$ s'identifie à $u$.

3.5. - On dira qu'un foncteur $F: I \rightarrow \mathcal{C}_{\text {da }}$ de source une petite catégorie $I$ est un système prérigide (resp. un système rigide) s'il satisfait aux conditions suivantes :

(a) pour tout objet $i$ de $I$, le complexe dirigé augmenté $F(i)$ est à base ;

(b) pour tout morphisme $f: i \rightarrow i^{\prime}$ de $I$, le morphisme $F(f): F(i) \rightarrow F\left(i^{\prime}\right)$ est prérigide (resp. rigide).

Proposition 3.6. - Si F $: I \rightarrow \mathcal{C}_{\mathrm{da}}$ est un système prérigide, alors le complexe dirigé augmenté $\lim _{i \in I} F(i)$ est à base. Plus précisément, l'ensemble gradué

$$
\left(\underset{i \in I}{\lim _{i \in I}} B(i)_{n}\right)_{n \geqslant 0}
$$

où $\left(B(i)_{n}\right)_{n \geqslant 0}$ désigne la base de $F(i)$, fournit une base de ce complexe dirigé augmenté. En particulier, les morphismes canoniques $F\left(i_{0}\right) \rightarrow \underline{\lim }_{i \in I} F(i)$, pour $i_{0}$ un objet de I, sont prérigides.

Démonstration. - Fixons $n \geqslant 0$. Par hypothèse, on a

$$
F(i)_{n} \simeq \mathbb{Z}^{\left(B(i)_{n}\right)} \quad \text { et } \quad F(i)_{n}^{*} \simeq \mathbb{N}^{\left(B(i)_{n}\right)} .
$$


Puisque $F$ est un système prérigide, le foncteur $i \mapsto F(i)_{n}$ se factorise par le foncteur $\mathbb{Z}$-module libre. Or celui-ci commute aux limites inductives et on a donc

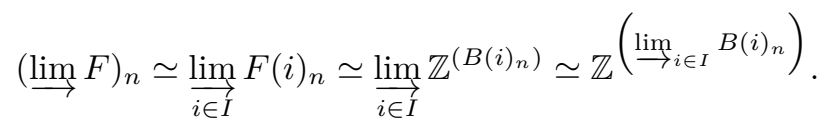

Par ailleurs, $\left(\lim _{\longrightarrow} F\right)_{n}^{*}$ est le sous-monoïde engendré par les $\mathbb{N}^{\left(B(i)_{n}\right)}$ qui n'est autre que $\mathbb{N}\left(\lim _{i \in I} B(i)_{n}\right)$, d'où le résultat.

3.7. - On dira qu'un foncteur $F: I \rightarrow \mathcal{C}_{\text {da }}$ de source une petite catégorie $I$ est un système de Steiner (resp. un système de Steiner fort) si $F$ satisfait aux conditions suivantes :

(a) $F$ est un système rigide;

(b) pour tout objet $i$ de $I$, le complexe $F(i)$ est de Steiner (resp. de Steiner fort);

(c) le complexe $\lim _{i \in I} F(i)$ est de Steiner (resp. de Steiner fort);

(d) pour tout objet $i_{0}$ de $I$, le morphisme canonique $F\left(i_{0}\right) \rightarrow \lim _{i \in I} F(i)$ est rigide. Si de plus la petite catégorie $I$ est connexe, on parlera de système de Steiner connexe (resp. de système de Steiner fort connexe).

Théorème 3.8. - Le foncteur $\nu: \mathcal{C}_{\mathrm{da}} \rightarrow \infty$-Cat commute aux limites inductives des systèmes de Steiner.

Démonstration. - Soit $F: I \rightarrow \mathcal{C}_{\text {da }}$ un système de Steiner. Il s'agit de montrer que le morphisme canonique

$$
\underset{i \in I}{\lim _{i \in I}} \nu(F(i)) \rightarrow \nu\left(\underset{i \in I}{\lim _{i \in I}} F(i)\right)
$$

est un isomorphisme de $\infty$-catégories. Pour ce faire, nous allons appliquer la proposition 1.6. En vertu du théorème 2.12, les $\infty$-catégories $\nu(F(i))$, pour $i$ un objet de $I$, et $\nu\left(\lim _{\longrightarrow} F\right)$ sont engendrées librement au sens des polygraphes par les bases de $F(i)$ et $\underset{\lim F}{\longrightarrow}$ respectivement (ou, plus précisément, par les atomes associés à ces bases). Par hypothèse, pour tout objet $i_{0}$ de $I$, le morphisme canonique $F\left(i_{0}\right) \rightarrow \underline{\lim } F$ est rigide. Cela implique la condition $(a)$ de la proposition 1.6. De même, le fait que, par hypothèse, pour tout morphisme $i \rightarrow i^{\prime}$ de $I$, le morphisme $F(i) \rightarrow F\left(i^{\prime}\right)$ est rigide entraîne la condition $(b)$. Enfin, la condition $(c)$ résulte de la proposition 3.6. On peut donc appliquer la proposition 1.6, ce qui achève la démonstration.

Dans la suite de ce chapitre, on va dégager des conditions suffisantes permettant d'appliquer ce théorème.

Lemme 3.9. - Soit $f: K \rightarrow L$ un monomorphisme prérigide entre complexes dirigés augmentés à base. Alors, pour tout élément homogène $x$ de $K$, on a

$$
f\left(x_{-}\right)=f(x)_{-} \quad \text { et } \quad f\left(x_{+}\right)=f(x)_{+} .
$$


Démonstration. - Écrivons $x=x_{+}-x_{-}$. Il s'agit de montrer que $f\left(x_{+}\right)-f\left(x_{-}\right)$est la décomposition $f(x)=f(x)_{+}-f(x)_{-}$, c'est-à-dire que les coefficients apparaissant dans l'écriture de $f\left(x_{+}\right)$et $f\left(x_{-}\right)$selon la base de $L$ sont positifs, et que les supports de $f\left(x_{+}\right)$et $f\left(x_{-}\right)$sont disjoints. Le premier point est immédiat et le second résulte du fait que $f$ est un monomorphisme prérigide, d'où le résultat.

Proposition 3.10. — Un monomorphisme prérigide entre complexes dirigés augmentés à base est rigide.

Démonstration. - Soit $f: K \rightarrow L$ un monomorphisme prérigide entre complexes dirigés augmentés à base. Il s'agit de montrer que, pour tout élément $b$ de la base de $K$, on a $f\left(\langle b\rangle_{k}^{\varepsilon}\right)=\langle f(b)\rangle_{k}^{\varepsilon}$, pour $\varepsilon=0,1$ et $0 \leqslant k \leqslant|b|$. Par définition, les $\langle b\rangle_{k}^{\varepsilon}$ sont obtenus en itérant la différentielle et les opérations $x \mapsto x_{-}$ou $x \mapsto x_{+}$selon la valeur de $\varepsilon$. Or, le morphisme $f$ est compatible aux différentielles par définition et aux opérations $x \mapsto x_{-}$et $x \mapsto x_{+}$en vertu du lemme précédent, d'où le résultat.

3.11. - On dira qu'un foncteur $F: I \rightarrow \mathcal{E} n s$ d'une petite catégorie $I$ vers la catégorie des ensembles est séparant $\mathrm{si}$, pour tout objet $i_{0}$ de $I$, l'application canonique $F\left(i_{0}\right) \rightarrow \lim _{i \in I} F(i)$ est une injection.

On dira qu'un foncteur $F: I \rightarrow \mathcal{C}_{\mathrm{da}}$ est un système séparant s'il satisfait aux conditions suivantes :

(a) $F$ est un système prérigide;

(b) pour tout $n \geqslant 0$, le foncteur $I \rightarrow \mathcal{E}$ ns qui envoie $i$ sur la base de $F(i)_{n}$ est séparant.

Proposition 3.12. - Si $F: I \rightarrow \mathcal{C}_{\mathrm{da}}$ est un système séparant, alors, pour tout objet $i_{0}$ de $I$, le morphisme canonique $F\left(i_{0}\right) \rightarrow \lim _{i \in I} F(i)$ est un monomorphisme rigide.

Démonstration. - Fixons un objet $i_{0}$ de $I$. On a déjà vu (proposition 3.6) que le morphisme $F\left(i_{0}\right) \rightarrow \underline{\lim } F$ est prérigide. En vertu de la proposition 3.10, il s'agit donc de montrer que ce morphisme est un monomorphisme. Notons $\left(B(i)_{n}\right)_{n \geqslant 0}$ la base du complexe $F(i)$. En vertu de la proposition 3.6,

$$
\left(\underset{i \in I}{\lim _{i \in I}} B(i)_{n}\right)_{n \geqslant 0}
$$

est une base du complexe $\lim _{\longrightarrow} F$. Il s'agit donc de montrer que, pour tout $n \geqslant 0$, le morphisme

$$
\mathbb{Z}^{\left(B\left(i_{0}\right)_{n}\right)} \rightarrow \mathbb{Z}^{\left(\lim _{i \in I} B(i)_{n}\right)}
$$

est un monomorphisme. Mais, puisque le système $F$ est séparant, l'application $B\left(i_{0}\right)_{n} \rightarrow \underline{\lim }_{i \in I} B(i)_{n}$ est une injection et on obtient le résultat puisque le foncteur $\mathbb{Z}$-module libre préserve les monomorphismes. 
Proposition 3.13. - Soit $F: I \rightarrow \mathcal{C}_{\mathrm{da}}$ un système séparant. Pour tout morphisme $f: i \rightarrow i^{\prime}$ de $I$, le morphisme $F(f): F(i) \rightarrow F\left(i^{\prime}\right)$ est un monomorphisme. En particulier, un tel système est rigide.

Démonstration. - On a un triangle commutatif

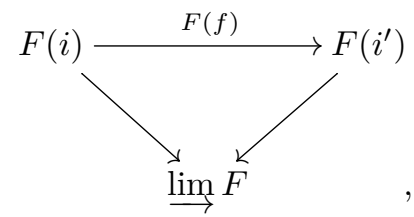

où, en vertu de la proposition précédente, les deux flèches obliques sont des monomorphismes. On en déduit qu'il en est de même de $F(f)$. La seconde assertion résulte de la première en vertu de la proposition 3.10 .

Proposition 3.14. - Si $F: I \rightarrow \mathcal{C}_{\mathrm{da}}$ est un système séparant à valeurs dans les complexes dirigés augmentés à base unitaire, alors le complexe $\lim _{i \in I} F(i)$ est à base unitaire.

Démonstration. - Notons $\left(B(i)_{n}\right)_{n \geqslant 0}$ la base du complexe $F(i)$. En vertu de la proposition 3.6, le complexe $\lim _{\longrightarrow} F$ est à base et sa base est

$$
\left(\underset{i \in I}{\lim _{i \in I}} B(i)_{n}\right)_{n \geqslant 0} .
$$

Fixons $n \geqslant 0$. Soit $b$ un élément de $\lim _{i \in I} B(i)_{n}$. Il s'agit de montrer qu'on a

$$
e\left(\langle b\rangle_{0}^{0}\right)=1 \quad \text { et } \quad e\left(\langle b\rangle_{0}^{1}\right)=1,
$$

où $e$ est l'augmentation de $\lim _{\longrightarrow} F$. L'élément $b$ provient d'un élément $b_{0}$ de $B\left(i_{0}\right)_{n}$

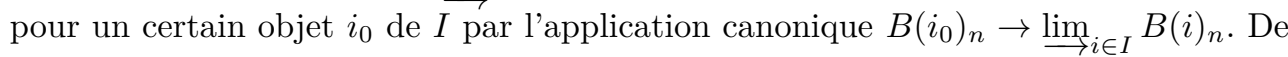
plus, puisque $B\left(i_{0}\right)$ est à base unitaire, on a

$$
e\left(i_{0}\right)\left(\left\langle b_{0}\right\rangle_{0}^{0}\right)=1 \quad \text { et } \quad e\left(i_{0}\right)\left(\left\langle b_{0}\right\rangle_{0}^{1}\right)=1,
$$

où $e\left(i_{0}\right)$ désigne l'augmentation de $F\left(i_{0}\right)$. En vertu de la proposition 3.12 , le morphisme $F\left(i_{0}\right) \rightarrow \underline{\lim }_{i \in I} F(i)$ est rigide, ce qui permet de conclure.

Proposition 3.15. - Soit $F: I \rightarrow \mathcal{C}_{\mathrm{da}}$ un foncteur satisfaisant aux conditions suivantes :

(a) F est un système séparant;

(b) pour tout objet $i$ de I, le complexe $F(i)$ est de Steiner (resp. de Steiner fort);

(c) le complexe $\lim _{i \in I} F(i)$ est à base sans boucle (resp. à base fortement sans boucle).

Alors $F$ est un système de Steiner (resp. un système de Steiner fort). En particulier, le foncteur $\nu: \mathcal{C}_{\mathrm{da}} \rightarrow \infty$-C at commute à la limite inductive de $F$. 
Démonstration. - En vertu de la proposition 3.13, la condition ( $a$ ) entraîne que $F$ est un système rigide. Par ailleurs, d'après la proposition 3.14, dont les hypothèses découlent des conditions $(a)$ et $(b)$, le complexe $\lim _{\longrightarrow} F$ est à base unitaire. La condition $(c)$ implique donc que ce complexe est de Steiner (resp. de Steiner fort). Enfin, la proposition 3.12, dont l'hypothèse est précisément la condition $(a)$, montre que, pour tout objet $i_{0}$ de $I$, le morphisme canonique $F\left(i_{0}\right) \rightarrow \lim F$ est rigide, ce qui achève de prouver la première assertion. La seconde résulte de la première et du théorème 3.8 .

Terminons ce chapitre par l'étude du cas des sommes amalgamées.

3.16. - Soit $f: K \rightarrow L$ un morphisme de complexes dirigés augmentés à base. On dira que $f$ est une inclusion rigide ordonnée si

(a) $f$ est un monomorphisme rigide;

(b) si $x$ et $y$ sont des éléments de la base de $K$, alors on a

$$
x \leqslant_{\mathbb{N}} y \quad \text { si et seulement si } f(x) \leqslant_{\mathbb{N}} f(y),
$$

où $\leqslant_{\mathbb{N}}$ désigne la relation de préordre du paragraphe 2.13 .

Notons que, en vertu du lemme 3.9, le sens direct dans l'équivalence de la condition $(b)$ est automatique si la condition $(a)$ est satisfaite; le contenu de cette condition se trouve donc dans l'implication réciproque.

Lemme 3.17. - Soit $K$ un complexe dirigé augmenté à base fortement sans boucle pour lequel la relation d'ordre $\leqslant_{\mathbb{N}}$ est totale. Alors tout monomorphisme rigide de $K$ vers un complexe dirigé augmenté à base fortement sans boucle est une inclusion rigide ordonnée.

Démonstration. - Cela résulte immédiatement du fait que si $i: E \rightarrow F$ est une injection croissante d'un ensemble totalement ordonné $E$ vers un ensemble ordonné $F$, alors, pour $x$ et $y$ dans $E$, on a $x \leqslant y$ si et seulement si $i(x) \leqslant i(y)$.

Lemme 3.18. - Soit

$$
K \stackrel{i}{\longleftarrow} M \stackrel{j}{\longrightarrow} L
$$

un diagramme de complexes dirigés augmentés à base fortement sans boucle et d'inclusions rigides ordonnées. Alors la somme amalgamée $K \amalg_{M} L$ est un complexe à base fortement sans boucle et les morphismes canoniques $K \rightarrow K \amalg_{M} L$ et $L \rightarrow K \amalg_{M} L$ sont des inclusions rigides ordonnées. 
Démonstration. - La proposition 3.6 et la description explicite des sommes amalgamées ensemblistes entraînent immédiatement que ce diagramme, vu comme un foncteur de $I=\bullet \leftarrow \bullet \rightarrow \bullet$ vers $\mathcal{C}_{\mathrm{da}}$, est un système séparant. Il résulte donc de la proposition 3.12 que les morphismes canoniques

$$
K \rightarrow K \amalg_{M} L \quad \text { et } \quad L \rightarrow K \amalg_{M} L
$$

sont des monomorphismes rigides. On va considérer dans la suite de cette démonstration les monomorphismes en jeu comme des inclusions.

Montrons que le complexe $K \amalg_{M} L$ est fortement sans boucle. En vertu de la proposition 3.6, la base de ce complexe est la somme amalgamée des bases de $K$ et $L$ au-dessous de celle de $M$. On définit une relation $\preccurlyeq$ sur cette base en posant

$$
x \preccurlyeq y \stackrel{\text { déf }}{\Longleftrightarrow} \begin{cases}\text { ou } & x, y \text { sont dans la base de } K \text { et } x \leqslant_{\mathbb{N}}^{K} y \\ & x, y \text { sont dans la base de } L \text { et } x \leqslant_{\mathbb{N}}^{L} y,\end{cases}
$$

où on a noté $\leqslant_{\mathbb{N}}^{K}$ et $\leqslant_{\mathbb{N}}^{L}$ la relation d'ordre du paragraphe 2.13 pour $K$ et $L$ respectivement. Notons que si $x$ et $y$ sont à la fois dans la base de $K$ et celle de $L$, cela signifie qu'ils sont dans la base de $M$ et le fait que $i$ et $j$ sont des inclusions rigides ordonnées montre qu'on a

$$
x \preccurlyeq y \quad \text { si et seulement si } x \leqslant_{\mathbb{N}}^{M} y \text {. }
$$

En particulier, si $x$ et $y$ sont dans la base de $K$, on a

$$
x \preccurlyeq y \quad \text { si et seulement si } x \leqslant_{\mathbb{N}}^{K} y \text {. }
$$

De même, si $x$ et $y$ sont dans la base de $L$, on a

$$
x \preccurlyeq y \quad \text { si et seulement si } x \leqslant_{\mathbb{N}}^{L} y .
$$

La clôture transitive de la relation $\preccurlyeq$ n'est autre que la relation de préordre $\leqslant_{\mathbb{N}}$ du paragraphe 2.13 pour $K \amalg_{M} L$. En effet, cela résulte du fait que pour $x$ et $y$ deux éléments de la base de $K \amalg_{M} L$, si $x$ appartient à $\operatorname{supp}\left(d(y)_{-}\right)$ou si $y$ appartient à $\operatorname{supp}\left(d(x)_{+}\right)$, alors $x$ et $y$ sont soit tous les deux dans la base de $K$, soit tous les deux dans la base de $L$. Pour conclure, il suffit donc de montrer que la relation $\preccurlyeq$ est sans cycle non trivial. Soit donc

$$
x_{0} \preccurlyeq x_{1} \preccurlyeq \cdots \preccurlyeq x_{n}=x_{0}
$$

un cycle non trivial, c'est-à-dire tel que $n \geqslant 2$, qu'on va supposer minimal. Par symétrie, on peut supposer que $x_{0}$ est dans la base de $K$. La relation $\leqslant_{\mathbb{N}}^{K}$ étant sans cycle non trivial, il existe un élément du cycle qui n'est pas dans la base de $K$ et est donc dans celle de $L$. Soit $i$ le plus petit indice tel que $x_{i}$ ne soit pas dans la base de $K$. On a nécessairement $0<i<n$. Puisqu'on a $x_{i-1} \preccurlyeq x_{i} \preccurlyeq x_{i+1}$ et que $x_{i}$ n'est pas dans la base de $K$, les éléments $x_{i-1}$ et $x_{i+1}$ sont nécessairement dans la base de $L$. Les éléments $x_{i-1} \preccurlyeq x_{i} \preccurlyeq x_{i+1}$ sont donc tous les trois dans la base de $L$. 
Si $n=2$, cela montre que $x_{0} \preccurlyeq x_{1} \preccurlyeq x_{2}$ est un cycle non trivial pour la relation $\leqslant_{\mathbb{N}}^{L}$, ce qui est exclu par hypothèse. Si $n>2$, par transitivité de la relation $\leqslant_{\mathbb{N}}^{L}$, on obtient un cycle non trivial plus court en supprimant $x_{i}$ de notre cycle qui était pourtant supposé minimal, ce qui est absurde.

Montrons maintenant que $K \rightarrow K \amalg_{M} L$ est une inclusion rigide ordonnée (le cas de $L \rightarrow K \amalg_{M} L$ en résultera par symétrie). Il s'agit donc de montrer que si on a

$$
x=x_{0} \preccurlyeq x_{1} \preccurlyeq \cdots \preccurlyeq x_{n}=y
$$

avec $x$ et $y$ dans la base de $K$, alors on a $x \leqslant_{\mathbb{N}}^{K} y$, c'est-à-dire $x \preccurlyeq y$. Ceci se démontre par un argument similaire à celui qu'on a utilisé pour montrer que $\preccurlyeq$ est sans cycle non trivial : on considère une chaîne minimale pour laquelle on n'a pas $x \preccurlyeq y$; elle doit nécessairement contenir un élément qui n'est pas dans la base de $K$; on en déduit une chaîne plus courte et donc une contradiction.

Théorème 3.19. — Un diagramme

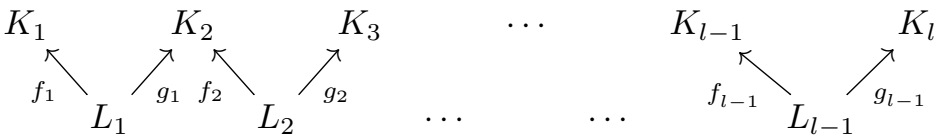

de complexes de Steiner forts dont les morphismes sont des inclusions rigides ordonnées est un système de Steiner fort. En particulier, la limite inductive d'un tel diagramme est un complexe de Steiner fort et le foncteur $\nu: \mathcal{C}_{\mathrm{da}} \rightarrow \infty$-Cat commute à cette limite inductive.

Démonstration. - En décomposant la limite inductive du diagramme en une somme amalgamée itérée, le lemme précédent et le stabilité des inclusions rigides ordonnées par composition impliquent que la limite inductive du diagramme est un complexe à base fortement sans boucle. La proposition 3.6 et la description explicite des sommes amalgamées itérées ensemblistes entraînent que ce diagramme est un système séparant. Les hypothèses de la proposition 3.15 sont donc satisfaites et on conclut en invoquant cette proposition.

Corollaire 3.20. - Soit

$$
K \longleftarrow M \longrightarrow L
$$

un diagramme de complexes de Steiner forts et de monomorphismes rigides. On suppose que la relation d'ordre $\leqslant_{\mathbb{N}}$ sur la base de $M$ est totale. Alors $K \amalg_{M} L$ est un complexe de Steiner fort et le morphisme canonique $\nu(K) \amalg_{\nu(M)} \nu(L) \rightarrow \nu\left(K \amalg_{M} L\right)$ est un isomorphisme.

Démonstration. — En vertu du lemme 3.17, les morphismes du diagramme sont des inclusions rigides ordonnées et le résultat découle donc du théorème précédent. 


\section{CHAPITRE 4}

\section{LA CATÉGORIE $\Theta$ DE JOYAL}

Ce chapitre est consacré à des rappels sur la catégorie $\Theta$, introduite par Joyal dans $[\mathbf{2 3}]$.

4.1. - Pour $i \geqslant 0$, on notera $\mathrm{D}_{i}$ la $\infty$-catégorie qui coreprésente le foncteur $\mathrm{FI}_{i}: \infty$-Cat $\rightarrow \mathcal{E}$ ns qui envoie une $\infty$-catégorie $C$ sur l'ensemble $C_{i}$ de ses $i$-flèches. Cette $\infty$-catégorie est en fait une $i$-catégorie. Elle admet une unique $i$-flèche non triviale qu'on appellera sa cellule principale. Pour tout $k$ tel que $0 \leqslant k<i$, la $i$-catégorie $\mathrm{D}_{i}$ admet exactement deux $k$-flèches non triviales qui sont la source et le but itérés en dimension $k$ de sa cellule principale. Voici les graphes sous-jacents (sans les identités) de $\mathrm{D}_{i}$ pour $i=0,1,2,3$ :

$$
\mathrm{D}_{0}=\bullet, \mathrm{D}_{1}=\bullet \longrightarrow, \quad \mathrm{D}_{2}=\bullet \overbrace{\downarrow}^{\bullet} \text { et } \mathrm{D}_{3}=\bullet \sqrt{\|} \bullet
$$

Il sera parfois utile d'étendre la notation $\mathrm{D}_{i}$ au cas $i=-1$ en convenant que $\mathrm{D}_{-1}$ est la $\infty$-catégorie vide.

Pour $i>0$, on a des $\infty$-foncteurs $\sigma_{i}, \tau_{i}: \mathrm{D}_{i-1} \rightarrow \mathrm{D}_{i}$ qui coreprésentent respectivement les transformations naturelles source et but $\mathrm{FI}_{i} \rightarrow \mathrm{FI}_{i-1}$. Explicitement, $\sigma_{i}: \mathrm{D}_{i-1} \rightarrow \mathrm{D}_{i}$ (resp. $\tau_{i}: \mathrm{D}_{i-1} \rightarrow \mathrm{D}_{i}$ ) envoie la cellule principale de $\mathrm{D}_{i-1}$ sur la source (resp. le but) de la cellule principale de $\mathrm{D}_{i}$.

Pour $i \geqslant j \geqslant 0$, on notera $\sigma_{j}^{i}, \tau_{j}^{i}: \mathrm{D}_{j} \rightarrow \mathrm{D}_{i}$ les $\infty$-foncteurs définis par

$$
\sigma_{j}^{i}=\sigma_{i} \cdots \sigma_{j+2} \sigma_{j+1} \quad \text { et } \quad \tau_{j}^{i}=\tau_{i} \cdots \tau_{j+2} \tau_{j+1} .
$$

4.2. - Soient $l \geqslant 1$ et $i_{1}, \ldots, i_{l}, j_{1}, \ldots, j_{l-1}$ des entiers positifs vérifiant les inégalités

$$
i_{k}>j_{k}<i_{k+1}, \quad \text { pour } 0<k<l .
$$

On associe à ces entiers le diagramme

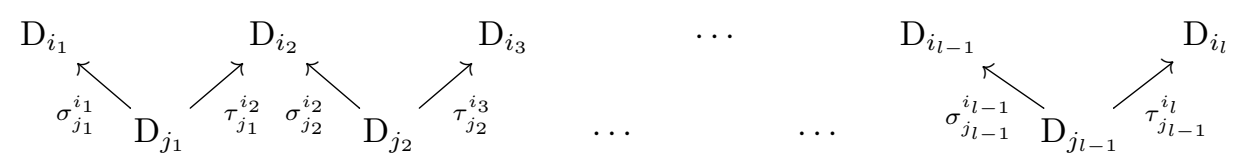


dans $\infty$-Cat. On appellera somme globulaire la limite inductive d'un tel diagramme et on la notera simplement

$$
\mathrm{D}_{i_{1}} \amalg_{\mathrm{D}_{j_{1}}} \cdots \amalg_{\mathrm{D}_{j_{l-1}}} \mathrm{D}_{i_{l}} .
$$

Les $\infty$-catégories obtenues de cette manière seront appelées des schémas de composition globulaires.

4.3. - Pour $i \geqslant 0$, on notera

$$
\kappa_{i}: \mathrm{D}_{i+1} \rightarrow \mathrm{D}_{i}
$$

le $\infty$-foncteur coreprésentant la transformation naturelle identité $\mathrm{FI}_{i} \rightarrow \mathrm{FI}_{i+1}$. Concrètement, $\kappa_{i}$ envoie la cellule principale de $\mathrm{D}_{i+1}$ sur l'identité de la cellule principale de $\mathrm{D}_{i}$. Pour $i \geqslant j \geqslant 0$, on notera $\kappa_{i}^{j}: \mathrm{D}_{i} \rightarrow \mathrm{D}_{j}$ le $\infty$-foncteur défini par

$$
\kappa_{i}^{j}=\kappa_{j} \cdots \kappa_{i-2} \kappa_{i-1} \text {. }
$$

Pour $i>j \geqslant 0$, on notera

$$
\nabla_{j}^{i}: \mathrm{D}_{i} \rightarrow \mathrm{D}_{i} \amalg_{\mathrm{D}_{j}} \mathrm{D}_{i}
$$

le $\infty$-foncteur qui coreprésente la transformation naturelle $*_{j}^{i}: \mathrm{Fl}_{i} \times_{\mathrm{FI}_{j}} \mathrm{Fl}_{i} \rightarrow \mathrm{Fl}_{i}$ de $j$-composition des $i$-flèches. Concrètement, $\nabla_{j}^{i}$ envoie la cellule principale de $\mathrm{D}_{i}$ sur le $j$-composé des deux $i$-flèches de $\mathrm{D}_{i} \amalg_{\mathrm{D}_{j}} \mathrm{D}_{i}$ correspondant aux cellules principales des deux copies de $\mathrm{D}_{i}$.

4.4. - La catégorie $\Theta$ de Joyal est la sous-catégorie pleine de $\infty$-Cat formée des schémas de composition globulaires. On notera $\Theta_{+}$la sous-catégorie pleine de $\infty$-C at dont les objets sont les schémas de composition globulaires ainsi que la $\infty$-catégorie vide.

Remarque 4.5. - La catégorie $\Theta$ a été introduite par Joyal dans $[\mathbf{2 3}]$ avec un point de vue différent de celui adopté ici. C'est Berger [10] et Makkai-Zawadowski [29] qui ont prouvé indépendamment l'équivalence des deux définitions.

Proposition 4.6. - La catégorie $\Theta$ (et donc la catégorie $\Theta_{+}$) est une sous-catégorie dense de $\infty$-Cat. Autrement dit, si $C$ est une $\infty$-catégorie, le morphisme canonique

$$
\lim _{(S, S \rightarrow C) \in \Theta / C} S \longrightarrow C
$$

est un isomorphisme de $\infty$-catégories.

Démonstration. - Voir le théorème 1.12 de [10] ou le théorème 4.10 de [43] appliqué à l'exemple 4.18 .

Proposition 4.7. - Soit $J$ une partie de $\mathbb{N}^{*}$. Pour tout objet $S$ de $\Theta_{+}$, la $\infty$-catégorie $D_{J}(S)$ (voir le paragraphe 1.8) est isomorphe à un objet de $\Theta_{+}$. 
Démonstration. - Cela résulte, par exemple, du fait que les $\infty$-catégories isomorphes à un objet de $\Theta$ peuvent être caractérisées de manière intrinsèque à la catégorie $\infty$ - $\mathcal{C}$ at munie du foncteur d'oubli vers les $\infty$-graphes (voir par exemple [43, exemple 4.18]). On donnera une démonstration directe dans $[\mathbf{9}]$ basée sur la description de $\Theta$ en termes de produit en couronnes de [11] (voir également [17]).

4.8. - La catégorie $\Theta^{\circ}$ est naturellement munie d'un objet $\infty$-catégorie interne. Plus précisément, les objets

$$
\mathrm{D}_{i}, \quad \text { pour } i \geqslant 0,
$$

et les morphismes

$$
\begin{array}{rlrl}
\sigma_{i}: \mathrm{D}_{i-1} \rightarrow \mathrm{D}_{i}, & & \text { pour } i>0, \\
\tau_{i}: \mathrm{D}_{i-1} \rightarrow \mathrm{D}_{i}, & & \text { pour } i>0, \\
\kappa_{i}: \mathrm{D}_{i+1} \rightarrow \mathrm{D}_{i}, & \text { pour } i \geqslant 0, \\
\nabla_{j}^{i}: \mathrm{D}_{i} \rightarrow \mathrm{D}_{i} \amalg_{\mathrm{D}_{j}} \mathrm{D}_{i}, & \text { pour } i>j \geqslant 0,
\end{array}
$$

de $\Theta$, correspondant respectivement aux cellules, sources, buts, unités et compositions, vérifient les axiomes duaux à ceux des $\infty$-catégories. Il en résulte que tout préfaisceau $X: \Theta^{\circ} \rightarrow \mathcal{E}$ ns sur $\Theta$ qui envoie les sommes globulaires sur des produits globulaires, au sens où, pour toute somme globulaire $\mathrm{D}_{i_{1}} \amalg_{\mathrm{D}_{j_{1}}} \cdots \amalg_{\mathrm{D}_{j_{l-1}}} \mathrm{D}_{i_{l}}$, l'application canonique

$$
F\left(\mathrm{D}_{i_{1}} \amalg_{\mathrm{D}_{j_{1}}} \cdots \amalg_{\mathrm{D}_{j_{l-1}}} \mathrm{D}_{i_{l}}\right) \rightarrow F\left(\mathrm{D}_{i_{1}}\right) \times_{F\left(\mathrm{D}_{j_{1}}\right)} \cdots \times_{F\left(\mathrm{D}_{j_{l-1}}\right)} F\left(\mathrm{D}_{i_{l}}\right)
$$

est une bijection, définit une $\infty$-catégorie. On notera $X\left(\mathrm{D}_{\bullet}\right)$ cette $\infty$-catégorie. L'ensemble de ses $i$-cellules, pour $i \geqslant 0$, est $X\left(\mathrm{D}_{i}\right)$ et les sources, buts, unités et compositions sont donnés par les applications $X\left(\sigma_{i}\right), X\left(\tau_{i}\right), X\left(\kappa_{i}\right)$ et $X\left(\nabla_{j}^{i}\right)$ pour des valeurs de $i$ et $j$ convenables. De plus, tout morphisme $f: X \rightarrow Y$ entre des préfaisceaux sur $\Theta$ envoyant les sommes globulaires sur des produits globulaires définit un $\infty$-foncteur $f_{\mathrm{D}_{\bullet}}: X\left(\mathrm{D}_{\bullet}\right) \rightarrow Y\left(\mathrm{D}_{\bullet}\right)$ entre les $\infty$-catégories associées. Ainsi, en notant $\widehat{\Theta}_{\text {glob }}$ la sous-catégorie pleine de $\widehat{\Theta}$ formée des préfaisceaux envoyant les sommes globulaires sur des produits globulaires, on obtient un foncteur

$$
\widehat{\Theta}_{\text {glob }} \rightarrow \infty-\mathcal{C} a t \text {. }
$$

Nous n'en aurons pas besoin dans la suite de ce texte mais on peut vérifier facilement que ce foncteur est une équivalence de catégories, un quasi-inverse étant induit par le nerf cellulaire $\infty$-C $a t \rightarrow \widehat{\Theta}$, foncteur « nerf » associé à l'inclusion $\Theta \hookrightarrow \infty-\mathcal{C}$ at.

4.9. - La catégorie $\Theta_{+}$possède une propriété analogue à celle de $\Theta$ exposée au paragraphe précédent. Soit $X_{+}:\left(\Theta_{+}\right)^{\circ} \rightarrow \mathcal{E} n s$ un préfaisceau sur $\Theta_{+}$qui envoie les sommes globulaires sur des produits globulaires, c'est-à-dire tel que sa restriction $X$ à $\Theta$ envoie les sommes globulaires sur des produits globulaires au sens du paragraphe précédent. En vertu de ce même paragraphe, un tel préfaisceau définit une $\infty$-catégorie $X\left(\mathrm{D}_{\bullet}\right)$. De plus, les morphismes $\varnothing \rightarrow \mathrm{D}_{i}$ de $\Theta_{+}$, pour $i \geqslant 0$, permettent 
de munir cette $\infty$-catégorie d'un $\infty$-foncteur vers l'ensemble $X_{+}(\varnothing)$, vu comme une $\infty$-catégorie dont les $i$-cellules sont triviales pour $i>0$. On notera $X_{+}\left(\mathrm{D}_{\bullet}\right)$ cette $\infty$-catégorie au-dessus de $X_{+}(\varnothing)$. Si $x$ est un élément de $X_{+}(\varnothing)$, on notera $X_{+}\left(\mathrm{D}_{\bullet}\right)_{x}$ la fibre en $x$ du $\infty$-foncteur $X\left(\mathrm{D}_{\bullet}\right) \rightarrow X_{+}(\varnothing)$. On a ainsi

$$
X(\mathrm{D} \bullet)=\coprod_{x \in X_{+}(\varnothing)} X_{+}\left(\mathrm{D}_{\bullet}\right)_{x}
$$

Si $f_{+}: X_{+} \rightarrow Y_{+}$est un morphisme entre préfaisceaux sur $\Theta_{+}$envoyant les sommes globulaires sur des produits globulaires, la restriction $f$ de $f_{+}$à $\Theta$ définit un $\infty$-foncteur $f_{\mathrm{D}_{\bullet}}: X\left(\mathrm{D}_{\bullet}\right) \rightarrow Y\left(\mathrm{D}_{\bullet}\right)$ rendant le carré

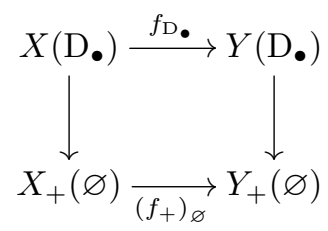

commutatif. En particulier, si $x$ appartient à $X_{+}(\varnothing)$ et $y=\left(f_{+}\right)_{\varnothing}(x)$, alors on dispose d'un $\infty$-foncteur $X_{+}\left(\mathrm{D}_{\bullet}\right)_{x} \rightarrow Y_{+}\left(\mathrm{D}_{\bullet}\right)_{y}$.

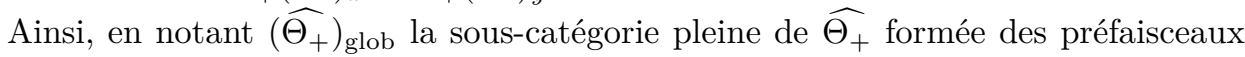
envoyant les sommes globulaires sur des produits globulaires, on obtient un foncteur

$$
\left(\widehat{\Theta_{+}}\right)_{\text {glob }} \rightarrow \infty-\mathcal{C} a t \downarrow \mathcal{E} n s,
$$

où $\infty$ - $\mathcal{C} a t \downarrow \mathcal{E} n s$ désigne la catégorie des $\infty$-catégories au-dessus d'un ensemble. Nous n'en aurons pas besoin dans la suite de ce texte mais on peut déduire facilement de l'énoncé analogue pour $\Theta$ que ce foncteur est une équivalence de catégories.

Nous allons maintenant étudier l'image de $\Theta$ par le foncteur $\lambda: \infty-\mathcal{C} a t \rightarrow \mathcal{C}_{\text {da }}$.

4.10. - Fixons $i \geqslant 0$ et considérons la $i$-catégorie $\mathrm{D}_{i}$. Notons $x$ sa cellule principale. Pour $k$ tel que $0 \leqslant k<i$, les $k$-flèches non triviales de $\mathrm{D}_{i}$ sont exactement $s_{k}(x)$ et $t_{k}(x)$. Ces cellules seront également notées $x_{k}^{0}$ et $x_{k}^{1}$. De même, on notera parfois $x_{i}^{0}, x_{i}^{1}$ ou $x_{i}$ la cellule $x$.

On vérifie immédiatement que le complexe dirigé augmenté $\lambda\left(\mathrm{D}_{i}\right)$ peut se décrire de la manière suivante. Pour $k \geqslant 0$, on a

$$
\lambda\left(\mathrm{D}_{i}\right)_{k}= \begin{cases}\mathbb{Z}^{\left(\left\{x_{k}^{0}, x_{k}^{1}\right\}\right)} & \text { si } 0 \leqslant k<i, \\ \mathbb{Z}^{\left(\left\{x_{i}\right\}\right)} & \text { si } k=i, \\ 0 & \text { si } k>i .\end{cases}
$$

On identifiera souvent les cellules non triviales de $\mathrm{D}_{i}$ avec les éléments de la base de $\lambda\left(\mathrm{D}_{i}\right)$. De plus, on posera parfois $x_{k}^{0}=0=x_{k}^{1}$ pour $k>i$. Pour $k$ tel que $0<k \leqslant i$, la différentielle envoie $x_{k}^{0}$ et $x_{k}^{1}$ sur $x_{k-1}^{1}-x_{k-1}^{0}$. Les sous-monoïdes $\lambda\left(\mathrm{D}_{i}\right)_{k}^{*}$ sont les 
sous-monoïdes engendrés par les bases canoniques et l'augmentation est donnée par la somme des coefficients. Le complexe dirigé augmenté $\lambda\left(\mathrm{D}_{i}\right)$ est donc à base.

Soient $k$ tel que $0<k \leqslant i$ et $\varepsilon=0,1$. On vérifie immédiatement qu'on a

$$
\left\langle x_{k}^{\varepsilon}\right\rangle_{l}^{\eta}=x_{l}^{\eta}
$$

pour $\eta=0,1$ et $l$ tel que $0 \leqslant l<k$ (voir le paragraphe 2.8 pour la notation $\langle z\rangle$ ). En particulier, on a

$$
\left\langle x_{k}^{\varepsilon}\right\rangle_{0}^{0}=x_{0}^{0} \quad \text { et } \quad\left\langle x_{k}^{\varepsilon}\right\rangle_{0}^{1}=x_{0}^{1}
$$

et la base de $\lambda\left(D_{i}\right)$ est unitaire.

Par ailleurs, la relation de préordre $\leqslant_{\mathbb{N}}$ sur $\lambda\left(D_{i}\right)$ est l'ordre total

$$
x_{0}^{0} \leqslant_{\mathbb{N}} x_{1}^{0} \leqslant_{\mathbb{N}} \cdots \leqslant_{\mathbb{N}} x_{i-1}^{0} \leqslant_{\mathbb{N}} x \leqslant_{\mathbb{N}} x_{i-1}^{1} \leqslant_{\mathbb{N}} \cdots \leqslant_{\mathbb{N}} x_{1}^{1} \leqslant_{\mathbb{N}} x_{0}^{1},
$$

ce qui prouve que la base de $\lambda\left(D_{i}\right)$ est fortement sans boucle. Le complexe $\lambda\left(D_{i}\right)$ est donc un complexe de Steiner fort.

4.11. - Fixons deux entiers positifs $i$ et $j$. On notera $x$ et $y$ les cellules principales respectives de $\mathrm{D}_{j}$ et $\mathrm{D}_{i}$.

Supposons $i \geqslant j$. Le morphisme $\lambda\left(\sigma_{j}^{i}\right): \lambda\left(\mathrm{D}_{j}\right) \rightarrow \lambda\left(\mathrm{D}_{i}\right)$ est donné par

$$
\begin{aligned}
x_{k}^{\varepsilon} \mapsto y_{k}^{\varepsilon}, \quad \text { pour } 0 \leqslant k<j \text { et } \varepsilon=0,1, \\
x_{j} \mapsto y_{j}^{0} .
\end{aligned}
$$

De même, le morphisme $\lambda\left(\tau_{j}^{i}\right): \lambda\left(\mathrm{D}_{j}\right) \rightarrow \lambda\left(\mathrm{D}_{i}\right)$ est donné par

$$
\begin{aligned}
& x_{k}^{\varepsilon} \mapsto y_{k}^{\varepsilon}, \quad \text { pour } 0 \leqslant k<j \text { et } \varepsilon=0,1, \\
& x_{j} \mapsto y_{j}^{1} .
\end{aligned}
$$

Supposons maintenant $i \leqslant j$. Le morphisme $\lambda\left(\kappa_{j}^{i}\right): \lambda\left(\mathrm{D}_{j}\right) \rightarrow \lambda\left(\mathrm{D}_{i}\right)$ est donné par

$$
x_{k}^{\varepsilon} \mapsto y_{k}^{\varepsilon}, \quad \text { pour } 0 \leqslant k \leqslant j \text { et } \varepsilon=0,1 \text {. }
$$

Ainsi, $x_{k}^{0}$ et $x_{k}^{1}$, pour $k=i$, sont envoyés sur $y_{i}$ et, pour $k>i$, sur 0 .

Soient maintenant $i>j \geqslant 0$ deux entiers. Considérons le morphisme de cocomposition $\nabla_{j}^{i}: \mathrm{D}_{i} \rightarrow \mathrm{D}_{i} \amalg_{\mathrm{D}_{j}} \mathrm{D}_{i}$. Notons $x$ la cellule principale de la source $\mathrm{D}_{i}$ de $\nabla_{j}^{i}$ et $y$ et $z$ les cellules principales des deux copies de $\mathrm{D}_{i}$ apparaissant de gauche à droite dans $\mathrm{D}_{i} \amalg_{\mathrm{D}_{j}} \mathrm{D}_{i}$, vues comme des cellules de $\mathrm{D}_{i} \amalg_{\mathrm{D}_{j}} \mathrm{D}_{i}$. On utilisera les notations du paragraphe 4.10 pour la cellule $x$ et on les étendra de la manière évidente à $y$ et $z$ : on notera $y_{k}^{\varepsilon}$ et $z_{k}^{\varepsilon}$, pour $0 \leqslant k \leqslant i$ et $\varepsilon=0,1$, les sources et buts itérés de $y$ et $z$, et on identifiera ces cellules avec l'élément de $\lambda\left(D_{i} \amalg_{D_{j}} D_{i}\right)$ qu'elles définissent. Puisque $s_{j}(y)=t_{j}(z)$, on a

$$
y_{j}^{0}=z_{j}^{1} \quad \text { et } \quad y_{k}^{\varepsilon}=z_{k}^{\varepsilon} \quad \text { pour } 0 \leqslant k<j \text { et } \varepsilon=0,1 .
$$


On vérifie que les $y_{i}^{\varepsilon}, z_{i}^{\varepsilon}$, pour $0 \leqslant k \leqslant i$ et $\varepsilon=0,1$, modulo les relations ci-dessus et les relations triviales $y_{i}^{0}=y_{i}^{1}$ et $z_{i}^{0}=z_{i}^{1}$, forment une base de $\lambda\left(\mathrm{D}_{i} \amalg_{\mathrm{D}_{j}} \mathrm{D}_{i}\right)$. Avec ces notations, le morphisme $\lambda\left(\nabla_{j}^{i}\right)$ est donné par

$$
\begin{array}{ll}
x_{k}^{0} \mapsto z_{k}^{0}, & \text { pour } 0 \leqslant k \leqslant j, \\
x_{k}^{1} \mapsto y_{k}^{1}, & \text { pour } 0 \leqslant k \leqslant j, \\
x_{k}^{\varepsilon} \mapsto y_{k}^{\varepsilon}+z_{k}^{\varepsilon}, & \text { pour } j<k \leqslant i \text { et } \varepsilon=0,1 .
\end{array}
$$

4.12. - Soit $S=\mathrm{D}_{i_{1}} \amalg_{\mathrm{D}_{j_{1}}} \cdots \amalg_{\mathrm{D}_{j_{l-1}}} \mathrm{D}_{i_{l}}$ un schéma de composition globulaire. Par définition, la $\infty$-catégorie $S$ est limite inductive du foncteur $F_{S}: I_{S} \rightarrow \infty$-Cat associé au diagramme décrit au paragraphe 4.2. On a donc un isomorphisme

$$
\lambda(S) \simeq \lambda\left(\mathrm{D}_{i_{1}}\right) \amalg_{\lambda\left(\mathrm{D}_{j_{1}}\right)} \cdots \amalg_{\lambda\left(\mathrm{D}_{j_{l-1}}\right)} \lambda\left(\mathrm{D}_{i_{l}}\right),
$$

où le membre de droite est la limite inductive du foncteur $G_{S}: I_{S} \stackrel{F_{S}}{\rightarrow} \infty$ - $\mathcal{C} a \stackrel{\lambda}{\rightarrow} \mathcal{C}_{\text {da }}$. Cet isomorphisme s'étend au cas $S=\varnothing$ en considérant les uniques foncteurs $F_{\varnothing}: \varnothing \rightarrow \infty$-Cat et $G_{\varnothing}: \varnothing \rightarrow \mathcal{C}_{\mathrm{da}}$.

Proposition 4.13. - Pour tout objet $S$ de $\Theta_{+}$, le foncteur $G_{S}$ est un système de Steiner fort. En particulier, le complexe $\lambda(S)$ est un complexe de Steiner fort. De plus, le morphisme d'adjonction $S \rightarrow \nu \lambda(S)$ est un isomorphisme.

Démonstration. - Il est immédiat que les morphismes

$$
\lambda\left(\sigma_{j}^{i}\right), \lambda\left(\tau_{j}^{i}\right): \lambda\left(\mathrm{D}_{j}\right) \rightarrow \lambda\left(\mathrm{D}_{i}\right), \quad \text { pour } i>j \geqslant 0,
$$

sont des inclusions rigides ordonnées. Le foncteur $G_{S}: I_{S} \rightarrow \mathcal{C}_{\mathrm{da}}$ vérifie donc les hypothèses du théorème 3.19 , ce qui entraîne la première assertion, ainsi que le fait que $\lambda(S)$ est un complexe de Steiner fort. Ce même théorème donne de plus un isomorphisme canonique

$$
\nu \lambda(S) \simeq \nu \lambda\left(\mathrm{D}_{i_{1}}\right) \amalg_{\nu \lambda\left(\mathrm{D}_{j_{1}}\right)} \cdots \amalg_{\nu \lambda\left(\mathrm{D}_{j_{l-1}}\right)} \nu \lambda\left(\mathrm{D}_{i_{l}}\right) .
$$

Pour obtenir la dernière assertion, il suffit donc de montrer que, pour tout $k \geqslant 0$, le morphisme d'adjonction $\mathrm{D}_{k} \rightarrow \nu \lambda\left(\mathrm{D}_{k}\right)$ est un isomorphisme, ce qui se vérifie facilement par un calcul direct.

Remarque 4.14. - Le fait que $\lambda(S)$, pour $S$ un schéma de composition, est un complexe de Steiner fort et que le morphisme d'adjonction $S \rightarrow \nu \lambda(S)$ est un isomorphisme est dû à Steiner [34]. On renvoie à ce même texte pour une étude plus complète des liens entre complexes dirigés augmentés et schémas de composition globulaires. 


\section{CHAPITRE 5}

\section{EXTENSION DE STRUCTURES DE CATÉGORIE MONOÏDALE À LA DAY}

Le but de ce chapitre est d'étendre un théorème dû à Day d'extension de structures de catégorie monoïdale bifermée au cadre plus général des catégories monoïdales localement bifermées, qu'on introduit dans le chapitre.

5.1. - Soit $\mathcal{D}$ une sous-catégorie pleine d'une catégorie $\mathcal{C}$. Rappelons qu'on dit que $\mathcal{D}$ est une sous-catégorie dense de $\mathcal{C}$ si tout objet de $\mathcal{C}$ est limite inductive canonique d'objets de $\mathcal{D}$. Cela signifie la chose suivante. Soit $X$ un objet de $\mathcal{C}$. Notons $\mathcal{D} / X$ la catégorie dont les objets sont les couples $(d, f: d \rightarrow X)$, où $d$ est un objet de $\mathcal{D}$ et $f$ un morphisme de $\mathcal{C}$, et dont les morphismes entre deux tels couples $(d, f)$ et $\left(d^{\prime}, f^{\prime}\right)$ sont les morphismes $g: d \rightarrow d^{\prime}$ de $\mathcal{C}$ tels que $f^{\prime} g=f$. On a un foncteur canonique $\mathcal{D} / X \rightarrow \mathcal{C}$ qui envoie $(d, d \rightarrow X)$ sur $d$. Ce foncteur est muni d'une transformation naturelle canonique vers le foncteur constant de valeur $X$. On dit que $X$ est limite inductive canonique d'objets de $\mathcal{D}$ si cette transformation naturelle est un cône inductif universel ou, autrement dit, si la limite inductive de ce foncteur existe et le morphisme canonique

$$
\lim _{(d, d \rightarrow X) \in \mathcal{D} / X} d \longrightarrow X
$$

est un isomorphisme.

On vérifie facilement que si une sous-catégorie pleine $\mathcal{D}$ de $\mathcal{C}$ contient une souscatégorie pleine dense dans $\mathcal{C}$, alors $\mathcal{D}$ est dense dans $\mathcal{C}$.

Rappelons enfin que si la sous-catégorie pleine $\mathcal{D}$ est une petite sous-catégorie $D$, alors $D$ est dense dans $\mathcal{C}$ si et seulement si le foncteur de $\mathcal{C}$ vers les préfaisceaux $\widehat{D}$ sur $D$ défini par

$$
X \longmapsto\left(d \mapsto \operatorname{Hom}_{\mathcal{C}}(d, X)\right)
$$

est pleinement fidèle. 
5.2. - Soit $\mathcal{C}$ une catégorie monoïdale de produit tensoriel $\otimes$. On rappelle que $\mathcal{C}$ est dite fermée à droite si, pour tout objet $Y$ de $\mathcal{C}$, le foncteur $\bullet \otimes Y: \mathcal{C} \rightarrow \mathcal{C}$ admet un adjoint à droite. De même, on dit que $\mathcal{C}$ est fermée à gauche si, pour tout objet $X$ de $\mathcal{C}$, le foncteur $X \otimes \bullet: \mathcal{C} \rightarrow \mathcal{C}$ admet un adjoint à droite. On dit que $\mathcal{C}$ est bifermée si elle est à la fois fermée à gauche et à droite.

En vertu d'un résultat classique sur les adjonctions paramétrées (voir par exemple $[\mathbf{2 8}$, chapitre IV, section 7 , théorème 3$])$, si $\mathcal{C}$ est fermée à droite, il existe un foncteur $\underline{\operatorname{Hom}}_{\mathcal{C}}^{\mathrm{d}}: \mathcal{C}^{\circ} \times \mathcal{C} \rightarrow \mathcal{C}$ et des bijections

$$
\operatorname{Hom}_{\mathcal{C}}(X \otimes Y, Z) \simeq \operatorname{Hom}_{\mathcal{C}}\left(X, \underline{\operatorname{Hom}}_{\mathcal{C}}^{\mathrm{d}}(Y, Z)\right),
$$

naturelles en $X, Y$ et $Z$ dans $\mathcal{C}$. De même, si $\mathcal{C}$ est fermée à gauche, il existe un foncteur $\underline{\operatorname{Hom}}_{\mathcal{C}}^{\mathrm{g}}: \mathcal{C}^{\circ} \times \mathcal{C} \rightarrow \mathcal{C}$ et des bijections

$$
\operatorname{Hom}_{\mathcal{C}}(X \otimes Y, Z) \simeq \operatorname{Hom}_{\mathcal{C}}\left(Y, \underline{\operatorname{Hom}}_{\mathcal{C}}^{\mathrm{g}}(X, Z)\right),
$$

naturelles en $X, Y$ et $Z$ dans $\mathcal{C}$.

Théorème 5.3 (Day). - Soient $\mathcal{C}$ une catégorie complète et cocomplète, et $\mathcal{D}$ une sous-catégorie pleine de $\mathcal{C}$ munie d'une structure de catégorie monö̈dale. On suppose qu'il existe une petite sous-catégorie pleine de $\mathcal{D}$ dense dans $\mathcal{C}$, des foncteurs

$$
H, H^{\prime}: \mathcal{D}^{\circ} \times \mathcal{C} \rightarrow \mathcal{C}
$$

et des bijections

$$
\operatorname{Hom}_{\mathcal{C}}\left(S, H\left(S^{\prime}, Z\right)\right) \simeq \operatorname{Hom}_{\mathcal{C}}\left(S \otimes S^{\prime}, Z\right) \simeq \operatorname{Hom}_{\mathcal{C}}\left(S^{\prime}, H^{\prime}(S, Z)\right),
$$

naturelles en $S, S^{\prime}$ dans $\mathcal{D}$ et $Z$ dans $\mathcal{C}$.

Alors il existe une et une seule structure monö̈dale sur $\mathcal{C}$ (à unique isomorphisme monoïdal près) donnée par un produit tensoriel commutant aux petites limites inductives en chaque variable et pour laquelle le foncteur d'inclusion $\mathcal{D} \hookrightarrow \mathcal{C}$ s'étend en un foncteur monoïdal. De plus, cette structure monoïdale est bifermée.

Démonstration. - Soit $D$ une petite sous-catégorie pleine de $\mathcal{D}$ dense dans $\mathcal{C}$. On va commencer par montrer que si $S$ et $S^{\prime}$ sont deux objets de $\mathcal{D}$, on a un isomorphisme canonique

$$
S \otimes S^{\prime} \simeq \lim _{\substack{s \rightarrow \overrightarrow{S \in D} D / S \\ s^{\prime} \rightarrow S^{\prime} \in D / S^{\prime}}} s \otimes s^{\prime},
$$

où la limite inductive, comme toutes les limites inductives dans cette preuve, est calculée dans $\mathcal{C}$. On a, pour tout objet $Z$ de $\mathcal{C}$,

$$
\begin{aligned}
& \operatorname{Hom}_{\mathcal{C}}\left(\lim _{s \rightarrow S}\left(s \otimes S^{\prime}\right), Z\right) \simeq \lim _{s \rightarrow S} \operatorname{Hom}_{\mathcal{C}}\left(s \otimes S^{\prime}, Z\right) \\
& \simeq \lim _{s \rightarrow S} \operatorname{Hom}_{\mathcal{C}}\left(s, H\left(S^{\prime}, Z\right)\right)
\end{aligned}
$$


CHAPITRE 5. EXTENSION DE STRUCTURES DE CATÉGORIE MONOÏDALE À LA DAY $\mathbf{5 1}$

$$
\begin{aligned}
& \simeq \operatorname{Hom}_{\mathcal{C}}\left(\underset{s \rightarrow S}{\left.\lim s, H\left(S^{\prime}, Z\right)\right)}\right. \\
& \simeq \operatorname{Hom}_{\mathcal{C}}\left(S, H\left(S^{\prime}, Z\right)\right) \\
& \simeq \operatorname{Hom}_{\mathcal{C}}\left(S \otimes S^{\prime}, Z\right),
\end{aligned}
$$

d'où un isomorphisme canonique

$$
S \otimes S^{\prime} \simeq \underset{s \rightarrow S}{\lim _{s \rightarrow S}}\left(s \otimes S^{\prime}\right)
$$

De même, en utilisant $H^{\prime}$, on obtient un isomorphisme canonique

$$
S \otimes S^{\prime} \simeq \underset{s^{\prime} \rightarrow S^{\prime}}{\lim _{\longrightarrow}}\left(S \otimes s^{\prime}\right)
$$

On a donc

$$
S \otimes S^{\prime} \simeq \underset{s \rightarrow S}{\lim }\left(s \otimes S^{\prime}\right) \simeq \underset{s \rightarrow S}{\lim _{s^{\prime} \rightarrow S^{\prime}}} \underset{\lim _{\longrightarrow}}{\longrightarrow} \otimes s^{\prime}
$$

d'où l'isomorphisme annoncé.

Étendons maintenant le produit tensoriel de $\mathcal{D}$ à $\mathcal{C}$. On est contraint de poser, pour $X$ et $Y$ dans $\mathcal{C}$,

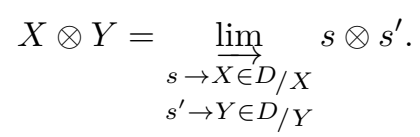

Le paragraphe précédent montre qu'on prolonge bien ainsi le produit tensoriel de $\mathcal{D}$. De même, on va étendre les foncteurs $H$ et $H^{\prime}$ en des foncteurs $\mathcal{C}^{\circ} \times \mathcal{C} \rightarrow \mathcal{C}$. Pour $X$ et $Y$ des objets de $\mathcal{C}$, on pose

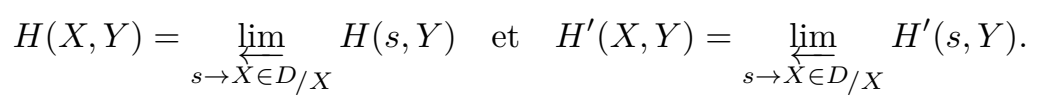

On va montrer qu'on a des bijections

$$
\operatorname{Hom}_{\mathcal{C}}(X, H(Y, Z)) \simeq \operatorname{Hom}_{\mathcal{C}}(X \otimes Y, Z) \simeq \operatorname{Hom}_{\mathcal{C}}\left(Y, H^{\prime}(X, Z)\right)
$$

naturelles en $X, Y$ et $Z$ dans $\mathcal{C}$. Faisons-le pour la bijection de gauche, celle de droite se traitant de manière analogue. On a

$$
\begin{aligned}
& \operatorname{Hom}_{\mathcal{C}}(X \otimes Y, Z) \simeq \operatorname{Hom}_{\mathcal{C}}\left(\underset{s \rightarrow X, s^{\prime} \rightarrow Y}{\lim _{X, Y}} s \otimes s^{\prime}, Z\right) \\
& \simeq \lim _{s \rightarrow X, s^{\prime} \rightarrow Y} \operatorname{Hom}_{\mathcal{C}}\left(s \otimes s^{\prime}, Z\right) \\
& \simeq \lim _{s \rightarrow \overleftarrow{X, s^{\prime}} \rightarrow Y} \operatorname{Hom}_{\mathcal{C}}\left(s, H\left(s^{\prime}, Z\right)\right) \\
& \simeq \operatorname{Hom}_{\mathcal{C}}\left(\lim _{s \rightarrow X} s, \lim _{s^{\prime} \rightarrow Y} H\left(s^{\prime}, Z\right)\right) \\
& \simeq \operatorname{Hom}_{\mathcal{C}}(X, H(Y, Z)) \text {, }
\end{aligned}
$$


ce qu'il fallait démontrer. Ceci montre que le foncteur $\otimes: \mathcal{C} \times \mathcal{C} \rightarrow \mathcal{C}$ commute aux limites inductives en chaque variable.

Pour conclure, il suffit de vérifier que la structure monoïdale sur $\mathcal{D}$ induit une structure monoïdale sur la catégorie $\mathcal{C}$ munie du produit qu'on vient de définir. Notons $I$ l'unité du produit tensoriel de $\mathcal{D}$. On a, pour tout objet $X$ de $\mathcal{C}$,

$$
I \otimes X \simeq I \otimes \lim _{s \rightarrow X} s \simeq \lim _{s \rightarrow X}(I \otimes s) \simeq \lim _{s \rightarrow X} s \simeq X
$$

et, de même, $X \otimes I \simeq X$. Enfin, en utilisant le fait que le produit sur $\mathcal{C}$ commute aux limites inductives en chaque variable, on obtient, pour tous objets $X, Y$ et $Z$ de $\mathcal{C}$, les isomorphismes

$$
\begin{aligned}
(X \otimes Y) \otimes Z & \simeq{\underset{s \rightarrow X, s^{\prime} \rightarrow Y, s^{\prime \prime} \rightarrow Z}{\lim _{s \rightarrow X, s^{\prime} \rightarrow Y, s^{\prime \prime} \rightarrow Z}}\left(s \otimes s^{\prime}\right) \otimes s^{\prime \prime}} s \otimes\left(s^{\prime} \otimes s^{\prime \prime}\right) \simeq X \otimes(Y \otimes Z),
\end{aligned}
$$

ce qui achève la démonstration.

Remarque 5.4. - Le résultat ci-dessus, purement formel, est attribué à Day par Street dans [40]. Les références données par celui-ci sont [19] et [20].

Le but de la suite du chapitre est de généraliser le théorème précédent en un énoncé qui puisse s'appliquer à des produits tensoriels dont on suppose uniquement qu'ils commutent aux limites inductives connexes.

5.5. - Soit $\mathcal{C}$ une catégorie monoïdale. Une coaugmentation à gauche de $\mathcal{C}$ est la donnée d'un endofoncteur $M$ de $\mathcal{C}$ et de morphismes

$$
M(X) \stackrel{\iota_{1}}{\rightarrow} X \otimes Y,
$$

naturels en $X$ et $Y$ dans $\mathcal{C}$. Plus formellement, on demande que $\iota_{1}$ soit une transformation naturelle

$$
M p \stackrel{\iota_{1}}{\longrightarrow} \otimes
$$

de foncteurs de $\mathcal{C} \times \mathcal{C}$ vers $\mathcal{C}$, où $p: \mathcal{C} \times \mathcal{C} \rightarrow \mathcal{C}$ désigne la première projection. De même, une coaugmentation à droite de $\mathcal{C}$ est la donnée d'un endofoncteur $N$ de $\mathcal{C}$ et de morphismes

$$
X \otimes Y \stackrel{\iota_{2}}{\longleftarrow} N(Y)
$$

naturels en $X$ et $Y$ dans $\mathcal{C}$. Plus formellement, on demande que $\iota_{2}$ soit une transformation naturelle

$$
\otimes \stackrel{\iota_{2}}{\longleftarrow} N q
$$

de foncteurs de $\mathcal{C} \times \mathcal{C}$ vers $\mathcal{C}$, où $q: \mathcal{C} \times \mathcal{C} \rightarrow \mathcal{C}$ désigne la seconde projection. Enfin, on appellera bicoaugmentation de $\mathcal{C}$ la donnée d'une coaugmentation à gauche et d'une coaugmentation à droite

$$
M(X) \stackrel{\iota_{1}}{\longrightarrow} X \otimes Y \stackrel{\iota_{2}}{\longleftarrow} N(Y)
$$


Par abus de langage, on dira parfois que $M$ est une coaugmentation à gauche, sousentendant ainsi la transformation naturelle $\iota_{1}$. De même pour les coaugmentations à droite et les bicoaugmentations.

Exemple 5.6. — Soit $\mathcal{C}$ une catégorie monoïdale possédant un objet initial $\varnothing$. Il existe deux bicoaugmentations naturelles sur $\mathcal{C}$ :

(a) la bicoaugmentation triviale : $M$ et $N$ sont les foncteurs constants de valeur $\varnothing$ et, pour tous objets $X$ et $Y$ de $\mathcal{C}$, les morphismes

$$
\varnothing \stackrel{\iota_{1}}{\longrightarrow} X \otimes Y \stackrel{\iota_{2}}{\longleftarrow} \varnothing
$$

sont l'unique morphisme $\varnothing \rightarrow X \otimes Y$;

(b) la bicoaugmentation pseudo-locale : $M$ et $N$ sont les foncteurs $\bullet \otimes \varnothing$ et $\varnothing \otimes \bullet$ respectivement et, pour tous objets $X$ et $Y$ de $\mathcal{C}$, les morphismes

$$
X \otimes \varnothing \stackrel{\iota_{1}}{\longrightarrow} X \otimes Y \stackrel{\iota_{2}}{\longleftarrow} \varnothing \otimes Y
$$

sont induits par fonctorialité par les morphismes $\varnothing \rightarrow Y$ et $\varnothing \rightarrow X$.

Notons que si la catégorie monoïdale $\mathcal{C}$ est bifermée, ces deux bicoaugmentations sont isomorphes en un sens évident.

Par ailleurs, si l'unité de la catégorie monoïdale $\mathcal{C}$ est l'objet initial $\varnothing$, alors la bicoaugmentation pseudo-locale induit une bicoaugmentation isomorphe :

(c) la bicoaugmentation locale : $M$ et $N$ sont l'endofoncteur identité de $\mathcal{C}$ et, pour tous objets $X$ et $Y$ de $\mathcal{C}$, les morphismes

$$
X \stackrel{\iota_{1}}{\longrightarrow} X \otimes Y \stackrel{\iota_{2}}{\longleftarrow} Y
$$

sont induits, à partir de la bicoaugmentation pseudo-locale, par les contraintes d'unité $X \simeq X \otimes \varnothing$ et $\varnothing \otimes Y \simeq Y$.

5.7. - Soit $\mathcal{C}$ une catégorie monoïdale munie d'une coaugmentation à droite $N$. On dira que $\mathcal{C}$ est localement fermée à droite si, pour tout objet $Y$ de $\mathcal{C}$, le foncteur

$$
\begin{aligned}
\mathcal{C} & \rightarrow N(Y) \backslash \mathcal{C} \\
X & \mapsto\left(X \otimes Y, \iota_{2}: N(Y) \rightarrow X \otimes Y\right)
\end{aligned}
$$

admet un adjoint à droite. De même, si $\mathcal{C}$ est munie d'une coaugmentation à gauche $M$, on dira que $\mathcal{C}$ est localement fermée à gauche si, pour tout objet $X$ de $\mathcal{C}$, le foncteur

$$
\begin{aligned}
\mathcal{C} & \rightarrow M(X) \backslash \mathcal{C} \\
Y & \mapsto\left(X \otimes Y, \iota_{1}: M(X) \rightarrow X \otimes Y\right)
\end{aligned}
$$

admet un adjoint à droite. Enfin, si $\mathcal{C}$ est munie d'une bicoaugmentation, on dira que $\mathcal{C}$ est localement bifermée si elle est localement fermée à gauche et à droite.

Si $\mathcal{C}$ est une catégorie monoïdale d'unité un objet initial, on appliquera parfois le vocabulaire précédent sans préciser les coaugmentations auxquelles on fait référence. On utilisera alors implicitement la bicoaugmentation locale de l'exemple 5.6. 
Si une catégorie monoïdale coaugmentée à droite $\mathcal{C}$ est localement fermée à droite, alors, pour tout objet $Y$ de $\mathcal{C}$, le foncteur $\bullet \otimes Y: \mathcal{C} \rightarrow \mathcal{C}$ commute aux limites inductives connexes. En effet, celui-ci se décompose en

$$
\mathcal{C} \rightarrow N(Y) \backslash \mathcal{C} \stackrel{U}{\rightarrow} \mathcal{C}
$$

où le premier foncteur est le foncteur canonique déjà considéré et $U$ est le foncteur d'oubli. Or, puisque $\mathcal{C}$ est localement fermée à droite, ce premier foncteur admet un adjoint à droite. On obtient l'assertion puisque le second commute toujours aux limites inductives connexes (voir par exemple [31, proposition 3.3.8]). De même, si $\mathcal{C}$ est localement fermée à gauche, alors, pour tout objet $X$ de $\mathcal{C}$, le foncteur $X \otimes \bullet: \mathcal{C} \rightarrow \mathcal{C}$ commute aux limites inductives connexes. Ainsi, si $\mathcal{C}$ est localement bifermée, son produit tensoriel commute aux limites inductives connexes en chaque variable.

Remarque 5.8. - Notons que si $\mathcal{C}$ est une catégorie monö̈dale avec un objet initial $\varnothing$ munie de la bicoaugmentation triviale (voir l'exemple 5.6), alors $\mathcal{C}$ est bifermée si et seulement si elle est localement bifermée.

Remarque 5.9. - Soit $\mathcal{C}$ une catégorie monoïdale d'unité un objet initial $\varnothing$. On munit $\mathcal{C}$ de la bicoaugmentation locale. On a vu que si $\mathcal{C}$ est localement bifermée, alors le produit tensoriel de $\mathcal{C}$ commute aux limites inductives connexes en chaque variable. La réciproque est également vraie si on suppose que la catégorie $\mathcal{C}$ est localement présentable. En effet, on vérifie tout d'abord que, pour tout objet $Y$ de $\mathcal{C}$, le foncteur $\mathcal{C} \rightarrow Y \backslash \mathcal{C}$ du paragraphe 5.7 commute aux limites inductives. Cela résulte du fait qu'il commute aux limites inductives connexes puisque le foncteur d'oubli $U: Y \backslash \mathcal{C} \rightarrow \mathcal{C}$ est conservatif et commute aux limites inductives connexes, et du fait qu'il envoie l'objet initial $\varnothing$ de $\mathcal{C}$ sur $\left(\varnothing \otimes Y, \iota_{2}\right) \simeq\left(Y, 1_{Y}\right)$ qui est un objet initial de $Y \backslash \mathcal{C}$. Par ailleurs, puisque la catégorie $\mathcal{C}$ est localement présentable, il en est de même de la catégorie $Y \backslash \mathcal{C}$. Or, il est bien connu qu'un foncteur entre catégories localement présentables qui commute aux limites inductives admet un adjoint à droite. Ceci montre que $\mathcal{C}$ est localement fermée à droite. On montre de même que $\mathcal{C}$ est localement fermée à gauche. Nous n'utiliserons pas ce résultat dans la suite de ce texte.

5.10. - Soit $F: \mathcal{C} \rightarrow \mathcal{D}$ un foncteur. On notera $\widetilde{\mathrm{FI}}(F)$ la catégorie dont les objets sont les triplets $(X, f: F(X) \rightarrow Y, Y)$, où $X$ est un objet de $\mathcal{C}, Y$ un objet de $\mathcal{D}$ et $f: F(X) \rightarrow Y$ un morphisme de $\mathcal{D}$, et dont les morphismes de $(X, f, Y)$ vers $\left(X^{\prime}, f^{\prime}, Y^{\prime}\right)$ sont les couples $(g, h)$, où $g: X^{\prime} \rightarrow X$ est un morphisme de $\mathcal{C}$ et $h: Y \rightarrow Y^{\prime}$ un morphisme de $\mathcal{D}$ tels que le carré

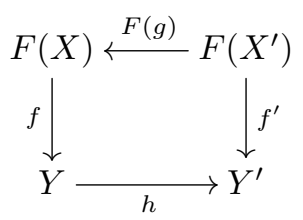


soit commutatif.

Notons que, dans le cas où $F$ est le foncteur constant de valeur un objet initial de $\mathcal{D}$, la catégorie $\widetilde{\mathrm{F}}(F)$ est canoniquement isomorphe à la catégorie $\mathcal{C}^{\circ} \times \mathcal{D}$.

5.11. - Soit $\mathcal{C}$ une catégorie monoïdale munie d'une coaugmentation à droite $N$. On notera $\widetilde{\mathrm{FI}} \mathrm{d}_{\mathrm{d}}(\mathcal{C})$ la catégorie $\widetilde{\mathrm{FI}}(N: \mathcal{C} \rightarrow \mathcal{C})$. Une variante du théorème sur les adjonctions paramétrées déjà cité montre que, si $\mathcal{C}$ est localement fermée à droite, alors il existe un foncteur

$$
H: \widetilde{\mathrm{F}}_{\mathrm{d}}(\mathcal{C}) \rightarrow \mathcal{C}
$$

et des bijections

$$
\operatorname{Hom}_{N(Y) \backslash \mathcal{C}}\left(\left(X \otimes Y, \iota_{2}\right),(Z, g)\right) \simeq \operatorname{Hom}_{\mathcal{C}}(X, H(Y, g, Z))
$$

naturelles en $X$ dans $\mathcal{C}$ et $(Y, g, Z)$ dans $\widetilde{\mathrm{F}}_{\mathrm{d}}(\mathcal{C})$.

De même, si $\mathcal{C}$ est munie d'une coaugmentation à gauche $M$, on notera $\widetilde{F}_{\mathrm{g}}(\mathcal{C})$ la catégorie $\widetilde{\mathrm{F}}(M: \mathcal{C} \rightarrow \mathcal{C})$ et on montre que, si $\mathcal{C}$ est localement fermée à gauche, alors il existe un foncteur

$$
H^{\prime}: \widetilde{\mathrm{F}}_{\mathrm{g}}(\mathcal{C}) \rightarrow \mathcal{C}
$$

et des bijections

$$
\operatorname{Hom}_{M(X) \backslash \mathcal{C}}\left(\left(X \otimes Y, \iota_{1}\right),(Z, f)\right) \simeq \operatorname{Hom}_{\mathcal{C}}\left(Y, H^{\prime}(X, f, Z)\right),
$$

naturelles en $Y$ dans $\mathcal{C}$ et $(X, f, Z)$ dans $\widetilde{\mathrm{F}}_{\mathrm{g}}(\mathcal{C})$.

Lemme 5.12. - Soient $F, G: I \rightarrow \mathcal{C}$ deux foncteurs de source une petite catégorie $I$ et de but une catégorie cocomplète $\mathcal{C}$, et $\alpha: F \rightarrow G$ une transformation naturelle. Alors, pour tout objet $Z$ de $\mathcal{C}$ et tout cône inductif $\left(f_{i}: F(i) \rightarrow Z\right)_{i \in I}$ de $F$ vers $Z$, on a une bijection canonique

$$
\operatorname{Hom}_{\longrightarrow} F \backslash \mathcal{C}\left(\left(\lim _{\longrightarrow} G, \lim _{\longrightarrow} \alpha\right),(Z, f)\right) \simeq \lim _{i \in I} \operatorname{Hom}_{F(i) \backslash \mathcal{C}}\left(\left(G(i), \alpha_{i}\right),\left(Z, f_{i}\right)\right),
$$

où $f: \underline{\lim } F \rightarrow Z$ désigne le morphisme déduit du cône inductif $\left(f_{i}\right)_{i \in I}$.

Démonstration. - Un élément du membre de droite est donné par une famille de morphismes $\left(g_{i}: G(i) \rightarrow Z\right)_{i \in I}$ satisfaisant $g_{i} \alpha_{i}=f_{i}$ pour $i$ dans $I$ et qui est de plus dans la limite projective en question, c'est-à-dire qui est telle que, pour tout $h: i \rightarrow i^{\prime}$ dans $I$, on ait $g_{i^{\prime}} G(h)=g_{i}$. Cette dernière condition signifie exactement que $\left(g_{i}\right)_{i \in I}$ est un cône inductif. On peut donc associer à la famille $\left(g_{i}\right)_{i \in I}$ un foncteur $\lim _{\longrightarrow} G \rightarrow Z$. Dire que ce foncteur est au-dessous de $\lim _{\longrightarrow} F$ signifie exactement qu'on a $\vec{g}_{i} \alpha_{i}=f_{i}$ pour tout $i$ dans $I$, d'où l'assertion.

Théorème 5.13. - Soit $\mathcal{C}$ une catégorie complète et cocomplète munie de deux endofoncteurs $M, N: \mathcal{C} \rightarrow \mathcal{C}$ commutant aux petites limites inductives connexes. Soit $\mathcal{D}$ une sous-catégorie pleine de $\mathcal{C}$, stable par les endofoncteurs $M$ et $N$, munie d'une 
structure de catégorie monö̈dale et d'une bicoaugmentation dont les endofoncteurs sous-jacents sont les restrictions de $M$ et $N$ à $\mathcal{D}$. On suppose que $\mathcal{D}$ contient un objet initial de $\mathcal{C}$, qu'il existe une petite sous-catégorie pleine de $\mathcal{D}$ dense dans $\mathcal{C}$, des foncteurs

$$
H: \widetilde{\mathrm{FI}}_{d}(\mathcal{D}, \mathcal{C}) \rightarrow \mathcal{C} \quad \text { et } \quad H^{\prime}: \widetilde{\mathrm{FI}}_{g}(\mathcal{D}, \mathcal{C}) \rightarrow \mathcal{C}
$$

où on a posé

$$
\widetilde{\mathrm{FI}}_{d}(\mathcal{D}, \mathcal{C})=\widetilde{\mathrm{FI}}\left(N_{\mid \mathcal{D}}: \mathcal{D} \rightarrow \mathcal{C}\right) \quad \text { et } \quad \widetilde{\mathrm{FI}}_{g}(\mathcal{D}, \mathcal{C})=\widetilde{\mathrm{FI}}\left(M_{\mid \mathcal{D}}: \mathcal{D} \rightarrow \mathcal{C}\right)
$$

des bijections

$$
\operatorname{Hom}_{N\left(S^{\prime}\right) \backslash \mathcal{C}}\left(\left(S \otimes S^{\prime}, \iota_{2}\right),(Z, g)\right) \simeq \operatorname{Hom}_{\mathcal{C}}\left(S, H\left(S^{\prime}, g, Z\right)\right),
$$

naturelles en $S$ dans $\mathcal{D}$ et $\left(S^{\prime}, g, Z\right)$ dans $\widetilde{\mathrm{FI}}_{d}(\mathcal{D}, \mathcal{C})$, et des bijections

$$
\operatorname{Hom}_{M(S) \backslash \mathcal{C}}\left(\left(S \otimes S^{\prime}, \iota_{1}\right),(Z, f)\right) \simeq \operatorname{Hom}_{\mathcal{C}}\left(S^{\prime}, H^{\prime}(S, f, Z)\right),
$$

naturelles en $S^{\prime}$ dans $\mathcal{D}$ et $(S, f, Z)$ dans $\widetilde{\mathrm{FI}}_{g}(\mathcal{D}, \mathcal{C})$.

Alors il existe une et une seule structure de catégorie monoïdale sur $\mathcal{C}$ (à unique isomorphisme monoïdal près) donnée par un produit tensoriel commutant aux petites limites inductives connexes en chaque variable et pour laquelle le foncteur d'inclusion $\mathcal{D} \hookrightarrow \mathcal{C}$ s'étend en un foncteur monoïdal. De plus, cette structure de catégorie monö̈dale sur $\mathcal{C}$ admet une unique bicoaugmentation d'endofoncteurs sous-jacents $M$ et $N$ prolongeant la bicoaugmentation de $\mathcal{D}$, et la structure est localement bifermée pour cette bicoaugmentation.

Démonstration. - Soit $D$ une petite sous-catégorie pleine de $\mathcal{D}$ dense dans $\mathcal{C}$ contenant un objet initial $\varnothing$ de $\mathcal{C}$. On va commencer par montrer que si $S$ et $S^{\prime}$ sont deux objets de $\mathcal{D}$, on a un isomorphisme canonique

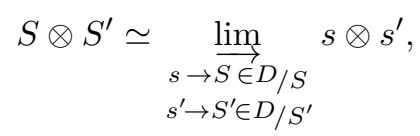

où la limite inductive, comme toutes les limites inductives dans cette preuve, est calculée dans $\mathcal{C}$ (ou dans une tranche de $\mathcal{C}$ ). Notons que ces limites inductives sont connexes puisque $D$ admet un objet initial. On a, pour tout objet $(Z, g)$ de $N\left(S^{\prime}\right) \backslash \mathcal{C}$,

$$
\begin{aligned}
& \operatorname{Hom}_{N\left(S^{\prime}\right) \backslash \mathcal{C}}\left(\underset{s \rightarrow S}{\lim _{s \rightarrow S}}\left(s \otimes S^{\prime}, \iota_{2}\right),(Z, g)\right) \simeq \lim _{s \rightarrow S} \operatorname{Hom}_{N\left(S^{\prime}\right) \backslash \mathcal{C}}\left(\left(s \otimes S^{\prime}, \iota_{2}\right),(Z, g)\right) \\
& \simeq \lim _{s \rightarrow S} \operatorname{Hom}_{\mathcal{C}}\left(s, H\left(S^{\prime}, g, Z\right)\right)
\end{aligned}
$$

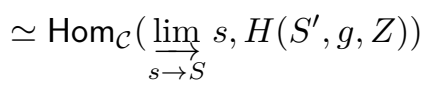

$$
\begin{aligned}
& \simeq \operatorname{Hom}_{\mathcal{C}}\left(S, H\left(S^{\prime}, g, Z\right)\right) \\
& \simeq \operatorname{Hom}_{N\left(S^{\prime}\right) \backslash \mathcal{C}}\left(\left(S \otimes S^{\prime}, \iota_{2}\right),(Z, g)\right),
\end{aligned}
$$


d'où un isomorphisme canonique

$$
\left(S \otimes S^{\prime}, \iota_{2}\right) \simeq \underset{s \rightarrow S}{\lim }\left(s \otimes S^{\prime}, \iota_{2}\right)
$$

dans $N\left(S^{\prime}\right) \backslash \mathcal{C}$ et donc un isomorphisme canonique

$$
S \otimes S^{\prime} \simeq \underset{s \rightarrow S}{\lim _{s \rightarrow S}}\left(s \otimes S^{\prime}\right)
$$

dans $\mathcal{C}$ puisque la limite inductive en jeu est connexe et que le foncteur d'oubli $N\left(S^{\prime}\right) \backslash \mathcal{C} \rightarrow \mathcal{C}$ commute aux limites inductives connexes. De même, en utilisant $H^{\prime}$, on obtient des isomorphismes canoniques

$$
\left(S \otimes S^{\prime}, \iota_{1}\right) \simeq \underset{s^{\prime} \rightarrow S^{\prime}}{\lim _{P}}\left(S \otimes s^{\prime}, \iota_{1}\right)
$$

dans $N(S) \backslash C$ et

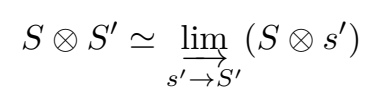

dans $\mathcal{C}$. On a donc

$$
S \otimes S^{\prime} \simeq \lim _{s \rightarrow S}\left(s \otimes S^{\prime}\right) \simeq \lim _{s \rightarrow S} \underset{s^{\prime} \rightarrow S^{\prime}}{\lim _{\rightarrow}} s \otimes s^{\prime}
$$

d'où l'isomorphisme annoncé.

Étendons maintenant le produit tensoriel de $\mathcal{D}$ à $\mathcal{C}$. Puisque pour tout objet $Z$ de $\mathcal{C}$, la limite inductive canonique

$$
\lim _{s \rightarrow \overrightarrow{Z \in D / Z}} s \simeq Z
$$

est connexe, on est contraint de poser, pour $X$ et $Y$ dans $\mathcal{C}$,

$$
X \otimes Y=\lim _{\substack{s \rightarrow \overrightarrow{X \in D} / X \\ s^{\prime} \rightarrow Y \in D / Y}} s \otimes s^{\prime} .
$$

Le paragraphe précédent montre qu'on prolonge bien ainsi le produit tensoriel de $\mathcal{D}$. Par ailleurs, puisque l'endofoncteur $M$ commute aux petites limites inductives connexes, la transformation naturelle $\iota_{1}$ induit des morphismes

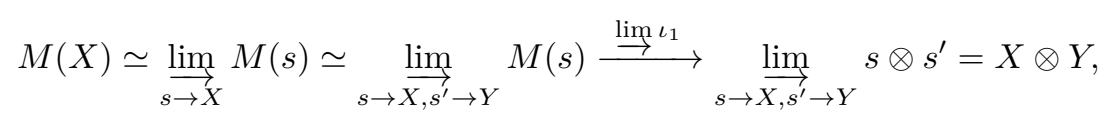

naturels en $X$ et $Y$ dans $\mathcal{C}$, qu'on notera également $\iota_{1}$. De même, la transformation naturelle $\iota_{2}$ induit des morphismes

$$
X \otimes Y \stackrel{\iota_{2}}{\longleftarrow} N(Y),
$$

naturels en $X$ et $Y$ dans $\mathcal{C}$. Il résulte également du paragraphe précédent que ces morphismes $\iota_{1}$ et $\iota_{2}$ prolongent ceux définis sur $\mathcal{D}$. Étendons maintenant les foncteurs $H$ et $H^{\prime}$ en des foncteurs $\widetilde{\mathrm{Fl}}_{\mathrm{d}}(\mathcal{C}) \rightarrow \mathcal{C}$ et $\widetilde{\mathrm{Fl}}_{\mathrm{g}}(\mathcal{C}) \rightarrow \mathcal{C}$ respectivement. Pour 
$(X, g: N(X) \rightarrow Y, Y)$ un objet de $\widetilde{\mathrm{FI}}_{\mathrm{d}}(\mathcal{C})$ et $(X, f: M(X) \rightarrow Z, Z)$ un objet de $\widetilde{\mathrm{F}} \mathrm{g}(\mathcal{C})$, on pose

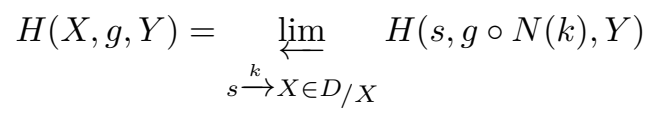

et

$$
H^{\prime}(X, f, Z)=\lim _{s \stackrel{h}{\rightarrow} X \in D / X} H^{\prime}(s, f \circ M(h), Z) .
$$

On va montrer qu'on a des bijections

$$
\operatorname{Hom}_{N(Y) \backslash \mathcal{C}}\left(\left(X \otimes Y, \iota_{2}\right),(Z, g)\right) \simeq \operatorname{Hom}_{\mathcal{C}}(X, H(Y, g, Z)),
$$

naturelles en $X$ dans $\mathcal{C}$ et $(Y, g, Z)$ dans $\widetilde{\mathrm{FI}}_{\mathrm{d}}(\mathcal{C})$, et des bijections

$$
\operatorname{Hom}_{M(X) \backslash \mathcal{C}}\left(\left(X \otimes Y, \iota_{1}\right),(Z, f)\right) \simeq \operatorname{Hom}_{\mathcal{C}}\left(Y, H^{\prime}(X, f, Z)\right),
$$

naturelles en $Y$ dans $\mathcal{C}$ et $(X, f, Z)$ dans $\widetilde{\mathrm{FI}}_{\mathrm{g}}(\mathcal{C})$. Faisons-le pour la première famille de bijections, la seconde se traitant de manière analogue. On a

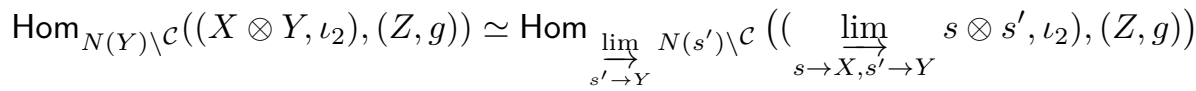

$$
\begin{aligned}
& \simeq \lim _{s \rightarrow X} \operatorname{Hom} \underset{s^{\prime} \rightarrow Y}{\lim _{\rightarrow} N\left(s^{\prime}\right) \backslash \mathcal{C}}\left(\left(\underset{s^{\prime} \rightarrow Y}{\lim _{\longrightarrow}} s \otimes s^{\prime}, \underset{s^{\prime} \rightarrow Y}{\lim _{\longrightarrow}} \iota_{2}\right),(Z, g)\right) \\
& \simeq \lim _{s \rightarrow X, s^{\prime} \stackrel{k}{\longrightarrow} Y} \operatorname{Hom}_{N\left(s^{\prime}\right) \backslash \mathcal{C}}\left(\left(s \otimes s^{\prime}, \iota_{2}\right),(Z, g \circ N(k))\right) \\
& s \rightarrow X, s^{\prime} \rightarrow Y
\end{aligned}
$$

(en vertu du lemme 5.12)

$$
\begin{aligned}
\simeq \quad \underbrace{}_{s \rightarrow X, s^{\prime}} \stackrel{k}{\rightarrow} Y \\
\simeq \operatorname{Hom}_{\mathcal{C}}\left(\underset{s \rightarrow X}{\lim _{\mathcal{C}}} s,{\underset{\lim }{s^{\prime}}}_{\stackrel{\lim }{\rightarrow} Y} H\left(s^{\prime}, g \circ N(k), Z\right)\right) \\
\simeq \operatorname{Hom}_{\mathcal{C}}(X, H(Y, g, Z)),
\end{aligned}
$$

ce qu'on voulait démontrer. Ceci montre que le foncteur $\otimes: \mathcal{C} \times \mathcal{C} \rightarrow \mathcal{C}$ commute aux limites inductives connexes en chaque variable.

Pour conclure, il suffit de vérifier que la structure monoïdale sur $\mathcal{D}$ induit une structure monoïdale sur $\mathcal{C}$ munie du produit tensoriel qu'on vient de définir. On procède exactement comme dans la preuve du théorème 5.3.

Corollaire 5.14. - Soient $\mathcal{C}$ une catégorie complète et cocomplète, et $\mathcal{D}$ une souscatégorie pleine de $\mathcal{C}$ munie d'une structure monö̈dale d'unité un objet initial de $\mathcal{C}$ et contenant une petite sous-catégorie pleine dense dans $\mathcal{C}$. On suppose qu'il existe des foncteurs

$$
H: \widetilde{\mathrm{FI}}_{d}(\mathcal{D}, \mathcal{C}) \rightarrow \mathcal{C} \quad \text { et } \quad H^{\prime}: \widetilde{\mathrm{FI}}_{g}(\mathcal{D}, \mathcal{C}) \rightarrow \mathcal{C}
$$


où on a posé

$$
\widetilde{\mathrm{FI}}_{d}(\mathcal{D}, \mathcal{C})=\widetilde{\mathrm{F}}(\mathcal{D} \hookrightarrow \mathcal{C})=\widetilde{\mathrm{F}}_{g}(\mathcal{D}, \mathcal{C})
$$

des bijections

$$
\operatorname{Hom}_{S^{\prime} \backslash \mathcal{C}}\left(\left(S \otimes S^{\prime}, \iota_{2}\right),(Z, g)\right) \simeq \operatorname{Hom}_{\mathcal{C}}\left(S, H\left(S^{\prime}, g, Z\right)\right)
$$

naturelles en $S$ dans $\mathcal{D}$ et $\left(S^{\prime}, g, Z\right)$ dans $\widetilde{\mathrm{FI}}_{d}(\mathcal{D}, \mathcal{C})$, et des bijections

$$
\operatorname{Hom}_{S \backslash \mathcal{C}}\left(\left(S \otimes S^{\prime}, \iota_{1}\right),(Z, f)\right) \simeq \operatorname{Hom}_{\mathcal{C}}\left(S^{\prime}, H^{\prime}(S, f, Z)\right),
$$

naturelles en $S^{\prime}$ dans $\mathcal{D}$ et $(S, f, Z)$ dans $\widetilde{\mathrm{FI}}_{g}(\mathcal{D}, \mathcal{C})$.

Alors il existe une et une seule structure de catégorie monoïdale sur $\mathcal{C}$ (à unique isomorphisme monoïdal près) donnée par un produit tensoriel commutant aux petites limites inductives connexes en chaque variable et pour laquelle le foncteur d'inclusion $\mathcal{D} \hookrightarrow \mathcal{C}$ s'étend en un foncteur monö̈dal. De plus, cette structure monoïdale est localement bifermée.

Démonstration. - On applique le théorème précédent pour $M$ et $N$ l'endofoncteur identité et pour la bicoaugmentation locale de l'exemple 5.6.

Remarque 5.15. - On peut également obtenir le théorème 5.3 comme corollaire du théorème 5.13. Si $\mathcal{D}$ contient un objet initial de $\mathcal{C}$, il suffit d'appliquer ce théorème à $\mathcal{D}$ munie de la bicoaugmentation triviale (voir l'exemple 5.6). Si $\mathcal{D}$ ne contient pas d'objet initial, il suffit de faire de même en remplaçant $\mathcal{D}$ par la catégorie $\mathcal{D}^{\prime}$ obtenue en ajoutant à $\mathcal{D}$ un objet initial de $\mathcal{C}$. On vérifie alors qu'on peut prolonger la structure de catégorie monoïdale et les foncteurs $H$ et $H^{\prime}$ à $\mathcal{D}^{\prime}$ en posant $\varnothing \otimes S \simeq \varnothing \simeq S \otimes \varnothing$ pour tout $S$ dans $\mathcal{D}$ et $H(\varnothing, Z)=*=H^{\prime}(\varnothing, Z)$, où $*$ est un objet final de $\mathcal{C}$, pour tout $Z$ dans $\mathcal{C}$. 



\section{CHAPITRE 6}

\section{JOINT ET TRANCHES $\infty$-CATÉGORIQUES}

Le but de ce chapitre est de définir le joint et les tranches $\infty$-catégoriques. Pour ce faire, on commence par définir un joint pour les complexes de Steiner forts qu'on étend aux $\infty$-catégories grâce à un théorème à la Day établi dans le chapitre précédent.

6.1. - Soient $K$ et $L$ deux complexes de chaînes (de groupes abéliens en degrés positifs, selon notre convention du paragraphe 2.1). Rappelons que leur produit tensoriel $K \otimes L$ est défini de la manière suivante. On pose

$$
(K \otimes L)_{p}=\bigoplus_{\substack{i+j=p \\ i \geqslant 0, j \geqslant 0}} K_{i} \otimes L_{j}, \quad \text { pour } p \geqslant 0,
$$

et, pour $x$ dans $K_{i}$ et $y$ dans $L_{j}$ avec $i+j>0$,

$$
d(x \otimes y)=d(x) \otimes y+(-1)^{|x|} x \otimes d(y),
$$

où $|x|$ désigne le degré de $x$, c'est-à-dire $i$. Ce produit tensoriel définit une structure de catégorie monoïdale sur la catégorie des complexes de chaînes. L'unité est donnée par le complexe concentré en degré 0 de valeur $\mathbb{Z}$. On dispose d'un morphisme $\gamma_{K, L}: K \otimes L \rightarrow L \otimes K$ envoyant $x \otimes y$, pour $x$ un élément homogène de $K$ et $y$ un élément homogène de $L$, sur $(-1)^{|x||y|} y \otimes x$ qui définit une symétrie pour cette structure de catégorie monoïdale. De plus, cette catégorie monoïdale symétrique est fermée.

Ce produit tensoriel s'étend aux complexes de chaînes augmentés de la manière suivante. Si $K$ et $L$ sont deux complexes de chaînes augmentés, on définit une augmentation sur $K \otimes L$ en posant

$$
e(x \otimes y)=e(x) e(y)
$$

pour $x$ dans $K_{0}$ et $y$ dans $L_{0}$. On obtient ainsi une structure de catégorie monoïdale symétrique fermée sur la catégorie des complexes de chaînes augmentés. L'unité est l'unité du produit tensoriel des complexes de chaînes munie de l'augmentation identité $1_{\mathbb{Z}}: \mathbb{Z} \rightarrow \mathbb{Z}$. 
6.2. - Dans [32, exemple 3.10], Steiner étend le produit tensoriel des complexes de chaînes augmentés aux complexes dirigés augmentés de la manière suivante. Si $K$ et $L$ sont deux complexes dirigés augmentés, le complexe de chaînes augmenté sous-jacent à $K \otimes L$ est le produit tensoriel des complexes de chaînes augmentés sous-jacents à $K$ et $L$ et, pour $p \geqslant 0$, le sous-monoïde de positivité $(K \otimes L)_{p}^{*}$ est le sous-monoïde de $(K \otimes L)_{p}$ engendré par les éléments de la forme $x \otimes y$, avec $x$ dans $K_{i}^{*}, y$ dans $L_{j}^{*}$ et $i+j=p$.

Steiner montre [32, exemple 3.10 et p. 198] qu'on obtient ainsi une structure de catégorie monoïdale bifermée (voir le paragraphe 5.2) sur la catégorie des complexes dirigés augmentés. L'unité $\underline{\mathbb{Z}}^{\prime}$ est définie de la manière suivante : le complexe sousjacent est le complexe concentré en degré 0 de valeur $\mathbb{Z}$, l'augmentation est l'identité $1_{\mathbb{Z}}$ et on a $\underline{\mathbb{Z}}_{0}^{\prime *}=\mathbb{N}$. On vérifie facilement que $\underline{\mathbb{Z}}^{\prime}$ est un objet final de la catégorie des complexes dirigés augmentés décents (voir le paragraphe 2.17). Notons par ailleurs que $\nu\left(\underline{\mathbb{Z}}^{\prime}\right)$ est la $\infty$-catégorie finale. On prendra garde que cette structure de catégorie monoïdale n'est pas symétrique. De fait, la symétrie $\gamma_{K, L}$ du paragraphe 6.1 n'induit pas un morphisme de complexes dirigés augmentés car elle ne respecte pas les sousmonoïdes de positivité à cause des signes apparaissant dans sa définition. De plus, on peut exhiber des complexes dirigés augmentés $K$ et $L$ tels que $K \otimes L$ et $L \otimes K$ ne soient pas isomorphes (par exemple $K=\lambda\left(\mathrm{D}_{1}\right)$ et $L=\lambda\left(\mathrm{D}_{2}\right)$ ).

6.3. - Soit $K$ un complexe de chaînes augmenté de différentielle $d$ et d'augmentation $e$. On notera $\Sigma K$ le complexe de chaînes de différentielle $d^{\prime}$ défini par

$$
(\Sigma K)_{p}= \begin{cases}\mathbb{Z} & \text { si } p=0, \\ K_{p-1} & \text { si } p>0,\end{cases}
$$

et

$$
d_{p}^{\prime}= \begin{cases}e & \text { si } p=1 \\ d_{p-1} & \text { si } p>1\end{cases}
$$

On appellera ce complexe la suspension de $K$.

Il est immédiat que $\Sigma$ définit un isomorphisme de la catégorie des complexes de chaînes augmentés vers la sous-catégorie non pleine de la catégorie des complexes de chaînes formée des complexes $K$ tels que $K_{0}=\mathbb{Z}$ et des morphismes $f$ tels que $f_{0}=1_{\mathbb{Z}}$. On notera $\Sigma^{-1}$ l'inverse du foncteur $\Sigma$.

Remarque 6.4. - Si on considère un complexe de chaînes augmenté $K$ comme un complexe de chaînes muni d'un morphisme vers le complexe concentré en degré 0 de valeur $\mathbb{Z}$, en suspendant ce morphisme avec la convention de signe usuelle, on obtient un complexe de chaînes qui diffère du complexe $\Sigma K$ qu'on a décrit ci-dessus : ce complexe s'obtient en multipliant par -1 les différentielles $d_{p}^{\prime}$, pour $p>1$, du complexe $\Sigma K$. Notre choix de signe est dicté par une compatibilité aux orientaux de Street (voir la remarque 7.11 ). 
6.5. - Soient $K$ et $L$ deux complexes de chaînes augmentés. On définit un nouveau complexe de chaînes augmenté $K \star L$, appelé le joint de $K$ et $L$, par la formule

$$
K \star L=\Sigma^{-1}(\Sigma K \otimes \Sigma L) .
$$

(Cette formule a un sens puisque $(\Sigma K \otimes \Sigma L)_{0}=\mathbb{Z} \otimes \mathbb{Z} \simeq \mathbb{Z}$.)

On obtient ainsi un foncteur

$$
(K, L) \mapsto K \star L .
$$

Les contraintes d'associativité et d'unité du produit tensoriel des complexes de chaînes induisent des contraintes pour le joint. Le joint définit donc une structure de catégorie monoïdale sur la catégorie des complexes de chaînes augmentés. L'unité est le complexe nul (muni de l'unique augmentation possible).

Explicitement, pour $p \geqslant 0$, on a

$$
(K \star L)_{p}=\bigoplus_{\substack{i+1+j=p \\ i \geqslant-1, j \geqslant-1}} K_{i} \otimes L_{j},
$$

où on a posé $K_{-1}=\mathbb{Z}$ et $L_{-1}=\mathbb{Z}$. Ainsi, on a

$$
(K \star L)_{p} \simeq K_{p} \oplus\left(K_{p-1} \otimes L_{0}\right) \oplus \cdots \oplus\left(K_{0} \otimes L_{p-1}\right) \oplus L_{p} .
$$

On notera $\varnothing$ le générateur positif de $K_{-1}$ et $L_{-1}$. Si $x$ est dans $K_{i}$ et $y$ est dans $L_{j}$ avec $i+1+j=p$, on notera $x \star y$ l'élément de $(K \star L)_{p}$ correspondant.

La différentielle du complexe $K \star L$ est donnée par

$$
d(x \star y)=d x \star y+(-1)^{|x|+1} x \star d y,
$$

où on a posé

pour $z$ de degré 0 et

$$
d z=e(z) \varnothing
$$

$$
d(\varnothing)=0 \quad \text { et } \quad|\varnothing|=-1 .
$$

En particulier, on a

$$
d(x \star \varnothing)=d x \star \varnothing \quad \text { et } \quad d(\varnothing \star y)=\varnothing \star d y .
$$

Enfin, l'augmentation de $K \star L$ est donnée par

$$
e(x \star \varnothing)=e(x) \text { et } \quad e(\varnothing \star y)=e(y),
$$

pour $x$ dans $K_{0}$ et $y$ dans $L_{0}$

6.6. - Soit $K$ un complexe dirigé augmenté. On notera $\Sigma K$ le complexe dirigé augmenté défini par la suspension du complexe de chaînes augmenté sous-jacent à $K$ (voir le paragraphe 6.3), les sous-monoïdes de positivité

$$
(\Sigma K)_{p}^{*}= \begin{cases}\mathbb{N} & \text { si } p=0, \\ K_{p-1}^{*} & \text { si } p>0,\end{cases}
$$


et l'augmentation nulle $e=\mathbb{Z} \stackrel{0}{\rightarrow} \mathbb{Z}$.

On obtient ainsi un foncteur $\Sigma: \mathcal{C}_{\mathrm{da}} \rightarrow \mathcal{C}_{\mathrm{da}}$. Il est immédiat que ce foncteur définit un isomorphisme de la catégorie des complexes dirigés augmentés vers la souscatégorie non pleine $\mathcal{C}_{\mathrm{da}}^{\Sigma}$ de la catégorie des complexes dirigés augmentés formée des complexes $K$ tels que $K_{0}=\mathbb{Z}, K_{0}^{*}=\mathbb{N}$ et d'augmentation nulle, et des morphismes $f$ tels que $f_{0}=1_{\mathbb{Z}}$. On notera $\Sigma^{-1}$ l'inverse du foncteur $\Sigma$.

6.7. - Soient $K$ et $L$ deux complexes dirigés augmentés. On définit un nouveau complexe dirigé augmenté $K \star L$, appelé le joint de $K$ et $L$, par la formule

$$
K \star L=\Sigma^{-1}(\Sigma K \otimes \Sigma L) .
$$

(Cette formule a un sens puisque $(\Sigma K \otimes \Sigma L)_{0}=\mathbb{Z} \otimes \mathbb{Z} \simeq \mathbb{Z},(\Sigma K \otimes \Sigma L)_{0}^{*}=\mathbb{N} \otimes \mathbb{N} \subset \mathbb{Z}$ vaut $\mathbb{N}$ et l'augmentation de ce complexe est nulle car produit de deux augmentations nulles.)

Explicitement, le complexe de chaînes augmenté sous-jacent à $K \star L$ est le joint des complexes de chaînes sous-jacents à $K$ et $L$ et, si $p \geqslant 0$, le sous-monoïde de positivité $(K \star L)_{p}^{*}$ est le sous-monoïde engendré par les éléments de la forme $x \star y$, avec $x$ dans $K_{i}^{*}$ et $y$ dans $L_{j}^{*}$, où $i$ et $j$ satisfont $i \geqslant-1, j \geqslant-1$ et $i+1+j=p$, en convenant que $K_{-1}^{*}=\mathbb{N}$ et $L_{-1}^{*}=\mathbb{N}$.

On obtient ainsi un foncteur

$$
\begin{aligned}
\mathcal{C}_{\text {da }} \times \mathcal{C}_{\text {da }} & \rightarrow \mathcal{C}_{\text {da }} \\
(K, L) & \mapsto K \star L
\end{aligned}
$$

qui définit une structure de catégorie monoïdale sur la catégorie des complexes dirigés augmentés. L'unité est le complexe nul muni de l'unique augmentation et des uniques sous-monoïdes de positivité possibles. On notera $\varnothing$ ce complexe dirigé augmenté. Cette notation est justifiée par le fait que $\varnothing$ est un objet initial de la catégorie des complexes dirigés augmentés. De plus, la $\infty$-catégorie $\nu(\varnothing)$ est la $\infty$-catégorie vide. On prendra garde que cette structure monoïdale n'est pas symétrique.

Remarque 6.8. - Cette opération joint est le pendant dans le monde des complexes dirigés augmentés du joint des complexes de parité défini par Street dans [37, section 5].

Proposition 6.9. - Soit $K$ un complexe dirigé augmenté. Alors les endofoncteurs de la catégorie des complexes dirigés augmentés

$$
L \mapsto K \star L \quad \text { et } \quad L \mapsto L \star K
$$

commutent aux limites inductives connexes.

Démonstration. — La formule

$$
K \star L=\Sigma^{-1}(\Sigma K \otimes \Sigma L)
$$


montre que, pour établir le résultat, il suffit de vérifier que les foncteurs $\Sigma K \otimes$ - $\otimes \Sigma L$ et le foncteur d'inclusion $\mathcal{C}_{\mathrm{da}}^{\Sigma} \hookrightarrow \mathcal{C}_{\mathrm{da}}$ (voir le paragraphe 6.6) commutent aux limites inductives connexes. Les deux premiers foncteurs commutent à toutes les limites inductives puisqu'ils admettent des adjoints à droite. En ce qui concerne le troisième foncteur, il suffit de vérifier que la limite inductive dans $\mathcal{C}_{\mathrm{da}}$ d'un diagramme connexe à valeurs dans $\mathcal{C}_{\mathrm{da}}^{\Sigma}$ est dans $\mathcal{C}_{\mathrm{da}}^{\Sigma}$; cela résulte du fait que les limites inductives se calculent degré par degré dans $\mathcal{C}_{\mathrm{da}}$ et du fait que la limite inductive d'un diagramme connexe constant de valeur $\mathbb{Z}$ vaut $\mathbb{Z}$.

Proposition 6.10. — Soient $K$ et $L$ deux complexes dirigés augmentés. Les applications

$$
\begin{aligned}
(K \star L)_{p} & \rightarrow(L \star K)_{p} \\
x \star y & \mapsto y \star x,
\end{aligned}
$$

pour $p \geqslant 0$, définissent un isomorphisme

$$
(K \star L)^{\mathrm{op}} \simeq L^{\mathrm{op}} \star K^{\mathrm{op}} .
$$

Démonstration. - La compatibilité aux augmentations, aux sous-monoïdes de positivité et la bijectivité sont évidentes. Il s'agit donc de vérifier la compatibilité aux différentielles. Notons $s: x \star y \mapsto y \star x$ l'application de l'énoncé. Pour tout élément homogène de $K \star L$ de la forme $x \star y$ et de degré au moins 1 , on a, en notant $d^{\prime}$ les différentielles dual impair,

$$
\begin{aligned}
s d^{\prime}(x \star y) & =(-1)^{|x|+1+|y|} s d(x \star y) \\
& =(-1)^{|x|+1+|y|} s\left(d x \star y+(-1)^{|x|+1} x \star d y\right) \\
& =(-1)^{|x|+1+|y|} y \star d x+(-1)^{|y|} d y \star x
\end{aligned}
$$

et

$$
\begin{aligned}
d s(x \star y) & =d(y \star x)=d^{\prime} y \star x+(-1)^{|y|+1} y \star d^{\prime} x \\
& =(-1)^{|y|} d y \star x+(-1)^{|y|+1+|x|} y \star d x \\
& =(-1)^{|x|+1+|y|} y \star d x+(-1)^{|y|} d y \star x,
\end{aligned}
$$

d'où le résultat.

Remarque 6.11. - Outre la dualité triviale, la dualité considérée dans l'énoncé précédent est la seule dualité pour laquelle on ait un isomorphisme de ce type. En particulier, on n'a pas d'isomorphismes

$$
(K \star L)^{\mathrm{co}} \simeq L^{\mathrm{co}} \star K^{\mathrm{co}} \quad \text { ou } \quad(K \star L)^{\circ} \simeq K^{\circ} \star L^{\circ} .
$$

De fait, la preuve précédente ne s'adapte pas aux dualités paire et totale. Dans cette preuve, et avec ses notations, on a utilisé le fait que l'égalité $d^{\prime} z=(-1)^{|z|} d z$ reste valable quand $z$ est de degré 0 puisque dans cette formule, par convention, les 
différentielles en degré 0 sont les augmentations, et que les dualités préservent les augmentations (voir le paragraphe 2.18). Mais si $d^{\prime \prime}$ et $d^{\prime \prime \prime}$ désignent les différentielles dual pair et dual total, les égalités $d^{\prime \prime} z=(-1)^{|z|+1} d z$ et $d^{\prime \prime \prime} z=-d z$ sont fausses lorsque $z$ est de degré 0 .

6.12. - Soient $K$ et $L$ deux complexes dirigés augmentés. Les morphismes canoniques $\varnothing \rightarrow K$ et $\varnothing \rightarrow L$ induisent des morphismes

$$
K \simeq K \star \varnothing \longrightarrow K \star L \longleftarrow \varnothing \star L \simeq L
$$

qu'on notera $\iota_{1}$ et $\iota_{2}$. On a donc des morphismes

$$
K \stackrel{\iota_{1}}{\longrightarrow} K \star L \stackrel{\iota_{2}}{\longleftarrow} L .
$$

(Ce sont les morphismes de la bicoaugmentation locale associée au joint, voir l'exemple 5.6.) Explicitement, pour tout élément homogène $x$ de $K$ et tout élément homogène $y$ de $L$, on a

$$
\iota_{1}(x)=x \star \varnothing \quad \text { et } \quad \iota_{2}(y)=\varnothing \star y .
$$

6.13. - Soient $K$ et $L$ des complexes dirigés augmentés admettant des bases $X$ et $Y$ respectivement. Il est immédiat que le complexe dirigé augmenté $K \star L$ admet pour base l'ensemble

$$
X \star Y=\{x \star \varnothing \mid x \in X\} \cup\{x \star y \mid x \in X, y \in Y\} \cup\{\varnothing \star y \mid y \in Y\} .
$$

Lemme 6.14. - Soient $K$ et $L$ deux complexes dirigés augmentés. Pour tout élément homogène de $K \star L$ de la forme $x \star y$, tout $r \geqslant 0$ et $\varepsilon=0,1$, on a

$$
\langle x \star y\rangle_{r}^{\varepsilon}=\sum_{\substack{p+1+q=r \\-1 \leqslant p \leqslant|x|,-1 \leqslant q \leqslant|y|}}\langle x\rangle_{p}^{\varepsilon} \star\langle y\rangle_{q}^{p+1+\varepsilon \bmod 2}
$$

où on a posé $\langle z\rangle_{-1}^{0}=\left(e\left(\langle z\rangle_{0}^{0}\right) \varnothing\right)_{-}$et $\langle z\rangle_{-1}^{1}=\left(e\left(\langle z\rangle_{0}^{1}\right) \varnothing\right)_{+}$pour z un élément homogène de $K$ ou $L$, et $\langle\varnothing\rangle_{-1}^{\varepsilon}=\varnothing$ pour $\varepsilon=0,1$.

Démonstration. - Observons tout d'abord que si $z$ est un élément homogène de $K$ ou $L$, en ajoutant la convention $d_{0}=e$ à nos précédentes conventions, on a $d\left(\langle z\rangle_{r}^{\varepsilon}\right)=\langle z\rangle_{r-1}^{1}-\langle z\rangle_{r-1}^{0}$ pour tout $r \geqslant 0$ et $\varepsilon=0,1$. (Rappelons qu'on a convenu que $\langle z\rangle_{r}^{\varepsilon}=0$ pour $\varepsilon=0,1$ dès que $r>|z|$.) De plus, cette égalité reste valable pour $z=\varnothing$ si l'on convient que, pour $\varepsilon=0,1$, en plus de $\langle\varnothing\rangle_{-1}^{\varepsilon}=\varnothing$, on a $\langle\varnothing\rangle_{r}^{\varepsilon}=0$ pour $r \geqslant 0$.

Démontrons maintenant le lemme. Pour $r>|x \star y|=|x|+1+|y|$, les deux membres de l'égalité sont nuls. Supposons donc $r \leqslant|x \star y|$. On va démontrer le résultat par récurrence descendante sur $r$ de $|x \star y|$ à 0 . Pour $r=|x \star y|$, les deux membres de 
l'égalité valent $x \star y$. Supposons le résultat vrai pour $r+1$ et montrons-le pour $r$. Par hypothèse de récurrence, on $\mathrm{a}$, en sous-entendant les « $\bmod 2 »$,

$$
\begin{aligned}
d\left(\langle x \star y\rangle_{r+1}^{0}\right) & =\sum_{\substack{p^{\prime}+1+q^{\prime}=r+1 \\
-1 \leqslant p^{\prime} \leqslant|x|,-1 \leqslant q^{\prime} \leqslant|y|}} d\left(\langle x\rangle_{p^{\prime}}^{0} \star\langle y\rangle_{q^{\prime}}^{p^{\prime}+1}\right) \\
& =\sum_{\substack{p^{\prime}+1+q^{\prime}=r+1 \\
-1 \leqslant p^{\prime} \leqslant|x|,-1 \leqslant q^{\prime} \leqslant|y|}}\left(d\left(\langle x\rangle_{p^{\prime}}^{0}\right) \star\langle y\rangle_{q^{\prime}}^{p^{\prime}+1}+(-1)^{p^{\prime}+1}\langle x\rangle_{p^{\prime}}^{0} \star d\left(\langle y\rangle_{q^{\prime}}^{p^{\prime}+1}\right)\right) \\
& =\sum_{\substack{p^{\prime}+1+q^{\prime}=r+1 \\
-1 \leqslant p^{\prime} \leqslant|x|,-1 \leqslant q^{\prime} \leqslant|y|}} d\left(\langle x\rangle_{p^{\prime}}^{0}\right) \star\langle y\rangle_{q^{\prime}}^{p^{\prime}+1}+\sum_{\substack{p^{\prime}+1+q^{\prime}=r+1 \\
-1 \leqslant p^{\prime} \leqslant|x|,-1 \leqslant q^{\prime} \leqslant|y|}}(-1)^{p^{\prime}+1}\langle x\rangle_{p^{\prime}}^{0} \star d\left(\langle y\rangle_{q^{\prime}}^{p^{\prime}+1}\right) \\
& =\sum_{\substack{p+1+q=r \\
-2 \leqslant p \leqslant|x|-1,-1 \leqslant q \leqslant|y|}} d\left(\langle x\rangle_{p+1}^{0}\right) \star\langle y\rangle_{q}^{p}+\sum_{\substack{p+1+q=r \\
-1 \leqslant p \leqslant|x|,-2 \leqslant q \leqslant|y|-1}}^{p+1}\langle x\rangle_{p}^{0} \star d\left(\langle y\rangle_{q+1}^{p+1}\right)
\end{aligned}
$$

(en posant $p=p^{\prime}-1$ et $q=q^{\prime}$ dans le terme de gauche et $p=p^{\prime}$ et $q=q^{\prime}-1$ dans celui de droite)

$$
=\sum_{\substack{p+1+q=r \\-1 \leqslant p \leqslant|x|,-1 \leqslant q \leqslant|y|}} d\left(\langle x\rangle_{p+1}^{0}\right) \star\langle y\rangle_{q}^{p}+\sum_{\substack{p+1+q=r \\-1 \leqslant p \leqslant|x|,-1 \leqslant q \leqslant|y|}}(-1)^{p+1}\langle x\rangle_{p}^{0} \star d\left(\langle y\rangle_{q+1}^{p+1}\right)
$$

(car on a, d'une part, $d\left(\langle x\rangle_{-1}^{0}\right)=0$ et $d\left(\langle x\rangle_{|x|+1}^{0}\right)=0$ et, d'autre part, $d\left(\langle y\rangle_{-1}^{p+1}\right)=0$ et $\left.d\left(\langle y\rangle_{|y|+1}^{p+1}\right)=0\right)$

$$
=\sum_{\substack{p+1+q=r \\-1 \leqslant p \leqslant|x|,-1 \leqslant q \leqslant|y|}}\left(d\left(\langle x\rangle_{p+1}^{0}\right) \star\langle y\rangle_{q}^{p}+(-1)^{p+1}\langle x\rangle_{p}^{0} \star d\left(\langle y\rangle_{q+1}^{p+1}\right)\right) .
$$

Or, si $p$ est pair, la quantité sous le signe somme vaut

$$
\begin{aligned}
& d\left(\langle x\rangle_{p+1}^{0}\right) \star\langle y\rangle_{q}^{p}+(-1)^{p+1}\langle x\rangle_{p}^{0} \star d\left(\langle y\rangle_{q+1}^{p+1}\right) \\
& \quad=\left(\langle x\rangle_{p}^{1} \star\langle y\rangle_{q}^{0}-\langle x\rangle_{p}^{0} \star\langle y\rangle_{q}^{0}\right)-\left(\langle x\rangle_{p}^{0} \star\langle y\rangle_{q}^{1}-\langle x\rangle_{p}^{0} \star\langle y\rangle_{q}^{0}\right) \\
& \quad=\langle x\rangle_{p}^{1} \star\langle y\rangle_{q}^{0}-\langle x\rangle_{p}^{0} \star\langle y\rangle_{q}^{1}
\end{aligned}
$$

et, si $p$ est impair,

$$
\begin{aligned}
& d\left(\langle x\rangle_{p+1}^{0}\right) \star\langle y\rangle_{q}^{p}+(-1)^{p+1}\langle x\rangle_{p}^{0} \star d\left(\langle y\rangle_{q+1}^{p+1}\right) \\
& \quad=\left(\langle x\rangle_{p}^{1} \star\langle y\rangle_{q}^{1}-\langle x\rangle_{p}^{0} \star\langle y\rangle_{q}^{1}\right)+\left(\langle x\rangle_{p}^{0} \star\langle y\rangle_{q}^{1}-\langle x\rangle_{p}^{0} \star\langle y\rangle_{q}^{0}\right) \\
& \quad=\langle x\rangle_{p}^{1} \star\langle y\rangle_{q}^{1}-\langle x\rangle_{p}^{0} \star\langle y\rangle_{q}^{0} .
\end{aligned}
$$

Ainsi, on a

$$
d\left(\langle x \star y\rangle_{r+1}^{0}\right)=\sum_{\substack{p+1+q=r \\-1 \leqslant p \leqslant|x|,-1 \leqslant q \leqslant|y|}}\left(\langle x\rangle_{p}^{1} \star\langle y\rangle_{q}^{p}-\langle x\rangle_{p}^{0} \star\langle y\rangle_{q}^{p+1}\right)
$$


et donc

$$
\langle x \star y\rangle_{r}^{0}=\sum_{\substack{p+1+q=r \\-1 \leqslant p \leqslant|x|,-1 \leqslant q \leqslant|y|}}\langle x\rangle_{p}^{0} \star\langle y\rangle_{q}^{p+1} \text { et }\langle x \star y\rangle_{p}^{1}=\sum_{\substack{p+1+q=r \\-1 \leqslant p \leqslant|x|,-1 \leqslant q \leqslant|y|}}\langle x\rangle_{p}^{1} \star\langle y\rangle_{q}^{p},
$$

ce qu'on voulait démontrer.

Proposition 6.15. — Si $K$ et $L$ sont des complexes dirigés augmentés à base unitaire, alors il en est de même de $K \star L$.

Démonstration. - Notons $X$ et $Y$ les bases respectives des complexes $K$ et $L$. Soient $x$ un élément de $X$ et $y$ un élément de $Y$. En vertu du lemme précédent, on a

$$
\begin{aligned}
\langle x \star y\rangle_{0}^{0} & =\langle x\rangle_{-1}^{0} \star\langle y\rangle_{0}^{0}+\langle x\rangle_{0}^{0} \star\langle y\rangle_{-1}^{1} \\
& =\left(e\left(x_{0}^{0}\right) \varnothing\right)_{-} \star\langle y\rangle_{0}^{0}+\langle x\rangle_{0}^{0} \star\left(e\left(y_{0}^{1}\right) \varnothing\right)_{+} \\
& =\varnothing_{-} \star\langle y\rangle_{0}^{0}+\langle x\rangle_{0}^{0} \star \varnothing_{+} \\
& =0 \star\langle y\rangle_{0}^{0}+\langle x\rangle_{0}^{0} \star \varnothing \\
& =\langle x\rangle_{0}^{0} \star \varnothing,
\end{aligned}
$$

et, par un calcul similaire,

$$
\langle x \star y\rangle_{0}^{1}=\varnothing \star\langle y\rangle_{0}^{1}
$$

On en déduit les égalités

$$
e\left(\langle x \star y\rangle_{0}^{0}\right)=e\left(\langle x\rangle_{0}^{0}\right)=1 \quad \text { et } \quad e\left(\langle x \star y\rangle_{0}^{1}\right)=e\left(\langle y\rangle_{0}^{1}\right)=1 .
$$

On a par ailleurs, pour $\varepsilon=0,1$,

$$
\langle x \star \varnothing\rangle_{0}^{\varepsilon}=\langle x\rangle_{0}^{\varepsilon} \star \varnothing \quad \text { et } \quad\langle\varnothing \star y\rangle_{0}^{\varepsilon}=\varnothing \star\langle y\rangle_{0}^{\varepsilon},
$$

et donc

$$
e\left(\langle x \star \varnothing\rangle_{0}^{\varepsilon}\right)=e\left(\langle x\rangle_{0}^{\varepsilon}\right)=1 \quad \text { et } \quad e\left(\langle\varnothing \star y\rangle_{0}^{\varepsilon}\right)=e\left(\langle y\rangle_{0}^{\varepsilon}\right)=1,
$$

ce qui achève de prouver que le complexe $K \star L$ est à base unitaire.

Lemme 6.16. - Soient $f: K \rightarrow K^{\prime}$ et $g: L \rightarrow L^{\prime}$ des morphismes entre complexes dirigés augmentés à base. Considérons un élément homogène de $K \star L$ de la forme $x \star y$. On suppose qu'il existe un élément homogène de $K^{\prime} \star L^{\prime}$ de la forme $x^{\prime} \star y^{\prime}$ tel que

(a) si $x$ et $y$ sont différents de $\varnothing$, alors il en est de même de $x^{\prime}$ et $y^{\prime}$, et on a

$$
f(\langle x\rangle)=\left\langle x^{\prime}\right\rangle \quad \text { et } g(\langle y\rangle)=\left\langle y^{\prime}\right\rangle \text {, }
$$

(b) si $x=\varnothing$, alors

(c) si $y=\varnothing$, alors

$$
x^{\prime}=\varnothing \quad \text { et } \quad g(\langle y\rangle)=\left\langle y^{\prime}\right\rangle,
$$

$$
f(\langle x\rangle)=\left\langle x^{\prime}\right\rangle \quad \text { et } \quad y^{\prime}=\varnothing .
$$

Alors on a

$$
(f \star g)(\langle x \star y\rangle)=\left\langle x^{\prime} \star y^{\prime}\right\rangle .
$$


Démonstration. - Pour tout $k \geqslant 0$ et $\varepsilon=0,1$, on a

$$
(f \star g)\left(\langle x \star y\rangle_{k}^{\varepsilon}\right)=(f \star g)\left(\sum_{\substack{p+1+q=k \\-1 \leqslant p \leqslant|x|,-1 \leqslant q \leqslant|y|}}\langle x\rangle_{p}^{\varepsilon} \star\langle y\rangle_{q}^{p+1+\varepsilon \bmod 2}\right)
$$

(en vertu du lemme 6.14 et avec ses conventions)

$$
\begin{aligned}
= & \sum_{\substack{p+1+q=k \\
-1 \leqslant p \leqslant|x|,-1 \leqslant q \leqslant|y|}} f\left(\langle x\rangle_{p}^{\varepsilon}\right) \star g\left(\langle y\rangle_{q}^{p+1+\varepsilon \bmod 2}\right) \\
& (\text { en convenant que } m(\varnothing)=\varnothing \text { pour } m=f, g) \\
= & \sum_{\substack{p+1+q=k \\
-1 \leqslant p \leqslant\left|x^{\prime}\right|,-1 \leqslant q \leqslant\left|y^{\prime}\right|}}\left\langle x^{\prime}\right\rangle_{p}^{\varepsilon} \star\left\langle y^{\prime}\right\rangle_{q}^{p+1+\varepsilon \bmod 2,},
\end{aligned}
$$

la dernière égalité découlant du fait que, d'une part, on a $f\left(\langle x\rangle_{p}^{\varepsilon}\right)=0$ si $p>\left|x^{\prime}\right|$ et $g\left(\langle y\rangle_{q}^{\varepsilon^{\prime}}\right)=0$ si $q>\left|y^{\prime}\right|$ et, d'autre part, nos hypothèses entraînent les égalités $f\left(\langle x\rangle_{-1}^{\varepsilon}\right)=\left\langle x^{\prime}\right\rangle_{-1}^{\varepsilon}$ et $g\left(\langle y\rangle_{-1}^{\varepsilon^{\prime}}\right)=\left\langle y^{\prime}\right\rangle_{-1}^{\varepsilon^{\prime}}$ (toujours avec les conventions du lemme 6.14). Une seconde application du lemme 6.14 permet de conclure.

Proposition 6.17. - Si $f: K \rightarrow K^{\prime}$ et $g: L \rightarrow L^{\prime}$ sont deux morphismes rigides (voir le paragraphe 3.2) entre complexes dirigés augmentés à base, alors leur joint $f \star g: K \star L \rightarrow K^{\prime} \star L^{\prime}$ est également rigide.

Démonstration. - Cela résulte immédiatement du lemme précédent.

Proposition 6.18. - Soient $f: K \rightarrow K^{\prime}$ et $g: L \rightarrow L^{\prime}$ des morphismes entre complexes dirigés augmentés à base unitaire et $x \star y$ un élément de la base de $K \star L$. Supposons qu'il existe un élément $x^{\prime} \star y^{\prime}$ de la base de $K^{\prime} \star L^{\prime}$ tel que

(a) si $x$ et $y$ sont différents de $\varnothing$, alors il en est de même de $x^{\prime}$ et $y^{\prime}$, et on a

$$
\nu(f)(\langle x\rangle)=1_{\left\langle x^{\prime}\right\rangle} \quad \text { et } \quad \nu(g)(\langle y\rangle)=1_{\left\langle y^{\prime}\right\rangle},
$$

(b) si $x=\varnothing$, alors

$$
x^{\prime}=\varnothing \quad \text { et } \quad \nu(g)(\langle y\rangle)=1_{\left\langle y^{\prime}\right\rangle},
$$

(c) si $y=\varnothing$, alors

$$
\nu(f)(\langle x\rangle)=1_{\left\langle x^{\prime}\right\rangle} \text { et } y^{\prime}=\varnothing,
$$

où 1 , dans les égalités ci-dessus, désigne une identité itérée (éventuellement 0 fois). Alors on a

$$
\nu(f \star g)(\langle x \star y\rangle)=1_{\left\langle x^{\prime} \star y^{\prime}\right\rangle} .
$$

En particulier, lorsque $x^{\prime}=f(x)$ et $y^{\prime}=g(y)$ vérifient les hypothèses ci-dessus (en convenant que $m(\varnothing)=\varnothing$ pour $m=f, g)$, on a

$$
\nu(f \star g)(\langle x \star y\rangle)=\langle f(x) \star g(y)\rangle .
$$

Démonstration. — Cela résulte immédiatement du lemme 6.16. 
Proposition 6.19. - Soient $K$ et $L$ deux complexes dirigés augmentés à base unitaire et $x \star y$ un élément de la base de $K \star L$, avec $x$ de degré $i$ et $y$ de degré $j$.

(a) Supposons $i \geqslant 0$ et $j \geqslant 0$, et notons z la cellule principale de $\mathrm{D}_{i}$ et $t$ celle de $\mathrm{D}_{j}$. Alors le diagramme

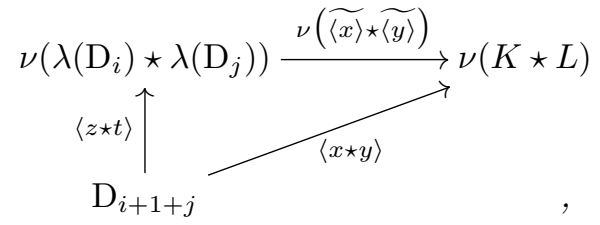

ò̀ $\widetilde{\langle x\rangle}: \lambda\left(\mathrm{D}_{i}\right) \rightarrow K$ et $\widetilde{\langle y\rangle}: \lambda\left(\mathrm{D}_{j}\right) \rightarrow L$ désignent les morphismes transposés de $\langle x\rangle: \mathrm{D}_{i} \rightarrow \nu(K)$ et $\langle y\rangle: \mathrm{D}_{j} \rightarrow \nu(L)$ respectivement, est commutatif.

(b) Si $i=-1$, de sorte que $x=\varnothing$ et $\mathrm{D}_{i}=\varnothing$, alors, en notant toujours $t$ la cellule principale de $\mathrm{D}_{j}$, le triangle ci-dessus commute pour $z=\varnothing$ et $\widetilde{\langle x\rangle}$ l'unique morphisme de $\lambda\left(\mathrm{D}_{i}\right)=\varnothing$ vers $\nu(K)$.

(c) Si $j=-1$, de sorte que $y=\varnothing$ et $\mathrm{D}_{j}=\varnothing$, alors, en notant toujours $z$ la cellule principale de $\mathrm{D}_{i}$, le triangle ci-dessus commute pour $t=\varnothing$ et $\widetilde{\langle y\rangle}$ l'unique morphisme de $\lambda\left(\mathrm{D}_{j}\right)=\varnothing$ vers $\nu(L)$.

Démonstration. - Il s'agit de montrer qu'on a

$$
\nu(\widetilde{\langle x\rangle} \star \widetilde{\langle y\rangle})(\langle z \star t\rangle)=\langle x \star y\rangle .
$$

Cela résulte de la proposition précédente puisque, par définition, si $i \geqslant 0$, on a $\nu(\widetilde{\langle x\rangle})(\langle z\rangle)=\langle x\rangle$ et, si $j \geqslant 0$, on a $\nu(\widetilde{\langle y\rangle})(\langle t\rangle)=\langle y\rangle$.

Proposition 6.20. — Si K et $L$ sont des complexes dirigés augmentés décents (voir le paragraphe 2.17) à base fortement sans boucle, alors il en est de même de $K \star L$. De plus, les morphismes

$$
\iota_{1}: K \rightarrow K \star L \quad \text { et } \quad \iota_{2}: L \rightarrow K \star L
$$

sont des inclusions rigides ordonnées.

Démonstration. - Soit $M$ un complexe dirigé augmenté décent admettant une base $Z$. On rappelle (voir le paragraphe 2.13 ) qu'on note $\leqslant_{\mathbb{N}}$ la plus petite relation de préordre sur $Z$ satisfaisant

$$
x \leqslant_{\mathbb{N}} y \quad \text { si } \quad x \in \operatorname{supp}\left(d(y)_{-}\right) \text {ou } y \in \operatorname{supp}\left(d(x)_{+}\right),
$$

où, par convention, $d(z)=0$ si $z$ est dans $Z_{0}$. De même, on notera $\leqslant_{\mathbb{N}}^{\varnothing}$ la plus petite relation de préordre sur $Z \cup\{\varnothing\}$ satisfaisant

$$
x \leqslant_{\mathbb{N}}^{\varnothing} y \quad \text { si } \quad x \in \operatorname{supp}\left(d(y)_{-}\right) \text {ou } y \in \operatorname{supp}\left(d(x)_{+}\right),
$$

où cette fois-ci on a convenu que $d(z)_{+}=e(z) \varnothing$ et $d(z)_{-}=0$ si $z$ est dans $Z_{0}$, et que $d(\varnothing)_{+}=0$ et $d(\varnothing)_{-}=0$. Par définition, la relation $\leqslant_{\mathbb{N}}^{\varnothing}$ est la plus petite relation 
de préordre prolongeant $\leqslant_{\mathbb{N}}$ et vérifiant $z \leqslant_{\mathbb{N}}^{\varnothing} \varnothing$ pour tout $z$ dans $Z$ de degré 0 tel que $e(z)>0$. On en déduit immédiatement que la relation de préordre $\leqslant_{\mathbb{N}}^{\varnothing}$ est une relation d'ordre si et seulement si la relation de préordre $\leqslant_{\mathbb{N}}$ en est une.

Démontrons maintenant la proposition. Il est immédiat que le complexe $K \star L$ est décent et il s'agit de montrer qu'il est à base unitaire. Notons $X$ et $Y$ les bases respectives de $K$ et $L$. On définit une relation sur la base $X \star Y$ de $K \star L$ en posant

$$
x \star y \preccurlyeq x^{\prime} \star y^{\prime} \Longleftrightarrow \begin{cases}\text { ou } & x<_{\mathbb{N}}^{\varnothing} x^{\prime} \\ \text { ou } & x=x^{\prime},|x| \text { est impair et } y \leqslant_{\mathbb{N}}^{\varnothing} y^{\prime} \\ & x=x^{\prime},|x| \text { est pair et } y \geqslant_{\mathbb{N}}^{\varnothing} y^{\prime} .\end{cases}
$$

Par hypothèse, les relations $\leqslant_{\mathbb{N}}$ sur $K$ et $L$ sont des relations d'ordre et il en est donc de même des relations $\leqslant_{\mathbb{N}}^{\varnothing}$. On en déduit facilement que la relation $\preccurlyeq$ est également une relation d'ordre.

Soient $x \star y$ et $x^{\prime} \star y^{\prime}$ dans $X \star Y$. Montrons que la relation $\preccurlyeq$ vérifie la propriété

$$
x \star y \in \operatorname{supp}\left(d\left(x^{\prime} \star y^{\prime}\right)_{-}\right) \text {ou } x^{\prime} \star y^{\prime} \in \operatorname{supp}\left(d(x \star y)_{+}\right) \quad \Rightarrow \quad x \star y \preccurlyeq x^{\prime} \star y^{\prime},
$$

ce qui établira que la relation de préordre $\leqslant_{\mathbb{N}}$ du complexe $K \star L$ est une relation d'ordre. Supposons tout d'abord que $x \star y$ appartienne au support de $d\left(x^{\prime} \star y^{\prime}\right)_{-}$. Puisqu'on a

$$
\begin{aligned}
d\left(x^{\prime} \star y^{\prime}\right)_{-} & =\left(d\left(x^{\prime}\right) \star y^{\prime}+(-1)^{\left|x^{\prime}\right|+1} x^{\prime} \star d\left(y^{\prime}\right)\right)_{-} \\
& =d\left(x^{\prime}\right)_{-} \star y^{\prime}+x^{\prime} \star d\left(y^{\prime}\right)_{(-1)^{\left|x^{\prime}\right|},}
\end{aligned}
$$

la deuxième égalité résultant de la non-simplification de termes pour des raisons de bidegrés, cela signifie que $x \star y$ appartient au support d'un des deux termes de cette somme. Si $x \star y$ appartient au support de $d\left(x^{\prime}\right)_{-} \star y^{\prime}$, cela signifie que $x$ appartient

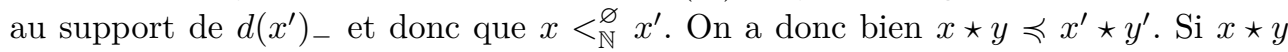
appartient au support de $x^{\prime} \star d\left(y^{\prime}\right)_{(-1)^{\left|x^{\prime}\right|}}$, cela signifie que $x=x^{\prime}$ et que $y$ appartient au support de $d\left(y^{\prime}\right)_{(-1)^{\mid x^{\prime}} \mid}$. Ainsi, si $\left|x^{\prime}\right|$ est impair, on a $y \leqslant_{\mathbb{N}}^{\varnothing} y^{\prime}$ et, si $\left|x^{\prime}\right|$ est pair, on a $y^{\prime} \leqslant_{\mathbb{N}}^{\varnothing} y$. On a donc dans tous les cas $x \star y \preccurlyeq x^{\prime} \star y^{\prime}$, ce qu'on voulait montrer. Si maintenant $x^{\prime} \star y^{\prime}$ appartient au support de $d(x \star y)_{+}$, on conclut de manière analogue en utilisant la formule

$$
d(x \star y)_{+}=d(x)_{+} \star y+x \star d(y)_{(-1)^{|x|+1}} .
$$

Ceci achève de montrer que $K \star L$ est à base unitaire.

Montrons enfin que le morphisme $\iota_{1}: K \rightarrow K \star L$ est une inclusion rigide ordonnée, le morphisme $\iota_{2}: L \rightarrow K \star L$ se traitant de manière analogue. Il est immédiat que ce morphisme est une inclusion rigide. Soient $x$ et $x^{\prime}$ dans la base de $K$. Il s'agit de montrer l'implication

$$
x \star \varnothing \leqslant_{\mathbb{N}} x^{\prime} \star \varnothing \Rightarrow x \leqslant_{\mathbb{N}} x^{\prime} .
$$


Or on a

$$
x \star \varnothing \leqslant \mathbb{N} x^{\prime} \star \varnothing \quad \Rightarrow \quad x \star \varnothing \preccurlyeq x^{\prime} \star \varnothing \quad \text { et } \quad x \star \varnothing \preccurlyeq x^{\prime} \star \varnothing \quad \Leftrightarrow \quad x \leqslant \mathbb{N} x^{\prime},
$$

d'où le résultat.

Corollaire 6.21. — Si K et L sont des complexes de Steiner forts, alors il en est de même de $K \star L$. De plus, les morphismes

$$
\iota_{1}: K \rightarrow K \star L \quad \text { et } \quad \iota_{2}: L \rightarrow K \star L
$$

sont des inclusions rigides ordonnées.

Démonstration. - Cela résulte des propositions 6.15 et 6.20 .

Remarque 6.22. - En vertu du corollaire précédent (et du fait que le complexe dirigé augmenté $\varnothing$ est de Steiner fort), la catégorie des complexes de Steiner forts est une sous-catégorie monoïdale de la catégorie monoïdale des complexes dirigés augmentés définie par le joint.

Proposition 6.23. - Soient $K$ un complexe de Steiner fort et $F: I \rightarrow \mathcal{C}_{\mathrm{da}}$ un système de Steiner fort connexe (voir le paragraphe 3.7). Alors le foncteur

$$
\begin{aligned}
K \star F: I & \rightarrow \mathcal{C}_{\mathrm{da}} \\
i & \mapsto K \star F(i)
\end{aligned}
$$

est un système de Steiner fort.

Démonstration. - Le foncteur $F$ étant un système rigide, il en est de même du foncteur $K \star F: i \mapsto K \star F(i)$ en vertu de la proposition 6.17. Par ailleurs, puisque d'après le corollaire 6.21 les complexes de Steiner forts sont stables par joint, le foncteur $K \star F$ est à valeurs dans les complexes de Steiner forts. Enfin, le foncteur $K \star \bullet$ commutant aux limites inductives connexes (voir la proposition 6.9), le morphisme canonique

$$
\underset{i \in I}{\lim _{i \in I}}(K \star F(i)) \rightarrow K \star \underset{i \in I}{\lim _{i \in I}} F(i)
$$

est un isomorphisme de complexes dirigés augmentés et, pour tout objet $i_{0}$ de $I$, le morphisme canonique $K \star F\left(i_{0}\right) \rightarrow \lim _{i \in I}(K \star F(i))$ s'identifie à travers cet isomorphisme au joint $K \star F\left(i_{0}\right) \rightarrow K \star \lim _{i \in I} F(i)$ de $K$ et du morphisme canonique associé à $F$. On en déduit le résultat en invoquant de nouveau la proposition 6.17 et le corollaire 6.21 .

Corollaire 6.24. — Si K est un complexe de Steiner fort, alors le foncteur

$$
\begin{aligned}
\mathcal{C}_{\mathrm{da}} & \rightarrow \infty-\mathcal{C} a t \\
L & \mapsto \nu(K \star L)
\end{aligned}
$$

commute aux limites inductives des systèmes de Steiner forts connexes. 
Démonstration. - Puisque le foncteur $\nu$ commute aux limites inductives des systèmes de Steiner forts (théorème 3.8), l'assertion résulte de la proposition précédente.

Corollaire 6.25. — Soit $K$ un complexe de Steiner fort. Alors le foncteur

$$
\begin{aligned}
\Theta_{+} & \rightarrow \infty-\mathcal{C} a t \\
S & \mapsto \nu(K \star \lambda(S))
\end{aligned}
$$

commute aux sommes globulaires.

Démonstration. - Cela résulte du corollaire précédent puisque, en vertu de la proposition 4.13, les sommes globulaires proviennent de systèmes de Steiner forts (qui sont bien sûr connexes).

6.26. - Fixons $K$ un complexe de Steiner fort. Soient $C$ une $\infty$-catégorie et $u: \nu(K) \rightarrow C$ un $\infty$-foncteur. Nous allons définir une $\infty$-catégorie $u \backslash C$. Il résulte du corollaire précédent que le foncteur

$$
\begin{aligned}
\left(\Theta_{+}\right)^{\circ} & \rightarrow \mathcal{E} n s \\
S & \mapsto \operatorname{Hom}_{\infty-\mathcal{C} a t}(\nu(K \star \lambda(S)), C)
\end{aligned}
$$

envoie les sommes globulaires sur des produits globulaires au sens du paragraphe 4.9. Ainsi, en vertu de ce même paragraphe et avec ces notations, ce foncteur définit une $\infty$-catégorie $\operatorname{Hom}_{\infty \text {-Cat }}\left(\nu\left(K \star \lambda\left(\mathrm{D}_{\bullet}\right)\right), C\right)$ au-dessus de l'ensemble

$$
\operatorname{Hom}_{\infty-\mathcal{C} a t}(\nu(K \star \lambda(\varnothing)), C)=\operatorname{Hom}_{\infty-\mathcal{C} a t}(\nu(K \star \varnothing), C) \simeq \operatorname{Hom}_{\infty-\mathcal{C} a t}(\nu(K), C) .
$$

On définit la $\infty$-catégorie $u \backslash C$ comme la fibre en $u$ de cette $\infty$-catégorie au-dessus de $\operatorname{Hom}_{\infty-\mathcal{C} a t}(\nu(K), C)$. Autrement dit, avec les notations du paragraphe 4.9, on pose

$$
u \backslash C=\operatorname{Hom}_{\infty-\mathcal{C} a t}\left(\nu\left(K \star \lambda\left(\mathrm{D}_{\bullet}\right)\right), C\right)_{u} .
$$

Explicitement, les $i$-flèches de $u \backslash C$ sont les $\infty$-foncteurs $\nu\left(K \star \lambda\left(\mathrm{D}_{i}\right)\right) \rightarrow C$ rendant le triangle

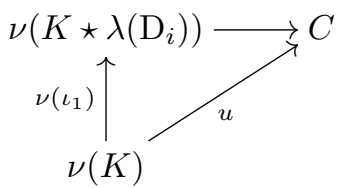

commutatif.

Notons qu'un morphisme $f: K \rightarrow K^{\prime}$ entre complexes de Steiner forts induit, pour toute $\infty$-catégorie $C$, une application

$$
\operatorname{Hom}_{\infty-\mathcal{C} a t}\left(\nu\left(K^{\prime} \star \lambda(S)\right), C\right) \rightarrow \operatorname{Hom}_{\infty-\mathcal{C} a t}(\nu(K \star \lambda(S)), C),
$$


naturelle en $S$ dans $\Theta_{+}$. Ainsi, toujours en vertu du paragraphe 4.9, pour tout triangle commutatif

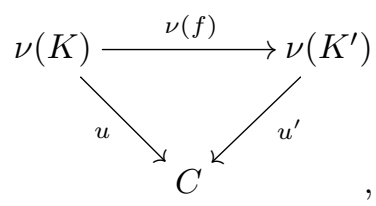

on obtient un $\infty$-foncteur $u^{\prime} \backslash C \rightarrow u \backslash C$.

Proposition 6.27. - Fixons $K$ un complexe de Steiner fort. Pour tout complexe de Steiner fort $L$, toute $\infty$-catégorie $C$ et tout $\infty$-foncteur $u: \nu(K) \rightarrow C$, on a une bijection

$$
\operatorname{Hom}_{\nu(K) \backslash \infty-\mathcal{C} a t}\left(\left(\nu(K \star L), \nu\left(\iota_{1}\right)\right),(C, u)\right) \simeq \operatorname{Hom}_{\infty-\mathcal{C} a t}(\nu(L), u \backslash C),
$$

naturelle en $L$ et $u$.

Démonstration. - Si $M$ est un complexe de Steiner fort et $t$ est un élément de la base de $M$ de degré $i \geqslant 0$, on notera, pour simplifier,

$$
\begin{aligned}
t_{j}^{\varepsilon} & =\langle t\rangle_{j}^{\varepsilon} \quad \text { pour } 0 \leqslant j \leqslant i \quad \text { (voir le paragraphe 2.8), } \\
t_{-1}^{0} & =0 \quad \text { et } \quad t_{-1}^{1}=\varnothing .
\end{aligned}
$$

On notera par ailleurs $\varnothing_{-1}^{0}=\varnothing$ et $\varnothing_{-1}^{1}=\varnothing$.

On va produire des fonctions

$$
\varphi: \operatorname{Hom}_{\nu(K) \backslash \infty-\mathcal{C} a t}\left(\left(\nu(K \star L), \nu\left(\iota_{1}\right)\right),(C, u)\right) \rightarrow \operatorname{Hom}_{\infty-\mathcal{C} a t}(\nu(L), u \backslash C)
$$

et

$$
\psi: \operatorname{Hom}_{\infty-\mathcal{C} a t}(\nu(L), u \backslash C) \rightarrow \operatorname{Hom}_{\nu(K) \backslash \infty-\mathcal{C} a t}\left(\left(\nu(K \star L), \nu\left(\iota_{1}\right)\right),(C, u)\right)
$$

inverses l'une de l'autre.

Commençons par $\varphi$. Soit $F: \nu(K \star L) \rightarrow C$ un $\infty$-foncteur au-dessous de $\nu(K)$. On définit un $\infty$-foncteur $\varphi(F): \nu(L) \rightarrow u \backslash C$ de la manière suivante. Soit $y: \mathrm{D}_{i} \rightarrow \nu(L)$, pour $i \geqslant 0$, une $i$-flèche de $\nu(L)$. On doit lui associer une $i$-flèche $\varphi(F)(y)$ de $u \backslash C$, c'est-à-dire un $\infty$-foncteur $\nu\left(K \star \lambda\left(\mathrm{D}_{i}\right)\right) \rightarrow C$ au-dessous de $\nu(K)$. On pose

$$
\varphi(F)(y)=\nu\left(K \star \lambda\left(\mathrm{D}_{i}\right)\right) \stackrel{\nu(K \star \tilde{y})}{\longrightarrow} \nu(K \star L) \stackrel{F}{\rightarrow} C,
$$

où on a noté $\tilde{y}: \lambda\left(\mathrm{D}_{i}\right) \rightarrow L$ le transposé de $y: \mathrm{D}_{i} \rightarrow \nu(L)$. Ce $\infty$-foncteur étant le composé de deux $\infty$-foncteurs au-dessous de $\nu(K)$, il est bien au-dessous de $\nu(K)$. Le fait que $\varphi(F)$ soit bien un $\infty$-foncteur résulte de la naturalité en $\mathrm{D}_{i}$, et plus généralement en $S$ dans $\Theta$, du $\infty$-foncteur $\varphi(F)(y)$. En particulier, pour $x$ un élément de la base de $K$ ou $x=\varnothing, y$ un élément de la base de $L$ de degré $i$ et $z$ la cellule principale de $\mathrm{D}_{i}$, on a, pour $j$ tel que $-1 \leqslant j \leqslant i$ et $\varepsilon=0,1$,

$$
\varphi(F)(\langle y\rangle)\left(\left\langle x \star z_{j}^{\varepsilon}\right\rangle\right)=F\left(\left\langle x \star y_{j}^{\varepsilon}\right\rangle\right) .
$$


De plus, cette formule détermine $\varphi(F)$ de manière unique puisque les $\infty$-catégories $\nu(L)$ et $\nu\left(K \star \lambda\left(\mathrm{D}_{i}\right)\right)$ sont engendrées librement au sens des polygraphes par leurs atomes en vertu du théorème 2.12 .

Définissons maintenant $\psi$. Soit $G: \nu(L) \rightarrow u \backslash C$ un $\infty$-foncteur. Il s'agit de définir un $\infty$-foncteur $\psi(G): \nu(K \star L) \rightarrow C$ au-dessous de $\nu(K)$. En vertu du théorème 2.12, la $\infty$-catégorie $\nu(K \star L)$ est engendrée librement au sens des polygraphes par ses atomes. Il suffit donc de définir $\psi(G)$ sur les atomes de $\nu(K \star L)$ et de vérifier les compatibilités aux sources et aux buts. Soit $x \star y$ dans la base de $K \star L$ avec $x$ de degré $i$ et $y$ de degré $j$. On pose

$$
\psi(G)(\langle x \star y\rangle)=\quad \mathrm{D}_{i+1+j} \stackrel{\left\langle x \star z_{j}^{\prime}\right\rangle}{\longrightarrow} \nu\left(K \star \lambda\left(\mathrm{D}_{j}\right)\right) \stackrel{G(\langle y\rangle)}{\longrightarrow} C,
$$

où $z^{\prime}$ désigne la cellule principale de $\mathrm{D}_{j}$ si $j \geqslant 0$, et $z^{\prime}=\varnothing$ si $j=-1$ et, par convention, $G(\langle\varnothing\rangle): \nu(K) \rightarrow C$ désigne $u$.

Vérifions maintenant les compatibilités aux sources et aux buts. Fixons $m \geqslant 0$ et supposons que les formules ci-dessus définissent bien un $m$-foncteur. Il s'agit de montrer que, pour tous $x$ et $y$ comme ci-dessus avec $i+1+j=m+1$, on a

$$
\psi(G)(s(\langle x \star y\rangle))=s(\psi(G)(\langle x \star y\rangle)) \quad \text { et } \quad \psi(G)(t(\langle x \star y\rangle))=t(\psi(G)(\langle x \star y\rangle)) .
$$

Montrons la première égalité, la seconde se démontrant de manière analogue. Notons $z$ et $z^{\prime}$ les cellules principales respectives de $\mathrm{D}_{i}$ et $\mathrm{D}_{j}$, en convenant que $z=\varnothing$ si $i=-1$ et $z^{\prime}=\varnothing$ si $j=-1$. Puisque la $\infty$-catégorie $\nu\left(\lambda\left(\mathrm{D}_{i}\right) \star \lambda\left(\mathrm{D}_{j}\right)\right)$ est engendrée librement au sens des polygraphes par ses atomes, en vertu de la proposition 1.5, ses atomes l'engendrent également par compositions et il existe donc une formule $\chi$ exprimant la source de $\left\langle z_{i} \star z_{j}^{\prime}\right\rangle$ en fonction des $\left\langle z_{k}^{\varepsilon} \star z_{l}^{\prime \varepsilon^{\prime}}\right\rangle$ avec $-1 \leqslant k \leqslant i,-1 \leqslant l \leqslant j$, $0 \leqslant k+1+l<i+1+j, \varepsilon=0,1$ et $\varepsilon^{\prime}=0,1$. On notera $\chi\left[\left\langle z_{k}^{\varepsilon} \star z_{l}^{\prime \varepsilon^{\prime}}\right\rangle\right]$ l'évaluation de la formule $\chi$ en les éléments $z_{k}^{\varepsilon} \star z_{l}^{\prime \varepsilon^{\prime}}$. On a donc $s\left(\left\langle z_{i} \star z_{j}^{\prime}\right\rangle\right)=\chi\left[\left\langle z_{k}^{\varepsilon} \star z_{l}^{\prime \varepsilon^{\prime}}\right\rangle\right]$. Plus généralement, il résulte de la proposition 6.19 que la même formule $\chi$ permet de calculer la source d'un atome $\langle m \star n\rangle$, où $m$ est de degré $i$ et $n$ de degré $j$, d'un joint quelconque de complexes dirigés augmentés à base unitaire $M$ et $N$. On obtient ainsi

$$
\begin{aligned}
\psi(G)(s(\langle x \star y\rangle))= & \psi(G)\left(\chi\left[\left\langle x_{k}^{\varepsilon} \star y_{l}^{\varepsilon^{\prime}}\right\rangle\right]\right) \\
= & \chi\left[\psi(G)\left(\left\langle x_{k}^{\varepsilon} \star y_{l}^{\varepsilon^{\prime}}\right\rangle\right)\right] \\
& (\operatorname{car} \psi(G) \text { est un } m \text {-foncteur et } k+1+l<i+1+j=m+1) \\
= & \chi\left[G\left(\left\langle y_{l}^{\varepsilon^{\prime}}\right\rangle\right)\left(\left\langle x_{k}^{\varepsilon} \star z_{l}^{\prime \prime}\right\rangle\right)\right],
\end{aligned}
$$

où $z^{\prime \prime}$ désigne la cellule principale de $\mathrm{D}_{l}$ (en convenant toujours que $z^{\prime \prime}=\varnothing$ si $l=-1$ ), cette dernière égalité résultant de la définition de $\psi(G)$. Par ailleurs, on a

$$
G\left(\left\langle y_{l}^{\varepsilon^{\prime}}\right\rangle\right)\left(\left\langle x_{k}^{\varepsilon} \star z_{l}^{\prime \prime}\right\rangle\right)=G(\langle y\rangle)\left(\left\langle x_{k}^{\varepsilon} \star z_{l}^{\prime \varepsilon^{\prime}}\right\rangle\right) .
$$


En effet, si $l=-1$, c'est une conséquence des égalités

$$
G(\varnothing)\left(\left\langle x_{k}^{\varepsilon} \star \varnothing\right\rangle\right)=u\left(\left\langle x_{k}^{\varepsilon}\right\rangle\right)=G(\langle y\rangle)\left(\left\langle x_{k}^{\varepsilon} \star \varnothing\right\rangle\right)
$$

et, pour $l \geqslant 0$ et $\varepsilon^{\prime}=0$, cela résulte du calcul suivant :

$$
\begin{aligned}
G\left(\left\langle y_{l}^{0}\right\rangle\right)\left(\left\langle x_{k}^{\varepsilon} \star z_{l}^{\prime \prime}\right\rangle\right) & =G\left(s_{l}(\langle y\rangle)\right)\left(\left\langle x_{k}^{\varepsilon} \star z_{l}^{\prime \prime}\right\rangle\right) \\
& =s_{l}(G(\langle y\rangle))\left(\left\langle x_{k}^{\varepsilon} \star z_{l}^{\prime \prime}\right\rangle\right) \\
& =G(\langle y\rangle)\left(\nu\left(K \star \lambda\left(\sigma_{l}^{i}\right)\right)\left(\left\langle x_{k}^{\varepsilon} \star z_{l}^{\prime \prime}\right\rangle\right)\right)
\end{aligned}
$$

(par définition des sources des cellules de $u \backslash C$ )

$$
=G(\langle y\rangle)\left(\left\langle x_{k}^{\varepsilon} \star z_{l}^{\prime 0}\right\rangle\right)
$$

la dernière égalité étant conséquence de la proposition 6.18 puisque $\sigma_{l}^{i}\left(\left\langle z_{l}^{\prime \prime}\right\rangle\right)=\left\langle z_{l}^{\prime 0}\right\rangle$. La démonstration dans le cas $\varepsilon^{\prime}=1$ est analogue. En insérant cette égalité dans notre calcul précédent, on obtient

$$
\begin{aligned}
\psi(G)(s(\langle x \star y\rangle))= & \chi\left[G\left(\left\langle y_{l}^{\varepsilon^{\prime}}\right\rangle\right)\left(\left\langle x_{k}^{\varepsilon} \star z_{l}^{\prime \prime}\right\rangle\right)\right] \\
= & \chi\left[G(\langle y\rangle)\left(\left\langle x_{k}^{\varepsilon} \star z_{l}^{\prime \varepsilon^{\prime}}\right\rangle\right)\right] \\
= & G(\langle y\rangle)\left(\chi\left[\left\langle x_{k}^{\varepsilon} \star z_{l}^{\varepsilon^{\prime}}\right\rangle\right]\right) \\
& (\operatorname{car} G(\langle y\rangle) \text { est un } \infty \text {-foncteur }) \\
= & G(\langle y\rangle)\left(s\left(\left\langle x \star z_{j}^{\prime}\right\rangle\right)\right) \\
= & s\left(G(\langle y\rangle)\left(\left\langle x \star z_{j}^{\prime}\right\rangle\right)\right) \\
= & s(\psi(G)(\langle x \star y\rangle)),
\end{aligned}
$$

la dernière égalité résultant de la définition de $\psi(G)$, ce qui achève de montrer que $\psi(G)$ est bien un $\infty$-foncteur. Ce $\infty$-foncteur $\psi(G)$ est bien au-dessous de $\nu(K)$. En effet, par définition, pour $x$ un élément de la base de $K$, on a $\psi(G)(\langle x \star \varnothing\rangle)=u(\langle x\rangle)$. Le $\infty$-foncteur $\psi(G) \iota_{1}: \nu(K) \rightarrow C$ coïncide donc avec le $\infty$-foncteur $u$ sur les atomes et on obtient le résultat puisque $\nu(K)$ est engendrée librement au sens des polygraphes par ses atomes en vertu du théorème 2.12.

Enfin, vérifions que $\varphi$ et $\psi$ sont bien des bijections inverses l'une de l'autre. Soient $F: \nu(K \star L) \rightarrow C$ au-dessous de $\nu(K)$ et $G: \nu(L) \rightarrow u \backslash C$ deux $\infty$-foncteurs. On a, avec les notations précédentes,

$$
\psi \varphi(F)(\langle x \star y\rangle)=\varphi(F)(\langle y\rangle)\left(\left\langle x \star z_{j}^{\prime}\right\rangle\right)=F(\langle x \star y\rangle)
$$

et

$$
\varphi \psi(G)(\langle y\rangle)\left(\left\langle x \star z_{j}^{\varepsilon}\right\rangle\right)=\psi(G)\left(\left\langle x \star y_{j}^{\varepsilon}\right\rangle\right)=G(\langle y\rangle)\left(\left\langle x \star z_{j}^{\varepsilon}\right\rangle\right) .
$$

Les $\infty$-foncteurs $\psi \varphi(F)$ et $F$ (resp. les $\infty$-foncteurs $\varphi \psi(G)$ et $G$ ) coïncident donc sur les atomes et sont donc égaux, ce qu'il fallait démontrer. 
6.28. - Soit $L$ un complexe de Steiner fort. On montre de même que le foncteur

$$
\begin{aligned}
\mathcal{C}_{\mathrm{da}} & \rightarrow \infty-\mathcal{C} a t \\
K & \mapsto \nu(K \star L)
\end{aligned}
$$

commute aux limites inductives des systèmes de Steiner forts connexes. On en déduit que le foncteur

$$
\begin{aligned}
\left(\Theta_{+}\right)^{\circ} & \rightarrow \mathcal{E} n s \\
S & \mapsto \operatorname{Hom}_{\infty-\mathcal{C} a t}(\nu(\lambda(S) \star L), C)
\end{aligned}
$$

envoie les sommes globulaires sur des produits globulaires. On définit alors, comme dans le paragraphe 6.26, pour toute $\infty$-catégorie $C$ et tout $\infty$-foncteur $v: \nu(L) \rightarrow C$, une $\infty$-catégorie $C / v$ en posant

$$
C / v=\operatorname{Hom}_{\infty-\mathcal{C} a t}\left(\nu\left(\lambda\left(\mathrm{D}_{\bullet}\right) \star L\right), C\right)_{v} .
$$

(La décoration « co » dans cette notation sera expliquée dans le paragraphe 6.31 et surtout dans la remarque 6.37.) On montre, comme dans la proposition précédente, que, pour tout complexe de Steiner fort $K$, toute $\infty$-catégorie $C$ et tout $\infty$-foncteur $v: \nu(L) \rightarrow C$, on a une bijection

$$
\operatorname{Hom}_{\nu(L) \backslash \infty-\mathcal{C} a t}\left(\left(\nu(K \star L), \nu\left(\iota_{2}\right)\right),(C, v)\right) \simeq \operatorname{Hom}_{\infty-\mathcal{C} a t}(\nu(K), C / v),
$$

naturelle en $K$ et $v$.

Théorème 6.29. - Il existe une et une seule (à unique isomorphisme monö̈dal près) structure de catégorie monö̈dale sur $\infty$-Cat de produit

$$
\begin{aligned}
\star: \infty-\mathcal{C} a t \times \infty-\mathcal{C} a t & \rightarrow \infty-\mathcal{C} a t \\
(A, B) & \mapsto A \star B
\end{aligned}
$$

ayant les deux propriétés suivantes:

(a) le foncteur $\nu_{\mid \mathcal{S} t_{\mathrm{f}}}: \mathcal{S}_{\mathrm{f}} \rightarrow \infty$-Cat, où la catégorie des complexes de Steiner forts $\mathcal{S} t_{\mathrm{f}}$ est munie de la structure de catégorie monoïdale définie par le joint, s'étend en un foncteur monoïdal;

(b) le foncteur $\star: \infty$-C at $\times \infty$-Cat $\rightarrow \infty$-Cat commute aux petites limites inductives connexes en chaque variable.

De plus, cette structure monö̈dale est localement bifermée (au sens du paragraphe 5.7).

Démonstration. - Le théorème résulte du corollaire 5.14 appliqué à $\mathcal{C}=\infty$-C $a t$ et $\mathcal{D}$ la catégorie des $\infty$-catégories de Steiner fortes (voir le paragraphe 2.15) munie du joint (par l'identification de cette sous-catégorie à celle des complexes de Steiner forts), la petite sous-catégorie dense étant la catégorie $\Theta_{+}$. En effet, les hypothèses de ce corollaire sont précisément le contenu de la proposition 6.27 et du paragraphe 6.28. 
6.30. — On appellera joint le produit monoïdal

$$
\star: \infty-\mathcal{C} a t \times \infty-\mathcal{C} a t \rightarrow \infty-\mathcal{C} a t
$$

défini par le théorème précédent. Si $K$ et $L$ sont des complexes de Steiner forts, on a, en vertu de ce même théorème, un isomorphisme canonique

$$
\nu(K) \star \nu(L) \simeq \nu(K \star L) .
$$

En particulier, si $S$ et $T$ sont deux objets de $\Theta_{+}$, puisqu'en vertu de la proposition 4.13 on a $S \simeq \nu \lambda(S)$ et $T \simeq \nu \lambda(T)$, on obtient un isomorphisme canonique

$$
S \star T \simeq \nu(\lambda(S) \star \lambda(T)) .
$$

Plus généralement, si $A$ et $B$ sont deux $\infty$-catégories, on a des isomorphismes canoniques

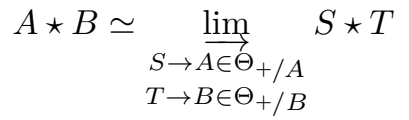

$$
\begin{aligned}
& \simeq \lim _{\substack{S \rightarrow \overrightarrow{A \in \Theta}_{+} / A \\
T \rightarrow B \in \Theta_{+} / B}} \nu(\lambda(S) \star \lambda(T)) .
\end{aligned}
$$

En effet, puisque la catégorie $\Theta_{+}$est dense dans $\infty$-C $a t$ et contient la $\infty$-catégorie vide, toute $\infty$-catégorie est limite inductive canonique connexe d'objets de $\Theta_{+}$. La formule résulte alors du fait que le joint commute aux limites inductives connexes en chaque argument.

L'unité du joint est l'image par $\nu$ du complexe dirigé augmenté $\varnothing$, c'est-à-dire la $\infty$-catégorie vide qu'on notera également $\varnothing$. Si $A$ est une $\infty$-catégorie, on a donc

$$
A \star \varnothing \simeq A \simeq \varnothing \star A .
$$

Si $A$ et $B$ sont deux $\infty$-catégories, on notera

$$
A \stackrel{\iota_{1}}{\longrightarrow} A \star B \stackrel{\iota_{2}}{\longleftarrow} B
$$

les $\infty$-foncteurs

$$
A \simeq A \star \varnothing \longrightarrow A \star B \longleftarrow \varnothing \star B \simeq B
$$

où les flèches pointant vers $A \star B$ sont induites par les $\infty$-foncteurs $\varnothing \rightarrow B$ et $\varnothing \rightarrow A$. (Ce sont les $\infty$-foncteurs de la bicoaugmentation locale associée au joint, voir l'exemple 5.6.)

6.31. - En vertu du théorème 6.29 , la structure de catégorie monoïdale définie par le joint est localement bifermée. Cela signifie exactement que les foncteurs

$$
\begin{aligned}
\infty-\mathcal{C} a t & \rightarrow A \backslash \infty-\mathcal{C} a t \\
B & \mapsto\left(A \star B, \iota_{1}: A \rightarrow A \star B\right)
\end{aligned}
$$


et

$$
\begin{aligned}
\infty-\mathcal{C} a t & \rightarrow B \backslash \infty-\mathcal{C} a t \\
A & \mapsto\left(A \star B, \iota_{2}: B \rightarrow A \star B\right)
\end{aligned}
$$

admettent des adjoints à droite. On obtient donc des couples de foncteurs adjoints

$$
\begin{aligned}
\infty-\mathcal{C} a t & \rightarrow A \backslash \infty \text { - Cat, } & A \backslash \infty \text { - Cat } & \rightarrow \infty \text { - Cat } \\
B & \mapsto\left(A \star B, \iota_{1}\right) & (C, A \stackrel{u}{\rightarrow} C) & \mapsto u \backslash C
\end{aligned}
$$

et

$$
\begin{aligned}
\infty-\mathcal{C} a t & \rightarrow B \backslash \infty \text { - C at, } & B \backslash \infty \text { - Cat } & \rightarrow \infty \text { - C at. } \\
A & \mapsto\left(A \star B, \iota_{2}\right) & (C, B \stackrel{v}{\rightarrow} C) & \mapsto C / v
\end{aligned}
$$

Ainsi, si $A$ et $B$ sont des $\infty$-catégories et $u: A \rightarrow C$ et $v: B \rightarrow C$ des $\infty$-foncteurs, on a des bijections naturelles

$$
\begin{aligned}
& \operatorname{Hom}_{A \backslash \infty-\mathcal{C} a t}\left(\left(A \star B, \iota_{1}\right),(C, u)\right) \simeq \operatorname{Hom}_{\infty-\mathcal{C} a t}(B, u \backslash C), \\
& \operatorname{Hom}_{B \backslash \infty-\mathcal{C} a t}\left(\left(A \star B, \iota_{2}\right),(C, v)\right) \simeq \operatorname{Hom}_{\infty-\mathcal{C} a t}(A, C / v) .
\end{aligned}
$$

Si $C$ est une $\infty$-catégorie et $u: A \rightarrow C$ est un $\infty$-foncteur, on appellera la $\infty$-catégorie $u \backslash C$ la tranche de $C$ au-dessous de $u$. Si $v: B \rightarrow C$ est un $\infty$-foncteur, on réservera la notation $C / v$ et la terminologie « tranche de $C$ au-dessus de $v$ » à une variante de $C \stackrel{\text { co }}{v}$ qu'on introduira dans la remarque 6.37 .

Notons que, dans le cas où la source de $u$ est de la forme $\nu(K)$ pour $K$ un complexe de Steiner fort, la $\infty$-catégorie $u \backslash C$ que l'on vient d'introduire coïncide, en vertu de la proposition 6.27, avec celle définie dans le paragraphe 6.26. De même, pour la $\infty$-catégorie $C / v$ et la $\infty$-catégorie définie au paragraphe 6.28 .

Remarque 6.32. - La tranche $u \backslash C$ définie au paragraphe précédent est une tranche généralisée au sens où on prend la tranche de $C$ au-dessous d'un $\infty$-foncteur. Dans le cas où la source du $\infty$-foncteur $u$ est la $\infty$-catégorie finale, la donnée de $u$ devient équivalente à celle d'un objet de $C$ et on se trouve alors dans le cadre usuel des tranches. Dans le chapitre 9, on décrira explicitement ces tranches au-dessous d'un objet et on vérifiera que notre définition est compatible avec les définitions usuelles quand $C$ est une 1-catégorie ou une 2-catégorie.

6.33. - Fixons $A$ une $\infty$-catégorie. Soient $C$ une $\infty$-catégorie et $u: A \rightarrow C$ un $\infty$-foncteur. Par adjonction, pour $i \geqslant 0$, on a

$$
\operatorname{Hom}_{\infty-\mathcal{C} a t}\left(\mathrm{D}_{i}, u \backslash C\right) \simeq \operatorname{Hom}_{A \backslash \infty-\mathcal{C} a t}\left(\left(A \star \mathrm{D}_{i}, \iota_{1}\right),(C, u)\right)
$$


Ainsi, une $i$-flèche de $u \backslash C$ est donnée par un $\infty$-foncteur $A \star \mathrm{D}_{i} \rightarrow C$ faisant commuter le triangle

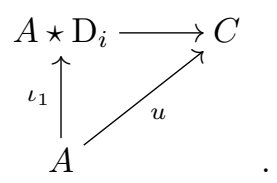

Notons qu'avec les notations du paragraphe 4.9 (voir également le paragraphe 6.26), on a un isomorphisme canonique

$$
u \backslash C \simeq \operatorname{Hom}_{\infty-\mathcal{C} a t}\left(A \star \mathrm{D}_{\bullet}, C\right)_{u} .
$$

Proposition 6.34. - Les foncteurs

$$
\lambda: \infty-\mathcal{C} a t \rightarrow \mathcal{C}_{\mathrm{da}} \quad \text { et } \quad \nu: \mathcal{C}_{\mathrm{da}} \rightarrow \infty-\mathcal{C} a t
$$

sont monoïdal et monoïdal lax respectivement, les catégories $\infty$-Cat et $\mathcal{C}_{\mathrm{da}}$ étant toutes deux munies des structures de catégorie monoïdale définies par le joint.

Démonstration. - Par adjonction, il suffit de montrer que le foncteur $\lambda$ est monoïdal. On a évidemment $\lambda(\varnothing)=\varnothing$. Par ailleurs, si $A$ et $B$ sont deux $\infty$-catégories, on a, en désignant par $S$ et $T$ des objets de $\Theta_{+}$,

$$
\begin{aligned}
& \lambda(A \star B) \simeq \lambda\left(\lim _{S \rightarrow \overrightarrow{A, T} \rightarrow B} \nu(\lambda(S) \star \lambda(T))\right) \\
& \text { (en vertu du paragraphe 6.30) } \\
& \simeq \lim _{S \rightarrow \overrightarrow{A, T} \rightarrow B} \lambda \nu(\lambda(S) \star \lambda(T)) \\
& \text { (car le foncteur } \lambda \text { est un adjoint à gauche) } \\
& \simeq \lim _{S \rightarrow \overrightarrow{A, T} \rightarrow B} \lambda(S) \star \lambda(T) \\
& \text { d'après la proposition } 4.13 \text { et le corollaire } 6.21 \text { ) }
\end{aligned}
$$

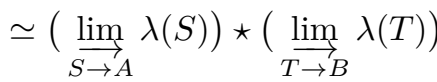

$$
\begin{aligned}
& \text { (en vertu de la proposition 6.9) }
\end{aligned}
$$

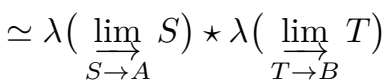

$$
\begin{aligned}
& \simeq \lambda(A) \star \lambda(B),
\end{aligned}
$$$$
\text { (en vertu du théorème } 2.11 \text { puisque } \lambda(S) \star \lambda(T) \text { est de Steiner fort }
$$

d'où le résultat.

Proposition 6.35. - Soient $A$ et $B$ deux $\infty$-catégories. On a un isomorphisme naturel canonique

$$
(A \star B)^{\mathrm{op}} \simeq B^{\mathrm{op}} \star A^{\mathrm{op}}
$$


Démonstration. - Commençons par observer que si $S$ est un objet de $\Theta_{+}$, alors le complexe dirigé augmenté $\lambda(S)^{\text {op }}$ est un complexe de Steiner fort. En effet, d'après les propositions 2.19 et 4.7 , on a $\lambda(S)^{\mathrm{op}} \simeq \lambda\left(S^{\mathrm{op}}\right) \simeq \lambda(T)$, pour un certain $T$ dans $\Theta_{+}$, et on conclut en vertu de la proposition 4.13. En particulier, si $S$ et $T$ sont deux objets de $\Theta_{+}$, en vertu du paragraphe 6.30, on a

$$
\nu\left(\lambda(T)^{\mathrm{op}} \star \lambda(S)^{\mathrm{op}}\right) \simeq \nu\left(\lambda(T)^{\mathrm{op}}\right) \star \nu\left(\lambda(S)^{\mathrm{op}}\right) .
$$

Ceci étant établi, considérons deux $\infty$-catégories $A$ et $B$. On a, en désignant par $S$ et $T$ des objets de $\Theta_{+}$,

$$
\begin{aligned}
& (A \star B)^{\mathrm{op}} \simeq\left(\lim _{S \rightarrow \overrightarrow{A, T} \rightarrow B} \nu(\lambda(S) \star \lambda(T))\right)^{\mathrm{op}} \\
& \text { (en vertu du paragraphe 6.30) } \\
& \simeq \lim _{S \rightarrow \overrightarrow{A, T} \rightarrow B} \nu(\lambda(S) \star \lambda(T))^{\mathrm{op}} \\
& \text { (puisque } C \mapsto C^{\text {op }} \text { est une équivalence de catégories) } \\
& \simeq \lim _{S \rightarrow \overrightarrow{A, T} \rightarrow B} \nu\left((\lambda(S) \star \lambda(T))^{\mathrm{op}}\right) \\
& \text { (en vertu de la proposition 2.19) } \\
& \simeq \lim _{S \rightarrow \overrightarrow{A, T} \rightarrow B} \nu\left(\lambda(T)^{\mathrm{op}} \star \lambda(S)^{\mathrm{op}}\right) \\
& \text { (en vertu de la proposition 6.10) } \\
& \simeq \lim _{S \rightarrow \overrightarrow{A, T} \rightarrow B} \nu\left(\lambda(T)^{\mathrm{op}}\right) \star \nu\left(\lambda(S)^{\mathrm{op}}\right) \\
& \text { (en vertu du paragraphe préliminaire à cette preuve) }
\end{aligned}
$$

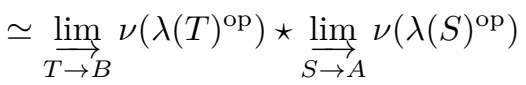

$$
\begin{aligned}
& \text { (en vertu du théorème 6.29) } \\
& \simeq\left(\lim _{T \rightarrow B} \nu \lambda(T)\right)^{\mathrm{op}} \star\left(\lim _{S \rightarrow A} \nu \lambda(S)\right)^{\mathrm{op}} \\
& \text { (par une nouvelle application de la proposition 2.19) } \\
& \simeq(\underset{T \rightarrow B}{\lim } T)^{\mathrm{op}} \star\left(\lim _{S \rightarrow A} S\right)^{\mathrm{op}} \\
& \text { (en vertu de la proposition 4.13) } \\
& \simeq B^{\mathrm{op}} \star A^{\mathrm{op}} \text {, }
\end{aligned}
$$

ce qu'il fallait démontrer.

Proposition 6.36. - Soient $C$ une $\infty$-catégorie et $v: B \rightarrow C$ un $\infty$-foncteur. On a un isomorphisme canonique

$$
C / v \simeq\left(v^{\mathrm{op}} \backslash C^{\mathrm{op}}\right)^{\mathrm{op}}
$$


Démonstration. - En effet, pour toute $\infty$-catégorie $A$, on a des isomorphismes naturels

$$
\begin{aligned}
& \operatorname{Hom}_{\infty-\mathcal{C} a t}\left(A,\left(v^{\mathrm{op}} \backslash C^{\mathrm{op}}\right)^{\mathrm{op}}\right) \simeq \operatorname{Hom}_{\infty-\mathcal{C} a t}\left(A^{\mathrm{op}}, v^{\mathrm{op}} \backslash C^{\mathrm{op}}\right) \\
& \simeq \mathrm{Hom}_{B^{\mathrm{op}} \backslash \infty-\mathcal{C} a t}\left(\left(B^{\mathrm{op}} \star A^{\mathrm{op}}, \iota_{1}^{\mathrm{op}}\right),\left(C^{\mathrm{op}}, v^{\mathrm{op}}\right)\right) \\
& \text { (par adjonction) } \\
& \simeq \mathrm{Hom}_{B^{\mathrm{op}} \backslash \infty-\mathcal{C} a t}\left(\left((A \star B)^{\mathrm{op}}, \iota_{2}^{\mathrm{op}}\right),\left(C^{\mathrm{op}}, v^{\mathrm{op}}\right)\right) \\
& \text { (en vertu de la proposition précédente) } \\
& \simeq \operatorname{Hom}_{B \backslash \infty-\mathcal{C} a t}\left(\left(A \star B, \iota_{2}\right),(C, v)\right) \\
& \simeq \operatorname{Hom}_{\infty-\mathcal{C} a t}(A, C \stackrel{\text { co }}{ }(v) \text {, }
\end{aligned}
$$

d'où le résultat.

Remarque 6.37. - Le foncteur

$$
\begin{aligned}
& \infty-\mathcal{C} a t \times \infty \text { - Cat } \rightarrow \infty \text { - Cat } \\
&(A, B) \quad \mapsto\left(B^{\circ} \star A^{\circ}\right)^{\circ},
\end{aligned}
$$

qu'on appellera le joint dual, définit une structure de catégorie monoïdale sur $\infty$ - $\mathcal{C}$ at distincte de celle définie par le joint (et de celle définie par $(A, B) \mapsto B \star A)$. Notons $A \star^{\prime} B=\left(B^{\circ} \star A^{\circ}\right)^{\circ}$ ce produit tensoriel. Cette structure de catégorie monoïdale est également localement bifermée et on dispose donc de couples de foncteurs adjoints

$$
\begin{aligned}
\infty-\mathcal{C} a t & \rightarrow A \backslash \infty \text { - Cat } & A \backslash \infty \text { - Cat } & \rightarrow \infty \text { - Cat } \\
B & \mapsto\left(A \star^{\prime} B, \iota_{1}^{\prime}\right) & (C, A \stackrel{u}{\rightarrow} C) & \mapsto u \backslash C
\end{aligned}
$$

et

$$
\begin{aligned}
\infty-\mathcal{C} a t & \rightarrow B \backslash \infty-\mathcal{C} a t, & B \backslash \infty-\mathcal{C} a t & \rightarrow \infty-\mathcal{C} a t, \\
A & \mapsto\left(A \star^{\prime} B, \iota_{2}^{\prime}\right) & (C, B \stackrel{v}{\rightarrow} C) & \mapsto C / v
\end{aligned}
$$

où

$$
A \stackrel{\iota_{1}^{\prime}}{\longrightarrow} A \star^{\prime} B \stackrel{\iota_{2}^{\prime}}{\longleftarrow} B
$$

désigne la bicoaugmentation locale (voir l'exemple 5.6). Explicitement, avec des notations évidentes, on a

$$
\iota_{1, A, B}^{\prime}=\iota_{2, B^{\circ}, A^{\circ}}^{\circ} \quad \text { et } \quad \iota_{2, A, B}^{\prime}=\iota_{1, B^{\circ}, A^{\circ} .}^{\circ}
$$

Ainsi, si $A$ et $B$ sont des $\infty$-catégories et $u: A \rightarrow C$ et $v: B \rightarrow C$ des $\infty$-foncteurs, on a des bijections naturelles

$$
\begin{aligned}
& \operatorname{Hom}_{A \backslash \infty-\mathcal{C} a t}\left(\left(A \star^{\prime} B, \iota_{1}^{\prime}\right),(C, u)\right) \simeq \operatorname{Hom}_{\infty-\mathcal{C} a t}(B, u \backslash C), \\
& \operatorname{Hom}_{B \backslash \infty-\mathcal{C} a t}\left(\left(A \star^{\prime} B, \iota_{2}^{\prime}\right),(C, v)\right) \simeq \operatorname{Hom}_{\infty-\mathcal{C} a t}(A, C / v) .
\end{aligned}
$$


On vérifie immédiatement qu'on a des isomorphismes canoniques

$$
C / u \simeq\left(C^{\mathrm{co}} / u^{\mathrm{co}}\right)^{\mathrm{co}} \text { et } \quad \stackrel{\mathrm{co}}{v} \backslash C \simeq\left(v^{\mathrm{co}} \backslash C^{\mathrm{co}}\right)^{\mathrm{co}},
$$

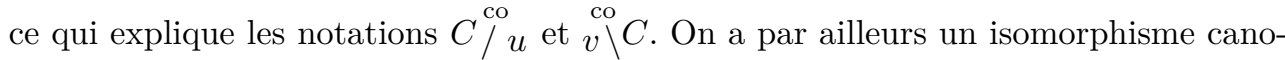
nique

$$
C / u \simeq\left(u^{\circ} \backslash C^{\circ}\right)^{\circ}
$$

On appellera la $\infty$-catégorie $C / u$ la tranche de $C$ au-dessus de $u$. Le choix de privilégier la $\infty$-catégorie $C / u$ par rapport à $C / u$ est dicté par les meilleures propriétés formelles dont dispose $C / u$.

6.38. - Soit

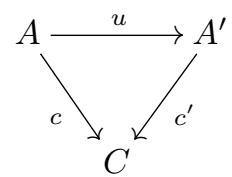

un triangle commutatif de $\infty$-foncteurs. On va définir un $\infty$-foncteur

$$
u^{*}: c^{\prime} \backslash C \rightarrow c \backslash C \text {. }
$$

Soit $B$ un $\infty$-catégorie. En vertu du lemme de Yoneda, il suffit de définir une application

$$
\operatorname{Hom}_{\infty-\mathcal{C} a t}\left(B, c^{\prime} \backslash C\right) \rightarrow \operatorname{Hom}_{\infty-\mathcal{C} a t}(B, c \backslash C),
$$

naturelle en $B$, ou encore, par adjonction, une application

$$
\operatorname{Hom}_{A^{\prime} \backslash \infty-\mathcal{C} a t}\left(\left(A^{\prime} \star B, \iota_{1}\right),\left(C, c^{\prime}\right)\right) \rightarrow \operatorname{Hom}_{A \backslash \infty-\mathcal{C} a t}\left(\left(A \star B, \iota_{1}\right),(C, c)\right) .
$$

Il est immédiat, par naturalité de $\iota_{1}$, que la précomposition par

$$
u \star B: A \star B \rightarrow A^{\prime} \star B
$$

fournit une telle application. La naturalité en $B$ résulte de la fonctorialité du joint et on obtient donc bien un $\infty$-foncteur $u^{*}: c^{\prime} \backslash C \rightarrow c \backslash C$.

Ce $\infty$-foncteur $u^{*}: c^{\prime} \backslash C \rightarrow c \backslash C$ peut se décrire de manière alternative comme suit. Par fonctorialité du joint, le $\infty$-foncteur $u$ induit une application

$$
\operatorname{Hom}_{\infty-\mathcal{C} a t}\left(A^{\prime} \star S, C\right) \rightarrow \mathrm{Hom}_{\infty-\mathcal{C} a t}(A \star S, C),
$$

naturelle en $S$ dans $\infty$-Cat. En vertu du paragraphe 4.9, cette transformation naturelle induit un $\infty$-foncteur

$$
c^{\prime} \backslash C \simeq \operatorname{Hom}_{\infty-\mathcal{C} a t}\left(A^{\prime} \star \mathrm{D}_{\bullet}, C\right)_{c^{\prime}} \rightarrow \operatorname{Hom}_{\infty-\mathcal{C} a t}\left(A \star \mathrm{D}_{\bullet}, C\right)_{c} \simeq c \backslash C
$$

qui n'est autre que $u^{*}$.

Puisque $\varnothing \star B$, pour $B$ une $\infty$-catégorie, est canoniquement isomorphe à $B$, il résulte de l'adjonction définissant la $\infty$-catégorie $c \backslash C$ que celle-ci est canoniquement 
isomorphe à $C$ lorsque $c: \varnothing \rightarrow A$ est l'unique morphisme de source la $\infty$-catégorie vide et de but $A$. On obtient donc un $\infty$-foncteur

$$
c^{\prime} \backslash C \rightarrow C
$$

qu'on appellera $\infty$-foncteur d'oubli.

En revenant au cas général, notons que le $\infty$-foncteur $u^{*}: c^{\prime} \backslash C \rightarrow c \backslash C$ est au-dessus de $C$. Autrement dit, le triangle

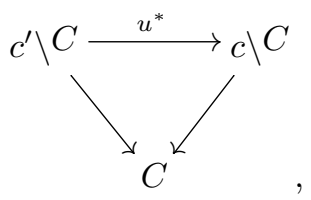

où les flèches obliques sont les $\infty$-foncteurs d'oubli, est commutatif. 


\section{CHAPITRE 7}

\section{UNE APPLICATION : CONSTRUCTION DU NERF DE STREET}

Dans ce chapitre, on montre comment le joint permet de définir facilement les orientaux et le nerf de Street [36].

7.1. - On notera $\boldsymbol{\Delta}$ la catégorie des simplexes. Rappelons que ses objets sont les ensembles ordonnés

$$
\Delta_{n}=\{0 \leqslant 1 \leqslant \cdots \leqslant n\}, \quad \text { pour } n \geqslant 0,
$$

et que ses morphismes sont les applications croissantes (au sens large) entre ceux-ci. La catégorie des simplexes augmentée $\boldsymbol{\Delta}_{+}$se définit de la même manière en ajoutant l'ensemble ordonné $\Delta_{-1}=\varnothing$. On considérera souvent $\boldsymbol{\Delta}$ et $\boldsymbol{\Delta}_{+}$comme des souscatégories pleines de la catégorie $\mathcal{C}$ at des petites catégories et donc comme des souscatégories pleines de $\infty$-C at. Avec ces conventions, la catégorie $\boldsymbol{\Delta}$ devient une souscatégorie pleine de la catégorie $\Theta$ de Joyal et la catégorie $\boldsymbol{\Delta}_{+}$une sous-catégorie pleine de $\Theta_{+}$.

La catégorie $\boldsymbol{\Delta}_{+}$admet $\Delta_{-1}$ comme objet initial et la somme disjointe ensembliste

$$
\left(\Delta_{m}, \Delta_{n}\right) \mapsto \Delta_{m} \amalg \Delta_{n}=\Delta_{m+1+n}
$$

induit une structure de catégorie monoïdale sur $\boldsymbol{\Delta}_{+}$d'objet unité $\Delta_{-1}$. On munira souvent implicitement la catégorie $\boldsymbol{\Delta}_{+}$de la structure de catégorie monoïdale définie par la somme.

Enfin, on rappelle que la catégorie des ensembles simpliciaux est la catégorie $\widehat{\boldsymbol{\Delta}}$ des préfaisceaux sur la catégorie $\boldsymbol{\Delta}$.

7.2. - Considérons la $\infty$-catégorie $\Delta_{0}$, qui n'est autre que la $\infty$-catégorie finale. Cette $\infty$-catégorie est munie d'une et une seule structure de monoïde dans la catégorie monoïdale $(\infty-\mathcal{C} a t, \star, \varnothing)$. En effet, on a des uniques $\infty$-foncteurs

$$
\Delta_{0} \star \Delta_{0} \rightarrow \Delta_{0} \quad \text { et } \quad \varnothing \rightarrow \Delta_{0}
$$


et ceux-ci vérifient trivialement les axiomes des monoïdes. Or, la donnée d'une structure de monoïde sur $\Delta_{0}$ est équivalente à celle d'un foncteur monoïdal $\boldsymbol{\Delta}_{+} \rightarrow \infty$ - $\mathcal{C}$ at envoyant $\Delta_{0}$ sur $\Delta_{0}$ (voir par exemple [28, chapitre VII, section 5]), défini à unique isomorphisme monoïdal près. Il existe donc un unique (à unique isomorphisme monoïdal près) foncteur monoïdal

$$
\mathcal{O}_{+}: \boldsymbol{\Delta}_{+} \rightarrow \infty-\mathcal{C} a t
$$

envoyant $\Delta_{0}$ sur $\Delta_{0}$. Pour $n \geqslant-1$, on notera $\mathcal{O}_{n}$ l'image de $\Delta_{n}$ par ce foncteur. Explicitement, on a

$$
\mathcal{O}_{n}=\Delta_{0} \star \cdots \star \Delta_{0}
$$

où $\Delta_{0}$ apparait $n+1$ fois dans le membre de droite. Par restriction, on obtient un foncteur

et donc un foncteur

$$
\mathcal{O}: \Delta \rightarrow \infty-\mathcal{C} a t
$$

$$
N_{\infty}: \infty-\mathcal{C} a t \rightarrow \widehat{\boldsymbol{\Delta}}
$$

défini par

$$
C \mapsto\left(\Delta_{n} \mapsto \operatorname{Hom}_{\infty-\mathcal{C} a t}\left(\mathcal{O}_{n}, C\right)\right)
$$

Le but de la suite de ce chapitre est de démontrer que le foncteur $\mathcal{O}: \boldsymbol{\Delta} \rightarrow \infty$-Cat n'est autre que l'objet cosimplicial de Street défini dans [36] et que le foncteur $N_{\infty}: \infty-\mathcal{C} a t \rightarrow \widehat{\boldsymbol{\Delta}}$ est donc le nerf de Street. En particulier, on obtiendra que $\mathcal{O}_{n}$, pour $n \geqslant 0$, est le $n$-ième oriental de Street. Voici une représentation graphique des premiers orientaux :

$$
\begin{aligned}
& \mathcal{O}_{0}=\mathrm{D}_{0}=\{0\}, \quad \mathcal{O}_{1}=\mathrm{D}_{1}=0 \longrightarrow 1, \quad \mathcal{O}_{2}=\overbrace{0 \longrightarrow 1}^{\searrow},
\end{aligned}
$$

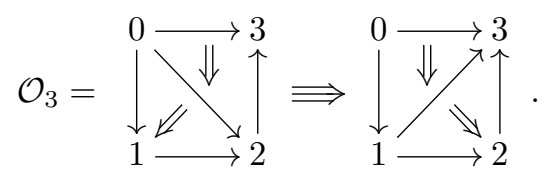

Notons qu'une description analogue du $n$-ième oriental en termes du joint des complexes de parités apparaît déjà chez Street [37, section 6].

7.3. - On va définir, selon Steiner [32], un foncteur $c: \boldsymbol{\Delta}_{+} \rightarrow \mathcal{C}_{\mathrm{da}}$. Fixons $m \geqslant-1$ et décrivons $c\left(\Delta_{m}\right)$. Le complexe de chaînes sous-jacent à $c\left(\Delta_{m}\right)$ est le complexe normalisé associé à l'ensemble simplicial $\Delta_{m}$. Explicitement, pour $n \geqslant 0$, on a

$$
c\left(\Delta_{m}\right)_{n}=\mathbb{Z}^{\left(B_{n}\right)},
$$

où

$$
B_{n}=\left\{\left(i_{0}, \ldots, i_{n}\right) \mid 0 \leqslant i_{0}<\cdots<i_{n} \leqslant m\right\}
$$


Pour $n \geqslant 1$, la différentielle $d_{n}: \mathbb{Z}^{\left(B_{n}\right)} \rightarrow \mathbb{Z}^{\left(B_{n-1}\right)}$ est donnée par

$$
d_{n}\left(i_{0}, \ldots, i_{n}\right)=\sum_{k=0}^{n}(-1)^{k}\left(i_{0}, \ldots, \widehat{i_{k}}, \ldots, i_{n}\right),
$$

où on a posé $\left(i_{0}, \ldots, \widehat{i_{k}}, \ldots, i_{n}\right)=\left(i_{0}, \ldots, i_{k-1}, i_{k+1}, \ldots, i_{n}\right)$. Enfin, pour $n \geqslant 0$, les sous-monoïdes de positivité sont les

$$
c\left(\Delta_{m}\right)_{n}^{*}=\mathbb{N}^{\left(B_{n}\right)}
$$

et l'augmentation $e: \mathbb{Z}^{\left(B_{0}\right)} \rightarrow \mathbb{Z}$ est donnée par la somme des coefficients.

On vérifie immédiatement qu'on obtient bien ainsi un foncteur $\boldsymbol{\Delta}_{+} \rightarrow \mathcal{C}_{\mathrm{da}}$.

Remarque 7.4. - Le foncteur du paragraphe précédent est la restriction à $\boldsymbol{\Delta}_{+}$ d'un foncteur $c: \widehat{\boldsymbol{\Delta}} \rightarrow \mathcal{C}_{\text {da }}$ qui est étudié dans [6] (voir notamment la section 5).

Proposition 7.5 (Steiner). - Pour tout $m \geqslant-1$, le complexe dirigé augmenté $c\left(\Delta_{m}\right)$ est un complexe de Steiner fort.

Démonstration. - La preuve est esquissée dans [32, exemple 3.8]. Pour une preuve détaillée (et s'appliquant non seulement à $\Delta_{m}$ mais également à n'importe quel complexe simplicial), voir [6, théorème 8.6].

Lemme 7.6. - Pour tous $m, n \geqslant-1$, on a un isomorphisme canonique

$$
c\left(\Delta_{m}\right) \star c\left(\Delta_{n}\right) \simeq c\left(\Delta_{m+1+n}\right),
$$

naturel en $\Delta_{m}$ et $\Delta_{n}$ dans $\boldsymbol{\Delta}_{+}$.

Démonstration. - Par récurrence, il suffit de traiter le cas $m=0$. Dans ce cas, on définit un isomorphisme $\chi: c\left(\Delta_{0}\right) \star c\left(\Delta_{n}\right) \rightarrow c\left(\Delta_{1+n}\right)$ par

$$
\begin{aligned}
\varnothing \star\left(i_{0}, \ldots, i_{k}\right) & \mapsto\left(i_{0}+1, \ldots, i_{k}+1\right) \\
(0) \star \varnothing & \mapsto(0) \\
(0) \star\left(i_{0}, \ldots, i_{k}\right) & \mapsto\left(0, i_{0}+1, \ldots, i_{k}+1\right) .
\end{aligned}
$$

Il est immédiat que cette application définit une bijection de la base du complexe $c\left(\Delta_{0}\right) \star c\left(\Delta_{n}\right)$ sur celle de $c\left(\Delta_{1+n}\right)$ et qu'elle est compatible aux augmentations et aux sous-monoïdes de positivité. Pour conclure, il suffit donc de vérifier la compatibilité à la différentielle. Soit $\left(i_{0}, \ldots, i_{k}\right)$ un élément de la base de $c\left(\Delta_{n}\right)$. La compatibilité à la différentielle pour les éléments de la forme $\varnothing \star\left(i_{0}, \ldots, i_{k}\right)$ est évidente. Pour ceux de la forme $(0) \star\left(i_{0}, \ldots, i_{k}\right)$, on a

$$
\begin{aligned}
\chi(d((0) & \left.\left.\star\left(i_{0}, \ldots, i_{k}\right)\right)\right) \\
\quad= & \chi\left(\varnothing \star\left(i_{0}, \ldots, i_{k}\right)-(0) \star d\left(i_{0}, \ldots, i_{k}\right)\right) \\
& =\left(i_{0}+1, \ldots, i_{k}+1\right)-\sum_{l=0}^{k}(-1)^{l} \chi\left((0) \star\left(i_{0}, \ldots, \widehat{i_{l}}, \ldots, i_{k}\right)\right)
\end{aligned}
$$




$$
\begin{aligned}
& =\left(i_{0}+1, \ldots, i_{k}+1\right)+\sum_{l=0}^{k}(-1)^{l+1}\left(0, i_{0}+1, \ldots \widehat{i_{l}+1}, \ldots, i_{k}+1\right) \\
& =\left(i_{0}+1, \ldots, i_{k}+1\right)+\sum_{l=1}^{k+1}(-1)^{l}\left(0, i_{0}+1, \ldots, \widehat{i_{l-1}+1}, \ldots, i_{k}+1\right) \\
& =d\left(0, i_{0}+1, \ldots, i_{k}+1\right) \\
& =d\left(\chi\left((0) \star\left(i_{0}, \ldots, i_{k}\right)\right)\right),
\end{aligned}
$$

ce qui achève la démonstration.

Remarque 7.7. - En particulier, pour $m \geqslant-1$, on a

$$
c\left(\Delta_{m}\right) \simeq c\left(\Delta_{0}\right) \star \cdots \star c\left(\Delta_{0}\right),
$$

où $c\left(\Delta_{0}\right)$ apparaît $m+1$ fois dans le membre de droite. Les complexes $c\left(\Delta_{-1}\right)$ et $c\left(\Delta_{0}\right)$ étant trivialement des complexes de Steiner forts, le fait que les $c\left(\Delta_{m}\right)$ sont des complexes de Steiner forts (proposition 7.5) résulte donc aussi de la stabilité des complexes de Steiner forts par le joint (corollaire 6.21).

Proposition 7.8. - Pour tous $m, n \geqslant-1$, on a un isomorphisme canonique

$$
\nu\left(c\left(\Delta_{m}\right)\right) \star \nu\left(c\left(\Delta_{n}\right)\right) \simeq \nu\left(c\left(\Delta_{m+1+n}\right)\right),
$$

naturel en $\Delta_{m}$ et $\Delta_{n}$ dans $\boldsymbol{\Delta}_{+}$.

Démonstration. - Cela résulte immédiatement de la proposition précédente, du fait que les $c\left(\Delta_{p}\right)$ sont des complexes de Steiner forts (proposition 7.5) et du caractère monoïdal du joint sur les complexes de Steiner forts (voir le théorème 6.29).

Théorème 7.9. - Le foncteur $\mathcal{O}_{+}: \Delta \rightarrow \infty$-Cat est canoniquement isomorphe au composé

$$
\boldsymbol{\Delta}_{+} \stackrel{c}{\rightarrow} \mathcal{C}_{\mathrm{da}} \stackrel{\nu}{\rightarrow} \infty \text { - } \mathcal{C} a t .
$$

En particulier, pour tout $n \geqslant-1$, on a $\mathcal{O}_{n} \simeq \nu\left(c\left(\Delta_{n}\right)\right)$.

Démonstration. - Le foncteur $\mathcal{O}_{+}: \boldsymbol{\Delta}_{+} \rightarrow \infty$-C at étant caractérisé par le fait qu'il est monoïdal et envoie $\Delta_{0}$ sur $\Delta_{0}$, il suffit de vérifier que le foncteur $\nu c: \boldsymbol{\Delta}_{+} \rightarrow \infty$-C at vérifie ces deux propriétés. Or, il est immédiat qu'on a bien $\nu c\left(\Delta_{0}\right) \simeq \Delta_{0}$ et le caractère monoïdal du foncteur $\nu c$ est donné par la proposition précédente, d'où le résultat.

Corollaire 7.10. - Les foncteurs

$$
\mathcal{O}: \Delta \rightarrow \infty \text {-Cat } \quad \text { et } \quad N_{\infty}: \infty \text {-Cat } \rightarrow \widehat{\boldsymbol{\Delta}}
$$

sont isomorphes à l'objet cosimplicial de Street et au nerf de Street définis dans [36].

Démonstration. - En vertu du théorème précédent, le foncteur $\mathcal{O}: \boldsymbol{\Delta} \rightarrow \infty$-Cat est isomorphe au foncteur $\Delta_{n} \mapsto \nu\left(c\left(\Delta_{n}\right)\right)$ qui est l'objet cosimplicial de Street d'après [33, théorème 3.2$]$, d'où le résultat. 
Remarque 7.11. - Dans ce chapitre, on a produit l'objet cosimplicial de Street et le nerf de Street à partir de la structure de catégorie monoïdale sur $\infty$-C $a t$ définie par le joint. Si on avait utilisé la structure de catégorie monoïdale de la remarque 6.37 , on aurait produit l'objet cosimplicial $\Delta_{n} \mapsto\left(\mathcal{O}_{n}\right)^{\text {co }}$ et le nerf $C \mapsto N_{\infty}\left(C^{\mathrm{co}}\right)$. Quant à la convention de signe exposée dans la remarque 6.4, elle aurait mené à l'objet cosimplicial $\Delta_{n} \mapsto\left(\mathcal{O}_{n}\right)^{\circ}$ et au nerf $C \mapsto N_{\infty}\left(C^{\circ}\right)$.

7.12. - Rappelons brièvement la définition du joint simplicial (voir par exemple $[\mathbf{2 4}$, section 3]). La structure de catégorie monoïdale sur $\boldsymbol{\Delta}_{+}$définie au paragraphe 7.1 s'étend par limites inductives canoniques en une structure de catégorie monoïdale sur la catégorie des ensembles simpliciaux augmentés $\widehat{\boldsymbol{\Delta}_{+}}$. On vérifie que la catégorie des ensembles simpliciaux forme une sous-catégorie monoïdale de cette structure, où on a considéré qu'un ensemble simplicial est un ensemble simplicial augmenté en l'augmentant au-dessus de $\Delta_{0}$ de l'unique manière possible. On obtient ainsi un foncteur

$$
\begin{aligned}
\widehat{\boldsymbol{\Delta}} \times \widehat{\boldsymbol{\Delta}} & \rightarrow \widehat{\boldsymbol{\Delta}} \\
(X, Y) & \mapsto X \star Y
\end{aligned}
$$

qu'on appelle le joint simplicial. On vérifie immédiatement que l'unité de cette structure est l'ensemble simplicial vide. Par ailleurs, on montre que le joint simplicial commute aux limites inductives connexes en chaque variable. Ainsi, en vertu de la remarque 5.9, le joint simplicial définit une structure monoïdale localement bifermée (voir le paragraphe 5.7).

Proposition 7.13. - Les foncteurs

$$
c_{\infty}: \widehat{\boldsymbol{\Delta}} \rightarrow \infty-\mathcal{C} \text { at } \quad \text { et } \quad N_{\infty}: \infty-\mathcal{C} a t \rightarrow \widehat{\boldsymbol{\Delta}},
$$

où $c_{\infty}$ désigne l'adjoint à gauche de $N_{\infty}$, sont monoïdal et monoïdal lax respectivement, les catégories $\widehat{\boldsymbol{\Delta}}$ et $\infty$-Cat étant toutes deux munies des structures de catégorie monoïdale définies par le joint.

Démonstration. - Par adjonction, il suffit de montrer que le foncteur $c_{\infty}$ est monoïdal. On a évidemment $c_{\infty}(\varnothing)=\varnothing$. Produisons maintenant la contrainte de compatibilité aux produits monoïdaux. Puisque $c_{\infty}$ commute aux limites inductives et que les deux foncteurs joint commutent aux limites inductives connexes en chaque variable (l'un en vertu du théorème 6.29 et l'autre en vertu du paragraphe précédent), les foncteurs

$$
(X, Y) \mapsto c_{\infty}(X) \star c_{\infty}(Y) \text { et } \quad(X, Y) \mapsto c_{\infty}(X \star Y)
$$

de $\widehat{\boldsymbol{\Delta}} \times \widehat{\boldsymbol{\Delta}}$ dans $\infty$-Cat commutent tout deux aux limites inductives connexes en chaque variable. Par ailleurs, pour $m \geqslant-1$ et $n \geqslant-1$, on a, en vertu du théorème 7.9, des 
isomorphismes naturels

$$
c_{\infty}\left(\Delta_{m}\right) \star c_{\infty}\left(\Delta_{n}\right) \simeq \mathcal{O}_{m} \star \mathcal{O}_{n} \simeq \mathcal{O}_{m+1+n} \simeq c_{\infty}\left(\Delta_{m+1+n}\right) \simeq c_{\infty}\left(\Delta_{m} \star \Delta_{n}\right),
$$

où $\Delta_{-1}$ désigne l'ensemble simplicial vide. On obtient le résultat puisque tout ensemble simplicial est limite inductive canonique de $\Delta_{p}$ avec $p \geqslant-1$ et que cette limite inductive est connexe.

Remarque 7.14. - Un ingrédient clé à la démonstration de notre théorème A de Quillen $\infty$-catégorique dans [7] et [8] est un résultat de compatibilité (partielle) du nerf de Street aux tranches $\infty$-catégoriques et simpliciales, ces dernières étant définies par les adjoints à droite associés à la structure de catégorie monoïdale localement bifermée définie par le joint simplicial. On renvoie à [8, proposition 2.9] pour un énoncé précis et une preuve de ce résultat, ainsi qu'à [7, proposition 4.6] pour une preuve qui n'utilise pas le joint dans le cas particulier des tranches au-dessous d'un objet. 


\section{CHAPITRE 8}

\section{JOINT ET TRANCHES n-CATÉGORIQUES}

Le but de ce chapitre est de montrer que le joint $\infty$-catégorique induit par troncation un joint $n$-catégorique, et de montrer que pour $n=1$ on obtient le joint catégorique usuel.

Dans tout le chapitre, on fixe un entier $n \geqslant 0$.

8.1. - On notera $\Theta_{n}$ la sous-catégorie pleine de la catégorie $n$-Cat des $n$-catégories formée des objets de $\Theta$ qui sont des $n$-catégories. De même, on notera $\left(\Theta_{n}\right)_{+}$la sous-catégorie pleine de la catégorie $n$-Cat formée des objets de $\Theta_{+}$qui sont des $n$-catégories.

On vérifie facilement que, si $S$ est un objet de $\Theta$, alors $\tau_{\leqslant n}^{\mathrm{i}}(S)$ (voir le paragraphe 1.2) est un objet de $\Theta_{n}$. En particulier, le foncteur $\tau_{\leqslant n}^{\mathrm{i}}: \infty$-C $a t \rightarrow n$-C $a t$ induit un foncteur $\Theta \rightarrow \Theta_{n}$ qui fournit un adjoint à gauche au foncteur d'inclusion $\Theta_{n} \hookrightarrow \Theta$. La catégorie $\Theta_{n}$ est donc une sous-catégorie réflexive de $\Theta$. De même, la catégorie $\left(\Theta_{n}\right)_{+}$est une sous-catégorie réflexive de $\Theta_{+}$.

Proposition 8.2. - La catégorie $\Theta_{n}$ (et donc la catégorie $\left(\Theta_{n}\right)_{+}$) est une souscatégorie dense de n-Cat. Autrement dit, si $C$ est une n-catégorie, le morphisme canonique

$$
\lim _{(S, S \rightarrow C) \in \Theta_{n} / C} S \longrightarrow C
$$

est un isomorphisme de n-catégories.

Démonstration. - En vertu de la proposition 4.6, on obtient un isomorphisme en remplaçant $\Theta_{n}$ par $\Theta$ dans la formule ci-dessus et il suffit donc de montrer que l'inclusion $\Theta_{n} / C \hookrightarrow \Theta / C$ est un foncteur cofinal. Or, il résulte immédiatement du fait que $\Theta_{n}$ est un sous-catégorie réflexive de $\Theta$ que ce foncteur est un adjoint à droite, d'où le résultat. 
Lemme 8.3. - Soient $p, q \geqslant 0$ deux entiers. Si $K$ est un complexe dirigé augmenté de dimension $p$ et $L$ un complexe dirigé augmenté de dimension $q$, alors leur joint $K \star L$ est un complexe dirigé augmenté de dimension $p+1+q$.

Démonstration. - Cela résulte immédiatement de la formule explicite définissant le joint des complexes dirigés augmentés (voir les paragraphes 6.5 et 6.7).

Lemme 8.4. - Soient $S$ un objet de $\left(\Theta_{p}\right)_{+}$et $T$ un objet de $\left(\Theta_{q}\right)_{+}$, où $p$ et $q$ sont des entiers positifs. Alors le joint de $S$ et $T$ est une $(p+1+q)$-catégorie.

Démonstration. - En vertu du paragraphe 6.30, on a $S \star T \simeq \nu(\lambda(S) \star \lambda(T))$. Puisque les foncteurs $\lambda$ et $\nu$ se restreignent en des foncteurs entre $r$-catégories et complexes dirigés augmentés de dimension au plus $r$, le résultat est conséquence du lemme précédent.

Proposition 8.5. - Soient $p, q \geqslant 0$ deux entiers. Si $C$ est une p-catégorie et $D$ une q-catégorie, alors on a un isomorphisme canonique

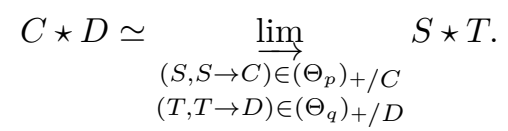

En particulier, le joint de $C$ et $D$ est une $(p+1+q)$-catégorie.

Démonstration. - En vertu de la proposition 8.2, pour $r=p, q$, la catégorie $\left(\Theta_{r}\right)_{+}$ est dense dans $r$-C at et on a donc

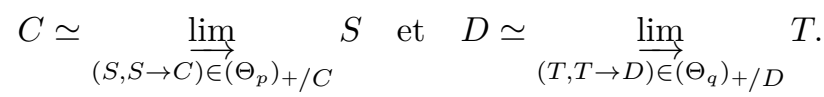

On obtient alors la formule de l'énoncé en utilisant la commutation du joint aux limites inductives connexes en chaque variable (voir le théorème 6.29). Ainsi, en vertu du lemme précédent, le joint de $C$ et $D$ est limite inductive de $(p+1+q)$-catégories et est donc une $(p+1+q)$-catégorie.

8.6. - Soient $C$ et $D$ deux $n$-catégories. On appelle joint $n$-catégorique de $C$ et $D$ la $n$-catégorie

$$
C \star_{n} D=\tau_{\leqslant n}^{\mathrm{i}}(C \star D) .
$$

Cette opération définit un foncteur

$$
\begin{aligned}
n-\mathcal{C} a t \times n-\mathcal{C} a t & \rightarrow n-\mathcal{C} a t \\
(C, D) & \mapsto C \star_{n} D .
\end{aligned}
$$

Si $C$ est une $n$-catégorie, on a

$$
\varnothing \star_{n} C=\tau_{\leqslant n}^{\mathrm{i}}(\varnothing \star C) \simeq \tau_{\leqslant n}^{\mathrm{i}}(C)=C
$$

et, de même,

$$
C \star_{n} \varnothing \simeq C
$$


On notera, comme dans le cas de $\infty$ - $\mathcal{C} a t$

$$
C \stackrel{\iota_{1}}{\longrightarrow} C \star_{n} D \stackrel{\iota_{2}}{\longleftarrow} D
$$

les $\infty$-foncteurs canoniques.

Le but des énoncés suivants est d'établir que le joint $n$-catégorique définit une structure de catégorie monoïdale sur $n$-Cat.

Lemme 8.7. - Soient $K$ et $L$ deux complexes dirigés augmentés. Les morphismes canoniques $K \rightarrow \tau_{\leqslant n}^{\mathrm{i}}(K)$ et $L \rightarrow \tau_{\leqslant n}^{\mathrm{i}}(L)$ induisent un isomorphisme

$$
\tau_{\leqslant n}^{\mathrm{i}}(K \star L) \simeq \tau_{\leqslant n}^{\mathrm{i}}\left(\tau_{\leqslant n}^{\mathrm{i}}(K) \star \tau_{\leqslant n}^{\mathrm{i}}(L)\right) .
$$

Démonstration. - Il s'agit de montrer que pour tout $r$ tel que $0 \leqslant r \leqslant n$, l'application canonique

$$
\alpha_{r}: \tau_{\leqslant n}^{\mathrm{i}}(K \star L)_{r} \rightarrow \tau_{\leqslant n}^{\mathrm{i}}\left(\tau_{\leqslant n}^{\mathrm{i}}(K) \star \tau_{\leqslant n}^{\mathrm{i}}(L)\right)_{r}
$$

est une bijection. Si $r<n$, cette application n'est autre que l'application canonique

$$
(K \star L)_{r} \rightarrow\left(\tau_{\leqslant n}^{\mathrm{i}}(K) \star \tau_{\leqslant n}^{\mathrm{i}}(L)\right)_{r} .
$$

Or, la description explicite du joint (voir les paragraphes 6.5 et 6.7) montre que cette application est l'identité puisque $\tau_{\leqslant n}^{\mathrm{i}}(K)_{p}=K_{p}$ et $\tau_{\leqslant n}^{\mathrm{i}}(L)_{q}=L_{q}$ pour $p, q \leqslant r<n$. Traitons maintenant le cas $r=n$. Par définition, $\tau_{\leqslant n}^{\mathrm{i}}(K \star L)_{n}$ est le quotient $\mathrm{du}$ groupe abélien

par le sous-groupe

$$
\left(\bigoplus_{\substack{p+1+q=n \\ n>p \geqslant-1, n>q \geqslant-1}}\left(K_{p} \otimes L_{q}\right)\right) \oplus K_{n} \oplus L_{n}
$$

$$
\left(\sum_{\substack{p^{\prime}+1+q^{\prime}=n+1 \\ n>p^{\prime} \geqslant-1, n>q^{\prime} \geqslant-1}} d\left(K_{p^{\prime}} \otimes L_{q^{\prime}}\right)\right)+d\left(K_{n} \otimes L_{0}\right)+d\left(K_{0} \otimes L_{n}\right)+d\left(K_{n+1}\right)+d\left(L_{n+1}\right)
$$

et $\tau_{\leqslant n}^{\mathrm{i}}\left(\tau_{\leqslant n}^{\mathrm{i}}(K) \star \tau_{\leqslant n}^{\mathrm{i}}(L)\right)_{n}$ le quotient du groupe abélien

$$
\left(\bigoplus_{\substack{p+1+q=n \\ n>p \geqslant-1, n>q \geqslant-1}}\left(K_{p} \otimes L_{q}\right)\right) \oplus K_{n} / d\left(K_{n+1}\right) \oplus L_{n} / d\left(L_{n+1}\right)
$$

par le sous-groupe

$$
\left(\sum_{\substack{p^{\prime}+1+q^{\prime}=n+1 \\ n>p^{\prime} \geqslant-1, n>q^{\prime} \geqslant-1}} d\left(K_{p^{\prime}} \otimes L_{q^{\prime}}\right)\right)+d\left(K_{n} / d\left(K_{n+1}\right) \otimes L_{0}\right)+d\left(K_{0} \otimes L_{n} / d\left(L_{n+1}\right)\right)
$$

(modulo un ajustement mineur pour traiter le cas $n=0$ ). Puisque

$$
d\left(K_{n} / d\left(K_{n+1}\right) \otimes L_{0}\right)=d\left(K_{n} \otimes L_{0}\right) \quad \text { et } \quad d\left(K_{0} \otimes L_{n} / d\left(L_{n+1}\right)\right)=d\left(K_{0} \otimes L_{n}\right),
$$

le morphisme canonique du premier quotient vers le second est un isomorphisme, ce qu'il fallait démontrer. 
Proposition 8.8. - Soient $C$ et $D$ deux $\infty$-catégories. Les $\infty$-foncteurs canoniques $C \rightarrow \tau_{\leqslant n}^{\mathrm{i}}(C)$ et $D \rightarrow \tau_{\leqslant n}^{\mathrm{i}}(D)$ induisent un isomorphisme

$$
\tau_{\leqslant n}^{\mathrm{i}}(C \star D) \simeq \tau_{\leqslant n}^{\mathrm{i}}\left(\tau_{\leqslant n}^{\mathrm{i}}(C) \star \tau_{\leqslant n}^{\mathrm{i}}(D)\right) .
$$

Démonstration. - Commençons par démontrer le résultat dans le cas où $C=S$ et $D=T$ sont des objets de $\Theta_{+}$. Observons tout d'abord que si $U$ est un objet de $\Theta_{+}$, alors $\tau_{\leqslant n}^{\mathrm{i}}(\lambda(U))$ est un complexe de Steiner fort. En effet, en vertu de la proposition 2.22 , on a $\tau_{\leqslant n}^{\mathrm{i}}(\lambda(U))=\lambda\left(\tau_{\leqslant n}^{\mathrm{i}}(U)\right)$ et, la $n$-catégorie $\tau_{\leqslant n}^{\mathrm{i}}(U)$ étant un objet de $\Theta_{+}$, on conclut en vertu de la proposition 4.13. On a donc

$$
\begin{aligned}
\tau_{\leqslant n}^{\mathrm{i}}(S \star T) \simeq & \tau_{\leqslant n}^{\mathrm{i}} \nu(\lambda(S) \star \lambda(T)) \\
& (\text { en vertu du paragraphe } 6.30) \\
\simeq & \nu \tau_{\leqslant n}^{\mathrm{i}}(\lambda(S) \star \lambda(T)) \\
& (\text { en vertu de la proposition } 2.23) \\
\simeq & \nu \tau_{\leqslant n}^{\mathrm{i}}\left(\tau_{\leqslant n}^{\mathrm{i}}(\lambda(S)) \star \tau_{\leqslant n}^{\mathrm{i}}(\lambda(T))\right) \\
& (\text { en vertu du lemme précédent }) \\
\simeq & \tau_{\leqslant n}^{\mathrm{i}} \nu\left(\tau_{\leqslant n}^{\mathrm{i}}(\lambda(S)) \star \tau_{\leqslant n}^{\mathrm{i}}(\lambda(T))\right) \\
\simeq & \tau_{\leqslant n}^{\mathrm{i}}\left(\nu \tau_{\leqslant n}^{\mathrm{i}}(\lambda(S)) \star \nu \tau_{\leqslant n}^{\mathrm{i}}(\lambda(T))\right) \\
& \left(\operatorname{puisque} \tau_{\leqslant n}^{\mathrm{i}}(\lambda(U)) \text { pour } U=S, T \text { est de Steiner fort }\right) \\
\simeq & \tau_{\leqslant n}^{\mathrm{i}}\left(\tau_{\leqslant n}^{\mathrm{i}} \nu(\lambda(S)) \star \tau_{\leqslant n}^{\mathrm{i}} \nu(\lambda(T))\right) \\
\simeq & \tau_{\leqslant n}^{\mathrm{i}}\left(\tau_{\leqslant n}^{\mathrm{i}}(S) \star \tau_{\leqslant n}^{\mathrm{i}}(T)\right),
\end{aligned}
$$

où le dernier isomorphisme résulte de la proposition 4.13 .

Passons au cas général. On a, en désignant par $S$ et $T$ des objets de $\Theta_{+}$,

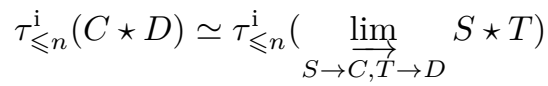

$$
\begin{aligned}
& \text { (en vertu du paragraphe 6.30) } \\
& \simeq \lim _{S \rightarrow \overrightarrow{C, T} \rightarrow D} \tau_{\leqslant n}^{\mathrm{i}}(S \star T) \\
& \text { (puisque } \tau_{\leqslant n}^{\mathrm{i}} \text { est un adjoint à gauche) } \\
& \simeq \lim _{S \rightarrow \overrightarrow{C, T} \rightarrow D} \tau_{\leqslant n}^{\mathrm{i}}\left(\tau_{\leqslant n}^{\mathrm{i}}(S) \star \tau_{\leqslant n}^{\mathrm{i}}(T)\right) \\
& \text { (en vertu du cas précédent) } \\
& \simeq \tau_{\leqslant n}^{\mathrm{i}}\left(\tau_{\leqslant n}^{\mathrm{i}}\left(\underset{S \rightarrow C}{\lim _{S \rightarrow C}} S\right) \star \tau_{\leqslant n}^{\mathrm{i}}\left(\underset{T \rightarrow D}{\lim _{T \rightarrow D}} T\right)\right) \\
& \text { (en vertu du théorème 6.29) } \\
& \simeq \tau_{\leqslant n}^{\mathrm{i}}\left(\tau_{\leqslant n}^{\mathrm{i}}(C) \star \tau_{\leqslant n}^{\mathrm{i}}(D)\right),
\end{aligned}
$$


ce qu'il fallait démontrer.

Proposition 8.9. - La structure de catégorie monö̈dale sur $\infty$-Cat définie par le joint induit une structure de catégorie monoïdale sur n-C $\mathcal{C}$ t pour le joint n-catégorique.

Démonstration. - Cela résulte formellement de la proposition précédente. En effet, si $A, B$ et $C$ sont trois $n$-catégories, on a

$$
\begin{aligned}
A \star_{n}\left(B \star_{n} C\right) & =\tau_{\leqslant n}^{\mathrm{i}}\left(A \star \tau_{\leqslant n}^{\mathrm{i}}(B \star C)\right) \\
& \simeq \tau_{\leqslant n}^{\mathrm{i}}\left(\tau_{\leqslant n}^{\mathrm{i}}(A) \star \tau_{\leqslant n}^{\mathrm{i}}(B \star C)\right) \\
& \simeq \tau_{\leqslant n}^{\mathrm{i}}(A \star(B \star C)),
\end{aligned}
$$

les deux isomorphismes résultant de la proposition précédente. De même, on a

$$
\left(A \star_{n} B\right) \star_{n} C \simeq \tau_{\leqslant n}^{\mathrm{i}}((A \star B) \star C),
$$

et la contrainte d'associativité du joint induit donc une contrainte d'associativité pour le joint $n$-catégorique. Par ailleurs, on a vérifié au paragraphe 8.6 qu'on a

$$
\varnothing \star_{n} C \simeq C \text { et } C \star_{n} \varnothing \simeq C,
$$

d'où le résultat.

Lemme 8.10. - Pour toute $\infty$-catégorie $A$ et pour tout $i \geqslant n$, le $\infty$-foncteur $\kappa_{i}^{n}: \mathrm{D}_{i} \rightarrow \mathrm{D}_{n}$ coreprésentant l'identité (voir le paragraphe 4.3) induit un isomorphisme

$$
\tau_{\leqslant n}^{\mathrm{i}}\left(A \star \mathrm{D}_{i}\right) \simeq \tau_{\leqslant n}^{\mathrm{i}}\left(A \star \mathrm{D}_{n}\right)
$$

Démonstration. - Le $n$-tronqué intelligent du $\infty$-foncteur $\kappa_{i}^{n}: \mathrm{D}_{i} \rightarrow \mathrm{D}_{n}$ étant un isomorphisme, l'assertion résulte de la proposition 8.8 .

Proposition 8.11. - Si $C$ est une n-catégorie et $u: A \rightarrow C$ est un $\infty$-foncteur, alors les $\infty$-catégories $u \backslash C$ et $C / u$ sont des $n$-catégories.

Démonstration. - Par dualité, il suffit de montrer l'assertion pour la $\infty$-catégorie $u \backslash C$. Par définition, les $i$-flèches de cette $\infty$-catégorie sont les $\infty$-foncteurs $A \star \mathrm{D}_{i} \rightarrow C$ au-dessous de $A$. Puisque $C$ est une $n$-catégorie, de tels $\infty$-foncteurs ne dépendent que du $n$-tronqué intelligent $\tau_{\leqslant n}^{\mathrm{i}}\left(A \star \mathrm{D}_{i}\right)$. Fixons $i \geqslant n$. En vertu du lemme précédent, le $\infty$-foncteur $\mathrm{D}_{i+1} \rightarrow \mathrm{D}_{i}$ coreprésentant l'identité induit un isomorphisme sur ces $n$-tronqués. L'application identité $(u \backslash C)_{i} \rightarrow(u \backslash C)_{i+1}$, induite par $\kappa_{i}$, est donc une bijection, d'où le résultat.

Proposition 8.12. - Soient $A$ et $B$ des n-catégories. On a des couples de foncteurs adjoints

$$
\begin{aligned}
n-\mathcal{C} a t & \rightarrow A \backslash n \text {-C } a t, & A \backslash n \text {-C } a t & \rightarrow n-\mathcal{C} a t \\
B & \mapsto\left(A \star_{n} B, \iota_{1}\right) & (C, A \stackrel{u}{\rightarrow} C) & \mapsto u \backslash C
\end{aligned}
$$


et

$$
\begin{aligned}
n-\mathcal{C} a t & \rightarrow B \backslash n \text {-C } a t, & B \backslash n \text {-C } a t & \rightarrow n \text {-C at. } \\
A & \mapsto\left(A \star_{n} B, \iota_{2}\right) & (C, B \stackrel{v}{\rightarrow} C) & \mapsto C / v
\end{aligned}
$$

Démonstration. - Notons tout d'abord que l'énoncé est bien défini puisque, en vertu de la proposition précédente, les $\infty$-catégories $u \backslash C$ et $C / v$ sont des $n$-catégories. Par ailleurs, on a

$$
\begin{aligned}
\operatorname{Hom}_{n-\mathcal{C} a t}(B, u \backslash C) \simeq & \operatorname{Hom}_{\infty-\mathcal{C} a t}(B, u \backslash C) \\
\simeq & \operatorname{Hom}_{A \backslash \infty-\mathcal{C} a t}\left(\left(A \star B, \iota_{1}\right),(C, u)\right) \\
& \quad(\text { par adjonction définissant les tranches }) \\
\simeq & \operatorname{Hom}_{\tau_{\leqslant n}^{\mathrm{i}}(A) \backslash \infty-\mathcal{C} a t}\left(\left(\tau_{\leqslant n}^{\mathrm{i}}(A \star B), \tau_{\leqslant n}^{\mathrm{i}}\left(\iota_{1}\right)\right),(C, u)\right) \\
& \quad(\operatorname{par} \operatorname{adjonction}) \\
\simeq & \operatorname{Hom}_{A \backslash \infty-\mathcal{C} a t}\left(\left(A \star_{n} B, \iota_{1}\right),(C, u)\right) \\
\simeq & \operatorname{Hom}_{A \backslash n-\mathcal{C} a t}\left(\left(A \star_{n} B, \iota_{1}\right),(C, u)\right),
\end{aligned}
$$

ce qui établit la première adjonction. La deuxième s'en déduit par dualité.

Corollaire 8.13. — La structure de catégorie monoïdale sur n-Cat définie par le joint n-catégorique est localement bifermée. En particulier, le joint n-catégorique commute aux limites inductives connexes en chaque variable.

Démonstration. — Cela découle immédiatement de la proposition précédente.

Remarque 8.14. - On peut déduire par dualité des résultats analogues pour le joint dual (voir la remarque 6.37). En particulier, le joint dual induit une structure de catégorie monoïdale localement bifermée sur $n$-C at.

Dans la suite du chapitre, nous allons comparer le joint $n$-catégorique dans le cas $n=1$ avec le joint catégorique classique tel qu'exposé, par exemple, dans la section 3.1 de $[\mathbf{2 5}]$.

8.15. - Soient $C$ et $D$ deux catégories. Le joint catégorique classique est la catégorie $C \star_{1}^{\mathrm{c}} D$ définie de la manière suivante :

- on pose $\mathrm{Ob}\left(C \star_{1}^{\mathrm{c}} D\right)=\mathrm{Ob}(C) \coprod \mathrm{Ob}(D)$;

- pour tous $x$ et $y$ dans $\mathrm{Ob}\left(C \star_{1}^{\mathrm{c}} D\right)$, on pose

$$
\operatorname{Hom}_{C \star_{1}^{\mathrm{c} D}}(x, y)= \begin{cases}\operatorname{Hom}_{C}(x, y) & \text { si } x \text { et } y \text { sont dans } \mathrm{Ob}(C), \\ \operatorname{Hom}_{D}(x, y) & \text { si } x \text { et } y \text { sont dans } \mathrm{Ob}(D), \\ \varnothing & \text { si } x \text { est dans } \mathrm{Ob}(C) \text { et } y \text { dans } \mathrm{Ob}(D), \\ \varnothing & \text { si } x \text { est dans } \mathrm{Ob}(D) \text { et } y \text { dans } \mathrm{Ob}(C) ;\end{cases}
$$

- la composition et les identités sont définies de la manière évidente. 
On obtient ainsi un foncteur $\star_{1}^{c}: \mathcal{C}$ at $\times \mathcal{C} a t \rightarrow \mathcal{C}$ at. On vérifie que ce foncteur définit une structure de catégorie monoïdale sur $\mathcal{C} a t$ d'unité la catégorie initiale $\varnothing$. Fixons maintenant $A$ et $B$ deux catégories. En utilisant le fait que $\varnothing$ est l'unité de la structure monoïdale, on obtient des foncteurs

$$
A \stackrel{\iota_{1}}{\longrightarrow} A \star_{1}^{\mathrm{c}} B \stackrel{\iota_{2}}{\longleftarrow} B,
$$

et donc des foncteurs

$$
\begin{aligned}
\mathcal{C} a t & \rightarrow A \backslash \mathcal{C} a t \\
B & \mapsto\left(A \star_{1}^{\mathrm{c}} B, \iota_{1}: A \rightarrow A \star_{1}^{\mathrm{c}} B\right)
\end{aligned}
$$

et

$$
\begin{aligned}
\mathcal{C} a t & \rightarrow B \backslash \mathcal{C} a t \\
A & \mapsto\left(A \star_{1}^{\mathrm{c}} B, \iota_{2}: B \rightarrow A \star_{1}^{\mathrm{c}} B\right) .
\end{aligned}
$$

Ces foncteurs admettent des adjoints à droite qu'on appellera les tranches catégoriques classiques au-dessous et au-dessus. En particulier, le foncteur joint catégorique classique commute aux limites inductives connexes en chaque variable. Dans le cas où $A$ et $B$ sont la catégorie finale, on retrouve les tranches usuelles $c \backslash C$ et $C / c$ au-dessous ou au-dessus d'un objet $c$ de $C$.

Lemme 8.16. - Soit $p \geqslant-1$. On a un isomorphisme canonique $\tau_{\leqslant 1}^{\mathrm{i}}\left(\mathcal{O}_{p}\right) \simeq \Delta_{p}$.

Démonstration. - En vertu du théorème 7.9 , on a $\mathcal{O}_{p} \simeq \nu c\left(\Delta_{p}\right)$ et donc, d'après la proposition $2.23, \tau_{\leqslant 1}^{\mathrm{i}}\left(\mathcal{O}_{p}\right) \simeq \tau_{\leqslant 1}^{\mathrm{i}} \nu c\left(\Delta_{p}\right) \simeq \nu \tau_{\leqslant 1}^{\mathrm{i}} c\left(\Delta_{p}\right)$. Par ailleurs, puisque $\Delta_{p}$ appartient à $\Theta_{+}$, on a $\Delta_{p} \simeq \nu \lambda\left(\Delta_{p}\right)$ en vertu de la proposition 4.13. Pour conclure, il suffit donc de définir un isomorphisme $\tau_{\leqslant 1}^{\mathrm{i}} c\left(\Delta_{p}\right) \rightarrow \lambda\left(\Delta_{p}\right)$, ce qui est immédiat.

Lemme 8.17. - Pour tous $p, q \geqslant-1$, on a un isomorphisme canonique

$$
\Delta_{p} \star_{1} \Delta_{q} \simeq \Delta_{p} \star_{1}^{c} \Delta_{q}
$$

naturel en $\Delta_{p}$ et $\Delta_{q}$ dans $\boldsymbol{\Delta}_{+}$.

Démonstration. - On vérifie immédiatement qu'on a $\Delta_{p} \star_{1}^{\mathrm{c}} \Delta_{q} \simeq \Delta_{p+1+q}$. Par ailleurs, on a

$$
\begin{aligned}
\Delta_{p} \star_{1} \Delta_{q}= & \tau_{\leqslant 1}^{\mathrm{i}}\left(\Delta_{p} \star \Delta_{q}\right) \\
\simeq & \tau_{\leqslant 1}^{\mathrm{i}}\left(\tau_{\leqslant 1}^{\mathrm{i}}\left(\mathcal{O}_{p}\right) \star \tau_{\leqslant 1}^{\mathrm{i}}\left(\mathcal{O}_{q}\right)\right) \\
& \quad(\text { en vertu du lemme précédent }) \\
\simeq & \tau_{\leqslant 1}^{\mathrm{i}}\left(\mathcal{O}_{p} \star \mathcal{O}_{q}\right) \\
& \quad(\text { en vertu de la proposition } 8.8) \\
\simeq & \tau_{\leqslant 1}^{\mathrm{i}}\left(\mathcal{O}_{p+1+q}\right) \\
\simeq & \Delta_{p+1+q},
\end{aligned}
$$

d'où le résultat. 
Proposition 8.18. — Le joint 1-catégorique et le joint catégorique classique sont canoniquement isomorphes. Autrement dit, on a un isomorphisme canonique

$$
C \star_{1} D \simeq C \star_{1}^{c} D
$$

naturel en $C$ et $D$ dans $\mathcal{C} a t$.

Démonstration. - Le lemme précédent donne un isomorphisme canonique

$$
C \star_{1} D \rightarrow C \star_{1}^{\mathrm{c}} D
$$

pour $C$ et $D$ dans $\boldsymbol{\Delta}_{+}$. Or toute catégorie est limite inductive connexe d'objets de $\boldsymbol{\Delta}_{+}$ et les deux foncteurs commutent aux limites inductives connexes en chaque variable, d'où le résultat.

Corollaire 8.19. - Si $C$ est une catégorie et $u: A \rightarrow C$ est un foncteur, alors les catégories $u \backslash C$ et $C / u$ sont les tranches catégoriques classiques.

Démonstration. - En vertu du cas $n=1$ de la proposition 8.12 et de la proposition précédente, les tranches $u \backslash C$ et $C / u$ vérifient les mêmes propriétés universelles que les tranches catégoriques classiques (voir le paragraphe 8.15), d'où le résultat.

Remarque 8.20. - Si $C$ est une 1-catégorie et $u: A \rightarrow C$ est un 1-foncteur, alors la catégorie $C / u$ coïncide avec la $\infty$-catégorie $C / u$ de la remarque 6.37 (qui est donc une catégorie). Cela résulte immédiatement du fait que $D^{\text {co }}=D$ si $D$ est une 1-catégorie. 


\section{CHAPITRE 9}

\section{DESCRIPTION EXPLICITE DES TRANCHES AU-DESSOUS D'UN OBJET}

Soient $C$ une $\infty$-catégorie et $c$ un objet de $C$. En considérant $c$ comme un $\infty$-foncteur $\mathrm{D}_{0} \rightarrow C$, le paragraphe 6.31 permet de définir une $\infty$-catégorie $c \backslash C$. Le but de ce chapitre est de décrire explicitement cette $\infty$-catégorie.

Dans ce chapitre, on utilisera librement les conventions sur les formules de composition de cellules dans une $\infty$-catégorie qu'on a fixées au paragraphe 1.1, ainsi que les notations relatives aux atomes des complexes dirigés augmentés à base unitaire introduites au paragraphe 2.8 .

9.1. - Fixons $i \geqslant 0$. Nous allons commencer par décrire la $(i+1)$-catégorie $\mathrm{D}_{0} \star \mathrm{D}_{i}$. On notera $a$ l'unique objet de $\mathrm{D}_{0}$ et $x$ la cellule principale de $\mathrm{D}_{i}$. En vertu de la proposition 4.13 et du paragraphe 6.30 , on a

$$
\mathrm{D}_{0} \star \mathrm{D}_{i} \simeq \nu\left(\lambda\left(\mathrm{D}_{0}\right) \star \lambda\left(\mathrm{D}_{i}\right)\right) .
$$

Par ailleurs, les paragraphes 4.10 et 6.13 montrent que le complexe $\lambda\left(D_{0}\right) \star \lambda\left(D_{i}\right)$ a pour base l'ensemble formé des

$$
a \star \varnothing, \quad \varnothing \star x_{k}^{\varepsilon}, \quad a \star x_{k}^{\varepsilon},
$$

où $k$ varie entre 0 et $i$, et $\varepsilon=0,1$ (en se souvenant que $x_{i}^{0}=x_{i}^{1}$ ).

Proposition 9.2. - Pour tout $k$ tel que $0<k \leqslant i$ et $\varepsilon=0,1$, on a

$$
s\left(\left\langle\varnothing \star x_{k}^{\varepsilon}\right\rangle\right)=\left\langle\varnothing \star x_{k-1}^{0}\right\rangle \quad \text { et } \quad t\left(\left\langle\varnothing \star x_{k}^{\varepsilon}\right\rangle\right)=\left\langle\varnothing \star x_{k-1}^{1}\right\rangle .
$$

Démonstration. — Cela résulte immédiatement de l'égalité

$$
d\left(\varnothing \star x_{k}^{\varepsilon}\right)=\varnothing \star d\left(x_{k}^{\varepsilon}\right)=\varnothing \star x_{k-1}^{1}-\varnothing \star x_{k-1}^{0} .
$$

Lemme 9.3. - Pour tout $k$ tel que $0 \leqslant k \leqslant i$, tout $l$ tel que $0 \leqslant l<k+1$ et $\varepsilon=0,1$, on $a$

$$
\left\langle a \star x_{k}^{\varepsilon}\right\rangle_{l}^{0}=a \star x_{l-1}^{1}
$$


et

$$
\left\langle a \star x_{k}^{\varepsilon}\right\rangle_{l}^{1}=\varnothing \star x_{l}^{\eta}+a \star x_{l-1}^{0},
$$

où $\eta$ vaut $\varepsilon$ si $k=l$ et 1 sinon. En particulier, on a

$$
\left\langle a \star x_{k}^{\varepsilon}\right\rangle_{0}^{0}=a \star \varnothing \quad \text { et }\left\langle a \star x_{k}^{\varepsilon}\right\rangle_{0}^{1}=\varnothing \star x_{0}^{\eta} .
$$

Démonstration. - En vertu du lemme 6.14 (et avec ses conventions), on a

$$
\left\langle a \star x_{k}^{\varepsilon}\right\rangle_{l}^{0}=\langle a\rangle_{-1}^{0} \star\left\langle x_{k}^{\varepsilon}\right\rangle_{l}^{0}+\langle a\rangle_{0}^{0} \star\left\langle x_{k}^{\varepsilon}\right\rangle_{l-1}^{1}=a \star x_{l-1}^{1}
$$

et

$$
\left\langle a \star x_{k}^{\varepsilon}\right\rangle_{l}^{1}=\langle a\rangle_{-1}^{1} \star\left\langle x_{k}^{\varepsilon}\right\rangle_{l}^{1}+\langle a\rangle_{0}^{1} \star\left\langle x_{k}^{\varepsilon}\right\rangle_{l-1}^{0}=\varnothing \star x_{l}^{\eta}+a \star x_{l-1}^{0},
$$

ce qu'on voulait démontrer.

Proposition 9.4. - Pour tout $k$ tel que $0 \leqslant k \leqslant i$ et $\varepsilon=0,1$, on a

$$
s\left(\left\langle a \star x_{k}^{\varepsilon}\right\rangle\right)=\left\langle a \star x_{k-1}^{1}\right\rangle
$$

et

$$
t\left(\left\langle a \star x_{k}^{\varepsilon}\right\rangle\right)=\left\langle\varnothing \star x_{k}^{\varepsilon}\right\rangle *_{0}\left\langle a \star x_{0}^{0}\right\rangle *_{1} \cdots *_{k-1}\left\langle a \star x_{k-1}^{0}\right\rangle .
$$

Démonstration. - C'est le cas $l=k$ du lemme plus général suivant.

Lemme 9.5. - Pour tous $k, l$ tels que $0 \leqslant l \leqslant k \leqslant i$ et $\varepsilon=0,1$, on a

$$
s_{l}\left(\left\langle a \star x_{k}^{\varepsilon}\right\rangle\right)=\left\langle a \star x_{l-1}^{1}\right\rangle
$$

et

$$
t_{l}\left(\left\langle a \star x_{k}^{\varepsilon}\right\rangle\right)=\left\langle\varnothing \star x_{l}^{\eta}\right\rangle *_{0}\left\langle a \star x_{0}^{0}\right\rangle *_{1} \cdots *_{l-1}\left\langle a \star x_{l-1}^{0}\right\rangle,
$$

où $\eta$ vaut $\varepsilon$ si $k=l$ et 1 sinon.

Démonstration. - En vertu du lemme 9.3, on a $\left\langle a \star x_{k}^{\varepsilon}\right\rangle_{l}^{0}=a \star x_{l-1}^{1}$ et donc $s_{l}\left(\left\langle a \star x_{k}^{\varepsilon}\right\rangle\right)=\left\langle a \star x_{l-1}^{1}\right\rangle$ par définition des atomes et de leurs sources.

Démontrons la deuxième égalité par récurrence sur $l$. Pour $l=0$, en vertu du lemme 9.3, on a $\left\langle a \star x_{k}^{\varepsilon}\right\rangle_{0}^{1}=\varnothing \star x_{0}^{\eta}$ et donc $t_{0}\left(\left\langle a \star x_{k}^{\varepsilon}\right\rangle\right)=\left\langle\varnothing \star x_{0}^{\eta}\right\rangle$. Supposons maintenant l'égalité démontrée au rang $l-1$ et montrons-la au rang $l \leqslant k$. Par la proposition 9.2 et l'hypothèse de récurrence, on a

$$
\begin{aligned}
& s_{l-1}\left(\left\langle\varnothing \star x_{l}^{\eta}\right\rangle *_{0}\left\langle a \star x_{0}^{0}\right\rangle *_{1} \cdots *_{l-2}\left\langle a \star x_{l-2}^{0}\right\rangle\right) \\
& \quad=\left\langle\varnothing \star x_{l-1}^{0}\right\rangle *_{0}\left\langle a \star x_{0}^{0}\right\rangle *_{1} \cdots *_{l-2}\left\langle a \star x_{l-2}^{0}\right\rangle \\
& \quad=t_{l-1}\left(\left\langle a \star x_{l-1}^{0}\right\rangle\right)
\end{aligned}
$$

et la cellule

$$
u=\left(\left\langle\varnothing \star x_{l}^{\eta}\right\rangle *_{0}\left\langle a \star x_{0}^{0}\right\rangle *_{1} \cdots *_{l-2}\left\langle a \star x_{l-2}^{0}\right\rangle\right) *_{l-1}\left\langle a \star x_{l-1}^{0}\right\rangle
$$

est donc bien définie. Par ailleurs, en utilisant le lemme 9.3, on obtient

$$
u_{l}=\varnothing \star x_{l}^{\eta}+a \star x_{l-1}^{0}=\left\langle a \star x_{k}^{\varepsilon}\right\rangle_{l}^{1}=t_{l}\left(\left\langle a \star x_{k}^{\varepsilon}\right\rangle\right)_{l} .
$$


Pour conclure, il suffit donc de montrer qu'on a

$$
s\left(t_{l}\left(\left\langle a \star x_{k}^{\varepsilon}\right\rangle\right)\right)=s(u) \quad \text { et } \quad t\left(t_{l}\left(\left\langle a \star x_{k}^{\varepsilon}\right\rangle\right)\right)=t(u) .
$$

Or, en utilisant la première égalité de l'énoncé, on a

$$
s\left(t_{l}\left(\left\langle a \star x_{k}^{\varepsilon}\right\rangle\right)\right)=s_{l-1}\left(\left\langle a \star x_{k}^{\varepsilon}\right\rangle\right)=\left\langle a \star x_{l-2}^{1}\right\rangle=s_{l-1}\left(\left\langle a \star x_{l-1}^{0}\right\rangle\right)=s(u)
$$

et, en utilisant l'hypothèse de récurrence, on a

$$
\begin{aligned}
t\left(t_{l}\left(\left\langle a \star x_{k}^{\varepsilon}\right\rangle\right)\right) & =t_{l-1}\left(\left\langle a \star x_{k}^{\varepsilon}\right\rangle\right) \\
& =\left\langle\varnothing \star x_{l-1}^{1}\right\rangle *_{0}\left\langle a \star x_{0}^{0}\right\rangle *_{1} \cdots *_{l-2}\left\langle a \star x_{l-2}^{0}\right\rangle \\
& =t_{l-1}\left(\left\langle\varnothing \star x_{l}^{\eta}\right\rangle *_{0}\left\langle a \star x_{0}^{0}\right\rangle *_{1} \cdots *_{l-2}\left\langle a \star x_{l-2}^{0}\right\rangle \star_{l-1}\left\langle a \star x_{l-1}^{0}\right\rangle\right) \\
& =t(u),
\end{aligned}
$$

d'où le résultat.

Proposition 9.6. - Soit $C$ une $\infty$-catégorie. Fixons

- $c$ un objet de $C$;

- $d$ une $i$-flèche de $C$;

- pour tout $k$ tel que $0 \leqslant k \leqslant i$ et $\varepsilon=0,1, \alpha_{k}^{\varepsilon}$ une $(k+1)$-flèche de $C$, avec $\alpha_{i}^{0}=\alpha_{i}^{1}$, vérifiant les égalités

$$
s\left(\alpha_{k}^{\varepsilon}\right)=\left\{\begin{array}{ll}
c & \text { si } k=0, \\
\alpha_{k-1}^{1} & \text { si } k>0,
\end{array} \quad \text { et } t\left(\alpha_{k}^{\varepsilon}\right)=d_{k}^{\varepsilon} *_{0} \alpha_{0}^{0} *_{1} \cdots *_{k-1} \alpha_{k-1}^{0},\right.
$$

où on a posé

$$
d_{k}^{\varepsilon}= \begin{cases}s_{k}(d) & \text { si } \varepsilon=0, \\ t_{k}(d) & \text { si } \varepsilon=1 .\end{cases}
$$

Alors il existe un et un seul $\infty$-foncteur $h: \mathrm{D}_{0} \star \mathrm{D}_{i} \rightarrow C$ tel que

$$
c=h(\langle a \star \varnothing\rangle), \quad d=h\left(\left\langle\varnothing \star x_{i}\right\rangle\right) \quad \text { et } \quad \alpha_{k}^{\varepsilon}=h\left(\left\langle a \star x_{k}^{\varepsilon}\right\rangle\right),
$$

pour tout $k$ tel que $0 \leqslant k \leqslant i$ et $\varepsilon=0,1$.

Démonstration. - En vertu du théorème 2.12, la $\infty$-catégorie $\nu\left(\lambda\left(\mathrm{D}_{0}\right) \star \lambda\left(\mathrm{D}_{i}\right)\right)$, isomorphe à la $\infty$-catégorie $\mathrm{D}_{0} \star \mathrm{D}_{i}$, est engendrée librement par ses atomes au sens des polygraphes. Cela signifie que la donnée d'un $\infty$-foncteur $h: \mathrm{D}_{0} \star \mathrm{D}_{i} \rightarrow C$ est équivalente à donnée de

$$
c=h(\langle a \star \varnothing\rangle), \quad d_{k}^{\varepsilon}=h\left(\left\langle\varnothing \star x_{k}^{\varepsilon}\right\rangle\right) \quad \text { et } \quad \alpha_{k}^{\varepsilon}=h\left(\left\langle a \star x_{k}^{\varepsilon}\right\rangle\right),
$$

pour $0 \leqslant k \leqslant i$ et $\varepsilon=0,1$, avec $d_{i}^{0}=d_{i}^{1}$ et $\alpha_{i}^{0}=\alpha_{i}^{1}$, compatibles aux sources et aux buts. En vertu de la proposition 9.4, cette compatibilité pour les atomes de la forme $\left\langle a \star x_{k}^{\varepsilon}\right\rangle$ s'exprime par les égalités de l'énoncé. Pour les atomes de la forme 
$\varnothing \star x_{k}^{\varepsilon}$ avec $0<k \leqslant i$, en vertu de la proposition 9.2 , ces compatibilités sont données par les égalités

$$
s\left(d_{k}^{\varepsilon}\right)=d_{k-1}^{0} \quad \text { et } \quad t\left(d_{k}^{\varepsilon}\right)=d_{k-1}^{1}
$$

Or, la donnée de $d_{k}^{\varepsilon}$ satisfaisant à ces égalités est équivalente à celle de $d=d_{i}^{0}=d_{i}^{1}$, d'où le résultat.

9.7. - Soient $C$ une $\infty$-catégorie et $c$ un objet de $C$. Par définition (voir le paragraphe 6.33), les $i$-flèches de $c \backslash C$ correspondent aux $\infty$-foncteurs $h: \mathrm{D}_{0} \star \mathrm{D}_{i} \rightarrow C$ rendant le triangle

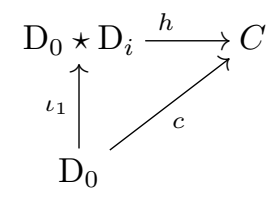

commutatif. En vertu de la proposition précédente, un tel $\infty$-foncteur est déterminé par un couple $(d, \alpha)$, où $d$ est une $i$-flèche de $C$ et $\alpha$ est une famille de cellules $\alpha_{k}^{\varepsilon}$ de $C$, pour $0 \leqslant k \leqslant i$ et $\varepsilon=0,1$, avec $\alpha_{i}^{0}=\alpha_{i}^{1}$,

$$
\begin{aligned}
& \alpha_{0}^{\varepsilon}: c \rightarrow d_{0}^{\varepsilon}, \quad 1 \text {-flèche, } \\
& \alpha_{k}^{\varepsilon}: \alpha_{k-1}^{1} \rightarrow d_{k}^{\varepsilon} *_{0} \alpha_{0}^{0} *_{1} \cdots *_{k-1} \alpha_{k-1}^{0}, \quad(k+1) \text {-flèche, } \text { pour } 0<k \leqslant i,
\end{aligned}
$$

où on a posé

$$
d_{k}^{\varepsilon}= \begin{cases}s_{k}(d) & \text { si } \varepsilon=0 \\ t_{k}(d) & \text { si } \varepsilon=1\end{cases}
$$

Dans la suite de ce chapitre, on identifiera les $i$-flèches de $c \backslash C$ avec de tels couples $(d, \alpha)$. On notera $\alpha_{i}$ pour $\alpha_{i}^{0}=\alpha_{i}^{1}$.

Voici une représentation graphique des objets, des 1-flèches et des 2-flèches de $c \backslash C$ :
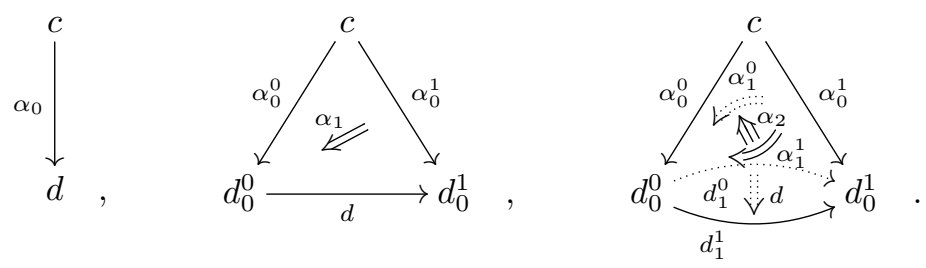

Le but de la suite de ce chapitre est de décrire la structure de $\infty$-catégorie de $c \backslash C$ en termes des $(d, \alpha)$. 
9.8. - En vertu de l'adjonction définissant la $\infty$-catégorie $c \backslash C$, les sources, buts, identités et compositions de cette $\infty$-catégorie sont induits par les $\infty$-foncteurs

$$
\begin{array}{cl}
\mathrm{D}_{0} \star \sigma_{i}: \mathrm{D}_{0} \star \mathrm{D}_{i-1} \rightarrow \mathrm{D}_{0} \star \mathrm{D}_{i} & \text { pour } i \geqslant 1, \\
\mathrm{D}_{0} \star \tau_{i}: \mathrm{D}_{0} \star \mathrm{D}_{i-1} \rightarrow \mathrm{D}_{0} \star \mathrm{D}_{i} & \text { pour } i \geqslant 1, \\
\mathrm{D}_{0} \star \kappa_{i}: \mathrm{D}_{0} \star \mathrm{D}_{i+1} \rightarrow \mathrm{D}_{0} \star \mathrm{D}_{i} & \text { pour } i \geqslant 0, \\
\mathrm{D}_{0} \star \nabla_{j}^{i}: \mathrm{D}_{0} \star \mathrm{D}_{i} \rightarrow\left(\mathrm{D}_{0} \star \mathrm{D}_{i}\right) \amalg_{\mathrm{D}_{0} \star \mathrm{D}_{j}}\left(\mathrm{D}_{0} \star \mathrm{D}_{i}\right) & \text { pour } i>j \geqslant 0,
\end{array}
$$

où $\sigma_{i}, \tau_{i}, \kappa_{i}$ et $\nabla_{j}^{i}$ désignent les $\infty$-foncteurs des paragraphes 4.1 et 4.3 , et où on a identifié $\left(\mathrm{D}_{0} \star \mathrm{D}_{i}\right) \amalg_{\mathrm{D}_{0} \star \mathrm{D}_{j}}\left(\mathrm{D}_{0} \star \mathrm{D}_{i}\right)$ et $\mathrm{D}_{0} \star\left(\mathrm{D}_{i} \amalg_{\mathrm{D}_{j}} \mathrm{D}_{i}\right)$.

Nous allons commencer par décrire concrètement les morphismes $\mathrm{D}_{0} \star \sigma_{i}, \mathrm{D}_{0} \star \tau_{i}$ et $\mathrm{D}_{0} \star \kappa_{i}$. On note toujours a l'unique objet de $\mathrm{D}_{0}$.

Proposition 9.9. - Fixons $i \geqslant 1$ et notons $x$ la cellule principale de $\mathrm{D}_{i-1}$ et y celle de $\mathrm{D}_{i}$. Alors le $\infty$-foncteur $\mathrm{D}_{0} \star \sigma_{i}: \mathrm{D}_{0} \star \mathrm{D}_{i-1} \rightarrow \mathrm{D}_{0} \star \mathrm{D}_{i}$ est donné par

$$
\begin{aligned}
\langle a \star \varnothing\rangle & \mapsto\langle a \star \varnothing\rangle, \\
\left\langle\varnothing \star x_{k}^{\varepsilon}\right\rangle & \mapsto\left\langle\varnothing \star y_{k}^{\varepsilon}\right\rangle \quad \text { pour } 0 \leqslant k<i-1 \text { et } \varepsilon=0,1, \\
\left\langle\varnothing \star x_{i-1}\right\rangle & \mapsto\left\langle\varnothing \star y_{i-1}^{0}\right\rangle, \\
\left\langle a \star x_{k}^{\varepsilon}\right\rangle & \mapsto\left\langle a \star y_{k}^{\varepsilon}\right\rangle \\
\left\langle a \star x_{i-1}\right\rangle & \mapsto\left\langle a \star y_{i-1}^{0}\right\rangle .
\end{aligned}
$$

De même, le $\infty$-foncteur $\mathrm{D}_{0} \star \tau_{i}: \mathrm{D}_{0} \star \mathrm{D}_{i-1} \rightarrow \mathrm{D}_{0} \star \mathrm{D}_{i}$ est donné par

$$
\begin{aligned}
\langle a \star \varnothing\rangle & \mapsto\langle a \star \varnothing\rangle, \\
\left\langle\varnothing \star x_{k}^{\varepsilon}\right\rangle & \mapsto\left\langle\varnothing \star y_{k}^{\varepsilon}\right\rangle \quad \text { pour } 0 \leqslant k<i-1 \text { et } \varepsilon=0,1, \\
\left\langle\varnothing \star x_{i-1}\right\rangle & \mapsto\left\langle\varnothing \star y_{i-1}^{1}\right\rangle, \\
\left\langle a \star x_{k}^{\varepsilon}\right\rangle & \mapsto\left\langle a \star y_{k}^{\varepsilon}\right\rangle \\
\left\langle a \star x_{i-1}\right\rangle & \mapsto\left\langle a \star y_{i-1}^{1}\right\rangle .
\end{aligned}
$$

Démonstration. - Cela résulte immédiatement de la proposition 6.18 et des formules

$$
\sigma_{i}\left(\left\langle x_{k}^{\varepsilon}\right\rangle\right)=\left\{\begin{array}{ll}
\left\langle y_{k}^{\varepsilon}\right\rangle & \text { si } 0 \leqslant k<i-1, \\
\left\langle y_{i-1}^{0}\right\rangle & \text { si } k=i-1,
\end{array} \quad \text { et } \quad \tau_{i}\left(\left\langle x_{k}^{\varepsilon}\right\rangle\right)= \begin{cases}\left\langle y_{k}^{\varepsilon}\right\rangle & \text { si } 0 \leqslant k<i-1, \\
\left\langle y_{i-1}^{1}\right\rangle & \text { si } k=i-1,\end{cases}\right.
$$

pour $\varepsilon=0,1$. 
Proposition 9.10. - Fixons $i \geqslant 0$ et notons $x$ la cellule principale de $\mathrm{D}_{i+1}$ et $y$ celle de $\mathrm{D}_{i}$. Alors le $\infty$-foncteur $\mathrm{D}_{0} \star \kappa_{i}: \mathrm{D}_{0} \star \mathrm{D}_{i+1} \rightarrow \mathrm{D}_{0} \star \mathrm{D}_{i}$ est donné par

$$
\begin{array}{rlrl}
\langle a \star \varnothing\rangle & \mapsto\langle a \star \varnothing\rangle, & & \\
\left\langle\varnothing \star x_{k}^{\varepsilon}\right\rangle & \mapsto\left\langle\varnothing \star y_{k}^{\varepsilon}\right\rangle & & \text { pour } 0 \leqslant k<i \text { et } \varepsilon=0,1, \\
\left\langle\varnothing \star x_{i}^{\varepsilon}\right\rangle & \mapsto\left\langle\varnothing \star y_{i}\right\rangle & \text { pour } \varepsilon=0,1, \\
\left\langle\varnothing \star x_{i+1}\right\rangle & \mapsto 1_{\left\langle\varnothing \star y_{i}\right\rangle}, & & \\
\left\langle a \star x_{k}^{\varepsilon}\right\rangle & \mapsto\left\langle a \star y_{k}^{\varepsilon}\right\rangle & & \text { pour } 0 \leqslant k<i \text { et } \varepsilon=0,1, \\
\left\langle a \star x_{i}^{\varepsilon}\right\rangle & \mapsto\left\langle a \star y_{i}\right\rangle & & \text { pour } \varepsilon=0,1, \\
\left\langle a \star x_{i+1}\right\rangle & \mapsto 1_{\left\langle a \star y_{i}\right\rangle} . & &
\end{array}
$$

Démonstration. - Cela résulte immédiatement de la proposition 6.18 et de la formule

$$
\kappa_{i}\left(\left\langle x_{k}^{\varepsilon}\right\rangle\right)= \begin{cases}\left\langle y_{k}^{\varepsilon}\right\rangle & \text { si } 0 \leqslant k<i \\ \left\langle y_{i}\right\rangle & \text { si } k=i \\ 1_{\left\langle y_{i}\right\rangle} & \text { si } k=i+1\end{cases}
$$

pour $\varepsilon=0,1$.

9.11. - Fixons maintenant $j$ tel que $0 \leqslant j<i$. Nous allons décrire explicitement le $\infty$-foncteur

$$
\mathrm{D}_{0} \star \nabla_{j}^{i}: \mathrm{D}_{0} \star \mathrm{D}_{i} \rightarrow \mathrm{D}_{0} \star \mathrm{D}_{i} \amalg_{\mathrm{D}_{0} \star \mathrm{D}_{j}} \mathrm{D}_{0} \star \mathrm{D}_{i} .
$$

Nous noterons $x$ la cellule principale de l'objet $\mathrm{D}_{i}$ apparaissant dans la source de $\mathrm{D}_{0} \star \nabla_{j}^{i}$, et $y$ et $z$ les cellules principales des objets $\mathrm{D}_{i}$ apparaissant de gauche à droite dans le but de $\mathrm{D}_{0} \star \nabla_{j}^{i}$.

En vertu du paragraphe 4.11 et avec ses notations, une base du complexe dirigé augmenté

$$
\lambda\left(\mathrm{D}_{0} \star \mathrm{D}_{i} \amalg_{\mathrm{D}_{0} \star \mathrm{D}_{j}} \mathrm{D}_{0} \star \mathrm{D}_{i}\right) \simeq \lambda\left(\mathrm{D}_{0}\right) \star \lambda\left(\mathrm{D}_{i} \amalg_{\mathrm{D}_{j}} \mathrm{D}_{i}\right)
$$

est donnée par les

$$
\langle a \star \varnothing\rangle, \quad\left\langle\varnothing \star y_{i}^{\varepsilon}\right\rangle, \quad\left\langle\varnothing \star z_{i}^{\varepsilon}\right\rangle, \quad\left\langle a \star y_{i}^{\varepsilon}\right\rangle, \quad\left\langle a \star z_{i}^{\varepsilon}\right\rangle,
$$

pour $0 \leqslant k \leqslant i$ et $\varepsilon=0,1$, modulo les identifications

$$
y_{j}^{0}=z_{j}^{1}, \quad y_{k}^{\varepsilon}=z_{k}^{\varepsilon} \quad \text { pour } 0 \leqslant k<j \text { et } \varepsilon=0,1,
$$

ainsi que les identifications triviales $y_{i}^{0}=y_{i}^{1}$ et $z_{i}^{0}=z_{i}^{1}$.

Lemme 9.12. - Pour tout $k$ tel que $j<k \leqslant i$ et $\varepsilon=0,1$, la $(k+1)$-flèche

$$
\left\langle\varnothing \star y_{j+1}^{\eta}\right\rangle *_{0}\left\langle a \star z_{0}^{0}\right\rangle *_{1} \cdots *_{j-1}\left\langle a \star z_{j-1}^{0}\right\rangle *_{j}\left\langle a \star z_{k}^{\varepsilon}\right\rangle *_{j+1}\left\langle a \star y_{k}^{\varepsilon}\right\rangle
$$

de $\mathrm{D}_{0} \star \mathrm{D}_{i} \amalg_{\mathrm{D}_{0} \star \mathrm{D}_{j}} \mathrm{D}_{0} \star \mathrm{D}_{i}$, où $\eta$ vaut $\varepsilon$ si $k=j+1$ et 1 sinon, est bien définie. 
Démonstration. — En vertu de la proposition 9.4, la $j$-flèche

$$
\left\langle\varnothing \star z_{j}^{1}\right\rangle *_{0}\left\langle a \star z_{0}^{0}\right\rangle *_{1} \cdots *_{j-1}\left\langle a \star z_{j-1}^{0}\right\rangle
$$

est bien définie. Puisque, pour $l \leqslant j-1$, on a

$$
s_{l}\left(\left\langle\varnothing \star y_{j+1}^{\eta}\right\rangle\right)=\left\langle\varnothing \star y_{l}^{0}\right\rangle=\left\langle\varnothing \star z_{l}^{0}\right\rangle=s_{l}\left(\left\langle\varnothing \star z_{j}^{1}\right\rangle\right),
$$

on en déduit que la cellule

$$
\left\langle\varnothing \star y_{j+1}^{\eta}\right\rangle *_{0}\left\langle a \star z_{0}^{0}\right\rangle *_{1} \cdots *_{j-1}\left\langle a \star z_{j-1}^{0}\right\rangle
$$

est également bien définie. De plus, on a

$$
\begin{aligned}
s_{j}(\langle\varnothing & \left.\left.\star y_{j+1}^{\eta}\right\rangle *_{0}\left\langle a \star z_{0}^{0}\right\rangle *_{1} \cdots *_{j-1}\left\langle a \star z_{j-1}^{0}\right\rangle\right) \\
& =\left\langle\varnothing \star y_{j}^{0}\right\rangle *_{0}\left\langle a \star z_{0}^{0}\right\rangle *_{1} \cdots *_{j-1}\left\langle a \star z_{j-1}^{0}\right\rangle \\
& =\left\langle\varnothing \star z_{j}^{1}\right\rangle *_{0}\left\langle a \star z_{0}^{0}\right\rangle *_{1} \cdots *_{j-1}\left\langle a \star z_{j-1}^{0}\right\rangle \\
& =t_{j}\left(\left\langle a \star z_{k}^{\varepsilon}\right\rangle\right),
\end{aligned}
$$

la dernière égalité résultant du lemme 9.5, ce qui montre que la cellule

$$
\left\langle\varnothing \star y_{j+1}^{\eta}\right\rangle *_{0}\left\langle a \star z_{0}^{0}\right\rangle *_{1} \cdots *_{j-1}\left\langle a \star z_{j-1}^{0}\right\rangle *_{j}\left\langle a \star z_{k}^{\varepsilon}\right\rangle
$$

est bien définie. Enfin, on a

$$
\begin{aligned}
s_{j+1}( & \left.\left\langle\varnothing \star y_{j+1}^{\eta}\right\rangle *_{0}\left\langle a \star z_{0}^{0}\right\rangle *_{1} \cdots *_{j-1}\left\langle a \star z_{j-1}^{0}\right\rangle *_{j}\left\langle a \star z_{k}^{\varepsilon}\right\rangle\right) \\
= & \left\langle\varnothing \star y_{j+1}^{\eta}\right\rangle *_{0}\left\langle a \star z_{0}^{0}\right\rangle *_{1} \cdots *_{j-1}\left\langle a \star z_{j-1}^{0}\right\rangle *_{j} s_{j+1}\left(\left\langle a \star z_{k}^{\varepsilon}\right\rangle\right) \\
= & \left\langle\varnothing \star y_{j+1}^{\eta}\right\rangle *_{0}\left\langle a \star z_{0}^{0}\right\rangle *_{1} \cdots *_{j-1}\left\langle a \star z_{j-1}^{0}\right\rangle *_{j}\left\langle a \star z_{j}^{1}\right\rangle \\
& \quad(\text { en vertu du lemme 9.5) } \\
= & \left\langle\varnothing \star y_{j+1}^{\eta}\right\rangle *_{0}\left\langle a \star y_{0}^{0}\right\rangle *_{1} \cdots *_{j-1}\left\langle a \star y_{j-1}^{0}\right\rangle *_{j}\left\langle a \star y_{j}^{0}\right\rangle \\
= & t_{j+1}\left(\left\langle a \star y_{k}^{\varepsilon}\right\rangle\right),
\end{aligned}
$$

la dernière égalité résultant de nouveau du lemme 9.5, ce qui achève de montrer que la cellule de l'énoncé est bien définie.

Lemme 9.13. - Pour tout $k$ tel que $j<k \leqslant i, \varepsilon=0,1$ et pour tout $l$ tel que $0 \leqslant l \leqslant k+1$, on $a$

$$
\left(\mathrm{D}_{0} \star \nabla_{j}^{i}\right)\left(\left\langle a \star x_{k}^{\varepsilon}\right\rangle\right)_{l}^{0}= \begin{cases}a \star y_{l-1}^{1} & \text { si } 0 \leqslant l \leqslant j+1, \\ a \star y_{l-1}^{1}+a \star z_{l-1}^{1} & \text { si } j+1<l<k+1, \\ a \star y_{l-1}^{\varepsilon}+a \star z_{l-1}^{\varepsilon} & \text { si } l=k+1,\end{cases}
$$


106 CHAPITRE 9. DESCRIPTION EXPLICITE DES TRANCHES AU-DESSOUS D'UN OBJET

et

$$
\left(\mathrm{D}_{0} \star \nabla_{j}^{i}\right)\left(\left\langle a \star x_{k}^{\varepsilon}\right\rangle\right)_{l}^{1}= \begin{cases}\varnothing \star y_{l}^{1}+a \star z_{l-1}^{0} & \text { si } 0 \leqslant l \leqslant j, \\ \varnothing \star y_{l}^{\eta}+\varnothing \star z_{l}^{\eta}+a \star z_{l-1}^{0} & \text { si } l=j+1, \\ \varnothing \star y_{l}^{\eta}+\varnothing \star z_{l}^{\eta}+a \star y_{l-1}^{0}+a \star z_{l-1}^{0} & \text { si } j+1<l<k+1, \\ a \star y_{l-1}^{\varepsilon}+a \star z_{l-1}^{\varepsilon} & \text { si } l=k+1,\end{cases}
$$

où $\eta$ vaut $\varepsilon$ si $l=k$ et 1 sinon.

Démonstration. - Montrons la première égalité. On a, en utilisant le lemme 9.3,

$$
\begin{aligned}
\left(\mathrm{D}_{0} \star \nabla_{j}^{i}\right)\left(\left\langle a \star x_{k}^{\varepsilon}\right\rangle\right)_{l}^{0} & =\lambda\left(\mathrm{D}_{0} \star \nabla_{j}^{i}\right)\left(\left\langle a \star x_{k}^{\varepsilon}\right\rangle_{l}^{0}\right) \\
& =\lambda\left(\mathrm{D}_{0} \star \nabla_{j}^{i}\right)\left(a \star x_{l-1}^{\delta}\right) \\
& =a \star \lambda\left(\nabla_{j}^{i}\right)\left(x_{l-1}^{\delta}\right),
\end{aligned}
$$

où $\delta$ vaut $\varepsilon$ si $l=k+1$ et 1 sinon, et on obtient l'égalité par la description de $\lambda\left(\nabla_{j}^{i}\right)$ donnée au paragraphe 4.11 .

Montrons la seconde. Le cas $l=k+1$ s'obtient comme ci-dessus. Pour $l<k+1$, en utilisant de nouveau le lemme 9.3 , on a

$$
\begin{aligned}
\left(\mathrm{D}_{0} \star \nabla_{j}^{i}\right)\left(\left\langle a \star x_{k}^{\varepsilon}\right\rangle\right)_{l}^{1} & =\lambda\left(\mathrm{D}_{0} \star \nabla_{j}^{i}\right)\left(\left\langle a \star x_{k}^{\varepsilon}\right\rangle_{l}^{1}\right) \\
& =\lambda\left(\mathrm{D}_{0} \star \nabla_{j}^{i}\right)\left(\varnothing \star x_{l}^{\eta}+a \star x_{l-1}^{0}\right) \\
& =\varnothing \star \lambda\left(\nabla_{j}^{i}\right)\left(x_{l}^{\eta}\right)+a \star \lambda\left(\nabla_{j}^{i}\right)\left(x_{l-1}^{0}\right)
\end{aligned}
$$

et on obtient le résultat en utilisant de nouveau la description de $\lambda\left(\nabla_{j}^{i}\right)$ donnée au paragraphe 4.11 .

Proposition 9.14. - Le $\infty$-foncteur $\mathrm{D}_{0} \star \nabla_{j}^{i}: \mathrm{D}_{0} \star \mathrm{D}_{i} \rightarrow \mathrm{D}_{0} \star \mathrm{D}_{i} \amalg_{\mathrm{D}_{0} \star \mathrm{D}_{j}} \mathrm{D}_{0} \star \mathrm{D}_{i}$ est donné par

$$
\begin{aligned}
\langle a \star \varnothing\rangle & \mapsto\langle a \star \varnothing\rangle, & & \\
\left\langle\varnothing \star x_{k}^{0}\right\rangle & \mapsto\left\langle\varnothing \star z_{k}^{0}\right\rangle & & \text { pour } 0 \leqslant k \leqslant j, \\
\left\langle\varnothing \star x_{k}^{1}\right\rangle & \mapsto\left\langle\varnothing \star y_{k}^{1}\right\rangle & & \text { pour } 0 \leqslant k \leqslant j, \\
\left\langle\varnothing \star x_{k}^{\varepsilon}\right\rangle & \mapsto\left\langle\varnothing \star y_{k}^{\varepsilon}\right\rangle *_{j}\left\langle\varnothing \star z_{k}^{\varepsilon}\right\rangle & & \text { pour } j<k \leqslant i \text { et } \varepsilon=0,1, \\
\left\langle a \star x_{k}^{0}\right\rangle & \mapsto\left\langle a \star z_{k}^{0}\right\rangle & & \text { pour } 0 \leqslant k \leqslant j, \\
\left\langle a \star x_{k}^{1}\right\rangle & \mapsto\left\langle a \star y_{k}^{1}\right\rangle & & \text { pour } 0 \leqslant k \leqslant j, \\
\left\langle a \star x_{k}^{\varepsilon}\right\rangle & \mapsto u_{k}^{\varepsilon} & & \text { pour } j<k \leqslant i \text { et } \varepsilon=0,1,
\end{aligned}
$$

où

$$
u_{k}^{\varepsilon}=\left\langle\varnothing \star y_{j+1}^{\eta}\right\rangle *_{0}\left\langle a \star z_{0}^{0}\right\rangle *_{1} \cdots *_{j-1}\left\langle a \star z_{j-1}^{0}\right\rangle *_{j}\left\langle a \star z_{k}^{\varepsilon}\right\rangle *_{j+1}\left\langle a \star y_{k}^{\varepsilon}\right\rangle,
$$

avec $\eta$ valant $\varepsilon$ si $k=j+1$ et 1 sinon. 
Démonstration. - Le cas des atomes de la forme $\left\langle\varnothing \star x_{k}^{\varepsilon}\right\rangle$ résulte de la naturalité de $\iota_{2}$ et, plus précisément, de la commutativité du carré

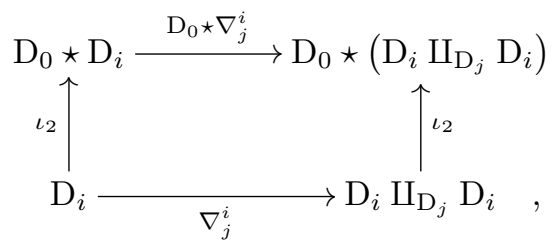

ainsi que de la description explicite du $\infty$-foncteur $\nabla_{j}^{i}: \mathrm{D}_{i} \rightarrow \mathrm{D}_{i} \amalg_{\mathrm{D}_{j}} \mathrm{D}_{i}$ (voir le paragraphe 4.3$)$.

Le cas de l'atome $\langle a \star \varnothing\rangle$ et des atomes de la forme $\left\langle a \star x_{k}^{\varepsilon}\right\rangle$ avec $0 \leqslant k \leqslant j$ est conséquence de la proposition 6.18 et des formules

$$
\nabla_{j}^{i}\left(\left\langle x_{k}^{0}\right\rangle\right)=\left\langle z_{k}^{0}\right\rangle \quad \text { et } \quad \nabla_{j}^{i}\left(\left\langle x_{k}^{1}\right\rangle\right)=\left\langle y_{k}^{1}\right\rangle,
$$

pour $0 \leqslant k \leqslant j$.

Enfin, traitons le cas des atomes de la forme $\left\langle a \star x_{k}^{\varepsilon}\right\rangle$ avec $k>j$. Soient $l$ tel que $0 \leqslant l \leqslant k+1$ et $\varepsilon^{\prime}=0,1$. Il s'agit de montrer l'égalité $\left(\mathrm{D}_{0} \star \nabla_{j}^{i}\right)\left(\left\langle a \star x_{k}^{\varepsilon}\right\rangle\right)_{l}^{\varepsilon^{\prime}}=\left(u_{k}^{\varepsilon}\right)_{l}^{\varepsilon^{\prime}}$. Le membre de gauche a été calculé dans le lemme 9.13. Calculons celui de droite.

Si $l \leqslant j+1$ (et donc $l \leqslant k$ ), on a, en utilisant le lemme 9.3,

$$
\left(u_{k}^{\varepsilon}\right)_{l}^{0}=\left(s_{l}\left(u_{k}^{\varepsilon}\right)\right)_{l}=\left\langle a \star y_{k}^{\varepsilon}\right\rangle_{l}^{0}=a \star y_{l-1}^{1} .
$$

Si $j+1<l \leqslant k+1$, on a

$$
\left(u_{k}^{\varepsilon}\right)_{l}^{\varepsilon^{\prime}}=\left\langle a \star z_{k}^{\varepsilon}\right\rangle_{l}^{\varepsilon^{\prime}}+\left\langle a \star y_{k}^{\varepsilon}\right\rangle_{l}^{\varepsilon^{\prime}}
$$

et donc, pour $l=k+1$,

$$
\left(u_{k}^{\varepsilon}\right)_{l}^{\varepsilon^{\prime}}=a \star z_{k}^{\varepsilon}+a \star y_{k}^{\varepsilon}
$$

et, pour $j+1<l<k+1$, en vertu du lemme 9.3,

$$
\left(u_{k}^{\varepsilon}\right)_{l}^{0}=a \star z_{l-1}^{1}+a \star y_{l-1}^{1} \quad \text { et } \quad\left(u_{k}^{\varepsilon}\right)_{l}^{1}=\varnothing \star z_{l}^{\eta}+a \star z_{l-1}^{0}+\varnothing \star y_{l}^{\eta}+a \star y_{l-1}^{0} .
$$

Si $l=j+1$, on a

$$
t_{l}\left(u_{k}^{\varepsilon}\right)=t_{l}\left(\left\langle\varnothing \star y_{j+1}^{\eta}\right\rangle *_{0}\left\langle a \star z_{0}^{0}\right\rangle *_{1} \cdots *_{j-1}\left\langle a \star z_{j-1}^{0}\right\rangle *_{j}\left\langle a \star z_{k}^{\varepsilon}\right\rangle\right)
$$

et donc, toujours en utilisant le lemme 9.3,

$$
\left(u_{k}^{\varepsilon}\right)_{l}^{1}=\left\langle\varnothing \star y_{j+1}^{\eta}\right\rangle_{l}^{1}+\left\langle a \star z_{k}^{\varepsilon}\right\rangle_{l}^{1}=\varnothing \star y_{l}^{\eta}+\varnothing \star z_{l}^{\eta}+a \star z_{l-1}^{0} .
$$

Enfin, si $l<j+1$, on a

$$
t_{l}\left(u_{k}^{\varepsilon}\right)=t_{l}\left(\left\langle\varnothing \star y_{j+1}^{\eta}\right\rangle *_{0}\left\langle a \star z_{0}^{0}\right\rangle *_{1} \cdots *_{l-1}\left\langle a \star z_{l-1}^{0}\right\rangle\right)
$$

et donc

$$
\left(u_{k}^{\varepsilon}\right)_{l}^{1}=\left\langle\varnothing \star y_{j+1}^{\eta}\right\rangle_{l}^{1}+\left\langle a \star z_{l-1}^{0}\right\rangle_{l}^{1}=\varnothing \star y_{l}^{1}+a \star z_{l-1}^{0} .
$$

On a bien retrouvé dans tous les cas la valeur de $\left(\mathrm{D}_{0} \star \nabla_{j}^{i}\right)\left(\left\langle a \star x_{k}^{\varepsilon}\right\rangle\right)_{l}^{\varepsilon^{\prime}}$ obtenue dans le lemme 9.13 , ce qui achève la démonstration. 
Proposition 9.15. - Soient $C$ une $\infty$-catégorie et c un objet de $C$. Fixons une $i$-flèche $(d, \alpha)$ de $c \backslash C$.

(a) Si $i \geqslant 1$, on a $s(d, \alpha)=(s(d), \gamma)$, ò̀

$$
\begin{aligned}
\gamma_{k}^{\varepsilon} & =\alpha_{k}^{\varepsilon} \quad \text { pour } 0 \leqslant k<i-1 \text { et } \varepsilon=0,1, \\
\gamma_{i-1} & =\alpha_{i-1}^{0} .
\end{aligned}
$$

(b) Si $i \geqslant 1$, on a $t(d, \alpha)=(t(d), \gamma)$, où

$$
\begin{aligned}
\gamma_{k}^{\varepsilon} & =\alpha_{k}^{\varepsilon} \quad \text { pour } 0 \leqslant k<i-1 \text { et } \varepsilon=0,1, \\
\gamma_{i-1} & =\alpha_{i-1}^{1} .
\end{aligned}
$$

(c) Si $i \geqslant 0$, on a $1_{(d, \alpha)}=\left(1_{d}, \gamma\right)$, ò̀

$$
\begin{aligned}
\gamma_{k}^{\varepsilon} & =\alpha_{k}^{\varepsilon} & & \text { pour } 0 \leqslant k<i \text { et } \varepsilon=0,1, \\
\gamma_{i}^{\varepsilon} & =\alpha_{i} & & \text { pour } \varepsilon=0,1, \\
\gamma_{i+1} & =1_{\alpha_{i}} . & &
\end{aligned}
$$

Soit $(e, \beta)$ une seconde $i$-flèche de $c \backslash C$.

(d) $S i(d, \alpha)$ et $(e, \beta)$ sont $j$-composables pour un $j$ tel que $0 \leqslant j<i$, alors on a $(d, \alpha) *_{j}(e, \beta)=\left(d *_{j} e, \gamma\right)$, ò

$$
\begin{array}{ll}
\gamma_{k}^{0}=\beta_{k}^{0} & \text { pour } 0 \leqslant k \leqslant j, \\
\gamma_{k}^{1}=\alpha_{k}^{1} & \text { pour } 0 \leqslant k \leqslant j
\end{array}
$$

et

$$
\gamma_{k}^{\varepsilon}=d_{j+1}^{\eta} *_{0} \beta_{0}^{0} *_{1} \cdots *_{j-1} \beta_{j-1}^{0} *_{j} \beta_{k}^{\varepsilon} *_{j+1} \alpha_{k}^{\varepsilon}
$$

pour $j<k \leqslant i$ et $\varepsilon=0,1$, où $\eta$ vaut $\varepsilon$ si $k=j+1$ et 1 sinon.

Démonstration. - Ces formules sont la traduction, à travers la bijection de la proposition 9.6 et du paragraphe 9.7, des formules obtenues dans les propositions 9.9, 9.10 et 9.14 .

Remarque 9.16. - Il résulte de la description de $c \backslash C$ obtenue dans la proposition précédente que cette $\infty$-catégorie est isomorphe à une sous- $\infty$-catégorie pleine de la $\infty$-catégorie $H C$ des cylindres dans $C$ introduite par Métayer dans [30] (voir notre remarque B.1.16 pour une définition abstraite de $H C$ ). Plus précisément, cette $\infty$-catégorie est la fibre en $c$ du $\infty$-foncteur $H C \rightarrow C$ qui envoie un cylindre sur sa « cellule source ». La $\infty$-catégorie $c \backslash C$ apparaît également dans [4] où elle est notée $\Lambda(C, c)$.

Remarque 9.17. - Si $C$ est une $n$-catégorie, il résulte immédiatement de la proposition 9.15 que la $\infty$-catégorie $c \backslash C$ est une $n$-catégorie. On retrouve ainsi un cas particulier de la proposition 8.11. 
9.18. - Soient $C$ une 2-catégorie et $c$ un objet de $C$. En vertu des résultats de ce chapitre, en utilisant des notations adaptées au cas de la dimension 2, on obtient la description suivante de la 2-catégorie $c \backslash C$. Les objets de $c \backslash C$ sont les couples $(d, f)$, où $d$ est un objet de $C$ et $f: c \rightarrow d$ est une 1-flèche de $C$ :

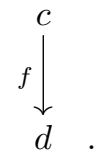

Si $(d, f)$ et $\left(d^{\prime}, f^{\prime}\right)$ sont deux objets de $c \backslash C$, une 1-flèche du premier vers le second est un couple $(g, \alpha)$, où $g: d \rightarrow d^{\prime}$ est une 1-flèche de $C$ et $\alpha: f^{\prime} \rightarrow g f$ une 2-flèche de $C$ :

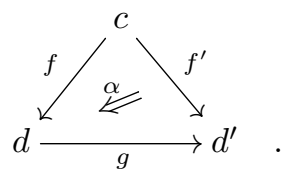

Enfin, si $(g, \alpha)$ et $\left(g^{\prime}, \alpha^{\prime}\right)$ sont deux telles 1-flèches, une 2-flèche de la première vers la seconde est une 2-flèche $\gamma: g \rightarrow g^{\prime}$ satisfaisant $\left(\gamma *_{0} f\right) *_{1} \alpha=\alpha^{\prime}$ :

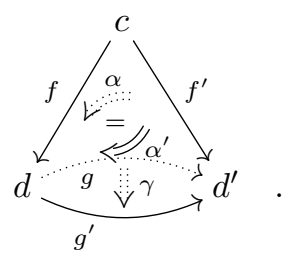

Par ailleurs, si $(d, f)$ est un objet et $(g, \alpha)$ est une 1-flèche de $c \backslash C$, on a

$$
1_{(d, f)}=\left(1_{d}, 1_{f}\right) \text { et } 1_{(g, \alpha)}=1_{g} .
$$

Si $(g, \alpha):(d, f) \rightarrow\left(d^{\prime}, f^{\prime}\right)$ et $\left(g^{\prime}, \alpha^{\prime}\right):\left(d^{\prime}, f^{\prime}\right) \rightarrow\left(d^{\prime \prime}, f^{\prime \prime}\right)$ sont deux 1-flèches composables de $c \backslash C$, on a

$$
\left(g^{\prime}, \alpha^{\prime}\right) *_{0}(g, \alpha)=\left(g^{\prime} g,\left(g^{\prime} *_{0} \alpha\right) *_{1} \alpha^{\prime}\right) .
$$

Enfin, les compositions horizontales et verticales des 2-flèches de $c \backslash C$ sont héritées de celles de $C$, ce qui achève de décrire la 2-catégorie $c \backslash C$.

Remarque 9.19. - La description de $c \backslash C$ donnée dans le paragraphe précédent montre que cette 2-catégorie est la 2-catégorie $c / / C$ introduite par Bullejos et Cegarra dans [13, section 2.1], 2-catégorie qui est, à une dualité près, un cas particulier de la construction des 2-catégories « comma » définie par Gray dans [22, paragraphe I.2.5]. C'est par contre notre 2-catégorie $c \backslash C$ qui est noté $c / / C$ par Cegarra dans [14]. Dans [15], Chiche appelle les 2-catégories $c \backslash C$ et $c \backslash C$ la optranche lax de $C$ audessous de $c$ (voir sa définition 1.4.5) et la optranche colax de $C$ au-dessous de $c$ (voir sa définition 1.4.7). 
110 CHAPITRE 9. DESCRIPTION EXPLICITE DES TRANCHES AU-DESSOUS D'UN OBJET

Remarque 9.20. - Lorsque $C$ est une 1-catégorie, la description explicite de $c \backslash C$ qu'on a donnée dans le cas où $C$ est une 2-catégorie montre que $c \backslash C$ est la tranche 1-catégorique usuelle. On retrouve ainsi un cas particulier du corollaire 8.19. 


\section{CHAPITRE 10}

\section{FONCTORIALITÉS DES TRANCHES : RÉSULTATS POUR LES COMPLEXES}

Dans ce chapitre, on définit des tranches pour les complexes dirigés augmentés. Ces tranches sont définies par des formules ad hoc dont on montre ensuite qu'elles sont imposées par une relation d'adjonction. Elles sont par ailleurs compatibles aux tranches $\infty$-catégoriques introduites dans le chapitre 6 lorsqu'on se restreint aux complexes de Steiner forts. Une partie importante du chapitre est consacrée à l'étude des propriétés de fonctorialité et de 2-fonctorialité de ces tranches pour les complexes dirigés augmentés.

\subsection{Tranches pour les complexes dirigés augmentés}

10.1.1. - Fixons $K$ un complexe dirigé augmenté. Soient $M$ un complexe dirigé augmenté et $g: K \rightarrow M$ un morphisme. On va définir un complexe dirigé augmenté $g \backslash M$. On conviendra des égalités suivantes :

$$
K_{-1}=\mathbb{Z}, \quad M_{-1}=\mathbb{Z}, \quad K_{-1}^{*}=\mathbb{N}, \quad M_{-1}^{*}=\mathbb{N}, \quad d_{0}=e \quad \text { et } \quad g_{-1}=1_{\mathbb{Z}}
$$

et, pour $j \leqslant-2$,

$$
K_{j}=0, \quad M_{j}=0, \quad d_{j+1}=0 \quad \text { et } \quad g_{j}=0 .
$$

On définit $(g \backslash M)_{0}$ comme le sous-ensemble

$$
(g \backslash M)_{0} \subset \prod_{j \geqslant-1} \operatorname{Hom}_{\mathcal{A} b}\left(K_{j}, M_{j+1}\right)
$$

constitué des éléments $\left(u_{j}\right)_{j \geqslant-1}$ vérifiant, pour $j \geqslant 0$,

$$
(-1)^{j+1}\left(d_{j+1} u_{j}-u_{j-1} d_{j}\right)=e\left(u_{-1}(1)\right) \cdot g_{j} .
$$

Notons qu'en convenant que $u_{j}=0$ pour tout $j \leqslant-2$, l'égalité ci-dessus reste vraie pour tout $j$ dans $\mathbb{Z}$ : pour $j=-1$, on obtient l'égalité $e u_{-1}=e\left(u_{-1}(1)\right) .1_{\mathbb{Z}}$ et, pour 
$j \leqslant-2$, l'égalité $0=0$. Pour $i \geqslant 1$, on pose

$$
(g \backslash M)_{i}=\prod_{j \geqslant-1} \operatorname{Hom}_{\mathcal{A} b}\left(K_{j}, M_{i+j+1}\right) .
$$

Pour $i>0$, on définit la différentielle

$$
d_{i}:(g \backslash M)_{i} \rightarrow(g \backslash M)_{i-1}
$$

en envoyant $u=\left(u_{j}\right)_{j \geqslant-1}$ sur $d_{i}(u)=\left(d_{i}(u)_{j}\right)_{j \geqslant-1}$ défini par, pour $j \geqslant-1$,

$$
d_{i}(u)_{j}=(-1)^{j+1}\left(d_{i+j+1} u_{j}-u_{j-1} d_{j}\right) .
$$

Notons que pour $j=-1$, la formule se simplifie, en vertu de nos conventions, en $d_{i}(u)_{-1}=d_{i} u_{-1}$. Par ailleurs, en étendant cette formule à tout $j$ dans $\mathbb{Z}$, en convenant à nouveau que $u_{j}=0$ pour $j \leqslant-2$, on obtient bien que $d_{i}(u)_{j}=0$ pour $j \leqslant-2$.

On définit l'augmentation

$$
e:(g \backslash M)_{0} \rightarrow \mathbb{Z}
$$

en envoyant $\left(u_{j}\right)_{j \geqslant-1}$ sur $e\left(u_{-1}(1)\right)$.

Enfin, les sous-monoïdes de positivité, pour $i \geqslant 0$, sont les sous-ensembles

$$
(g \backslash M)_{i}^{*} \subset(g \backslash M)_{i}
$$

constitués des $\left(u_{j}\right)_{j \geqslant-1}$ tels que, pour tout $j \geqslant-1$, on ait l'inclusion

$$
u_{j}\left(K_{j}^{*}\right) \subset M_{i+j+1}^{*} .
$$

Proposition 10.1.2. — Fixons $K$ un complexe dirigé augmenté. Soient $M$ un complexe dirigé augmenté et $g: K \rightarrow M$ un morphisme. Alors $g \backslash M$ est bien un complexe dirigé augmenté.

Démonstration. - Commençons par montrer que $d_{1}$ est bien à valeurs dans $(g \backslash M)_{0}$. Soit $u=\left(u_{j}\right)_{j \geqslant-1}$ un élément de $(g \backslash M)_{1}$. Il s'agit de montrer que, pour tout $j \geqslant 0$, on a

$$
(-1)^{j+1}\left(d_{j+1} d_{1}(u)_{j}-d_{1}(u)_{j-1} d_{j}\right)=e\left(d_{1}(u)_{-1}(1)\right) \cdot g_{j} .
$$

Plus généralement, pour tout $i \geqslant 1$, tout $u=\left(u_{j}\right)_{j \geqslant-1}$ dans $(g \backslash M)_{i}$ et tout $j \geqslant-1$, on a

$$
\begin{aligned}
(-1)^{j+1} & \left(d_{i+j} d_{i}(u)_{j}-d_{i}(u)_{j-1} d_{j}\right) \\
& =(-1)^{j+1}\left[\left(d_{i+j}(-1)^{j+1}\left(d_{i+j+1} u_{j}-u_{j-1} d_{j}\right)-(-1)^{j}\left(d_{i+j} u_{j-1}-u_{j-2} d_{j-1}\right) d_{j}\right]\right. \\
& =-d_{i+j} u_{j-1} d_{j}+d_{i+j} u_{j-1} d_{j} \\
& =0 .
\end{aligned}
$$

En particulier, pour $i=1$, on a

$$
(-1)^{j+1}\left(d_{j+1} d_{1}(u)_{j}-d_{1}(u)_{j-1} d_{j}\right)=0 .
$$

Pour $j=-1$, on trouve

$$
e d_{1}(u)_{-1}=0
$$


et on a donc bien, pour tout $j \geqslant 0$,

$$
(-1)^{j+1}\left(d_{j+1} d_{1}(u)_{j}-d_{1}(u)_{j-1} d_{j}\right)=e\left(d_{1}(u)_{-1}(1)\right) \cdot g_{j} .
$$

Montrons maintenant que, pour tout $i \geqslant 2$, on a $d_{i-1} d_{i}=0$. Soit donc $u=\left(u_{j}\right)_{j \geqslant-1}$ un élément de $(g \backslash M)_{i}$. On a, pour tout $j \geqslant-1$,

$$
\left(d_{i-1} d_{i}(u)\right)_{j}=(-1)^{j+1}\left(d_{i+j} d_{i}(u)_{j}-d_{i}(u)_{j-1} d_{j}\right)=0,
$$

la dernière égalité résultant du calcul ci-dessus, d'où l'assertion.

Enfin, la relation $e d_{1}(u)_{-1}=0$ trouvée précédemment montre qu'on a bien $e d_{1}=0$, d'où le résultat.

10.1.3. - Fixons $K$ un complexe dirigé augmenté. Soit $f: M \rightarrow M^{\prime}$ un morphisme de complexes dirigés augmentés au-dessous de $K$, c'est-à-dire un triangle commutatif

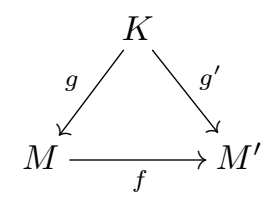

de morphismes de complexes dirigés augmentés. On vérifie immédiatement qu'on associe à $f$ un morphisme de complexes dirigés augmentés $g \backslash M \rightarrow g^{\prime} \backslash M^{\prime}$ en envoyant, pour tout $i \geqslant 0$, un élément $\left(u_{j}\right)_{j \geqslant-1}$ de $(g \backslash M)_{i}$ sur l'élément $\left(f u_{j}\right)_{j \geqslant-1} \operatorname{de}\left(g^{\prime} \backslash M^{\prime}\right)_{i}$. On définit ainsi un foncteur

$$
\begin{aligned}
K \backslash \mathcal{C}_{\mathrm{da}} & \rightarrow \mathcal{C}_{\mathrm{da}} \\
(M, K \stackrel{g}{\rightarrow} M) & \mapsto g \backslash M .
\end{aligned}
$$

Proposition 10.1.4. — Fixons $K$ un complexe dirigé augmenté. Les foncteurs

$$
\begin{array}{rlrl}
\mathcal{C}_{\mathrm{da}} & \rightarrow K \backslash \mathcal{C}_{\mathrm{da}} & K \backslash \mathcal{C}_{\mathrm{da}} & \rightarrow \mathcal{C}_{\mathrm{da}} \\
L & \mapsto\left(K \star L, \iota_{1}\right) & (M, K \stackrel{g}{\rightarrow} M) & \mapsto g \backslash M
\end{array}
$$

forment un couple de foncteurs adjoints.

Démonstration. - Soient $L$ un complexe dirigé augmenté et $M$ un complexe dirigé augmenté muni d'un morphisme $g: K \rightarrow M$. On va produire des fonctions

$$
\begin{aligned}
& \varphi: \operatorname{Hom}_{K \backslash \mathcal{C}_{\mathrm{da}}}\left(\left(K \star L, \iota_{1}\right),(M, g)\right) \rightarrow \operatorname{Hom}_{\mathcal{C}_{\mathrm{da}}}(L, g \backslash M), \\
& \psi: \operatorname{Hom}_{\mathcal{C}_{\mathrm{da}}}(L, g \backslash M) \rightarrow \operatorname{Hom}_{K \backslash \mathcal{C}_{\mathrm{da}}}\left(\left(K \star L, \iota_{1}\right),(M, g)\right),
\end{aligned}
$$

naturelles en $L$ et $(M, g)$, inverses l'une de l'autre.

Commençons par $\varphi$. Soit $F: K \star L \rightarrow M$ un morphisme au-dessous de $K$. On définit un morphisme $\varphi(F): L \rightarrow g \backslash M$ de la manière suivante. Soient $j \geqslant 0$ et $y$ dans $L_{j}$. On doit définir un élément $\varphi(F)_{j}(y)$ de $(g \backslash M)_{j}$ et donc, pour tout $i \geqslant-1$, un morphisme $\varphi(F)_{j}(y)_{i}: K_{i} \rightarrow M_{i+j+1}$. Pour $x$ dans $K_{i}$, on pose

$$
\varphi(F)_{j}(y)_{i}(x)=F_{i+1+j}(x \star y),
$$


en convenant, pour $i=-1$, d'identifier 1 dans $K_{-1}=\mathbb{Z}$ avec $\varnothing$. Vérifions tout d'abord que, pour $j=0, \varphi(F)_{0}(y)$ est bien dans $(g \backslash M)_{0}$. Il s'agit de voir que, pour tout $i \geqslant 0$, on a

$$
(-1)^{i+1}\left(d_{i+1} \varphi(F)_{0}(y)_{i}-\varphi(F)_{0}(y)_{i-1} d_{i}\right)=e\left(\varphi(F)_{0}(y)_{-1}(1)\right) \cdot g_{i} .
$$

Plus généralement, pour tout $j \geqslant 0$ et tout $i \geqslant-1$, on a

$$
\begin{aligned}
(-1)^{i+1} & \left(d_{i+j+1} \varphi(F)_{j}(y)_{i}-\varphi(F)_{j}(y)_{i-1} d_{i}\right)(x) \\
& =(-1)^{i+1}\left(d_{i+j+1} F_{i+j+1}(x \star y)-F_{i+j}\left(d_{i} x \star y\right)\right) \\
& =(-1)^{i+1}\left(F_{i+j} d_{i+j+1}(x \star y)-F_{i+j}\left(d_{i} x \star y\right)\right) \\
& =(-1)^{i+1} F_{i+j}\left(d_{i+j+1}(x \star y)-d_{i} x \star y\right) \\
& =(-1)^{i+1} F_{i+j}\left((-1)^{i+1} x \star d_{j} y\right) \\
& =F_{i+j}\left(x \star d_{j} y\right) .
\end{aligned}
$$

En revenant au cas $j=0$, on a donc

$$
\begin{aligned}
(-1)^{i+1} & \left(d_{i+1} \varphi(F)_{0}(y)_{i}-\varphi(F)_{0}(y)_{i-1} d_{i}\right)(x) \\
& =F_{i}(x \star e(y) \varnothing) \\
& =e(y) F_{i}(x \star \varnothing) \\
& =e(y) g_{i}(x)
\end{aligned}
$$

puisque $F$ est au-dessous de $K$. D'où l'assertion puisque

$$
e\left(\varphi(F)_{0}(y)_{-1}(1)\right)=e F_{j}(\varnothing \star y)=e(y) .
$$

Vérifions maintenant la compatibilité aux différentielles. Pour tout $j \geqslant 1$ et tout $i \geqslant-1$, on a

$$
\begin{aligned}
\left(d_{j} \varphi(F)_{j}(y)\right)_{i}(x)= & (-1)^{i+1}\left(d_{i+j+1} \varphi(F)_{j}(y)_{i}-\varphi(F)_{j}(y)_{i-1} d_{i}\right)(x) \\
= & F_{i+j}\left(x \star d_{j} y\right) \\
& (\text { par le calcul du début de la preuve }) \\
= & \varphi(F)_{j-1}\left(d_{j} y\right)_{i}(x),
\end{aligned}
$$

d'où la compatibilité aux différentielles. La compatibilité aux augmentations résulte d'un calcul précédent :

$$
e\left(\varphi(F)_{0}(y)\right)=e\left(\varphi(F)_{0}(y)_{-1}(1)\right)=e(y) .
$$

La compatibilité aux sous-monoïdes de positivité étant évidente, on a bien établi que $\varphi(F): L \rightarrow g \backslash M$ est un morphisme.

Définissons maintenant $\psi$. Soit $G: L \rightarrow g \backslash M$ un morphisme. Il s'agit de définir un morphisme $\psi(G): K \star L \rightarrow M$ au-dessous de $K$. Soient $x$ un élément de $K_{i}$ et $y$ un élément de $L_{j}$ avec $i \geqslant-1, j \geqslant-1$ et $i+1+j \geqslant 0$. On pose

$$
\psi(G)_{i+1+j}(x \star y)=G_{j}(y)_{i}(x),
$$


en convenant que $G_{-1}(\varnothing)_{i}=g_{i}$. Vérifions que $\psi(G)$ est bien un morphisme au-dessous de $K$. La compatibilité aux sous-monoïdes de positivité est évidente. Il est immédiat que, pour tout $i \geqslant 0$, le morphisme $\psi(G)_{i}$ est bien au-dessous de $K_{i}$; en effet, pour $x$ dans $K_{i}$, on a

$$
\psi(G)_{i}(x \star \varnothing)=G_{-1}(\varnothing)_{i}(x)=g_{i}(x) .
$$

Ainsi, pour $i=0$, on a

$$
e \psi(G)_{0}(x \star \varnothing)=e g_{0}(x)=e(x) .
$$

Par ailleurs, pour $y$ dans $L_{0}$, on a

$$
e\left(\psi(G)_{0}(\varnothing \star y)\right)=e\left(G_{0}(y)_{-1}(1)\right)=e G_{0}(y)=e(y),
$$

ce qui montre la compatibilité de $\psi(G)_{0}$ aux augmentations. Montrons la compatibilité aux différentielles. Soient donc $x$ dans $K_{i}$ et $y$ dans $L_{j}$ avec $i \geqslant-1, j \geqslant-1$ et $i+1+j \geqslant 1$. Si $j=-1$, de sorte qu'on peut supposer $y=\varnothing$, on a

$$
d_{i} \psi(G)_{i}(x \star \varnothing)=d_{i} g_{i}(x)=g_{i-1} d_{i}(x)=\psi(G)_{i-1}\left(d_{i} x \star \varnothing\right)=\psi(G)_{i-1} d_{i}(x \star \varnothing) .
$$

Si $j=0$, on a

$$
\begin{aligned}
d_{i+1} \psi(G)_{i+1}(x \star y)= & d_{i+1} G_{0}(y)_{i}(x) \\
= & \left(G_{0}(y)_{i-1} d_{i}+(-1)^{i+1} e\left(G_{0}(y)_{-1}(1)\right) g_{i}\right)(x) \\
& \left(\text { par définition de }(g \backslash M)_{0}\right) \\
= & G_{0}(y)_{i-1}\left(d_{i}(x)\right)+(-1)^{i+1} e(y) g_{i}(x)
\end{aligned}
$$

(par compatibilité de $G$ aux augmentations)

$$
=\psi(G)_{i}\left(d_{i} x \star y\right)+(-1)^{i+1} \psi(G)_{i}(x \star e(y) \varnothing)
$$

$\left(\operatorname{car} \psi(G)_{i}\right.$ est au-dessous de $\left.K\right)$

$$
\begin{aligned}
& =\psi(G)_{i}\left(d_{i} x \star y+(-1)^{i+1} x \star e(y) \varnothing\right) \\
& =\psi(G)_{i} d_{i+1}(x \star y) .
\end{aligned}
$$

Enfin, si $j \geqslant 1$, on a

$$
\begin{aligned}
d_{i+j+1} \psi(G)_{i+j+1}(x \star y)= & d_{i+j+1} G_{j}(y)_{i}(x) \\
= & \left(G_{j}(y)_{i-1} d_{i}+(-1)^{i+1}\left(d_{j}\left(G_{j}(y)\right)\right)_{i}\right)(x) \\
& \left(\text { par définition de } d_{j}\left(G_{j}(y)\right)\right) \\
= & G_{j}(y)_{i-1}\left(d_{i} x\right)+(-1)^{i+1}\left(G_{j-1}\left(d_{j} y\right)\right)_{i}(x) \\
= & \psi(G)_{i+j}\left(d_{i} x \star y\right)+(-1)^{i+1} \psi(G)_{i+j}\left(x \star d_{j} y\right) \\
= & \psi(G)_{i+j}\left(d_{i} x \star y+(-1)^{i+1} x \star d_{j} y\right) \\
= & \psi(G)_{i+j} d_{i+j+1}(x \star y),
\end{aligned}
$$

ce qui achève de montrer que $\psi(G): K \star L \rightarrow M$ est bien un morphisme au-dessous de $K$. 
Pour conclure, il suffit de vérifier que les applications $\varphi$ et $\psi$ sont bien inverses l'une de l'autre, ce qui est immédiat.

10.1.5. - Fixons $L$ un complexe dirigé augmenté. Soient $M$ un complexe dirigé augmenté et $g: L \rightarrow M$ un morphisme. On définit un complexe dirigé augmenté $M / g$ en posant

$$
M / g=\left(g^{\mathrm{cop}} \backslash M^{\mathrm{op}}\right)^{\mathrm{op}} .
$$

On laisse le soin au lecteur de décrire explicitement ce complexe dirigé augmenté. On définit ainsi un foncteur

$$
\begin{aligned}
L \backslash \mathcal{C}_{\mathrm{da}} & \rightarrow \mathcal{C}_{\mathrm{da}} \\
(M, L \stackrel{g}{\rightarrow} M) & \mapsto M / g .
\end{aligned}
$$

Proposition 10.1.6. — Fixons L un complexe dirigé augmenté. Les foncteurs

$$
\begin{array}{rlrl}
\mathcal{C}_{\mathrm{da}} & \rightarrow L \backslash \mathcal{C}_{\mathrm{da}} & L \backslash \mathcal{C}_{\mathrm{da}} & \rightarrow \mathcal{C}_{\mathrm{da}} \\
K & \mapsto\left(K \star L, \iota_{2}\right) & (M, L \stackrel{g}{\rightarrow} M) & \mapsto M / g
\end{array}
$$

forment un couple de foncteurs adjoints.

Démonstration. - Le résultat se déduit par dualité des propositions 10.1.4 et 6.10 par un argument semblable à celui de la preuve de l'assertion analogue pour les $\infty$-catégories (proposition 6.36).

Corollaire 10.1.7. - La structure de catégorie monö̈dale sur $\mathcal{C}_{\mathrm{da}}$ définie par le joint est localement bifermée.

Démonstration. — Cela résulte des propositions 10.1.4 et 10.1.6.

Remarque 10.1.8. - On aurait pu montrer le corollaire précédent sans décrire explicitement les adjoints à droite. En effet, celui-ci résulte de la remarque 5.9 puisque la catégorie $\mathcal{C}_{\mathrm{da}}$ est localement présentable et que le joint commute aux limites inductives connexes en chaque variable (voir la proposition 6.9).

Proposition 10.1.9. — Fixons $K$ un complexe dirigé augmenté. Soient $M$ un complexe dirigé augmenté et $g: K \rightarrow M$ un morphisme. Alors, on dispose d'un morphisme canonique

$$
\nu(g \backslash M) \rightarrow \nu(g) \backslash \nu(M),
$$

où $\nu(g) \backslash \nu(M)$ désigne la tranche $\infty$-catégorique introduite au paragraphe 6.31. De plus, lorsque $K$ est un complexe de Steiner fort, ce morphisme est un isomorphisme.

Démonstration. - La sous-catégorie $\Theta$ étant dense dans $\infty$-C at (proposition 4.6), il suffit de définir une application

$$
\operatorname{Hom}_{\infty-\mathcal{C} a t}(S, \nu(g \backslash M)) \rightarrow \operatorname{Hom}_{\infty-\mathcal{C} a t}(S, \nu(g) \backslash \nu(M)),
$$


naturelle en $S$ dans $\Theta$, et de montrer que celle-ci est bijective lorsque $K$ est un complexe de Steiner fort. Soit donc $S$ dans $\Theta$. En vertu de la proposition 4.13, on a un isomorphisme naturel $S \simeq \nu(\lambda(S))$. En notant $L$ le complexe de Steiner fort $\lambda(S)$, on a des applications naturelles

$$
\begin{aligned}
& \operatorname{Hom}_{\infty-\mathcal{C} a t}(S, \nu(g \backslash M)) \simeq \operatorname{Hom}_{\infty-\mathcal{C} a t}(\nu(L), \nu(g \backslash M)) \\
& \simeq \operatorname{Hom}_{\mathcal{C}_{\mathrm{da}}}(\lambda \nu(L), g \backslash M) \\
& \text { (par adjonction) } \\
& \simeq \operatorname{Hom}_{\mathcal{C}_{\mathrm{da}}}(L, g \backslash M) \\
& \text { (en vertu du théorème 2.11) } \\
& \simeq \operatorname{Hom}_{K \backslash \mathcal{C}_{\mathrm{da}}}\left(\left(K \star L, \iota_{1}\right),(M, g)\right) \\
& \text { (par adjonction) } \\
& \stackrel{\alpha}{\rightarrow} \operatorname{Hom}_{\nu(K) \backslash \infty-\mathcal{C} a t}\left(\left(\nu(K \star L), \nu\left(\iota_{1}\right)\right),(\nu(M), \nu(g))\right) \\
& \stackrel{\beta}{\rightarrow} \operatorname{Hom}_{\nu(K) \backslash \infty-\mathcal{C} a t}\left(\left(\nu(K) \star \nu(L), \iota_{1}\right),(\nu(M), \nu(g))\right) \\
& \simeq \operatorname{Hom}_{\infty-\mathcal{C} a t}(\nu(L), \nu(g) \backslash \nu(M)) \\
& \text { (par adjonction) } \\
& \simeq \operatorname{Hom}_{\infty-\mathcal{C} a t}(S, \nu(g) \backslash \nu(M)),
\end{aligned}
$$

où l'application $\alpha$ est induite par le foncteur $\nu$ et l'application $\beta$ par la contrainte du foncteur monoïdal lax $\nu$ (voir la proposition 6.34), d'où le morphisme recherché. Notons que l'application $\alpha$ se factorise en

$$
\begin{aligned}
& \operatorname{Hom}_{K \backslash \mathcal{C}_{\mathrm{da}}}\left(\left(K \star L, \iota_{1}\right),(M, g)\right) \rightarrow \operatorname{Hom}_{\lambda \nu(K) \backslash \mathcal{C}_{\mathrm{da}}}\left(\left(\lambda \nu(K \star L), \lambda \nu\left(\iota_{1}\right)\right),(M, g)\right) \\
& \simeq \operatorname{Hom}_{\nu(K) \backslash \infty-\mathcal{C} a t}\left(\left(\nu(K \star L), \nu\left(\iota_{1}\right)\right),(\nu(M), \nu(g))\right) .
\end{aligned}
$$

Ainsi, si $K$ est un complexe de Steiner fort, cette application est une bijection en vertu du théorème 2.11 et du corollaire 6.21. Par ailleurs, toujours sous l'hypothèse que $K$ est un complexe de Steiner fort, en vertu du théorème 6.29 , l'application $\beta$ est également une bijection, d'où le résultat.

\subsection{Morphisme associé à un triangle}

10.2.1. - Dans cette section, on fixe

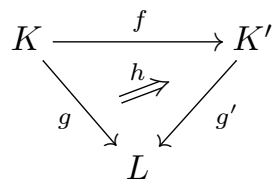

un triangle dans la catégorie des complexes dirigés augmentés commutant à une antihomotopie $h$ de $g$ vers $g^{\prime} f$ près. On suppose de plus que le complexe $L$ est décent 
(voir le paragraphe 2.17). On va définir un morphisme de complexes dirigés augmentés $(f, h)^{*}: g^{\prime} \backslash L \rightarrow g \backslash L$.

On conviendra que, pour tout $i \leqslant-1$, on a

$$
h_{i}=0
$$

Pour tout $i \geqslant 0$, on définit un morphisme $(f, h)_{i}^{*}:\left(g^{\prime} \backslash L\right)_{i} \rightarrow(g \backslash L)_{i}$ en envoyant $u^{\prime}=\left(u_{j}^{\prime}: K_{j}^{\prime} \rightarrow L_{i+1+j}\right)_{j \geqslant-1}$ sur $(f, h)_{i}^{*}\left(u^{\prime}\right)=\left((f, h)_{i}^{*}\left(u^{\prime}\right)_{j}\right)_{j \geqslant-1}$ défini par, pour tout $j \geqslant-1$,

$$
(f, h)_{i}^{*}\left(u^{\prime}\right)_{j}= \begin{cases}u_{j}^{\prime} f_{j}+e\left(u_{-1}^{\prime}(1)\right) . h_{j} & \text { si } i=0 \\ u_{j}^{\prime} f_{j} & \text { sinon. }\end{cases}
$$

(On vérifiera dans la preuve de la proposition suivante que $(f, h)_{0}^{*}$ est bien à valeurs dans $(g \backslash L)_{0}$.) Afin d'avoir une formule uniforme en $i$, on étendra parfois l'augmentation $e$ de $L$ à tout élément homogène de $L$ en posant $e(z)=0$ si $z$ est un élément homogène de degré non nul. Avec cette convention, pour tout $i \geqslant 0$, on a

$$
(f, h)_{i}^{*}\left(u^{\prime}\right)_{j}=u_{j}^{\prime} f_{j}+e\left(u_{-1}^{\prime}(1)\right) \cdot h_{j}
$$

Notons qu'en vertu de nos précédentes conventions cette égalité reste vraie pour tout $j$ dans $\mathbb{Z}$. Enfin, remarquons qu'on a $(f, h)_{i}^{*}\left(u^{\prime}\right)_{-1}=u_{-1}^{\prime}$.

Proposition 10.2.2. - Les morphismes $(f, h)_{i}^{*}$, pour $i \geqslant 0$, définissent un morphisme de complexes dirigés augmentés $(f, h)^{*}: g^{\prime} \backslash L \rightarrow g \backslash L$.

Démonstration. - Commençons par vérifier que le morphisme $(f, h)_{0}^{*}$ est bien à valeurs dans $(g \backslash L)_{0}$. Soit donc $u^{\prime}=\left(u_{j}^{\prime}\right)_{j \geqslant-1}$ un élément de $\left(g^{\prime} \backslash L\right)_{0}$. Par définition, en posant $z=u_{-1}^{\prime}(1)=(f, h)_{0}^{*}\left(u^{\prime}\right)_{-1}(1)$, on a, pour tout $j$ dans $\mathbb{Z}$,

$$
d_{j+1} u_{j}^{\prime}-u_{j-1}^{\prime} d_{j}=(-1)^{j+1} e(z) g_{j}^{\prime}
$$

Pour tout $j \geqslant-1$, on a

$$
\begin{aligned}
d_{j+1}(f, h)_{0}^{*}\left(u^{\prime}\right)_{j}-(f, h)_{0}^{*}\left(u^{\prime}\right)_{j-1} d_{j} & =d_{j+1}\left(u_{j}^{\prime} f_{j}+e(z) h_{j}\right)-\left(u_{j-1}^{\prime} f_{j-1}+e(z) h_{j-1}\right) d_{j} \\
& =\left(d_{j+1} u_{j}^{\prime}-u_{j-1}^{\prime} d_{j}\right) f_{j}+e(z)\left(d_{j+1} h_{j}-h_{j-1} d_{j}\right) \\
& =(-1)^{j+1} e(z) g_{j}^{\prime} f_{j}+(-1)^{j} e(z)\left(g_{j}^{\prime} f_{j}-g_{j}\right) \\
& =(-1)^{j+1} e(z) g_{j},
\end{aligned}
$$

ce qui montre que $(f, h)_{0}^{*}\left(u^{\prime}\right)$ appartient à $(g \backslash L)_{0}$.

La compatibilité de $(f, h)^{*}$ à l'augmentation est évidente et la compatibilité aux sous-monoïdes de positivité résulte du fait que $L$ est décent. Il nous reste à montrer la compatibilité aux différentielles. Soient donc $i \geqslant 1$ et $u^{\prime}=\left(u_{j}^{\prime}\right)_{j \geqslant-1}$ un élément 
de $\left(g^{\prime} \backslash L\right)_{i}$. Pour tout $j \geqslant-1$, on a

$$
\begin{aligned}
d_{i}(f, h)_{i}^{*}\left(u^{\prime}\right)_{j} & =(-1)^{j+1}\left(d_{i+j+1}(f, h)_{i}^{*}\left(u^{\prime}\right)_{j}-(f, h)_{i}^{*}\left(u^{\prime}\right)_{j-1} d_{j}\right) \\
& =(-1)^{j+1}\left(d_{i+j+1} u_{j}^{\prime} f_{j}-u_{j-1}^{\prime} f_{j-1} d_{j}\right) \\
& =(-1)^{j+1}\left(d_{i+j+1} u_{j}^{\prime}-u_{j-1}^{\prime} d_{j}\right) f_{j} \\
& =d_{i}\left(u^{\prime}\right)_{j} f_{j} \\
& =(f, h)_{i-1}^{*}\left(d_{i}\left(u^{\prime}\right)\right)_{j},
\end{aligned}
$$

la dernière égalité étant également valable quand $i=1$ puisqu'on a

$$
e\left(d_{1}\left(u^{\prime}\right)_{-1}(1)\right)=e d_{1}\left(u^{\prime}\right)=0,
$$

d'où le résultat.

10.2.3. - Un cas particulier important est celui où le triangle

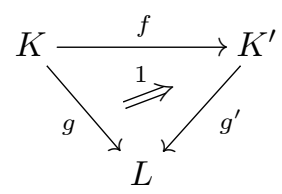

est commutatif et l'antihomotopie $h$ est l'identité de $g^{\prime} f=g$ (voir le paragraphe 2.30). Dans ce cas, en vertu de la proposition précédente, on obtient un morphisme $(f, 1)^{*}: g^{\prime} \backslash L \rightarrow g \backslash L$ qu'on notera plus simplement

$$
f^{*}: g^{\prime} \backslash L \rightarrow g \backslash L .
$$

Proposition 10.2.4. - Soit

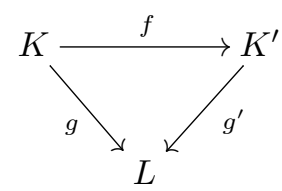

un triangle commutatif dans la catégorie des complexes dirigés augmentés. Alors le carré

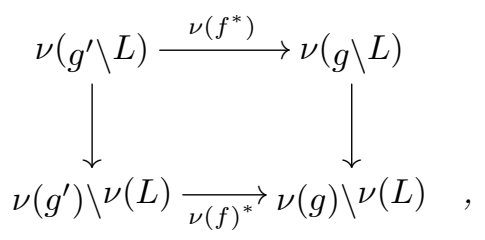

où les morphismes verticaux sont ceux de la proposition 10.1 .9 et le foncteur $\nu(f)^{*}$ celui du paragraphe 6.38 , est commutatif. 
Démonstration. - Il s'agit de démontrer que les deux $\infty$-foncteurs allant du coin supérieur gauche au coin inférieur droit coïncident sur les cellules ou, autrement dit, que pour tout $l \geqslant 0$, le carré

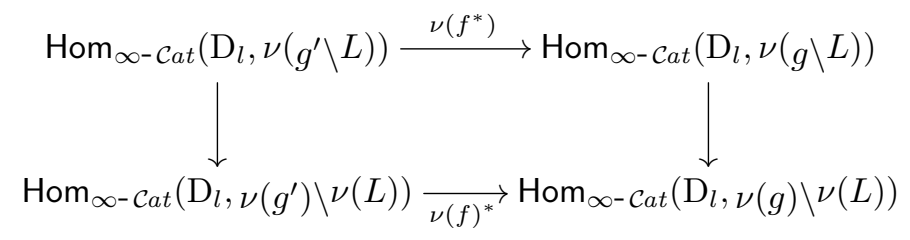

est commutatif. Par définition de $\nu(f)^{*}$ et des morphismes canoniques (voir la preuve de la proposition 10.1.9), cela revient à montrer que le carré

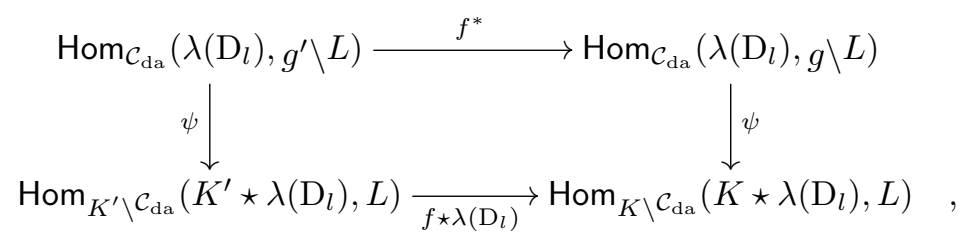

où $f^{*}$ désigne la postcomposition par $f^{*}, f \star \lambda\left(\mathrm{D}_{l}\right)$ la précomposition par $f \star \lambda\left(\mathrm{D}_{l}\right)$ et $\psi$ les isomorphismes de (la preuve de) la proposition 10.1.4, est commutatif. Soit donc $G: \lambda\left(\mathrm{D}_{l}\right) \rightarrow g^{\prime} \backslash L$ un morphisme. Soient $x$ dans $K_{i}$ et $y$ dans $\lambda\left(\mathrm{D}_{l}\right)_{j}$ avec $i \geqslant-1$, $j \geqslant-1$ et $i+1+j \geqslant 0$. Si $j \geqslant 0$, on a

$$
\psi\left(f^{*} G\right)(x \star y)=\left(f^{*} G\right)(y)_{i}(x)=G(y)_{i}(f(x))=\psi(G)(f(x) \star y),
$$

la première et la dernière égalité résultant de la définition de $\psi$ et la deuxième de la définition de $f^{*}$. Si $j=-1$, on peut supposer $y=\varnothing$ et on a

$$
\psi\left(f^{*} G\right)(x \star \varnothing)=g(x)=g^{\prime} f(x)=\psi(G)(f(x) \star \varnothing),
$$

la première et la dernière égalité résultant encore une fois de la définition de $\psi$, d'où le résultat.

10.2.5. - Un cas particulier du paragraphe 10.2.3 est celui d'un triangle commutatif

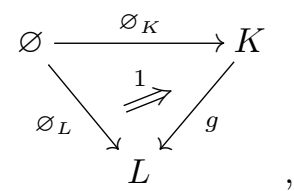

où $\varnothing_{K}$ et $\varnothing_{L}$ désignent les uniques morphismes de source $\varnothing$ et de buts respectifs $K$ et $L$, et 1 désigne l'antihomotopie identité de $\varnothing_{L}$. En vertu de ce même paragraphe, on obtient un morphisme

$$
\varnothing_{K}^{*}: g \backslash L \rightarrow \varnothing_{L} \backslash L
$$


On vérifie immédiatement que le morphisme de $\varnothing_{L} \backslash L$ vers $L$ qui, pour $i \geqslant 0$, envoie un élément $\left(u_{j}\right)_{j \geqslant-1}$ de $\left(\varnothing_{L} \backslash L\right)_{i}$ sur $u_{-1}(1)$ dans $L_{i}$ est un isomorphisme. On obtient donc un morphisme

$$
g \backslash L \rightarrow L
$$

qu'on appellera morphisme d'oubli. Explicitement, ce morphisme envoie, pour $i \geqslant 0$, un élément $\left(u_{j}\right)_{j \geqslant-1}$ de $(g \backslash L)_{i}$ sur $u_{-1}(1)$ dans $L_{i}$.

Proposition 10.2.6. - Le morphisme $(f, h)^{*}: g^{\prime} \backslash L \rightarrow g \backslash L$ est au-dessus de $L$. Autrement dit, le triangle

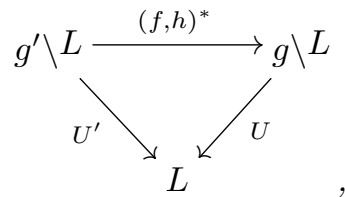

où $U$ et $U^{\prime}$ désignent les morphismes d'oubli, est commutatif.

Démonstration. - Pour tout $i \geqslant 0$ et tout $u^{\prime}=\left(u_{j}^{\prime}\right)_{j \geqslant-1}$ dans $\left(g^{\prime} \backslash L\right)_{i}$, on a

$$
U(f, h)^{*}\left(u^{\prime}\right)=u_{-1}^{\prime}(1)=U^{\prime}\left(u^{\prime}\right),
$$

d'où l'assertion.

\subsection{Fonctorialité des morphismes associés aux triangles}

10.3.1. - Soit $g: K \rightarrow L$ un morphisme de complexes dirigés augmentés, où le complexe $L$ est décent. Formons le triangle

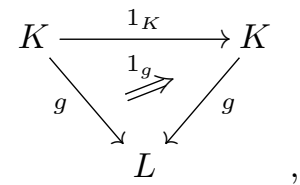

où $1_{g}$ désigne l'antihomotopie identité de $g$. À partir de ce triangle, en vertu de la proposition 10.2.2, on obtient un morphisme $\left(1_{K}, 1_{g}\right)^{*}: g \backslash L \rightarrow g \backslash L$.

Proposition 10.3.2. - On $a\left(1_{K}, 1_{g}\right)^{*}=1_{g \backslash L}$.

Démonstration. - Cela résulte immédiatement de la définition de $\left(1_{K}, 1_{g}\right)^{*}$.

10.3.3. - Considérons maintenant un diagramme

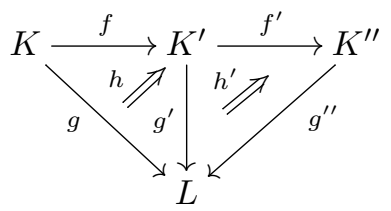


de complexes dirigés augmentés, où $h$ et $h^{\prime}$ sont des antihomotopies de $g$ vers $g^{\prime} f$ et de $g^{\prime}$ vers $g^{\prime \prime} f^{\prime}$ respectivement. On suppose toujours le complexe $L$ décent.

En composant ce diagramme, on obtient un triangle

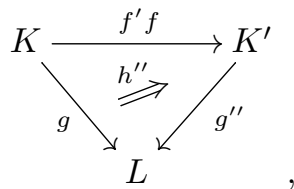

où $h^{\prime \prime}$ est l'antihomotopie $h^{\prime} f+h$ (voir les paragraphes 2.31 et 2.32) En vertu de la proposition 10.2 .2 , on obtient donc un triangle

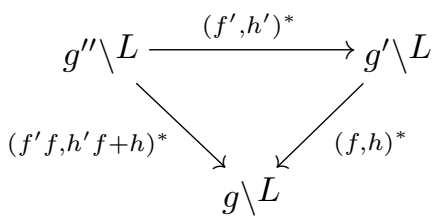

de complexes dirigés augmentés.

La proposition suivante affirme que ce triangle est commutatif.

Proposition 10.3.4. - On $a(f, h)^{*}\left(f^{\prime}, h^{\prime}\right)^{*}=\left(f^{\prime} f, h^{\prime} f+h\right)^{*}$.

Démonstration. - Soient $i \geqslant 0$ et $u^{\prime \prime}=\left(u_{j}^{\prime \prime}\right)_{j \geqslant-1}$ un élément de $\left(g^{\prime \prime} \backslash L\right)_{i}$. En posant $z=u_{-1}^{\prime \prime}(1)$ et en utilisant le fait que chacun des morphismes du triangle de l'énoncé est au-dessus de $L$, pour tout $j \geqslant-1$, on obtient

$$
\begin{aligned}
(f, h)_{i}^{*}\left(f^{\prime}, h^{\prime}\right)_{i}^{*}\left(u^{\prime \prime}\right)_{j} & =\left(f^{\prime}, h^{\prime}\right)_{i}^{*}\left(u^{\prime \prime}\right)_{j} f_{j}+e(z) h_{j} \\
& =\left(u_{j}^{\prime \prime} f_{j}^{\prime}+e(z) h_{j}^{\prime}\right) f_{j}+e(z) h_{j} \\
& =u_{j}^{\prime \prime} f_{j}^{\prime} f_{j}+e(z)\left(h_{j}^{\prime} f_{j}+h_{j}\right) \\
& =\left(f^{\prime} f, h^{\prime} f+h\right)_{i}^{*}\left(u^{\prime \prime}\right)_{j},
\end{aligned}
$$

d'où le résultat.

\subsection{Homotopie associée à un cône}

10.4.1. - Dans cette section, on fixe un diagramme

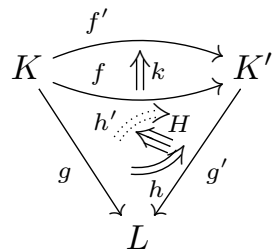


de complexes dirigés augmentés, où $h$ et $h^{\prime}$ sont des antihomotopies de source $g$ et de buts respectifs $g^{\prime} f$ et $g^{\prime} f^{\prime}, k$ est une antihomotopie de $f$ vers $f^{\prime}$ et $H$ est une 2 -antihomotopie (voir le paragraphe 2.28) de $g^{\prime} k+h$ vers $h^{\prime}$. On suppose le complexe $L$ décent.

À partir de ces données, en vertu de la proposition 10.2.2, on obtient deux morphismes

$$
(f, h)^{*},\left(f^{\prime}, h^{\prime}\right)^{*}: g^{\prime} \backslash L \rightarrow g \backslash L .
$$

On va définir une homotopie $(k, H)^{*}$ de $\left(f^{\prime}, h^{\prime}\right)^{*}$ vers $(f, h)^{*}$. Pour tout $i \geqslant 0$, on définit un morphisme $(k, H)_{i}^{*}:\left(g^{\prime} \backslash L\right)_{i} \rightarrow(g \backslash L)_{i+1}$ en posant, pour tout $u^{\prime}=\left(u_{j}\right)_{j \geqslant-1}$ dans $\left(g^{\prime} \backslash L\right)_{i}$ et tout $j \geqslant-1$,

$$
(k, H)_{i}^{*}\left(u^{\prime}\right)_{j}= \begin{cases}u_{j+1}^{\prime} k_{j}+e\left(u_{-1}^{\prime}(1)\right) \cdot H_{j} & \text { si } i=0, \\ u_{j+1}^{\prime} k_{j} & \text { sinon. }\end{cases}
$$

Comme dans la section 10.2, on étendra parfois l'augmentation $e$ de $L$ à tout élément homogène de $L$ en posant $e(z)=0$ si $z$ est un élément homogène de degré non nul. Avec cette convention, pour $i \geqslant 0$, on a

$$
(k, H)_{i}^{*}\left(u^{\prime}\right)_{j}=u_{j+1}^{\prime} k_{j}+e\left(u_{-1}^{\prime}(1)\right) \cdot H_{j} .
$$

On conviendra que, pour tout $i \leqslant-1$, on a

$$
H_{i}=0 .
$$

Pour $j=-1$, en vertu de cette convention ainsi que de nos précédentes conventions, on trouve $(k, H)_{i}^{*}\left(u^{\prime}\right)_{-1}=0$. Plus généralement, on a $(k, H)_{i}^{*}\left(u^{\prime}\right)_{j}=0$ pour tout $j \leqslant-1$ et l'égalité $(k, H)_{i}^{*}\left(u^{\prime}\right)_{j}=u_{j+1}^{\prime} k_{j}+e\left(u_{-1}^{\prime}(1)\right) . H_{j}$ est donc valable pour tout $j$ dans $\mathbb{Z}$.

Proposition 10.4.2. - Les morphismes $(k, H)_{i}^{*}:\left(g^{\prime} \backslash L\right)_{i} \rightarrow(g \backslash L)_{i+1}$, pour $i \geqslant 0$, définissent une homotopie de $\left(f^{\prime}, h^{\prime}\right)^{*}$ vers $(f, h)^{*}$.

Démonstration. - La compatibilité aux sous-monoïdes de positivité est évidente. Vérifions que $(k, H)^{*}$ est bien une homotopie de complexes de chaînes de $\left(f^{\prime}, h^{\prime}\right)^{*}$ vers $(f, h)^{*}$. Soient $i \geqslant 0$ et $u^{\prime}=\left(u_{j}^{\prime}\right)_{j \geqslant-1}$ dans $\left(g^{\prime} \backslash L\right)_{i}$. Si $i \geqslant 1$, pour tout $j \geqslant-1$, on a

$$
\begin{gathered}
\left(d_{i+1}(k, H)_{i}^{*}+(k, H)_{i-1}^{*} d_{i}\right)\left(u^{\prime}\right)_{j}=(-1)^{j+1}\left(d_{i+j+2}(k, H)_{i}^{*}\left(u^{\prime}\right)_{j}-(k, H)_{i}^{*}\left(u^{\prime}\right)_{j-1} d_{j}\right) \\
+\left(d_{i}\left(u^{\prime}\right)_{j+1} k_{j}+e\left(d_{i}\left(u^{\prime}\right)_{-1}(1)\right) H_{j}\right) \\
=(-1)^{j+1}\left(d_{i+j+2}(k, H)_{i}^{*}\left(u^{\prime}\right)_{j}-(k, H)_{i}^{*}\left(u^{\prime}\right)_{j-1} d_{j}\right) \\
\quad+d_{i}\left(u^{\prime}\right)_{j+1} k_{j} \\
\left(\text { puisque } e d_{i}=0 \text { pour tout } i \geqslant 1\right) \\
=(-1)^{j+1}\left(d_{i+j+2} u_{j+1}^{\prime} k_{j}-u_{j}^{\prime} k_{j-1} d_{j}\right) \\
+(-1)^{j+2}\left(d_{i+j+2} u_{j+1}^{\prime}-u_{j}^{\prime} d_{j+1}\right) k_{j}
\end{gathered}
$$




$$
\begin{aligned}
& =(-1)^{j+1} u_{j}^{\prime}\left(d_{j+1} k_{j}-k_{j-1} d_{j}\right) \\
& =-u_{j}^{\prime}\left(f_{j}^{\prime}-f_{j}\right) \\
& =u_{j}^{\prime} f_{j}-u_{j}^{\prime} f_{j}^{\prime} \\
& =\left((f, h)_{i}^{*}-\left(f^{\prime}, h^{\prime}\right)_{i}^{*}\right)\left(u^{\prime}\right)_{j} .
\end{aligned}
$$

Si maintenant $i=0$, de sorte qu'on a, pour tout $j$ dans $\mathbb{Z}$,

$$
d_{j+1} u_{j}^{\prime}-u_{j-1}^{\prime} d_{j}=(-1)^{j+1} e(z) g_{j}^{\prime},
$$

où on a posé $z=u_{-1}(1)$, alors, pour tout $j \geqslant-1$, on a

$$
\begin{aligned}
\left(d_{1}(k, H)_{0}^{*}\right)\left(u^{\prime}\right)_{j}= & (-1)^{j+1}\left(d_{j+2}(k, H)_{0}^{*}\left(u^{\prime}\right)_{j}-(k, H)_{0}^{*}\left(u^{\prime}\right)_{j-1} d_{j}\right) \\
= & (-1)^{j+1}\left[d_{j+2}\left(u_{j+1}^{\prime} k_{j}+e(z) H_{j}\right)-\left(u_{j}^{\prime} k_{j-1}+e(z) H_{j-1}\right) d_{j}\right] \\
= & (-1)^{j+1}\left[d_{j+2} u_{j+1}^{\prime} k_{j}+e(z)\left(d_{j+2} H_{j}-H_{j-1} d_{j}\right)-u_{j}^{\prime} k_{j-1} d_{j}\right] \\
= & (-1)^{j+1}\left[\left(u_{j}^{\prime} d_{j+1}+(-1)^{j+2} e(z) g_{j+1}^{\prime}\right) k_{j}\right. \\
& \left.\quad+(-1)^{j} e(z)\left(h_{j}^{\prime}-\left(g_{j+1}^{\prime} k_{j}+h_{j}\right)\right)-u_{j}^{\prime} k_{j-1} d_{j}\right] \\
= & (-1)^{j+1}\left[\left(u_{j}^{\prime} d_{j+1} k_{j}-u_{j}^{\prime} k_{j-1} d_{j}+(-1)^{j} e(z)\left(h_{j}^{\prime}-h_{j}\right)\right]\right. \\
= & (-1)^{j+1} u_{j}^{\prime}\left(d_{j+1} k_{j}-k_{j-1} d_{j}\right)-e(z)\left(h_{j}^{\prime}-h_{j}\right) \\
= & -u_{j}^{\prime}\left(f_{j}^{\prime}-f_{j}\right)-e(z)\left(h_{j}^{\prime}-h_{j}\right) \\
= & \left(u_{j}^{\prime} f_{j}+e(z) h_{j}\right)-\left(u_{j}^{\prime} f_{j}^{\prime}+e(z) h_{j}^{\prime}\right) \\
= & \left((f, h)_{0}^{*}-\left(f^{\prime}, h^{\prime}\right)_{0}^{*}\right)\left(u^{\prime}\right)_{j},
\end{aligned}
$$

d'où le résultat.

Proposition 10.4.3. - L'homotopie $(k, H)^{*}$ de $\left(f^{\prime}, h^{\prime}\right)^{*}$ vers $(f, h)^{*}$, qui sont deux morphismes de $g^{\prime} \backslash L$ vers $g \backslash L$ au-dessus de $L$, est au-dessus de $L$ au sens où, en notant $U: g \backslash L \rightarrow L$ et $U^{\prime}: g^{\prime} \backslash L \rightarrow L$ les morphismes d'oubli, on a $U(k, H)^{*}=1_{U^{\prime}}$.

Démonstration. - Pour tout $i \geqslant 0$ et $u^{\prime}=\left(u_{j}^{\prime}\right)_{j \geqslant-1}$ dans $\left(g^{\prime} \backslash L\right)_{i}$, on a

$$
U(k, H)^{*}\left(u^{\prime}\right)=\left((k, H)^{*}\left(u^{\prime}\right)_{-1}\right)(1)=0=1_{U^{\prime}}\left(u^{\prime}\right),
$$

d'où l'assertion.

\subsection{Fonctorialités des morphismes associés aux cônes}

10.5.1. - Considérons un diagramme

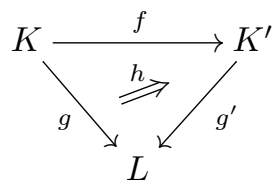


de complexes dirigés augmentés, où $h$ est une antihomotopie de $g$ vers $g^{\prime} f$. On suppose le complexe $L$ décent.

À partir de ce diagramme, on peut former un diagramme

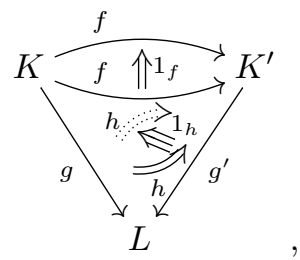

où $1_{f}$ désigne l'antihomotopie identité de $f$ et $1_{h}$ la 2 -antihomotopie identité de $h$ (voir le paragraphe 2.30). On obtient donc, en vertu de la proposition 10.4.2, une homotopie $\left(1_{f}, 1_{h}\right)^{*}$ de $(f, h)^{*}$ vers $(f, h)^{*}$.

Proposition 10.5.2. - On a $\left(1_{f}, 1_{h}\right)^{*}=1_{(f, h)^{*}}$.

Démonstration. - Cela résulte immédiatement de la définition de $\left(1_{f}, 1_{h}\right)^{*}$.

10.5.3. - Considérons maintenant un diagramme

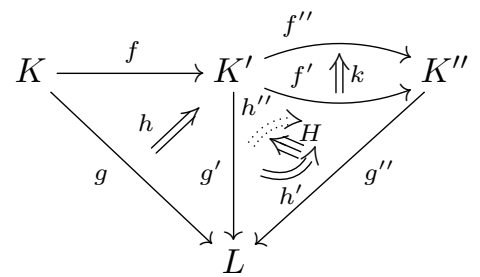

de complexes dirigés augmentés, où $h, h^{\prime}$ et $h^{\prime \prime}$ sont des antihomotopies de $g$ vers $g^{\prime} f$, de $g^{\prime}$ vers $g^{\prime \prime} f^{\prime}$ et de $g^{\prime}$ vers $g^{\prime \prime} f^{\prime \prime}$ respectivement, $k$ est une antihomotopie de $f^{\prime}$ vers $f^{\prime \prime}$ et $H$ est une 2 -antihomotopie de $g^{\prime \prime} k+h^{\prime}$ vers $h^{\prime \prime}$. On suppose toujours le complexe $L$ décent.

À partir de ces données, en vertu des propositions 10.2 .2 et 10.4.2, on obtient un diagramme

$$
g^{\prime \prime} \backslash L \underset{\left(f^{\prime}, h^{\prime}\right)^{*}}{\stackrel{\left(f^{\prime \prime}, h^{\prime \prime}\right)^{*}}{\Downarrow_{(k, H)^{*}}}} g^{\prime} \backslash L \stackrel{(f, h)^{*}}{\longrightarrow} g \backslash L .
$$

En vertu de la proposition 10.3.4, on a

$$
(f, h)^{*}\left(f^{\prime \prime}, h^{\prime \prime}\right)^{*}=\left(f^{\prime \prime} f, h^{\prime \prime} f+h\right)^{*} \quad \text { et } \quad(f, h)^{*}\left(f^{\prime}, h^{\prime}\right)^{*}=\left(f^{\prime} f, h^{\prime} f+h\right)^{*},
$$

et en composant ce diagramme on obtient donc une homotopie $(f, h)^{*}(k, H)^{*}$ de $\left(f^{\prime \prime} f, h^{\prime \prime} f+h\right)^{*}$ vers $\left(f^{\prime} f, h^{\prime} f+h\right)^{*}$. 
Par ailleurs, en composant le diagramme de départ, on obtient un diagramme

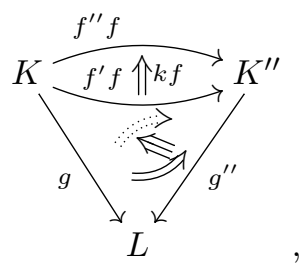

où la 2-flèche courbée de devant est l'antihomotopie $h^{\prime} f+h$, la 2-flèche courbée de derrière, en pointillé, l'antihomotopie $h^{\prime \prime} f+h$ et la 3 -flèche est la 2-antihomotopie $H f+1_{h}$ (voir les paragraphes 2.31 et 2.33) de $\left(g^{\prime \prime} k+h^{\prime}\right) f+h=g^{\prime \prime}(k f)+\left(h^{\prime} f+h\right)$ vers $h^{\prime \prime} f+h$. Pour tout $n \geqslant 0$, on a $\left(H f+1_{h}\right)_{n}=(H f)_{n}$ et on désignera plus simplement cette 2-antihomotopie par $H f$. On obtient donc, toujours en vertu de la proposition 10.4.2, une homotopie $(k f, H f)^{*}$ de $\left(f^{\prime \prime} f, h^{\prime \prime} f+h\right)^{*}$ vers $\left(f^{\prime} f, h^{\prime} f+h\right)^{*}$.

Proposition 10.5.4. - On $a(f, h)^{*}(k, H)^{*}=(k f, H f)^{*}$.

Démonstration. - Soient $i \geqslant 0$ et $u^{\prime \prime}=\left(u_{j}^{\prime \prime}\right)_{j \geqslant-1}$ un élément de $\left(g^{\prime \prime} \backslash L\right)_{i}$. Pour tout $j \geqslant-1$, en posant $z=u_{-1}^{\prime \prime}(1)$, on a

$$
\begin{aligned}
\left((f, h)^{*}(k, H)^{*}\right)_{i}\left(u^{\prime \prime}\right)_{j} & =(f, h)_{i+1}^{*}(k, H)_{i}^{*}\left(u^{\prime \prime}\right)_{j} \\
& =(k, H)_{i}^{*}\left(u^{\prime \prime}\right)_{j} f_{j} \\
& =u_{j+1}^{\prime \prime} k_{j} f_{j}+e(z) H_{j} f_{j} \\
& =(k f, H f)_{i}^{*}\left(u^{\prime \prime}\right)_{j},
\end{aligned}
$$

d'où le résultat.

10.5.5. — De même, considérons un diagramme

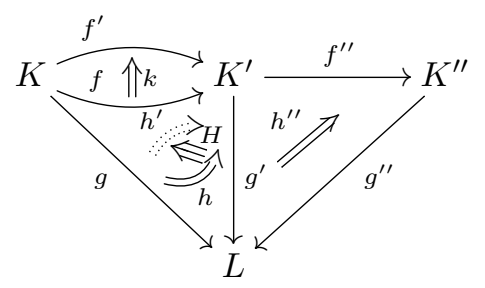

de complexes dirigés augmentés, où $h, h^{\prime}$ et $h^{\prime \prime}$ sont des antihomotopies de $g$ vers $g^{\prime} f$, de $g$ vers $g^{\prime} f^{\prime}$ et de $g^{\prime}$ vers $g^{\prime \prime} f^{\prime \prime}$ respectivement, $k$ est une antihomotopie de $f$ vers $f^{\prime}$ et $H$ est une 2-antihomotopie de $g^{\prime} k+h$ vers $h^{\prime}$. On suppose toujours le complexe $L$ décent. 
À partir de ces données, on obtient un diagramme

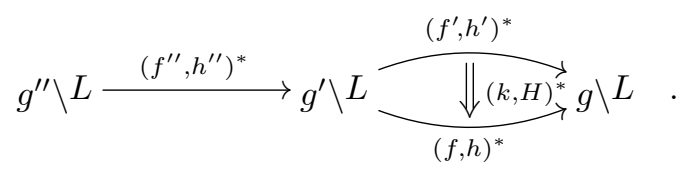

En vertu de la proposition 10.3.4, on a

$$
\left(f^{\prime}, h^{\prime}\right)^{*}\left(f^{\prime \prime}, h^{\prime \prime}\right)^{*}=\left(f^{\prime \prime} f^{\prime}, h^{\prime \prime} f^{\prime}+h^{\prime}\right)^{*} \text { et }(f, h)^{*}\left(f^{\prime \prime}, h^{\prime \prime}\right)^{*}=\left(f^{\prime \prime} f, h^{\prime \prime} f+h\right)^{*},
$$

et en composant ce diagramme on obtient donc une homotopie $(k, H)^{*}\left(f^{\prime \prime}, h^{\prime \prime}\right)^{*}$ de $\left(f^{\prime \prime} f^{\prime}, h^{\prime \prime} f^{\prime}+h^{\prime}\right)^{*}$ vers $\left(f^{\prime \prime} f, h^{\prime \prime} f+h\right)^{*}$.

Par ailleurs, en composant le diagramme de départ, on obtient un diagramme

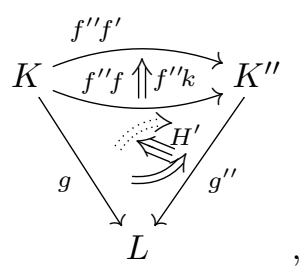

où la 2-flèche courbée de devant est l'antihomotopie $h^{\prime \prime} f+h$, la 2-flèche courbée de derrière, en pointillé, l'antihomotopie $h^{\prime \prime} f^{\prime}+h^{\prime}$ et la 3-flèche est la 2-antihomotopie $H^{\prime}=\left(1_{h^{\prime \prime} f^{\prime}}+H\right)+\left(h^{\prime \prime} k+1_{h}\right)$ (où les " $+»$ de gauche et de droite désignent l'opération définie au paragraphe 2.33, celui du milieu celle définie au paragraphe 2.32 et $h^{\prime \prime} k^{\prime}$ la 2-antihomotopie du paragraphe 2.34). Ceci a bien un sens puisque, $h^{\prime \prime} k+1_{h}$ étant une 2-antihomotopie de $\left(\left(g^{\prime \prime} f^{\prime \prime}\right) k+h^{\prime \prime} f\right)+h$ vers $\left(h^{\prime \prime} f+g^{\prime} k\right)+h$ et $1_{h^{\prime \prime} f^{\prime}}+H$ une 2-antihomotopie de $h^{\prime \prime} f^{\prime}+\left(g^{\prime} k+h\right)$ vers $h^{\prime \prime} f^{\prime}+h^{\prime}, H^{\prime}$ est une 2-antihomotopie de $g^{\prime \prime}\left(f^{\prime \prime} k\right)+\left(h^{\prime \prime} f+h\right)$ vers $h^{\prime \prime} f^{\prime}+h^{\prime}$. On désignera plus simplement $H^{\prime}$ par $H+h^{\prime \prime} k$. On obtient donc une homotopie $\left(f^{\prime \prime} k, H+h^{\prime \prime} k\right)^{*}$ de $\left(f^{\prime \prime} f^{\prime}, h^{\prime \prime} f^{\prime}+h^{\prime}\right)^{*}$ vers $\left(f^{\prime \prime} f, h^{\prime \prime} f+h\right)^{*}$.

Proposition 10.5.6. - On a $(k, H)^{*}\left(f^{\prime \prime}, h^{\prime \prime}\right)^{*}=\left(f^{\prime \prime} k, H+h^{\prime \prime} k\right)^{*}$.

Démonstration. - Soient $i \geqslant 0$ et $u^{\prime \prime}=\left(u_{j}^{\prime \prime}\right)_{j \geqslant-1}$ un élément de $\left(g^{\prime \prime} \backslash L\right)_{i}$. Pour tout $j \geqslant-1$, en posant $z=u_{-1}^{\prime \prime}(1)$, on a

$$
\begin{aligned}
\left((k, H)^{*}\left(f^{\prime \prime}, h^{\prime \prime}\right)^{*}\right)_{i}\left(u^{\prime \prime}\right)_{j} & =(k, H)_{i}^{*}\left(f^{\prime \prime}, h^{\prime \prime}\right)_{i}^{*}\left(u^{\prime \prime}\right)_{j} \\
& =\left(f^{\prime \prime}, h^{\prime \prime}\right)_{i}^{*}\left(u^{\prime \prime}\right)_{j+1} k_{j}+e(z) H_{j} \\
& =\left(u_{j+1}^{\prime \prime} f_{j+1}^{\prime \prime}+e(z) h_{j+1}^{\prime \prime}\right) k_{j}+e(z) H_{j} \\
& =u_{j+1}^{\prime \prime} f_{j+1}^{\prime \prime} k_{j}+e(z)\left(H_{j}+h_{j+1}^{\prime \prime} k_{j}\right) \\
& =\left(f^{\prime \prime} k, H+h^{\prime \prime} k\right)_{i}^{*}\left(u^{\prime \prime}\right)_{j},
\end{aligned}
$$

d'où le résultat. 
10.5.7. - Enfin, considérons un diagramme

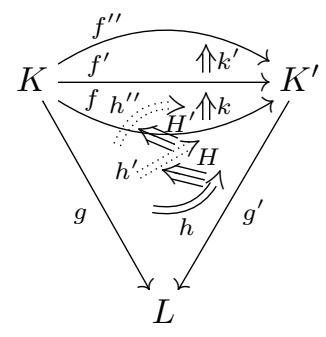

de complexes dirigés augmentés, où $h, h^{\prime}$ et $h^{\prime \prime}$ sont des antihomotopies de $g$ vers $g^{\prime} f$, $g^{\prime} f^{\prime}$ et $g^{\prime} f^{\prime \prime}$ respectivement, $k$ et $k^{\prime}$ sont des antihomotopies de $f$ vers $f^{\prime}$ et $f^{\prime}$ vers $f^{\prime \prime}$ respectivement et $H$ et $H^{\prime}$ sont des 2-antihomotopies de $g^{\prime} k+h$ vers $h^{\prime}$ et de $g^{\prime} k^{\prime}+h^{\prime}$ vers $h^{\prime \prime}$ respectivement. On suppose toujours le complexe $L$ décent.

À partir de ces données, en vertu de la proposition 10.4.2, on obtient un diagramme

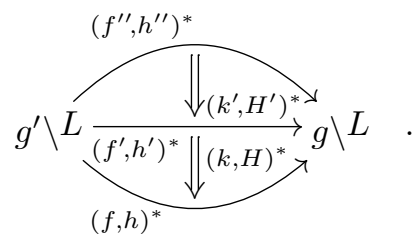

En composant ce diagramme on obtient une homotopie $(k, H)^{*}+\left(k^{\prime}, H^{\prime}\right)^{*} \operatorname{de}\left(f^{\prime \prime}, h^{\prime \prime}\right)^{*}$ vers $(f, h)^{*}$.

Par ailleurs, en composant le diagramme de départ, on obtient un diagramme

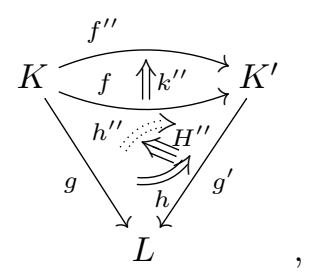

où $k^{\prime \prime}$ désigne l'antihomotopie $k^{\prime}+k$ et $H^{\prime \prime}$ la 2-antihomotopie $H^{\prime}+\left(1_{g^{\prime}} k^{\prime}+H\right)$ (où le " + » de gauche désigne l'opération définie au paragraphe 2.32 et celui de droite celle définie au paragraphe 2.33). Ceci a un sens puisque, $1_{g^{\prime} k^{\prime}}+H$ étant une 2-antihomotopie de $g^{\prime} k^{\prime}+\left(g^{\prime} k+h\right)$ vers $g^{\prime} k^{\prime}+h^{\prime}$ et $H^{\prime}$ une 2-antihomotopie de $g^{\prime} k^{\prime}+h^{\prime}$ vers $h^{\prime \prime}, H^{\prime \prime}$ est une 2-antihomotopie de $g^{\prime}\left(k^{\prime}+k\right)+h$ vers $h^{\prime \prime}$. On désignera plus simplement $H^{\prime \prime}$ par $H^{\prime}+H$. On obtient donc, toujours en vertu de la proposition 10.4.2, une homotopie $\left(H^{\prime}+H, k^{\prime}+k\right)^{*}$ de $\left(f^{\prime \prime}, h^{\prime \prime}\right)^{*}$ vers $(f, h)^{*}$.

Proposition 10.5.8. - On a $(k, H)^{*}+\left(k^{\prime}, H^{\prime}\right)^{*}=\left(k^{\prime}+k, H^{\prime}+H\right)^{*}$. 
Démonstration. - Soient $i \geqslant 0$ et $u^{\prime}=\left(u_{j}^{\prime}\right)_{j \geqslant-1}$ un élément de $\left(g^{\prime} \backslash L\right)_{i}$. Pour tout $j \geqslant-1$, en posant $z=u_{-1}^{\prime}(1)$, on a

$$
\begin{aligned}
\left((k, H)^{*}+\left(k^{\prime}, H^{\prime}\right)^{*}\right)_{i}\left(u^{\prime}\right)_{j} & =(k, H)_{i}^{*}\left(u^{\prime}\right)_{j}+\left(k^{\prime}, H^{\prime}\right)_{i}^{*}\left(u^{\prime}\right)_{j} \\
& =\left(u_{j+1}^{\prime} k_{j}+e(z) H_{j}\right)+\left(u_{j+1}^{\prime} k_{j}^{\prime}+e(z) H_{j}^{\prime}\right) \\
& =u_{j+1}^{\prime}\left(k_{j}^{\prime}+k_{j}\right)+e(z)\left(H_{j}^{\prime}+H_{j}\right) \\
& =\left(k^{\prime}+k, H^{\prime}+H\right)_{i}^{*}\left(u^{\prime}\right)_{j}
\end{aligned}
$$

d'où le résultat. 



\section{CHAPITRE 11}

\section{FONCTORIALITÉS DES TRANCHES : RÉSULTATS POUR LES $\infty$-CATÉGORIES}

Fixons $C$ une $\infty$-catégorie. Les tranches définies dans le chapitre 6 permettent d'associer à tout $\infty$-foncteur $u: A \rightarrow C$ une $\infty$-catégorie $u \backslash C$. Le but de ce chapitre est de montrer, sous des hypothèses techniques que l'on conjecture être superflues, que cette correspondance s'étend en une correspondance

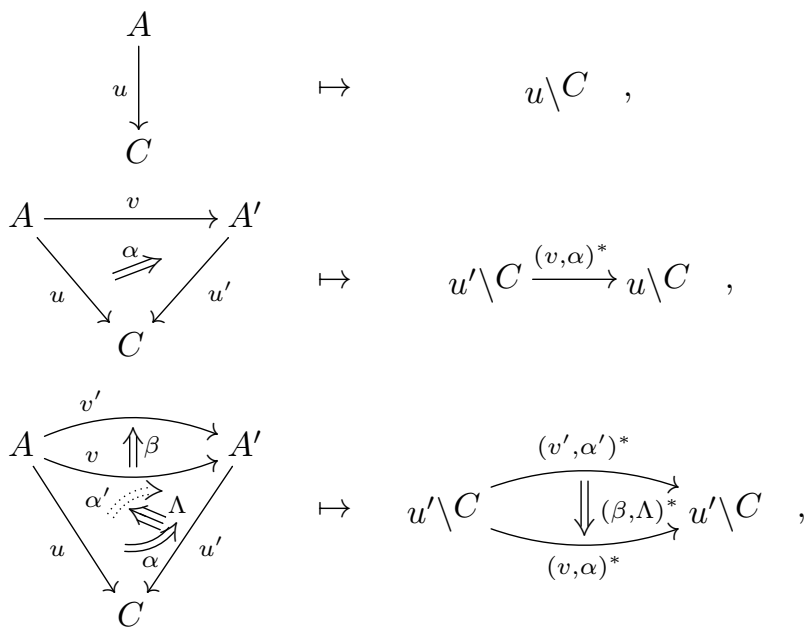

où les 2-flèches et 3-flèches à gauche du signe « $\mapsto »$ sont des transformations lax et des 2-transformations lax respectivement, et la 2-flèche à droite de ce signe est une transformation oplax. Plus généralement, on conjecture que cette correspondance s'étend en un $\infty$-foncteur de Gray (voir la conjecture C.27).

\subsection{Un lemme pour se ramener aux complexes dirigés augmentés}

Le but de cette section est de démontrer un lemme technique qui nous permettra de ramener les fonctorialités des tranches pour les $\infty$-catégories aux fonctorialités 
analogues pour les complexes dirigés augmentés qu'on a établies dans le chapitre précédent.

11.1.1. - Dans cette section, et uniquement dans celle-ci, nous utiliserons la théorie du produit tensoriel de Gray telle qu'elle est rappelée dans l'appendice A. Si $C$ et $D$ sont deux $\infty$-catégories, nous noterons $C \otimes D$ leur produit tensoriel (de Gray). Rappelons (voir l'appendice B et plus particulièrement le corollaire B.2.6) que la donnée d'une transformation oplax entre deux $\infty$-foncteurs de $C$ vers $D$ correspond à celle qu'un $\infty$-foncteur $\mathrm{D}_{1} \otimes C \rightarrow D$, où $\mathrm{D}_{1}$ désigne la $\infty$-catégorie du paragraphe 4.1.

11.1.2. - Dans cette section, on fixe

$$
K \stackrel{g}{\longrightarrow} L \stackrel{g^{\prime}}{\longleftarrow} K^{\prime}
$$

deux morphismes de complexes dirigés augmentés et $S$ un complexe dirigé augmenté.

À partir de ces données, on définit deux foncteurs de $L \backslash \mathcal{C}_{\mathrm{da}}$ vers $\mathcal{C}_{\mathrm{da}}$ :

$$
(M, L \stackrel{a}{\rightarrow} M) \mapsto S \otimes a g^{\prime} \backslash M \quad \text { et } \quad(M, L \stackrel{a}{\rightarrow} M) \mapsto a g \backslash M ;
$$

ainsi que deux foncteurs de $\nu(L) \backslash \infty$-Cat vers $\infty$-Cat :

$$
(C, \nu(L) \stackrel{b}{\rightarrow} C) \mapsto \nu(S) \otimes b \nu\left(g^{\prime}\right) \backslash C \quad \text { et } \quad(C, \nu(L) \stackrel{b}{\rightarrow} C) \mapsto b \nu(g) \backslash C .
$$

Lemme 11.1.3. - On suppose que $K, K^{\prime}, L$ et $S$ sont des complexes de Steiner forts et que $g^{\prime}$ est une inclusion rigide ordonnée (voir le paragraphe 3.16). Alors, pour tout morphisme

$$
\alpha_{(M, a)}: S \otimes a g^{\prime} \backslash M \rightarrow a g \backslash M,
$$

naturel en $(M, a)$ dans $L \backslash \mathcal{C}_{\mathrm{da}}$, il existe un et un seul $\infty$-foncteur

$$
\beta_{(C, b)}: \nu(S) \otimes b \nu\left(g^{\prime}\right) \backslash C \rightarrow b \nu(g) \backslash C,
$$

naturel en $(C, b)$ dans $\nu(L) \backslash \infty$-Cat, tel que, pour tout complexe de Steiner fort $M$ muni d'un morphisme $a: L \rightarrow M$, le diagramme

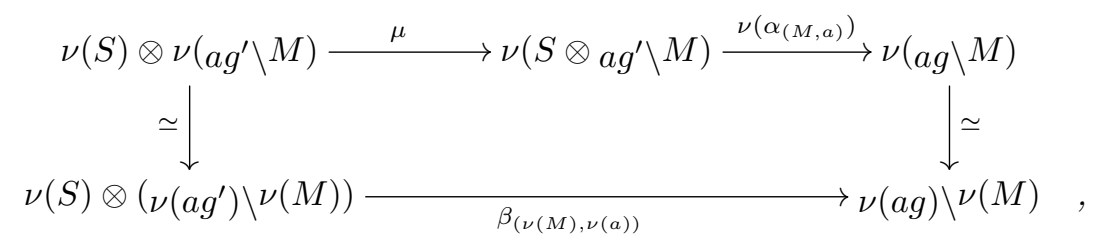

où les isomorphismes verticaux proviennent de ceux de la proposition 10.1 .9 et $\mu$ désigne la contrainte du foncteur monoïdal lax $\nu$ (voir la proposition A.19), soit commutatif. 
Démonstration. - Par adjonction, la donnée d'un $\infty$-foncteur

$$
\beta_{(C, b)}: \nu(S) \otimes b \nu\left(g^{\prime}\right) \backslash C \rightarrow b \nu(g) \backslash C
$$

est équivalente à celle d'un $\infty$-foncteur

$$
b \nu\left(g^{\prime}\right) \backslash C \rightarrow \underline{\operatorname{Hom}}_{\text {lax }}(\nu(S), b \nu(g) \backslash C),
$$

où $\underline{\text { Hom }}_{\text {lax }}$ désigne le Hom interne à gauche du produit tensoriel, introduit au paragraphe A.18. Par ailleurs, la sous-catégorie pleine de $\infty$-C at formée des $\infty$-catégories de Steiner fortes étant dense dans $\infty$ - $\mathcal{C}$ at (car elle contient $\Theta$, elle-même dense dans $\infty$-Cat d'après la proposition 4.6), la donnée d'un tel $\infty$-foncteur est équivalente à celle d'une application

$$
\operatorname{Hom}_{\infty-\mathcal{C} a t}\left(\nu(T), b \nu\left(g^{\prime}\right) \backslash C\right) \rightarrow \operatorname{Hom}_{\infty-\mathcal{C} a t}\left(\nu(T), \underline{\operatorname{Hom}}_{l a x}(\nu(S), b \nu(g) \backslash C)\right),
$$

naturelle en $T$ dans la sous-catégorie pleine $\mathcal{S} t_{\mathrm{f}}$ de $\mathcal{C}_{\text {da }}$ formée des complexes de Steiner forts (en vertu de la pleine fidélité du foncteur $\nu$ restreint aux complexes de Steiner forts, voir le théorème 2.11). Par adjonction, la donnée d'une telle application correspond à celle d'une application

$$
\operatorname{Hom}_{\infty-\mathcal{C} a t}\left(\nu(T), b \nu\left(g^{\prime}\right) \backslash C\right) \rightarrow \operatorname{Hom}_{\infty-\mathcal{C} a t}(\nu(S) \otimes \nu(T), b \nu(g) \backslash C),
$$

ou, de nouveau par adjonction (voir le paragraphe 6.31), à celle d'une application

$$
\begin{aligned}
\operatorname{Hom}_{\nu\left(K^{\prime}\right) \backslash \infty-\mathcal{C} a t}\left(\left(\nu\left(K^{\prime}\right) \star \nu(T), \iota_{1}\right),\left(C, b \nu\left(g^{\prime}\right)\right)\right) \\
\rightarrow \operatorname{Hom}_{\nu(K) \backslash \infty-\mathcal{C} a t}\left(\left(\nu(K) \star(\nu(S) \otimes \nu(T)), \iota_{1}\right),(C, b \nu(g))\right),
\end{aligned}
$$

ou encore, en vertu de la propriété universelle de la somme amalgamée, à celle d'une application

$$
\begin{aligned}
\operatorname{Hom}_{\nu(L) \backslash \infty-\mathcal{C} a t} & \left(\left(\nu(L) \amalg_{\nu\left(K^{\prime}\right)}\left(\nu\left(K^{\prime}\right) \star \nu(T)\right), \varepsilon_{1}\right),(C, b)\right) \\
\rightarrow & \operatorname{Hom}_{\nu(L) \backslash \infty-\mathcal{C} a t}\left(\left(\nu(L) \amalg_{\nu(K)}(\nu(K) \star(\nu(S) \otimes \nu(T))), \varepsilon_{1}\right),(C, b)\right),
\end{aligned}
$$

où $\varepsilon_{1}$ désigne la première inclusion canonique dans une somme amalgamée. Puisqu'on demande que le $\infty$-foncteur $\beta_{(C, b)}$ soit naturel en $(C, b)$ dans $\nu(L) \backslash \infty$-C at, en vertu du lemme de Yoneda, la donnée de l'application ci-dessus pour tout $(C, b)$ est équivalente à celle d'un $\infty$-foncteur

$$
\nu(L) \amalg_{\nu(K)}(\nu(K) \star(\nu(S) \otimes \nu(T))) \rightarrow \nu(L) \amalg_{\nu\left(K^{\prime}\right)}\left(\nu\left(K^{\prime}\right) \star \nu(T)\right)
$$

au-dessous de $\nu(L)$, naturel en $T$ dans $\mathcal{S} t_{\mathrm{f}}$, ou, ce qui revient au même, d'un $\infty$-foncteur

$$
\nu(K) \star(\nu(S) \otimes \nu(T)) \rightarrow \nu(L) \amalg_{\nu\left(K^{\prime}\right)}\left(\nu\left(K^{\prime}\right) \star \nu(T)\right)
$$


faisant commuter le carré

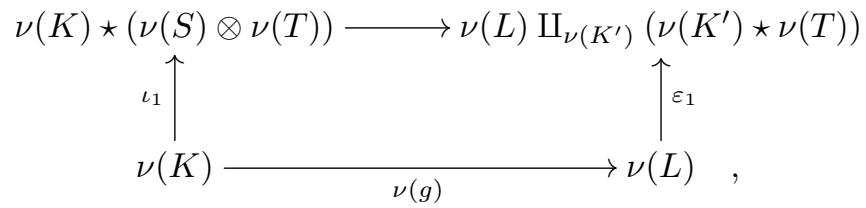

toujours naturel en $T$ dans $\mathcal{S} t_{\mathrm{f}}$.

En vertu de la stabilité des complexes de Steiner forts par produit tensoriel et joint (proposition A.3 et corollaire 6.21) et de la compatibilité du foncteur $\nu$ à ces opérations (théorèmes A.15 et 6.29), le complexe $K \star(S \otimes T)$ est un complexe de Steiner fort et on a un isomorphisme canonique

$$
\nu(K) \star(\nu(S) \otimes \nu(T)) \simeq \nu(K \star(S \otimes T)) .
$$

De même, le complexe $K^{\prime} \star T$ est un complexe de Steiner fort et on a un isomorphisme canonique

$$
\nu\left(K^{\prime}\right) \star \nu(T) \simeq \nu\left(K^{\prime} \star T\right) .
$$

De plus, puisque, d'une part, le morphisme $g^{\prime}: K^{\prime} \rightarrow L$ est une inclusion rigide ordonnée par hypothèse et, d'autre part, le morphisme $\iota_{1}: K^{\prime} \rightarrow K^{\prime} \star T$ est une inclusion rigide ordonnée en vertu du corollaire 6.21, le théorème 3.19 entraîne que la somme amalgamée $L \amalg_{K^{\prime}}\left(K^{\prime} \star T\right)$ est un complexe de Steiner fort et qu'on a un isomorphisme canonique

$$
\nu(L) \amalg_{\nu\left(K^{\prime}\right)}\left(\nu\left(K^{\prime}\right) \star \nu(T)\right) \simeq \nu\left(L \amalg_{K^{\prime}}\left(K^{\prime} \star T\right)\right) .
$$

Ainsi, puisque le foncteur $\nu$ restreint aux complexes de Steiner forts est pleinement fidèle, la donnée du $\infty$-foncteur $\beta_{(C, b)}$, naturel en $(C, b)$ dans $\nu(L) \backslash \infty$-Cat, est équivalente à celle d'un morphisme

$$
\alpha_{T}^{\prime}: K \star(S \otimes T) \rightarrow L \amalg_{K^{\prime}}\left(K^{\prime} \star T\right),
$$

naturel en $T$ dans $\mathcal{S} t_{\mathrm{f}}$, faisant commuter le carré

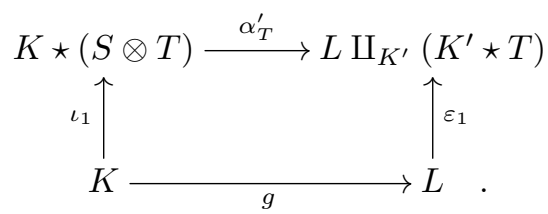

Nous allons montrer qu'un morphisme

$$
\alpha_{(M, a)}: S \otimes a g^{\prime} \backslash M \rightarrow a g \backslash M,
$$

naturel en $(M, a)$ dans $L \backslash \mathcal{C}_{\mathrm{da}}$, permet de définir un morphisme $\alpha_{T}^{\prime}$ comme ci-dessus. En effet, par adjonction, le morphisme $\alpha_{(M, a)}$ correspond à un morphisme

$$
a g^{\prime} \backslash M \rightarrow \underline{\operatorname{Hom}_{\mathcal{C}_{\mathrm{da}}}^{\mathrm{g}}}(S, a g \backslash M),
$$


où $\underline{\mathrm{Hom}}_{\mathcal{C}_{\mathrm{da}}}^{\mathrm{g}}$ désigne le Hom interne à gauche du produit tensoriel de $\mathcal{C}_{\mathrm{da}}$ (qui existe en vertu du paragraphe 6.2). On dispose donc d'une application

$$
\operatorname{Hom}_{\mathcal{C}_{\mathrm{da}}}\left(T, a g^{\prime} \backslash M\right) \rightarrow \operatorname{Hom}_{\mathcal{C}_{\mathrm{da}}}\left(T, \underline{\operatorname{Hom}}_{\mathcal{C}_{\mathrm{da}}^{\mathrm{g}}}^{\mathrm{g}}(S, a g \backslash M)\right),
$$

naturelle en $T$ dans $\mathcal{C}_{\text {da }}$, ainsi qu'en $(M, a)$ dans $L \backslash \mathcal{C}_{\text {da }}$. Cette application correspond, par adjonction, à une application

$$
\operatorname{Hom}_{\mathcal{C}_{\mathrm{da}}}\left(T, a g^{\prime} \backslash M\right) \rightarrow \operatorname{Hom}_{\mathcal{C}_{\mathrm{da}}}(S \otimes T, a g \backslash M),
$$

ou, de nouveau par adjonction (voir la proposition 10.1.4), à une application

$$
\operatorname{Hom}_{K^{\prime} \backslash \mathcal{C}_{\mathrm{da}}}\left(\left(K^{\prime} \star T, \iota_{1}\right),\left(M, a g^{\prime}\right)\right) \rightarrow \operatorname{Hom}_{K \backslash \mathcal{C}_{\mathrm{da}}}\left(\left(K \star(S \otimes T), \iota_{1}\right),(M, a g)\right),
$$

ou encore, en vertu de la propriété universelle de la somme amalgamée, à celle d'une application

$$
\begin{aligned}
\operatorname{Hom}_{L \backslash \mathcal{C}_{\mathrm{da}}}\left(\left(L \amalg_{K^{\prime}}\left(K^{\prime} \star T\right), \varepsilon_{1}\right),(M, a)\right) \\
\quad \rightarrow \operatorname{Hom}_{L \backslash \mathcal{C}_{\mathrm{da}}}\left(\left(L \amalg_{K}(K \star(S \otimes T)), \varepsilon_{1}\right),(M, a)\right) .
\end{aligned}
$$

Ainsi, le lemme de Yoneda fournit un morphisme

$$
L \amalg_{K}(K \star(S \otimes T)) \rightarrow L \amalg_{K^{\prime}}\left(K^{\prime} \star T\right)
$$

au-dessous de $L$ ou, ce qui revient au même, un morphisme

$$
\alpha_{T}^{\prime}: K \star(S \otimes T) \rightarrow L \amalg_{K^{\prime}}\left(K^{\prime} \star T\right)
$$

faisant commuter le carré

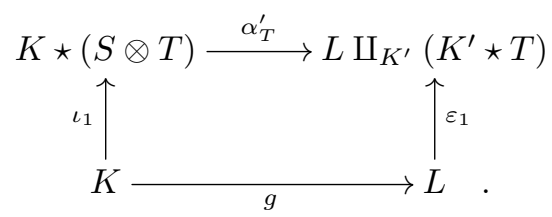

Ce morphisme est naturel en $T$ dans $\mathcal{C}_{\text {da }}$.

Ainsi, on a associé à tout morphisme $\alpha_{(M, a)}$, naturel en $(M, a)$ dans $L \backslash \mathcal{C}_{\text {da }}$, un morphisme $\alpha_{T}^{\prime}$ naturel en $T$ dans $\mathcal{S} t_{\mathrm{f}}$ (et même dans $\mathcal{C}_{\mathrm{da}}$ ). Or, on a montré que la donnée d'un tel $\alpha_{T}^{\prime}$ est équivalente à celle d'un $\infty$-foncteur $\beta_{(C, b)}$, naturel en $(C, b)$ dans $\nu(L) \backslash \infty$ - $\mathcal{C}$ at. On vérifie que la manière dont est défini $\beta_{(C, b)}$ à partir de $\alpha_{T}^{\prime}$, lui-même défini à partir de $\alpha_{(M, a)}$, s'exprime par la commutativité du diagramme de l'énoncé, d'où le résultat.

Remarque 11.1.4. - La construction du lemme précédent est naturelle en $S$ au sens suivant. Si

$$
\alpha_{1}: S_{1} \otimes a g^{\prime} \backslash M \rightarrow a g \backslash M \quad \text { et } \quad \alpha_{2}: S_{2} \otimes a g^{\prime} \backslash M \rightarrow a g \backslash M
$$


sont deux morphismes comme dans l'énoncé du lemme (en particulier, $S_{1}$ et $S_{2}$ sont des complexes de Steiner forts) et que $f: S_{1} \rightarrow S_{2}$ est un morphisme de complexes dirigés augmentés rendant le diagramme

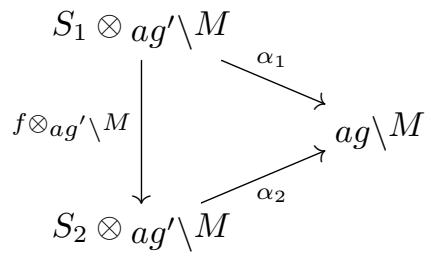

commutatif, alors le diagramme

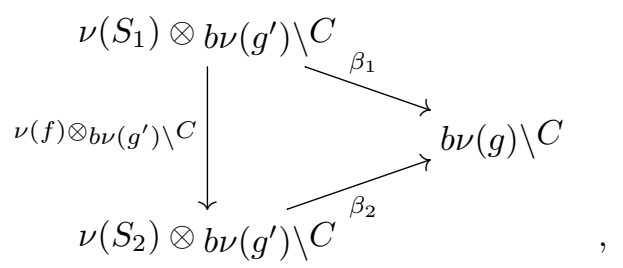

où $\beta_{1}$ et $\beta_{2}$ sont les $\infty$-foncteurs associés par le lemme à $\alpha_{1}$ et $\alpha_{2}$ respectivement, est également commutatif. Cela résulte immédiatement de la propriété d'unicité du $\infty$-foncteur $\beta_{1}$ donnée par le lemme.

Les deux énoncés qui suivent sont des reformulations de ce lemme dans les cas $S=\lambda\left(\mathrm{D}_{0}\right)$ et $S=\lambda\left(\mathrm{D}_{1}\right)$, où $\mathrm{D}_{0}$ et $\mathrm{D}_{1}$ désignent les $\infty$-catégories du paragraphe 4.1, cas qui seront les seuls que nous utiliserons dans ce texte. Nous supposons, comme dans le lemme, que les complexes $K, K^{\prime}$ et $L$ sont des complexes de Steiner forts et que le morphisme $g^{\prime}$ est une inclusion rigide ordonnée.

Corollaire 11.1.5. - Pour tout morphisme

$$
\alpha_{(M, a)}: a g^{\prime} \backslash M \rightarrow a g \backslash M,
$$

naturel en $(M, a)$ dans $L \backslash \mathcal{C}_{\mathrm{da}}$, il existe un et un seul $\infty$-foncteur

$$
\beta_{(C, b)}: b \nu\left(g^{\prime}\right) \backslash C \rightarrow b \nu(g) \backslash C,
$$

naturel en $(C, b)$ dans $\nu(L) \backslash \infty$-Cat, tel que, pour tout complexe de Steiner fort $M$ muni d'un morphisme $a: L \rightarrow M$, le carré

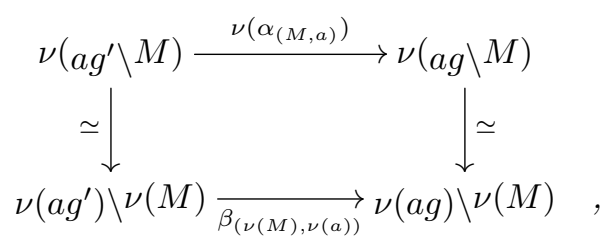

où les isomorphismes verticaux sont ceux de la proposition 10.1.9, soit commutatif. 
Démonstration. - C'est ce qu'on obtient en reformulant le cas $S=\lambda\left(\mathrm{D}_{0}\right) \mathrm{du}$ lemme précédent en utilisant le fait que $\lambda\left(\mathrm{D}_{0}\right)$ et $\nu\left(\lambda\left(\mathrm{D}_{0}\right)\right) \simeq \mathrm{D}_{0}$ sont les unités des produits tensoriels sur $\mathcal{C}_{\text {da }}$ et $\infty$-C at respectivement (voir les paragraphes 6.1 et A.16).

Corollaire 11.1.6. - Pour toute homotopie

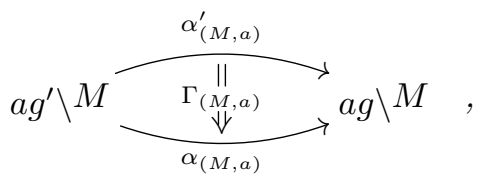

naturelle en $(M, a)$ dans $L \backslash \mathcal{C}_{\mathrm{da}}$, il existe une et une seule transformation oplax

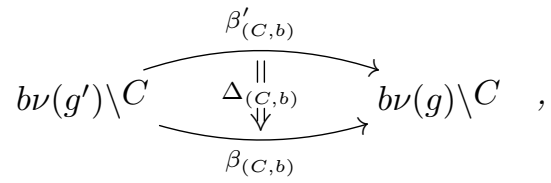

naturelle en $(C, b)$ dans $\nu(L) \backslash \infty$-Cat, telle que, pour tout complexe de Steiner fort $M$ muni d'un morphisme $a: L \rightarrow M$, le diagramme

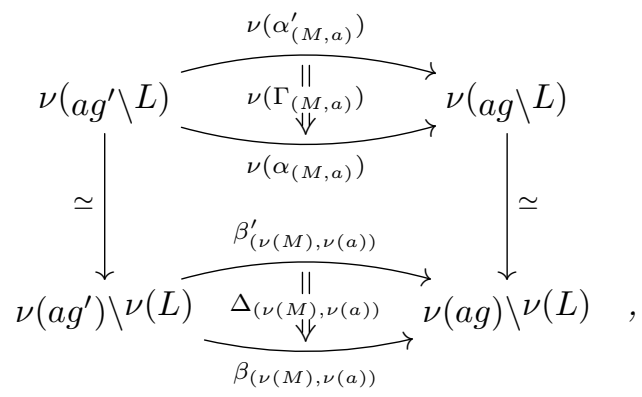

où les isomorphismes verticaux sont ceux de la proposition 10.1 .9 et $\nu\left(\Gamma_{(M, a)}\right)$ désigne la transformation oplax associée à l'homotopie $\Gamma_{(M, a)}$ (voir le paragraphe B.4.6), soit commutatif.

Démonstration. - C'est ce qu'on obtient en reformulant le cas $S=\lambda\left(\mathrm{D}_{1}\right)$ du lemme précédent en utilisant la description des homotopies en termes de produit tensoriel par $\lambda\left(D_{1}\right)$ (voir le paragraphe B.4.1), la description des transformations oplax et de leurs composés avec un $\infty$-foncteur en termes de produit tensoriel par $\nu\left(\lambda\left(D_{1}\right)\right) \simeq D_{1}$ (voir le corollaire B.2.6 et la proposition B.2.11) et la description de la transformation oplax associée à une homotopie (voir le paragraphe B.4.6).

Remarque 11.1.7. - La naturalité en $(C, b)$ dans $\nu(L) \backslash \infty$-C at de la transformation oplax $\Delta_{(C, b)}$ du corollaire précédent peut s'exprimer de la manière suivante. Pour tout 
triangle commutatif

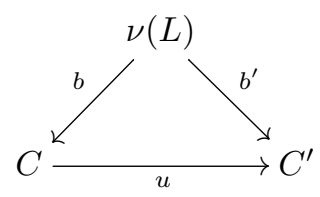

de $\infty$-Cat, le diagramme

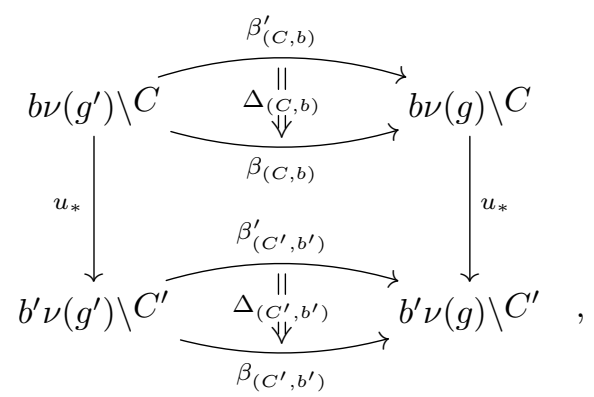

où les $\infty$-foncteurs verticaux sont ceux induits par $u$ vu comme morphisme de $\nu\left(K^{\prime}\right) \backslash \infty$ - Cat et de $\nu(K) \backslash \infty$-C at respectivement, est commutatif au sens où on a

$$
u_{*} * \Delta_{(C, b)}=\Delta_{\left(C^{\prime}, b^{\prime}\right)} * u_{*} .
$$

La naturalité de l'homotopie $\Gamma_{(M, a)}$ en $(M, a)$ dans $L \backslash \mathcal{C}_{\mathrm{da}}$ peut s'exprimer de manière analogue.

\section{2. $\infty$-foncteur associé à un triangle}

11.2.1. - Dans cette section, on fixe

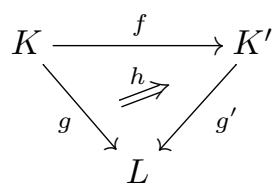

un triangle dans la catégorie des complexes dirigés augmentés commutant à une antihomotopie $h$ de $g$ vers $g^{\prime} f$ près.

Soit $C$ une $\infty$-catégorie munie d'un $\infty$-foncteur $b: \nu(L) \rightarrow C$. On souhaite associer à ces données un $\infty$-foncteur

$$
(f, h, b)^{*}: b \nu\left(g^{\prime}\right) \backslash C \rightarrow b \nu(g) \backslash C
$$

au-dessus de $C$. Nous y parviendrons sous l'hypothèse que les complexes $K, K^{\prime}$ et $L$ sont des complexes de Steiner forts et que le morphisme $g^{\prime}$ est une inclusion rigide ordonnée (voir le paragraphe 3.16). 
Notons que lorsque $C=\nu(M)$ pour $M$ un complexe dirigé augmenté et $b=\nu(a)$ pour $a: L \rightarrow M$, on dispose bien d'un tel $\infty$-foncteur. En effet, on a un triangle

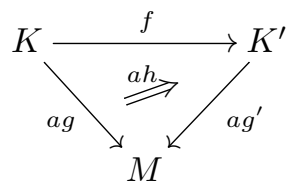

et donc, en vertu de la proposition 10.2.2, un $\infty$-foncteur

$$
\nu\left((f, a h)^{*}\right): \nu\left(a g^{\prime} \backslash M\right) \rightarrow \nu(a g \backslash M)
$$

qui, à travers les isomorphismes de la proposition 10.1.9, définit un $\infty$-foncteur comme souhaité.

Théorème 11.2.2. - On suppose que $K, K^{\prime}$ et $L$ sont des complexes de Steiner forts et que $g$ est une inclusion rigide ordonnée. Soit $C$ une $\infty$-catégorie munie d'un $\infty$-foncteur $b: \nu(L) \rightarrow C$. Il existe un et un seul $\infty$-foncteur

$$
(f, h, b)^{*}: b \nu\left(g^{\prime}\right) \backslash C \rightarrow b \nu(g) \backslash C,
$$

naturel en $(C, b)$ dans $\nu(L) \backslash \infty$-Cat, tel que, pour tout complexe de Steiner fort $M$ muni d'un morphisme $a: L \rightarrow M$, le carré

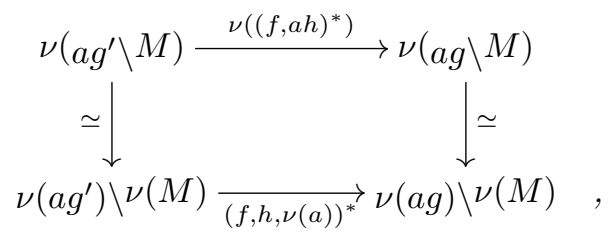

où les isomorphismes verticaux sont ceux de la proposition 10.1.9, soit commutatif.

Démonstration. — C'est ce qu'affirme le corollaire 11.1.5 pour

$$
\alpha_{(M, a)}=(f, a h)^{*}: a g^{\prime} \backslash M \rightarrow a g \backslash M,
$$

dont la naturalité résulte immédiatement des formules du paragraphe 10.2.1.

Remarque 11.2.3. - En déroulant la preuve du théorème précédent (et donc celle du lemme 11.1.3) et en utilisant les formules du paragraphe 10.2.1 définissant le morphisme $(f, a h)^{*}: a g^{\prime} \backslash M \rightarrow a g \backslash M$, on peut décrire le $\infty$-foncteur $(f, h, b)^{*}$ de la manière suivante. Soit $T$ un complexe de Steiner fort. Il suffit de décrire l'application

$$
\operatorname{Hom}_{\infty-\mathcal{C} a t}\left(\nu(T), b \nu\left(g^{\prime}\right) \backslash C\right) \rightarrow \operatorname{Hom}_{\infty-\mathcal{C} a t}(\nu(T), b \nu(g) \backslash C)
$$


induite par $(f, h, b)^{*}$. Or, on a des bijections naturelles

$$
\begin{aligned}
\operatorname{Hom}_{\infty-\mathcal{C} a t}\left(\nu(T), b \nu\left(g^{\prime}\right) \backslash C\right) & \simeq \operatorname{Hom}_{\nu(L) \backslash \infty-\mathcal{C} a t}\left(\left(\nu\left(L \amalg_{K^{\prime}}\left(K^{\prime} \star T\right)\right), \nu\left(\varepsilon_{1}\right)\right),(C, b)\right) \\
& \subset \operatorname{Hom}_{\infty-\mathcal{C} a t}\left(\nu\left(L \amalg_{K^{\prime}}\left(K^{\prime} \star T\right)\right), C\right), \\
\operatorname{Hom}_{\infty-\mathcal{C} a t}(\nu(T), b \nu(g) \backslash C) & \simeq \operatorname{Hom}_{\nu(K) \backslash \infty-\mathcal{C} a t}\left(\left(\nu(K \star T), \nu\left(\iota_{1}\right)\right),(C, b \nu(g))\right) \\
& \subset \operatorname{Hom}_{\infty-\mathcal{C} a t}(\nu(K \star T), C),
\end{aligned}
$$

où $\varepsilon_{1}$ désigne la première inclusion canonique, et l'application

$$
\operatorname{Hom}_{\infty-\mathcal{C} a t}\left(\nu(T), b \nu\left(g^{\prime}\right) \backslash C\right) \rightarrow \operatorname{Hom}_{\infty-\mathcal{C} a t}(\nu(T), b \nu(g) \backslash C)
$$

est induite par le morphisme

$$
(f, h)_{*}: K \star T \rightarrow L \amalg_{K^{\prime}}\left(K^{\prime} \star T\right)
$$

défini de la manière suivante :

$$
(f, h)_{*}(x \star y)= \begin{cases}g(x) & \text { si } y=\varnothing, \\ f(x) \star y+e(y) h(x) & \text { sinon, }\end{cases}
$$

où on convient que $f(\varnothing)=\varnothing, h(\varnothing)=0$ et $e(y)=0$ si $y$ n'est pas de degré 0 . Nous n'utiliserons pas cette description de $(f, h, b)^{*}$ dans la suite de ce texte.

11.2.4. - Considérons le cas particulier d'un triangle commutatif

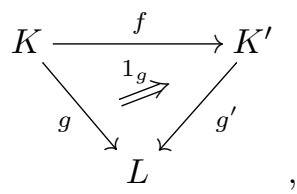

de complexes dirigés augmentés, c'est-à-dire le cas où $h$ est l'antihomotopie identité de $g=g^{\prime} f$. On suppose que les complexes $K, K^{\prime}$ et $L$ sont des complexes de Steiner forts et que le morphisme $g^{\prime}$ est une inclusion rigide ordonnée.

Soit $C$ une $\infty$-catégorie munie d'un $\infty$-foncteur $b: \nu(L) \rightarrow C$. On pose

$$
c=b \nu(g) \quad \text { et } \quad c^{\prime}=b \nu\left(g^{\prime}\right) .
$$

En vertu du théorème 11.2.2, on dispose d'un $\infty$-foncteur

$$
\left(f, 1_{g}, b\right)^{*}: c^{\prime} \backslash C \rightarrow c \backslash C .
$$

Par ailleurs, en considérant le triangle commutatif

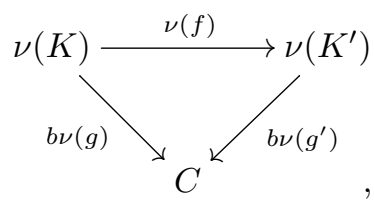


obtenu un appliquant le foncteur $\nu$ et en composant par $b: \nu(L) \rightarrow C$, on obtient, en vertu du paragraphe 6.38 , un $\infty$-foncteur

$$
\nu(f)^{*}: c^{\prime} \backslash C \rightarrow c \backslash C .
$$

Proposition 11.2.5. - Les $\infty$-foncteurs $\left(f, 1_{g}, b\right)^{*}$ et $\nu(f)^{*}$ du paragraphe ci-dessus coïncident.

Démonstration. - Il résulte de la définition de $\nu(f)^{*}: c^{\prime} \backslash C \rightarrow c \backslash C$ (paragraphe 6.38) que ce $\infty$-foncteur est naturel en $(C, b)$ dans $\nu(L) \backslash \infty$-C $a t$. Ainsi, en vertu de la caractérisation du $\infty$-foncteur $(f, 1, b)^{*}$ donnée par le théorème 11.2.2, il suffit de vérifier, pour tout complexe de Steiner fort $M$ muni d'un morphisme $a: L \rightarrow M$, la commutativité du carré

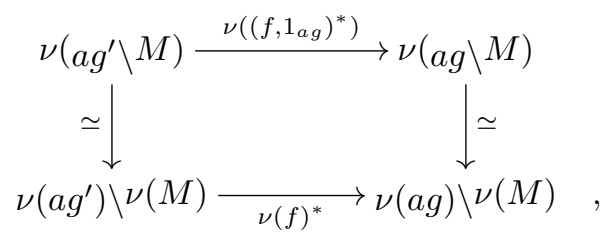

où les isomorphismes verticaux sont ceux de la proposition 10.1.9, ce qui résulte de la proposition 10.2.4.

Remarque 11.2.6. - Si l'on ne suppose pas que le morphisme $g^{\prime}$ est une inclusion rigide ordonnée, le théorème 11.2.2 ne fournit par de $\infty$-foncteur $\left(f, 1_{g}, b\right)^{*}$. Dans ce cas, il sera parfois commode de définir ce $\infty$-foncteur par l'égalité de la proposition précédente :

$$
\left(f, 1_{g}, b\right)^{*}=\nu(f)^{*} .
$$

Remarque 11.2.7. - Il résulte de la proposition 11.2.5 et de la convention de la remarque précédente que, si $g: K \rightarrow L$ est un morphisme entre complexes de Steiner forts, en considérant le triangle

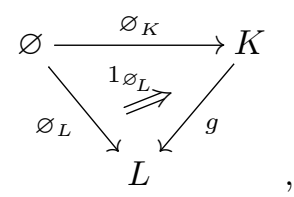

où $\varnothing_{K}$ et $\varnothing_{L}$ désignent les uniques morphismes de source $\varnothing$ et de buts respectifs $K$ et $L$, le $\infty$-foncteur

$$
\left(\varnothing_{K}, 1_{\varnothing_{L}}, b\right)^{*}: c \backslash C \rightarrow \varnothing \backslash C \simeq C
$$

est le $\infty$-foncteur d'oubli du paragraphe 6.38 . 


\subsection{Fonctorialité des $\infty$-foncteurs associés aux triangles}

11.3.1. - Soient $g: K \rightarrow L$ une inclusion rigide ordonnée entre complexes de Steiner forts et $C$ une $\infty$-catégorie munie d'un $\infty$-foncteur $b: \nu(L) \rightarrow C$. On pose $c=b \nu(g)$.

Formons, comme dans le paragraphe 10.3.1, le triangle commutatif

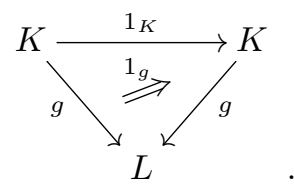

À partir de ce triangle, en vertu du théorème 11.2.2, on obtient un $\infty$-foncteur $\left(1_{K}, 1_{g}, b\right)^{*}: c \backslash C \rightarrow c \backslash C$.

Proposition 11.3.2. - On $a\left(1_{K}, 1_{g}, b\right)^{*}=1_{c \backslash} C$.

Démonstration. - En vertu de la proposition 11.2.5, on a $\left(1_{K}, 1_{g}, b\right)^{*}=\nu\left(1_{K}\right)^{*}$, où $\nu\left(1_{K}\right)^{*}$ est le $\infty$-foncteur du paragraphe 6.38. Or, il est immédiat que $\nu\left(1_{K}\right)^{*}$ est le $\infty$-foncteur identité.

11.3.3. - Considérons maintenant un diagramme

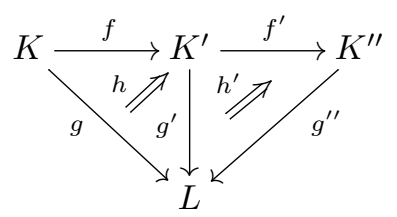

de complexes dirigés augmentés, où $h$ et $h^{\prime}$ sont des antihomotopies de $g$ vers $g^{\prime} f$ et de $g^{\prime}$ vers $g^{\prime \prime} f^{\prime}$ respectivement. On suppose que les complexes $K, K^{\prime}, K^{\prime \prime}$ et $L$ sont des complexes de Steiner forts et que les morphismes $g^{\prime}$ et $g^{\prime \prime}$ sont des inclusions rigides ordonnées.

Soit $C$ une $\infty$-catégorie munie d'un $\infty$-foncteur $b: \nu(L) \rightarrow C$. On pose

$$
c=b \nu(g), \quad c^{\prime}=b \nu\left(g^{\prime}\right) \quad \text { et } \quad c^{\prime \prime}=b \nu\left(g^{\prime \prime}\right) .
$$

En composant le diagramme ci-dessus, on obtient un triangle

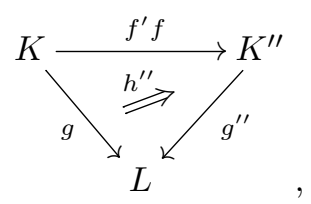


où $h^{\prime \prime}$ est l'antihomotopie $h^{\prime} f+h$. En vertu du théorème 11.2.2, on obtient donc un triangle

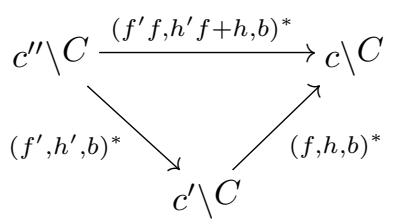

de $\infty$-foncteurs.

La proposition suivante affirme que ce triangle est commutatif.

Proposition 11.3.4. - On a $(f, h, b)^{*}\left(f^{\prime}, h^{\prime}, b\right)^{*}=\left(f^{\prime} f, h^{\prime} f+h, b\right)^{*}$.

Démonstration. - Par définition, les $\infty$-foncteurs $(f, h, b)^{*}$ et $\left(f^{\prime}, h^{\prime}, b\right)^{*}$ sont naturels en $(C, b)$ et il en est donc de même de leur composé $(f, h, b)^{*}\left(f^{\prime}, h^{\prime}, b\right)^{*}$. Ainsi, en vertu de la caractérisation du $\infty$-foncteur $\left(f^{\prime} f, h^{\prime} f+h, b\right)^{*}$ donnée par le théorème 11.2.2, il suffit de vérifier l'égalité recherchée lorsque $C=\nu(M)$ pour $M$ un complexe de Steiner fort et $b=\nu(a)$ pour $a: L \rightarrow M$. Or, cette égalité résulte de la proposition 10.3.4 appliquée au diagramme

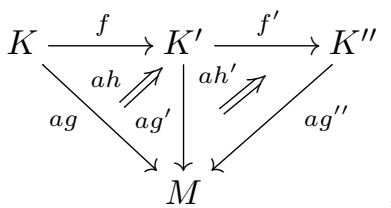

Remarque 11.3.5. - Si $h$ est l'antihomotopie $1_{g}$, alors, avec le convention énoncée dans la remarque 11.2.6, la conclusion de la proposition précédente reste valable sans supposer que $g^{\prime}$ est une inclusion rigide ordonnée. En effet, dans la preuve de la proposition, cette hypothèse sur $g^{\prime}$ n'est utilisée que pour justifier l'existence de $(f, h, b)^{*}$ et sa naturalité en $(C, b)$.

Proposition 11.3.6. - Le $\infty$-foncteur

$$
(f, h, b)^{*}: c^{\prime} \backslash C \rightarrow c \backslash C
$$

du théorème 11.2.2 est au-dessus de C. Autrement dit, le triangle

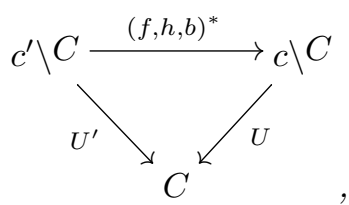

où $U$ et $U^{\prime}$ désignent les $\infty$-foncteurs d'oubli, est commutatif. 
Démonstration. - En appliquant la proposition 11.3.4 sous la forme donnée par la remarque précédente au diagramme

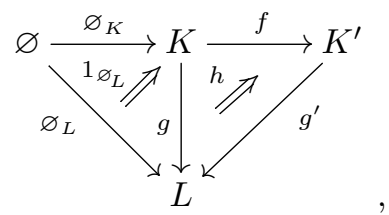

où $\varnothing_{M}$, pour $M$ un complexe dirigé augmenté, désigne l'unique morphisme de $\varnothing$ vers $M$, on obtient l'égalité

$$
\left(\varnothing_{K}, 1_{\varnothing_{L}}, b\right)^{*}(f, h, b)^{*}=\left(\varnothing_{K^{\prime}}, 1_{\varnothing_{L}}, b\right)^{*} .
$$

Or, en vertu de la remarque 11.2.7, on a

$$
\left(\varnothing_{K}, 1_{\varnothing_{L}}, b\right)^{*}=U \quad \text { et } \quad\left(\varnothing_{K^{\prime}}, 1_{\varnothing_{L}}, b\right)^{*}=U^{\prime},
$$

d'où le résultat.

\subsection{Transformation oplax associée à un cône}

11.4.1. - Dans cette section, on fixe un diagramme

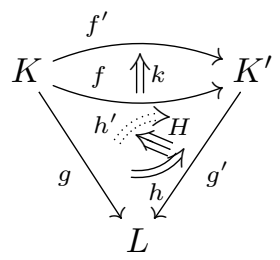

de complexes dirigés augmentés, où $h$ et $h^{\prime}$ sont des antihomotopies de source $g$ et de buts respectifs $g^{\prime} f$ et $g^{\prime} f^{\prime}, k$ est une antihomotopie de $f$ vers $f^{\prime}$ et $H$ est une 2-antihomotopie de $g^{\prime} k+h$ vers $h^{\prime}$. On suppose que les complexes $K, K^{\prime}$ et $L$ sont des complexes de Steiner forts et que $g^{\prime}$ est une inclusion rigide ordonnée.

Soit $C$ une $\infty$-catégorie munie d'un $\infty$-foncteur $b: \nu(L) \rightarrow C$. En vertu du théorème 11.2.2 et de la proposition 11.3.6, on peut associer à ces données des $\infty$-foncteurs

$$
(f, h, b)^{*},\left(f^{\prime}, h^{\prime}, b\right)^{*}: b \nu\left(g^{\prime}\right) \backslash C \rightarrow b \nu(g) \backslash C
$$

au-dessus de $C$. Nous allons définir une transformation oplax

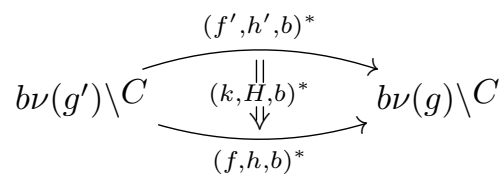

au-dessus de $C$. 
Notons que lorsque $C=\nu(M)$ pour $M$ un complexe dirigé augmenté et $b=\nu(a)$ pour $a: L \rightarrow M$, on dispose bien d'une telle transformation oplax. En effet, on a un diagramme

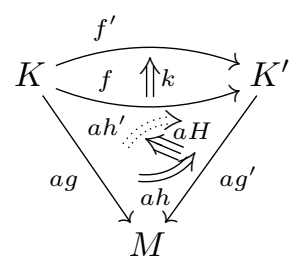

et donc, en vertu de la proposition 10.4.2, une homotopie

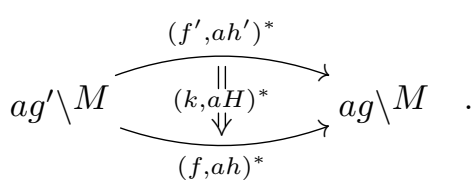

Or, en vertu du paragraphe B.4.6, cette homotopie induit une transformation oplax

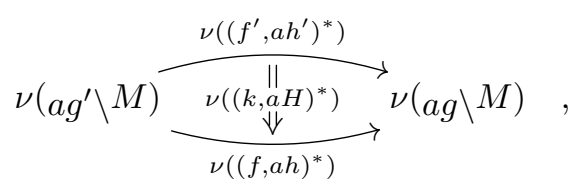

transformation oplax qui, à travers les isomorphismes de la proposition 10.1.9, définit une transformation oplax comme souhaitée.

Théorème 11.4.2. — Soit $C$ une $\infty$-catégorie munie d'un $\infty$-foncteur $b: \nu(L) \rightarrow C$. Il existe une et une seule transformation oplax

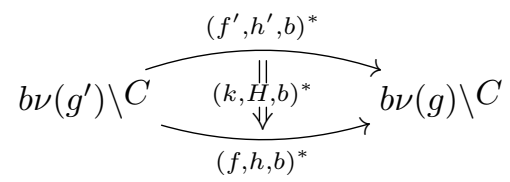

naturelle en $(C, b)$ dans $\nu(L) \backslash \infty$-Cat (voir la remarque 11.1.7), telle que, pour tout complexe de Steiner fort $M$ muni d'un morphisme $a: L \rightarrow M$, le diagramme

$$
\nu\left(a g^{\prime} \backslash M\right) \frac{\nu\left(\left(f^{\prime}, a h^{\prime}\right)^{*}\right)}{\frac{\substack{\| \\ \Downarrow}}{\nu\left((k, a H)^{*}\right)}} \nu(a g \backslash M)
$$


où les isomorphismes verticaux sont ceux de la proposition 10.1 .9 et $\nu\left((k, a H)^{*}\right)$ désigne la transformation oplax associée à l'homotopie $(k, a H)^{*}$ par la construction du paragraphe B.4.6, soit commutatif.

Démonstration. — C'est ce qu'affirme le corollaire 11.1.6 pour

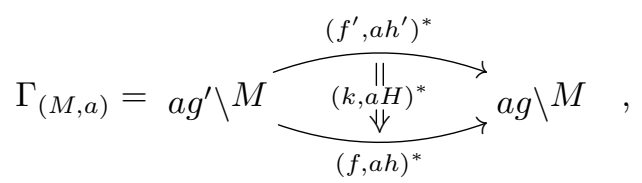

dont la naturalité résulte immédiatement des formules du paragraphe 10.4.1.

Remarque 11.4.3. - En déroulant la preuve du théorème précédent (et donc celle du lemme 11.1.3) et en utilisant les formules du paragraphe 10.4.1 définissant l'homotopie $(k, a H)^{*}$, on peut décrire la transformation oplax $(k, H, b)^{*}$, ou plus précisément le $\infty$-foncteur $b \nu\left(g^{\prime}\right) \backslash C \rightarrow \underline{\operatorname{Hom}}_{\text {lax }}\left(\mathrm{D}_{1}, b \nu(g) \backslash C\right)$ qui lui est associé (voir la proposition B.2.5), de la manière suivante. Soit $T$ un complexe de Steiner fort. Il suffit de décrire l'application

$$
\operatorname{Hom}_{\infty-\mathcal{C} a t}\left(\nu(T), b \nu\left(g^{\prime}\right) \backslash C\right) \rightarrow \operatorname{Hom}_{\infty-\mathcal{C} a t}\left(\nu(T), \underline{\operatorname{Hom}}_{l a x}\left(\mathrm{D}_{1}, b \nu(g) \backslash C\right)\right)
$$

induite par ce $\infty$-foncteur. Or, on a des bijections naturelles

$$
\operatorname{Hom}_{\infty-\mathcal{C} a t}\left(\nu(T), b \nu\left(g^{\prime}\right) \backslash C\right) \simeq \operatorname{Hom}_{\nu(L) \backslash \infty-\mathcal{C} a t}\left(\left(\nu\left(L \amalg_{K^{\prime}}\left(K^{\prime} \star T\right)\right), \nu\left(\varepsilon_{1}\right)\right),(C, b)\right),
$$

où $\varepsilon_{1}$ désigne la première inclusion canonique, et

$$
\begin{aligned}
\operatorname{Hom}_{\infty}-\mathcal{C} a t & \left(\nu(T), \underline{\operatorname{Hom}}_{\text {lax }}\left(\mathrm{D}_{1}, b \nu(g) \backslash C\right)\right) \\
& \simeq \operatorname{Hom}_{\nu(K) \backslash \infty-\mathcal{C} a t}\left(\left(\nu\left(K \star\left(\lambda\left(\mathrm{D}_{1}\right) \otimes T\right)\right), \nu\left(\iota_{1}\right)\right),(C, b \nu(g))\right),
\end{aligned}
$$

et l'application

$$
\operatorname{Hom}_{\infty-\mathcal{C} a t}\left(\nu(T), b \nu\left(g^{\prime}\right) \backslash C\right) \rightarrow \operatorname{Hom}_{\infty-\mathcal{C} a t}\left(\nu(T), \underline{\operatorname{Hom}}_{l a x}\left(\mathrm{D}_{1}, b \nu(g) \backslash C\right)\right)
$$

est induite par le morphisme

$$
(k, H)_{*}: K \star\left(\lambda\left(\mathrm{D}_{1}\right) \otimes T\right) \rightarrow L \amalg_{K^{\prime}}\left(K^{\prime} \star T\right)
$$

défini de la manière suivante :

$$
\begin{aligned}
(k, H)_{*}(x \star \varnothing) & =g(x), \\
(k, H)_{*}\left(x \star\left(d_{0}^{0} \otimes y\right)\right) & =f^{\prime}(x) \star y+e(y) h^{\prime}(x), \\
(k, H)_{*}\left(x \star\left(d_{0}^{1} \otimes y\right)\right) & =f(x) \star y+e(y) h(x), \\
(k, H)_{*}\left(x \star\left(d_{1} \otimes y\right)\right) & =k(x) \star y+e(y) H(x),
\end{aligned}
$$

où $d$ désigne la cellule principale de $\mathrm{D}_{1}$ et où, en plus des conventions du paragraphe 11.2.3, on convient que $k(\varnothing)=0$ et que $H(\varnothing)=0$. Nous n'utiliserons pas cette description de $(k, H, b)^{*}$ dans la suite de ce texte. 


\subsection{Fonctorialités des transformations oplax associées aux cônes}

11.5.1. - Considérons un diagramme

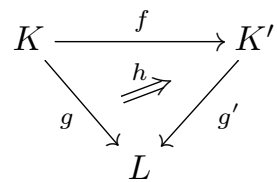

de complexes dirigés augmentés, où $h$ est une antihomotopie de $g$ vers $g^{\prime} f$. On suppose que les complexes $K, K^{\prime}$ et $L$ sont des complexes de Steiner forts et que le morphisme $g^{\prime}$ est une inclusion rigide ordonnée.

Soit $C$ une $\infty$-catégorie munie d'un $\infty$-foncteur $b: \nu(L) \rightarrow C$. On pose

$$
c=b \nu(g) \quad \text { et } \quad c^{\prime}=b \nu\left(g^{\prime}\right) .
$$

À partir de ce diagramme, on peut former, comme dans le paragraphe 10.5.1, le diagramme

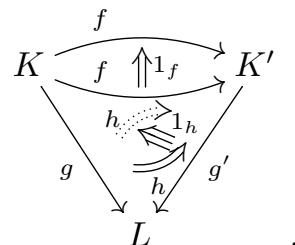

On obtient donc, en vertu du théorème 11.4.2, une transformation oplax $\left(1_{f}, 1_{h}, b\right)^{*}$ de $(f, h, b)^{*}$ vers $(f, h, b)^{*}$.

Proposition 11.5.2. - On $a\left(1_{f}, 1_{h}, b\right)^{*}=1_{(f, h, b)^{*}}$.

Démonstration. - Le $\infty$-foncteur $(f, h, b)^{*}$ est naturel en $(C, b)$ par définition et il en est donc de même de sa transformation oplax identité $1_{(f, h, b)^{*}}$ (cela résulte formellement du fait que les transformations oplax identité sont des identités pour la composition horizontale par un $\infty$-foncteur). Ainsi, en vertu de la caractérisation de la transformation oplax $\left(1_{f}, 1_{h}, b\right)^{*}$ donnée par le théorème 11.4.2, il suffit de démontrer l'égalité recherchée lorsque $C=\nu(M)$ pour $M$ un complexe de Steiner fort et $b=\nu(a)$ pour $a: L \rightarrow M$. Or, cela résulte de la proposition 10.5.2 appliquée au diagramme

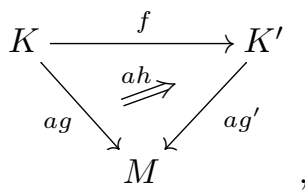

ainsi que de la proposition B.4.9 qui affirme que $\nu$ envoie une homotopie identité sur une transformation oplax identité. 
11.5.3. - Considérons maintenant un diagramme

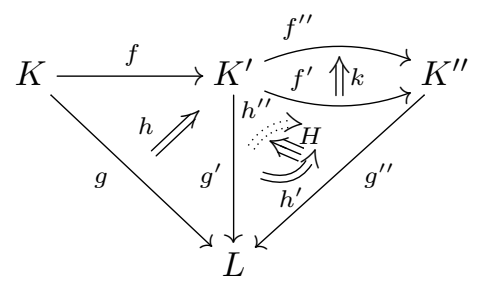

de complexes dirigés augmentés, où $h, h^{\prime}$ et $h^{\prime \prime}$ sont des antihomotopies de $g$ vers $g^{\prime} f$, de $g^{\prime}$ vers $g^{\prime \prime} f^{\prime}$ et de $g^{\prime}$ vers $g^{\prime \prime} f^{\prime \prime}$ respectivement, $k$ est une antihomotopie de $f^{\prime}$ vers $f^{\prime \prime}$ et $H$ est une 2-antihomotopie de $g^{\prime \prime} k+h^{\prime}$ vers $h^{\prime \prime}$. On suppose que les complexes $K$, $K^{\prime}, K^{\prime \prime}$ et $L$ sont des complexes de Steiner forts et que les morphismes $g^{\prime}$ et $g^{\prime \prime}$ sont des inclusions rigides ordonnées.

Soit $C$ une $\infty$-catégorie munie d'un $\infty$-foncteur $b: \nu(L) \rightarrow C$. On pose

$$
c=b \nu(g), \quad c^{\prime}=b \nu\left(g^{\prime}\right) \quad \text { et } \quad c^{\prime \prime}=b \nu\left(g^{\prime \prime}\right) .
$$

À partir de ces données, comme dans le paragraphe 10.5.3 et avec les mêmes notations, en utilisant les théorèmes 11.2.2 et 11.4.2, ainsi que la proposition 11.3.4, on obtient deux transformations oplax

$$
(f, h, b)^{*} *(k, H, b)^{*} \quad \text { et } \quad(k f, H f, b)^{*}
$$

de $\left(f^{\prime \prime} f, h^{\prime \prime} f+h, b\right)^{*}$ vers $\left(f^{\prime} f, h^{\prime} f+h, b\right)^{*}$, qui sont deux $\infty$-foncteurs de $c^{\prime \prime} \backslash C$ vers $c \backslash C$.

Proposition 11.5.4. - On a $(f, h, b)^{*} *(k, H, b)^{*}=(k f, H f, b)^{*}$.

Démonstration. - Le $\infty$-foncteur $(f, h, b)^{*}$ et la transformation oplax $(k, H, b)^{*}$ sont naturels en $(C, b)$ par définition et il en est donc de même de la transformation oplax composée $(f, h, b)^{*} *(k, H, b)^{*}$ (cela résulte formellement de l'associativité de la composition horizontale d'un $\infty$-foncteur et d'une transformation oplax). Ainsi, en raisonnant comme dans la preuve de la proposition précédente, on se ramène au cas où $C=\nu(M)$ pour $M$ un complexe de Steiner fort et $b=\nu(a)$ pour $a: L \rightarrow M$. Or, ce cas résulte de la proposition 10.5.4 appliquée au diagramme

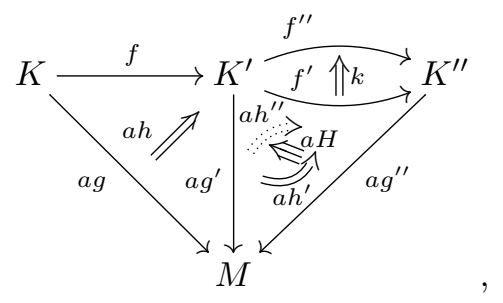


ainsi que de la proposition B.4.8 affirmant que $\nu$ envoie le composé horizontal d'un morphisme et d'une homotopie sur le composé horizontal du $\infty$-foncteur et de la transformation oplax associés.

Remarque 11.5.5. - Pour les mêmes raisons que celles données dans la remarque 11.3.5, si $h$ est l'antihomotopie $1_{g}$, la conclusion de la proposition précédente reste valable sans supposer que $g^{\prime}$ est une inclusion rigide ordonnée.

Proposition 11.5.6. - La transformation oplax $(k, H, b)^{*}$ du théorème 11.4.2 de $\left(f^{\prime}, h^{\prime}, b\right)^{*}$ vers $(f, h, b)^{*}$ est au-dessus de $C$. Autrement dit, on a l'égalité $U *(k, H, b)^{*}=1_{U^{\prime}}$, où $U: c \backslash C \rightarrow C$ et $U^{\prime}: c^{\prime} \backslash C \rightarrow C$ désignent les $\infty$-foncteurs d'oubli.

Démonstration. - En appliquant la proposition 11.5.4 sous la forme donnée par la remarque précédente au diagramme

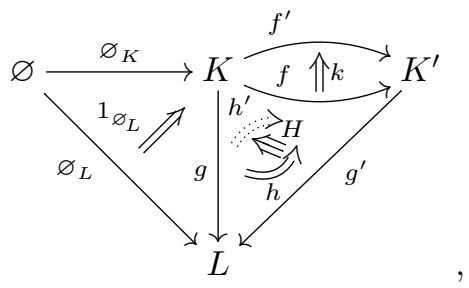

où $\varnothing_{M}$, pour $M$ un complexe dirigé augmenté, désigne l'unique morphisme de $\varnothing$ vers $M$, on obtient l'égalité

$$
\left(\varnothing_{K}, 1_{\varnothing_{L}}, b\right)^{*} *(k, H, b)^{*}=\left(1_{\varnothing_{K^{\prime}}}, 1_{1_{\varnothing_{L}}}, b\right)^{*} .
$$

Or, en vertu de la remarque 11.2.7 et de la proposition 11.5.2, on a

$$
\left(\varnothing_{K}, 1_{\varnothing_{L}}, b\right)^{*}=U \quad \text { et } \quad\left(1_{\varnothing_{K^{\prime}}}, 1_{1_{\varnothing_{L}}}, b\right)^{*}=1_{\left(\varnothing_{K^{\prime}}, 1_{\varnothing_{L}}, b\right)^{*}}=1_{U^{\prime}},
$$

d'où le résultat.

11.5.7. - De même, considérons un diagramme

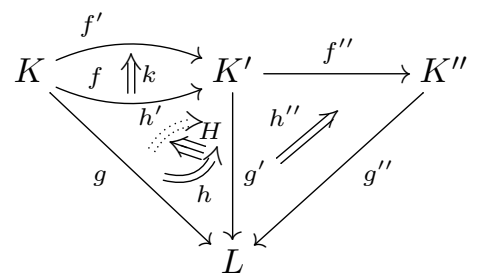

de complexes dirigés augmentés, où $h, h^{\prime}$ et $h^{\prime \prime}$ sont des antihomotopies de $g$ vers $g^{\prime} f$, de $g$ vers $g^{\prime} f^{\prime}$ et de $g^{\prime}$ vers $g^{\prime \prime} f^{\prime \prime}$ respectivement, $k$ est une antihomotopie de $f$ vers $f^{\prime}$ et $H$ est une 2-antihomotopie de $g^{\prime} k+h$ vers $h^{\prime}$. On suppose toujours que les complexes 
$K, K^{\prime}, K^{\prime \prime}$ et $L$ sont des complexes de Steiner forts et que les morphismes $g^{\prime}$ et $g^{\prime \prime}$ sont des inclusions rigides ordonnées.

Soit $C$ une $\infty$-catégorie munie d'un $\infty$-foncteur $b: \nu(L) \rightarrow C$. On pose

$$
c=b \nu(g), \quad c^{\prime}=b \nu\left(g^{\prime}\right) \quad \text { et } \quad c^{\prime \prime}=b \nu\left(g^{\prime \prime}\right) .
$$

À partir de ces données, comme dans le paragraphe 10.5.5 et avec les mêmes notations, en utilisant les théorèmes 11.2.2 et 11.4.2, ainsi que la proposition 11.3.4, on obtient deux transformations oplax

$$
(k, H, b)^{*} *\left(f^{\prime \prime}, h^{\prime \prime}, b\right)^{*} \text { et }\left(f^{\prime \prime} k, H+h^{\prime \prime} k, b\right)^{*}
$$

$\operatorname{de}\left(f^{\prime \prime} f^{\prime}, h^{\prime \prime} f^{\prime}+h^{\prime}, b\right)^{*}$ vers $\left(f^{\prime \prime} f, h^{\prime \prime} f+h, b\right)^{*}$.

Proposition 11.5.8. - On a $(k, H, b)^{*} *\left(f^{\prime \prime}, h^{\prime \prime}, b\right)^{*}=\left(f^{\prime \prime} k, H+h^{\prime \prime} k, b\right)^{*}$.

Démonstration. - La démonstration est une adaptation immédiate de la démonstration de la proposition 11.5.4, l'usage de la proposition 10.5.4 étant remplacé par celui de la proposition 10.5.6.

11.5.9. - Enfin, considérons un diagramme

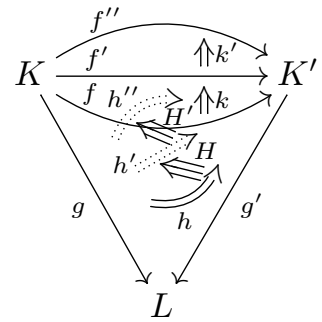

de complexes dirigés augmentés, où $h, h^{\prime}$ et $h^{\prime \prime}$ sont des antihomotopies de $g$ vers $g^{\prime} f$, $g^{\prime} f^{\prime}$ et $g^{\prime} f^{\prime \prime}$ respectivement, $k$ et $k^{\prime}$ sont des antihomotopies de $f$ vers $f^{\prime}$ et de $f^{\prime}$ vers $f^{\prime \prime}$ respectivement et $H$ et $H^{\prime}$ sont des 2-antihomotopies de $g^{\prime} k+h$ vers $h^{\prime}$ et de $g^{\prime} k^{\prime}+h^{\prime}$ vers $h^{\prime \prime}$ respectivement. On suppose que les complexes $K, K^{\prime}$ et $L$ sont des complexes de Steiner forts et que le morphisme $g^{\prime}$ est une inclusion rigide ordonnée.

Soit $C$ une $\infty$-catégorie munie d'un $\infty$-foncteur $b: \nu(L) \rightarrow C$. On pose

$$
c=b \nu(g) \quad \text { et } \quad c^{\prime}=b \nu\left(g^{\prime}\right) .
$$

À partir de ces données, comme dans le paragraphe 10.5.7 et avec les mêmes notations, en utilisant le théorème 11.4.2, on obtient deux transformations oplax

$$
(k, H, b)^{*} \circ\left(k^{\prime}, H^{\prime}, b\right)^{*} \text { et } \quad\left(k^{\prime}+k, H^{\prime}+H, b\right)^{*}
$$

de $\left(f^{\prime \prime}, h^{\prime \prime}, b\right)^{*}$ vers $(f, h, b)^{*}$ (la composition verticale des transformations oplax est définie dans le paragraphe B.3.2).

Proposition 11.5.10. - On a $(k, H, b)^{*} \circ\left(k^{\prime}, H^{\prime}, b\right)^{*}=\left(k^{\prime}+k, H^{\prime}+H, b\right)^{*}$. 
Démonstration. - Les transformations oplax $(k, H, b)^{*}$ et $\left(k^{\prime}, H^{\prime}, b\right)^{*}$ sont naturelles en $(C, b)$ par définition et il en est donc de même de la transformation oplax composée $(k, H, b)^{*} \circ\left(k^{\prime}, H^{\prime}, b\right)^{*}$ (cela résulte de la compatibilité entre la composition horizontale par un $\infty$-foncteur et la composition verticale des transformations oplax, voir l'appendice $\mathrm{C}$ qui montre que les $\infty$-catégories, $\infty$-foncteurs et transformations oplax forment une sesquicatégorie). Ainsi, en raisonnant comme dans les démonstrations précédentes, on se ramène au cas où $C=\nu(M)$ pour $M$ un complexe de Steiner fort et $b=\nu(a)$ pour $a: L \rightarrow M$. Or, ce cas résulte de la proposition 10.5.8 appliquée au diagramme

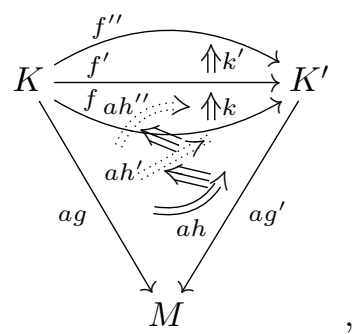

où les 3-flèches sont, du bas vers le haut, les 2-antihomotopies $a H$ et $a H^{\prime}$, ainsi que de la proposition B.4.10 affirmant que $\nu$ envoie le composé horizontal de deux homotopies sur le composé horizontal des deux transformations oplax associées. 



\section{APPENDICE A}

\section{PRODUIT TENSORIEL $\infty$-CATÉGORIQUE}

Le but de cet appendice est d'introduire le produit tensoriel de Gray et les Hom internes à gauche et à droite correspondants, et de démontrer leurs principales propriétés. La définition du produit tensoriel que nous adoptons est inspirée d'idées de Steiner [32, Section 7].

A.1. - On rappelle qu'on a défini au paragraphe 6.2 , selon Steiner, le produit tensoriel de deux complexes dirigés augmentés et qu'on obtient ainsi une structure de catégorie monoïdale bifermée sur la catégorie des complexes dirigés augmentés.

Si $K$ et $L$ sont deux complexes dirigés augmentés admettant des bases $X$ et $Y$ respectivement, on vérifie immédiatement que le complexe dirigé augmenté $K \otimes L$ admet pour base l'ensemble

$$
X \otimes Y=\{x \otimes y \mid x \in X, y \in Y\} .
$$

Lemme A.2 (Steiner). — Soient $K$ et L des complexes dirigés augmentés à base. Pour tout élément homogène $x$ de $K$ et tout élément homogène $y$ de $L$, tout $r \geqslant 0$ et $\varepsilon=0,1$, on $a$

$$
\langle x \otimes y\rangle_{r}^{\varepsilon}=\sum_{\substack{p+q=r \\ 0 \leqslant p \leqslant|x|, 0 \leqslant q \leqslant|y|}}\langle x\rangle_{p}^{\varepsilon} \otimes\langle y\rangle_{q}^{p+\varepsilon \bmod 2} .
$$

Démonstration. - La formule est donnée dans [32, exemple 3.10] dans le cas où $x$ et $y$ sont dans la base de $K$ et $L$ respectivement. Elle se démontre par récurrence, essentiellement comme la formule de notre lemme 6.14

Proposition A.3 (Steiner). — Si K et L sont des complexes dirigés augmentés à base unitaire (resp. à base fortement sans boucle), alors il en est de même de $K \otimes L$. En particulier, si $K$ et $L$ sont des complexes de Steiner forts, alors il en est de même de $K \otimes L$. 
Démonstration. - Voir [32, exemple 3.10].

Remarque A.4. - En vertu de la proposition précédente (et du fait que le complexe dirigé augmenté $\underline{\mathbb{Z}}^{\prime}$ du paragraphe 6.2 est de Steiner fort), la catégorie des complexes de Steiner forts est une sous-catégorie monoïdale de la catégorie monoïdale des complexes dirigés augmentés définie par le produit tensoriel.

Lemme A.5. - Soient $f: K \rightarrow K^{\prime}$ et $g: L \rightarrow L^{\prime}$ des morphismes entre complexes dirigés augmentés à base et soient $x$ un élément homogène de $K$ et $y$ un élément homogène de $L$. On suppose qu'il existe un élément homogène $x^{\prime}$ de $K$ et un élément homogène $y^{\prime}$ de L tels qu'on ait

$$
f(\langle x\rangle)=\left\langle x^{\prime}\right\rangle \quad \text { et } \quad g(\langle y\rangle)=\left\langle y^{\prime}\right\rangle .
$$

Alors on a

$$
(f \otimes g)(\langle x \otimes y\rangle)=\left\langle x^{\prime} \otimes y^{\prime}\right\rangle .
$$

Démonstration. - La preuve est une adaptation immédiate de la preuve de l'assertion analogue pour le joint (lemme 6.16) en remplaçant l'usage de la formule du lemme 6.14 par celui de la formule du lemme A.2.

Proposition A.6. - Si $f: K \rightarrow K^{\prime}$ et $g: L \rightarrow L^{\prime}$ sont deux morphismes rigides (voir le paragraphe 3.2 ) entre complexes dirigés augmentés à base, alors leur produit tensoriel $f \otimes g: K \otimes L \rightarrow K^{\prime} \otimes L^{\prime}$ est également rigide.

Démonstration. — Cela résulte immédiatement du lemme précédent.

Proposition A.7. - Soient $f: K \rightarrow K^{\prime}$ et $g: L \rightarrow L^{\prime}$ des morphismes entre complexes dirigés augmentés à base unitaire et soient $x$ un élément de la base de $K$ et y un élément de la base de $L$. On suppose qu'il existe un élément $x^{\prime}$ de la base de $K^{\prime}$ et un élément $y^{\prime}$ de la base de $L^{\prime}$ tels qu'on ait

$$
\nu(f)(\langle x\rangle)=1_{\left\langle x^{\prime}\right\rangle} \quad \text { et } \quad \nu(g)(\langle y\rangle)=1_{\left\langle y^{\prime}\right\rangle},
$$

où 1 désigne une identité itérée (éventuellement 0 fois). Alors on a

$$
\nu(f \otimes g)(\langle x \otimes y\rangle)=1_{\left\langle x^{\prime} \otimes y^{\prime}\right\rangle} .
$$

En particulier, lorsque $x^{\prime}=f(x)$ et $y^{\prime}=g(y)$ vérifient les hypothèses ci-dessus, on a

$$
\nu(f \otimes g)(\langle x \otimes y\rangle)=\langle f(x) \otimes g(y)\rangle .
$$

Démonstration. - Cela résulte immédiatement du lemme A.5. 
Proposition A.8. - Soient $K$ et $L$ deux complexes dirigés augmentés à base unitaire, $x$ un élément de la base de $K$ de degré $i$ et y un élément de la base de $L$ de degré $j$. Notons $z$ la cellule principale de $\mathrm{D}_{i}$ et $t$ celle de $\mathrm{D}_{j}$. Alors le diagramme

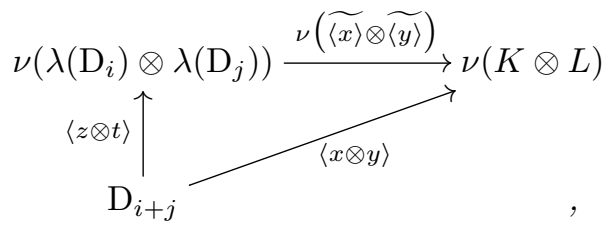

où $\widetilde{\langle x\rangle}: \lambda\left(\mathrm{D}_{i}\right) \rightarrow K$ et $\widetilde{\langle y\rangle}: \lambda\left(\mathrm{D}_{j}\right) \rightarrow L$ désignent les transposés de $\langle x\rangle: \mathrm{D}_{i} \rightarrow \nu(K)$ et $\langle y\rangle: \mathrm{D}_{j} \rightarrow \nu(L)$ respectivement, est commutatif.

Démonstration. - Il s'agit de montrer qu'on a

$$
\nu(\widetilde{\langle x\rangle} \otimes \widetilde{\langle y\rangle})(\langle z \otimes t\rangle)=\langle x \otimes y\rangle .
$$

Cela résulte de la proposition précédente puisque, par définition, on a

$$
\nu(\widetilde{\langle x\rangle})(\langle z\rangle)=\langle x\rangle \quad \text { et } \quad \nu(\widetilde{\langle y\rangle})(\langle t\rangle)=\langle y\rangle .
$$

Proposition A.9. - Soient $K$ un complexe de Steiner fort et $F: I \rightarrow \mathcal{C}_{\mathrm{da}}$ un système de Steiner fort (voir le paragraphe 3.7). Alors le foncteur

$$
\begin{aligned}
K \otimes F: I & \rightarrow \mathcal{C}_{\mathrm{da}} \\
i & \mapsto K \otimes F(i)
\end{aligned}
$$

est un système de Steiner fort.

Démonstration. - Le foncteur $F$ étant un système rigide, il en est de même du foncteur $K \otimes F: i \mapsto K \otimes F(i)$ en vertu de la proposition A.6. Par ailleurs, puisque d'après la proposition A.3 les complexes de Steiner forts sont stables par produit tensoriel, le foncteur $K \otimes F$ est à valeurs dans les complexes de Steiner forts. Enfin, le foncteur $K \otimes \bullet$ admettant un adjoint à droite, il commute aux limites inductives. Le morphisme canonique

$$
\underset{i \in I}{\lim _{i \in I}}(K \otimes F(i)) \rightarrow K \otimes \lim _{i \in I} F(i)
$$

est donc un isomorphisme de complexes dirigés augmentés et, pour tout objet $i_{0}$ de $I$, le morphisme canonique $K \otimes F\left(i_{0}\right) \rightarrow \lim _{i \in I}(K \otimes F(i))$ s'identifie à travers cet isomorphisme au produit tensoriel $K \otimes F\left(i_{0}\right) \rightarrow K \otimes \lim _{i \in I} F(i)$ de $K$ et du morphisme canonique associé à $F$. On en déduit le résultat en invoquant de nouveau les propositions A.3 et A.6.

Corollaire A.10. — Si K est un complexe de Steiner fort, alors le foncteur

$$
\begin{aligned}
\mathcal{C}_{\mathrm{da}} & \rightarrow \infty-\mathcal{C} a t \\
L & \mapsto \nu(K \otimes L)
\end{aligned}
$$


commute aux limites inductives des systèmes de Steiner forts.

Démonstration. - Puisque le foncteur $\nu$ commute aux limites inductives des systèmes de Steiner forts (théorème 3.8), l'assertion résulte de la proposition précédente.

Corollaire A.11. — Soit $K$ un complexe de Steiner fort. Alors le foncteur

$$
\begin{aligned}
& \Theta \rightarrow \infty \text {-Cat } \\
& S \mapsto \nu(K \otimes \lambda(S))
\end{aligned}
$$

commute aux sommes globulaires.

Démonstration. - Cela résulte du corollaire précédent puisqu'en vertu de la proposition 4.13, les sommes globulaires proviennent de systèmes de Steiner forts.

A.12. - Fixons $K$ un complexe de Steiner fort. Soit $C$ une $\infty$-catégorie. Nous allons définir une $\infty$-catégorie $\underline{\operatorname{Hom}}_{\operatorname{lax}}(\nu(K), C)$. Il résulte du corollaire précédent que le foncteur

$$
\begin{aligned}
\Theta^{\circ} & \rightarrow \mathcal{E} n s \\
S & \mapsto \operatorname{Hom}_{\infty-\mathcal{C} a t}(\nu(K \otimes \lambda(S)), C)
\end{aligned}
$$

envoie les sommes globulaires sur des produits globulaires, au sens du paragraphe 4.8. Ainsi, en vertu de ce même paragraphe, ce foncteur définit une $\infty$-catégorie et c'est cette $\infty$-catégorie qu'on notera $\underline{\operatorname{Hom}}_{\text {lax }}(\nu(K), C)$. Autrement dit, avec les notations du paragraphe 4.8 , on pose

$$
\underline{\operatorname{Hom}}_{\operatorname{lax}}(\nu(K), C)=\operatorname{Hom}_{\infty-\mathcal{C} a t}\left(\nu\left(K \otimes \lambda\left(\mathrm{D}_{\bullet}\right)\right), C\right) .
$$

Explicitement, les $i$-flèches de $\underline{\operatorname{Hom}}_{\text {lax }}(\nu(K), C)$ sont les $\infty$-foncteurs de $\nu\left(K \otimes \lambda\left(\mathrm{D}_{i}\right)\right)$ vers $C$.

Notons qu'un morphisme $f: K \rightarrow K^{\prime}$ entre complexes de Steiner forts induit, pour tout $\infty$-catégorie $C$, une application

$$
\operatorname{Hom}_{\infty-\mathcal{C} a t}\left(\nu\left(K^{\prime} \otimes \lambda(S)\right), C\right) \rightarrow \operatorname{Hom}_{\infty-\mathcal{C} a t}(\nu(K \otimes \lambda(S)), C),
$$

naturelle en $S$ dans $\Theta$. Ainsi, toujours en vertu du paragraphe 4.8, un tel morphisme induit un $\infty$-foncteur $\underline{\operatorname{Hom}}_{\text {lax }}\left(\nu\left(K^{\prime}\right), C\right) \rightarrow \underline{\operatorname{Hom}}_{\text {lax }}(\nu(K), C)$.

Proposition A.13. - Fixons $K$ un complexe de Steiner fort. Pour tout complexe de Steiner fort $L$ et toute $\infty$-catégorie $C$, on a une bijection

$$
\operatorname{Hom}_{\infty-\mathcal{C} a t}(\nu(K \otimes L), C) \simeq \operatorname{Hom}_{\infty-\mathcal{C} a t}\left(\nu(L), \underline{\operatorname{Hom}}_{\operatorname{lax}}(\nu(K), C)\right),
$$

naturelle en $L$ et $C$. 
Démonstration. - Si $M$ est un complexe de Steiner fort et $t$ est un élément de la base de $M$ de degré $i \geqslant 0$, on notera, pour simplifier, $t_{j}^{\varepsilon}=\langle t\rangle_{j}^{\varepsilon}$, pour $0 \leqslant j \leqslant i$ (voir le paragraphe 2.8 pour la notation $\langle t\rangle_{j}^{\varepsilon}$ ).

On va produire des fonctions

$$
\varphi: \operatorname{Hom}_{\infty-\mathcal{C} a t}(\nu(K \otimes L), C) \rightarrow \operatorname{Hom}_{\infty-\mathcal{C} a t}\left(\nu(L), \underline{\operatorname{Hom}}_{\operatorname{lax}}(\nu(K), C)\right)
$$

et

$$
\psi: \operatorname{Hom}_{\infty-\mathcal{C} a t}\left(\nu(L), \underline{\operatorname{Hom}}_{l a x}(\nu(K), C)\right) \rightarrow \operatorname{Hom}_{\infty-\mathcal{C} a t}(\nu(K \otimes L), C)
$$

inverses l'une de l'autre.

Commençons par $\varphi$. Soit $F: \nu(K \otimes L) \rightarrow C$ un $\infty$-foncteur. On définit un $\infty$-foncteur $\varphi(F): \nu(L) \rightarrow \underline{\operatorname{Hom}}_{\text {lax }}(\nu(K), C)$ de la manière suivante. Soit $y: \mathrm{D}_{i} \rightarrow \nu(L)$, pour $i \geqslant 0$, une $i$-flèche de $\nu(L)$. On doit lui associer une $i$-flèche $\varphi(F)(y)$ de $\underline{\operatorname{Hom}}_{\text {lax }}(\nu(K), C)$, c'est-à-dire un $\infty$-foncteur $\nu\left(K \otimes \lambda\left(\mathrm{D}_{i}\right)\right) \rightarrow C$. On pose

$$
\varphi(F)(y)=\nu\left(K \otimes \lambda\left(\mathrm{D}_{i}\right)\right) \stackrel{\nu(K \otimes \tilde{y})}{\longrightarrow} \nu(K \otimes L) \stackrel{F}{\rightarrow} C,
$$

où on a noté $\tilde{y}: \lambda\left(\mathrm{D}_{i}\right) \rightarrow L$ le transposé de $y: \mathrm{D}_{i} \rightarrow \nu(L)$. Le fait qu'on obtienne bien ainsi un $\infty$-foncteur résulte de la naturalité en $\mathrm{D}_{i}$, et plus généralement en $S$ dans $\Theta$, du $\infty$-foncteur $\varphi(F)(y)$. En particulier, pour $x$ un élément de degré $i$ de la base de $K, y$ un élément de la base de $L$ et $z$ la cellule principale de $\mathrm{D}_{i}$, on a, pour $j$ tel que $0 \leqslant j \leqslant i$ et $\varepsilon=0,1$,

$$
\varphi(F)(\langle y\rangle)\left(\left\langle x \otimes z_{j}^{\varepsilon}\right\rangle\right)=F\left(\left\langle x \otimes y_{j}^{\varepsilon}\right\rangle\right) .
$$

De plus, cette formule détermine $\varphi(F)$ de manière unique puisque les $\infty$-catégories $\nu(L)$ et $\nu\left(K \otimes \lambda\left(\mathrm{D}_{i}\right)\right)$ sont engendrées librement au sens des polygraphes par leurs atomes en vertu du théorème 2.12 .

Définissons maintenant $\psi$. Soit $G: \nu(L) \rightarrow \underline{\operatorname{Hom}}_{\operatorname{lax}}(\nu(K), C)$ un $\infty$-foncteur. Il s'agit de définir un $\infty$-foncteur $\psi(G): \nu(K \otimes L) \rightarrow C$. En vertu du théorème 2.12, la $\infty$-catégorie $\nu(K \otimes L)$ est engendrée librement au sens des polygraphes par ses atomes. Il suffit donc de définir $\psi(G)$ sur les atomes de $\nu(K \otimes L)$ et de vérifier les compatibilités aux sources et aux buts. Soient $x$ de degré $i$ dans la base de $K$ et $y$ de degré $j$ dans la base de $L$. On pose

$$
\psi(G)(\langle x \otimes y\rangle)=\quad \mathrm{D}_{i+j} \stackrel{\left\langle x \otimes z_{j}^{\prime}\right\rangle}{\longrightarrow} \nu\left(K \otimes \lambda\left(\mathrm{D}_{j}\right)\right) \stackrel{G(\langle y\rangle)}{\longrightarrow} C,
$$

où $z^{\prime}$ désigne la cellule principale de $\mathrm{D}_{j}$.

Vérifions maintenant les compatibilités aux sources et aux buts. Fixons $m \geqslant 0$ et supposons que les formules ci-dessus définissent bien un $m$-foncteur. Il s'agit de montrer que, pour tous $x$ et $y$ comme ci-dessus avec $i+j=m+1$, on a

$$
\psi(G)(s(\langle x \otimes y\rangle))=s(\psi(G)(\langle x \otimes y\rangle)) \quad \text { et } \quad \psi(G)(t(\langle x \otimes y\rangle))=t(\psi(G)(\langle x \otimes y\rangle)) .
$$


Montrons la première égalité, la seconde se démontrant de manière analogue. Notons $z$ et $z^{\prime}$ les cellules principales respectives de $\mathrm{D}_{i}$ et $\mathrm{D}_{j}$. Puisque la $\infty$-catégorie $\nu\left(\lambda\left(\mathrm{D}_{i}\right) \otimes \lambda\left(\mathrm{D}_{j}\right)\right)$ est engendrée librement au sens des polygraphes par ses atomes, en vertu de la proposition 1.5, ses atomes l'engendrent également par compositions et il existe donc une formule $\chi$ exprimant la source de $\left\langle z_{i} \otimes z_{j}^{\prime}\right\rangle$ en fonction des $\left\langle z_{k}^{\varepsilon} \otimes z_{l}^{\prime \varepsilon^{\prime}}\right\rangle$ avec $0 \leqslant k \leqslant i, 0 \leqslant l \leqslant j, k+l<i+j, \varepsilon=0,1$ et $\varepsilon^{\prime}=0,1$. On notera $\chi\left[\left\langle z_{k}^{\varepsilon} \otimes z_{l}^{\prime \varepsilon^{\prime}}\right\rangle\right]$ l'évaluation de la formule $\chi$ en les éléments $z_{k}^{\varepsilon} \otimes z_{l}^{\prime \varepsilon^{\prime}}$. On a donc $s\left(\left\langle z_{i} \otimes z_{j}^{\prime}\right\rangle\right)=\chi\left[\left\langle z_{k}^{\varepsilon} \otimes z_{l}^{\prime \varepsilon^{\prime}}\right\rangle\right]$. Plus généralement, il résulte de la proposition A.8 que la même formule $\chi$ permet de calculer la source d'un atome $\langle m \otimes n\rangle$, où $m$ est de degré $i$ et $n$ de degré $j$, d'un produit tensoriel quelconque de complexes dirigés augmentés à base unitaire $M$ et $N$. On obtient ainsi

$$
\begin{aligned}
\psi(G)(s(\langle x \otimes y\rangle))= & \psi(G)\left(\chi\left[\left\langle x_{k}^{\varepsilon} \otimes y_{l}^{\varepsilon^{\prime}}\right\rangle\right]\right) \\
= & \chi\left[\psi(G)\left(\left\langle x_{k}^{\varepsilon} \otimes y_{l}^{\varepsilon^{\prime}}\right\rangle\right)\right] \\
& (\operatorname{car} \psi(G) \text { est un } m \text {-foncteur et } k+l<i+j=m+1) \\
= & \chi\left[G\left(\left\langle y_{l}^{\varepsilon^{\prime}}\right\rangle\right)\left(\left\langle x_{k}^{\varepsilon} \otimes z_{l}^{\prime \prime}\right\rangle\right)\right],
\end{aligned}
$$

où $z^{\prime \prime}$ désigne la cellule principale de $\mathrm{D}_{l}$, cette dernière égalité résultant de la définition de $\psi(G)$. Par ailleurs, on a

$$
G\left(\left\langle y_{l}^{\varepsilon^{\prime}}\right\rangle\right)\left(\left\langle x_{k}^{\varepsilon} \otimes z_{l}^{\prime \prime}\right\rangle\right)=G(\langle y\rangle)\left(\left\langle x_{k}^{\varepsilon} \otimes z_{l}^{\prime \varepsilon^{\prime}}\right\rangle\right) .
$$

En effet, pour $\varepsilon^{\prime}=0$, on a

$$
\begin{aligned}
G\left(\left\langle y_{l}^{0}\right\rangle\right)\left(\left\langle x_{k}^{\varepsilon} \otimes z_{l}^{\prime \prime}\right\rangle\right)= & G\left(s_{l}(\langle y\rangle)\right)\left(\left\langle x_{k}^{\varepsilon} \otimes z_{l}^{\prime \prime}\right\rangle\right) \\
= & s_{l}(G(\langle y\rangle))\left(\left\langle x_{k}^{\varepsilon} \otimes z_{l}^{\prime \prime}\right\rangle\right) \\
= & G(\langle y\rangle)\left(\nu\left(K \otimes \lambda\left(\sigma_{l}^{i}\right)\right)\left(\left\langle x_{k}^{\varepsilon} \otimes z_{l}^{\prime \prime}\right\rangle\right)\right) \\
& \left(\text { par définition des sources des cellules de } \underline{\text { Hom }}_{\text {lax }}(\nu(K), C)\right) \\
= & G(\langle y\rangle)\left(\left\langle x_{k}^{\varepsilon} \otimes z_{l}^{\prime 0}\right\rangle\right),
\end{aligned}
$$

la dernière égalité étant conséquence de la proposition A.7 puisque $\sigma_{l}^{i}\left(\left\langle z_{l}^{\prime \prime}\right\rangle\right)=\left\langle z_{l}^{\prime 0}\right\rangle$. La démonstration dans le cas $\varepsilon^{\prime}=1$ est analogue. En insérant cette égalité dans notre calcul précédent, on obtient

$$
\begin{aligned}
\psi(G)(s(\langle x \otimes y\rangle))= & \chi\left[G\left(\left\langle y_{l}^{\varepsilon^{\prime}}\right\rangle\right)\left(\left\langle x_{k}^{\varepsilon} \otimes z_{l}^{\prime \prime}\right\rangle\right)\right] \\
= & \chi\left[G(\langle y\rangle)\left(\left\langle x_{k}^{\varepsilon} \otimes z_{l}^{\prime \varepsilon^{\prime}}\right\rangle\right)\right] \\
= & G(\langle y\rangle)\left(\chi\left[\left\langle x_{k}^{\varepsilon} \otimes z_{l}^{\varepsilon^{\prime}}\right\rangle\right]\right) \\
& (\operatorname{car} G(\langle y\rangle) \text { est un } \infty \text {-foncteur }) \\
= & G(\langle y\rangle)\left(s\left(\left\langle x \otimes z_{j}^{\prime}\right\rangle\right)\right) \\
= & s\left(G(\langle y\rangle)\left(\left\langle x \otimes z_{j}^{\prime}\right\rangle\right)\right) \\
= & s(\psi(G)(\langle x \otimes y\rangle)),
\end{aligned}
$$


la dernière égalité résultant de la définition de $\psi(G)$, ce qui achève de montrer que $\psi(G)$ est bien un $\infty$-foncteur.

Enfin, vérifions que $\varphi$ et $\psi$ sont bien des bijections inverses l'une de l'autre. Soient $F: \nu(K \otimes L) \rightarrow C$ et $G: \nu(L) \rightarrow \underline{\operatorname{Hom}}_{\text {lax }}(\nu(K), C)$ deux $\infty$-foncteurs. On a, avec les notations précédentes,

$$
\psi \varphi(F)(\langle x \otimes y\rangle)=\varphi(F)(\langle y\rangle)\left(\left\langle x \otimes z_{j}^{\prime}\right\rangle\right)=F(\langle x \otimes y\rangle)
$$

et

$$
\varphi \psi(G)(\langle y\rangle)\left(\left\langle x \otimes z_{j}^{\varepsilon}\right\rangle\right)=\psi(G)\left(\left\langle x \otimes y_{j}^{\varepsilon}\right\rangle\right)=G(\langle y\rangle)\left(\left\langle x \otimes z_{j}^{\varepsilon}\right\rangle\right) .
$$

Les $\infty$-foncteurs $\psi \varphi(F)$ et $F$ (resp. les $\infty$-foncteurs $\varphi \psi(G)$ et $G$ ) coïncident donc sur les atomes et sont donc égaux, ce qu'il fallait démontrer.

A.14. - Soit $L$ un complexe de Steiner fort. On montre de même que le foncteur

$$
\begin{aligned}
\mathcal{C}_{\mathrm{da}} & \rightarrow \infty \text {-Cat } \\
K & \mapsto \nu(K \otimes L)
\end{aligned}
$$

commute aux limites inductives des systèmes de Steiner forts. On en déduit que le foncteur

$$
\begin{aligned}
\Theta^{\circ} & \rightarrow \mathcal{E} n s \\
S & \mapsto \operatorname{Hom}_{\infty-\mathcal{C} a t}(\nu(\lambda(S) \otimes L), C)
\end{aligned}
$$

envoie les sommes globulaires sur des produits globulaires. On définit alors, comme dans le paragraphe A.12, pour toute $\infty$-catégorie $C$, une $\infty$-catégorie $\underline{\text { Hom }}_{\text {oplax }}(\nu(L), C)$ en posant

$$
\underline{\operatorname{Hom}}_{\text {oplax }}(\nu(L), C)=\operatorname{Hom}_{\infty-\mathcal{C} a t}\left(\nu\left(\lambda\left(\mathrm{D}_{\bullet}\right) \otimes L\right), C\right) .
$$

On montre, comme dans la proposition précédente, que, pour tout complexe de Steiner fort $K$ et toute $\infty$-catégorie $C$, on a une bijection

$$
\operatorname{Hom}_{\infty-\mathcal{C} a t}(\nu(K \otimes L), C) \simeq \operatorname{Hom}_{\infty-\mathcal{C} a t}\left(\nu(K), \underline{\operatorname{Hom}}_{\text {oplax }}(\nu(L), C)\right),
$$

naturelle en $K$ et $C$.

Théorème A.15. - Il existe une et une seule (à unique isomorphisme monö̈dal près) structure de catégorie monoïdale sur $\infty$-Cat de produit

$$
\begin{aligned}
& \otimes: \infty-\mathcal{C} a t \times \infty-\mathcal{C} a t \rightarrow \infty-\mathcal{C} a t \\
&(A, B) \quad \mapsto A \otimes B
\end{aligned}
$$

ayant les deux propriétés suivantes :

(a) le foncteur $\nu_{\mid S t_{\mathrm{f}}}: \mathcal{S} t_{\mathrm{f}} \rightarrow \infty$-Cat, où la catégorie des complexes de Steiner forts $\mathcal{S} t_{\mathrm{f}}$ est munie de la structure de catégorie monoïdale définie par le produit tensoriel, s'étend en un foncteur monö̈dal;

(b) le foncteur $\otimes: \infty$-Cat $\times \infty$-Cat $\rightarrow \infty$-Cat commute aux petites limites inductives en chaque variable. 
De plus, cette structure monoïdale est bifermée.

Démonstration. - L'assertion résulte du théorème 5.3 appliqué à $\mathcal{C}=\infty$ - $\mathcal{C}$ at et $\mathcal{D}$ la catégorie des $\infty$-catégories de Steiner fortes (voir le paragraphe 2.15) munie du produit tensoriel (par l'identification de cette sous-catégorie à celle des complexes de Steiner forts), la petite sous-catégorie dense de l'énoncé étant la catégorie $\Theta$. En effet, les hypothèses de ce théorème sont précisément le contenu de la proposition A.13 et du paragraphe A.14.

A.16. - On appellera produit tensoriel de Gray ou plus simplement produit tensoriel le produit monoïdal

$$
\otimes: \infty-\mathcal{C} a t \times \infty-\mathcal{C} a t \rightarrow \infty-\mathcal{C} a t
$$

défini dans le théorème précédent. Si $K$ et $L$ sont des complexes de Steiner forts, on a, en vertu de ce même théorème, un isomorphisme canonique

$$
\nu(K) \otimes \nu(L) \simeq \nu(K \otimes L) .
$$

En particulier, si $S$ et $T$ sont deux objets de $\Theta$, puisqu'en vertu de la proposition 4.13 on a $S \simeq \nu \lambda(S)$ et $T \simeq \nu \lambda(T)$, on obtient un isomorphisme canonique

$$
S \otimes T \simeq \nu(\lambda(S) \otimes \lambda(T)) .
$$

Plus généralement, si $A$ et $B$ sont deux $\infty$-catégories, on a des isomorphismes canoniques

$$
A \otimes B \simeq \lim _{\substack{S \rightarrow \overrightarrow{A \in \Theta} / A \\ T \rightarrow B \in \Theta / B}} S \otimes T \simeq \lim _{\substack{S \rightarrow \overrightarrow{A \in \Theta} / A \\ T \rightarrow B \in \Theta / B}} \nu(\lambda(S) \otimes \lambda(T)) .
$$

En effet, puisque la catégorie $\Theta$ est dense dans $\infty$-C $a t$, toute $\infty$-catégorie est limite inductive canonique d'objets de $\Theta$. La formule résulte alors du fait que le produit tensoriel commute aux limites inductives en chaque variable.

Notons que l'unité du produit tensoriel est l'image par $\nu$ du complexe dirigé augmenté $\underline{\mathbb{Z}}^{\prime}$ du paragraphe 6.2 , c'est-à-dire la $\infty$-catégorie finale $\mathrm{D}_{0}$. Si $A$ est une $\infty$-catégorie, on a donc

$$
A \otimes \mathrm{D}_{0} \simeq A \simeq \mathrm{D}_{0} \otimes A .
$$

Remarque A.17. - Le produit tensoriel défini au paragraphe précédent coïncide avec celui introduit par Al-Agl et Steiner dans [1] et étudié par Crans dans [18]. En effet, le produit tensoriel étudié dans ces textes commutant aux limites inductives en chaque variable, il suffit de vérifier que les deux produits tensoriels coïncident sur une sous-catégorie dense de $\infty$-Cat. On peut vérifier que c'est le cas pour la sous-catégorie pleine des cubes, c'est-à-dire des objets de la forme $\Delta_{1} \otimes \cdots \otimes \Delta_{1}$, où $\Delta_{1}$ apparaît $n$ fois avec $n \geqslant 0$. 
A.18. - En vertu du théorème A.15, la structure de catégorie monoïdale définie par le produit tensoriel est bifermée. On obtient donc des foncteurs

$$
\underline{\text { Hom }}_{\text {oplax }}: \infty-\mathcal{C} a t^{\circ} \times \infty-\mathcal{C} a t \rightarrow \infty-\mathcal{C} a t \quad \text { et } \quad \underline{\operatorname{Hom}}_{\text {lax }}: \infty-\mathcal{C} a t^{\circ} \times \infty-\mathcal{C} a t \rightarrow \infty-\mathcal{C} a t
$$

vérifiant, pour toutes $\infty$-catégories $A, B$ et $C$,

$$
\operatorname{Hom}_{\infty-\mathcal{C} a t}(A \otimes B, C) \simeq \operatorname{Hom}_{\infty-\mathcal{C} a t}\left(A, \underline{\operatorname{Hom}}_{\text {oplax }}(B, C)\right)
$$

et

$$
\operatorname{Hom}_{\infty-\mathcal{C} a t}(A \otimes B, C) \simeq \operatorname{Hom}_{\infty-\mathcal{C} a t}\left(B, \underline{\operatorname{Hom}}_{\text {lax }}(A, C)\right),
$$

et ce de manière naturelle en $A, B$ et $C$. En particulier, pour tout $i \geqslant 0$, on a

$$
\underline{\operatorname{Hom}}_{\text {oplax }}(A, B)_{i}=\operatorname{Hom}_{\infty-\mathcal{C} a t}\left(\mathrm{D}_{i} \otimes A, B\right)
$$

et

$$
\underline{\operatorname{Hom}}_{\text {lax }}(A, B)_{i}=\operatorname{Hom}_{\infty-\mathcal{C} a t}\left(A \otimes \mathrm{D}_{i}, B\right) .
$$

Notons que, dans le cas où $A$ est de la forme $\nu(K)$ pour $K$ un complexe de Steiner fort, la $\infty$-catégorie $\underline{\operatorname{Hom}}_{\text {lax }}(\nu(K), C)$ que l'on vient d'introduire coïncide, en vertu de la proposition A.13, avec celle définie dans le paragraphe A.12. De même pour le foncteur $\underline{\text { Hom }}_{\text {oplax }}$ défini au paragraphe A.14.

Proposition A.19. - Les foncteurs

$$
\lambda: \infty-\mathcal{C} a t \rightarrow \mathcal{C}_{\mathrm{da}} \quad \text { et } \quad \nu: \mathcal{C}_{\mathrm{da}} \rightarrow \infty-\mathcal{C} a t
$$

sont monoïdal et monoïdal lax respectivement, les catégories $\infty$-Cat et $\mathcal{C}_{\mathrm{da}}$ étant toutes deux munies des structures de catégorie monö̈dale définies par le produit tensoriel.

Démonstration. - La démonstration est une adaptation immédiate de la preuve de l'assertion analogue pour le joint (proposition 6.34).

Proposition A.20. - Soient $K$ et $L$ deux complexes dirigés augmentés. Les applications

$$
\begin{aligned}
(K \otimes L)_{p} & \rightarrow(L \otimes K)_{p} \\
x \otimes y & \mapsto y \otimes x \quad,
\end{aligned}
$$

pour $p \geqslant 0$, définissent des isomorphismes

$$
(K \otimes L)^{\mathrm{op}} \simeq L^{\mathrm{op}} \otimes K^{\mathrm{op}} \quad \text { et } \quad(K \otimes L)^{\mathrm{co}} \simeq L^{\mathrm{co}} \otimes K^{\mathrm{co}} .
$$

En particulier, les applications identité de $(K \otimes L)_{p}$, pour $p \geqslant 0$, définissent un isomorphisme

$$
(K \otimes L)^{\circ} \simeq K^{\circ} \otimes L^{\circ} .
$$


Démonstration. - Commençons par démontrer le résultat pour le dual impair. La compatibilité aux augmentations, aux sous-monoïdes de positivité et la bijectivité sont évidentes. Il s'agit donc de vérifier la compatibilité aux différentielles. Notons $s: x \otimes y \mapsto y \otimes x$ l'application de l'énoncé. Pour tout élément homogène de $K \otimes L$ de la forme $x \otimes y$ et de degré au moins 1 , on a, en notant $d^{\prime}$ les différentielles dual impair,

$$
\begin{aligned}
s d^{\prime}(x \otimes y) & =(-1)^{|x|+|y|} s d(x \otimes y) \\
& =(-1)^{|x|+|y|} s\left(d x \otimes y+(-1)^{|x|} x \otimes d y\right) \\
& =(-1)^{|x|+|y|} y \otimes d x+(-1)^{|y|} d y \otimes x
\end{aligned}
$$

et

$$
\begin{aligned}
d s(x \otimes y) & =d(y \otimes x)=d^{\prime} y \otimes x+(-1)^{|y|} y \otimes d^{\prime} x \\
& =(-1)^{|y|} d y \otimes x+(-1)^{|y|+|x|} y \otimes d x \\
& =(-1)^{|x|+|y|} y \otimes d x+(-1)^{|y|} d y \otimes x,
\end{aligned}
$$

d'où le premier isomorphisme. Le résultat pour le dual pair se démontre de manière analogue : en notant $d^{\prime}$ les différentielles dual pair, on a

$$
\begin{aligned}
s d^{\prime}(x \otimes y) & =(-1)^{|x|+|y|+1} s d(x \otimes y) \\
& =(-1)^{|x|+|y|+1} s\left(d x \otimes y+(-1)^{|x|} x \otimes d y\right) \\
& =(-1)^{|x|+|y|+1} y \otimes d x+(-1)^{|y|+1} d y \otimes x
\end{aligned}
$$

et

$$
\begin{aligned}
d s(x \otimes y) & =d(y \otimes x)=d^{\prime} y \otimes x+(-1)^{|y|} y \otimes d^{\prime} x \\
& =(-1)^{|y|+1} d y \otimes x+(-1)^{|y|+|x|+1} y \otimes d x \\
& =(-1)^{|x|+|y|+1} y \otimes d x+(-1)^{|y|+1} d y \otimes x,
\end{aligned}
$$

et on obtient ainsi le deuxième isomorphisme. Le troisième se déduit des deux premiers : l'application $s \circ s$, qui n'est autre que l'identité, définit un isomorphisme

$$
(A \otimes B)^{\circ}=\left((A \otimes B)^{\mathrm{op}}\right)^{\mathrm{co}} \simeq\left(B^{\mathrm{op}} \otimes A^{\mathrm{op}}\right)^{\mathrm{co}} \simeq\left(A^{\mathrm{op}}\right)^{\mathrm{co}} \otimes\left(B^{\mathrm{op}}\right)^{\mathrm{co}}=A^{\circ} \otimes B^{\circ} .
$$

Remarque A.21. - Outre la dualité triviale, les dualités considérées dans l'énoncé précédent sont les seules pour lesquelles on a des isomorphismes de ce type.

Proposition A.22. - Soient $A$ et $B$ deux $\infty$-catégories. On a des isomorphismes naturels canoniques

$$
(A \otimes B)^{\mathrm{op}} \simeq B^{\mathrm{op}} \otimes A^{\mathrm{op}} \quad \text { et } \quad(A \otimes B)^{\mathrm{co}} \simeq B^{\mathrm{co}} \otimes A^{\mathrm{co}} .
$$

En particulier, on a un isomorphisme naturel canonique

$$
(A \otimes B)^{\circ} \simeq A^{\circ} \otimes B^{\circ} .
$$


Démonstration. - La preuve est une adaptation immédiate de la preuve de l'assertion analogue pour le joint (proposition 6.35) en remplaçant l'usage de la proposition 6.10 par celui de la proposition A.20.

Proposition A.23. - Soient $A$ et $B$ deux $\infty$-catégories. On a des isomorphismes naturels canoniques

$$
\begin{aligned}
& \underline{\operatorname{Hom}}_{\text {oplax }}(A, B)^{\mathrm{op}} \simeq \underline{\operatorname{Hom}}_{\text {lax }}\left(A^{\mathrm{op}}, B^{\mathrm{op}}\right), \\
& \underline{\operatorname{Hom}}_{\text {oplax }}(A, B)^{\mathrm{co}} \simeq \underline{\operatorname{Hom}}_{\mathrm{lax}}\left(A^{\mathrm{co}}, B^{\mathrm{co}}\right), \\
& \underline{\operatorname{Hom}}_{\text {oplax }}(A, B)^{\circ} \simeq \underline{\operatorname{Hom}}_{\text {oplax }}\left(A^{\circ}, B^{\circ}\right) .
\end{aligned}
$$

Démonstration. - Notons $D$ la dualité paire ou impaire. Pour toute $\infty$-catégorie $C$, on a

$$
\begin{aligned}
\operatorname{Hom}_{\infty-\mathcal{C} a t}\left(C, D\left(\underline{\operatorname{Hom}}_{\mathrm{oplax}}(A, B)\right)\right) \simeq & \operatorname{Hom}_{\infty-\mathcal{C} a t}\left(D(C), \underline{\operatorname{Hom}}_{\mathrm{oplax}}(A, B)\right) \\
\simeq & \operatorname{Hom}_{\infty-\mathcal{C} a t}(D(C) \otimes A, B) \\
& (\operatorname{par} \operatorname{adjonction}) \\
\simeq & \operatorname{Hom}_{\infty-\mathcal{C} a t}(D(D(C) \otimes A), D(B)) \\
\simeq & \operatorname{Hom}_{\infty-\mathcal{C} a t}(D(A) \otimes C, D(B))
\end{aligned}
$$

(en vertu de la proposition précédente)

$\simeq \operatorname{Hom}_{\infty-\mathcal{C} a t}\left(C, \underline{\operatorname{Hom}}_{\text {lax }}(D(A), D(B))\right)$,

d'où les deux premiers isomorphismes. Le troisième en résulte :

$$
\begin{aligned}
\underline{\operatorname{Hom}}_{\mathrm{oplax}}(A, B)^{\circ} & =\left(\underline{\operatorname{Hom}}_{\mathrm{oplax}}(A, B)^{\mathrm{op}}\right)^{\mathrm{co}} \\
& \simeq \underline{\operatorname{Hom}}_{\mathrm{lax}}\left(A^{\mathrm{op}}, B^{\mathrm{op}}\right)^{\mathrm{co}} \\
& \simeq \underline{\operatorname{Hom}}_{\mathrm{oplax}}\left(\left(A^{\mathrm{op}}\right)^{\mathrm{co}},\left(B^{\mathrm{op}}\right)^{\mathrm{co}}\right) \\
& \simeq \underline{\operatorname{Hom}}_{\mathrm{oplax}}\left(A^{\circ}, B^{\circ}\right) .
\end{aligned}
$$

Proposition A.24. - Soient $p, q \geqslant 0$ deux entiers. Si A est une p-catégorie et $B$ une q-catégorie, alors on a un isomorphisme canonique

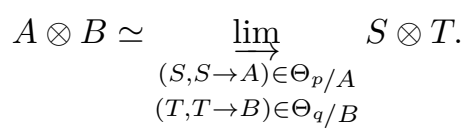

En particulier, le produit tensoriel de $A$ et $B$ est une $(p+q)$-catégorie.

Démonstration. - La preuve est une adaptation immédiate de la preuve de l'assertion analogue pour le joint (proposition 8.5). 
Dans la suite de cet appendice on fixe un entier $n \geqslant 0$.

A.25. - Soient $A$ et $B$ deux $n$-catégories. On appelle produit tensoriel $n$-catégorique de $A$ et $B$ la $n$-catégorie

$$
A \otimes_{n} B=\tau_{\leqslant n}^{\mathrm{i}}(A \otimes B) .
$$

Cette opération définit un foncteur

$$
\begin{aligned}
& n-\mathcal{C} a t \times n-\mathcal{C} a t \rightarrow n-\mathcal{C} a t \\
&(A, B) \quad \mapsto A \otimes_{n} B .
\end{aligned}
$$

Lemme A.26. - Soient $K$ et $L$ deux complexes dirigés augmentés. Les morphismes canoniques $K \rightarrow \tau_{\leqslant n}^{\mathrm{i}}(K)$ et $L \rightarrow \tau_{\leqslant n}^{\mathrm{i}}(L)$ induisent un isomorphisme

$$
\tau_{\leqslant n}^{\mathrm{i}}(K \otimes L) \simeq \tau_{\leqslant n}^{\mathrm{i}}\left(\tau_{\leqslant n}^{\mathrm{i}}(K) \otimes \tau_{\leqslant n}^{\mathrm{i}}(L)\right) .
$$

Démonstration. - La preuve est une adaptation facile de la preuve de l'assertion analogue pour le joint (lemme 8.7).

Proposition A.2\%. - Soient $A$ et $B$ deux $\infty$-catégories. Les $\infty$-foncteurs canoniques $A \rightarrow \tau_{\leqslant n}^{\mathrm{i}}(A)$ et $B \rightarrow \tau_{\leqslant n}^{\mathrm{i}}(B)$ induisent un isomorphisme

$$
\tau_{\leqslant n}^{\mathrm{i}}(A \otimes B) \simeq \tau_{\leqslant n}^{\mathrm{i}}\left(\tau_{\leqslant n}^{\mathrm{i}}(A) \otimes \tau_{\leqslant n}^{\mathrm{i}}(B)\right)
$$

Démonstration. - La preuve est une adaptation immédiate de la preuve de l'assertion analogue pour le joint (proposition 8.8).

Proposition A.28. — La structure de catégorie monö̈dale sur $\infty$-Cat définie par le produit tensoriel induit une structure de catégorie monö̈dale sur $n$-C $\mathcal{C}$ t pour le produit tensoriel $n$-catégorique.

Démonstration. - La preuve est une adaptation immédiate de la preuve de l'assertion analogue pour le joint (proposition 8.9).

Proposition A.29. - Soit $B$ une n-catégorie. Pour toute $\infty$-catégorie A, les $\infty$-catégories $\underline{\mathrm{Hom}}_{\mathrm{oplax}}(A, B)$ et $\underline{\mathrm{Hom}}_{\mathrm{lax}}(A, B)$ sont des $n$-catégories.

Démonstration. - La preuve est une adaptation immédiate de la preuve de l'assertion analogue pour le joint (proposition 8.11).

Proposition A.30. — La structure de catégorie monoïdale sur n-Cat définie par le produit tensoriel $n$-catégorique est bifermée, ses Hom internes étant donnés par les restrictions de $\underline{\mathrm{Hom}}_{\text {oplax }}$ et $\underline{\mathrm{Hom}}_{\text {lax }}$ à $n$-C at.

Démonstration. - La preuve est une adaptation immédiate de la preuve de l'assertion analogue pour le joint (corollaire 8.13). 
A.31. - Pour $n=1$, le produit tensoriel 1-catégorique n'est autre que le produit cartésien. En effet, le produit tensoriel 1-catégorique comme le produit cartésien commutant aux limites inductives en chaque variable, il suffit de démontrer que ces deux foncteurs coïncident sur une sous-catégorie dense de $\mathcal{C} a t$. On peut le vérifier pour la sous-catégorie pleine de $\mathcal{C}$ at formée des $\Delta_{1} \times \cdots \times \Delta_{1}$, où $\Delta_{1}$ apparaît $n$ fois avec $n \geqslant 0$. Il s'agit alors de montrer qu'on a $\tau_{\leqslant 1}^{\mathrm{i}}\left(\Delta_{1} \otimes \cdots \otimes \Delta_{1}\right)=\Delta_{1} \times \cdots \times \Delta_{1}$, ce qu'on vérifie sans difficulté en utilisant les complexes dirigés augmentés.

De même, si $n=2$, on peut démontrer que le produit tensoriel 2-catégorique est le produit tensoriel introduit par Gray dans [22]. Comme ci-dessus, il suffit de démontrer qu'on a $\tau_{\leqslant 2}^{\mathrm{i}}\left(\Delta_{1} \otimes \cdots \otimes \Delta_{1}\right) \simeq \Delta_{1} \otimes_{\mathrm{G}} \cdots \otimes_{\mathrm{G}} \Delta_{1}$, où $\otimes_{\mathrm{G}}$ désigne le produit tensoriel de [22], ce qu'on peut également faire en utilisant les complexes dirigés augmentés. 



\section{APPENDICE B}

\section{COMPLÉMENTS SUR LES TRANSFORMATIONS OPLAX}

Dans cet appendice, on montre que les transformations oplax entre $\infty$-foncteurs de $C$ vers $D$, définies par des formules explicites au paragraphe 1.9, sont en correspondance canonique avec les $\infty$-foncteurs de $\mathrm{D}_{1} \otimes C$ vers $D$. On étudie par ailleurs le lien entre $\mathrm{D}_{1} \otimes C, \mathrm{D}_{0} \star C$ et une suspension $\Sigma C$ de $C$.

\section{B.1. Description de la $\infty$-catégorie des cylindres}

Si $C$ est une $\infty$-catégorie, le paragraphe A.18 permet de définir une $\infty$-catégorie $\underline{\mathrm{Hom}}_{\text {lax }}\left(\mathrm{D}_{1}, C\right)$. Le but de cette section est de décrire explicitement cette $\infty$-catégorie.

B.1.1. - Fixons $i \geqslant 0$. Nous allons commencer par décrire la $(i+1)$-catégorie $\mathrm{D}_{1} \otimes \mathrm{D}_{i}$. On notera $a$ la cellule principale de $\mathrm{D}_{1}$ et $x$ celle de $\mathrm{D}_{i}$. En vertu de la proposition 4.13 et du paragraphe A.16, on a

$$
\mathrm{D}_{1} \otimes \mathrm{D}_{i} \simeq \nu\left(\lambda\left(\mathrm{D}_{1}\right) \otimes \lambda\left(\mathrm{D}_{i}\right)\right)
$$

Par ailleurs, les paragraphes 4.10 et A.1 montrent que le complexe $\lambda\left(D_{1}\right) \otimes \lambda\left(D_{i}\right)$ a pour base l'ensemble formé des

$$
a_{0}^{0} \otimes x_{k}^{\varepsilon}, \quad a_{0}^{1} \otimes x_{k}^{\varepsilon}, \quad a \otimes x_{k}^{\varepsilon},
$$

où $k$ varie entre 0 et $i$, et $\varepsilon=0,1$ (en se souvenant que $x_{i}^{0}=x_{i}^{1}$ ).

Proposition B.1.2. - Pour tout $k$ tel que $0<k \leqslant i, \varepsilon=0,1$ et $\varepsilon^{\prime}=0,1$, on a

$$
s\left(\left\langle a_{0}^{\varepsilon^{\prime}} \otimes x_{k}^{\varepsilon}\right\rangle\right)=\left\langle a_{0}^{\varepsilon^{\prime}} \otimes x_{k-1}^{0}\right\rangle \quad \text { et } \quad t\left(\left\langle a_{0}^{\varepsilon^{\prime}} \otimes x_{k}^{\varepsilon}\right\rangle\right)=\left\langle a_{0}^{\varepsilon^{\prime}} \otimes x_{k-1}^{1}\right\rangle .
$$

Démonstration. — Cela résulte immédiatement de l'égalité

$$
d\left(a_{0}^{\varepsilon^{\prime}} \otimes x_{k}^{\varepsilon}\right)=a_{0}^{\varepsilon^{\prime}} \otimes d\left(x_{k}^{\varepsilon}\right)=a_{0}^{\varepsilon^{\prime}} \otimes x_{k-1}^{1}-a_{0}^{\varepsilon^{\prime}} \otimes x_{k-1}^{0} .
$$

Lemme B.1.3. - Pour tout $k$ tel que $0 \leqslant k \leqslant i, \varepsilon=0,1$, tout l tel que $0 \leqslant l<k+1$ et $\varepsilon^{\prime}=0,1$, on $a$

$$
\left\langle a \otimes x_{k}^{\varepsilon}\right\rangle_{l}^{\varepsilon^{\prime}}=a_{0}^{\varepsilon^{\prime}} \otimes x_{l}^{\eta}+a \otimes x_{l-1}^{1-\varepsilon^{\prime}},
$$


où $\eta$ vaut $\varepsilon$ si $k=l$ et $\varepsilon^{\prime}$ sinon, en convenant que $x_{-1}^{0}=0=x_{-1}^{1}$. En particulier, on $a$

$$
\left\langle a \otimes x_{k}^{\varepsilon}\right\rangle_{0}^{\varepsilon^{\prime}}=a_{0}^{\varepsilon^{\prime}} \otimes x_{0}^{\eta}
$$

Démonstration. - En vertu du lemme A.2 et de la convention de l'énoncé pour le cas $l=0$, on a

$$
\left\langle a \otimes x_{k}^{\varepsilon}\right\rangle_{l}^{\varepsilon^{\prime}}=\langle a\rangle_{0}^{\varepsilon^{\prime}} \otimes\left\langle x_{k}^{\varepsilon}\right\rangle_{l}^{\varepsilon^{\prime}}+\langle a\rangle_{1}^{\varepsilon^{\prime}} \otimes\left\langle x_{k}^{\varepsilon}\right\rangle_{l-1}^{1-\varepsilon^{\prime}}=a_{0}^{\varepsilon^{\prime}} \otimes x_{l}^{\eta}+a \otimes x_{l-1}^{1-\varepsilon^{\prime}},
$$

ce qu'on voulait démontrer.

Proposition B.1.4. - Pour tout $k$ tel que $0 \leqslant k \leqslant i$ et $\varepsilon=0,1$, on a

$$
s\left(\left\langle a \otimes x_{k}^{\varepsilon}\right\rangle\right)=\left\langle a \otimes x_{k-1}^{1}\right\rangle *_{k-1} \cdots *_{1}\left\langle a \otimes x_{0}^{1}\right\rangle *_{0}\left\langle a_{0}^{0} \otimes x_{k}^{\varepsilon}\right\rangle
$$

et

$$
t\left(\left\langle a \otimes x_{k}^{\varepsilon}\right\rangle\right)=\left\langle a_{0}^{1} \otimes x_{k}^{\varepsilon}\right\rangle *_{0}\left\langle a \otimes x_{0}^{0}\right\rangle *_{1} \cdots *_{k-1}\left\langle a \otimes x_{k-1}^{0}\right\rangle .
$$

Démonstration. - C'est le cas $l=k$ du lemme plus général suivant.

Lemme B.1.5. - Pour tous $k, l$ tels que $0 \leqslant l \leqslant k \leqslant i$ et $\varepsilon=0,1$, on a

$$
s_{l}\left(\left\langle a \otimes x_{k}^{\varepsilon}\right\rangle\right)=\left\langle a \otimes x_{l-1}^{1}\right\rangle *_{l-1} \cdots *_{1}\left\langle a \otimes x_{0}^{1}\right\rangle *_{0}\left\langle a_{0}^{0} \otimes x_{l}^{\eta_{0}}\right\rangle
$$

et

$$
t_{l}\left(\left\langle a \otimes x_{k}^{\varepsilon}\right\rangle\right)=\left\langle a_{0}^{1} \otimes x_{l}^{\eta_{1}}\right\rangle *_{0}\left\langle a \otimes x_{0}^{0}\right\rangle *_{1} \cdots *_{l-1}\left\langle a \otimes x_{l-1}^{0}\right\rangle,
$$

où $\eta_{0}$ et $\eta_{1}$ valent $\varepsilon$ si $k=l$ et 0 et 1 respectivement sinon.

Démonstration. - On va démontrer les deux égalités simultanément par récurrence sur $l$. Pour $l=0$, en vertu du lemme B.1.3, pour $\varepsilon^{\prime}=0,1$, on a $\left\langle a \otimes x_{k}^{\varepsilon}\right\rangle_{0}^{\varepsilon^{\prime}}=a_{0}^{\varepsilon^{\prime}} \otimes x_{0}^{\eta_{\varepsilon^{\prime}}}$ et donc

$$
s_{0}\left(\left\langle a \otimes x_{k}^{\varepsilon}\right\rangle\right)=\left\langle a_{0}^{0} \otimes x_{0}^{\eta_{0}}\right\rangle \quad \text { et } \quad t_{0}\left(\left\langle a \otimes x_{k}^{\varepsilon}\right\rangle\right)=\left\langle a_{0}^{1} \otimes x_{0}^{\eta_{1}}\right\rangle .
$$

Supposons maintenant l'égalité démontrée au rang $l-1$ et montrons-la au rang $l \leqslant k$. Par la proposition B.1.2 et l'hypothèse de récurrence, on a

$$
\begin{aligned}
t_{l-1} & \left(\left\langle a \otimes x_{l-2}^{1}\right\rangle *_{l-2} \cdots *_{1}\left\langle a \otimes x_{0}^{1}\right\rangle *_{0}\left\langle a_{0}^{0} \otimes x_{l}^{\eta_{0}}\right\rangle\right) \\
& =\left\langle a \otimes x_{l-2}^{1}\right\rangle *_{l-2} \cdots *_{1}\left\langle a \otimes x_{0}^{1}\right\rangle *_{0}\left\langle a_{0}^{0} \otimes x_{l-1}^{1}\right\rangle \\
& =s_{l-1}\left(\left\langle a \otimes x_{l-1}^{1}\right\rangle\right)
\end{aligned}
$$

et

$$
\begin{aligned}
s_{l-1} & \left(\left\langle a_{0}^{1} \otimes x_{l}^{\eta_{1}}\right\rangle *_{0}\left\langle a \otimes x_{0}^{0}\right\rangle *_{1} \cdots *_{l-2}\left\langle a \otimes x_{l-2}^{0}\right\rangle\right) \\
& =\left\langle a_{0}^{1} \otimes x_{l-1}^{0}\right\rangle *_{0}\left\langle a \otimes x_{0}^{0}\right\rangle *_{1} \cdots *_{l-2}\left\langle a \otimes x_{l-2}^{0}\right\rangle \\
& =t_{l-1}\left(\left\langle a \otimes x_{l-1}^{0}\right\rangle\right)
\end{aligned}
$$

et les cellules

$$
\begin{aligned}
& u=\left\langle a \otimes x_{l-1}^{1}\right\rangle *_{l-1}\left(\left\langle a \otimes x_{l-2}^{1}\right\rangle *_{l-2} \cdots *_{1}\left\langle a \otimes x_{0}^{1}\right\rangle *_{0}\left\langle a_{0}^{0} \otimes x_{l}^{\eta_{0}}\right\rangle\right), \\
& v=\left(\left\langle a_{0}^{1} \otimes x_{l}^{\eta_{1}}\right\rangle *_{0}\left\langle a \otimes x_{0}^{0}\right\rangle *_{1} \cdots *_{l-2}\left\langle a \otimes x_{l-2}^{0}\right\rangle\right) *_{l-1}\left\langle a \otimes x_{l-1}^{0}\right\rangle
\end{aligned}
$$


sont donc bien définies. Par ailleurs, en utilisant le lemme B.1.3, on obtient

$$
u_{l}=a \otimes x_{l-1}^{1}+a_{0}^{0} \otimes x_{l}^{\eta_{0}}=s_{l}\left(\left\langle a \otimes x_{k}^{\varepsilon}\right\rangle\right)_{l}
$$

et de même

$$
v_{l}=a_{0}^{1} \otimes x_{l}^{\eta_{1}}+a \otimes x_{l-1}^{0}=t_{l}\left(\left\langle a \otimes x_{k}^{\varepsilon}\right\rangle\right)_{l} .
$$

Pour conclure, il suffit donc de montrer qu'on a, d'une part,

$$
s\left(s_{l}\left(\left\langle a \otimes x_{k}^{\varepsilon}\right\rangle\right)\right)=s(u) \quad \text { et } \quad t\left(s_{l}\left(\left\langle a \otimes x_{k}^{\varepsilon}\right\rangle\right)\right)=t(u)
$$

et, d'autre part,

$$
s\left(t_{l}\left(\left\langle a \otimes x_{k}^{\varepsilon}\right\rangle\right)\right)=s(v) \quad \text { et } \quad t\left(t_{l}\left(\left\langle a \otimes x_{k}^{\varepsilon}\right\rangle\right)\right)=t(v) .
$$

Or, en utilisant l'hypothèse de récurrence, on a

$$
\begin{aligned}
s\left(s_{l}\left(\left\langle a \otimes x_{k}^{\varepsilon}\right\rangle\right)\right) & =s_{l-1}\left(\left\langle a \otimes x_{k}^{\varepsilon}\right\rangle\right) \\
& =\left\langle a \otimes x_{l-2}^{1}\right\rangle *_{l-2} \cdots *_{1}\left\langle a \otimes x_{0}^{1}\right\rangle *_{0}\left\langle a_{0}^{0} \otimes x_{l-1}^{0}\right\rangle \\
& =s_{l-1}\left(\left\langle a \otimes x_{l-1}^{1}\right\rangle *_{l-1}\left\langle a \otimes x_{l-2}^{1}\right\rangle *_{l-2} \cdots *_{1}\left\langle a \otimes x_{0}^{1}\right\rangle *_{0}\left\langle a_{0}^{0} \otimes x_{l}^{\eta_{0}}\right\rangle\right) \\
& =s(u), \\
t\left(s_{l}\left(\left\langle a \otimes x_{k}^{\varepsilon}\right\rangle\right)\right) & =t_{l-1}\left(\left\langle a \otimes x_{k}^{\varepsilon}\right\rangle\right) \\
& =\left\langle a_{0}^{1} \otimes x_{l-1}^{1}\right\rangle *_{0}\left\langle a \otimes x_{0}^{0}\right\rangle *_{1} \cdots *_{l-2}\left\langle a \otimes x_{l-2}^{0}\right\rangle \\
& =t_{l-1}\left(\left\langle a \otimes x_{l-1}^{1}\right\rangle\right) \\
& =t_{l-1}\left(\left\langle a \otimes x_{l-1}^{1}\right\rangle *_{l-1}\left\langle a \otimes x_{l-2}^{1}\right\rangle *_{l-2} \cdots *_{1}\left\langle a \otimes x_{0}^{1}\right\rangle *_{0}\left\langle a_{0}^{0} \otimes x_{l}^{\eta_{0}}\right\rangle\right) \\
& =t(u), \\
s\left(t_{l}\left(\left\langle a \otimes x_{k}^{\varepsilon}\right\rangle\right)\right) & =s_{l-1}\left(\left\langle a \otimes x_{k}^{\varepsilon}\right\rangle\right) \\
& =\left\langle a \otimes x_{l-2}^{1}\right\rangle *_{l-2} \cdots *_{1}\left\langle a \otimes x_{0}^{1}\right\rangle *_{0}\left\langle a_{0}^{0} \otimes x_{l-1}^{0}\right\rangle \\
& =s_{l-1}\left(\left\langle a \otimes x_{l-1}^{0}\right\rangle\right) \\
& =s_{l-1}\left(\left\langle a_{0}^{1} \otimes x_{l}^{\eta_{1}}\right\rangle *_{0}\left\langle a \otimes x_{0}^{0}\right\rangle *_{1} \cdots *_{l-2}\left\langle a \otimes x_{l-2}^{0}\right\rangle *_{l-1}\left\langle a \otimes x_{l-1}^{0}\right\rangle\right) \\
& =s(v) \\
\text { et } & \\
t\left(t_{l}\left(\left\langle a \otimes x_{k}^{\varepsilon}\right\rangle\right)\right) & =t_{l-1}\left(\left\langle a \otimes x_{k}^{\varepsilon}\right\rangle\right) \\
& =\left\langle a_{0}^{1} \otimes x_{l-1}^{1}\right\rangle *_{0}\left\langle a \otimes x_{0}^{0}\right\rangle *_{1} \cdots *_{l-2}\left\langle a \otimes x_{l-2}^{0}\right\rangle \\
& =t_{l-1}\left(\left\langle a_{0}^{1} \otimes x_{l}^{\eta_{1}}\right\rangle *_{0}\left\langle a \otimes x_{0}^{0}\right\rangle *_{1} \cdots *_{l-2}\left\langle a \otimes x_{l-2}^{0}\right\rangle *_{l-1}\left\langle a \otimes x_{l-1}^{0}\right\rangle\right) \\
& =t(v),
\end{aligned}
$$

d'où le résultat.

Proposition B.1.6. - Soit $C$ une $\infty$-catégorie. Fixons

- $c$ et d deux $i$-flèches de $C$;

- pour tout $k$ tel que $0 \leqslant k \leqslant i$ et $\varepsilon=0,1, \alpha_{k}^{\varepsilon}$ une $(k+1)$-flèche de $C$, avec $\alpha_{i}^{0}=\alpha_{i}^{1}$, 
vérifiant les égalités

$$
s\left(\alpha_{k}^{\varepsilon}\right)=\alpha_{k-1}^{1} *_{k-1} \cdots *_{1} \alpha_{0}^{1} *_{0} c_{k}^{\varepsilon} \quad \text { et } \quad t\left(\alpha_{k}^{\varepsilon}\right)=d_{k}^{\varepsilon} *_{0} \alpha_{0}^{0} *_{1} \cdots *_{k-1} \alpha_{k-1}^{0},
$$

ò̀, pour $e=c, d$, on a posé

$$
e_{k}^{\varepsilon}= \begin{cases}s_{k}(e) & \text { si } \varepsilon=0, \\ t_{k}(e) & \text { si } \varepsilon=1 .\end{cases}
$$

Alors il existe un et un seul $\infty$-foncteur $h: \mathrm{D}_{1} \otimes \mathrm{D}_{i} \rightarrow C$ tel que

$$
c=h\left(\left\langle a_{0}^{0} \otimes x_{i}\right\rangle\right), \quad d=h\left(\left\langle a_{0}^{1} \otimes x_{i}\right\rangle\right) \quad \text { et } \quad \alpha_{k}^{\varepsilon}=h\left(\left\langle a \otimes x_{k}^{\varepsilon}\right\rangle\right),
$$

pour tout $k$ tel que $0 \leqslant k \leqslant i$ et $\varepsilon=0,1$.

Démonstration. — En vertu du théorème 2.12 , la $\infty$-catégorie $\nu\left(\lambda\left(\mathrm{D}_{1}\right) \otimes \lambda\left(\mathrm{D}_{i}\right)\right)$, isomorphe à la $\infty$-catégorie $\mathrm{D}_{1} \otimes \mathrm{D}_{i}$, est engendrée librement par ses atomes au sens des polygraphes. On conclut alors comme dans la preuve de la proposition 9.6 en utilisant les propositions B.1.2 et B.1.4.

B.1.7. - Soit $C$ une $\infty$-catégorie. Par définition (voir le paragraphe A.18), les $i$-flèches de $\underline{\operatorname{Hom}}_{\text {lax }}\left(\mathrm{D}_{1}, C\right)$ correspondent aux $\infty$-foncteurs $h: \mathrm{D}_{1} \otimes \mathrm{D}_{i} \rightarrow C$. En vertu de la proposition précédente, un tel $\infty$-foncteur est déterminé par un triplet $(c, d, \alpha)$, où $c$ et $d$ sont des $i$-flèches de $C$ et $\alpha$ est une famille de cellules $\alpha_{k}^{\varepsilon} \operatorname{de} C$, pour $0 \leqslant k \leqslant i$ et $\varepsilon=0,1$, avec $\alpha_{i}^{0}=\alpha_{i}^{1}$,

$$
\alpha_{k}^{\varepsilon}: \alpha_{k-1}^{1} *_{k-1} \cdots *_{1} \alpha_{0}^{1} *_{0} c_{k}^{\varepsilon} \rightarrow d_{k}^{\varepsilon} *_{0} \alpha_{0}^{0} *_{1} \cdots *_{k-1} \alpha_{k-1}^{0}, \quad(k+1) \text {-flèche, }
$$

où, pour $e=c, d$, on a posé

$$
e_{k}^{\varepsilon}= \begin{cases}s_{k}(e) & \text { si } \varepsilon=0 \\ t_{k}(e) & \text { si } \varepsilon=1\end{cases}
$$

Dans la suite de cette section, on identifiera les $i$-flèches de $\underline{\operatorname{Hom}_{\text {lax }}}\left(\mathrm{D}_{1}, C\right)$ avec de tels triplets $(c, d, \alpha)$. On notera $\alpha_{i}$ pour $\alpha_{i}^{0}=\alpha_{i}^{1}$.

Voici une représentation graphique des objets, des 1-flèches et des 2-flèches de $\underline{\mathrm{Hom}}_{\text {lax }}\left(\mathrm{D}_{1}, C\right)$ :
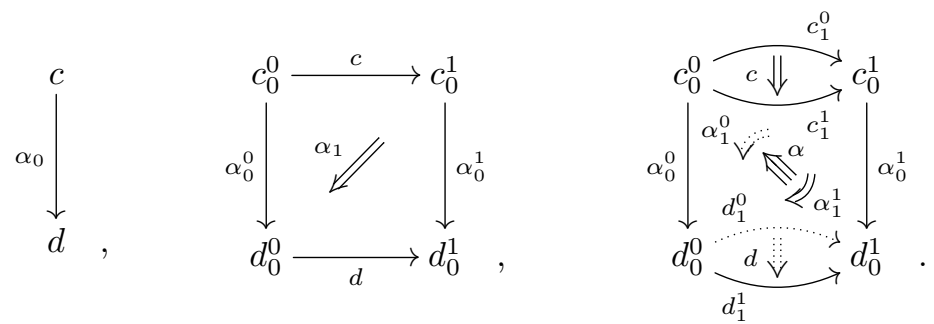

Le but de la suite de cette section est de décrire la structure de $\infty$-catégorie de $\underline{\operatorname{Hom}}_{\text {lax }}\left(\mathrm{D}_{1}, C\right)$ en termes des $(c, d, \alpha)$. 
B.1.8. - En vertu de l'adjonction définissant la $\infty$-catégorie $\underline{\operatorname{Hom}}_{\text {lax }}\left(\mathrm{D}_{1}, C\right)$, les sources, buts, identités et compositions de cette $\infty$-catégorie sont induits par les $\infty$-foncteurs

$$
\begin{array}{cl}
\mathrm{D}_{1} \otimes \sigma_{i}: \mathrm{D}_{1} \otimes \mathrm{D}_{i-1} \rightarrow \mathrm{D}_{1} \otimes \mathrm{D}_{i} & \text { pour } i \geqslant 1, \\
\mathrm{D}_{1} \otimes \tau_{i}: \mathrm{D}_{1} \otimes \mathrm{D}_{i-1} \rightarrow \mathrm{D}_{1} \otimes \mathrm{D}_{i} & \text { pour } i \geqslant 1, \\
\mathrm{D}_{1} \otimes \kappa_{i}: \mathrm{D}_{1} \otimes \mathrm{D}_{i+1} \rightarrow \mathrm{D}_{1} \otimes \mathrm{D}_{i} & \text { pour } i \geqslant 0, \\
\mathrm{D}_{1} \otimes \nabla_{j}^{i}: \mathrm{D}_{1} \otimes \mathrm{D}_{i} \rightarrow\left(\mathrm{D}_{1} \otimes \mathrm{D}_{i}\right) \amalg_{\mathrm{D}_{1} \otimes \mathrm{D}_{j}}\left(\mathrm{D}_{1} \otimes \mathrm{D}_{i}\right) & \text { pour } i>j \geqslant 0,
\end{array}
$$

où $\sigma_{i}, \tau_{i}, \kappa_{i}$ et $\nabla_{j}^{i}$ désignent les $\infty$-foncteurs des paragraphes 4.1 et 4.3 , et où on a identifié $\left(\mathrm{D}_{1} \otimes \mathrm{D}_{i}\right) \amalg_{\mathrm{D}_{1} \otimes \mathrm{D}_{j}}\left(\mathrm{D}_{1} \otimes \mathrm{D}_{i}\right)$ et $\mathrm{D}_{1} \otimes\left(\mathrm{D}_{i} \amalg_{\mathrm{D}_{j}} \mathrm{D}_{i}\right)$.

Nous allons commencer par décrire concrètement les morphismes $\mathrm{D}_{1} \otimes \sigma_{i}, \mathrm{D}_{1} \otimes \tau_{i}$ et $\mathrm{D}_{1} \otimes \kappa_{i}$. On note toujours $a$ la cellule principale de $\mathrm{D}_{1}$.

Proposition B.1.9. - Fixons $i \geqslant 1$ et notons $x$ la cellule principale de $\mathrm{D}_{i-1}$ et $y$ celle de $\mathrm{D}_{i}$. Alors le $\infty$-foncteur $\mathrm{D}_{1} \otimes \sigma_{i}: \mathrm{D}_{1} \otimes \mathrm{D}_{i-1} \rightarrow \mathrm{D}_{1} \otimes \mathrm{D}_{i}$ est donné par

$$
\begin{aligned}
\left\langle a_{0}^{\varepsilon^{\prime}} \otimes x_{k}^{\varepsilon}\right\rangle & \mapsto\left\langle a_{0}^{\varepsilon^{\prime}} \otimes y_{k}^{\varepsilon}\right\rangle & & \text { pour } 0 \leqslant k<i-1, \varepsilon=0,1 \text { et } \varepsilon^{\prime}=0,1, \\
\left\langle a_{0}^{\varepsilon^{\prime}} \otimes x_{i-1}\right\rangle & \mapsto\left\langle a_{0}^{\varepsilon^{\prime}} \otimes y_{i-1}^{0}\right\rangle & & \text { pour } \varepsilon^{\prime}=0,1, \\
\left\langle a \otimes x_{k}^{\varepsilon}\right\rangle & \mapsto\left\langle a \otimes y_{k}^{\varepsilon}\right\rangle & & \text { pour } 0 \leqslant k<i-1 \text { et } \varepsilon=0,1, \\
\left\langle a \otimes x_{i-1}\right\rangle & \mapsto\left\langle a \otimes y_{i-1}^{0}\right\rangle . & &
\end{aligned}
$$

De même, le $\infty$-foncteur $\mathrm{D}_{1} \otimes \tau_{i}: \mathrm{D}_{1} \otimes \mathrm{D}_{i-1} \rightarrow \mathrm{D}_{1} \otimes \mathrm{D}_{i}$ est donné par

$$
\begin{aligned}
\left\langle a_{0}^{\varepsilon^{\prime}} \otimes x_{k}^{\varepsilon}\right\rangle & \mapsto\left\langle a_{0}^{\varepsilon^{\prime}} \otimes y_{k}^{\varepsilon}\right\rangle & & \text { pour } 0 \leqslant k<i-1, \varepsilon=0,1 \text { et } \varepsilon^{\prime}=0,1, \\
\left\langle a_{0}^{\varepsilon^{\prime}} \otimes x_{i-1}\right\rangle & \mapsto\left\langle a_{0}^{\varepsilon^{\prime}} \otimes y_{i-1}^{1}\right\rangle & & \text { pour } \varepsilon^{\prime}=0,1, \\
\left\langle a \otimes x_{k}^{\varepsilon}\right\rangle & \mapsto\left\langle a \otimes y_{k}^{\varepsilon}\right\rangle & & \text { pour } 0 \leqslant k<i-1 \text { et } \varepsilon=0,1, \\
\left\langle a \otimes x_{i-1}\right\rangle & \mapsto\left\langle a \otimes y_{i-1}^{1}\right\rangle . & &
\end{aligned}
$$

Démonstration. - Cela résulte immédiatement de la proposition A.7 et des formules

$$
\sigma_{i}\left(\left\langle x_{k}^{\varepsilon}\right\rangle\right)=\left\{\begin{array}{ll}
\left\langle y_{k}^{\varepsilon}\right\rangle & \text { si } 0 \leqslant k<i-1, \\
\left\langle y_{i-1}^{0}\right\rangle & \text { si } k=i-1,
\end{array} \quad \text { et } \quad \tau_{i}\left(\left\langle x_{k}^{\varepsilon}\right\rangle\right)= \begin{cases}\left\langle y_{k}^{\varepsilon}\right\rangle & \text { si } 0 \leqslant k<i-1, \\
\left\langle y_{i-1}^{1}\right\rangle & \text { si } k=i-1,\end{cases}\right.
$$

pour $\varepsilon=0,1$. 
Proposition B.1.10. - Fixons $i \geqslant 0$ et notons $x$ la cellule principale de $\mathrm{D}_{i+1}$ et $y$ celle de $\mathrm{D}_{i}$. Alors le $\infty$-foncteur $\mathrm{D}_{1} \otimes \kappa_{i}: \mathrm{D}_{1} \otimes \mathrm{D}_{i+1} \rightarrow \mathrm{D}_{1} \otimes \mathrm{D}_{i}$ est donné par

$$
\begin{aligned}
\left\langle a_{0}^{\varepsilon^{\prime}} \otimes x_{k}^{\varepsilon}\right\rangle & \mapsto\left\langle a_{0}^{\varepsilon^{\prime}} \otimes y_{k}^{\varepsilon}\right\rangle & & \text { pour } 0 \leqslant k<i, \varepsilon=0,1 \text { et } \varepsilon^{\prime}=0,1, \\
\left\langle a_{0}^{\varepsilon^{\prime}} \otimes x_{i}^{\varepsilon}\right\rangle & \mapsto\left\langle a_{0}^{\varepsilon^{\prime}} \otimes y_{i}\right\rangle & & \text { pour } \varepsilon=0,1 \text { et } \varepsilon^{\prime}=0,1, \\
\left\langle a_{0}^{\varepsilon^{\prime}} \otimes x_{i+1}\right\rangle & \mapsto 1_{\left\langle a_{0}^{\varepsilon^{\prime}} \otimes y_{i}\right\rangle} & & \text { pour } \varepsilon^{\prime}=0,1, \\
\left\langle a \otimes x_{k}^{\varepsilon}\right\rangle & \mapsto\left\langle a \otimes y_{k}^{\varepsilon}\right\rangle & & \text { pour } 0 \leqslant k<i \text { et } \varepsilon=0,1, \\
\left\langle a \otimes x_{i}^{\varepsilon}\right\rangle & \mapsto\left\langle a \otimes y_{i}\right\rangle & & \text { pour } \varepsilon=0,1, \\
\left\langle a \otimes x_{i+1}\right\rangle & \mapsto 1_{\left\langle a \otimes y_{i}\right\rangle} . & &
\end{aligned}
$$

Démonstration. - Cela résulte immédiatement de la proposition A.7 et de la formule

$$
\kappa_{i}\left(\left\langle x_{k}^{\varepsilon}\right\rangle\right)= \begin{cases}\left\langle y_{k}^{\varepsilon}\right\rangle & \text { si } 0 \leqslant k<i \\ \left\langle y_{i}\right\rangle & \text { si } k=i \\ 1_{\left\langle y_{i}\right\rangle} & \text { si } k=i+1\end{cases}
$$

pour $\varepsilon=0,1$.

B.1.11. - Fixons maintenant $j$ tel que $0 \leqslant j<i$. Nous allons décrire explicitement le $\infty$-foncteur

$$
\mathrm{D}_{1} \otimes \nabla_{j}^{i}: \mathrm{D}_{1} \otimes \mathrm{D}_{i} \rightarrow \mathrm{D}_{1} \otimes \mathrm{D}_{i} \amalg_{\mathrm{D}_{1} \otimes \mathrm{D}_{j}} \mathrm{D}_{1} \otimes \mathrm{D}_{i} .
$$

Nous noterons $x$ la cellule principale de l'objet $\mathrm{D}_{i}$ apparaissant dans la source de $\mathrm{D}_{1} \otimes \nabla_{j}^{i}$, et $y$ et $z$ les cellules principales des objets $\mathrm{D}_{i}$ apparaissant de gauche à droite dans le but de $\mathrm{D}_{1} \otimes \nabla_{j}^{i}$.

En vertu du paragraphe 4.11 et avec ses notations, une base du complexe dirigé augmenté

$$
\lambda\left(\mathrm{D}_{1} \otimes \mathrm{D}_{i} \amalg_{\mathrm{D}_{1} \otimes \mathrm{D}_{j}} \mathrm{D}_{1} \otimes \mathrm{D}_{i}\right) \simeq \lambda\left(\mathrm{D}_{1}\right) \otimes \lambda\left(\mathrm{D}_{i} \amalg_{\mathrm{D}_{j}} \mathrm{D}_{i}\right)
$$

est donnée par les

$$
\left\langle a_{0}^{\varepsilon^{\prime}} \otimes y_{i}^{\varepsilon}\right\rangle, \quad\left\langle a_{0}^{\varepsilon^{\prime}} \otimes z_{i}^{\varepsilon}\right\rangle, \quad\left\langle a \otimes y_{i}^{\varepsilon}\right\rangle, \quad\left\langle a \otimes z_{i}^{\varepsilon}\right\rangle,
$$

pour $0 \leqslant k \leqslant i, \varepsilon=0,1$ et $\varepsilon^{\prime}=0,1$, modulo les identifications

$$
y_{j}^{0}=z_{j}^{1}, \quad y_{k}^{\varepsilon}=z_{k}^{\varepsilon} \quad \text { pour } 0 \leqslant k<j \text { et } \varepsilon=0,1,
$$

ainsi que les identifications triviales $y_{i}^{0}=y_{i}^{1}$ et $z_{i}^{0}=z_{i}^{1}$.

Lemme B.1.12. — Pour tout $k$ tel que $j<k \leqslant i$ et $\varepsilon=0$, 1, la $(k+1)$-flèche

$$
\begin{aligned}
& \left(\left\langle a_{0}^{1} \otimes y_{j+1}^{\eta_{1}}\right\rangle *_{0}\left\langle a \otimes z_{0}^{0}\right\rangle *_{1} \cdots *_{j-1}\left\langle a \otimes z_{j-1}^{0}\right\rangle *_{j}\left\langle a \otimes z_{k}^{\varepsilon}\right\rangle\right) \\
& \quad *_{j+1}\left(\left\langle a \otimes y_{k}^{\varepsilon}\right\rangle *_{j}\left\langle a \otimes y_{j-1}^{1}\right\rangle *_{j-1} \cdots *_{1}\left\langle a \otimes y_{0}^{1}\right\rangle *_{0}\left\langle a_{0}^{0} \otimes z_{j+1}^{\eta_{0}}\right\rangle\right)
\end{aligned}
$$

de $\mathrm{D}_{1} \otimes \mathrm{D}_{i} \amalg_{\mathrm{D}_{1} \otimes \mathrm{D}_{j}} \mathrm{D}_{1} \otimes \mathrm{D}_{i}$, où $\eta_{0}$ et $\eta_{1}$ valent $\varepsilon$ si $k=j+1$ et 0 et 1 respectivement sinon, est bien définie. 
Démonstration. - En vertu de la proposition B.1.4, la $j$-flèche

$$
\left\langle a \otimes y_{j-1}^{1}\right\rangle *_{j-1} \cdots *_{1}\left\langle a \otimes y_{0}^{1}\right\rangle *_{0}\left\langle a_{0}^{0} \otimes y_{j}^{0}\right\rangle
$$

est bien définie. Puisque, pour $l \leqslant j-1$, on a

$$
t_{l}\left(\left\langle a_{0}^{0} \otimes z_{j+1}^{\eta_{0}}\right\rangle\right)=\left\langle a_{0}^{0} \otimes z_{l}^{1}\right\rangle=\left\langle a_{0}^{0} \otimes y_{l}^{1}\right\rangle=t_{l}\left(\left\langle a_{0}^{0} \otimes y_{j}^{0}\right\rangle\right),
$$

on en déduit que la cellule

$$
\left\langle a \otimes y_{j-1}^{1}\right\rangle *_{j-1} \cdots *_{1}\left\langle a \otimes y_{0}^{1}\right\rangle *_{0}\left\langle a_{0}^{0} \otimes z_{j+1}^{\eta_{0}}\right\rangle
$$

est également bien définie. De plus, on a

$$
\begin{aligned}
t_{j}(\langle a & \left.\left.\otimes y_{j-1}^{1}\right\rangle *_{j-1} \cdots *_{1}\left\langle a \otimes y_{0}^{1}\right\rangle *_{0}\left\langle a_{0}^{0} \otimes z_{j+1}^{\eta_{0}}\right\rangle\right) \\
& =\left\langle a \otimes y_{j-1}^{1}\right\rangle *_{j-1} \cdots *_{1}\left\langle a \otimes y_{0}^{1}\right\rangle *_{0}\left\langle a_{0}^{0} \otimes z_{j}^{1}\right\rangle \\
& =\left\langle a \otimes y_{j-1}^{1}\right\rangle *_{j-1} \cdots *_{1}\left\langle a \otimes y_{0}^{1}\right\rangle *_{0}\left\langle a_{0}^{0} \otimes y_{j}^{0}\right\rangle \\
& =s_{j}\left(\left\langle a \otimes y_{k}^{\varepsilon}\right\rangle\right),
\end{aligned}
$$

la dernière égalité résultant du lemme B.1.5, ce qui montre que la cellule

$$
\left\langle a \otimes y_{k}^{\varepsilon}\right\rangle *_{j}\left\langle a \otimes y_{j-1}^{1}\right\rangle *_{j-1} \cdots *_{1}\left\langle a \otimes y_{0}^{1}\right\rangle *_{0}\left\langle a_{0}^{0} \otimes z_{j+1}^{\eta_{0}}\right\rangle
$$

est bien définie. On montre de même que la cellule

$$
\left\langle a_{0}^{1} \otimes y_{j+1}^{\eta_{1}}\right\rangle *_{0}\left\langle a \otimes z_{0}^{0}\right\rangle *_{1} \cdots *_{j-1}\left\langle a \otimes z_{j-1}^{0}\right\rangle *_{j}\left\langle a \otimes z_{k}^{\varepsilon}\right\rangle
$$

est bien définie. Enfin, on a

$$
\begin{aligned}
& s_{j+1}\left(\left\langle a_{0}^{1} \otimes y_{j+1}^{\eta_{1}}\right\rangle *_{0}\left\langle a \otimes z_{0}^{0}\right\rangle *_{1} \cdots *_{j-1}\left\langle a \otimes z_{j-1}^{0}\right\rangle *_{j}\left\langle a \otimes z_{k}^{\varepsilon}\right\rangle\right) \\
& =\left\langle a_{0}^{1} \otimes y_{j+1}^{\eta_{1}}\right\rangle *_{0}\left\langle a \otimes z_{0}^{0}\right\rangle *_{1} \cdots *_{j-1}\left\langle a \otimes z_{j-1}^{0}\right\rangle *_{j} s_{j+1}\left(\left\langle a \otimes z_{k}^{\varepsilon}\right\rangle\right) \\
& =\left\langle a_{0}^{1} \otimes y_{j+1}^{\eta_{1}}\right\rangle *_{0}\left\langle a \otimes z_{0}^{0}\right\rangle *_{1} \cdots *_{j-1}\left\langle a \otimes z_{j-1}^{0}\right\rangle \\
& \quad *_{j}\left\langle a \otimes z_{j}^{1}\right\rangle *_{j}\left\langle a \otimes z_{j-1}^{1}\right\rangle *_{j-1} \cdots *_{1}\left\langle a \otimes z_{0}^{1}\right\rangle *_{0}\left\langle a_{0}^{0} \otimes z_{j+1}^{\eta_{0}}\right\rangle,
\end{aligned}
$$

la dernière égalité résultant du lemme B.1.5, et

$$
\begin{aligned}
& t_{j+1}\left(\left\langle a \otimes y_{k}^{\varepsilon}\right\rangle *_{j}\left\langle a \otimes y_{j-1}^{1}\right\rangle *_{j-1} \cdots *_{1}\left\langle a \otimes y_{0}^{1}\right\rangle *_{0}\left\langle a_{0}^{0} \otimes z_{j+1}^{\eta_{0}}\right\rangle\right) t_{j+1}\left(\left\langle a \otimes y_{k}^{\varepsilon}\right\rangle\right) *_{j}\left\langle a \otimes y_{j-1}^{1}\right\rangle *_{j-1} \cdots *_{1}\left\langle a \otimes y_{0}^{1}\right\rangle *_{0}\left\langle a_{0}^{0} \otimes z_{j+1}^{\eta_{0}}\right\rangle \\
&=\left\langle a_{0}^{1} \otimes y_{j+1}^{\eta_{1}}\right\rangle *_{0}\left\langle a \otimes y_{0}^{0}\right\rangle *_{1} \cdots *_{j}\left\langle a \otimes y_{j}^{0}\right\rangle \\
& \quad *_{j}\left\langle a \otimes y_{j-1}^{1}\right\rangle *_{j-1} \cdots *_{1}\left\langle a \otimes y_{0}^{1}\right\rangle *_{0}\left\langle a_{0}^{0} \otimes z_{j+1}^{\eta_{0}}\right\rangle \\
& \quad \text { (en vertu du lemme B.1.5) } \\
&=\left\langle a_{0}^{1} \otimes y_{j+1}^{\eta_{1}}\right\rangle *_{0}\left\langle a \otimes y_{0}^{0}\right\rangle *_{1} \cdots *_{j-1}\left\langle a \otimes y_{j-1}^{0}\right\rangle \\
& \quad *_{j}\left\langle a \otimes y_{j}^{0}\right\rangle *_{j}\left\langle a \otimes y_{j-1}^{1}\right\rangle *_{j-1} \cdots *_{1}\left\langle a \otimes y_{0}^{1}\right\rangle *_{0}\left\langle a_{0}^{0} \otimes z_{j+1}^{\eta_{0}}\right\rangle \\
&=\left\langle a_{0}^{1} \otimes y_{j+1}^{\eta_{1}}\right\rangle *_{0}\left\langle a \otimes z_{0}^{0}\right\rangle *_{1} \cdots *_{j-1}\left\langle a \otimes z_{j-1}^{0}\right\rangle \\
& \quad *_{j}\left\langle a \otimes z_{j}^{1}\right\rangle *_{j}\left\langle a \otimes z_{j-1}^{1}\right\rangle *_{j-1} \cdots *_{1}\left\langle a \otimes z_{0}^{1}\right\rangle *_{0}\left\langle a_{0}^{0} \otimes z_{j+1}^{\eta_{0}}\right\rangle,
\end{aligned}
$$

ce qui achève de montrer que la cellule de l'énoncé est bien définie. 
Lemme B.1.13. - Pour tout $k$ tel que $j<k \leqslant i, \varepsilon=0,1$ et tout $l$ tel que $0 \leqslant l \leqslant k+1$, on $a$

$$
\begin{aligned}
\left(\mathrm{D}_{1} \otimes \nabla_{j}^{i}\right)\left(\left\langle a \otimes x_{k}^{\varepsilon}\right\rangle\right)_{l}^{0}= & \\
& \begin{cases}a_{0}^{0} \otimes z_{l}^{0}+a \otimes y_{l-1}^{1} & \text { si } 0 \leqslant l \leqslant j, \\
a_{0}^{0} \otimes y_{l}^{\eta_{0}}+a_{0}^{0} \otimes z_{l}^{\eta_{0}}+a \otimes y_{l-1}^{1} & \text { si } l=j+1, \\
a_{0}^{0} \otimes y_{l}^{\eta_{0}}+a_{0}^{0} \otimes z_{l}^{\eta_{0}}+a \otimes y_{l-1}^{1}+a \otimes z_{l-1}^{1} & \text { si } j+1<l<k+1, \\
a \otimes y_{l-1}^{\varepsilon}+a \otimes z_{l-1}^{\varepsilon} & \text { si } l=k+1,\end{cases}
\end{aligned}
$$

où $\eta_{0}$ vaut $\varepsilon$ si $l=k$ et 0 sinon, et

$\left(\mathrm{D}_{1} \otimes \nabla_{j}^{i}\right)\left(\left\langle a \otimes x_{k}^{\varepsilon}\right\rangle\right)_{l}^{1}=$

$$
\begin{cases}a_{0}^{1} \otimes y_{l}^{1}+a \otimes z_{l-1}^{0} & \text { si } 0 \leqslant l \leqslant j, \\ a_{0}^{1} \otimes y_{l}^{\eta_{1}}+a_{0}^{1} \otimes z_{l}^{\eta_{1}}+a \otimes z_{l-1}^{0} & \text { si } l=j+1, \\ a_{0}^{1} \otimes y_{l}^{\eta_{1}}+a_{0}^{1} \otimes z_{l}^{\eta_{1}}+a \otimes y_{l-1}^{0}+a \otimes z_{l-1}^{0} & \text { si } j+1<l<k+1, \\ a \otimes y_{l-1}^{\varepsilon}+a \otimes z_{l-1}^{\varepsilon} & \text { si } l=k+1,\end{cases}
$$

où $\eta_{1}$ vaut $\varepsilon$ si $l=k$ et 1 sinon.

Démonstration. - Soit $\varepsilon^{\prime} \in\{0,1\}$. Pour $l<k+1$, en utilisant le lemme B.1.3, on a

$$
\begin{aligned}
\left(\mathrm{D}_{1} \otimes \nabla_{j}^{i}\right)\left(\left\langle a \otimes x_{k}^{\varepsilon}\right\rangle\right)_{l}^{\varepsilon^{\prime}} & =\lambda\left(\mathrm{D}_{1} \otimes \nabla_{j}^{i}\right)\left(\left\langle a \otimes x_{k}^{\varepsilon}\right\rangle_{l}^{\varepsilon^{\prime}}\right) \\
& =\lambda\left(\mathrm{D}_{1} \otimes \nabla_{j}^{i}\right)\left(a_{0}^{\varepsilon^{\prime}} \otimes x_{l}^{\eta_{\varepsilon^{\prime}}}+a \otimes x_{l-1}^{1-\varepsilon^{\prime}}\right) \\
& =a_{0}^{\varepsilon^{\prime}} \otimes \lambda\left(\nabla_{j}^{i}\right)\left(x_{l}^{\eta_{\varepsilon^{\prime}}}\right)+a \otimes \lambda\left(\nabla_{j}^{i}\right)\left(x_{l-1}^{1-\varepsilon^{\prime}}\right)
\end{aligned}
$$

et on obtient le résultat en utilisant la description de $\lambda\left(\nabla_{j}^{i}\right)$ donnée au paragraphe 4.11 . Pour $l=k+1$, en utilisant de nouveau le lemme B.1.3, on a

$$
\left(\mathrm{D}_{1} \otimes \nabla_{j}^{i}\right)\left(\left\langle a \otimes x_{k}^{\varepsilon}\right\rangle\right)_{l}^{\varepsilon^{\prime}}=\lambda\left(\mathrm{D}_{1} \otimes \nabla_{j}^{i}\right)\left(a \otimes x_{k}^{\varepsilon}\right)=a \otimes \lambda\left(\nabla_{j}^{i}\right)\left(x_{k}^{\varepsilon}\right),
$$

et on conclut en invoquant de nouveau le paragraphe 4.11.

Proposition B.1.14. — Le $\infty$-foncteur $\mathrm{D}_{1} \otimes \nabla_{j}^{i}: \mathrm{D}_{1} \otimes \mathrm{D}_{i} \rightarrow \mathrm{D}_{1} \otimes \mathrm{D}_{i} \amalg_{\mathrm{D}_{1} \otimes \mathrm{D}_{j}} \mathrm{D}_{1} \otimes \mathrm{D}_{i}$ est donné par

$$
\begin{aligned}
\left\langle a_{0}^{\varepsilon^{\prime}} \otimes x_{k}^{0}\right\rangle & \mapsto\left\langle a_{0}^{\varepsilon^{\prime}} \otimes z_{k}^{0}\right\rangle & & \text { pour } 0 \leqslant k \leqslant j \text { et } \varepsilon^{\prime}=0,1, \\
\left\langle a_{0}^{\varepsilon^{\prime}} \otimes x_{k}^{1}\right\rangle & \mapsto\left\langle a_{0}^{\varepsilon^{\prime}} \otimes y_{k}^{1}\right\rangle & & \text { pour } 0 \leqslant k \leqslant j \text { et } \varepsilon^{\prime}=0,1, \\
\left\langle a_{0}^{\varepsilon^{\prime}} \otimes x_{k}^{\varepsilon}\right\rangle & \mapsto\left\langle a_{0}^{\varepsilon^{\prime}} \otimes y_{k}^{\varepsilon}\right\rangle *_{j}\left\langle a_{0}^{\varepsilon^{\prime}} \otimes z_{k}^{\varepsilon}\right\rangle & & \text { pour } j<k \leqslant i, \varepsilon=0,1 \text { et } \varepsilon^{\prime}=0,1, \\
\left\langle a \otimes x_{k}^{0}\right\rangle & \mapsto\left\langle a \otimes z_{k}^{0}\right\rangle & & \text { pour } 0 \leqslant k \leqslant j, \\
\left\langle a \otimes x_{k}^{1}\right\rangle & \mapsto\left\langle a \otimes y_{k}^{1}\right\rangle & & \text { pour } 0 \leqslant k \leqslant j, \\
\left\langle a \otimes x_{k}^{\varepsilon}\right\rangle & \mapsto u_{k}^{\varepsilon} & & \text { pour } j<k \leqslant i \text { et } \varepsilon=0,1,
\end{aligned}
$$


où

$$
\begin{aligned}
u_{k}^{\varepsilon}=( & \left.\left\langle a_{0}^{1} \otimes y_{j+1}^{\eta_{1}}\right\rangle *_{0}\left\langle a \otimes z_{0}^{0}\right\rangle *_{1} \cdots *_{j-1}\left\langle a \otimes z_{j-1}^{0}\right\rangle *_{j}\left\langle a \otimes z_{k}^{\varepsilon}\right\rangle\right) \\
& *_{j+1}\left(\left\langle a \otimes y_{k}^{\varepsilon}\right\rangle *_{j}\left\langle a \otimes y_{j-1}^{1}\right\rangle *_{j-1} \cdots *_{1}\left\langle a \otimes y_{0}^{1}\right\rangle *_{0}\left\langle a_{0}^{0} \otimes z_{j+1}^{\eta_{0}}\right\rangle\right),
\end{aligned}
$$

avec $\eta_{0}$ et $\eta_{1}$ valant $\varepsilon$ si $k=j+1$ et 0 et 1 respectivement sinon.

Démonstration. - Le cas des atomes de la forme $\left\langle a_{0}^{\varepsilon^{\prime}} \otimes x_{k}^{\varepsilon}\right\rangle$ résulte de la commutativité des carrés

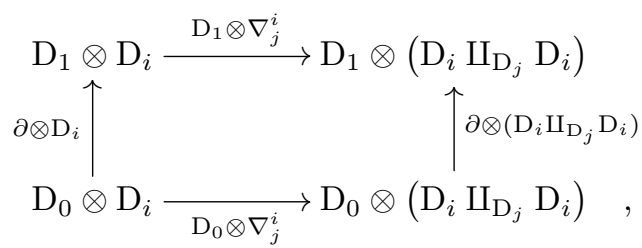

où $\partial$ vaut $\sigma_{1}: \mathrm{D}_{0} \rightarrow \mathrm{D}_{1}$ ou $\tau_{1}: \mathrm{D}_{0} \rightarrow \mathrm{D}_{1}$, ainsi que de la description explicite du $\infty$-foncteur $\nabla_{j}^{i}: \mathrm{D}_{i} \rightarrow \mathrm{D}_{i} \amalg_{\mathrm{D}_{j}} \mathrm{D}_{i}$ (voir le paragraphe 4.3).

Le cas des atomes de la forme $\left\langle a \otimes x_{k}^{\varepsilon}\right\rangle$ avec $0 \leqslant k \leqslant j$ est conséquence de la proposition A.7 et des formules

$$
\nabla_{j}^{i}\left(\left\langle x_{k}^{0}\right\rangle\right)=\left\langle z_{k}^{0}\right\rangle \quad \text { et } \quad \nabla_{j}^{i}\left(\left\langle x_{k}^{1}\right\rangle\right)=\left\langle y_{k}^{1}\right\rangle,
$$

pour $0 \leqslant k \leqslant j$.

Enfin, traitons le cas des atomes de la forme $\left\langle a \otimes x_{k}^{\varepsilon}\right\rangle$ avec $k>j$. Soient $l$ tel que $0 \leqslant l \leqslant k+1$ et $\varepsilon^{\prime}=0,1$. Il s'agit de montrer l'égalité $\left(\mathrm{D}_{1} \otimes \nabla_{j}^{i}\right)\left(\left\langle a \otimes x_{k}^{\varepsilon}\right\rangle\right)_{l}^{\varepsilon^{\prime}}=\left(u_{k}^{\varepsilon}\right)_{l}^{\varepsilon^{\prime}}$. Le membre de gauche a été calculé dans le lemme B.1.13. Calculons celui de droite.

Si $l \leqslant j$, on a

$$
s_{l}\left(u_{k}^{\varepsilon}\right)=s_{l}\left(\left\langle a \otimes y_{l-1}^{1}\right\rangle *_{l-1} \cdots *_{1}\left\langle a \otimes y_{0}^{1}\right\rangle *_{0}\left\langle a_{0}^{0} \otimes z_{j+1}^{\eta_{0}}\right\rangle\right)
$$

et donc

$$
\left(u_{k}^{\varepsilon}\right)_{l}^{0}=\left\langle a \otimes y_{l-1}^{1}\right\rangle_{l}^{0}+\left\langle a_{0}^{0} \otimes z_{j+1}^{\eta_{0}}\right\rangle_{l}^{0}=a \otimes y_{l-1}^{1}+a_{0}^{0} \otimes z_{l}^{0} .
$$

De même, on a

$$
\left(u_{k}^{\varepsilon}\right)_{l}^{1}=\left\langle a_{0}^{1} \otimes y_{j+1}^{\eta_{1}}\right\rangle_{l}^{1}+\left\langle a \otimes z_{l-1}^{0}\right\rangle_{l}^{1}=a_{0}^{1} \otimes y_{l}^{1}+a \otimes z_{l-1}^{0} .
$$

Si $l=j+1$, on a

$$
s_{l}\left(u_{k}^{\varepsilon}\right)=s_{l}\left(\left\langle a \otimes y_{k}^{\varepsilon}\right\rangle *_{j}\left\langle a \otimes y_{j-1}^{1}\right\rangle *_{j-1} \cdots *_{1}\left\langle a \otimes y_{0}^{1}\right\rangle *_{0}\left\langle a_{0}^{0} \otimes z_{j+1}^{\eta_{0}}\right\rangle\right)
$$

et donc, en utilisant le lemme B.1.3,

$$
\left(u_{k}^{\varepsilon}\right)_{l}^{0}=\left\langle a \otimes y_{k}^{\varepsilon}\right\rangle_{l}^{0}+\left\langle a_{0}^{0} \otimes z_{j+1}^{\eta_{0}}\right\rangle_{l}^{0}=a_{0}^{0} \otimes y_{l}^{\eta_{0}}+a \otimes y_{l-1}^{1}+a_{0}^{0} \otimes z_{l}^{\eta_{0}} .
$$

De même, on a

$$
\left(u_{k}^{\varepsilon}\right)_{l}^{1}=\left\langle a_{0}^{1} \otimes y_{j+1}^{\eta_{1}}\right\rangle_{l}^{1}+\left\langle a \otimes z_{k}^{\varepsilon}\right\rangle_{l}^{1}=a_{0}^{1} \otimes y_{l}^{\eta_{1}}+a_{0}^{1} \otimes z_{l}^{\eta_{1}}+a \otimes z_{l-1}^{0} .
$$

Si $j+1<l \leqslant k+1$, on a

$$
\left(u_{k}^{\varepsilon}\right)_{l}^{\varepsilon^{\prime}}=\left\langle a \otimes z_{k}^{\varepsilon}\right\rangle_{l}^{\varepsilon^{\prime}}+\left\langle a \otimes y_{k}^{\varepsilon}\right\rangle_{l}^{\varepsilon^{\prime}}
$$


et donc, pour $j+1<l<k+1$, toujours en vertu du lemme B.1.3,

$$
\left(u_{k}^{\varepsilon}\right)_{l}^{\varepsilon^{\prime}}=a_{0}^{\varepsilon^{\prime}} \otimes z_{l}^{\eta_{\varepsilon^{\prime}}}+a \otimes z_{l-1}^{1-\varepsilon^{\prime}}+a_{0}^{\varepsilon^{\prime}} \otimes y_{l}^{\eta_{\varepsilon^{\prime}}}+a \otimes y_{l-1}^{1-\varepsilon^{\prime}}
$$

et, pour $l=k+1$,

$$
\left(u_{k}^{\varepsilon}\right)_{l}^{\varepsilon^{\prime}}=a \otimes z_{k}^{\varepsilon}+a \otimes y_{k}^{\varepsilon} .
$$

On a bien retrouvé dans tous les cas la valeur de $\left(\mathrm{D}_{1} \otimes \nabla_{j}^{i}\right)\left(\left\langle a \otimes x_{k}^{\varepsilon}\right\rangle\right)_{l}^{\varepsilon^{\prime}}$ obtenue dans le lemme B.1.13, ce qui achève la démonstration.

Proposition B.1.15. - Soit $C$ une $\infty$-catégorie. Fixons une $i$-flèche $(c, d, \alpha)$ de $\underline{\operatorname{Hom}}_{\text {lax }}\left(\mathrm{D}_{1}, C\right)$.

(a) Si $i \geqslant 1$, on a $s(c, d, \alpha)=(s(c), s(d), \gamma)$, où

$$
\begin{aligned}
\gamma_{k}^{\varepsilon} & =\alpha_{k}^{\varepsilon} \quad \text { pour } 0 \leqslant k<i-1 \text { et } \varepsilon=0,1, \\
\gamma_{i-1} & =\alpha_{i-1}^{0} .
\end{aligned}
$$

(b) Si $i \geqslant 1$, on a $t(c, d, \alpha)=(t(c), t(d), \gamma)$, ò̀

$$
\begin{aligned}
\gamma_{k}^{\varepsilon} & =\alpha_{k}^{\varepsilon} \quad \text { pour } 0 \leqslant k<i-1 \text { et } \varepsilon=0,1, \\
\gamma_{i-1} & =\alpha_{i-1}^{1} .
\end{aligned}
$$

(c) Si $i \geqslant 0$, on a $1_{(c, d, \alpha)}=\left(1_{c}, 1_{d}, \gamma\right)$, où

$$
\begin{aligned}
\gamma_{k}^{\varepsilon} & =\alpha_{k}^{\varepsilon} & & \text { pour } 0 \leqslant k<i \text { et } \varepsilon=0,1, \\
\gamma_{i}^{\varepsilon} & =\alpha_{i} & & \text { pour } \varepsilon=0,1, \\
\gamma_{i+1} & =1_{\alpha_{i}} . & &
\end{aligned}
$$

Soit $(e, f, \beta)$ une seconde $i$-flèche de $\underline{\mathrm{Hom}}_{\text {lax }}\left(\mathrm{D}_{1}, C\right)$.

(d) $S i(c, d, \alpha)$ et $(e, f, \beta)$ sont $j$-composables pour un $j$ tel que $0 \leqslant j<i$, alors on a $(c, d, \alpha) *_{j}(e, f, \beta)=\left(c *_{j} e, d *_{j} f, \gamma\right)$, ò̀

$$
\begin{array}{rlrl}
\gamma_{k}^{0} & =\beta_{k}^{0} & & \text { pour } 0 \leqslant k \leqslant j, \\
\gamma_{k}^{1}=\alpha_{k}^{1} & \text { pour } 0 \leqslant k \leqslant j
\end{array}
$$

et

$$
\begin{aligned}
\gamma_{k}^{\varepsilon}=\left(d_{j+1}^{\eta_{1}} *_{0} \beta_{0}^{0} *_{1} \cdots *_{j-1} \beta_{j-1}^{0} *_{j} \beta_{k}^{\varepsilon}\right) & \\
& *_{j+1}\left(\alpha_{k}^{\varepsilon} *_{j} \alpha_{j-1}^{1} *_{j-1} \cdots *_{1} \alpha_{0}^{1} *_{0} e_{j+1}^{\eta_{0}}\right)
\end{aligned}
$$

pour $j<k \leqslant i$ et $\varepsilon=0,1$, où $\eta_{0}$ et $\eta_{1}$ valent $\varepsilon$ si $k=j+1$ et 0 et 1 respectivement sinon.

Démonstration. - Ces formules sont la traduction, à travers la bijection de la proposition B.1.6 et du paragraphe B.1.7, des formules obtenues dans les propositions B.1.9, B.1.10 et B.1.14. 
Remarque B.1.16. - Il résulte de la description de $\underline{\operatorname{Hom}}_{\text {lax }}\left(\mathrm{D}_{1}, C\right)$ obtenue dans la proposition précédente que cette $\infty$-catégorie est isomorphe à la $\infty$-catégorie $H C$ des cylindres dans $C$ introduite par Métayer dans [30] (voir également [27] pour une description alternative de cette $\infty$-catégorie).

\section{B.2. Transformations oplax et produit tensoriel}

B.2.1. - Fixons deux $\infty$-foncteurs $u, v: C \rightarrow D$. Le but de cette section est de montrer que les transformations oplax de $u$ vers $v$, telles que définies au paragraphe 1.9, sont en bijection avec les $\infty$-foncteurs $H: \mathrm{D}_{1} \otimes C \rightarrow D$ rendant commutatif le diagramme

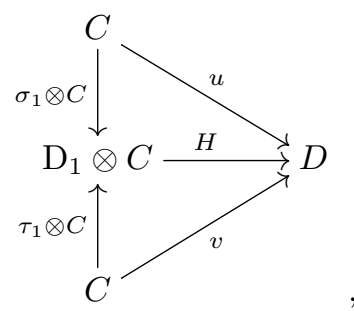

où, d'une part, on a identifié $C$ et $\mathrm{D}_{0} \otimes C$ et, d'autre part, $\sigma_{1}, \tau_{1}: \mathrm{D}_{0} \rightarrow \mathrm{D}_{1}$ désignent les $\infty$-foncteurs du paragraphe 4.1 .

B.2.2. - Par adjonction, un $\infty$-foncteur $H: \mathrm{D}_{1} \otimes C \rightarrow D$, comme dans le paragraphe précédent, correspond à un $\infty$-foncteur $K: C \rightarrow \underline{\operatorname{Hom}}_{\operatorname{lax}}\left(\mathrm{D}_{1}, D\right)$ rendant commutatif le diagramme de $\infty$-foncteurs

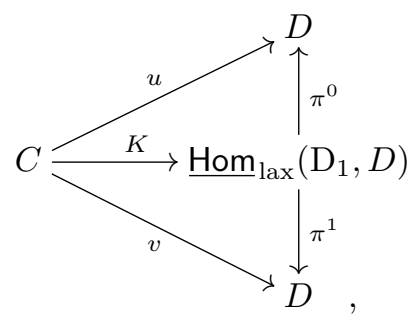

où on a posé

$$
\begin{aligned}
& \pi^{0}=\underline{\operatorname{Hom}}_{\text {lax }}\left(\sigma_{1}, D\right): \underline{\operatorname{Hom}}_{\text {lax }}\left(\mathrm{D}_{1}, D\right) \rightarrow \underline{\operatorname{Hom}}_{\text {lax }}\left(\mathrm{D}_{0}, D\right), \\
& \pi^{1}=\underline{\operatorname{Hom}}_{\operatorname{lax}}\left(\tau_{1}, D\right): \underline{\operatorname{Hom}}_{\text {lax }}\left(\mathrm{D}_{1}, D\right) \rightarrow \underline{\operatorname{Hom}}_{\operatorname{lax}}\left(\mathrm{D}_{0}, D\right)
\end{aligned}
$$

et identifié $\underline{\operatorname{Hom}}_{\operatorname{lax}}\left(\mathrm{D}_{0}, D\right)$ à $D$ (ce qui est licite puisque $\mathrm{D}_{0}$ est l'unité du produit tensoriel).

L'action du $\infty$-foncteur $\pi^{0}$ sur les $i$-flèches, pour $i \geqslant 0$, est par définition induite par le $\infty$-foncteur $\sigma_{1} \otimes \mathrm{D}_{i}: \mathrm{D}_{0} \otimes \mathrm{D}_{i} \rightarrow \mathrm{D}_{1} \otimes \mathrm{D}_{i}$. On en déduit qu'en utilisant la description 
des $i$-flèches de $\underline{\operatorname{Hom}}_{\text {lax }}\left(\mathrm{D}_{1}, D\right)$ donnée au paragraphe B.1.7, on a $\pi^{0}(c, d, \alpha)=c$. De même, on a $\pi^{1}(c, d, \alpha)=d$.

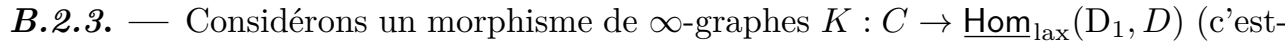
à-dire une application des cellules de $C$ vers les cellules de $\underline{\operatorname{Hom}}_{\text {lax }}\left(\mathrm{D}_{1}, D\right)$ qui préserve la dimension des cellules et leurs sources et buts) rendant commutatif le diagramme du paragraphe précédent. La commutativité de ce diagramme signifie exactement que si $x$ est une cellule de $C$, les deux premières composantes de $K(x)$ sont $u(x)$ et $v(x)$. Notons $\alpha(x)$ la troisième composante et $\alpha_{x}$ la $(i+1)$-flèche $\alpha(x)_{i}$, où $i$ est la dimension de $x$. Par définition, on a

$$
\alpha_{x}: \alpha(x)_{i-1}^{1} *_{i-1} \cdots *_{1} \alpha(x)_{0}^{1} *_{0} u(x) \rightarrow v(x) *_{0} \alpha(x)_{0}^{0} *_{1} \cdots *_{i-1} \alpha(x)_{i-1}^{0} .
$$

Or, en vertu de la proposition B.1.15 et de la compatibilité de $K$ aux sources, on a

$$
\alpha(x)_{l}^{0}=s_{l}(\alpha(x))_{l}=\alpha\left(s_{l}(x)\right)_{l}=\alpha_{s_{l}(x)}
$$

et, de même,

$$
\alpha(x)_{l}^{1}=\alpha_{t_{l}(x)},
$$

et donc

$$
\alpha_{x}: \alpha_{t_{i-1}(x)} *_{i-1} \cdots *_{1} \alpha_{t_{0}(x)} *_{0} u(x) \rightarrow v(x) *_{0} \alpha_{s_{0}(x)} *_{1} \cdots *_{i-1} \alpha_{s_{i-1}(x)} .
$$

Autrement dit, l'application $x \mapsto \alpha_{x}$ est une prétransformation oplax de $u$ vers $v$ au sens du paragraphe 1.9.

Proposition B.2.4. - Les morphismes de $\infty$-graphes $K: C \rightarrow \underline{\operatorname{Hom}}_{\operatorname{lax}}\left(\mathrm{D}_{1}, D\right)$ comme dans le paragraphe précédent sont en bijection avec les prétransformations oplax de $u$ vers $v$ via l'application définie au paragraphe précédent.

Démonstration. - Il suffit de construire une application inverse à l'application définie au paragraphe précédent. On vérifie facilement qu'on obtient un tel inverse en envoyant une prétransformation oplax $\alpha$ de $u$ vers $v$ sur le morphisme de $\infty$-graphes $K: C \rightarrow \underline{\operatorname{Hom}}_{\operatorname{lax}}\left(\mathrm{D}_{1}, D\right)$ défini par, pour $x$ une $i$-flèche de $C$, pour $i \geqslant 0$,

$$
K(x)=(u(x), v(x), \alpha(x)),
$$

où, pour $0 \leqslant l \leqslant i$, on a posé

$$
\alpha(x)_{l}^{0}=\alpha_{s_{l}(x)} \quad \text { et } \quad \alpha(x)_{l}^{1}=\alpha_{t_{l}(x)} .
$$

Proposition B.2.5. - Les $\infty$-foncteurs $K: C \rightarrow \underline{\operatorname{Hom}}_{\operatorname{lax}}\left(\mathrm{D}_{1}, D\right)$ comme dans le paragraphe B.2.2 sont en bijection avec les transformations oplax de $u$ vers $v$ via l'application définie au paragraphe B.2.3. 
Démonstration. - Soient $K: C \rightarrow \underline{\operatorname{Hom}}_{\operatorname{lax}}\left(\mathrm{D}_{1}, D\right)$ un tel $\infty$-foncteur, $\alpha(x)$ la troisième composante de $K(x)$ pour $x$ une cellule de $C$ et $\alpha$ la prétransformation oplax associée. Commençons par vérifier que $\alpha$ est une transformation oplax. Si $x$ est une $i$-flèche de $C$, pour un $i \geqslant 0$, on a, par fonctorialité de $K$,

$$
\left(u\left(1_{x}\right), v\left(1_{x}\right), \alpha\left(1_{x}\right)\right)=1_{(u(x), v(x), \alpha(x))}
$$

et donc, en vertu de la proposition B.1.15,

$$
\alpha\left(1_{x}\right)_{i+1}=1_{\alpha(x)_{i}},
$$

ou encore

$$
\alpha_{1_{x}}=1_{\alpha_{x}} .
$$

Si maintenant $x$ et $y$ sont deux $i$-flèches $j$-composables de $C$, pour $i>j \geqslant 0$, alors on a, toujours par fonctorialité de $K$,

$$
\left(u\left(x *_{j} y\right), v\left(x *_{j} y\right), \alpha\left(x *_{j} y\right)\right)=(u(x), v(x), \alpha(x)) *_{j}(u(y), v(y), \alpha(y)) .
$$

En vertu de la proposition B.1.15, on a donc

$$
\begin{array}{r}
\alpha\left(x *_{j} y\right)_{i}=\left(t_{j+1}(v(x)) *_{0} \alpha(y)_{0}^{0} *_{1} \cdots *_{j-1} \alpha(y)_{j-1}^{0} *_{j} \alpha(y)_{i}\right) \\
*_{j+1}\left(\alpha(x)_{i} *_{j} \alpha(x)_{j-1}^{1} *_{j-1} \cdots *_{1} \alpha(x)_{0}^{1} *_{0} s_{j+1}(u(y))\right),
\end{array}
$$

ou encore

$$
\begin{aligned}
& \alpha_{x *_{j} y}=\left(v\left(t_{j+1}(x)\right) *_{0} \alpha_{s_{0}(y)} *_{1} \cdots *_{j-1} \alpha_{s_{j-1}(y)} *_{j} \alpha_{y}\right) \\
& *_{j+1}\left(\alpha_{x} *_{j} \alpha_{t_{j-1}(x)} *_{j-1} \cdots *_{1} \alpha_{t_{0}(x)} *_{0} u\left(s_{j+1}(y)\right)\right),
\end{aligned}
$$

ce qui montre que $\alpha$ est bien une transformation oplax.

Pour conclure, en vertu de la proposition précédente, il nous suffit de montrer que si $K: C \rightarrow \underline{\operatorname{Hom}}_{\text {lax }}\left(\mathrm{D}_{1}, D\right)$ est un morphisme de $\infty$-graphes comme dans le paragraphe B.2.3 qui satisfait de plus aux relations $\left(\star_{1}\right)$ et $\left(\star_{2}\right)$, alors $K$ est un $\infty$-foncteur. Si $x$ est une $i$-flèche, pour un $i \geqslant 0$, on a, pour $k$ tel que $0 \leqslant k \leqslant i$,

$$
\alpha\left(1_{x}\right)_{k}^{0}=\alpha\left(s_{k}\left(1_{x}\right)\right)_{k}=\alpha\left(s_{k}(x)\right)_{k}=\alpha(x)_{k}^{0}
$$

et de même, on a $\alpha\left(1_{x}\right)_{k}^{1}=\alpha(x)_{k}^{1}$, ce qui, en vertu de la relation $\left(\star_{1}\right)$, montre la compatibilité aux identités (voir la proposition B.1.15). Vérifions maintenant la compatibilité aux compositions. Soient donc $x$ et $y$ deux $i$-flèches $j$-composables de $C$, pour $i>j \geqslant 0$. Un calcul similaire à celui qu'on vient de mener montre que si $0 \leqslant k \leqslant j$, on a

$$
\alpha\left(x *_{j} y\right)_{k}^{0}=\alpha(y)_{k}^{0} \quad \text { et } \quad \alpha\left(x *_{j} y\right)_{k}^{1}=\alpha(x)_{k}^{1} .
$$


Soit maintenant $k$ tel que $j<k<i$. En utilisant la relation $\left(\star_{2}\right)$, on obtient

$$
\begin{aligned}
& \alpha\left(x *_{j} y\right)_{k}^{0} \\
& =\alpha\left(s_{k}\left(x *_{j} y\right)\right)_{k}=\alpha\left(s_{k}(x) *_{j} s_{k}(y)\right)_{k} \\
& =\left(t_{j+1}\left(v\left(s_{k}(x)\right) *_{0} \alpha\left(s_{k}(y)\right)_{0}^{0} *_{1} \cdots *_{j-1} \alpha\left(s_{k}(y)\right)_{j-1}^{0} *_{j} \alpha\left(s_{k}(y)\right)_{k}\right)\right. \\
& \quad *_{j+1}\left(\alpha\left(s_{k}(x)\right)_{k} *_{j} \alpha\left(s_{k}(x)\right)_{j-1}^{1} *_{j-1} \cdots *_{1} \alpha\left(s_{k}(x)\right)_{0}^{1} *_{0} s_{j+1}\left(u\left(s_{k}(y)\right)\right)\right) \\
& =\left(t_{j+1} s_{k}(v(x)) *_{0} \alpha(y)_{0}^{0} *_{1} \cdots *_{j-1} \alpha(y)_{j-1}^{0} *_{j} \alpha(y)_{k}^{0}\right) \\
& \quad *_{j+1}\left(\alpha(x)_{k}^{0} *_{j} \alpha(x)_{j-1}^{1} *_{j-1} \cdots *_{1} \alpha(x)_{0}^{1} *_{0} s_{j+1}(u(y))\right) .
\end{aligned}
$$

Or, $t_{j+1} s_{k}(v(x))$ vaut $s_{j+1}(v(x))$ si $k=j+1$ et $t_{j+1}(v(x))$ sinon, et on obtient donc bien la valeur de $\alpha\left(x *_{j} y\right)_{k}^{0}$ attendue (voir la proposition B.1.15). Un calcul similaire montre l'assertion analogue pour $\alpha\left(x *_{j} y\right)_{j}^{1}$, ce qui achève de montrer que $K$ est un $\infty$-foncteur.

Corollaire B.2.6. - Les $\infty$-foncteurs $H: \mathrm{D}_{1} \otimes C \rightarrow D$ rendant commutatif le diagramme

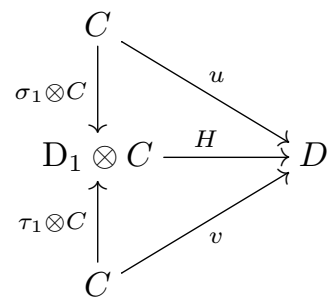

sont en bijection canonique avec les transformations oplax de $u$ vers $v$.

Démonstration. - Cela résulte immédiatement de la proposition précédente et du paragraphe B.2.2.

Corollaire B.2.7. - Les $\infty$-foncteurs $H: C \otimes \mathrm{D}_{1} \rightarrow D$ rendant commutatif le diagramme

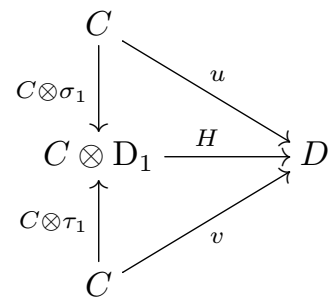

sont en bijection canonique avec les transformations lax de $u$ vers $v$. 
Démonstration. - En vertu de la proposition A.22, en appliquant la dualité paire au diagramme de l'énoncé on obtient le diagramme

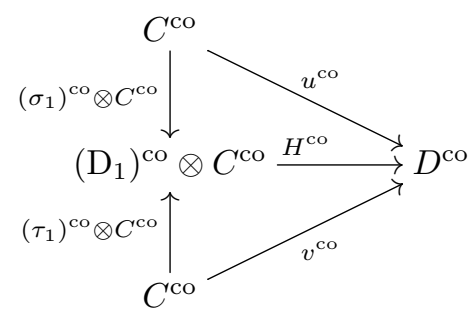

et, puisque $\left(\mathrm{D}_{1}\right)^{\mathrm{co}}=\mathrm{D}_{1},\left(\sigma_{1}\right)^{\mathrm{co}}=\sigma_{1}$ et $\left(\tau_{1}\right)^{\mathrm{co}}=\tau_{1}$, ce diagramme n'est autre que

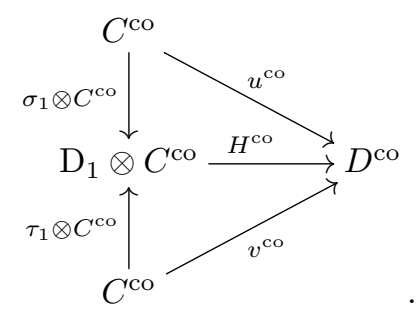

Le $\infty$-foncteur $H^{\text {co }}$ définit donc, en vertu du corollaire précédent, une transformation oplax de $u^{\text {co }}$ vers $v^{\text {co }}$, c'est-à-dire une transformation lax de $u$ vers $v$ (voir le paragraphe 1.9).

Remarque B.2.8. - Les deux corollaires précédents justifient les notations $\underline{\mathrm{Hom}}_{\text {oplax }}(C, D)$ et $\underline{\mathrm{Hom}}_{\text {lax }}(C, D)$. En effet, les 1-flèches de ces $\infty$-catégories sont respectivement les $\infty$-foncteurs $\mathrm{D}_{1} \otimes C \rightarrow D$ et les $\infty$-foncteurs $C \otimes \mathrm{D}_{1} \rightarrow D$ (voir le paragraphe A.18) et correspondent donc respectivement, en vertu de ces corollaires, aux transformations oplax et aux transformations lax entre $\infty$-foncteurs de $C$ vers $D$ (voir le paragraphe B.3.1 pour plus de détails).

Remarque B.2.9. - On peut facilement déduire la remarque 1.14 des corollaires précédents en utilisant les dualités de $\infty$ - $\mathcal{C}$ at (et notamment la proposition A.22).

Proposition B.2.10. - Soit $\alpha$ une transformation oplax de u vers $v$. Notons $H: \mathrm{D}_{1} \otimes C \rightarrow D$ le $\infty$-foncteur correspondant. Pour toute $i$-flèche $x$ de $C$, pour un $i \geqslant 0$, la $(i+1)$-flèche $\alpha_{x}$ est l'image par le $\infty$-foncteur

$$
\mathrm{D}_{1} \otimes \mathrm{D}_{i} \stackrel{\mathrm{D}_{1} \otimes x}{\longrightarrow} \mathrm{D}_{1} \otimes C \stackrel{H}{\rightarrow} D
$$

de l'unique $(i+1)$-flèche non triviale de $\mathrm{D}_{1} \otimes \mathrm{D}_{i}$.

Démonstration. - Notons $K: C \rightarrow \underline{\operatorname{Hom}}_{\text {lax }}\left(\mathrm{D}_{1}, D\right)$ le $\infty$-foncteur correspondant à $H$ par adjonction. En vertu de la proposition B.2.5, en notant $\alpha(x)$ la troisième 
composante de $K(x)$ (voir le paragraphe B.1.7), on a $\alpha_{x}=\alpha(x)_{i}$. Or, par adjonction, la $i$-flèche $K(x)$

correspond au composé

$$
\mathrm{D}_{i} \stackrel{x}{\rightarrow} C \stackrel{K}{\rightarrow} \underline{\operatorname{Hom}}_{\text {lax }}\left(\mathrm{D}_{1}, D\right)
$$

$$
\mathrm{D}_{1} \otimes \mathrm{D}_{i} \stackrel{\mathrm{D}_{1} \otimes x}{\longrightarrow} \mathrm{D}_{1} \otimes C \stackrel{H}{\rightarrow} D
$$

et, en vertu de la proposition B.1.6, la $(i+1)$-flèche $\alpha(x)_{i}$ est l'image par ce $\infty$-foncteur de l'unique $(i+1)$-flèche non triviale de $\mathrm{D}_{1} \otimes \mathrm{D}_{i}$, d'où le résultat.

Proposition B.2.11. - Soient $v_{0}, v_{1}: C \rightarrow D$ deux $\infty$-foncteurs et $\alpha$ une transformation oplax de $v_{0}$ vers $v_{1}$. Notons $H: \mathrm{D}_{1} \otimes C \rightarrow D$ le $\infty$-foncteur correspondant $\grave{a} \alpha$.

(a) Pour tout $\infty$-foncteur $u: B \rightarrow C$, la transformation oplax $\alpha * u$ (voir le paragraphe 1.16) correspond au $\infty$-foncteur

$$
\mathrm{D}_{1} \otimes B \stackrel{\mathrm{D}_{1} \otimes u}{\longrightarrow} \mathrm{D}_{1} \otimes C \stackrel{H}{\longrightarrow} D .
$$

(b) Pour tout $\infty$-foncteur $w: D \rightarrow E$, la transformation oplax $w * \alpha$ (voir également le paragraphe 1.16) correspond au $\infty$-foncteur

$$
\mathrm{D}_{1} \otimes C \stackrel{H}{\rightarrow} D \stackrel{w}{\rightarrow} E .
$$

Démonstration. - Commençons par démontrer la première assertion. Par adjonction, en notant $K: C \rightarrow \underline{\operatorname{Hom}}_{\text {lax }}\left(\mathrm{D}_{1}, C\right)$ le transposé de $H$, cela revient à montrer que la transformation oplax $\alpha * u$ correspond, dans la bijection de la proposition B.2.5, au $\infty$-foncteur

$$
B \stackrel{u}{\rightarrow} C \stackrel{K}{\rightarrow} \underline{\text { Hom }}_{\text {lax }}\left(\mathrm{D}_{1}, D\right) .
$$

Or, par définition (voir le paragraphe B.2.3), si $x$ est une cellule de $B$, la transformation oplax $\beta$ correspondant à $K u$ vérifie $\beta_{x}=\alpha_{u(x)}$ et on a donc bien $\beta=\alpha * u$.

De même, établir la seconde assertion revient à montrer que la transformation oplax $w * \alpha$ correspond, dans la bijection de la proposition B.2.5, au $\infty$-foncteur

$$
C \stackrel{K}{\longrightarrow} \underline{\operatorname{Hom}}_{\text {lax }}\left(\mathrm{D}_{1}, D\right) \stackrel{\mathrm{Hom}_{\text {lax }}\left(\mathrm{D}_{1}, w\right)}{\longrightarrow} \underline{\operatorname{Hom}}_{\text {lax }}\left(\mathrm{D}_{1}, E\right),
$$

ce qui se vérifie comme ci-dessus en utilisant de nouveau le paragraphe B.2.3.

\section{B.3. Composition verticale des transformations oplax}

Dans cette section, on fixe deux $\infty$-catégories $C$ et $D$.

B.3.1. - Considérons la $\infty$-catégorie $\underline{\mathrm{Hom}}_{\mathrm{oplax}}(C, D)$ (voir le paragraphe A.18). Par définition, pour $i \geqslant 0$, ses $i$-flèches sont les $\infty$-foncteurs $\mathrm{D}_{i} \otimes C \rightarrow D$. En particulier, modulo l'identification $C \simeq \mathrm{D}_{0} \otimes C$, ses objets sont les $\infty$-foncteurs $C \rightarrow D$ et ses 1-flèches les $\infty$-foncteurs $\mathrm{D}_{1} \otimes C \rightarrow D$, les objets source et but étant obtenus par 
précomposition par $\sigma_{1} \otimes C: C \rightarrow \mathrm{D}_{1} \otimes C$ et $\tau_{1} \otimes C: C \rightarrow \mathrm{D}_{1} \otimes C$ respectivement, en identifiant toujours $C$ et $\mathrm{D}_{0} \otimes C$. Ainsi, en vertu du corollaire B.2.6, les 1-flèches de $\underline{\operatorname{Hom}}_{\text {oplax }}(C, D)$ d'un $\infty$-foncteur $u: C \rightarrow D$ vers un $\infty$-foncteur $v: C \rightarrow D$ correspondent aux transformations oplax de $u$ vers $v$.

B.3.2. - En vertu du paragraphe précédent, la composition des 1-flèches de la $\infty$-catégorie $\underline{\mathrm{Hom}}_{\mathrm{oplax}}(C, D)$ définit une composition des transformations oplax. Plus précisément, fixons $u, v$ et $w$ trois $\infty$-foncteurs de $C$ vers $D$. Si $\alpha$ est une transformation oplax de $u$ vers $v$ et $\alpha^{\prime}$ une transformation oplax de $v$ vers $w$, alors on dispose d'une transformation oplax de $u$ vers $w$ qu'on notera $\alpha^{\prime} \circ \alpha$.

Cette composition peut se décrire de la manière suivante. Les transformations oplax $\alpha$ et $\alpha^{\prime}$ correspondent à des $\infty$-foncteurs $H: \mathrm{D}_{1} \otimes C \rightarrow D$ et $H^{\prime}: \mathrm{D}_{1} \otimes C \rightarrow D$. Par ailleurs, le $\infty$-foncteur $\nabla_{0}^{1}: \mathrm{D}_{1} \rightarrow \mathrm{D}_{1} \amalg_{\mathrm{D}_{0}} \mathrm{D}_{1}$ du paragraphe 4.3 induit un $\infty$-foncteur

$$
\nabla_{0}^{1} \otimes C: \mathrm{D}_{1} \otimes C \longrightarrow\left(\mathrm{D}_{1} \amalg_{\mathrm{D}_{0}} \mathrm{D}_{1}\right) \otimes C \simeq\left(\mathrm{D}_{1} \otimes C\right) \amalg_{C}\left(\mathrm{D}_{1} \otimes C\right) .
$$

La transformation oplax $\alpha^{\prime} \circ \alpha$ correspond alors au $\infty$-foncteur composé

$$
\mathrm{D}_{1} \otimes C \stackrel{\nabla_{0}^{1} \otimes C}{\longrightarrow}\left(\mathrm{D}_{1} \otimes C\right) \amalg_{C}\left(\mathrm{D}_{1} \otimes C\right) \stackrel{\left(H^{\prime}, H\right)}{\longrightarrow} D .
$$

B.3.3. - Soit $u: C \rightarrow D$ un $\infty$-foncteur. On peut voir $u$ comme un objet de $\underline{\text { Hom }}_{\text {oplax }}(C, D)$ et on dispose donc d'une 1-flèche $1_{u}$ de $\underline{\text { Hom }}_{\text {oplax }}(C, D)$ qu'on verra comme une transformation oplax de $u$ vers $u$. Explicitement, la transformation oplax $1_{u}$ correspond au $\infty$-foncteur

$$
\mathrm{D}_{1} \otimes C \stackrel{\kappa_{0} \otimes C}{\longrightarrow} C \stackrel{u}{\rightarrow} D
$$

induit par le $\infty$-foncteur $\kappa_{0}: \mathrm{D}_{1} \rightarrow \mathrm{D}_{0}$ du paragraphe 4.1 , où on a encore identifié $\mathrm{D}_{0} \otimes C$ et $C$. Par adjonction, elle correspond également, dans la bijection de la proposition B.2.5, au $\infty$-foncteur

$$
C \stackrel{\mathrm{Hom}_{\text {lax }}\left(\kappa_{0}, C\right)}{\longrightarrow} \underline{\operatorname{Hom}}_{\text {lax }}\left(\mathrm{D}_{1}, C\right) \stackrel{\mathrm{Hom}_{\text {lax }}\left(\mathrm{D}_{1}, u\right)}{\longrightarrow} \underline{\operatorname{Hom}}_{\text {lax }}\left(\mathrm{D}_{1}, D\right)
$$

où cette fois on a identifié $\underline{\operatorname{Hom}}_{\text {lax }}\left(\mathrm{D}_{0}, C\right)$ et $C$.

On a déjà défini, au paragraphe 1.15 , une transformation oplax $1_{u}$. Dans la suite de cette section, sauf mention du contraire, $1_{u}$ fera toujours référence à la transformation oplax que l'on vient d'introduire. On va montrer que les deux définitions sont équivalentes.

Proposition B.3.4. - Fixons $i \geqslant 0$ et notons a la cellule principale de $\mathrm{D}_{1}, b$ celle de $\mathrm{D}_{0}$ et $x$ celle de $\mathrm{D}_{i}$. Alors le $\infty$-foncteur $\kappa_{0} \otimes \mathrm{D}_{i}: \mathrm{D}_{1} \otimes \mathrm{D}_{i} \rightarrow \mathrm{D}_{0} \otimes \mathrm{D}_{i}$ est donné 
par

$$
\begin{aligned}
\left\langle a_{0}^{\varepsilon^{\prime}} \otimes x_{k}^{\varepsilon}\right\rangle & \mapsto\left\langle b \otimes x_{k}^{\varepsilon}\right\rangle & & \text { pour } 0 \leqslant k \leqslant i, \varepsilon=0,1 \text { et } \varepsilon^{\prime}=0,1, \\
\left\langle a \otimes x_{k}^{\varepsilon}\right\rangle & \mapsto 1_{\left\langle b \otimes x_{k}^{\varepsilon}\right\rangle} & & \text { pour } 0 \leqslant k \leqslant i \text { et } \varepsilon=0,1 .
\end{aligned}
$$

Démonstration. - Le cas des atomes de la forme $\left\langle a_{0}^{\varepsilon^{\prime}} \otimes x_{k}^{\varepsilon}\right\rangle$ résulte, par fonctorialité, des égalités $\kappa_{0} \sigma_{1}=1_{\mathrm{D}_{0}}=\kappa_{0} \tau_{1}$. Par ailleurs, puisque $\lambda\left(\kappa_{0}\right)(a)=0$, l'image d'un atome de la forme $\left\langle a \otimes x_{k}^{\varepsilon}\right\rangle$ est une identité. De plus, en utilisant le lemme B.1.3, on a

$$
\begin{aligned}
s\left(\left(\kappa_{0} \otimes \mathrm{D}_{i}\right)\left(\left\langle a \otimes x_{k}^{\varepsilon}\right\rangle\right)\right)_{k} & =\left(\kappa_{0} \otimes \mathrm{D}_{i}\right)\left(s\left(\left\langle a \otimes x_{k}^{\varepsilon}\right\rangle\right)\right)_{k} \\
& =\left(\lambda\left(\kappa_{0}\right) \otimes \lambda\left(\mathrm{D}_{i}\right)\right)\left(\left\langle a \otimes x_{k}^{\varepsilon}\right\rangle_{k}^{0}\right) \\
& =\left(\lambda\left(\kappa_{0}\right) \otimes \lambda\left(\mathrm{D}_{i}\right)\right)\left(a_{0}^{0} \otimes x_{k}^{\varepsilon}+a \otimes x_{k-1}^{1}\right) \\
& =b \otimes x_{k}^{\varepsilon},
\end{aligned}
$$

où la dernière égalité résulte des relations $\lambda\left(\kappa_{0}\right)\left(a_{0}^{0}\right)=b$ et $\lambda\left(\kappa_{0}\right)(a)=0$. Or, la seule $k$-flèche $y$ de $\mathrm{D}_{0} \otimes \mathrm{D}_{i} \simeq \mathrm{D}_{i}$ telle que $y_{k}=b \otimes x_{k}^{\varepsilon}$ est $\left\langle b \otimes x_{k}^{\varepsilon}\right\rangle$, d'où le résultat.

Proposition B.3.5. - Soit $u: C \rightarrow D$ un $\infty$-foncteur. Pour toute cellule $x$ de $C$, on a

$$
\left(1_{u}\right)_{x}=1_{u(x)}
$$

Démonstration. - En vertu de la proposition B.2.11, on peut supposer que $u$ est un $\infty$-foncteur identité. Si $x$ est une $i$-flèche de $C$, pour un $i \geqslant 0$, l'image de $x$ par le $\infty$-foncteur

$$
\underline{\operatorname{Hom}}_{\operatorname{lax}}\left(\kappa_{0}, C\right): C \rightarrow \underline{\operatorname{Hom}}_{\text {lax }}\left(\mathrm{D}_{1}, C\right)
$$

est obtenue en composant

$$
\mathrm{D}_{1} \otimes \mathrm{D}_{i} \stackrel{\kappa_{0} \otimes \mathrm{D}_{i}}{\longrightarrow} \mathrm{D}_{0} \otimes \mathrm{D}_{i} \simeq \mathrm{D}_{i} \stackrel{x}{\rightarrow} C .
$$

En vertu de la proposition précédente, cette image est $1_{x}$. On a donc $\left(1_{u}\right)_{x}=1_{x}$, ce qu'on voulait démontrer.

Remarque B.3.6. - La proposition précédente affirme que la transformation oplax $1_{u}$ définie dans cette section coïncide avec la transformation oplax $1_{u}$ définie au paragraphe 1.15 .

\section{B.4. Homotopies et transformations oplax}

B.4.1. - Soient $f, g: K \rightarrow L$ deux morphismes de complexes dirigés augmentés. La donnée d'une homotopie $h$ de $f$ vers $g$ est équivalente à celle d'un morphisme de 
complexes dirigés augmentés $H: \lambda\left(\mathrm{D}_{1}\right) \otimes K \rightarrow L$ rendant le diagramme

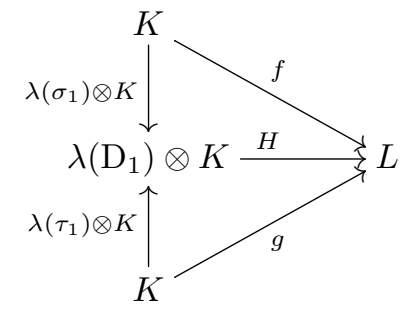

commutatif. En effet, l'assertion analogue pour les complexes de chaînes est bien connue et on vérifie immédiatement qu'elle s'étend aux complexes dirigés augmentés. Explicitement, en notant $a$ la cellule principale de $\mathrm{D}_{1}$, l'homotopie $h$ associée à $H$ est définie par $h(x)=H(a \otimes x)$ pour tout élément homogène $x$ de $K$.

Remarque B.4.2. - On déduit du paragraphe précédent, par dualité (en utilisant notamment la proposition A.20), comme dans la preuve du corollaire B.2.7, qu'une antihomotopie $h$ de $f$ vers $g$ correspond à un morphisme de complexes dirigés augmentés $H: K \otimes \lambda\left(\mathrm{D}_{1}\right) \rightarrow L$ rendant le diagramme

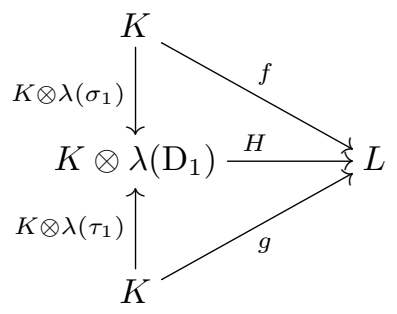

commutatif.

Remarque B.4.3. - Plus généralement, pour $n \geqslant 1$, on peut vérifier que la donnée d'une $n$-homotopie (voir le paragraphe 2.28) de $K$ vers $L$ correspond à la donnée d'un morphisme

$$
\lambda\left(\mathrm{D}_{n}\right) \otimes K \rightarrow L,
$$

les $(n-1)$-homotopies source et but correspondant aux morphismes obtenus en précomposant par

$$
\lambda\left(\sigma_{n}\right) \otimes K, \lambda\left(\tau_{n}\right) \otimes K: \lambda\left(\mathrm{D}_{n-1}\right) \otimes K \rightarrow \lambda\left(\mathrm{D}_{n}\right) \otimes K .
$$

On vérifie de même qu'on obtient l'assertion analogue pour les $n$-antihomotopies (voir également le paragraphe 2.28) en inversant les facteurs du produit tensoriel.

B.4.4. - Soient $f_{0}, f_{1}: K \rightarrow L$ deux morphismes de complexes dirigés augmentés et $h$ une homotopie de $f_{0}$ vers $f_{1}$. Notons $H: \lambda\left(\mathrm{D}_{1}\right) \otimes K \rightarrow L$ le morphisme correspondant. 
(a) Pour tout morphisme $g: J \rightarrow K$, l'homotopie $h g$ (voir le paragraphe 2.31) correspond au morphisme

$$
\lambda\left(\mathrm{D}_{1}\right) \otimes J \stackrel{\lambda\left(\mathrm{D}_{1}\right) \otimes g}{\longrightarrow} \lambda\left(\mathrm{D}_{1}\right) \otimes K \stackrel{H}{\rightarrow} L .
$$

En effet, par naturalité, un tel morphisme correspond bien à une homotopie de $f_{0} g$ vers $f_{1} g$ et, en notant a la cellule principale de $\mathrm{D}_{1}$, pour $x$ un élément homogène de $J$, on a $H\left(\lambda\left(\mathrm{D}_{1}\right) \otimes g\right)(a \otimes x)=H(a \otimes g(x))=h(g(x))$.

(b) Pour tout morphisme $g: L \rightarrow M$, l'homotopie $g h$ (voir également le paragraphe 2.31) correspond au morphisme

$$
\lambda\left(\mathrm{D}_{1}\right) \otimes K \stackrel{H}{\rightarrow} L \stackrel{g}{\rightarrow} M .
$$

En effet, un tel morphisme correspond bien à une homotopie de $g f_{0}$ vers $g f_{1}$ et, en notant $a$ la cellule principale de $\mathrm{D}_{1}$, pour $x$ un élément homogène de $K$, on a $g H(a, x)=g(h(x))$.

Par ailleurs, pour tout morphisme de complexes dirigés augmentés $f: K \rightarrow L$, l'homotopie $1_{f}$ (voir le paragraphe 2.30) correspond au morphisme

$$
\lambda\left(\mathrm{D}_{1}\right) \otimes K \stackrel{\lambda\left(\kappa_{0}\right) \otimes K}{\longrightarrow} K \stackrel{f}{\rightarrow} L,
$$

induit par le $\infty$-foncteur $\kappa_{0}: \mathrm{D}_{1} \rightarrow \mathrm{D}_{0}$ du paragraphe 4.1. En effet, par naturalité, un tel morphisme correspond bien à une homotopie de $f$ vers $f$ et, en notant $a$ la cellule principale de $\mathrm{D}_{1}$, pour $x$ un élément homogène de $K$, on a $f\left(\lambda\left(\kappa_{0}\right) \otimes K\right)(a \otimes x)=f(0 \otimes x)=0$.

B.4.5. - Soient $f_{0}, f_{1}, f_{2}: K \rightarrow L$ trois morphismes de complexes dirigés augmentés, $h$ une homotopie de $f_{0}$ vers $f_{1}$ et $h^{\prime}$ une homotopie de $f_{1}$ vers $f_{2}$. D'après le paragraphe B.4.1, ces homotopies correspondent à des morphismes $H: \lambda\left(\mathrm{D}_{1}\right) \otimes K \rightarrow L$ et $H^{\prime}: \lambda\left(\mathrm{D}_{1}\right) \otimes K \rightarrow L$ respectivement. Par ailleurs, comme dans le paragraphe B.3.2, on peut définir un morphisme $H^{\prime \prime}$ en composant

$$
\lambda\left(\mathrm{D}_{1}\right) \otimes K \stackrel{\lambda\left(\nabla_{0}^{1}\right) \otimes K}{\longrightarrow}\left(\lambda\left(\mathrm{D}_{1}\right) \otimes K\right) \amalg_{K}\left(\lambda\left(\mathrm{D}_{1}\right) \otimes K\right) \stackrel{\left(H^{\prime}, H\right)}{\longrightarrow} L .
$$

Le morphisme $H^{\prime \prime}$ correspond à une homotopie de $f_{0}$ vers $f_{2}$ qui n'est autre que l'homotopie $h^{\prime}+h$ (voir le paragraphe 2.32). En effet, en notant $a, b$ et $c$ les cellules principales des copies de $\lambda\left(\mathrm{D}_{1}\right)$ apparaissant, dans l'ordre, dans la source de $\lambda\left(\nabla_{0}^{1}\right) \otimes K$, puis de gauche à droite dans son but, on a, pour tout élément homogène $x$ de $K$,

$$
H^{\prime \prime}(a \otimes x)=\left(H^{\prime}, H\right)(b \otimes x+c \otimes x)=H^{\prime}(b \otimes x)+H(c \otimes x)=h^{\prime}(x)+h(x),
$$

d'où l'assertion.

B.4.6. - Soient $f, g: K \rightarrow L$ deux morphismes de complexes dirigés augmentés. À toute homotopie $h$ de $f$ vers $g$, on associe une transformation oplax $\nu(h)$ de $\nu(f)$ vers $\nu(g)$ de la manière suivante. L'homotopie $h$ correspond, en vertu du 
paragraphe B.4.1, à un morphisme $H: \lambda\left(\mathrm{D}_{1}\right) \otimes K \rightarrow L$. On obtient un $\infty$-foncteur $\mathrm{D}_{1} \otimes \nu(K) \rightarrow \nu(L)$ en composant

$$
\mathrm{D}_{1} \otimes \nu(K) \simeq \nu\left(\lambda\left(\mathrm{D}_{1}\right)\right) \otimes \nu(K) \rightarrow \nu\left(\lambda\left(\mathrm{D}_{1}\right) \otimes K\right) \stackrel{\nu(H)}{\longrightarrow} \nu(L),
$$

où la flèche du milieu est la contrainte du foncteur monoïdal lax $\nu$ (voir la proposition A.19). Ce $\infty$-foncteur correspond à son tour, cette fois en vertu du corollaire B.2.6, à une transformation oplax qui, par naturalité des flèches en jeu, va de $\nu(f)$ vers $\nu(g)$.

Proposition B.4.7. - Soient $f, g: K \rightarrow L$ deux morphismes de complexes dirigés augmentés avec $K$ un complexe de Steiner fort. Le foncteur $\nu$ induit une bijection entre les homotopies de $f$ vers $g$ et les transformations oplax de $\nu(f)$ vers $\nu(g)$.

Démonstration. - Par adjonction, la correspondance $h \mapsto \nu(h)$ du paragraphe précédent peut se décrire comme le composé

$$
\begin{aligned}
\operatorname{Hom}_{\mathcal{C}_{\mathrm{da}}}\left(\lambda\left(\mathrm{D}_{1}\right) \otimes K, L\right) & \rightarrow \operatorname{Hom}_{\mathcal{C}_{\mathrm{da}}}\left(\lambda \nu\left(\lambda\left(\mathrm{D}_{1}\right) \otimes K\right), L\right) \\
& \simeq \operatorname{Hom}_{\infty-\mathcal{C} a t}\left(\nu\left(\lambda\left(\mathrm{D}_{1}\right) \otimes K\right), \nu(L)\right) \\
& \rightarrow \operatorname{Hom}_{\infty-\mathcal{C} a t}\left(\nu\left(\lambda\left(\mathrm{D}_{1}\right)\right) \nu(K), \nu(L)\right) \\
& \simeq \operatorname{Hom}_{\infty-\mathcal{C} a t}\left(\mathrm{D}_{1} \otimes \nu(K), \nu(L)\right),
\end{aligned}
$$

où la première application est induite par la coünité du couple de foncteurs adjoints $(\lambda, \nu)$ et la troisième par la contrainte du foncteur monoïdal lax $\nu$. Mais, puisque $K$ est un complexe de Steiner fort, il en est de même de $\lambda\left(\mathrm{D}_{1}\right) \otimes K$ (voir la proposition A.3) et les théorèmes 2.11 et A.15 entraînent que ces deux applications sont des bijections, d'où le résultat.

Montrons maintenant la compatibilité aux compositions et identités de l'extension de $\nu$ aux homotopies.

Proposition B.4.8. - Soient $K$ et $L$ deux complexes dirigés augmentés et $h$ une homotopie entre morphismes de $K$ vers $L$.

(a) Pour tout morphisme de complexes dirigés augmentés $g: J \rightarrow K$, on a

$$
\nu(h g)=\nu(h) * \nu(g) .
$$

(b) Pour tout morphisme de complexes dirigés augmentés $g: L \rightarrow M$, on a

$$
\nu(g h)=\nu(g) * \nu(h) .
$$

Démonstration. - Notons $H: \lambda\left(\mathrm{D}_{1}\right) \otimes K \rightarrow L$ le morphisme correspondant à $h$. En vertu de la proposition B.2.11 et du paragraphe B.4.4, les transformations oplax de la première assertion correspondent aux deux $\infty$-foncteurs $\mathrm{D}_{1} \otimes \nu(J) \rightarrow \nu(L)$ qu'on 
peut définir à partir du diagramme

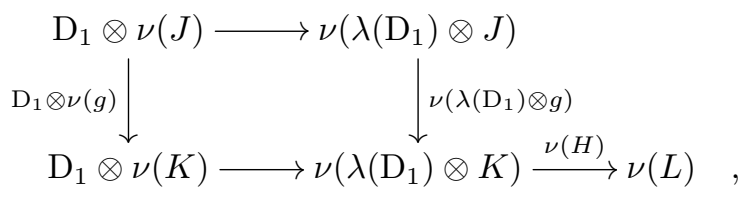

où les flèches non nommées sont la contrainte du foncteur monoïdal lax $\nu$. Or, le carré de ce diagramme est commutatif par naturalité, d'où la première assertion.

D'après les mêmes résultats, les transformations oplax de la seconde assertion correspondent aux deux manières de composer les trois $\infty$-foncteurs

$$
\mathrm{D}_{1} \otimes \nu(K) \rightarrow \nu\left(\lambda\left(\mathrm{D}_{1}\right) \otimes K\right) \stackrel{\nu(H)}{\longrightarrow} \nu(L) \stackrel{\nu(g)}{\longrightarrow} \nu(M)
$$

où, de nouveau, la flèche non nommée est la contrainte du foncteur monoïdal lax $\nu$, d'où le résultat.

Proposition B.4.9. - Soit $f: K \rightarrow L$ un morphisme de complexes dirigés augmentés. On a

$$
\nu\left(1_{f}\right)=1_{\nu(f)}
$$

Démonstration. - En vertu de la proposition B.3.5 et du paragraphe B.4.4, cela résulte de la commutativité, par naturalité, du diagramme

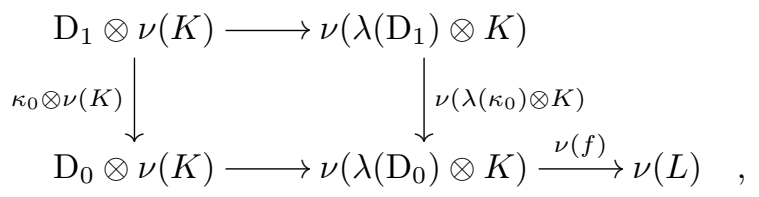

où les flèches non nommées sont la contrainte du foncteur monoïdal lax $\nu$.

Proposition B.4.10. - Soient $f_{0}, f_{1}, f_{2}: K \rightarrow L$ trois morphismes de complexes dirigés augmentés, $h$ une homotopie de $f_{0}$ vers $f_{1}$ et $h^{\prime}$ une homotopie de $f_{1}$ vers $f_{2}$. On a

$$
\nu\left(h^{\prime}+h\right)=\nu\left(h^{\prime}\right) \circ \nu(h) .
$$

Démonstration. - Notons $H, H^{\prime}: \lambda\left(\mathrm{D}_{1}\right) \otimes K \rightarrow L$ les morphismes correspondant respectivement à $h$ et $h^{\prime}$. En vertu des paragraphes B.3.2 et B.4.5, il suffit de montrer 
la commutativité du diagramme

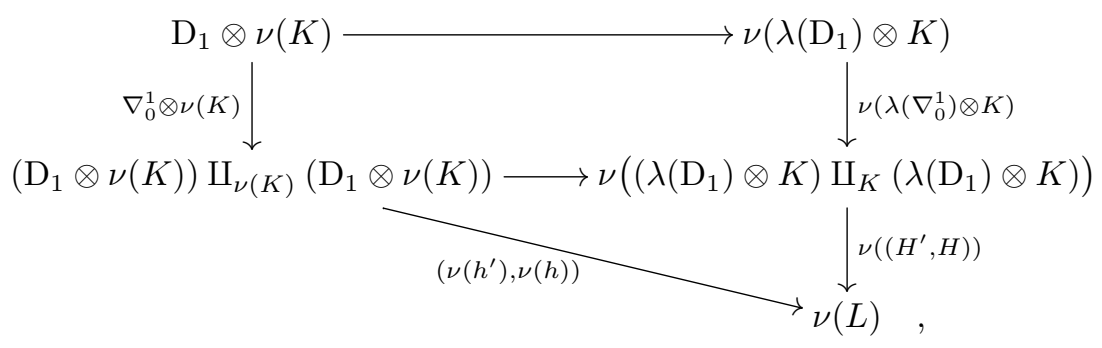

où les flèches non nommées sont induites par la contrainte du foncteur monoïdal lax $\nu$ et, plus précisément, pour celle du bas, par le morphisme

$$
\left(\mathrm{D}_{1} \amalg_{\mathrm{D}_{0}} \mathrm{D}_{1}\right) \otimes \nu(K) \rightarrow \nu\left(\lambda\left(\mathrm{D}_{1} \amalg_{\mathrm{D}_{0}} \mathrm{D}_{1}\right) \otimes K\right) .
$$

Or, le rectangle de ce diagramme est commutatif par naturalité. Pour conclure, il nous suffit donc de vérifier la commutativité du triangle. En précomposant ce triangle par les deux morphismes canoniques

$$
\mathrm{D}_{1} \otimes \nu(K) \rightarrow\left(\mathrm{D}_{1} \otimes \nu(K)\right) \amalg_{\nu(K)}\left(\mathrm{D}_{1} \otimes \nu(K)\right),
$$

on obtient deux triangles qui commutent par définition de $\nu(h)$ et $\nu\left(h^{\prime}\right)$, d'où le résultat.

Remarque B.4.11. - Dualement, si $h$ est une antihomotopie de complexes dirigés augmentés, on peut lui associer une transformation lax $\nu(h)$. Les propositions B.4.7, B.4.8, B.4.9 et B.4.10 entraînent, par dualité, leurs analogues pour les antihomotopies et les transformations lax.

\section{B.5. Joint et transformations oplax}

B.5.1. - Soient $C$ une $\infty$-catégorie et $c$ un objet de $C$. On définit un foncteur d'inclusion $c \backslash C \hookrightarrow \underline{\operatorname{Hom}}_{\text {lax }}\left(\mathrm{D}_{1}, C\right)$ de la manière suivante. Pour tout $i \geqslant 0$, si $(d, \alpha)$ est une $i$-flèche de $c \backslash C$ (voir le paragraphe 9.7), on lui associe la $i$-flèche $\left(1_{c}, d, \alpha\right)$ de $\underline{\operatorname{Hom}}_{\text {lax }}\left(\mathrm{D}_{1}, C\right)$ (voir le paragraphe B.1.7), où $1_{c}$ désigne l'identité itérée de $c$ en dimension $i$. Le fait qu'on obtienne bien ainsi une $i$-flèche de $\underline{\operatorname{Hom}}_{\operatorname{lax}}\left(\mathrm{D}_{1}, C\right)$ résulte des paragraphes 9.7 et B.1.7, ainsi que de la formule

$$
\alpha_{k-1}^{1} *_{k-1} \cdots *_{1} \alpha_{0}^{1} * 1_{c}=\alpha_{k-1}^{1} .
$$

Par ailleurs, la fonctorialité de cette correspondance découle des propositions 9.15 et B.1.15, ainsi que de la formule ci-dessus. 
Proposition B.5.2. - Pour toute $\infty$-catégorie $C$ et tout objet c de $C$, le carré

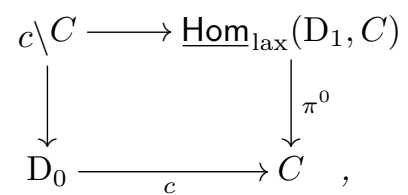

où la flèche horizontale du haut est le $\infty$-foncteur défini au paragraphe précédent et $\pi^{0}$ désigne le $\infty$-foncteur du paragraphe B.2.2, est cartésien.

Démonstration. - Cela résulte immédiatement de la description de l'inclusion $c \backslash C \hookrightarrow \underline{\text { Hom }}_{\text {lax }}\left(\mathrm{D}_{1}, C\right)$ donnée au paragraphe précédent.

Corollaire B.5.3. - Soient $C$ une $\infty$-catégorie et $c$ un objet de $C$. Pour toute $\infty$-catégorie $A$, on a une bijection naturelle entre les $\infty$-foncteurs $A \rightarrow c \backslash C$ et les transformations oplax entre $\infty$-foncteurs de $A$ vers $C$ de source le $\infty$-foncteur constant de valeur c. De plus, le but de la transformation oplax associée à un tel $\infty$-foncteur est le composé de $A \rightarrow c \backslash C \stackrel{U}{\rightarrow} C$, où $U$ désigne le $\infty$-foncteur d'oubli.

Démonstration. - La première assertion est conséquence immédiate de la proposition précédente et de la description des transformations oplax en termes de $\underline{\operatorname{Hom}}_{\text {lax }}\left(\mathrm{D}_{1}, C\right)$ (proposition B.2.5). La seconde résulte de la commutativité du triangle

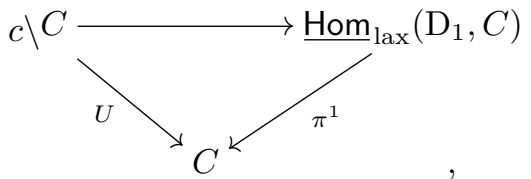

où $U$ désigne le $\infty$-foncteur d'oubli et $\pi^{1}$ le $\infty$-foncteur du paragraphe B.2.2.

Corollaire B.5.4. - Soient $C$ une $\infty$-catégorie et c un objet de $C$. Pour tout $i \geqslant 0$, les $i$-flèches de $c \backslash C$ sont en correspondance bijective canonique avec les couples $(d, \alpha)$, où $d$ est une $i$-flèche de $C$ et $\alpha$ est une transformation oplax du $\infty$-foncteur $\mathrm{D}_{i} \rightarrow C$ constant de valeur c vers le $\infty$-foncteur $d: \mathrm{D}_{i} \rightarrow C$.

Démonstration. - Cela résulte du corollaire précédent appliqué à $A=\mathrm{D}_{i}$.

B.5.5. - Soit $C$ une $\infty$-catégorie. On définit un $\infty$-foncteur $\mathrm{D}_{1} \otimes C \rightarrow \mathrm{D}_{0} \star C$ de la manière suivante. Si $A$ est une $\infty$-catégorie, en utilisant les adjonctions des paragraphes 6.31 et A.18, ainsi que le $\infty$-foncteur du paragraphe B.5.1, on obtient une application

$$
\begin{aligned}
& \operatorname{Hom}_{\infty-\mathcal{C} a t}\left(\mathrm{D}_{0} \star C, A\right) \stackrel{\sim}{\longrightarrow} \coprod_{a \in A_{0}} \operatorname{Hom}_{\infty-\mathcal{C} a t}(C, a \backslash A) \\
& \operatorname{Hom}_{\infty-\mathcal{C} a t}\left(C, \underline{\operatorname{Hom}}_{\text {lax }}\left(\mathrm{D}_{1}, A\right)\right) \stackrel{\sim}{\rightarrow} \operatorname{Hom}_{\infty-\mathcal{C} a t}\left(\mathrm{D}_{1} \otimes C, A\right),
\end{aligned}
$$


naturelle en $A$, et donc le $\infty$-foncteur recherché en vertu du lemme de Yoneda.

Corollaire B.5.6. - Pour toute $\infty$-catégorie C, le carré

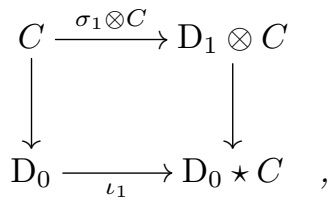

où la flèche verticale de droite est le $\infty$-foncteur défini au paragraphe précédent et $\sigma_{1}$ désigne le $\infty$-foncteur du paragraphe 4.1, est cocartésien.

Démonstration. - Si $A$ est une $\infty$-catégorie, en vertu des adjonctions utilisées dans le paragraphe précédent et de la proposition B.5.2, on a

$$
\begin{aligned}
\operatorname{Hom}_{\infty-\mathcal{C} a t}\left(\mathrm{D}_{0} \star C, A\right) & \simeq \coprod_{a \in A_{0}} \operatorname{Hom}_{\infty-\mathcal{C} a t}(C, a \backslash A) \\
& \simeq \coprod_{a \in A_{0}} \operatorname{Hom}_{\infty-\mathcal{C} a t}\left(C, \underline{\operatorname{Hom}}_{\operatorname{lax}}\left(\mathrm{D}_{1}, A\right)\right) \times_{\operatorname{Hom}_{\infty-\mathcal{C} a t}(C, A)}\{a\} \\
& \simeq \coprod_{a \in A_{0}} \operatorname{Hom}_{\infty-\mathcal{C} a t}\left(\mathrm{D}_{1} \otimes C, A\right) \times_{\operatorname{Hom}_{\infty-\mathcal{C} a t}(C, A)}\{a\} \\
& \simeq \operatorname{Hom}_{\infty-\mathcal{C} a t}\left(\mathrm{D}_{1} \otimes C, A\right) \times_{\operatorname{Hom}_{\infty-\mathcal{C} a t}(C, A)} \operatorname{Hom}_{\infty-\mathcal{C} a t}\left(\mathrm{D}_{0}, A\right) \\
& \simeq \operatorname{Hom}_{\infty-\mathcal{C} a t}\left(\left(\mathrm{D}_{1} \otimes C\right) \amalg_{C} \mathrm{D}_{0}, A\right),
\end{aligned}
$$

d'où le résultat.

\section{B.6. Suspension et transformations oplax}

B.6.1. - Soit $C$ une $\infty$-catégorie et soient $c$ et $d$ deux objets de $C$. On notera $\underline{\text { Hom }}_{C}(c, d)$ la $\infty$-catégorie des cellules de $C$ de 0 -source $c$ et de 0 -but $d$. On définit un foncteur d'inclusion $\underline{\operatorname{Hom}}_{C}(c, d)^{\circ} \hookrightarrow \underline{\operatorname{Hom}}_{\operatorname{lax}}\left(\mathrm{D}_{1}, C\right)$ de la manière suivante. Pour tout $i \geqslant 0$, on associe à une $i$-flèche $\operatorname{de} \underline{\operatorname{Hom}}_{C}(c, d)^{\circ}$, qui correspond par définition à une $(i+1)$-flèche $f$ de $C$ de 0 -source $c$ et de 0 -but $d$, la $i$-flèche $\left(1_{c}, 1_{d}, \alpha\right)$ de $\underline{\operatorname{Hom}}_{\text {lax }}\left(\mathrm{D}_{1}, C\right)$ (voir le paragraphe B.1.7), où $1_{c}$ et $1_{d}$ désignent les identités itérées respectives de $c$ et $d$ en dimension $i$, et où $\alpha$ est défini par

$$
\alpha_{k}^{0}=t_{k+1}(f) \quad \text { et } \quad \alpha_{k}^{1}=s_{k+1}(f),
$$

pour $0 \leqslant k \leqslant i$ (en particulier, on a $\alpha_{i}=f$ ). Le fait qu'on obtienne bien ainsi une $i$-flèche de $\underline{\operatorname{Hom}}_{\operatorname{lax}}\left(\mathrm{D}_{1}, C\right)$ résulte du paragraphe B.1.7, ainsi que des formules

$$
\alpha_{k-1}^{1} *_{k-1} \cdots *_{1} \alpha_{0}^{1} *_{0} 1_{c}=\alpha_{k-1}^{1} \quad \text { et } \quad 1_{d} *_{0} \alpha_{0}^{0} *_{1} \cdots *_{k-1} \alpha_{k-1}^{0}=\alpha_{k-1}^{0} .
$$

Par ailleurs, la fonctorialité de cette correspondance découle de la proposition B.1.15, ainsi que des formules ci-dessus. 
Proposition B.6.2. - Pour toute $\infty$-catégorie $C$ et tous objets $c$ et $d$ de $C$, le $\infty$-foncteur d'inclusion $\underline{\operatorname{Hom}}_{C}(c, d)^{\circ} \hookrightarrow{\underline{\mathrm{Hom}_{\text {lax }}}}\left(\mathrm{D}_{1}, C\right)$ induit un isomorphisme

$$
\underline{\operatorname{Hom}}_{C}(c, d)^{\circ} \simeq\{c\} \times_{C} \underline{\operatorname{Hom}}_{\text {lax }}\left(\mathrm{D}_{1}, C\right) \times_{C}\{d\}
$$

le membre de droite désignant la limite projective du diagramme

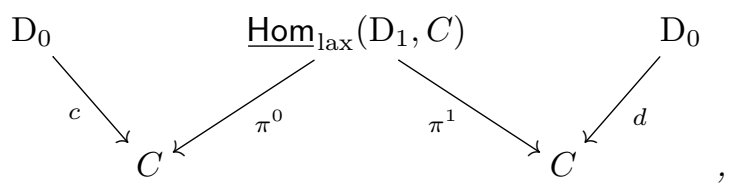

où $\pi^{0}$ et $\pi^{1}$ sont les $\infty$-foncteurs du paragraphe B.2.2.

Démonstration. - Cela résulte immédiatement de la description de l'inclusion $\underline{\operatorname{Hom}}_{C}(c, d)^{\circ} \hookrightarrow \underline{\text { Hom }}_{\text {lax }}\left(\mathrm{D}_{1}, C\right)$ donnée au paragraphe précédent.

Corollaire B.6.3. - Soit $C$ une $\infty$-catégorie et soient $c$ et d deux objets de $C$. Pour toute $\infty$-catégorie $A$, on a une bijection naturelle entre les $\infty$-foncteurs $A \rightarrow \underline{\operatorname{Hom}}_{C}(c, d)^{\circ}$ et les transformations oplax entre $\infty$-foncteurs de $A$ vers $C$ de source le $\infty$-foncteur constant de valeur $c$ et de but le $\infty$-foncteur constant de valeur $d$.

Démonstration. - L'assertion est conséquence immédiate de la proposition précédente et de la description des transformations oplax en termes de $\underline{\operatorname{Hom}}_{\text {lax }}\left(\mathrm{D}_{1}, C\right)$ (proposition B.2.5).

Corollaire B.6.4. - Soit $C$ une $\infty$-catégorie et soient c et d deux objets de $C$. Pour tout $i \geqslant 0$, les transformations oplax $d u \infty$-foncteur $\mathrm{D}_{i} \rightarrow C$ constant de valeur $c$ vers le $\infty$-foncteur $\mathrm{D}_{i} \rightarrow C$ constant de valeur $d$ sont en bijection naturelle avec les $(i+1)$-flèches de $C$ de 0 -source $c$ et de 0 -but d. De plus, si une transformation oplax $\alpha$ et une $(i+1)$-cellule $f$ de $C$ se correspondent dans cette bijection, alors, pour $i \geqslant 1$, les $i$-cellules source et but de $f$ correspondent aux transformations oplax $\alpha * \tau_{i}$ et $\alpha * \sigma_{i}$, où $\sigma_{i}$ et $\tau_{i}$ désignent les $\infty$-foncteurs du paragraphe 4.1 .

Démonstration. - La première assertion résulte de la bijection du corollaire précédent dans le cas $A=\mathrm{D}_{i}$ et la seconde de la naturalité de cette même bijection appliquée à $\sigma_{i}$ et $\tau_{i}$.

B.6.5. - Soit $C$ une $\infty$-catégorie. La suspension de $C$ est la $\infty$-catégorie $\Sigma C$ définie de la manière suivante :

- les objets de $\Sigma C$ sont 0 et 1 ; 
- si $x$ et $y$ sont deux objets de $\Sigma C$, on a

$$
\underline{\operatorname{Hom}}_{\Sigma C}(x, y)= \begin{cases}C^{\circ} & \text { si } x=0 \text { et } y=1 \\ * & \text { si } x=y \\ \varnothing & \text { sinon }\end{cases}
$$

- les compositions et les identités sont définies de la façon évidente.

On définit un $\infty$-foncteur $\mathrm{D}_{1} \otimes C \rightarrow \Sigma C$ comme suit. Soit $A$ une $\infty$-catégorie. On vérifie immédiatement que la donnée d'un $\infty$-foncteur de $\Sigma C$ vers $A$ correspond à la donnée de deux objets $a$ et $b$ de $A$, images respectives des objets 0 et 1 , et d'un $\infty$-foncteur de $\underline{\operatorname{Hom}}_{\Sigma C}(0,1)=C^{\circ}$ vers $\underline{\operatorname{Hom}}_{A}(a, b)$. Ainsi, on dispose de bijections naturelles

$$
\begin{aligned}
\operatorname{Hom}_{\infty-\mathcal{C} a t}(\Sigma C, A) & \simeq \coprod_{a, b \in A_{0}} \operatorname{Hom}_{\infty-\mathcal{C} a t}\left(C^{\circ}, \underline{\operatorname{Hom}}_{A}(a, b)\right) \\
& \simeq \coprod_{a, b \in A_{0}} \operatorname{Hom}_{\infty-\mathcal{C} a t}\left(C, \underline{\operatorname{Hom}}_{A}(a, b)^{\circ}\right) .
\end{aligned}
$$

En utilisant le $\infty$-foncteur du paragraphe B.6.1 et l'adjonction du paragraphe 6.31, on obtient donc une application

$$
\begin{aligned}
\operatorname{Hom}_{\infty-\mathcal{C} a t}(\Sigma C, A) & \stackrel{\sim}{\rightarrow} \coprod_{a, b \in A_{0}} \operatorname{Hom}_{\infty-\mathcal{C} a t}\left(C, \underline{\operatorname{Hom}}_{A}(a, b)^{\circ}\right) \\
& \operatorname{Hom}_{\infty-\mathcal{C} a t}\left(C, \underline{\operatorname{Hom}}_{\text {lax }}\left(\mathrm{D}_{1}, A\right)\right) \stackrel{\sim}{\rightarrow} \operatorname{Hom}_{\infty-\mathcal{C} a t}\left(\mathrm{D}_{1} \otimes C, A\right),
\end{aligned}
$$

naturelle en $A$, et donc un $\infty$-foncteur $\mathrm{D}_{1} \otimes C \rightarrow \Sigma C$ en vertu du lemme de Yoneda.

Corollaire B.6.6. - Pour toute $\infty$-catégorie $C$, le $\infty$-foncteur $\mathrm{D}_{1} \otimes C \rightarrow \Sigma C$ défini au paragraphe précédent induit un isomorphisme

$$
\Sigma C \simeq \mathrm{D}_{0} \amalg_{C}\left(\mathrm{D}_{1} \otimes C\right) \amalg_{C} \mathrm{D}_{0},
$$

le membre de droite désignant la limite inductive du diagramme

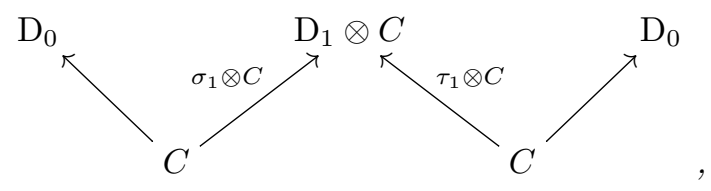

où $\sigma_{1}$ et $\tau_{1}$ sont les $\infty$-foncteurs du paragraphe 4.1. 
Démonstration. - Si $A$ est une $\infty$-catégorie, en vertu du paragraphe précédent et de la proposition B.6.2, on a

$$
\begin{aligned}
\mathrm{Hom}_{\infty-\mathcal{C} a t}(\Sigma C, A) \\
\simeq \coprod_{a, b \in A_{0}} \operatorname{Hom}_{\infty-\mathcal{C} a t}\left(C, \underline{\operatorname{Hom}}_{A}(a, b)^{\circ}\right) \\
\simeq \coprod_{a, b \in A_{0}}\{a\} \times_{\operatorname{Hom}_{\infty-\mathcal{C} a t}(C, A)} \operatorname{Hom}_{\infty-\mathcal{C} a t}\left(C, \underline{\operatorname{Hom}}_{\operatorname{lax}}\left(\mathrm{D}_{1}, A\right)\right) \times_{\mathrm{Hom}_{\infty-\mathcal{C} a t}(C, A)\{b\}} \\
\simeq \coprod_{a, b \in A_{0}}\{a\} \times_{\operatorname{Hom}_{\infty-\mathcal{C} a t}(C, A)} \operatorname{Hom}_{\infty-\mathcal{C} a t}\left(\mathrm{D}_{1} \otimes C, A\right) \times_{\operatorname{Hom}_{\infty-\mathcal{C} a t}(C, A)\{b\}} \\
\simeq \operatorname{Hom}_{\infty-\mathcal{C} a t}\left(\mathrm{D}_{0}, A\right) \times \operatorname{Hom}_{\infty-\mathcal{C} a t}\left(\mathrm{D}_{1} \otimes C, A\right) \times \operatorname{Hom}_{\infty-\mathcal{C} a t}\left(\mathrm{D}_{0}, A\right) \\
\simeq \operatorname{Hom}_{\infty-\mathcal{C} a t}(C, A) \\
\quad \operatorname{Hom}_{\infty-\mathcal{C} a t}\left(\mathrm{D}_{0} \amalg_{C}\left(\mathrm{D}_{1} \otimes C\right) \amalg_{C} \mathrm{D}_{0}, A\right),
\end{aligned}
$$

d'où le résultat. 


\section{APPENDICE C}

\section{FONCTORIALITÉS DES TRANCHES : CONJECTURES}

Le but de cet appendice est de dégager des conjectures de fonctorialité des tranches généralisant les résultats obtenus dans le chapitre 11. Pour formuler ces conjectures, on a besoin, entre autres, d'introduire les notions de $\infty$-sesquicatégorie, de $\infty$-catégorie de Gray (catégorie enrichie dans $\infty$-C at muni du produit tensoriel de Gray), de $\infty$-catégorie de Gray gauche, et de montrer que la sesquicatégorie des $\infty$-catégories, $\infty$-foncteurs et transformations oplax (resp. transformations lax) provient d'une $\infty$-catégorie de Gray $\infty$-Cat ${ }_{\text {oplax }}$ (resp. d'une $\infty$-catégorie de Gray gauche $\infty$-( Cat $\left._{\text {lax }}\right)$. On commence par développer les notions correspondantes dans un cadre monoïdal (non nécessairement symétrique) général.

$\boldsymbol{C . 1}$. - Soit $\mathcal{V}$ une catégorie munie d'un foncteur $P: \mathcal{V} \rightarrow \mathcal{E} n s$ à valeurs dans la catégorie des ensembles qu'on appellera foncteur points. Une $\mathcal{V}$-sesquicatégorie, ou sesquicatégorie enrichie dans $\mathcal{V}$, est une catégorie $\mathcal{C}$ munie d'un foncteur

$$
\underline{\operatorname{Hom}}_{\mathcal{C}}: \mathcal{C}^{\circ} \times \mathcal{C} \rightarrow \mathcal{V}
$$

et d'un isomorphisme de foncteurs

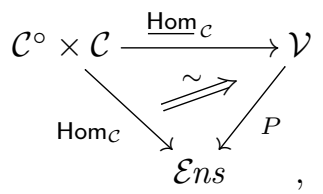

qui le plus souvent, pour simplifier, sera considéré comme étant l'identité. On dit que $\mathcal{C}$ est la catégorie sous-jacente à une telle $\mathcal{V}$-sesquicatégorie, que les objets de $\mathcal{C}$ sont ses objets et que, pour $x, y$ deux objets, $\underline{\operatorname{Hom}}_{\mathcal{C}}(x, y)$ est son objet de morphismes de $x$ vers $y$.

Si $\mathcal{V}$ est une catégorie monoïdale d'objet unité $I$, on dispose d'un foncteur points canonique

$$
P=\operatorname{Hom}_{\mathcal{V}}(I, \bullet): \mathcal{V} \rightarrow \mathcal{E} n s,
$$


d'où une notion de $\mathcal{V}$-sesquicatégorie. Toutes les $\mathcal{V}$-sesquicatégories considérées pour $\mathcal{V}$ une catégorie monoïdale seront relatives à ce foncteur points. On remarque que le produit tensoriel de la catégorie monoïdale ne joue aucun rôle dans cette définition.

Exemple C.2. - Si $\mathcal{V}$ est la catégorie des ensembles avec comme foncteur points le foncteur identité, une $\mathcal{V}$-sesquicatégorie n'est autre qu'une catégorie. Si $\mathcal{V}$ est la catégorie $\mathcal{C}$ at des petites catégories munie du foncteur points $\mathrm{Ob}: \mathcal{C}$ at $\rightarrow \mathcal{E}$ ns, alors on vérifie facilement qu'une $\mathcal{V}$-sesquicatégorie est ce qu'on appelle habituellement une sesquicatégorie (voir par exemple [39, section 2]).

C.3. - Une $\infty$-sesquicatégorie est une sesquicatégorie enrichie dans la catégorie $\infty$-Cat des $\infty$-catégories munie du foncteur points $\mathrm{Ob}: \infty$-C $a t \rightarrow \mathcal{E} n s$, associant à une $\infty$-catégorie l'ensemble de ses objets ou, de façon équivalente, est une sesquicatégorie enrichie dans la catégorie monoïdale $\infty$-C at munie du produit tensoriel défini, au choix, par le produit cartésien ou par le produit tensoriel de Gray (voir le paragraphe A.16), puisque dans ces deux cas l'objet unité est un objet final de $\infty$-C at.

Soit $\mathcal{C}$ une $\infty$-sesquicatégorie. Pour $i \geqslant 1$, une $i$-flèche de $\mathcal{C}$ est une $(i-1)$-flèche de la $\infty$-catégorie $\underline{\operatorname{Hom}}_{\mathcal{C}}(x, y)$, pour des objets $x, y$ de $\mathcal{C}$, et on dit que $x$ est sa 0 -source et $y$ son 0 -but. Pour $0<j \leqslant i$, sa $j$-source et son $j$-but sont respectivement sa $(j-1)$-source et son $(j-1)$-but comme cellule de $\underline{\operatorname{Hom}}_{\mathcal{C}}(x, y)$. La commutativité du triangle du paragraphe C.1 montre que les 1-flèches de $\mathcal{C}$ sont les flèches de sa catégorie sous-jacente. Pour $0 \leqslant k<i, j$, on dit qu'une $i$-flèche $\alpha$ de $\mathcal{C}$ et une $j$-flèche $\beta$ de $\mathcal{C}$ sont $k$-composables si la $k$-source de $\alpha$ est égale au $k$-but de $\beta$, et alors si $k>0$, leur $k$-composé $\alpha *_{k} \beta$ est défini comme étant leur $(k-1)$-composé dans la $\infty$-catégorie $\underline{\operatorname{Hom}}_{\mathcal{C}}(x, y)$, où $x$ et $y$ sont respectivement la 0 -source, nécessairement commune, et le 0 -but, nécessairement commun, de $\alpha$ et $\beta$. Si $k=0$, leur 0 -composé $\alpha *_{0} \beta$ n'est défini que si $i=1$ ou $j=1$. Dans ce cas, soient $x$ la 0 -source de $\beta, y$ son 0 -but, qui est alors égal à la 0 -source de $\alpha$, et $z$ le 0 -but de $\alpha$. Si $i=1$, le 0 -composé $\alpha *_{0} \beta$ est défini comme étant l'image de la cellule $\beta$ de $\underline{\operatorname{Hom}}_{\mathcal{C}}(x, y)$ par le $\infty$-foncteur

$$
\underline{\operatorname{Hom}}_{\mathcal{C}}(x, \alpha): \underline{\operatorname{Hom}}_{C}(x, y) \rightarrow \underline{\operatorname{Hom}}_{\mathcal{C}}(x, z)
$$

et, si $j=1$, comme étant l'image de la cellule $\alpha$ de $\underline{\operatorname{Hom}}_{\mathcal{C}}(y, z)$ par le $\infty$-foncteur

$$
\underline{\operatorname{Hom}}_{\mathcal{C}}(\beta, z): \underline{\operatorname{Hom}}_{C}(y, z) \rightarrow \underline{\operatorname{Hom}}_{\mathcal{C}}(x, z) .
$$

Si $x$ est un objet de $\mathcal{C}$, l'unité de $x$ est l'unité $1_{x}$ de $x$ dans la catégorie sous-jacente à $\mathcal{C}$ (qui est une 1 -flèche de $\mathcal{C}$ ) et si, pour $i>0, \alpha$ est une $i$-flèche de $\mathcal{C}$ de 0 -source $x$ et 0 -but $y$, l'unité de $\alpha$ est son unité $1_{\alpha}$ comme cellule de $\underline{\operatorname{Hom}}_{\mathcal{C}}(x, y)$ (qui est une $(i+1)$-flèche de $\mathcal{C})$. Ces données satisfont aux mêmes axiomes que les données correspondantes d'une $\infty$-catégorie, sauf la règle de Godement pour la 0 -composition (autrement dit, pour $i, j>1$, si $\alpha$ est une $i$-flèche de 1 -source $a_{0}$ et 1-but $a_{1}$ et $\beta$ une 
$j$-flèche de 1 -source $b_{0}$ et 1 -but $b_{1}$ et si $\alpha$ et $\beta$ sont 0 -composables

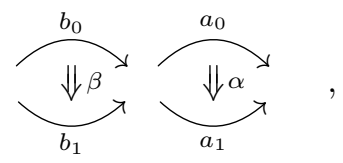

le composé $\left(\alpha *_{0} b_{1}\right) *_{1}\left(a_{0} *_{0} \beta\right)$ n'est pas en général égal au composé $\left(a_{1} *_{0} \beta\right) *_{1}\left(\alpha *_{0} b_{0}\right)$, ce qui empêche de définir le 0 -composé de $\alpha$ et $\beta$ ). Ces données soumises à ces axiomes fournissent une définition équivalente des $\infty$-sesquicatégories.

C.4. - Soient $C$ une $\infty$-catégorie et $c$ un objet de $C$. Dans la description explicite de la $\infty$-catégorie tranche $c \backslash C$ donnée dans le chapitre 9 , les seules 0 -compositions dans $C$ figurant dans les formules définissant les cellules de $c \backslash C$, ainsi que leurs compositions, sont de la forme $\alpha *_{0} \beta$, où $\alpha$ ou $\beta$ est une 1-flèche de $C$ (voir le paragraphe 9.7 et la proposition 9.15). De plus, si $(x, f: c \rightarrow x)$ et $\left(x^{\prime}, f^{\prime}: c \rightarrow x^{\prime}\right)$ sont deux objet de $c \backslash C$ et $i>1$, toute $i$-flèche non triviale de $C$ qui est une composante d'une cellule de la $\infty$-catégorie $\underline{\mathrm{Hom}}_{c \backslash} C\left((x, f),\left(x^{\prime}, f^{\prime}\right)\right)$ a comme 0 -source $c$ ou $x$ et comme 0 -but $x^{\prime}$. Ainsi, aucune utilisation de la règle de Godement pour la 0 -composition de $C$ n'intervient pour vérifier que les formules du paragraphe 9.7 et de la proposition 9.15 définissent une $\infty$-catégorie $\underline{\operatorname{Hom}}_{c \backslash} C\left((x, f),\left(x^{\prime}, f^{\prime}\right)\right)$.

On en déduit que si $\mathcal{C}$ est une $\infty$-sesquicatégorie, $c$ un objet de $\mathcal{C}$ et $f: c \rightarrow x$, $f^{\prime}: c \rightarrow x^{\prime}$ deux 1-flèches de $\mathcal{C}$ de source $c$, on peut définir une $\infty$-catégorie notée $\underline{\text { Hom }}_{c \backslash} \mathcal{C}\left((x, f),\left(x^{\prime}, f^{\prime}\right)\right)$ par les mêmes formules que dans le cas d'une $\infty$-catégorie $C$.

En revanche, il n'est pas possible en général d'assembler, pour $(x, f)$ et $\left(x^{\prime}, f^{\prime}\right)$ variables, les $\infty$-catégories $\underline{\operatorname{Hom}}_{c \backslash} \mathcal{C}\left((x, f),\left(x^{\prime}, f^{\prime}\right)\right)$ en une $\infty$-sesquicatégorie. En effet, étant donné une $\infty$-catégorie $C$ et un objet $c$ de $C$, on observe que dans la vérification du fait que, si $\alpha$ et $\beta$ sont une 2-flèche et une 1-flèche 0 -composables de $c \backslash C$, alors les formules de la proposition 9.15 définissant le composé $\alpha *_{0} \beta$ définissent bien une 2-flèche de $c \backslash C$, on utilise la règle de Godement pour la 0-composition de $C$.

De façon analogue, on peut définir une $\infty$-catégorie $\underline{\operatorname{Hom}}_{c \mathcal{C}}\left((x, f),\left(x^{\prime}, f^{\prime}\right)\right)$ et, pour $g: x \rightarrow c$ et $g^{\prime}: x^{\prime} \rightarrow c$ deux 1-flèches de $\mathcal{C}$ de but $c$, des $\infty$-catégories $\underline{\operatorname{Hom}}_{\mathcal{C} / c}\left((x, g),\left(x^{\prime}, g^{\prime}\right)\right)$ et $\underline{\text { Hom }}_{\mathcal{C}^{\text {co }}}\left((x, g),\left(x^{\prime}, g^{\prime}\right)\right)$ mais, par contre, pas de $\infty$-sesquicatégories associées. Pour pouvoir le faire, on a besoin d'une structure plus forte, celle de $\infty$-catégorie de Gray ou de $\infty$-catégorie de Gray gauche (voir le paragraphe C.16 et la conjecture C.24).

C.5. - Soit $\mathcal{V}$ une catégorie monoïdale de produit tensoriel $\otimes$ et d'objet unité $I$. On rappelle qu'une catégorie enrichie dans $\mathcal{V}$, ou plus simplement $\mathcal{V}$-catégorie, $\mathbb{C}$ est la donnée

- d'une classe $\mathrm{Ob}(\mathbb{C})$ dont les éléments sont appelés les objets de $\mathbb{C}$; 
- pour tous $x, y$ objets de $\mathbb{C}$, d'un objet $\underline{\operatorname{Hom}}_{\mathbb{C}}(x, y)$ de $\mathcal{V}$, appelé objet de morphismes de $x$ vers $y$;

- pour tous $x, y, z$ objets de $\mathbb{C}$, d'un morphisme

$$
\circ_{z, y, x}: \underline{\operatorname{Hom}}_{\mathbb{C}}(y, z) \otimes \underline{\operatorname{Hom}}_{\mathbb{C}}(x, y) \rightarrow \underline{\operatorname{Hom}}_{\mathbb{C}}(x, z)
$$

de $\mathcal{V}$, appelé morphisme de composition;

- pour tout $x$ objet de $\mathbb{C}$, d'un morphisme $\mathrm{id}_{x}: I \rightarrow \underline{\operatorname{Hom}}_{\mathbb{C}}(x, x)$ de $\mathcal{V}$, appelé morphisme d'unité,

ces données satisfaisant aux conditions usuelles d'associativité et d'unité, autrement dit rendant commutatifs les diagrammes

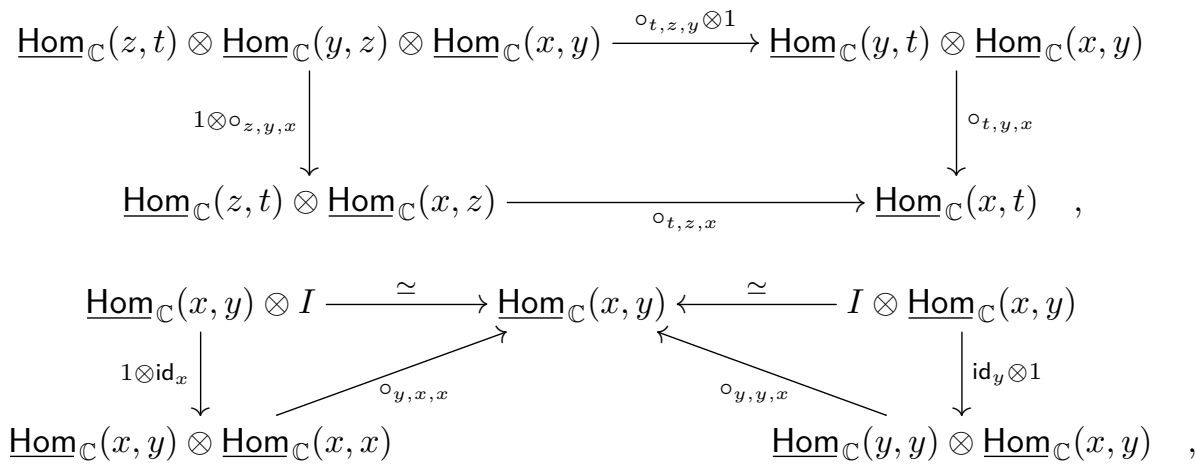

pour $x, y, z, t$ objets de $\mathbb{C}$ (où on omet pour simplifier les contraintes d'associativité et d'unité de $\mathcal{V}$ ).

Une $\mathcal{V}$-catégorie gauche est une catégorie enrichie dans la catégorie monoïdale transposée de $\mathcal{V}$, c'est-à-dire dans la catégorie monoïdale ayant même catégorie sousjacente et même unité que $\mathcal{V}$ mais comme produit tensoriel le foncteur

$$
\begin{aligned}
\mathcal{V} \times \mathcal{V} & \rightarrow \mathcal{V} \\
(X, Y) & \mapsto Y \otimes X,
\end{aligned}
$$

obtenu à partir de $\otimes$ en composant avec l'isomorphisme de symétrie du produit cartésien. Les notions de $\mathcal{V}$-catégorie et $\mathcal{V}$-catégorie gauche sont bien distinctes, sauf dans le cas où la catégorie monoïdale $\mathcal{V}$ est symétrique, auquel cas ces deux notions sont essentiellement équivalentes, la symétrie définissant une bijection entre $\mathcal{V}$-catégories et $\mathcal{V}$-catégories gauches.

Exemple $\boldsymbol{C . 6}$. - Une catégorie enrichie dans la catégorie monoïdale des ensembles, avec comme produit tensoriel le produit cartésien, n'est autre qu'une catégorie ordinaire.

C.7. - Soient $\mathcal{V}$ et $\mathcal{V}^{\prime}$ deux catégories monoïdales de produits tensoriels et unités $\otimes, I$ et $\otimes^{\prime}, I^{\prime}$ respectivement, et $F: \mathcal{V} \rightarrow \mathcal{V}^{\prime}$ un foncteur monoïdal lax. À toute 
$\mathcal{V}$-catégorie $\mathbb{C}$, le foncteur $F$ associe une $\mathcal{V}^{\prime}$-catégorie $\mathbb{C}^{\prime}=F_{*}(\mathbb{C})$, appelée image directe de $\mathbb{C}$ par $F$, définie comme suit :

- les objets de $\mathbb{C}^{\prime}$ sont les mêmes que ceux de $\mathbb{C}$;

- pour $x, y$ objets de $\mathbb{C}^{\prime}$, l'objet de morphismes de $x$ vers $y$ est $F\left(\underline{\operatorname{Hom}}_{\mathbb{C}}(x, y)\right)$;

- pour $x, y, z$ objets de $\mathbb{C}^{\prime}$, le morphisme de composition est le composé

$F\left(\underline{\operatorname{Hom}}_{\mathbb{C}}(y, z)\right) \otimes^{\prime} F\left(\underline{\operatorname{Hom}}_{\mathbb{C}}(x, y)\right) \rightarrow F\left(\underline{\operatorname{Hom}}_{\mathbb{C}}(y, z) \otimes \underline{\operatorname{Hom}}_{\mathbb{C}}(x, y)\right) \rightarrow F\left(\underline{\operatorname{Hom}}_{\mathbb{C}}(x, z)\right)$,

la flèche de gauche venant de la contrainte de composition du foncteur monoïdal lax $F$ et la flèche de droite étant $F\left(\circ_{z, y, x}\right)$;

- pour $x$ objet de $\mathbb{C}^{\prime}$, le morphisme d'unité est le composé

$$
I^{\prime} \rightarrow F(I) \rightarrow F\left(\underline{\operatorname{Hom}}_{\mathbb{C}}(x, x)\right),
$$

la flèche de gauche venant de la contrainte d'unité du foncteur monoïdal lax $F$ et la flèche de droite étant $F\left(\mathrm{id}_{x}\right)$.

Exemple C.8. - Soit $\mathcal{V}$ une catégorie monoïdale de produit tensoriel $\otimes$ et d'objet unité $I$. Le foncteur

$$
\operatorname{Hom}_{\mathcal{V}}(I, \bullet): \mathcal{V} \rightarrow \mathcal{E} n s
$$

s'enrichit en un foncteur monoïdal lax à valeurs dans la catégorie des ensembles munie du produit cartésien en définissant pour $X, Y$ objets de $\mathcal{V}$ la contrainte de composition

$$
\operatorname{Hom}_{\mathcal{V}}(I, X) \times \operatorname{Hom}_{\mathcal{V}}(I, Y) \rightarrow \operatorname{Hom}_{\mathcal{V}}(I, X \otimes Y)
$$

par

$$
(x: I \rightarrow X, y: I \rightarrow Y) \mapsto x \otimes y: I \simeq I \otimes I \rightarrow X \otimes Y
$$

et la contrainte d'unité

$$
\{*\} \rightarrow \operatorname{Hom}_{\mathcal{V}}(I, I) \quad \text { par } \quad * \mapsto 1_{I} .
$$

Pour toute $\mathcal{V}$-catégorie $\mathbb{C}$, l'image directe de $\mathbb{C}$ par ce foncteur monoïdal lax est, en vertu de l'exemple C.6, une catégorie ordinaire appelée catégorie sous-jacente à la $\mathcal{V}$-catégorie $\mathbb{C}$.

La catégorie sous-jacente à une $\mathcal{V}$-catégorie gauche $\mathbb{C}^{\prime}$ est la catégorie sous-jacente à $\mathbb{C}^{\prime}$, vue comme catégorie enrichie dans la catégorie monoïdale transposée de $\mathcal{V}$.

C.9. - Soient $\mathcal{V}$ une catégorie monoïdale de produit tensoriel $\otimes$ et d'objet unité $I$, et $\mathbb{C}$ une $\mathcal{V}$-catégorie de catégorie sous-jacente $\mathcal{C}$. On peut munir la catégorie $\mathcal{C}$ d'une structure canonique de $\mathcal{V}$-sesquicatégorie, appelée $\mathcal{V}$-sesquicatégorie sous-jacente à la $\mathcal{V}$-catégorie $\mathbb{C}$, comme suit. Le foncteur $\underline{\operatorname{Hom}}_{\mathcal{C}}: \mathcal{C}^{\circ} \times \mathcal{C} \rightarrow \mathcal{V}$ associe à un objet $(x, y)$ de $\mathcal{C}^{\circ} \times \mathcal{C}$ l'objet $\underline{\operatorname{Hom}}_{\mathbb{C}}(x, y)$ de $\mathcal{V}$. Si $f: x^{\prime} \rightarrow x$ et $g: y \rightarrow y^{\prime}$ sont des morphismes 
de $\mathcal{C}$, la flèche $\underline{\operatorname{Hom}}_{\mathcal{C}}(f, g)$ de $\mathcal{V}$ est définie par le diagramme commutatif

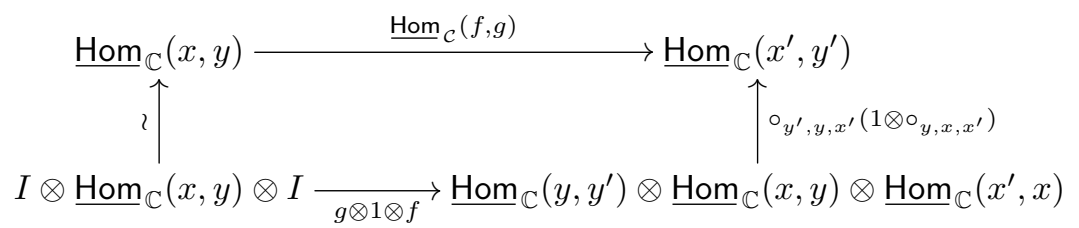

(où la flèche verticale de gauche vient de la contrainte d'unité de $\mathcal{V}$ et où on néglige les contraintes d'associativité). Il est alors immédiat que le triangle

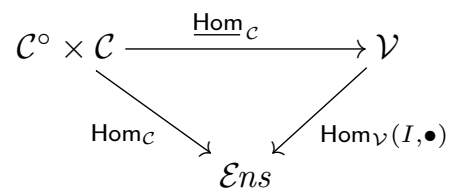

du paragraphe C.1 est strictement commutatif.

La $\mathcal{V}$-sesquicatégorie sous-jacente à une $\mathcal{V}$-catégorie gauche $\mathbb{C}^{\prime}$ est la $\mathcal{V}$-sesquicatégorie sous-jacente à $\mathbb{C}^{\prime}$, vue comme catégorie enrichie dans la catégorie monoïdale transposée de $\mathcal{V}$ (on rappelle que le produit tensoriel de $\mathcal{V}$ n'intervient pas dans la définition d'une $\mathcal{V}$-sesquicatégorie).

Exemple C.10. - Soit $\mathcal{V}$ une catégorie monoïdale fermée à droite (voir le paragraphe 5.2) de produit tensoriel $\otimes$ et d'objet unité $I$, de sorte qu'il existe un foncteur $\underline{\operatorname{Hom}_{\mathcal{V}}^{\mathrm{d}}}: \mathcal{V}^{\circ} \times \mathcal{V} \rightarrow \mathcal{V}$ et une bijection

$$
\operatorname{Hom}_{\mathcal{V}}(X \otimes Y, Z) \simeq \operatorname{Hom}_{\mathcal{V}}\left(X, \underline{\operatorname{Hom}}_{\mathcal{V}}^{\mathrm{d}}(Y, Z)\right)
$$

naturelle en $X, Y$ et $Z$ dans $\mathcal{V}$. On définit une $\mathcal{V}$-catégorie $\mathbb{V}^{\mathrm{d}}$ ayant mêmes objets que $\mathcal{V}$ et telle que $\underline{\operatorname{Hom}}_{\mathbb{V}^{d}}(X, Y)=\underline{\operatorname{Hom}}_{\mathcal{V}}^{\mathrm{d}}(X, Y)$ comme suit. La bijection ci-dessus, appliquée à $X=\underline{\operatorname{Hom}}_{\mathcal{V}}^{\mathrm{d}}(Y, Z)$, fournit un morphisme de $\mathcal{V}$, dit d'évaluation,

$$
\operatorname{ev}_{Z, Y}: \underline{\operatorname{Hom}}_{\mathcal{V}}^{\mathrm{d}}(Y, Z) \otimes Y \rightarrow Z
$$

correspondant par cette bijection à $1_{\underline{H o m}_{\mathcal{V}}^{\mathrm{d}}(Y, Z)}$. Le composé ev $\mathrm{v}_{Z, Y}\left(1 \otimes \mathrm{ev}_{Y, X}\right)$

$$
\underline{\operatorname{Hom}}_{\mathcal{V}}^{\mathrm{d}}(Y, Z) \otimes \underline{\operatorname{Hom}}_{\mathcal{V}}^{\mathrm{d}}(X, Y) \otimes X \rightarrow \underline{\operatorname{Hom}}_{\mathcal{V}}^{\mathrm{d}}(Y, Z) \otimes Y \rightarrow Z
$$

définit par adjonction le morphisme de composition. De même, l'isomorphisme $I \otimes X \simeq X$ définit par adjonction le morphisme d'unité $I \rightarrow \underline{\operatorname{Hom}}_{\mathcal{V}}^{\mathrm{d}}(X, X)$. Une vérification simple mais fastidieuse prouve les propriétés d'associativité et d'unité (voir par exemple $[\mathbf{2 6}]$ ). La catégorie sous-jacente à la $\mathcal{V}$-catégorie $\mathbb{V}^{\mathrm{d}}$ n'est autre que la catégorie sous-jacente à la catégorie monoïdale $\mathcal{V}$ et la $\mathcal{V}$-sesquicatégorie sous-jacente à $\mathbb{V}^{\mathrm{d}}$ est définie par le foncteur $\underline{\operatorname{Hom}}_{\mathcal{V}}^{\mathrm{d}}: \mathcal{V}^{\circ} \times \mathcal{V} \rightarrow \mathcal{V}$. 
Si la catégorie monoïdale $\mathcal{V}$, au lieu d'être fermée à droite est fermée à gauche, de sorte qu'il existe un foncteur $\operatorname{Hom}_{\mathcal{V}}^{\mathrm{g}}: \mathcal{V}^{\circ} \times \mathcal{V} \rightarrow \mathcal{V}$ et une bijection

$$
\operatorname{Hom}_{\mathcal{V}}(X \otimes Y, Z) \simeq \operatorname{Hom}_{\mathcal{V}}\left(Y, \underline{\operatorname{Hom}}_{\mathcal{V}}^{\mathrm{g}}(X, Z)\right)
$$

naturelle en $X, Y$ et $Z$ dans $\mathcal{V}$, il faut se garder de croire qu'on puisse définir en général une $\mathcal{V}$-catégorie ayant mêmes objets que $\mathcal{V}$ et dont l'objet de morphismes soit donné par $\underline{\operatorname{Hom}}_{\mathcal{V}}^{g}$. En revanche, en appliquant ce qui précède à la catégorie monoïdale $\mathcal{V}^{\prime}$ transposée de $\mathcal{V}$, on déduit l'existence d'une $\mathcal{V}$-catégorie gauche $\mathbb{V}^{\mathrm{g}}$ ayant mêmes objets que $\mathcal{V}$ et dont l'objet de morphismes est défini par $\underline{H o m}_{\mathcal{V}}^{\mathrm{g}}$. En effet, la catégorie monoïdale $\mathcal{V}^{\prime}$ est alors fermée à droite et, pour $X, Y$ objets de $\mathcal{V}$, on a

$$
\underline{\operatorname{Hom}}_{\mathcal{V}}^{\mathrm{g}}(X, Y)=\underline{\operatorname{Hom}}_{\mathcal{V}^{\prime}}^{\mathrm{d}}(X, Y) \text {. }
$$

La catégorie sous-jacente à cette $\mathcal{V}$-catégorie gauche est la catégorie sous-jacente à la catégorie monoïdale $\mathcal{V}$ et sa $\mathcal{V}$-sesquicatégorie sous-jacente est définie par le foncteur $\underline{\operatorname{Hom}}_{\mathcal{V}}^{\mathrm{g}}: \mathcal{V}^{\circ} \times \mathcal{V} \rightarrow \mathcal{V}$

C.11. - Soient $\mathcal{V}$ une catégorie monoïdale de produit tensoriel $\otimes$ et d'objet unité $I$, et $\mathbb{C}$ et $\mathbb{C}^{\prime}$ deux catégories enrichies dans $\mathcal{V}$ (voir le paragraphe C.5). On rappelle qu'un foncteur enrichi, ou plus simplement $\mathcal{V}$-foncteur, $\mathbb{F}$ de $\mathbb{C}$ vers $\mathbb{C}^{\prime}$ est la donnée

- d'une application $\mathbb{F}_{0}: \mathrm{Ob}(\mathbb{C}) \rightarrow \mathrm{Ob}\left(\mathbb{C}^{\prime}\right)$;

- pour tous $x, y$ objets de $\mathbb{C}$, d'un morphisme de $\mathcal{V}$

$$
\mathbb{F}_{y, x}: \underline{\operatorname{Hom}}_{\mathbb{C}}(x, y) \rightarrow \underline{\operatorname{Hom}}_{\mathbb{C}^{\prime}}\left(\mathbb{F}_{0}(x), \mathbb{F}_{0}(y)\right),
$$

ces données satisfaisant aux compatibilités usuelles aux compositions et aux unités, autrement dit rendant commutatifs les diagrammes
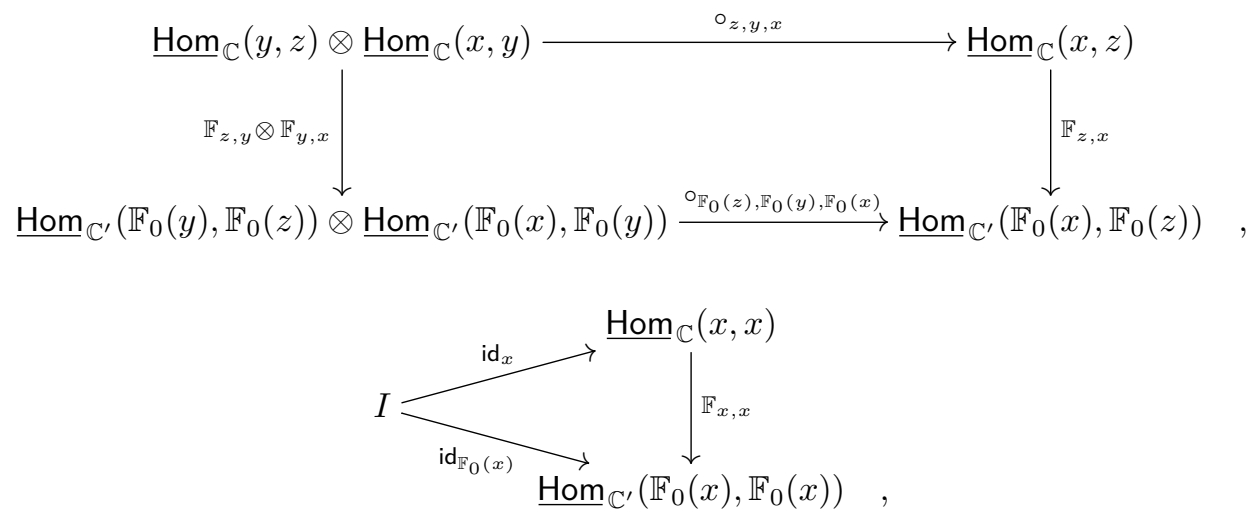

pour $x, y, z$ objets de $\mathbb{C}$.

Si $\mathbb{C}$ et $\mathbb{C}^{\prime}$ sont deux $\mathcal{V}$-catégories gauches, un $\mathcal{V}$-foncteur gauche de $\mathbb{C}$ vers $\mathbb{C}^{\prime}$ est un foncteur enrichi dans la catégorie monoïdale transposée de $\mathcal{V}$ de $\mathbb{C}$ vers $\mathbb{C}^{\prime}$. 
C.12. - Soient $\mathcal{V}$ une catégorie monoïdale d'objet unité $I, \mathbb{C}$ et $\mathbb{C}^{\prime}$ deux catégories enrichies dans $\mathcal{V}$ et $\mathbb{F}$ un foncteur enrichi de $\mathbb{C}$ vers $\mathbb{C}^{\prime}$. Le foncteur enrichi $\mathbb{F}$ induit un foncteur ordinaire $F$ de la catégorie $\mathcal{C}$ sous-jacente à $\mathbb{C}$ vers la catégorie $\mathcal{C}^{\prime}$ sous-jacente à $\mathbb{C}^{\prime}$, appelé foncteur sous-jacent à $\mathbb{F}$, défini comme suit. Sur les objets, le foncteur $F$ est défini par l'application $\mathbb{F}_{0}$. Si $f: x \rightarrow y$ est un morphisme de $\mathcal{C}$, autrement dit une flèche $f: I \rightarrow \underline{\operatorname{Hom}}_{\mathbb{C}}(x, y)$ de $\mathcal{V}$, le morphisme $F(f)$ de $\mathcal{C}^{\prime}$ est défini par le composé

$$
I \stackrel{f}{\longrightarrow} \underline{\operatorname{Hom}}_{\mathbb{C}}(x, y) \stackrel{\mathbb{F}_{y, x}}{\longrightarrow} \underline{\operatorname{Hom}}_{\mathbb{C}^{\prime}}\left(\mathbb{F}_{0}(x), \mathbb{F}_{0}(y)\right) .
$$

Le foncteur sous-jacent à un $\mathcal{V}$-foncteur gauche est son foncteur sous-jacent comme foncteur enrichi dans la catégorie monoïdale transposée de $\mathcal{V}$.

C.13. - Soient $\mathcal{V}$ une catégorie monoïdale et $\mathbb{C}$ une $\mathcal{V}$-catégorie. Pour toute partie de $\mathrm{Ob}(\mathbb{C})$, on définit une $\mathcal{V}$-catégorie, appelée sous-V-catégorie pleine de $\mathbb{C}$ définie par cette partie, dont la classe des objets est cette partie et dont les objets de morphismes, les morphismes de composition et les morphismes d'unité sont induits par ceux de $\mathbb{C}$. L'inclusion de cette partie dans la classe des objets de $\mathbb{C}$ s'enrichit en un $\mathcal{V}$-foncteur, appelé $\mathcal{V}$-foncteur d'inclusion. De même, si $\mathbb{C}$ est une $\mathcal{V}$-catégorie gauche, toute partie de $\mathrm{Ob}(\mathbb{C})$ définit une sous- $\mathcal{V}$-catégorie gauche pleine de $\mathbb{C}$ et un $\mathcal{V}$-foncteur gauche d'inclusion.

C.14. - Soit $\mathcal{V}$ une catégorie monoïdale. À une $\mathcal{V}$-catégorie $\mathbb{C}$, on associe une $\mathcal{V}$-catégorie gauche ${ }^{t} \mathbb{C}$, transposée de $\mathbb{C}$, ayant mêmes objets que $\mathbb{C}$ et, pour $x, y$ objets de $\mathbb{C}$, comme objet de morphismes de $x$ vers $y$

$$
\underline{\operatorname{Hom}}_{t \mathbb{C}}(x, y)=\underline{\operatorname{Hom}}_{\mathbb{C}}(y, x) .
$$

De même, à une $\mathcal{V}$-catégorie gauche $\mathbb{C}$, on associe de façon analogue une $\mathcal{V}$-catégorie transposée ${ }^{t} \mathbb{C}$. Pour $\mathbb{C}$ une $\mathcal{V}$-catégorie ou une $\mathcal{V}$-catégorie gauche, la catégorie sousjacente à ${ }^{t} \mathbb{C}$ est la catégorie opposée de la catégorie sous-jacente à $\mathbb{C}$ et on a ${ }^{t t} \mathbb{C}=\mathbb{C}$.

Si $\mathbb{C}$ et $\mathbb{C}^{\prime}$ sont deux $\mathcal{V}$-catégories (resp. deux $\mathcal{V}$-catégories gauches) et $\mathbb{F}: \mathbb{C} \rightarrow \mathbb{C}^{\prime}$ est un $\mathcal{V}$-foncteur (resp. un $\mathcal{V}$-foncteur gauche), on définit le $\mathcal{V}$-foncteur gauche (resp. le $\mathcal{V}$-foncteur) transposé ${ }^{t} \mathbb{F}:{ }^{t} \mathbb{C} \rightarrow{ }^{t} \mathbb{C}^{\prime}$ de la façon évidente.

Exemple C.15. - Soit $\mathcal{V}$ une catégorie monoïdale bifermée (voir le paragraphe 5.2) de produit tensoriel $\otimes$, de sorte qu'il existe des foncteurs

$$
\underline{\operatorname{Hom}}_{\mathcal{V}}^{\mathrm{d}}, \underline{\operatorname{Hom}}_{\mathcal{V}}^{\mathrm{g}}: \mathcal{V}^{\circ} \times \mathcal{V} \rightarrow \mathcal{V}
$$

et des bijections

$$
\operatorname{Hom}_{\mathcal{V}}\left(Y, \underline{\operatorname{Hom}}_{\mathcal{V}}^{\mathrm{g}}(X, Z)\right) \simeq \operatorname{Hom}_{\mathcal{V}}(X \otimes Y, Z) \simeq \operatorname{Hom}_{\mathcal{V}}\left(X, \underline{\operatorname{Hom}}_{\mathcal{V}}^{\mathrm{d}}(Y, Z)\right),
$$

naturelles en $X, Y$ et $Z$ dans $\mathcal{V}$, et soient $\mathbb{V}^{\mathrm{d}}$ la $\mathcal{V}$-catégorie et $\mathbb{V}^{\mathrm{g}}$ la $\mathcal{V}$-catégorie gauche associées (voir l'exemple C.10). Pour $X$ un objet de $\mathcal{V}$, les affirmations suivantes sont purement formelles (voir $[\mathbf{2 6}]$ ). 
(a) Il existe un $\mathcal{V}$-foncteur canonique $\mathbb{V}^{\mathrm{d}} \rightarrow \mathbb{V}^{\mathrm{d}}$ dont le foncteur sous-jacent est le foncteur $\bullet \otimes X: \mathcal{V} \rightarrow \mathcal{V}$.

(b) Il existe un $\mathcal{V}$-foncteur gauche canonique $\mathbb{V}^{\mathrm{g}} \rightarrow \mathbb{V}^{\mathrm{g}}$ dont le foncteur sous-jacent est le foncteur $X \otimes \bullet: \mathcal{V} \rightarrow \mathcal{V}$.

(c) Il existe un $\mathcal{V}$-foncteur canonique $\mathbb{V}^{\mathrm{d}} \rightarrow \mathbb{V}^{\mathrm{d}}$ dont le foncteur sous-jacent est le foncteur $\underline{\operatorname{Hom}}_{\mathcal{V}}^{\mathrm{d}}(X, \bullet): \mathcal{V} \rightarrow \mathcal{V}$.

(d) Il existe un $\mathcal{V}$-foncteur gauche canonique ${ }^{t} \mathbb{V}^{\mathrm{d}} \rightarrow \mathbb{V}^{\mathrm{g}}$ dont le foncteur sous-jacent est le foncteur $\underline{\operatorname{Hom}}_{\mathcal{V}}^{\mathrm{d}}(\bullet, X): \mathcal{V}^{\circ} \rightarrow \mathcal{V}$.

(e) Il existe un $\mathcal{V}$-foncteur gauche canonique $\mathbb{V}^{\mathrm{g}} \rightarrow \mathbb{V}^{\mathrm{g}}$ dont le foncteur sous-jacent est le foncteur $\underline{\operatorname{Hom}}_{\mathcal{V}}^{\mathrm{g}}(X, \bullet): \mathcal{V} \rightarrow \mathcal{V}$.

(f) Il existe un $\mathcal{V}$-foncteur canonique ${ }^{t} \mathbb{V}^{g} \rightarrow \mathbb{V}^{\mathrm{d}}$ dont le foncteur sous-jacent est le foncteur $\operatorname{Hom}_{\mathcal{V}}^{\mathrm{g}}(\bullet, X): \mathcal{V}^{\circ} \rightarrow \mathcal{V}$.

C.16. - Une $\infty$-catégorie de Gray est une catégorie enrichie dans la catégorie monoïdale des $\infty$-catégories avec comme produit tensoriel le produit tensoriel de Gray (voir le paragraphe A.16). Une $\infty$-catégorie de Gray gauche est la variante gauche de cette notion, autrement dit une catégorie enrichie dans la catégorie monoïdale des $\infty$-catégories avec comme produit tensoriel le foncteur

$$
(X, Y) \mapsto Y \otimes X \simeq\left(X^{\mathrm{op}} \otimes Y^{\mathrm{op}}\right)^{\mathrm{op}} \simeq\left(X^{\mathrm{co}} \otimes Y^{\mathrm{co}}\right)^{\mathrm{co}}
$$

(voir la proposition A.22). Le produit tensoriel de Gray dans $\infty$-Cat n'étant pas symétrique, les notions de $\infty$-catégorie de Gray et $\infty$-catégorie de Gray gauche ne sont pas équivalentes. Si $\mathbb{C}$ est une $\infty$-catégorie de Gray ou une $\infty$-catégorie de Gray gauche et $x, y$ deux objets de $\mathbb{C}$, alors par définition $\underline{\operatorname{Hom}}_{\mathbb{C}}(x, y)$ est une $\infty$-catégorie. On vérifie immédiatement que les objets de $\underline{\operatorname{Hom}}_{\mathbb{C}}(x, y)$ sont les morphismes de source $x$ et but $y$ dans la catégorie sous-jacente à $\mathbb{C}$ (voir l'exemple C.8). De plus, si $\mathcal{C}$ désigne la $\infty$-sesquicatégorie sous-jacente à $\mathbb{C}$, on a $\underline{\operatorname{Hom}}_{\mathcal{C}}(x, y)=\underline{\operatorname{Hom}}_{\mathbb{C}}(x, y)$ (voir les paragraphes C.3 et C.9). Pour $i \geq 1$, une $i$-flèche de $\mathbb{C}$ sera par définition une $i$-flèche de sa $\infty$-sesquicatégorie sous-jacente $\mathcal{C}$ et de même pour les sources, buts, compositions et unités.

Exemple $\boldsymbol{C . 1 7}$. - Le foncteur identité de $\infty$-Cat s'enrichit en un foncteur monoïdal lax de source $\infty$-Cat munie du produit tensoriel défini par le produit cartésien et de but $\infty$-Cat munie du produit tensoriel de Gray. Pour $X, Y$ deux $\infty$-catégories, la contrainte de composition est

$$
\left(1_{X} \otimes p_{Y}, p_{X} \otimes 1_{Y}\right): X \otimes Y \rightarrow X \times Y,
$$

où pour $Z$ une $\infty$-catégorie, $p_{Z}$ désigne l'unique flèche de $Z$ vers la $\infty$-catégorie finale, et la contrainte d'unité est l'identité (en vertu du paragraphe A.16, l'unité du produit tensoriel de Gray est la catégorie finale). Or, une catégorie enrichie dans la catégorie monoïdale des $\infty$-catégories avec comme produit tensoriel le produit cartésien est simplement une $\infty$-catégorie (non nécessairement petite mais localement petite). Ainsi, 
à une telle $\infty$-catégorie $\mathcal{C}$, on associe une $\infty$-catégorie de Gray $\mathbb{C}$, image directe de $\mathcal{C}$ par ce foncteur monoïdal lax, satisfaisant à l'égalité

$$
\underline{\operatorname{Hom}}_{\mathbb{C}}(x, y)=\underline{\operatorname{Hom}}_{\mathcal{C}}(x, y) .
$$

Exemple C.18. - On rappelle que la catégorie monoïdale $\infty$-Cat, munie du produit tensoriel de Gray, est bifermée (voir le théorème A.15), de sorte que dans les notations du paragraphe A.18, on a des bijections

$\operatorname{Hom}_{\infty-\mathcal{C} a t}\left(B, \underline{\operatorname{Hom}}_{\text {lax }}(A, C)\right) \simeq \operatorname{Hom}_{\infty-\mathcal{C} a t}(A \otimes B, C) \simeq \operatorname{Hom}_{\infty-\mathcal{C} a t}\left(A, \underline{\operatorname{Hom}}_{\text {oplax }}(B, C)\right)$ naturelles en les $\infty$-catégories $A, B, C$. En vertu de l'exemple C.10, on a donc une $\infty$-catégorie de Gray $\infty$-Cat ${ }_{\text {oplax }}$, appelée $\infty$-catégorie de Gray des $\infty$-catégories, dont les objets sont les $\infty$-catégories et telle que pour tout couple $A, B$ de $\infty$-catégories, l'objet de morphismes de $A$ vers $B$ soit $\underline{\operatorname{Hom}}_{\text {oplax }}(A, B)$. La catégorie sous-jacente à $\infty$-Cat ${ }_{\text {oplax }}$ n'est autre que $\infty$-C at. En vertu de la remarque B.2.8, les 2-flèches de $\infty$ - $\mathbb{C a t}_{\text {oplax }}$ sont les transformations oplax. Plus généralement, pour $i \geq 0$, les $(i+1)$-flèches de $\infty$-Cat ${ }_{\text {oplax }}$ sont appelées des $i$-transformations oplax (une 0 -transformation oplax est donc un $\infty$-foncteur et une 1-transformation oplax une transformation oplax ordinaire). De même, on a une $\infty$-catégorie de Gray gauche $\infty$ - $\mathbb{C a t}_{\text {lax }}$, appelée $\infty$-catégorie de Gray gauche des $\infty$-catégories, dont les objets sont les $\infty$-catégories et telle que pour tout couple $A, B$ de $\infty$-catégories, l'objet de morphismes de $A$ vers $B$ soit $\underline{\operatorname{Hom}}_{\text {lax }}(A, B)$. La catégorie sous-jacente à $\infty$-Cat lax $_{\text {lax }}$ est $\infty$-Cat, et les 2-flèches de $\infty$-Cat lax $_{\text {lax }}$ sont les transformations lax (voir la remarque B.2.8). Plus généralement, pour $i \geq 0$, les $(i+1)$-flèches de $\infty$-Cat ${ }_{\text {oplax }}$ sont appelées des $i$-transformations lax.

C.19. - Soient $\mathbb{C}$ et $\mathbb{C}^{\prime}$ deux $\infty$-catégories de Gray. Un $\infty$-foncteur de Gray de $\mathbb{C}$ vers $\mathbb{C}^{\prime}$ est un foncteur enrichi de $\mathbb{C}$ vers $\mathbb{C}^{\prime}$ (voir le paragraphe C.11). De même, si $\mathbb{C}$ et $\mathbb{C}^{\prime}$ sont deux $\infty$-catégories de Gray gauches, un $\infty$-foncteur de Gray gauche de $\mathbb{C}$ vers $\mathbb{C}^{\prime}$ est un foncteur enrichi de $\mathbb{C}$ vers $\mathbb{C}^{\prime}$.

C.20. - En vertu du paragraphe C.13, si $\mathbb{C}$ est une $\infty$-catégorie de Gray, toute partie de la classe des objets de $\mathbb{C}$ définit une sous- $\infty$-catégorie de Gray pleine de $\mathbb{C}$ et un $\infty$-foncteur de Gray d'inclusion. De même, si $\mathbb{C}$ est une $\infty$-catégorie de Gray gauche, toute partie de la classe des objets de $\mathbb{C}$ définit une sous- $\infty$-catégorie de Gray gauche pleine de $\mathbb{C}$ et un $\infty$-foncteur de Gray gauche d'inclusion.

C.21. - Soit $\mathbb{C}$ une $\infty$-catégorie de Gray. On définit une $\infty$-catégorie de Gray $\mathbb{C}^{\text {op }}$, ayant mêmes objets que $\mathbb{C}$, en posant

$$
\underline{\operatorname{Hom}}_{\mathbb{C}^{\text {op }}}(x, y)=\underline{\operatorname{Hom}}_{\mathbb{C}}(y, x)^{\text {co }},
$$

les morphismes de composition et d'unité étant définis de la manière évidente (en utilisant la proposition A.22), et deux $\infty$-catégories de Gray gauches $\mathbb{C}^{\text {co }}$ et $\mathbb{C}^{\circ}$, ayant 
également mêmes objets que $\mathbb{C}$, en posant

$$
\begin{aligned}
& \underline{\operatorname{Hom}}_{\mathbb{C}^{\mathrm{co}}}(x, y)=\underline{\operatorname{Hom}}_{\mathbb{C}}(x, y)^{\mathrm{op}}, \\
& \underline{\operatorname{Hom}}_{\mathbb{C}^{\circ}}(x, y)=\underline{\operatorname{Hom}}_{\mathbb{C}}(y, x)^{\circ}
\end{aligned}
$$

(voir la proposition A.22), les morphismes de composition et d'unité étant toujours définis de la manière évidente. En particulier, à une $\infty$-catégorie de Gray $\mathbb{C}$, on en associe trois autres $\mathbb{C}^{\text {op }},{ }^{t} \mathbb{C}^{\text {co }}$ et ${ }^{t} \mathbb{C}^{\circ}$ (voir le paragraphe C.14). On définit de façon analogue, pour $\mathbb{C}$ une $\infty$-catégorie de Gray gauche, une $\infty$-catégorie de Gray gauche $\mathbb{C}^{\text {op }}$ et deux $\infty$-catégories de Gray $\mathbb{C}^{\text {co }}$ et $\mathbb{C}^{\circ}$.

Pour $\mathbb{C}$ une $\infty$-catégorie de Gray ou une $\infty$-catégorie de Gray gauche, de catégorie sous-jacente $\mathcal{C}$, la catégorie sous-jacente à $\mathbb{C}^{\text {op }}$ et à $\mathbb{C}^{\circ}$ est $\mathcal{C}^{\circ}$, la catégorie sous-jacente à $\mathcal{C}^{\text {co }}$ est $\mathcal{C}$ et on a des égalités

$$
\begin{aligned}
& \mathbb{C}^{\text {op op }}=\mathbb{C}^{\text {co co }}=\mathbb{C}^{\circ \circ}=\mathbb{C}, \\
& \mathbb{C}^{\text {op co }}=\mathbb{C}^{\mathrm{co} \mathrm{op}}=\mathbb{C}^{\circ}, \\
& \mathbb{C}^{\text {op } \circ}=\mathbb{C}^{\circ \text { op }}=\mathbb{C}^{\mathrm{co}}, \\
& \mathbb{C}^{\mathrm{co} \circ}=\mathbb{C}^{\circ \text { co }}=\mathbb{C}^{\mathrm{op}} .
\end{aligned}
$$

Si $\mathbb{C}$ et $\mathbb{C}^{\prime}$ sont deux $\infty$-catégories de Gray et $\mathbb{F}: \mathbb{C} \rightarrow \mathbb{C}^{\prime}$ est un $\infty$-foncteur de Gray, on définit de la façon évidente un $\infty$-foncteur de Gray $\mathbb{F}^{\text {op }}: \mathbb{C}^{\text {op }} \rightarrow \mathbb{C}^{\prime o p}$ et des $\infty$-foncteurs de Gray gauches $\mathbb{F}^{\text {co }}: \mathbb{C}^{\mathrm{co}} \rightarrow \mathbb{C}^{\prime c o}$ et $\mathbb{F}^{\circ}: \mathbb{C}^{\circ} \rightarrow \mathbb{C}^{\prime \circ}$. De même, si $\mathbb{C}$ et $\mathbb{C}^{\prime}$ sont deux $\infty$-catégories de Gray gauches et $\mathbb{F}: \mathbb{C} \rightarrow \mathbb{C}^{\prime}$ est un $\infty$-foncteur de Gray gauche, on définit un $\infty$-foncteur de Gray gauche $\mathbb{F}^{\text {op }}: \mathbb{C}^{\text {op }} \rightarrow \mathbb{C}^{\prime o p}$ et des $\infty$-foncteurs de Gray $\mathbb{F}^{\text {co }}: \mathbb{C}^{\text {co }} \rightarrow \mathbb{C}^{\prime c o}$ et $\mathbb{F}^{\circ}: \mathbb{C}^{\circ} \rightarrow \mathbb{C}^{\prime \circ}$.

Remarque C.22. - Les isomorphismes naturels

$$
\begin{aligned}
\underline{\operatorname{Hom}}_{\text {oplax }}(A, B)^{\mathrm{op}} & \simeq \underline{\operatorname{Hom}}_{\text {lax }}\left(A^{\mathrm{op}}, B^{\mathrm{op}}\right), \\
\underline{\operatorname{Hom}}_{\mathrm{oplax}}(A, B)^{\mathrm{co}} & \simeq \underline{\operatorname{Hom}}_{\text {lax }}\left(A^{\mathrm{co}}, B^{\mathrm{co}}\right), \\
\underline{\operatorname{Hom}}_{\mathrm{oplax}}(A, B)^{\circ} & \simeq \underline{\operatorname{Hom}}_{\mathrm{oplax}}\left(A^{\circ}, B^{\circ}\right),
\end{aligned}
$$

pour $A, B$ des $\infty$-catégories (voir la proposition A.23), induisent des isomorphismes de $\infty$-catégories de Gray

$$
\begin{aligned}
& \left(\infty-\mathbb{C a t}_{\text {lax }}\right)^{\mathrm{co}} \stackrel{\text { op }}{\sim} \infty-\mathbb{C a t}_{\text {oplax }}, \\
& \left(\infty-\mathbb{C a t}_{\text {oplax }}\right)^{\text {op }} \stackrel{\text { co }}{\sim}{ }^{t}\left(\infty-\mathbb{C a t}_{\text {lax }}\right), \\
& \left(\infty-\mathbb{C a t}_{\text {lax }}\right)^{\circ} \stackrel{\text { o }}{\sim}{ }^{t}\left(\infty-\mathbb{C a t}_{\text {lax }}\right)
\end{aligned}
$$


et des isomorphismes de $\infty$-catégories de Gray gauches

$$
\begin{aligned}
& \left(\infty-\mathbb{C a t}_{\text {oplax }}\right)^{\text {co }} \stackrel{\text { op }}{\sim} \infty-\mathbb{C a t}_{\text {lax }}, \\
& \left(\infty-\mathbb{C a t}_{\text {lax }}\right)^{\text {op }} \stackrel{\text { co }}{\sim}{ }^{t}\left(\infty-\mathbb{C a t}_{\text {oplax }}\right), \\
& \left(\infty-\mathbb{C a t}_{\text {oplax }}\right)^{\circ} \stackrel{\text { o }}{\sim}{ }^{t}\left(\infty-\mathbb{C a t}_{\text {oplax }}\right)
\end{aligned}
$$

de foncteurs sous-jacents respectifs $C \mapsto C^{\mathrm{op}}, C \mapsto C^{\mathrm{co}}$ et $C \mapsto C^{\circ}$.

Exemple C.23. — En se souvenant que la catégorie monö̈dale $\infty$-Cat, munie du produit tensoriel de Gray, est bifermée (voir le théorème A.15), de sorte que dans les notations du paragraphe A.18 on a des bijections

$\operatorname{Hom}_{\infty-\mathcal{C} a t}\left(B, \underline{\operatorname{Hom}}_{\text {lax }}(A, C)\right) \simeq \operatorname{Hom}_{\infty-\mathcal{C} a t}(A \otimes B, C) \simeq \operatorname{Hom}_{\infty-\mathcal{C} a t}\left(A, \underline{\operatorname{Hom}}_{\text {oplax }}(B, C)\right)$ naturelles en les $\infty$-catégories $A, B$ et $C$, on obtient comme cas particulier de l'exemple C.15 les assertions suivantes pour $C$ une $\infty$-catégorie.

(a) Il existe un $\infty$-foncteur de Gray canonique $\infty$ - Cat $_{\text {oplax }} \rightarrow \infty$-Cat ${ }_{\text {oplax }}$ dont le foncteur sous-jacent est le foncteur $\bullet \otimes C: \infty$ - $\mathcal{C}$ at $\rightarrow \infty$ - $\mathcal{C}$ at.

(b) Il existe un $\infty$-foncteur de Gray gauche canonique $\infty$ - $\mathbb{C a t}_{\text {lax }} \rightarrow \infty$ - $\mathbb{C a t}_{\text {lax }}$ dont le foncteur sous-jacent est le foncteur $C \otimes \bullet: \infty$ - $\mathcal{C}$ at $\rightarrow \infty$-Cat.

(c) Il existe un $\infty$-foncteur de Gray canonique $\infty$ - $\mathbb{C a t}_{\text {oplax }} \rightarrow \infty$-Cat oplax $_{\text {dont le }}$ foncteur sous-jacent est le foncteur $\underline{\operatorname{Hom}}_{\text {oplax }}(C, \bullet): \infty$-C at $\rightarrow \infty$-Cat.

(d) Il existe un $\infty$-foncteur de Gray gauche canonique ${ }^{t} \infty$-Cat $\mathbb{C}_{\text {oplax }} \rightarrow \infty$-Cat ${ }_{\text {lax }}$ dont le foncteur sous-jacent est le foncteur $\underline{\operatorname{Hom}}_{\text {oplax }}(\bullet, C): \infty-\mathcal{C} a t^{\circ} \rightarrow \infty-\mathcal{C}$ at.

(e) Il existe un $\infty$-foncteur de Gray gauche canonique $\infty$ - $\mathbb{C a t}_{\text {lax }} \rightarrow \infty$ - $\mathbb{C a t}_{\text {lax }}$ dont le foncteur sous-jacent est le foncteur $\underline{\operatorname{Hom}}_{\text {lax }}(C, \bullet): \infty$-C at $\rightarrow \infty$-Cat.

(f) Il existe un $\infty$-foncteur de Gray canonique ${ }^{t} \infty$-Cat ${ }_{\text {lax }} \rightarrow \infty$-Cat ${ }_{\text {oplax }}$ dont le

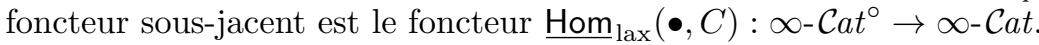

Conjecture C.24. - (a) Soient $\mathbb{C}$ une $\infty$-catégorie de Gray de $\infty$-sesquicatégorie sous-jacente $\mathcal{C}$ et $c$ un objet de $\mathbb{C}$.

(a.1) Il existe une $\infty$-catégorie de Gray canonique $\mathbb{C} / c$ dont les objets sont les couples $(x, g)$ formés d'un objet $x$ de $\mathbb{C}$ et d'une 1 -flèche $g: x \rightarrow c$ de $\mathbb{C}$, et telle que si $(x, g)$ et $\left(x^{\prime}, g^{\prime}\right)$ sont deux tels couples, on ait

$$
\underline{\operatorname{Hom}}_{\mathbb{C} / c}\left((x, g),\left(x^{\prime}, g^{\prime}\right)\right)=\underline{\operatorname{Hom}}_{\mathcal{C} / c}\left((x, g),\left(x^{\prime}, g^{\prime}\right)\right)
$$

(voir le paragraphe C.4)

(a.2) Il existe une $\infty$-catégorie de Gray canonique $c \backslash \mathbb{C}$ dont les objets sont les couples $(x, f)$ formés d'un objet $x$ de $\mathbb{C}$ et d'une 1-flèche $f: c \rightarrow x$ de $\mathbb{C}$, et telle que si $(x, f)$ et $\left(x^{\prime}, f^{\prime}\right)$ sont deux tels couples, on ait

$$
\underline{\text { Hom }}_{c \backslash \mathbb{C}}\left((x, f),\left(x^{\prime}, f^{\prime}\right)\right)=\underline{\text { Hom }}_{c \backslash \mathcal{C}}\left((x, f),\left(x^{\prime}, f^{\prime}\right)\right)
$$


(voir le paragraphe C.4).

(b) Soient $\mathbb{C}$ une $\infty$-catégorie de Gray gauche de $\infty$-sesquicatégorie sous-jacente $\mathcal{C}$ et $c$ un objet de $\mathbb{C}$.

(b.1) Il existe une $\infty$-catégorie de Gray gauche canonique $\mathbb{C} / c$ dont les objets sont les couples $(x, g)$ formés d'un objet $x$ de $\mathbb{C}$ et d'une 1 -flèche $g: x \rightarrow c$ de $\mathbb{C}$, et telle que si $(x, g)$ et $\left(x^{\prime}, g^{\prime}\right)$ sont deux tels couples, on ait

$$
\underline{\operatorname{Hom}}_{\mathbb{C} / c}^{\text {co }}\left((x, g),\left(x^{\prime}, g^{\prime}\right)\right)=\underline{\operatorname{Hom}}_{\mathcal{C} / c}^{\text {co }}\left((x, g),\left(x^{\prime}, g^{\prime}\right)\right)
$$

(voir le paragraphe C.4).

(b.2) Il existe une $\infty$-catégorie de Gray gauche canonique $c \backslash \mathbb{C}$ dont les objets sont les couples $(x, f)$ formés d'un objet $x$ de $\mathbb{C}$ et d'une 1 -flèche $f: c \rightarrow x$ de $\mathbb{C}$, et telle que si $(x, f)$ et $\left(x^{\prime}, f^{\prime}\right)$ sont deux tels couples, on ait

$$
\underline{\mathrm{Hom}}_{c \backslash \mathbb{C}}\left((x, f),\left(x^{\prime}, f^{\prime}\right)\right)=\underline{\operatorname{Hom}}_{c \backslash} \mathcal{C}\left((x, f),\left(x^{\prime}, f^{\prime}\right)\right)
$$

(voir le paragraphe C.4)

(c) $S i \mathbb{C}$ est une $\infty$-catégorie de Gray et c un objet de $\mathbb{C}$, on a des isomorphismes canoniques de $\infty$-catégories de Gray

$$
\begin{aligned}
& \mathbb{C} / c \simeq\left(\left.c\right|^{\mathrm{co}} \mathbb{C}^{\mathrm{op}}\right)^{\mathrm{op}} \simeq\left(\mathbb{C}^{\mathrm{co}} / c\right)^{\mathrm{co}} \simeq\left(c \mathbb{C}^{\mathrm{o}}\right)^{\circ}, \\
& c \backslash \mathrm{Co} \simeq\left(\mathbb{C}^{\mathrm{op}} / c\right)^{\mathrm{op}} \simeq\left(c \backslash \mathbb{C}^{\mathrm{co}}\right)^{\mathrm{co}} \simeq\left(\mathbb{C}^{\mathrm{o}} / c\right)^{\mathrm{co}}
\end{aligned}
$$

et, si $\mathbb{C}$ est une $\infty$-catégorie de Gray gauche et $c$ un objet de $\mathbb{C}$, on a des isomorphismes canoniques de $\infty$-catégories de Gray gauches

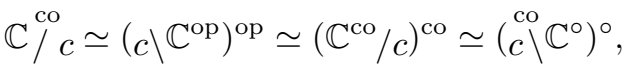

$$
\begin{aligned}
& c \backslash \mathbb{C} \simeq\left(\mathbb{C}^{\mathrm{op}} / \mathrm{co}\right)^{\mathrm{op}} \simeq\left(\mathrm{c} \mathrm{c}^{\mathrm{co}} \mathbb{C}^{\mathrm{co}}\right)^{\mathrm{co}} \simeq\left(\mathbb{C}^{\circ} / c\right)^{\circ},
\end{aligned}
$$

tous ces isomorphismes étant compatibles entre eux via les dualités $\bullet$ op, •co et $\bullet$.

Remarque C.25. - On remarque que pour démontrer cette conjecture, il suffit de démontrer une seule des assertions $(a .1),(a .2),(b .1)$ ou (b.2). En effet, il suffit alors de définir les autres «tranches» par les formules pertinentes de l'assertion $(c)$.

Exemple C.26. - On va s'intéresser plus spécialement au cas particulier de la

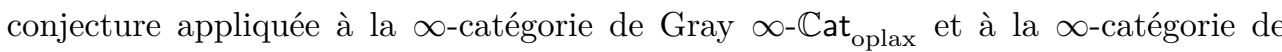
Gray gauche $\infty$ - $\mathbb{C a t}_{\text {lax }}$. Ainsi, conjecturalement, pour toute $\infty$-catégorie $C$, on dispose d'une $\infty$-catégorie de Gray $\infty$-Cat ${ }_{\text {oplax }} / C$ des $\infty$-catégories au-dessus de $C$ et d'une $\infty$-catégorie de Gray $C \backslash \infty$-Cat oplax $_{\text {des }} \infty$-catégories au-dessous de $C$. De même, on dispose d'une $\infty$-catégorie de Gray gauche $\infty$-Cat ${ }_{\text {lax }} / C$ des $\infty$-catégories au-dessus 
de $C$ et d'une $\infty$-catégorie de Gray gauche $C \backslash \infty$-Cat ${ }_{\text {lax }}$ des $\infty$-catégories au-dessous de $C$. On note

$$
\infty-\mathcal{C}_{\text {oplax }} / C, \quad C \backslash \infty-\mathcal{C} a t_{\text {oplax }}, \quad \infty-\mathcal{C} a t_{\text {lax }} / C \text { et } \quad C \backslash \infty-\mathcal{C} a t_{\text {lax }}
$$

les catégories sous-jacentes à

$$
\infty-\mathbb{C a t}_{\text {oplax }} / C, \quad \stackrel{C}{\text { co }} \infty-\mathbb{C a t}_{\text {oplax }}, \quad \infty-\mathbb{C a t}_{\text {lax }} / C \text { et } \quad C \backslash \infty-\mathbb{C a t}_{\text {lax }}
$$

respectivement. Les objets de $\infty$ - $\mathcal{C} a t_{\text {oplax }} / C$ et de $\infty$ - $\mathcal{C} a t_{\text {lax }} / C$ sont les mêmes que ceux de $\infty$-Cat $/ C$. De même, les objets de $C \backslash \infty$ - $\mathcal{C} a t_{\text {oplax }}$ et de $C \backslash \infty$ - $\mathcal{C} a t_{\text {lax }}$ sont les mêmes que ceux de $C \backslash \infty$ - $\mathcal{C} a t$. Dans le tableau suivant, on indique les 2-diagrammes définissant les morphismes d'un objet vers un objet « prime » dans ces quatre catégories :

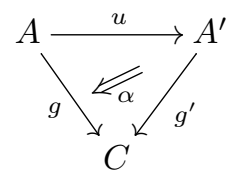

$$
\infty-\mathcal{C a t}_{\text {oplax }} / C
$$

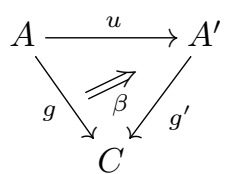

$\infty-\operatorname{Cat}_{\operatorname{lax}} \stackrel{\text { co }}{/} C$

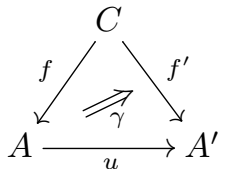

$C \backslash \infty-\mathcal{C a t}_{\text {oplax }}$

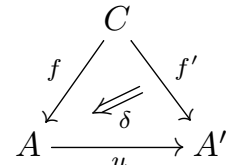

$C \backslash \infty-\mathcal{C a t}_{\text {lax }}$

$\alpha$ et $\gamma$ étant des transformations oplax et $\beta$ et $\delta$ des transformations lax. On vérifie aussitôt qu'on a des foncteurs d'inclusion canoniques

$$
\begin{aligned}
& \infty-\mathcal{C} a t / C \rightarrow \infty-\mathcal{C}_{\text {oplax }} / C, \quad \infty-\mathcal{C} a t / C \rightarrow \infty-{\mathcal{C} a t_{\text {lax }}}^{\text {co }} C, \\
& C \backslash \infty-\mathcal{C} a t \rightarrow C \backslash \infty-\mathcal{C} a t_{\text {oplax }}, \quad C \backslash \infty-\mathcal{C} a t \rightarrow C \backslash \infty-\mathcal{C} a t_{\text {lax }}
\end{aligned}
$$

(en admettant que les morphismes dans les catégories buts se composent de la manière évidente) induisant l'identité sur les objets et envoyant les triangles commutatifs sur les 2-triangles de même contour munis de la transformation oplax ou lax unité.

Conjecture C.27. - Soit $C$ une $\infty$-catégorie.

(a) Il existe un $\infty$-foncteur de Gray canonique $\infty$-Cat ${ }_{\text {oplax }} \rightarrow C \backslash \infty$-Cat ${ }_{\text {oplax }}$ dont le foncteur sous-jacent est le composé

$$
\infty-\mathcal{C} a t \rightarrow C \backslash \infty-\mathcal{C} a t \rightarrow C \backslash \infty-\mathcal{C} a t_{\text {oplax }}
$$

où la flèche de gauche est le foncteur

$$
\bullet \star C: A \mapsto\left(A \star C, i_{2}: C \rightarrow A \star C\right)
$$

et la flèche de droite le foncteur d'inclusion canonique.

(b) Il existe un $\infty$-foncteur de Gray gauche canonique $\infty-\mathbb{C a t}_{\text {lax }} \rightarrow C \backslash \infty$-Cat lax dont le foncteur sous-jacent est le composé

$$
\infty-\mathcal{C} a t \rightarrow C \backslash \infty-\mathcal{C} a t \rightarrow C \backslash \infty-\mathcal{C} a t_{\mathrm{lax}},
$$


où la flèche de gauche est le foncteur

$$
C \star \bullet: A \mapsto\left(C \star A, i_{1}: C \rightarrow C \star A\right)
$$

et la flèche de droite le foncteur d'inclusion canonique.

(c) Il existe un $\infty$-foncteur de Gray canonique $C \backslash \infty$-Cat ${ }_{\text {oplax }} \rightarrow \infty$-Cat ${ }_{\text {oplax }}$ dont le foncteur sous-jacent composé avec l'inclusion $C \backslash \infty-\mathcal{C}$ at $\rightarrow C \backslash \infty-\mathcal{C}$ at $t_{\text {oplax }}$ est le foncteur

$$
\begin{aligned}
C \backslash \infty-\mathcal{C} a t & \rightarrow \infty-\mathcal{C} a t \\
(A, f: C \rightarrow A) & \mapsto A / f .
\end{aligned}
$$

(d) Il existe un $\infty$-foncteur de Gray gauche canonique $\left(\infty-\mathbb{C a t}_{\mathrm{oplax}} / C\right)^{\circ} \rightarrow \infty$-Cat ${ }_{\text {lax }}$ dont le foncteur sous-jacent composé avec l'inclusion

$$
(\infty-\mathcal{C} a t / C)^{\circ} \rightarrow\left(\infty-\mathcal{C} a t_{\text {oplax }} / C\right)^{\circ}
$$

est le foncteur

$$
\begin{aligned}
(\infty-\mathcal{C} a t / C)^{\circ} & \rightarrow \infty-\mathcal{C} a t \\
(A, f: A \rightarrow C) & \mapsto C / f .
\end{aligned}
$$

(e) Il existe un $\infty$-foncteur de Gray gauche canonique $C \backslash \infty$ - $\mathbb{C a t}_{\text {lax }} \rightarrow \infty$-Cat lax $_{\text {ax }}$ dont le foncteur sous-jacent composé avec l'inclusion $C \backslash \infty$-Cat $\rightarrow C \backslash \infty$-Cat lax est le foncteur

$$
\begin{aligned}
C \backslash \infty-\mathcal{C} a t & \rightarrow \infty-\mathcal{C} a t \\
(A, f: C \rightarrow A) & \mapsto f \backslash A .
\end{aligned}
$$

(f) Il existe un $\infty$-foncteur de Gray canonique $\left(\infty-\mathbb{C a t}_{\mathrm{lax}}{ }^{\mathrm{co}} C\right)^{\circ} \rightarrow \infty$-Cat ${ }_{\text {oplax }}$ dont le foncteur sous-jacent composé avec l'inclusion $(\infty-\mathcal{C} a t / C)^{\circ} \rightarrow\left(\infty-\mathcal{C} a t_{\operatorname{lax}}{ }^{c o} C\right)^{\circ}$ est le foncteur

$$
\begin{aligned}
(\infty-\mathcal{C a t} / C)^{\circ} & \rightarrow \infty-\mathcal{C} a t \\
(A, f: A \rightarrow C) & \mapsto f \backslash C
\end{aligned}
$$

Remarque C.28. - Les assertions $(a)-(f)$ de la conjecture ci-dessus sont les analogues pour le joint des assertions formelles $(a)-(f)$ de l'exemple C.23 relatives au produit tensoriel de Gray. Par ailleurs, en utilisant les isomorphismes de la remarque C.22 ainsi que ceux de l'assertion $(c)$ de la conjecture C.24, on remarque que pour prouver l'assertion $(b)$ (resp. $(e)$, resp. $(f))$ de la conjecture C.27, il suffit de démontrer l'assertion $(a)$ (resp. $(c)$, resp. $(d)$ ) et réciproquement. De même, la conjecture C.27 est équivalente à la conjecture analogue pour le foncteur $\star^{\prime}$ et les « tranches $\gg C / f$

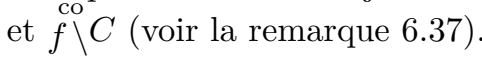


Remarque C.29. - L'assertion $(f)$ de la conjecture C.27 est une vaste généralisation des résultats du chapitre 11. Par exemple, si

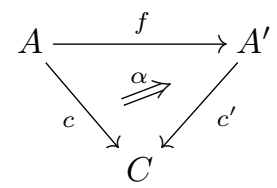

est un diagramme dans $\infty$ - $\mathcal{C} a t$, avec $\alpha$ une transformation lax, autrement dit un diagramme représentant une 1-flèche $(f, \alpha):(A, c) \rightarrow\left(A^{\prime}, c^{\prime}\right)$ de $\infty$ - $\mathbb{C a t}_{\text {lax }} / C$ (qui est une flèche de sa catégorie sous-jacente $\infty$-C $a t_{\text {lax }} / C$ ), en appliquant le foncteur $\left(\underset{\mathrm{co}}{\infty}-\mathcal{C} a t_{\mathrm{lax}} / C\right)^{\circ} \rightarrow \infty$-Cat sous-jacent au $\infty$-foncteur de Gray canonique $\left(\infty \text { - } \text { Cat }_{\text {lax }} / C\right)^{\circ} \rightarrow \infty$ - Cat $_{\text {oplax }}$, on obtient un $\infty$-foncteur $(f, \alpha)^{*}: c^{\prime} \backslash C \rightarrow c \backslash C$, généralisant celui du théorème 11.2.2. Par fonctorialité, on obtient une généralisation des résultats de la section 11.3. De même, si

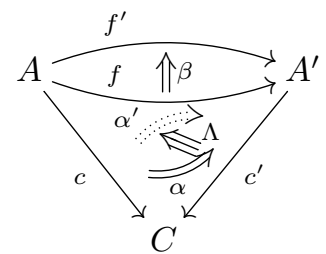

est un diagramme dans $\infty$-C at, avec $\alpha$, $\alpha^{\prime}$ et $\beta$ des transformations lax et $\Lambda$ une 2-transformation lax, autrement dit un diagramme représentant une 2-flèche $(\beta, \Lambda):(f, \alpha) \rightarrow\left(f^{\prime}, \alpha_{\text {co }}^{\prime}\right)$ de $\infty$ - $\mathbb{C a t}_{\text {lax }} / C$, en appliquant le $\infty$-foncteur de Gray canonique $\left(\infty \text {-Cat }_{\text {lax }} / C\right)^{\circ} \rightarrow \infty$ - Cat $_{\text {oplax }}$, on obtient une 2 -flèche de $\infty$ - $\mathbb{C a t}_{\text {oplax }}$, autrement dit une 2-transformation oplax de source $\left(f^{\prime}, h^{\prime}\right)^{*}$ et but $(f, h)^{*}$, généralisant ainsi le théorème 11.4.2. Enfin, une description plus précise de la $\infty$-sesquicatégorie sous-jacente à $\infty-\mathbb{C a t}_{\mathrm{lax}} / C$ fournirait une généralisation des résultats de fonctorialité de la section 11.5.

C.30. - Les conjectures C.24 et C.27 sont étayées par le fait que des conjectures analogues sont vraies pour les complexes dirigés augmentés. Plus précisément, on rappelle que la catégorie monoïdale $\mathcal{C}_{\mathrm{da}}$ des complexes dirigés augmentés, munie du produit tensoriel, est bifermée (voir le paragraphe 6.2), de sorte qu'il existe des foncteurs $\underline{\operatorname{Hom}}_{\mathcal{C}_{\mathrm{da}}}^{\mathrm{d}}, \underline{\text { Hom }}_{\mathcal{C}_{\mathrm{da}}}^{\mathrm{g}}: \mathcal{C}_{\mathrm{da}}^{\circ} \times \mathcal{C}_{\mathrm{da}} \rightarrow \mathcal{C}_{\mathrm{da}}$ et des bijections

$$
\operatorname{Hom}_{\mathcal{C}_{\mathrm{da}}}\left(L, \underline{\operatorname{Hom}}_{\mathcal{C}_{\mathrm{da}}}^{\mathrm{g}}(K, M)\right) \simeq \operatorname{Hom}_{\mathcal{C}_{\mathrm{da}}}(K \otimes L, M) \simeq \operatorname{Hom}_{\mathcal{C}_{\mathrm{da}}}\left(K, \underline{\operatorname{Hom}}_{\mathcal{C}_{\mathrm{da}}^{\mathrm{d}}}(L, M)\right),
$$

naturelles en les complexes dirigés augmentés $K, L, M$. En vertu de l'exemple C.10, on dispose donc d'une $\mathcal{C}_{\mathrm{da}}$-catégorie ayant mêmes objets que $\mathcal{C}_{\mathrm{da}}$ et dont l'objet de morphismes est défini par $\underline{\operatorname{Hom}}_{\mathcal{C}_{\mathrm{da}}}^{\mathrm{d}}$, et d'une $\mathcal{C}_{\mathrm{da}}$-catégorie gauche ayant également les mêmes objets que $\mathcal{C}_{\mathrm{da}}$ et dont l'objet de morphismes est défini par $\underline{\text { Hom }}_{\mathcal{C}_{\mathrm{da}}^{\mathrm{g}}}$. Comme 
le foncteur $\nu: \mathcal{C}_{\mathrm{da}} \rightarrow \infty$-C at s'enrichit en un foncteur monoïdal lax (voir la proposition A.19), on peut appliquer la construction de l'image directe du paragraphe C.7 pour obtenir une $\infty$-catégorie de Gray $\mathbb{C}_{\mathrm{d} a}^{\mathrm{d}}$ (resp. une $\infty$-catégorie de Gray gauche $\mathbb{C}_{\mathrm{d} a}^{\mathrm{g}}$ ) ayant comme objets les complexes dirigés augmentés et telle que pour tous objets $K, L$,

$$
\underline{\operatorname{Hom}}_{\mathbb{C}_{\mathrm{d} a}^{\mathrm{d}}}(K, L)=\nu\left(\underline{\operatorname{Hom}}_{\mathcal{C}_{\mathrm{da}}^{\mathrm{d}}}^{\mathrm{d}}(K, L)\right) \quad\left(\text { resp. } \quad \underline{\operatorname{Hom}}_{\mathbb{C}_{\mathrm{d} a}^{\mathrm{g}}}(K, L)=\nu\left(\underline{\operatorname{Hom}}_{\mathcal{C}_{\mathrm{da}}^{\mathrm{g}}}(K, L)\right)\right) .
$$

On remarque que, essentiellement par définition, pour $i \geq 0$, les $i$-flèches de $\underline{\text { Hom }}_{\mathbb{C}_{\mathrm{da}}^{\mathrm{d}}}(K, L)$ (resp. de $\underline{\text { Hom }}_{\mathbb{C}_{\mathrm{d} a}^{\mathrm{g}}}(K, L)$ ) sont les $i$-homotopies (resp. les $i$-antihomotopies) de complexes dirigés augmentés de $K$ vers $L$ (voir le paragraphe 2.28). En particulier, les objets de $\underline{\operatorname{Hom}}_{\mathbb{C}_{\mathrm{d} a}^{\mathrm{d}}}(K, L)$ ou de $\underline{\operatorname{Hom}}_{\mathbb{C}_{\mathrm{d} a}^{\mathrm{g}}}(K, L)$ sont les morphismes de $K$ vers $L$. De plus, il résulte facilement des théorèmes 2.11 et A.15 ainsi que des propriétés d'adjonction que si $K$ est un complexe de Steiner fort, on a des isomorphismes canoniques

$$
\underline{\operatorname{Hom}}_{\mathbb{C}_{\mathrm{d} a}^{\mathrm{d}}}(K, L) \simeq \underline{\operatorname{Hom}}_{\mathrm{oplax}}(\nu(K), \nu(L)) \text { et } \underline{\operatorname{Hom}}_{\mathbb{C}_{\mathrm{d} a}^{\mathrm{g}}}(K, L) \simeq \underline{\operatorname{Hom}}_{\operatorname{lax}}(\nu(K), \nu(L)) \text {. }
$$

En particulier, la sous- $\infty$-catégorie de Gray pleine de $\mathbb{C}_{\mathrm{d} a}^{\mathrm{d}}$ définie par les complexes de Steiner forts s'identifie à la sous- $\infty$-catégorie de Gray pleine de $\infty$-Cat ${ }_{\text {oplax }}$ définie par les $\infty$-catégories de Steiner fortes (voir le paragraphe 2.15), et de même pour les sous- $\infty$-catégories de Gray gauches pleines de $\mathbb{C}_{\mathrm{d} a}^{\mathrm{g}}$ et $\infty$-Cat ${ }_{\text {lax }}$ correspondantes.

Par ailleurs, pour tout complexe dirigé augmenté $L$ et tout couple de morphismes $g: K \rightarrow L, g: K^{\prime} \rightarrow L$ (resp. $f: L \rightarrow K, f^{\prime}: L \rightarrow K^{\prime}$ ) de $\mathcal{C}_{\text {da }}$ de but $L$ (resp. de source $L)$, on sait définir des complexes dirigés augmentés explicites

$$
\begin{aligned}
& \underline{\operatorname{Hom}}_{L}^{\mathrm{d}}\left((K, g),\left(K^{\prime}, g^{\prime}\right)\right) \text { et } \underline{\operatorname{Hom}}_{L}^{\mathrm{g}}\left((K, g),\left(K^{\prime}, g^{\prime}\right)\right) \\
\text { (resp. } & \underline{\operatorname{Hom}}_{\mathrm{d}}^{L}\left((K, f),\left(K^{\prime}, f^{\prime}\right)\right) \text { et } \underline{\left.\underline{\operatorname{Hom}}_{\mathrm{g}}^{L}\left((K, f),\left(K^{\prime}, f^{\prime}\right)\right)\right),}
\end{aligned}
$$

pouvant s'assembler pour former $\operatorname{deux} \mathcal{C}_{\mathrm{da}}$-catégories dont les objets sont les complexes dirigés augmentés respectivement au-dessus et au-dessous de $L$ et dont les objets de morphismes sont définis par $\underline{\operatorname{Hom}}_{L}^{\mathrm{d}}\left((K, g),\left(K^{\prime}, g^{\prime}\right)\right)$ et $\underline{\operatorname{Hom}}_{\mathrm{d}}^{L}\left((K, f),\left(K^{\prime}, f^{\prime}\right)\right)$, et deux $\mathcal{C}_{\mathrm{da}}$-catégories gauches dont les objets sont aussi respectivement les complexes dirigés augmentés au-dessus et au-dessous de $L$ et dont les objets de morphismes sont définis par $\underline{\operatorname{Hom}}_{L}^{\mathrm{g}}\left((K, g),\left(K^{\prime}, g^{\prime}\right)\right)$ et $\underline{\operatorname{Hom}}_{\mathrm{g}}^{L}\left((K, f),\left(K^{\prime}, f^{\prime}\right)\right)$. En appliquant la construction de l'image directe du paragraphe C.7 par le foncteur monoïdal lax $\nu$, on obtient deux $\infty$-catégories de Gray notées $\mathbb{C}_{\mathrm{d} a}^{\mathrm{d}} / L$ et $L \backslash \mathbb{C}_{\mathrm{d} a}^{\mathrm{d}}$ et deux $\infty$-catégories de Gray gauches notées $\mathbb{C}_{\mathrm{d} a}^{\mathrm{g}} / L$ et $L \backslash \mathbb{C}_{\mathrm{d} a}^{\mathrm{g}}$. On peut démontrer que celles-ci satisfont les affirmations de la conjecture C.24 relatives à la $\infty$-catégorie de Gray $\mathbb{C}_{\mathrm{d} a}^{\mathrm{d}}$ et à la $\infty$-catégorie de Gray gauche $\mathbb{C}_{\mathrm{d} a}^{\mathrm{g}}$. En particulier, cela implique le cas de la conjecture C.24 exposé dans l'exemple C.26 pour la sous- $\infty$-catégorie de Gray pleine de $\infty$-Cat oplax $_{\text {et la }}$ sous- $\infty$-catégorie de Gray gauche pleine de $\infty$-Cat ${ }_{\text {lax }}$ définies par les $\infty$-catégories de Steiner fortes. 
De plus, si le complexe dirigé augmenté $L$ est décent (voir le paragraphe 2.17), on peut définir explicitement les morphismes de complexes dirigés augmentés pertinents pour construire les $\infty$-foncteurs de Gray et $\infty$-foncteurs de Gray gauches correspondant à l'analogue de la conjecture C.27 pour les complexes dirigés augmentés. Ceci permet comme ci-dessus d'établir la conjecture C.27 si on se restreint aux $\infty$-catégories de Steiner fortes.

Ces résultats seront exposés plus en détails ailleurs. On espère pouvoir en déduire, par un argument de densité et une version « enrichie » de la généralisation du théorème de Day démontrée dans ce texte (théorème 5.13), la conjecture C.27 en toute généralité. 


\section{RÉFÉRENCES}

[1] F. A. Al-Agl \& R. Steiner - « Nerves of multiple categories », Proc. London Math. Soc. (3) 66 (1993), no. 1, p. 92-128.

[2] D. ArA - « Structures de catégorie de modèles à la Thomason sur la catégorie des 2-catégories strictes », Cah. Topol. Géom. Différ. Catég. 56 (2015), no. 2, p. $83-108$.

[3] _ _ A Quillen Theorem B for strict $\infty$-categories », J. Lond. Math. Soc. (2) $\mathbf{1 0 0}$ (2019), no. 2, p. 470-497.

[4] D. Ara, Y. Lafont \& F. MÉtayer - « Orientals as free algebras », en préparation.

[5] D. Ara \& G. Maltsiniotis - « Vers une structure de catégorie de modèles à la Thomason sur la catégorie des n-catégories strictes », Adv. Math. 259 (2014), p. $557-654$.

[6] _ _ « Le type d'homotopie de la $\infty$-catégorie associée à un complexe simplicial », Prépublication, arXiv:1503.02720v1, 2015.

[7] _ , «Un théorème A de Quillen pour les $\infty$-catégories strictes I : la preuve simpliciale », Adv. Math. 328 (2018), p. 446-500.

[8] _ _ « Un théorème A de Quillen pour les $\infty$-catégories strictes II : la preuve $\infty$-catégorique », High. Struct. 4 (2020), no. 1, p. 284-388.

[9] _ , « Comparaison des nerfs n-catégoriques », en préparation.

[10] C. Berger - «A cellular nerve for higher categories », Adv. Math. 169 (2002), no. 1 , p. $118-175$. 
[11] _ « Iterated wreath product of the simplex category and iterated loop spaces », Adv. Math. 213 (2007), no. 1, p. 230-270.

[12] R. Brown \& P. J. Higgins - « Tensor products and homotopies for $\omega$-groupoids and crossed complexes », J. Pure Appl. Algebra 47 (1987), no. 1, p. 1-33.

[13] M. Bullejos \& A. M. Cegarra - « On the geometry of 2-categories and their classifying spaces », K-Theory 29 (2003), no. 3, p. 211-229.

[14] A. M. Cegarra - « Homotopy fiber sequences induced by 2-functors », J. Pure Appl. Algebra 215 (2011), no. 4, p. 310-334.

[15] J. Chiche - « La théorie de l'homotopie des 2-catégories », Thèse, Université Paris Diderot - Paris 7, 2014, sous la direction de G. Maltsiniotis.

[16] _ « Un théorème A de Quillen pour les 2-foncteurs lax », Theory Appl. Categ. 30 (2015), p. 49-85.

[17] D.-C. Cisinski \& G. Maltsiniotis - « La catégorie $\Theta$ de Joyal est une catégorie test », J. Pure Appl. Algebra 215 (2011), no. 5, p. 962-982.

[18] S. CRANS - « On combinatorial models for higher dimensional homotopies », Thèse, Université d'Utrecht, 1995, sous la direction de I. Moerdijk et D. van Dalen.

[19] B. DAY - « On closed categories of functors », in Reports of the Midwest Category Seminar, IV, Lecture Notes in Mathematics, vol. 137, Springer, 1970, p. 1-38.

[20] _ « A reflection theorem for closed categories », J. Pure Appl. Algebra 2 (1972), no. 1, p. 1-11.

[21] A. GaGna - « Strict $n$-categories and augmented directed complexes model homotopy types », Adv. Math. 331 (2018), p. 542-564.

[22] J. W. GRAY - Formal category theory: adjointness for 2-categories, Lecture Notes in Mathematics, vol. 391, Springer-Verlag, 1974.

[23] A. Joyal - « Disks, duality and $\Theta$-categories », Prépublication, 1997.

[24] _ « Quasi-categories and Kan complexes », J. Pure Appl. Algebra 175 (2002), no. 1-3, p. 207-222, Special volume celebrating the 70th birthday of Professor Max Kelly.

[25] _ « The theory of quasi-categories and its applications », Prépublication, 2008. 
[26] G. M. KELLY - Basic concepts of enriched category theory, London Mathematical Society Lecture Note Series, vol. 64, Cambridge University Press, 1982.

[27] Y. LAFont \& F. MÉTAYER - « Polygraphic resolutions and homology of monoids », J. Pure Appl. Algebra 213 (2009), no. 6, p. 947-968.

[28] S. MAC LANE - Categories for the working mathematician, seconde éd., Graduate Texts in Mathematics, vol. 5, Springer-Verlag, 1998.

[29] M. Makkai \& M. Zawadowski - « Duality for simple $\omega$-categories and disks », Theory Appl. Categ. 8 (2001), p. 114-243.

[30] F. MÉtayer - « Resolutions by polygraphs », Theory Appl. Categ. 11 (2003), no. 7, p. $148-184$.

[31] E. RIEHL - Category theory in context, Aurora: Dover Modern Math Originals, Dover Publications, 2017.

[32] R. Steiner - «Omega-categories and chain complexes », Homology Homotopy Appl. 6 (2004), no. 1, p. 175-200.

[33] _ « Orientals », in Categories in algebra, geometry and mathematical physics, Contemp. Math., vol. 431, Amer. Math. Soc., 2007, p. 427-439.

[34] _ « Simple omega-categories and chain complexes », Homology Homotopy Appl. 9 (2007), no. 1, p. 451-465.

[35] _ « "Tensor products of $\omega$-categories », Communication privée, 2014.

[36] R. Street - « The algebra of oriented simplexes », J. Pure Appl. Algebra 49 (1987), no. 3, p. 283-335.

[37] _ « Parity complexes », Cah. Topol. Géom. Différ. Catég. 32 (1991), no. 4, p. 315-343.

[38] _ « Parity complexes: corrigenda », Cah. Topol. Géom. Différ. Catég. 35 (1994), no. 4, p. 359-361.

[39] _ _ « Categorical structures », in Handbook of algebra, Handb. Algebr., vol. 1, Elsevier, 1996, p. 529-577.

[40] - « Categorical and combinatorial aspects of descent theory », Appl. Categ. Structures 12 (2004), no. 5-6, p. 537-576.

[41] D. Verity - «Complicial sets characterising the simplicial nerves of strict w-categories », Mem. Amer. Math. Soc. 193 (2008), no. 905, p. xvi+184. 
[42] _ « «eak complicial sets. I. Basic homotopy theory», Adv. Math. 219 (2008), no. 4, p. 1081-1149.

[43] M. WeBER - «Familial 2-functors and parametric right adjoints », Theory Appl. Categ. 18 (2007), p. 665-732. 


\section{INDEX DES NOTATIONS}

$\mathrm{Ob}(\mathcal{C})$, p. $11, \S$ Notations et terminologie $\mathcal{C}^{\circ}$, p. $11, \S$ Notations et terminologie $\underline{\operatorname{Hom}}(\mathcal{C}, \mathcal{D})$,

p. $11, \S$ Notations et terminologie $\mathcal{E} n s$, p. $11, \S$ Notations et terminologie $\mathcal{A} b$, p. $11, \S$ Notations et terminologie $\widehat{A}$, p. $11, \S$ Notations et terminologie $\infty$-Cat, p. $13, \S 1.1$

$C_{i}$, p. $13, \S 1.1$

$s(x), s_{j}(x)$, p. $13, \S 1.1$

$t(x), t_{j}(x)$, p. $13, \S 1.1$

$x *_{j} y$, p. $13, \S 1.1$

$1_{x}$, p. $13, \S 1.1$

$n$-Cat, p. $14, \S 1.2$

$\tau_{\leqslant n}^{\mathrm{i}}(C), \tau_{\leqslant n}^{\mathrm{b}}(C)$, p. $14, \S 1.2$

$C^{\circ}, C^{\text {op }}, C^{\text {co }}$, p. $17, \S 1.8$

$D_{J}(C)$, p. $17, \S 1.8$

$\alpha_{x}$, p. $17, \S 1.9$

$1_{u}$, p. $19, \S 1.15$

$\alpha * u, u * \alpha$, p. $19, \S 1.16$

$|x|$, p. $21, \S 2.1$

$e: K_{0} \rightarrow \mathbb{Z}$, p. $21, \S 2.2$

$K_{n}^{*}$, p. $21, \S 2.2$

$\mathcal{C}_{\text {da }}$, p. $21, \S 2.2$

$\lambda(C)$, p. $22, \S 2.3$

$[x]$, p. $22, \S 2.3$

$\nu(K)$, p. $22, \S 2.4$

$x_{i}^{\varepsilon}, x_{i}$, p. $22, \S 2.4$

$\operatorname{supp}(x)$, p. $24, \S 2.7$

$x_{+}, x_{-}$, p. $24, \S 2.7$ $\langle x\rangle,\langle x\rangle_{i}^{\varepsilon}$, p. $24, \S 2.8$

$\leqslant_{i}$, p. $25, \S 2.9$

$\leqslant_{\mathbb{N}}$, p. $25, \S 2.13$

$\mathcal{S} t_{\mathrm{f}}$, p. $26, \S 2.15$

$D_{J}(K)$, p. $27, \S 2.18$

$K^{\circ}, K^{\mathrm{op}}, K^{\mathrm{co}}$, p. $27, \S 2.18$

$n-\mathcal{C}_{\text {da }}$, p. $28, \S 2.20$

$\tau_{\leqslant n}^{\mathrm{i}}(K), \tau_{\leqslant n}^{\mathrm{b}}(K)$, p. $28, \S 2.20$

$1_{f}, 1_{h}$, p. $33, \S 2.30$

$H g, g H$, p. $33, \S 2.31$

$H^{\prime}+H$, p. $33, \S 2.32$

$H^{\prime}+H$, p. $34, \S 2.33$

$h^{\prime} h$, p. $34, \S 2.34$

$\mathrm{D}_{i}$, p. $43, \S 4.1$

$\mathrm{FI}_{i}(C)$, p. $43, \S 4.1$

$\sigma_{i}: \mathrm{D}_{i-1} \rightarrow \mathrm{D}_{i}, \sigma_{i}^{j}: \mathrm{D}_{j} \rightarrow \mathrm{D}_{i}$, p. $43, \S 4.1$

$\tau_{i}: \mathrm{D}_{i-1} \rightarrow \mathrm{D}_{i}, \tau_{i}^{j}: \mathrm{D}_{j} \rightarrow \mathrm{D}_{i}$, p. $43, \S 4.1$

$\mathrm{D}_{i_{1}} \amalg_{\mathrm{D}_{j_{1}}} \cdots \amalg_{\mathrm{D}_{j_{l-1}}} \mathrm{D}_{i_{l}}$, p. $44, \S 4.2$

$\kappa_{i}: \mathrm{D}_{i+1} \rightarrow \mathrm{D}_{i}, \kappa_{i}^{j}: \mathrm{D}_{i} \rightarrow \mathrm{D}_{j}$, p. $44, \S 4.3$

$\nabla_{j}^{i}: \mathrm{D}_{i} \rightarrow \mathrm{D}_{i} \amalg_{\mathrm{D}_{j}} \mathrm{D}_{i}$, p. $44, \S 4.3$

$\Theta, \Theta_{+}$, p. $44, \S 4.4$

$X\left(\mathrm{D}_{\bullet}\right), f_{\mathrm{D} \bullet}$, p. $45, \S 4.8$

$\widehat{\Theta}_{\text {glob }}$, p. $45, \S 4.8$

$X_{+}(\mathrm{D} \bullet), X_{+}\left(\mathrm{D}_{\bullet}\right)_{x}$, p. $46, \S 4.9$

$\left(\widehat{\Theta_{+}}\right)_{\text {glob }}$, p. $46, \S 4.9$

$x_{k}^{\varepsilon}, x_{i}$, p. $46, \S 4.10$

$F_{S}: I_{S} \rightarrow \infty$-Cat, p. $48, \S 4.12$

$G_{S}: I_{S} \rightarrow \mathcal{C}_{\text {da }}$, p. $48, \S 4.12$

$\mathcal{D} / X$, p. $49, \S 5.1$ 
${\underline{\mathrm{Hom}^{\mathrm{d}}}}_{\mathcal{C}}^{\mathrm{d}},{\underline{\mathrm{Hom}^{\mathrm{g}}}}_{\mathcal{C}}^{\mathrm{g}}: \mathcal{C}^{\circ} \times \mathcal{C} \rightarrow \mathcal{C}$, p. $50, \S 5.2$

$\iota_{1}: M(X) \rightarrow X \otimes Y, \iota_{2}: N(Y) \rightarrow X \otimes Y$, p. $52, \S 5.5$

$\widetilde{\mathrm{F}}(F)$, p. $54, \S 5.10$

$\widetilde{\mathrm{FI}}_{\mathrm{d}}(\mathcal{C}), \widetilde{\mathrm{FI}}_{\mathrm{g}}(\mathcal{C})$, p. $55, \S 5.11$

$K \otimes L$, p. $61, \S 6.1$

$K \otimes L$, p. $62, \S 6.2$

$\underline{\mathbb{Z}}^{\prime}$, p. $62, \S 6.2$

$\Sigma K, \Sigma^{-1}$, p. $62, \S 6.3$

$K \star L$, p. $63, \S 6.5$

$x \star y$, p. $63, \S 6.5$

$\Sigma K, \Sigma^{-1}$, p. $63, \S 6.6$

$\mathcal{C}_{\text {da }}^{\Sigma}$, p. $64, \S 6.6$

$K \star L$, p. $64, \S 6.7$

$\iota_{1}: K \rightarrow K \star L, \iota_{2}: L \rightarrow K \star L$, p. $66, \S 6.12$

$X \star Y$, p. $66, \S 6.13$

$u \backslash C$, p. $73, \S 6.26$

$C / v$, p. $77, \S 6.28$

$A \star B$, p. $78, \S 6.30$

$\iota_{1}: A \rightarrow A \star B, \iota_{2}: B \rightarrow A \star B$, p. $78, \S 6.30$

$u \backslash C, C / u$, p. $79, \S 6.31$

$A \star^{\prime} B$, p. $82, \S 6.37$

$u \backslash C, C / u$, p. $82, \S 6.37$

$\iota_{1}^{\prime}: A \rightarrow A \star^{\prime} B, \iota_{2}^{\prime}: B \rightarrow A \star^{\prime} B$,

p. $82, \S 6.37$

$u^{*}: c^{\prime} \backslash C \rightarrow c \backslash C$, p. $83, \S 6.38$

$\boldsymbol{\Delta}, \boldsymbol{\Delta}_{+}$, p. $85, \S 7.1$

$\Delta_{n}$, p. $85, \S 7.1$

$\mathcal{O}: \boldsymbol{\Delta} \rightarrow \infty-\mathcal{C} a t, \mathcal{O}_{+}: \boldsymbol{\Delta}_{+} \rightarrow \infty-\mathcal{C} a t$,

p. $86, \S 7.2$

$\mathcal{O}_{n}$, p. $86, \S 7.2$

$N_{\infty}: \infty$-Cat $\rightarrow \widehat{\boldsymbol{\Delta}}$, p. $86, \S 7.2$

$c\left(\Delta_{m}\right)$, p. $86, \S 7.3$

$X \star Y$, p. $89, \S 7.12$

$\Theta_{n},\left(\Theta_{n}\right)_{+}$, p. $91, \S 8.1$

$A \star_{n} B$, p. $92, \S 8.6$

$\iota_{1}: A \rightarrow A \star_{n} B, \iota_{2}: B \rightarrow A \star_{n} B$,

p. $93, \S 8.6$

$A \star_{1}^{\mathrm{c}} B$, p. $96, \S 8.15$

$\iota_{1}: A \rightarrow A \star_{1}^{\mathrm{c}} B, \iota_{2}: B \rightarrow A \star_{1}^{\mathrm{c}} B$,

p. $97, \S 8.15$

$g \backslash M$, p. $111, \S 10.1 .1$

$M \stackrel{\text { co }}{/}$, p. $116, \S 10.1 .5$

$(f, h)^{*}: g^{\prime} \backslash L \rightarrow g \backslash L$, p. $118, \S 10.2 .1$

$f^{*}: g^{\prime} \backslash L \rightarrow g \backslash L$, p. 119, § 10.2.3

$(k, H)^{*}:\left(f^{\prime}, h^{\prime}\right)^{*} \Rightarrow(f, h)^{*}$, p. $123, \S 10.4 .1$ $(f, h, b)^{*}: b \nu\left(g^{\prime}\right) \backslash C \rightarrow b \nu(g) \backslash C$, p. $138, \S 11.2 .1$

$(k, H, b)^{*}:\left(f^{\prime}, h^{\prime}, b\right)^{*} \Rightarrow(f, h, b)^{*}$, p. $144, \S 11.4 .1$

$X \otimes Y$, p. $153, \S$ A. 1

$\underline{\operatorname{Hom}}_{\operatorname{lax}}(\nu(K), C)$, p. $156, \S$ A.12

$\underline{\operatorname{Hom}}_{\text {oplax }}(\nu(K), C)$, p. $159, \S$ A. 14

$A \otimes B$, p. $160, \S$ A.16

$\underline{\mathrm{Hom}}_{\text {oplax }}(A, B), \underline{\mathrm{Hom}}_{\text {lax }}(A, B)$, p. $161, \S \mathrm{A} .18$

$A \otimes_{n} B$, p. $164, \S$ A. 25

$\pi^{0}, \pi^{1}: \underline{\operatorname{Hom}}_{\text {lax }}\left(\mathrm{D}_{1}, C\right) \rightarrow C$, p. $177, \S$ B. 2.2

$\alpha^{\prime} \circ \alpha$, p. $183, \S$ B.3.2

$\nu(h): \nu(f) \Rightarrow \nu(g)$, p. $186, \S$ B. 4.6

Hom $_{C}(c, d)$, p. 191, § B.6.1

$\Sigma C$, p. $192, \S \mathrm{B} .6 .5$

$\underline{\mathrm{Hom}}_{\mathcal{C}}: \mathcal{C}^{\circ} \times \mathcal{C} \rightarrow \mathcal{V}$, p. $195, \S$ C.1

$\alpha *_{k} \beta$, p. 196, § C.3

$1_{x}, 1_{\alpha}$, p. $196, \S$ C. 3

$\underline{\mathrm{Hom}}_{c \backslash \mathcal{C}}, \underline{\mathrm{Hom}}_{c \backslash}{ }_{c}, \underline{\mathrm{Hom}}_{\mathcal{C} / c}, \underline{\mathrm{Hom}}_{\mathcal{C} / c}^{\text {co }}$ p. $197, \S$ C. 4

$\mathrm{Ob}(\mathbb{C})$, p. $197, \S$ C.5

$\underline{\mathrm{Hom}}_{\mathbb{C}}(x, y)$, p. $198, \S$ C.5

$\mathrm{o}_{z, y, x}: \underline{\mathrm{Hom}}_{\mathbb{C}}(y, z) \otimes \underline{\mathrm{Hom}}_{\mathbb{C}}(x, y) \rightarrow \underline{\operatorname{Hom}}_{\mathbb{C}}(x, z)$, p. $198, \S$ C. 5

$\mathrm{id}_{x}: I \rightarrow \underline{\operatorname{Hom}}_{\mathbb{C}}(x, x)$, p. $198, \S$ C.5

$F_{*}(\mathbb{C})$, p. $199, \S$ C.7

$\mathbb{V}^{\mathrm{d}}, \mathbb{V}^{\mathrm{g}}$, p. $200, \S \mathrm{C} .10$

$\mathbb{F}_{0}: \mathrm{Ob}(\mathbb{C}) \rightarrow \mathrm{Ob}\left(\mathbb{C}^{\prime}\right)$, p. $201, \S \mathrm{C} .11$

$\mathbb{F}_{y, x}: \underline{\operatorname{Hom}}_{\mathbb{C}}(x, y) \rightarrow \underline{\operatorname{Hom}}_{\mathbb{C}^{\prime}}\left(\mathbb{F}_{0}(x), \mathbb{F}_{0}(y)\right)$, p. $201, \S$ C.11

${ }^{t} \mathbb{C}$, p. $202, \S$ C. 14

$\infty-\mathbb{C a t}_{\text {oplax }}, \infty-\mathbb{C a t}_{\text {lax }}$, p. $204, \S$ C.18

$\mathbb{C}^{\text {op }}, \mathbb{C}^{\mathrm{co}}, \mathbb{C}^{\circ}$, p. $204, \S \mathrm{C} .21$

$\mathbb{C} / c, c \backslash \mathbb{C}, \mathbb{C} / c, c \backslash \mathbb{C}$, p. $206, \S$ C. 24

$\infty$ - Cat $_{\text {oplax }} / C, C \backslash \infty$-Cat ${ }_{\text {oplax }}$,

$\infty-\mathbb{C a t}_{\text {lax }} / C, C \backslash \infty-\mathbb{C a t}_{\text {lax }}$,

p. $207, \S$ C.26

$\infty-\mathcal{C} a t_{\text {oplax }} / C, \stackrel{C}{C \backslash \infty} \infty-\mathcal{C} a t_{\text {oplax }}$,

$\infty-\mathcal{C} a t_{\operatorname{lax}} / C, C \backslash \infty-\mathcal{C} a t_{\text {lax }}$,

p. $208, \S$ C.26

$\underline{\operatorname{Hom}}_{\mathcal{C}_{\mathrm{da}}}^{\mathrm{d}}(K, L), \underline{\operatorname{Hom}}_{\mathcal{C}_{\mathrm{da}}}^{\mathrm{g}}(K, L)$, p. $210, \S$ C.30

$\mathbb{C}_{\mathrm{d} a}^{\mathrm{d}}, \mathbb{C}_{\mathrm{d} a}^{\mathrm{g}}$, p. $211, \S \mathrm{C} .30$

$\mathbb{C}_{\mathrm{d} a}^{\mathrm{d}} / L, L \backslash \stackrel{\text { co }}{\mathbb{C}_{\mathrm{d} a}^{\mathrm{d}},}, \mathbb{C}_{\mathrm{d} a}^{\mathrm{g}} / L, L \backslash \mathbb{C}_{\mathrm{d} a}^{\mathrm{g}}$, p. $211, \S$ C. 30 


\section{INDEX TERMINOLOGIQUE}

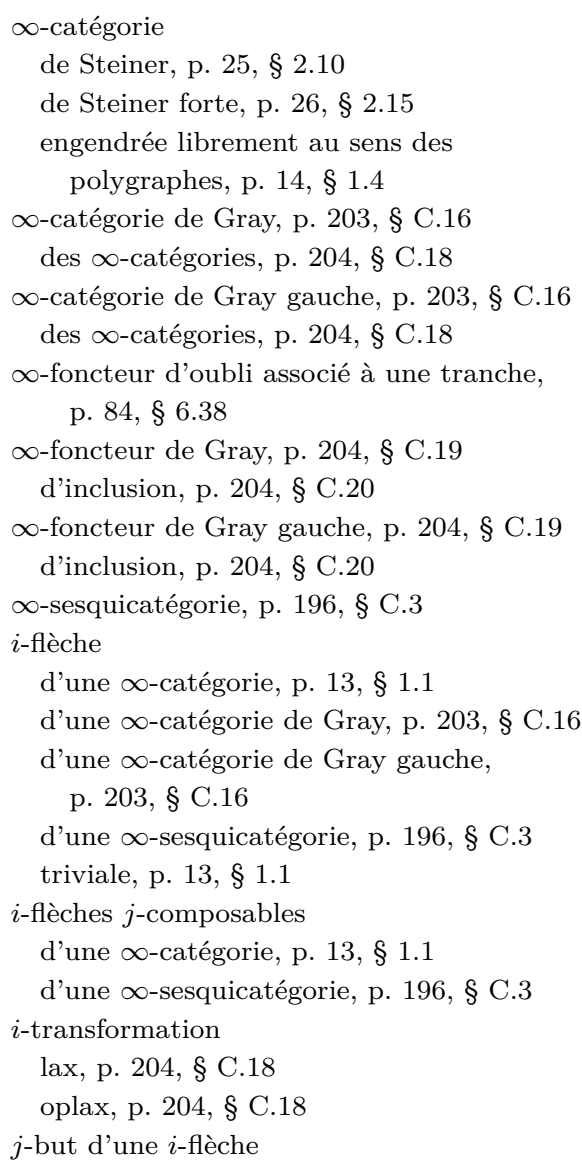

$\infty$-catégorie

engendrée librement au sens des

polygraphes, p. $14, \S 1.4$

des $\infty$-catégories, p. $204, \S$ C.18

-catégorie de Gray gauche, p. 203, § C.16

des $\infty$-catégories, p. 204, § C.18

p. $84, \S 6.38$

--foncteur de Gray, p. 204, § C.19

d'inclusion, p. 204, § C.20

d'inclusion, p. 204, § C.20

$\infty$-sesquicatégorie, p. 196, § C.3

$i$-flèche

d'une $\infty$-catégorie, p. $13, \S 1.1$

d'une $\infty$-catégorie de Gray, p. 203, § C.16

$\infty$-catégorie de Gray gauche,

triviale, p. $13, \S 1.1$

-flèches $j$-composables

d'une $\infty$-catégorie, p. $13, \S 1$.

-transformation

lax, p. 204, § C.18

$j$-but d'une $i$-flèche

d'une $\infty$-catégorie, p. $13, \S 1.1$

d'une $\infty$-sesquicatégorie, p. 196, § C.3

$j$-composé de $i$-flèches

d'une $\infty$-catégorie, p. $13, \S 1.1$

d'une $\infty$-sesquicatégorie, p. 196, § C.3

$j$-source d'une $i$-flèche

d'une $\infty$-catégorie, p. $13, \S 1.1$

d'une $\infty$-sesquicatégorie, p. 196, § C.3

$J$-dual

d'un complexe dirigé augmenté,

$$
\text { p. } 27, \S 2.18
$$

d'une $\infty$-catégorie, p. $17, \S 1.8$

$n$-antihomotopie, p. $32, \S 2.28$

$n$-homotopie, p. $32, \S 2.28$

$n$-homotopies parallèles, p. $32, \S 2.28$

$n$-tronqué

bête

d'un complexe dirigé augmenté, p. $28, \S 2.20$

d'une $\infty$-catégorie, p. $14, \S 1.2$

intelligent

d'un complexe dirigé augmenté, p. $28, \S 2.20$

d'une $\infty$-catégorie, p. $14, \S 1.2$

$\mathcal{V}$-catégorie, p. 197, § C.5

$\mathcal{V}$-catégorie gauche, p. 198, § C.5

$\mathcal{V}$-foncteur, p. 201, § C.11

d'inclusion, p. 202, § C.13

$\mathcal{V}$-foncteur gauche, p. 201, § C.11

d'inclusion, p. 202, § C.13 
$\mathcal{V}$-sesquicatégorie, p. 195, § C.1

sous-jacente

à une $\mathcal{V}$-catégorie, p. 199, § C.9

à une $\mathcal{V}$-catégorie gauche, p. $200, \S \mathrm{C} .9$

antihomotopie, p. $31, \S 2.26$

identité, p. 33, § 2.30

atome d'un complexe dirigé augmenté à base unitaire, p. $25, \S 2.8$

base d'un complexe dirigé augmenté, p. $23, \S 2.6$

fortement sans boucle, p. $25, \S 2.13$

sans boucle, p. $25, \S 2.9$

unitaire, p. $25, \S 2.8$

bicoaugmentation, p. $52, \S 5.5$

locale, p. $53, \S 5.6$

pseudo-locale, p. $53, \S 5.6$

triviale, p. $53, \S 5.6$

catégorie des simplexes, p. 85, §7.1

catégorie des simplexes augmentée, p. $85, \S 7.1$

catégorie enrichie, p. 197, § C.5

catégorie monoïdale

bifermée, p. 50, § 5.2

fermée à droite, p. $50, \S 5.2$

fermée à gauche, p. $50, \S 5.2$

localement bifermée, p. 53, § 5.7

localement fermée à droite, p. 53, § 5.7

localement fermée à gauche, p. 53, § 5.7

transposée, p. 198, § C.5

catégorie sous-jacente

à une $\mathcal{V}$-catégorie, p. 199, § C.8

à une $\mathcal{V}$-catégorie gauche, p. 199, § C.8

à une $\mathcal{V}$-sesquicatégorie, p. $195, \S$ C.1

cellule principale de $\mathrm{D}_{i}$, p. $43, \S 4.1$

coaugmentation

à droite, p. $52, \S 5.5$

à gauche, p. $52, \S 5.5$

complexe

de Steiner, p. $25, \S 2.10$

de Steiner fort, p. 26, § 2.15

complexe dirigé augmenté, p. 21, § 2.2

à base, p. $24, \S 2.6$

fortement sans boucle, p. $26, \S 2.13$

sans boucle, p. $25, \S 2.9$

unitaire, p. $25, \S 2.8$

décent, p. $26, \S 2.17$ degré d'un élément homogène d'un complexe de chaînes, p. $21, \S 2.1$

dimension d'un complexe dirigé augmenté, p. $28, \S 2.20$

dual

d'un complexe dirigé augmenté,

$$
\text { p. } 27, \S 2.18
$$

d'une $\infty$-catégorie, p. $17, \S 1.8$

dual impair

d'un complexe dirigé augmenté,

$$
\text { p. } 27, \S 2.18
$$

d'une $\infty$-catégorie, p. $17, \S 1.8$

dual pair

d'un complexe dirigé augmenté, p. $27, \S 2.18$

d'une $\infty$-catégorie, p. $17, \S 1.8$

ensemble multiplicatif de cellules, p. $14, \S 1.3$

ensemble simplicial, p. $85, \S 7.1$

envoyer les sommes globulaires sur des produits globulaires

pour un préfaisceau sur $\Theta$, p. $45, \S 4.8$

pour un préfaisceau sur $\Theta_{+}$, p. $45, \S 4.9$

foncteur enrichi, p. 201, § C.11

foncteur points, p. 195, § C.1

foncteur séparant, p. $38, \S 3.11$

homotopie

de complexes de chaînes, p. $31, \S 2.25$

de complexes dirigés augmentés,

$$
\text { p. } 31, \S 2.26
$$

identité, p. 33, § 2.30

image directe d'une $\mathcal{V}$-catégorie, p. $199, \S$ C.7

inclusion rigide ordonnée, p. 40, § 3.16

joint

$\infty$-catégorique, p. $78, \S 6.30$

dual, p. $82, \S 6.37$

$n$-catégorique, p. $92, \S 8.6$

de complexes dirigés augmentés, p. $64, \S 6.7$

catégorique classique, p. $96, \S 8.15$

de complexes de chaînes augmentés, p. $63, \S 6.5$

simplicial, p. 89, § 7.12

limite inductive canonique, p. $49, \S 5.1$

morphisme d'évaluation dans une catégorie monoïdale fermée à droite,

p. $200, \S$ C. 10 
morphisme d'oubli associé à une tranche, p. $121, \S 10.2 .5$

morphisme d'unité d'une $\mathcal{V}$-catégorie, p. $198, \S$ C.5

morphisme de complexes dirigés augmentés,

p. $21, \S 2.2$

prérigide, p. $35, \S 3.2$

rigide, p. $35, \S 3.2$

morphisme de composition d'une

$\mathcal{V}$-catégorie, p. $198, \S$ C.5

nerf cellulaire, p. $45, \S 4.8$

objet

d'une $\mathcal{V}$-catégorie, p. 197, § C.5

d'une $\mathcal{V}$-sesquicatégorie, p. 195, § C.1

objet de morphismes

d'une $\mathcal{V}$-catégorie, p. 198, § C.5

d'une $\mathcal{V}$-sesquicatégorie, p. 195, § C.1

prétransformation oplax, p. 17, § 1.9

produit tensoriel

$\infty$-catégorique, p. $160, \S$ A.16

$n$-catégorique, p. 164, § A.25

de Gray, p. 160, § A.16

schéma de composition globulaire,

p. $44, \S 4.2$

sesquicatégorie enrichie, p. 195, § C.1

somme globulaire, p. $44, \S 4.2$

sous- $\infty$-catégorie de Gray gauche pleine, p. $204, \S$ C.20

sous- $\infty$-catégorie de Gray pleine,

p. $204, \S$ C.20

sous- $\mathcal{V}$-catégorie gauche pleine,

p. $202, \S$ C.13

sous- $\mathcal{V}$-catégorie pleine, p. 202, § C.13

sous-catégorie dense, p. $49, \S 5.1$

sous-monoïde de positivité, p. 21 , 2.2

support d'un élément d'un groupe abélien

libre, p. $24, \S 2.7$ suspension

d'un complexe de chaînes augmenté, p. $62, \S 6.3$

d'un complexe dirigé augmenté, p. $63, \S 6.6$

d'une $\infty$-catégorie, p. 192, § B.6.5

système

de Steiner, p. 37, § 3.7

connexe, p. $37, \S 3.7$

de Steiner fort, p. 37, § 3.7

connexe, p. $37, \S 3.7$

prérigide, p. $36, \S 3.5$

rigide, p. $36, \S 3.5$

séparant, p. 38 , 33.11

tranche

$\infty$-catégorique

au-dessous, p. $79, \S 6.31$

au-dessus, p. $83, \S 6.37$

catégorique classique, p. $97, \S 8.15$

pour les complexes dirigés augmentés, p. $111, \S 10.1 .1$

transformation

lax, p. $18, \S 1.12$

oplax, p. 18, § 1.9

au-dessus d'une $\infty$-catégorie, p. $19, \S 1.17$

identité, p. 19, § 1.15

transposé

d'un $\mathcal{V}$-foncteur, p. 202, § C.14

d'un $\mathcal{V}$-foncteur gauche, p. 202, § C.14

transposée

d'une $\mathcal{V}$-catégorie, p. 202, § C.14

d'une $\mathcal{V}$-catégorie gauche, p. 202, § C.14

unité d'une cellule

d'une $\infty$-catégorie, p. $13, \S 1.1$

d'une $\infty$-sesquicatégorie, p. 196, § C.3 